\title{
WestVirginiaUniversity
}

THE RESEARCH REPOSITORY @ WVU

Graduate Theses, Dissertations, and Problem Reports

2006

\section{Web-based forensic information management system}

\author{
Parmjit Singh \\ West Virginia University
}

Follow this and additional works at: https://researchrepository.wvu.edu/etd

\section{Recommended Citation}

Singh, Parmjit, "Web-based forensic information management system" (2006). Graduate Theses, Dissertations, and Problem Reports. 2382.

https://researchrepository.wvu.edu/etd/2382

This Thesis is protected by copyright and/or related rights. It has been brought to you by the The Research Repository @ WVU with permission from the rights-holder(s). You are free to use this Thesis in any way that is permitted by the copyright and related rights legislation that applies to your use. For other uses you must obtain permission from the rights-holder(s) directly, unless additional rights are indicated by a Creative Commons license in the record and/ or on the work itself. This Thesis has been accepted for inclusion in WVU Graduate Theses, Dissertations, and Problem Reports collection by an authorized administrator of The Research Repository @ WVU. For more information, please contact researchrepository@mail.wvu.edu. 


\title{
WEB BASED FORENSIC INFORMATION MANAGEMENT SYSTEM
}

\author{
Parmjit Singh \\ Thesis Submitted to \\ The College of Engineering and Mineral Resources \\ at West Virginia University \\ in partial fulfillment of the requirements \\ for the degree of \\ Master of Science \\ in \\ Industrial Engineering
}

Dr. Rashpal S. Ahluwalia (Chair)

Dr. Warren R. Myers

Captain Ted Smith

Department of Industrial and Management Systems Engineering

Morgantown, West Virginia

2006

Keywords: Forensics, Information Management, .NET, Crime Lab, Crime Database 


\section{ABSTRACT \\ WEB BASED FORENSIC INFORMATION MANAGEMENT SYSTEM \\ Parmjit Singh}

The focus of this work was on the design and implementation of a web based forensic information management system for the West Virginia State Police Forensics Laboratory (WVSPFL). The software tool enables law enforcement agents to submit crime information to the Forensic Laboratory via a secure Internet connection. The tool also enables each of the nine units of WVSPFL to process the information electronically. Online electronic forms were created to mirror the existing paper based forms, making the transition easier. The existing processes were standardized and streamlined, there by minimizing information inconsistency. The crime information once gathered is automatically uploaded to a database. It can then be viewed and queried by any authorized law enforcement officer at anytime from anywhere. The software tool was implemented in ASP.NET with MS SQL Server as the database. The key benefits of the system are streamlining of information flow, around the clock availability of case information, and sharing of information between the various law enforcement agencies. 


\section{ACKNOWLEDGEMENTS}

I would like to thank Dr. Rashpal S. Ahluwalia and Dr. Warren Myers for their direction, assistance, and guidance. In particular, Dr. Ahluwalia's recommendations and suggestions have been invaluable for the project and software improvement. I also wish to thank Captain Ted Smith, Director, West Virginia State Police Laboratory, for providing information on the forensic laboratory and his continuous support throughout this project.

I also like to thank Mr. Max Houck for his support and encouragement. This project was supported in part by a grant 2001-RC-CX-K003, awarded by the National

Institute of Justice, Office of Justice Programs. The opinions, findings, and conclusions expressed in this thesis are those of the author and do not necessarily reflect the views of the Department of Justice.

Special thanks should be given to my student colleagues who helped me in many ways. Finally, words alone cannot express the thanks I owe to my parents and Nilam Kumari, my wife, for their encouragement and assistance. I also like to thank department of Industrial and Management Systems Engineering (IMSE), West Virginia University for giving me a chance to pursue my higher education. 


\section{TABLE OF CONTENTS}

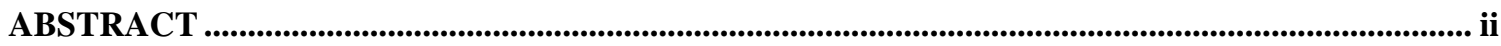

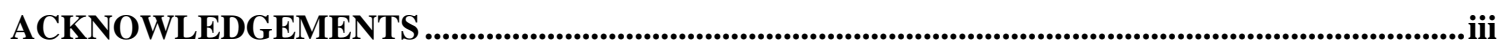

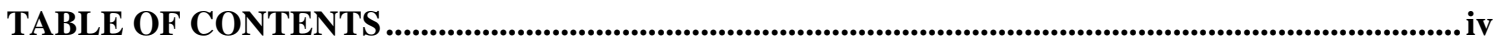

LIST OF FIGURES.................................................................................................................................... vi

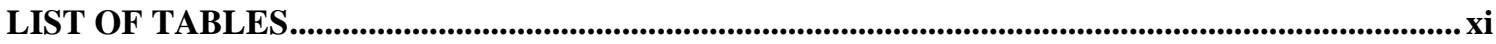

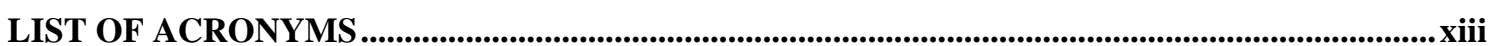

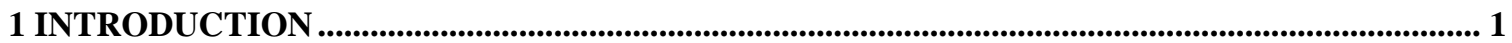

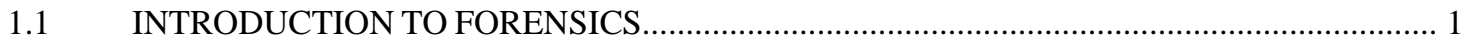

1.2 INTRODUCTION TO INFORMATION MANAGEMENT SYSTEM …................................. 1

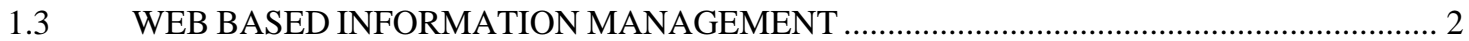

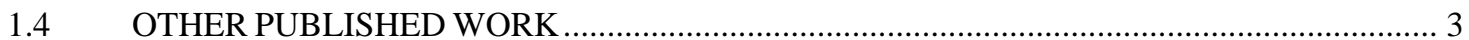

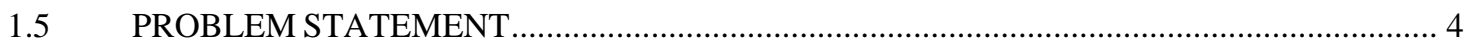

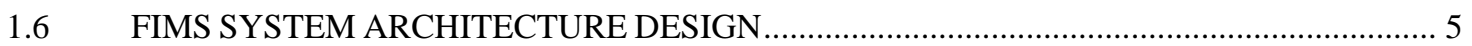

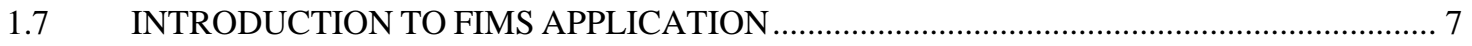

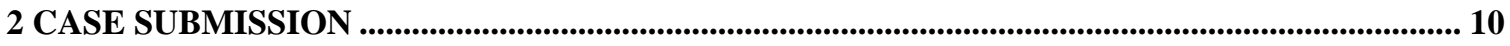

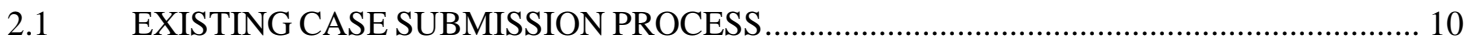

2.2 RE-ENGINEERING OF THE CASE SUBMISSION PROCESS …........................................ 13

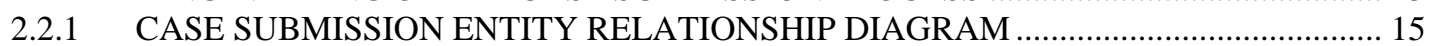

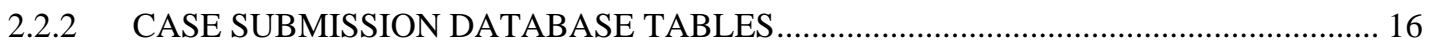

2.2.3 WORKING WITH THE CASE SUBMISSION MODULE............................................. 23

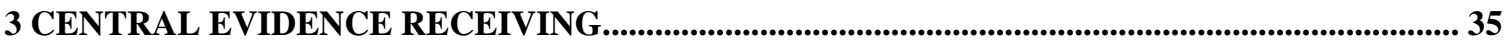

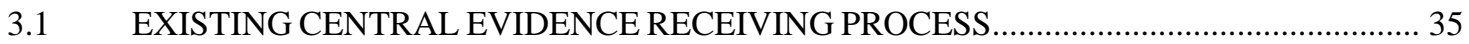

3.2 RE-ENGINEERING THE CENTRAL EVIDENCE RECEIVING PROCESS ......................... 38

3.2.1 CENTRAL EVIDENCE RECEIVING ENTITY RELATIONSHIP DIAGRAM.....................40

3.2.2 CENTRAL EVIDENCE RECEIVING DATABASE TABLES ....................................... 41

3.2.3 WORKING WITH CENTRAL EVIDENCE RECEIVING ............................................... 42

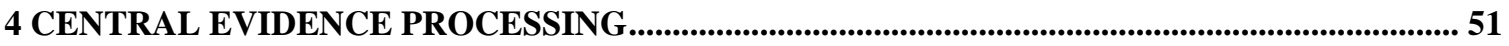

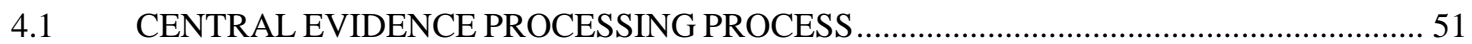

4.1.1 CENTRAL EVIDENCE PROCESSING ENTITY RELATIONSHIP DIAGRAM ............... 53

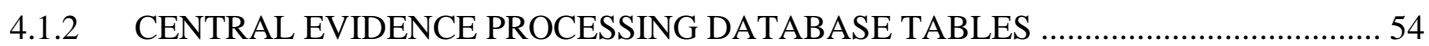

4.1.3 WORKING WITH CENTRAL EVIDENCE PROCESSING ............................................ 56

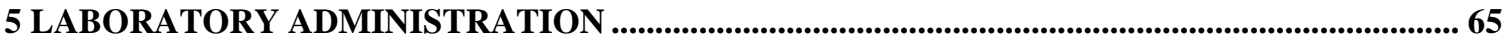

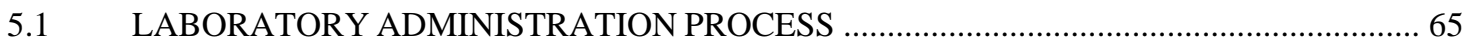

5.1.1 LABORATORY ADMINISTRATION ENTITY RELATIONSHIP DIAGRAM...................... 67

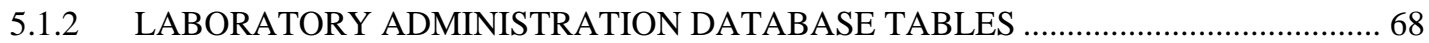

5.1.3 WORKING WITH LABORATORY ADMINISTRATION ............................................. 71

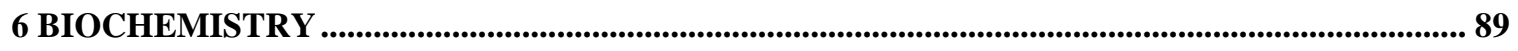

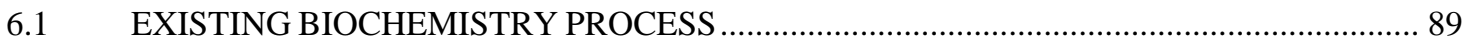

6.2 RE-ENGINEERING THE BIOCHEMISTRY PROCESS ..................................................... 91

6.2.1 BIOCHEMISTRY ENTITY RELATIONSHIP DIAGRAM............................................. 93

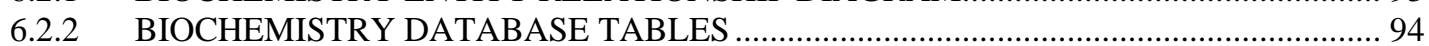

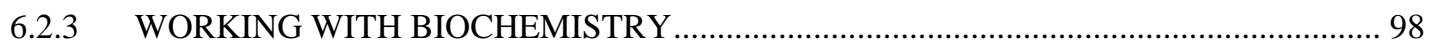

6.2.4 GENERATING ITEM AND SAMPLE REPORTS ………………….............................. 115

7 DRUG IDENTIFICATION ................................................................................................................... 119 


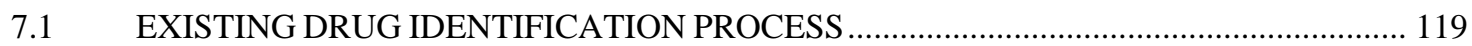

7.2 RE-ENGINEERING THE DRUG IDENTIFICATION PROCESS ........................................ 121

7.2.1 DRUG IDENTIFICATION ENTITY RELATIONSHIP DIAGRAM ................................. 123

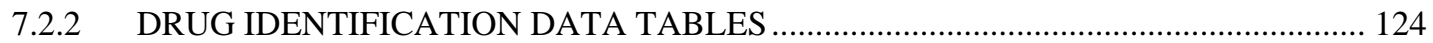

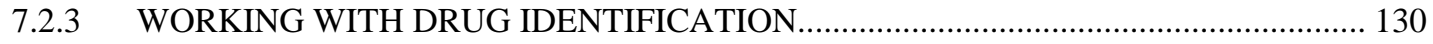

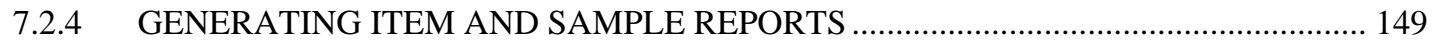

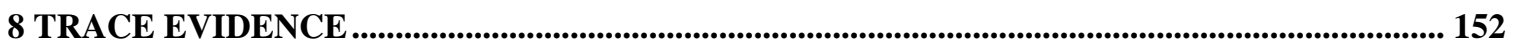

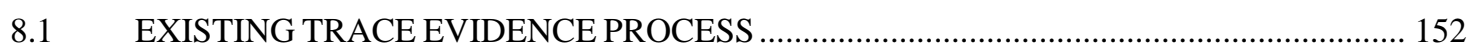

8.2 RE-ENGINEERING THE TRACE EVIDENCE PROCESS............................................... 154

8.2.1 TRACE EVIDENCE ENTITY RELATIONSHIP DIAGRAM ……............................... 156

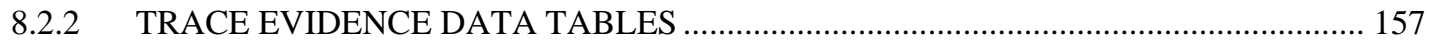

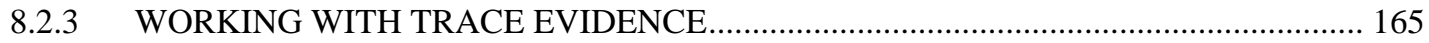

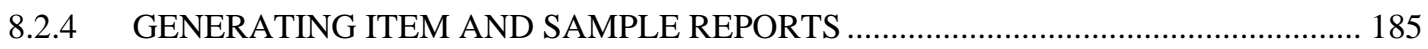

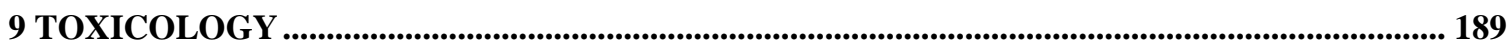

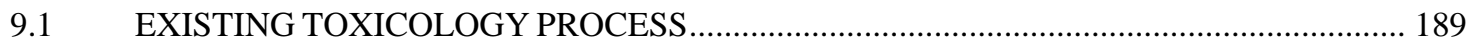

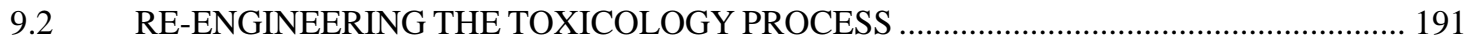

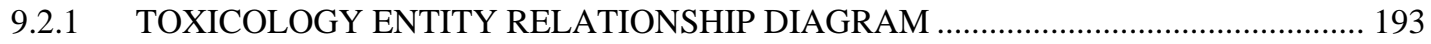

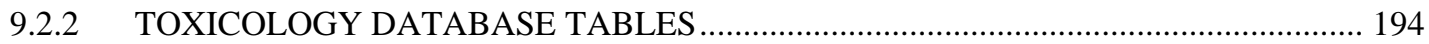

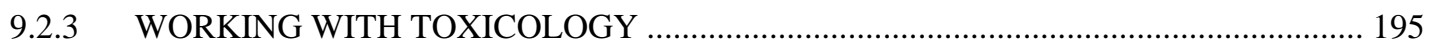

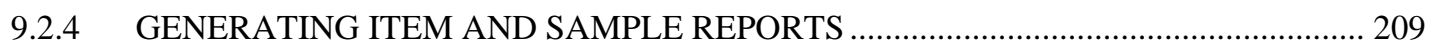

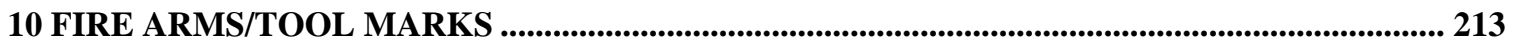

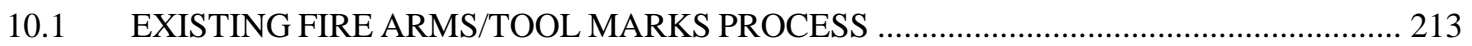

10.2 RE-ENGINEERING THE FIRE ARMS/TOOL MARKS PROCESS ……….......................... 215

10.2.1 FIRE ARMS/TOOL MARKS ENTITY RELATIONSHIP DIAGRAM ….................... 217

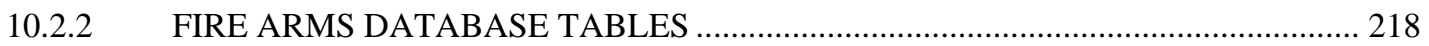

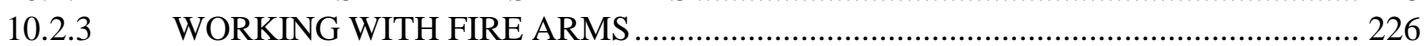

10.2.4 GENERATING ITEM AND SAMPLE REPORTS …............................................ 250

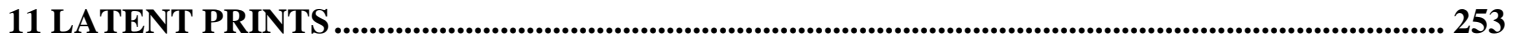

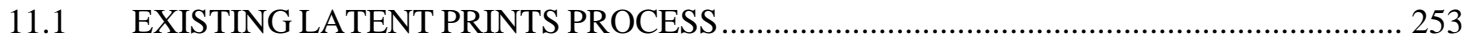

11.2 RE-ENGINEERING THE LATENT PRINTS PROCESS ................................................... 255

11.2.1 LATENT PRINTS ENTITY RELATIONSHIP DIAGRAM ……................................ 257

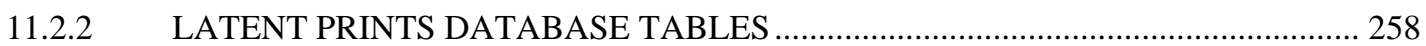

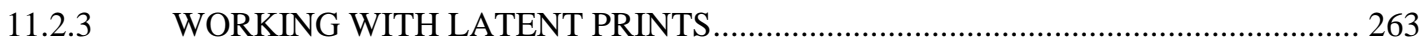

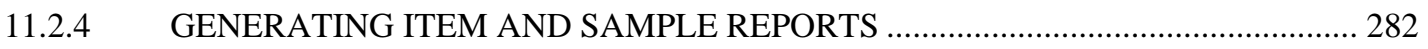

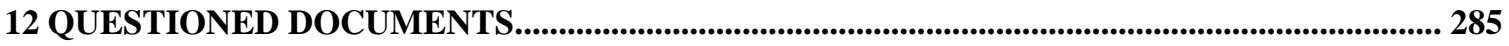

12.1 EXISTING QUESTIONED DOCUMENTS PROCESS ……............................................... 285

12.2 RE-ENGINEERING THE QUESTIONED DOCUMENTS PROCESS................................ 287

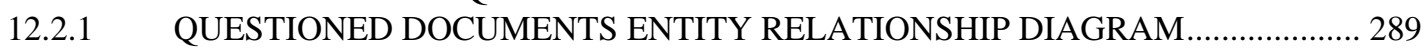

12.2.2 QUESTIONED DOCUMENTS DATABASE TABLES ……………............................. 290

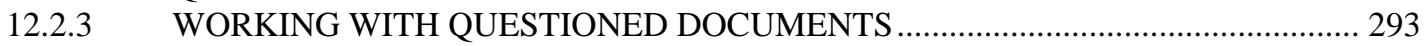

12.2.4 GENERATING ITEM AND SAMPLE REPORTS ……............................................... 309

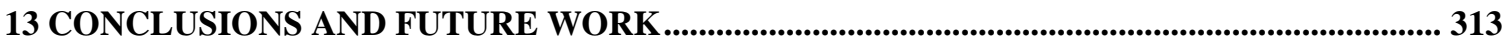

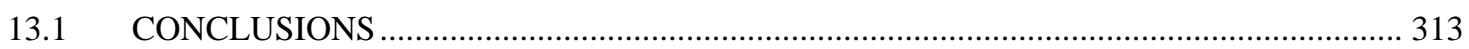

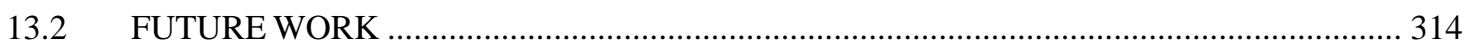

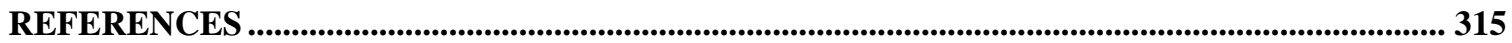




\section{LIST OF FIGURES}

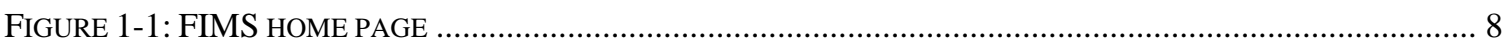

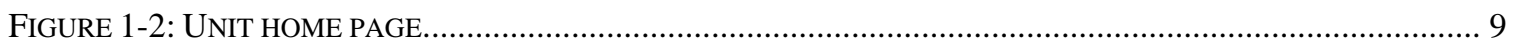

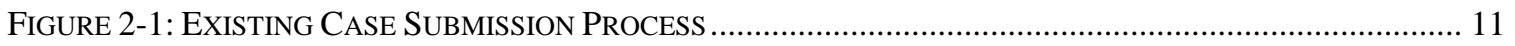

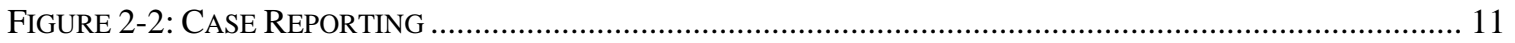

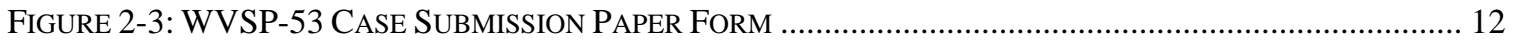

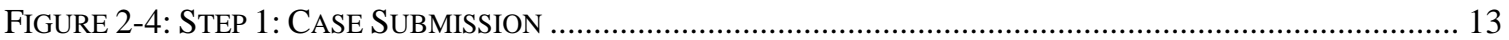

FigURE 2-5: STEP 15 RECEIVE PACKAGE \& FINAL CASE REPORT …......................................................... 14

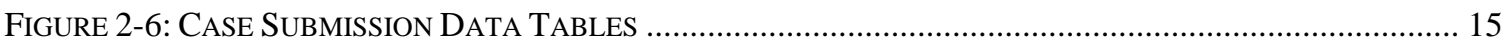

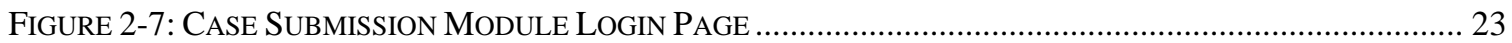

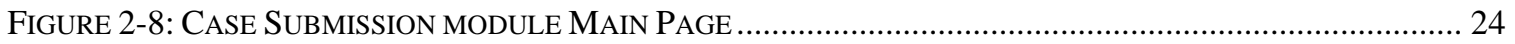

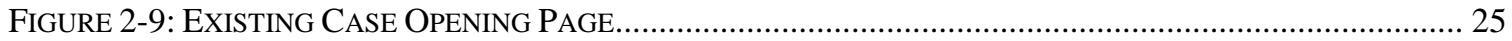

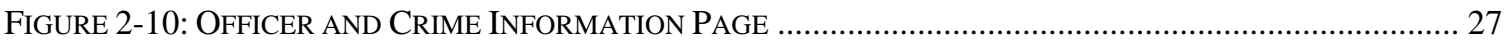

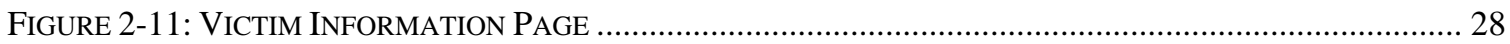

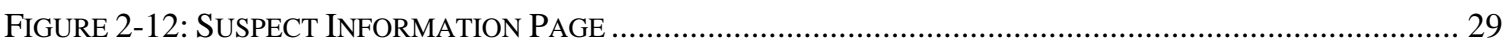

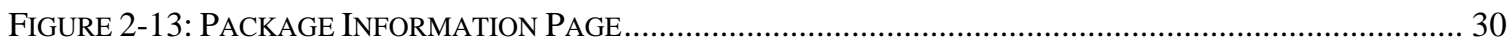

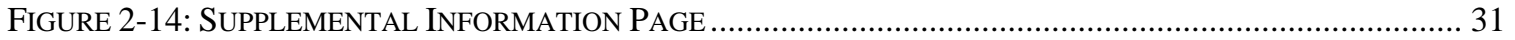

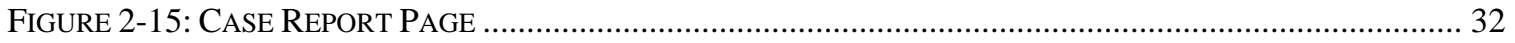

FIGURE 2-16: PDF VERSION OF CASE SUBMISSION REPORT ………........................................................... 33

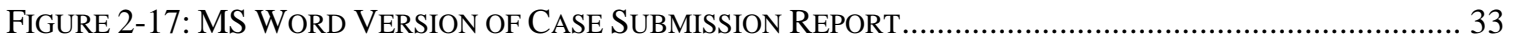

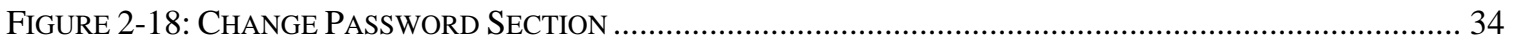

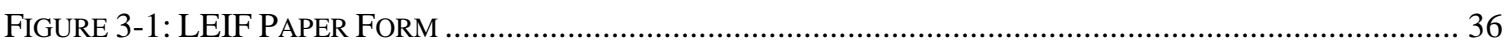

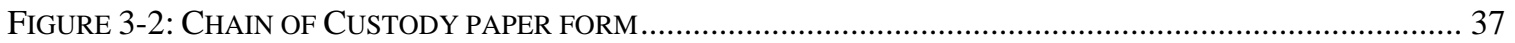

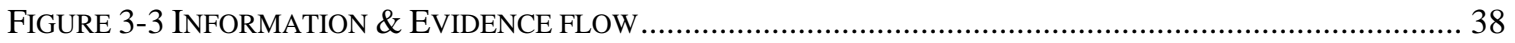

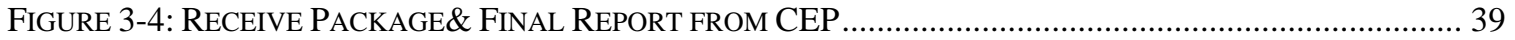

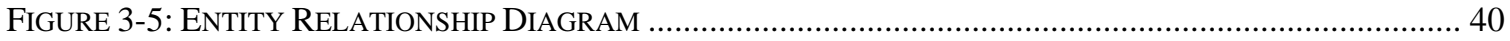

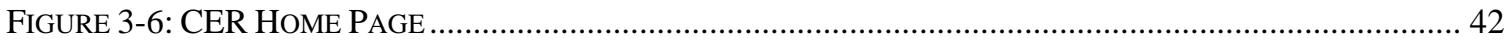

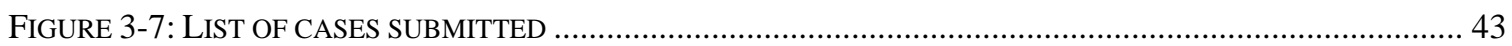

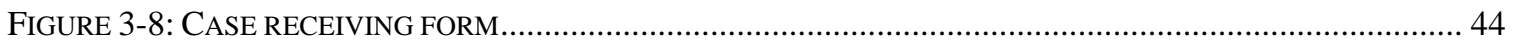

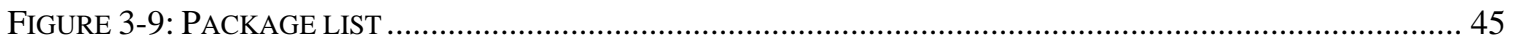

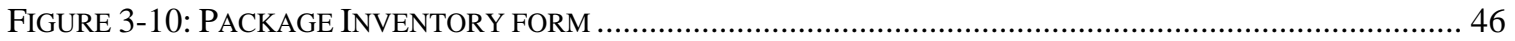

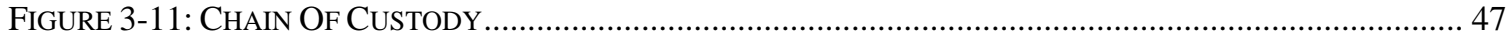

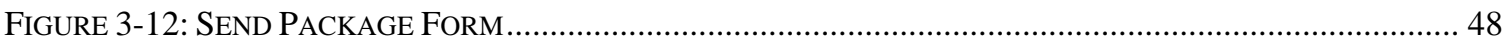

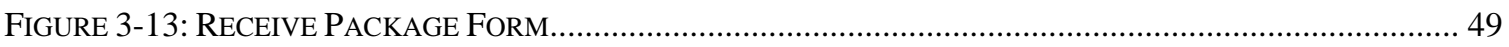

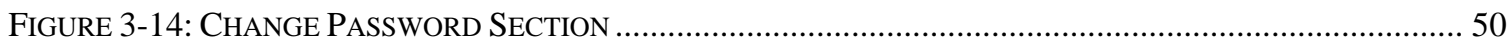

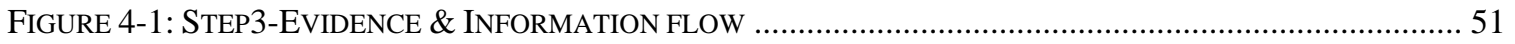

FIGURE 4-2: STEP 13- EVIDENCE \& INFORMATION FLOW ……….............................................................. 52

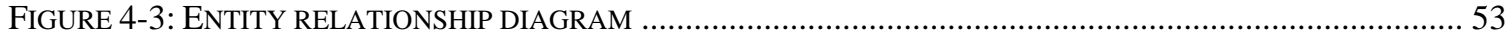

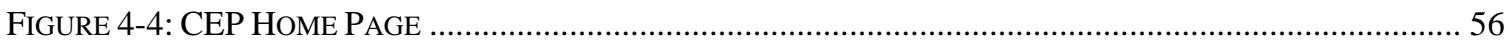

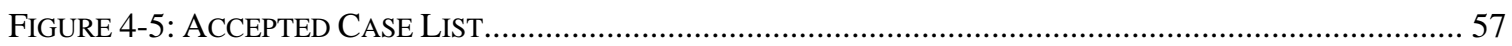

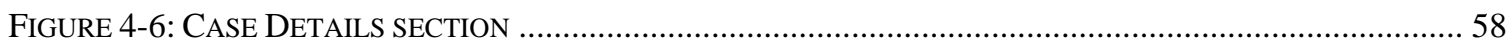

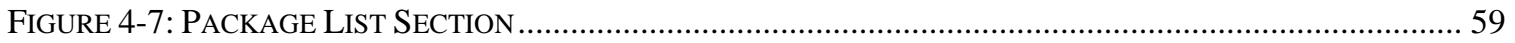

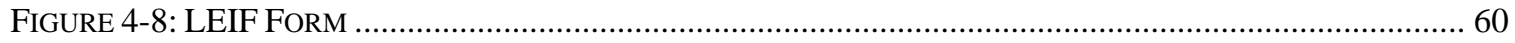

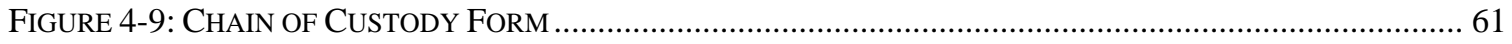

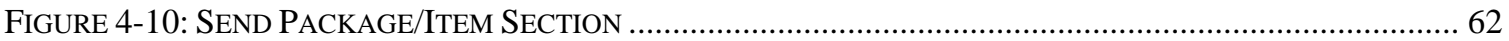

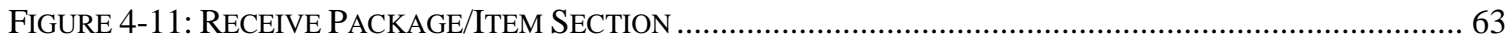

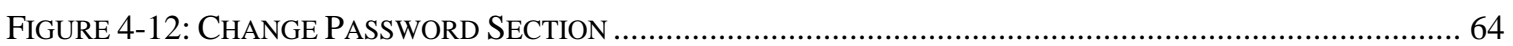

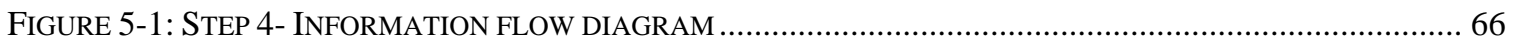

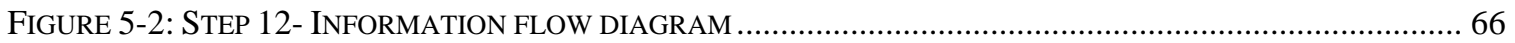

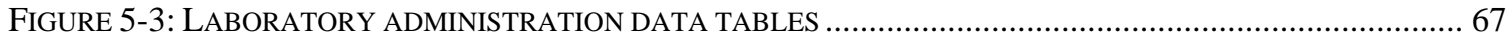

FIGURE 5-4: LABORATORY ADMINISTRATION LOGIN PAGE ...................................................................... 71

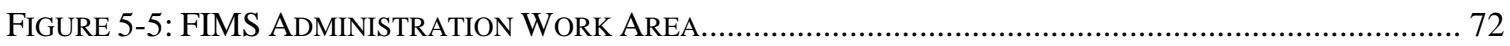

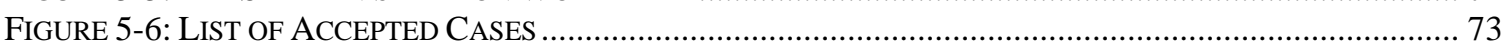

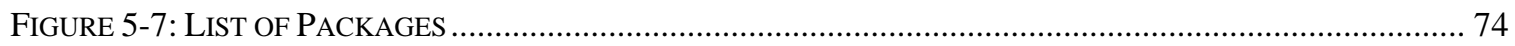

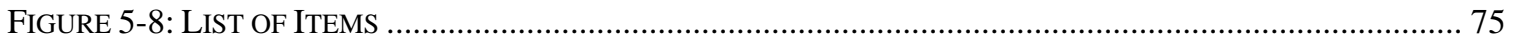




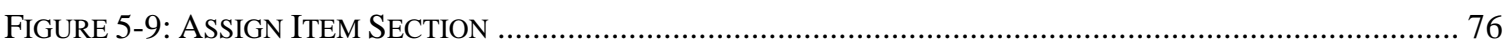

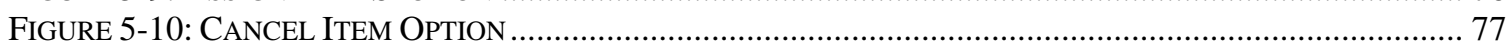

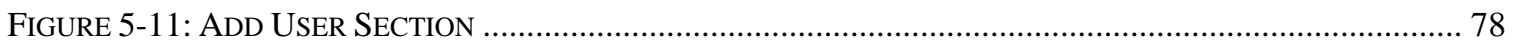

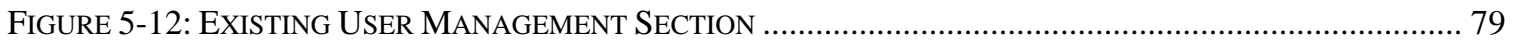

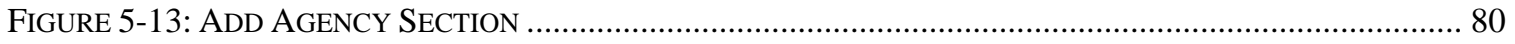

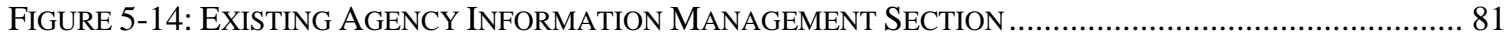

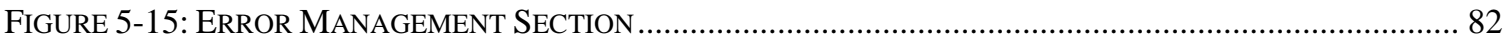

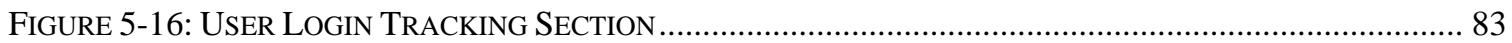

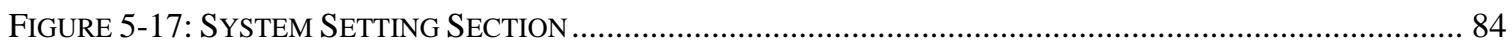

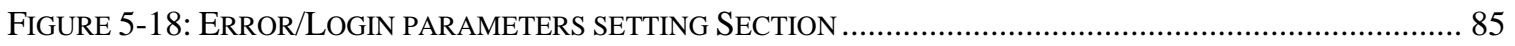

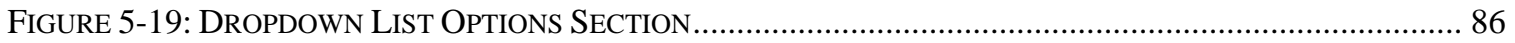

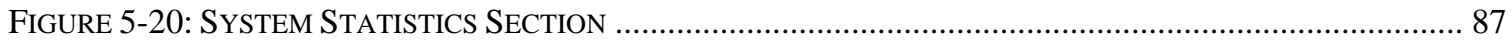

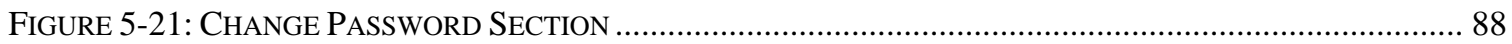

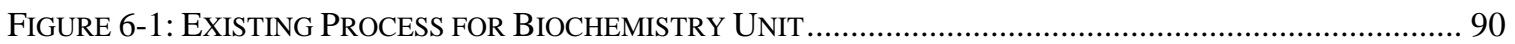

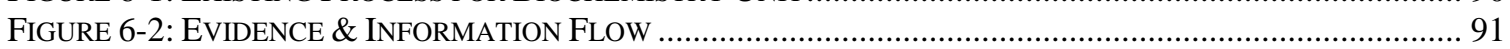

FiguRE 6-3: EvidENCE ITEM PROCESSING \& REPORT GENERATION ........................................................ 92

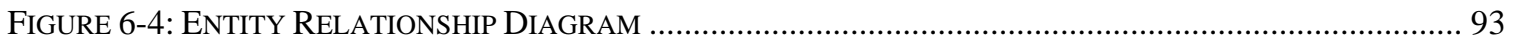

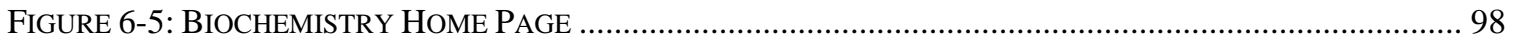

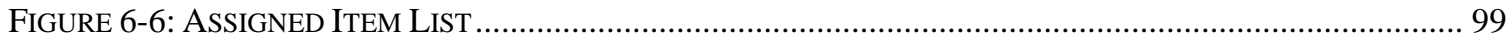

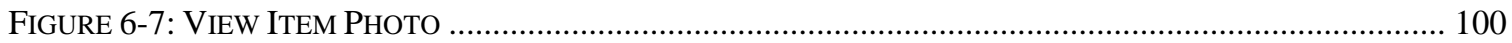

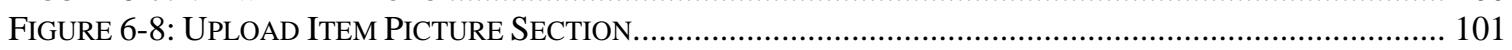

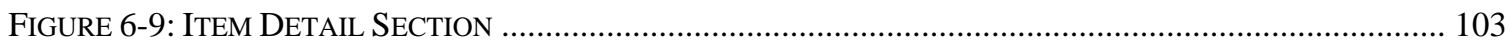

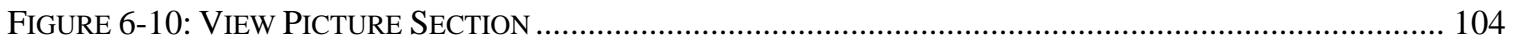

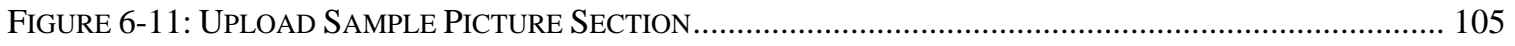

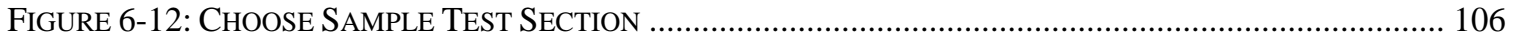

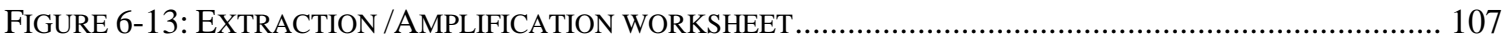

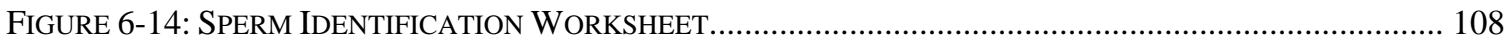

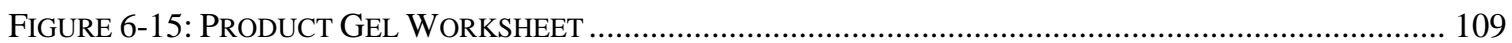

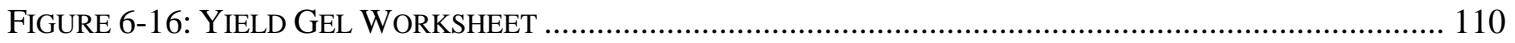

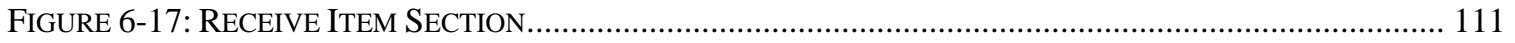

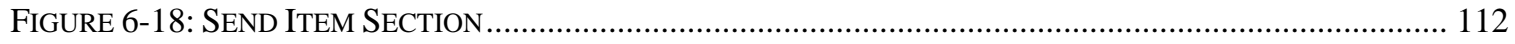

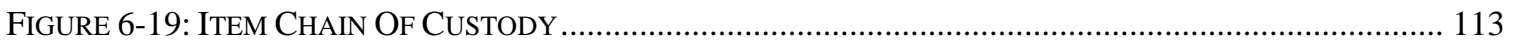

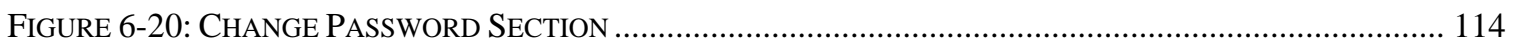

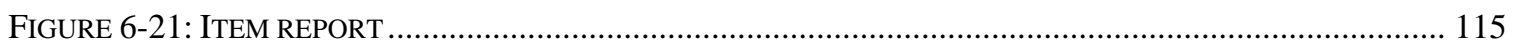

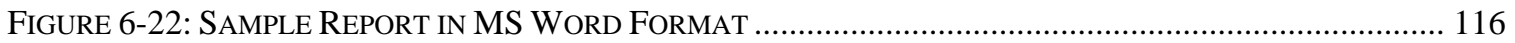

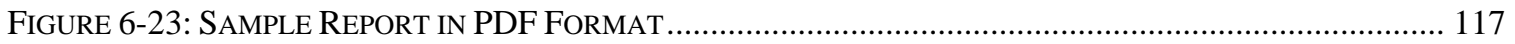

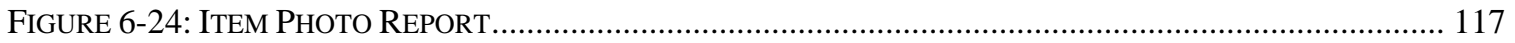

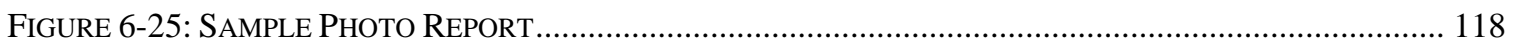

FigURE 7-1: EXISTING PROCESS FOR THE DRUG IDENTIFICATION UNIT .................................................... 120

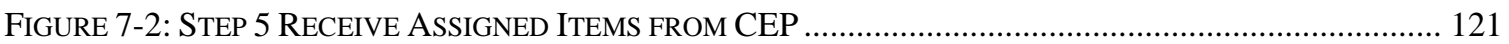

Figure 7-3: Detail Evidence ItEM PRocessing \& RePort GENERATION PROCEDURE ........................... 122

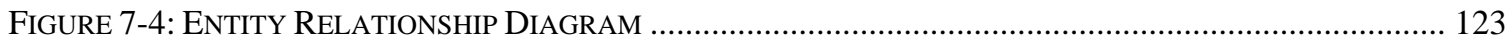

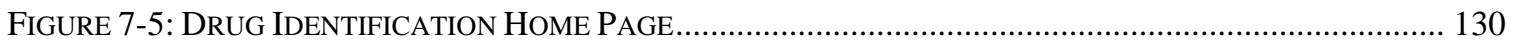

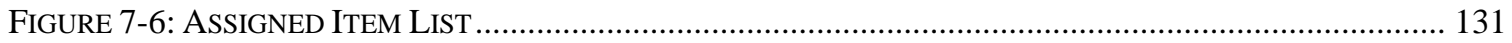

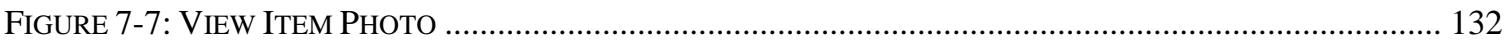

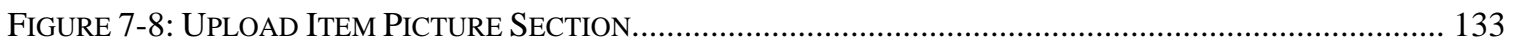

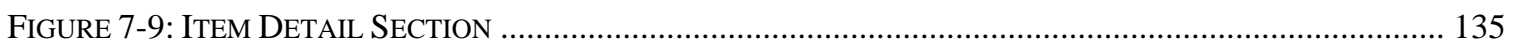

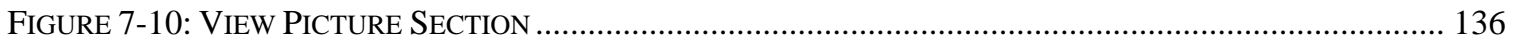

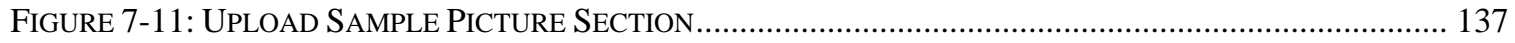

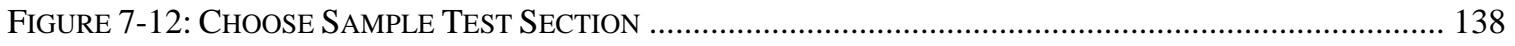

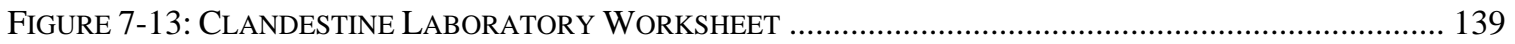

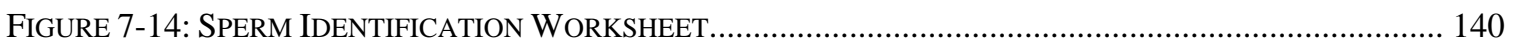

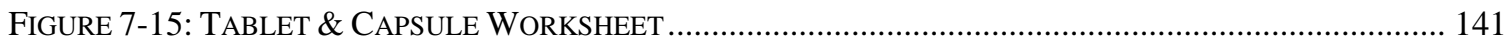

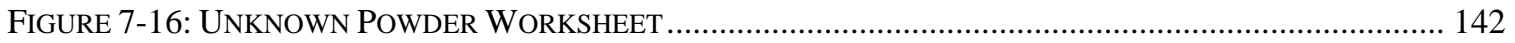

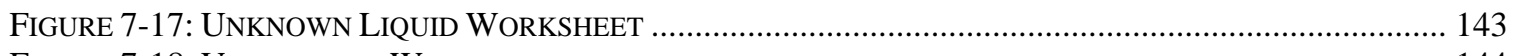

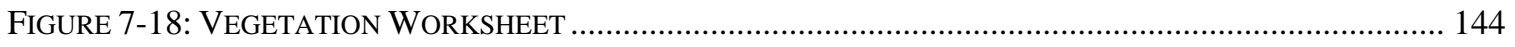




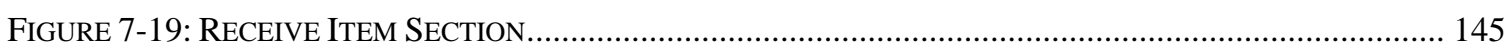

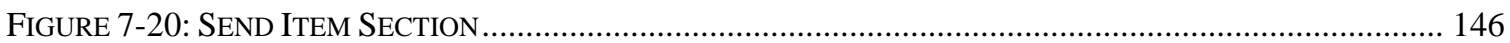

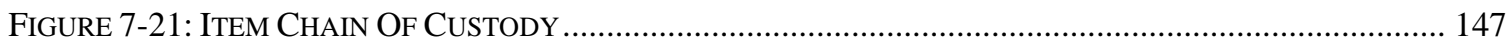

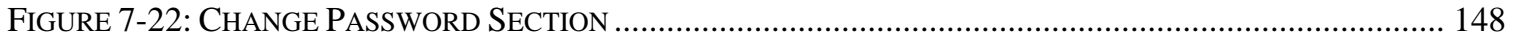

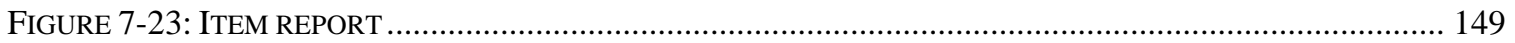

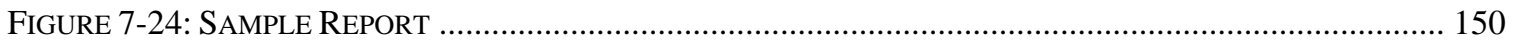

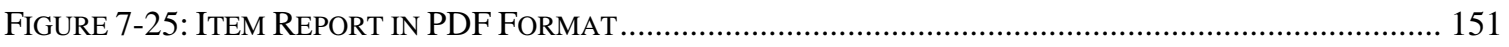

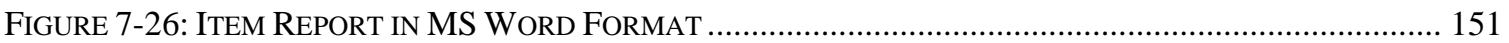

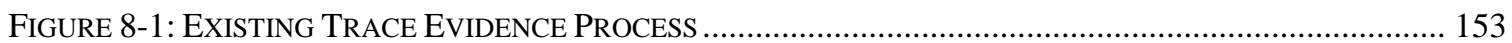

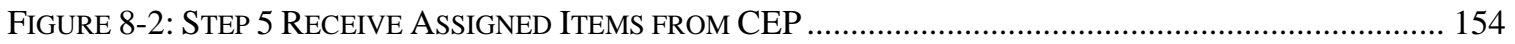

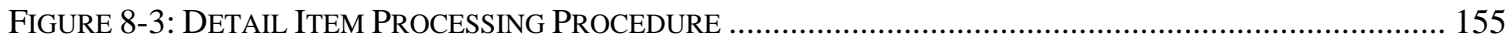

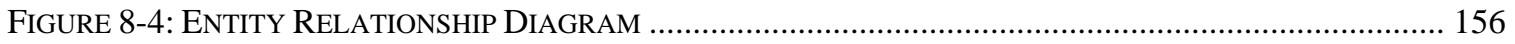

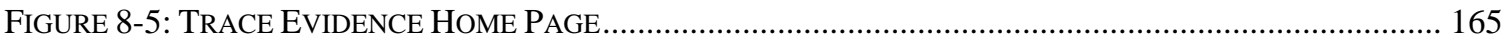

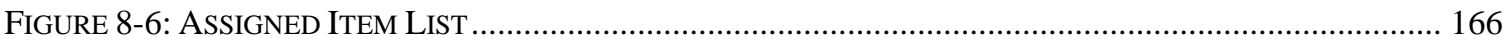

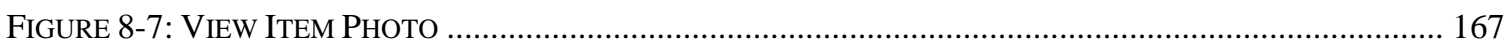

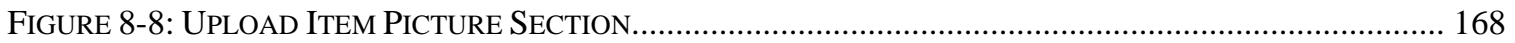

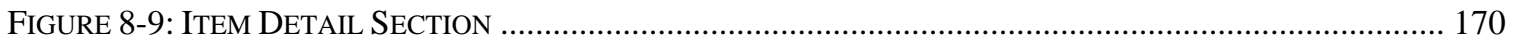

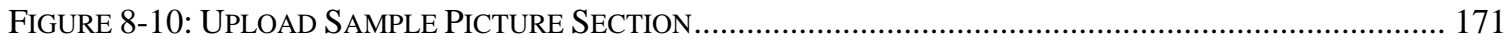

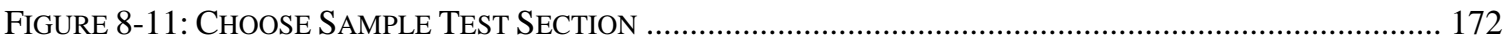

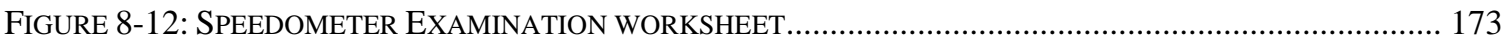

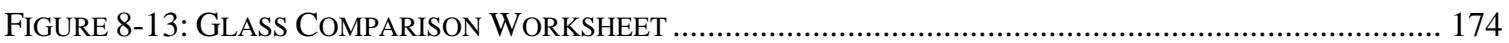

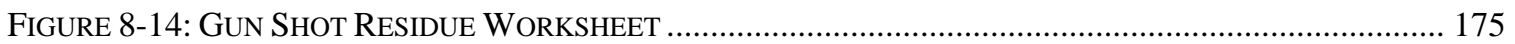

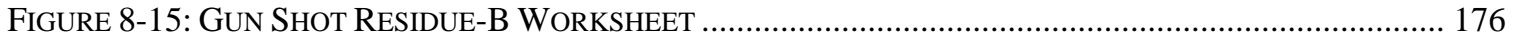

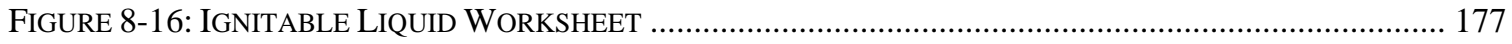

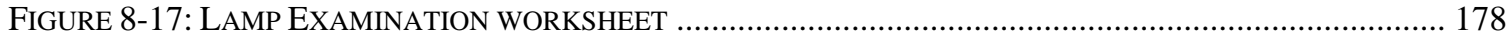

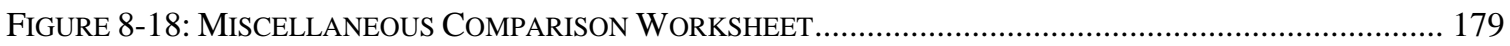

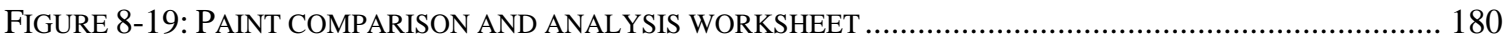

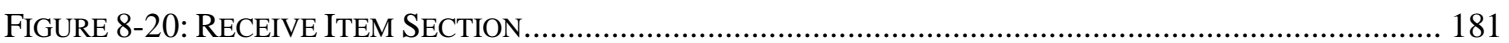

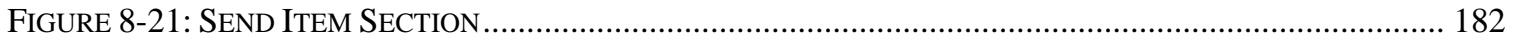

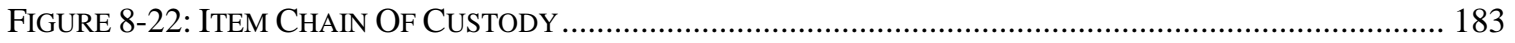

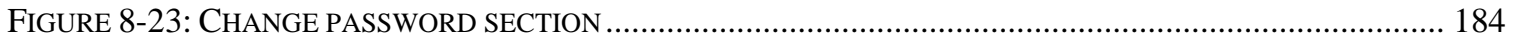

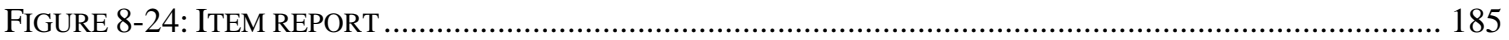

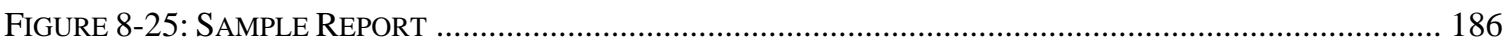

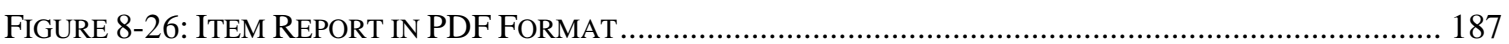

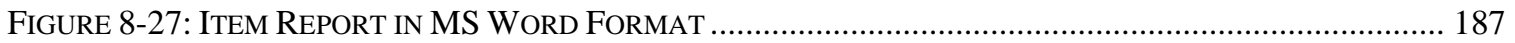

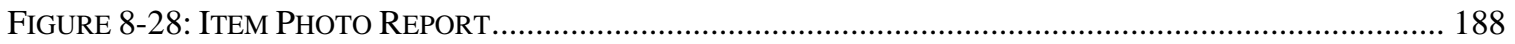

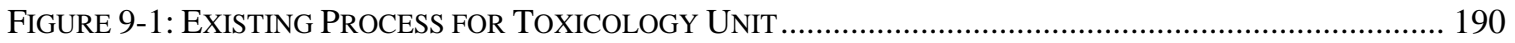

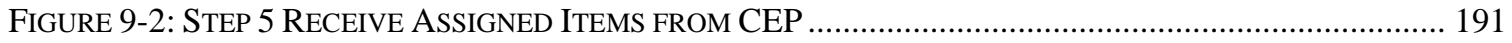

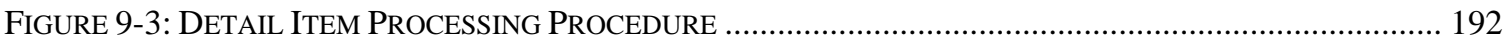

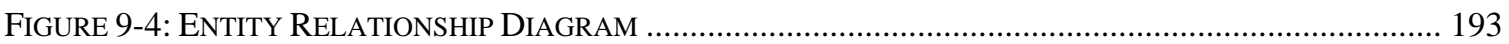

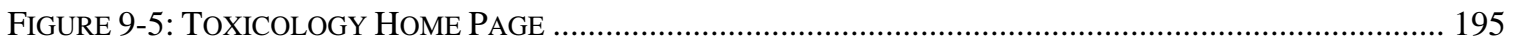

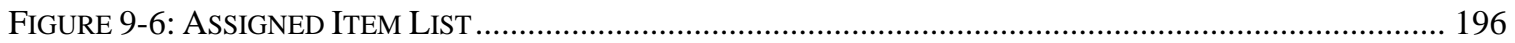

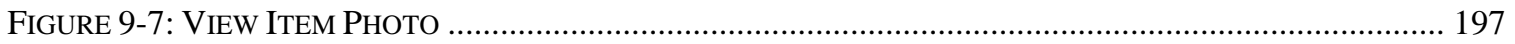

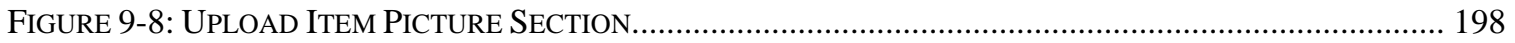

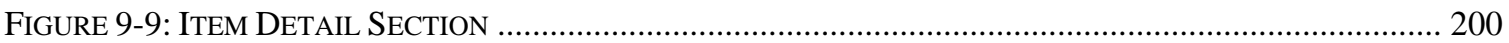

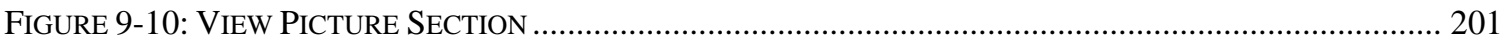

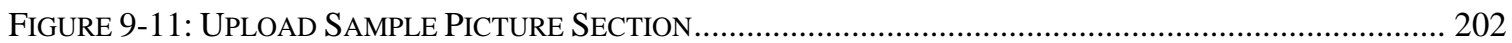

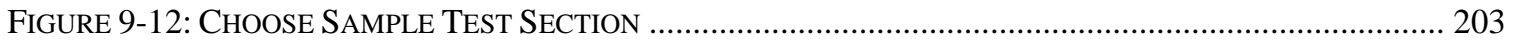

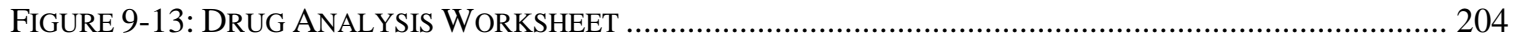

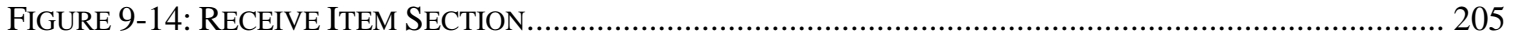

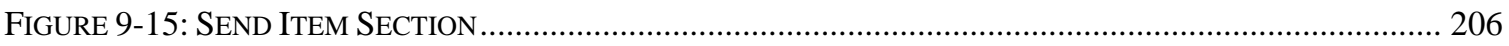

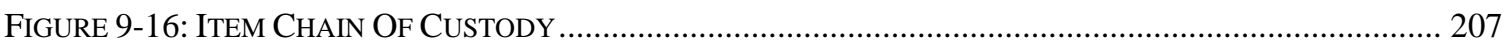

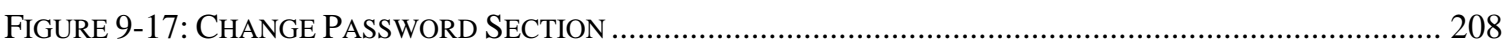

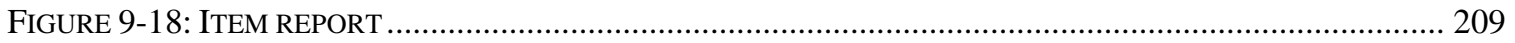

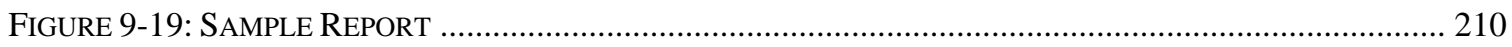

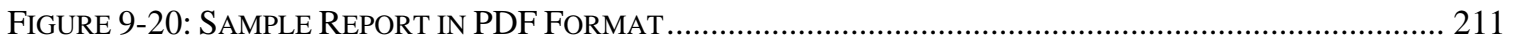




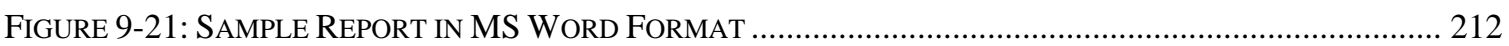

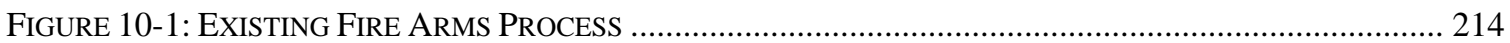

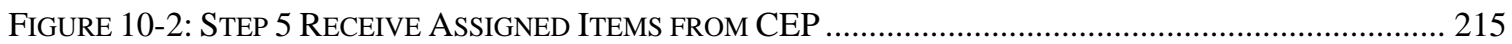

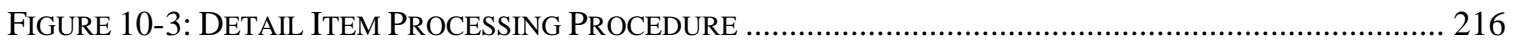

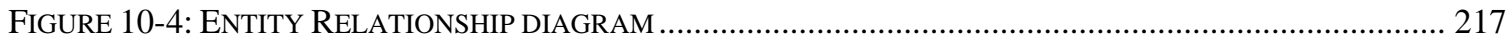

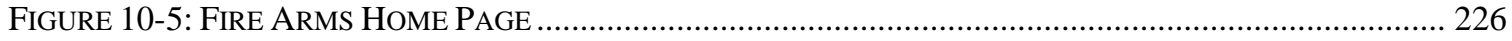

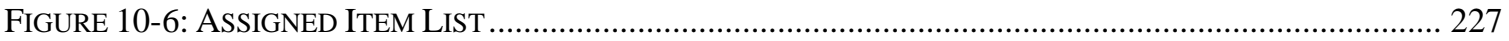

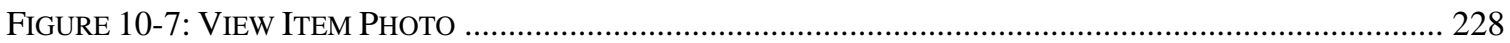

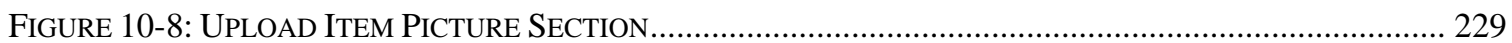

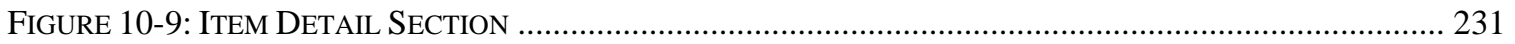

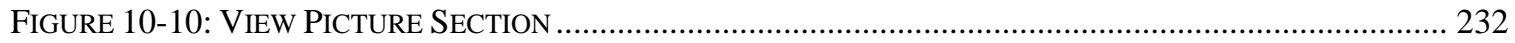

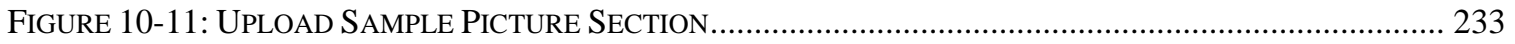

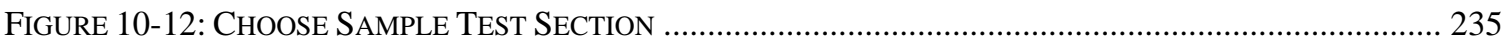

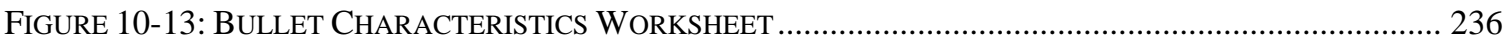

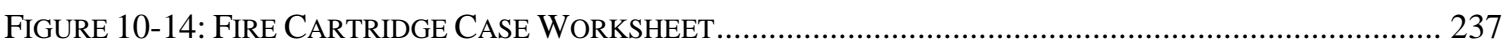

FigURE 10-15: CHEMICAL RESIDUE EXAMINATION WORKSHEET ………................................................ 238

FIGURE 10-16: COMPARISON/EXAMINATION WORKSHEET ……………................................................ 239

FIGURE 10-17: DISTANCE DETERMINATION WORKSHEET ………........................................................... 240

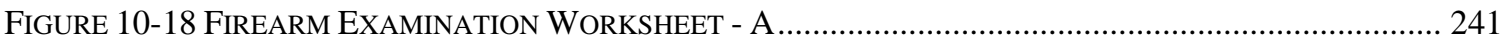

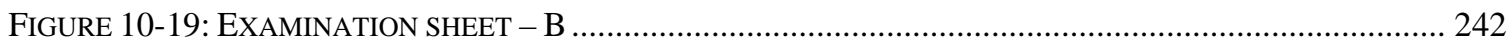

FIGURE 10-20: EXAMINATION SHEET (SHORT FORM) WORKSHEET ........................................................... 243

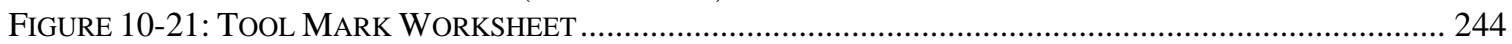

FigURE 10-22: VISUAL/MICROSCOPIC RESIDUE EXAMINATION WORKSHEET ............................................... 245

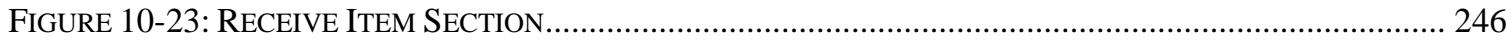

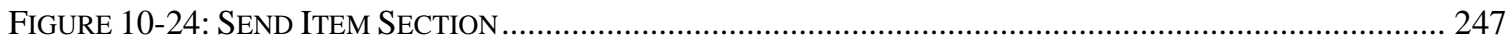

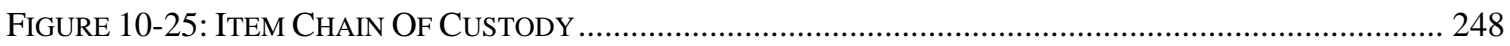

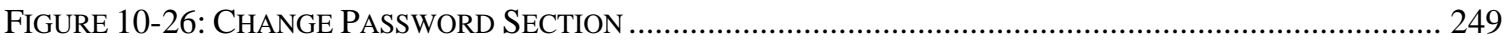

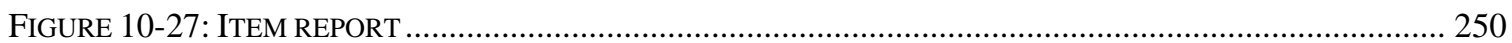

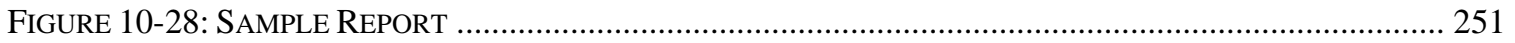

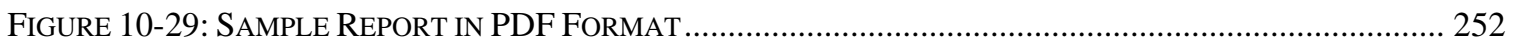

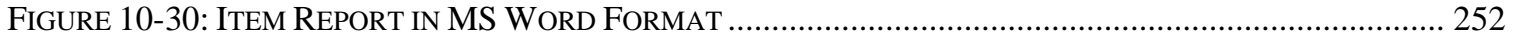

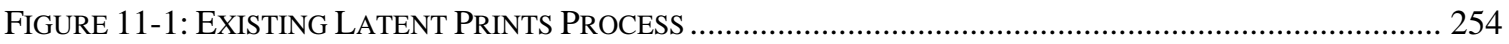

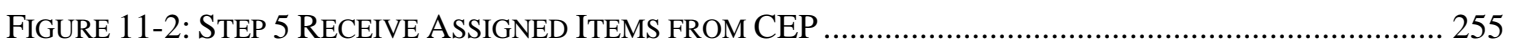

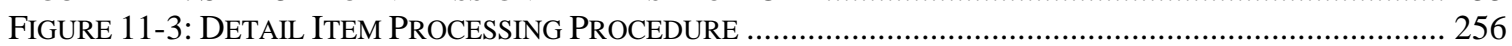

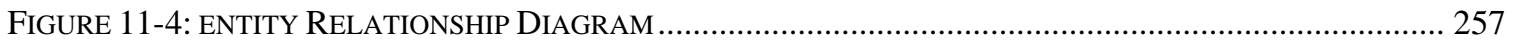

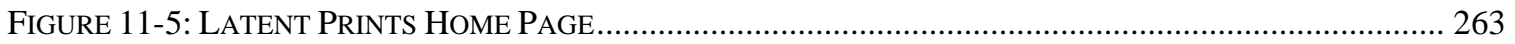

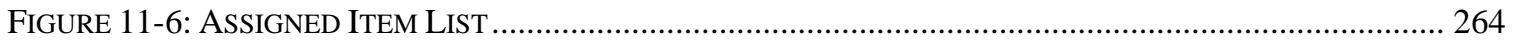

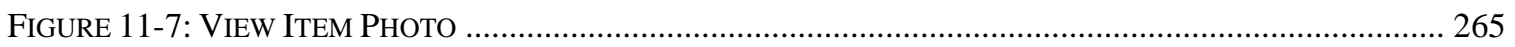

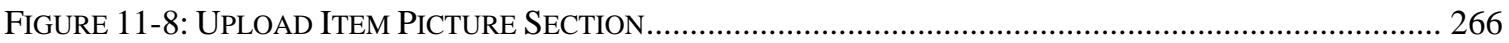

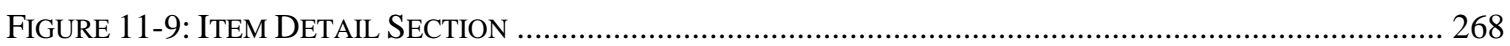

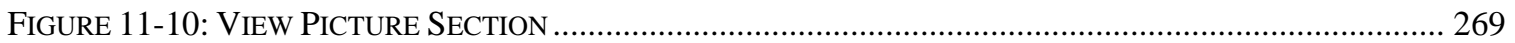

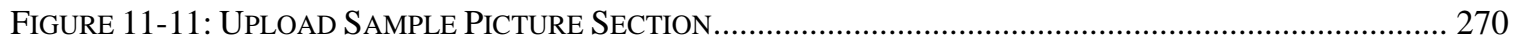

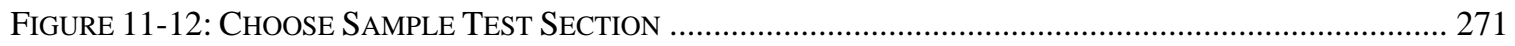

FIGURE 11-13: ACE ANALYSIS \& COMPARISON FOR INKED FINGER PRINTS WORKSHEET ........................... 272

FIGURE 11-14: AFIS \& IAFIS COMPARISON REFERENCE WORKSHEET ..................................................... 273

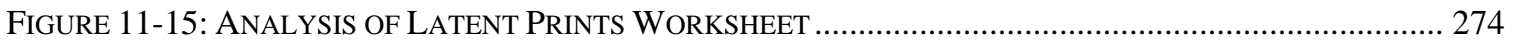

FIGURE 11-16: COMPARISON, EVALUATION, \& VERIFICATION WORKSHEET ………..................................... 275

FIGURE 11-17: ANALYSIS OF EXEMPLAR PRINTS WORKSHEET................................................................. 276

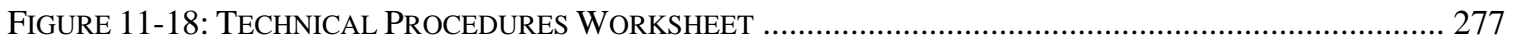

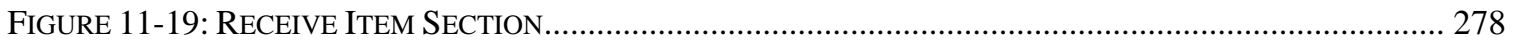

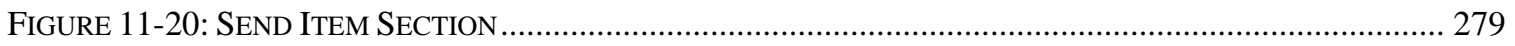

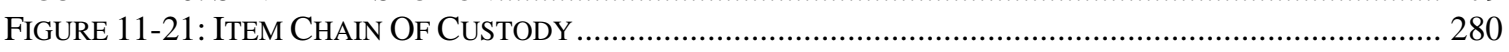

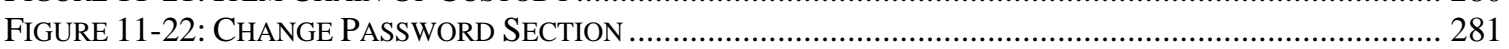

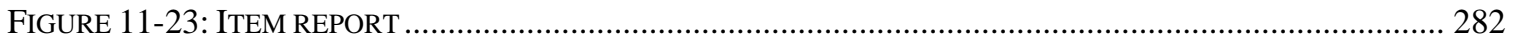

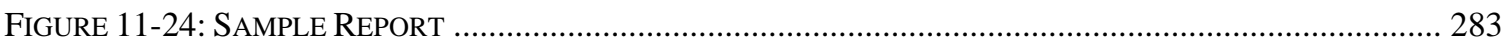

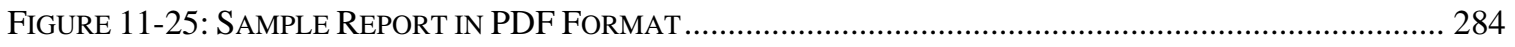




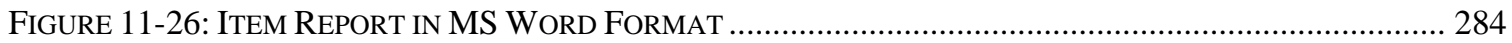

FigURE 12-1: EXISTING PROCESS FOR QUESTIONED DOCUMENTS UNIT …................................................ 286

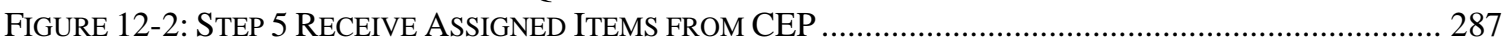

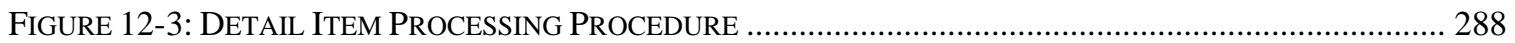

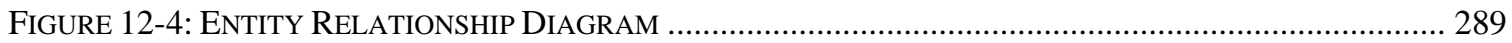

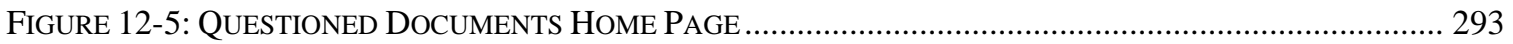

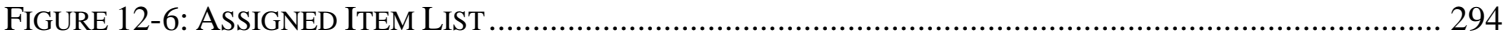

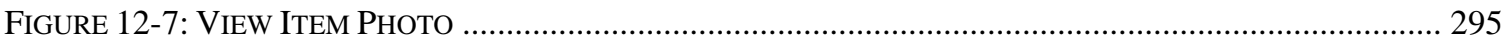

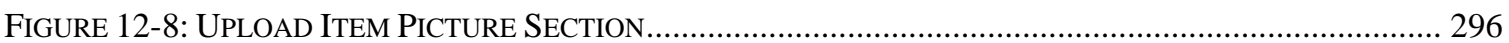

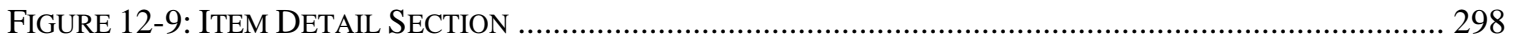

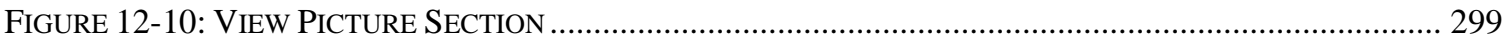

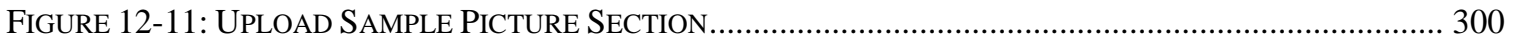

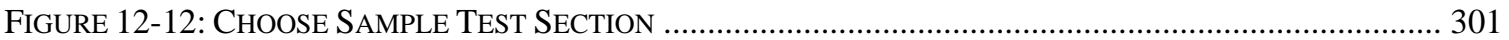

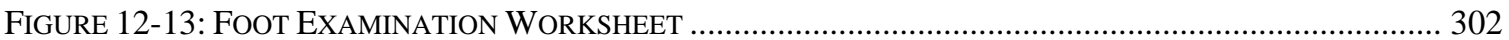

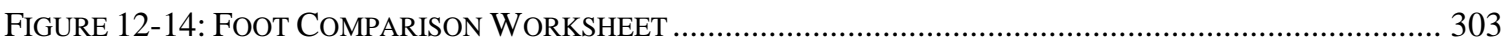

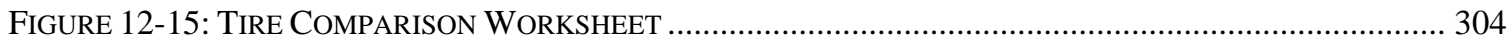

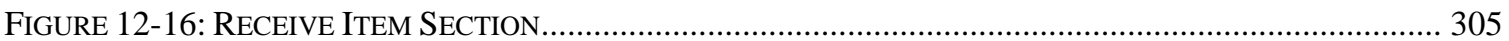

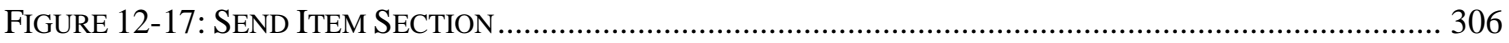

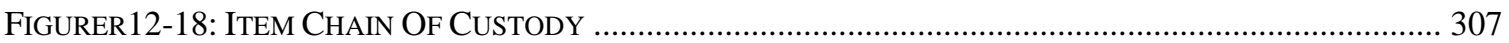

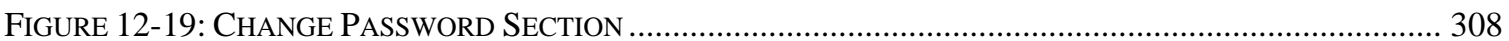

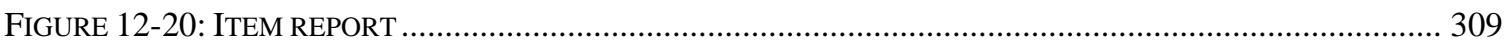

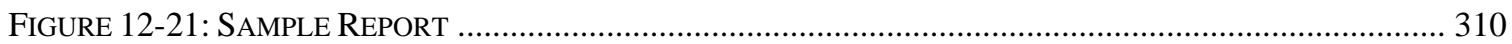

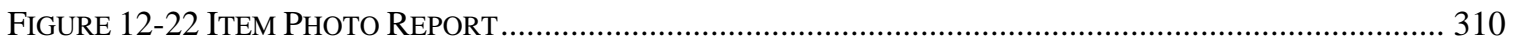

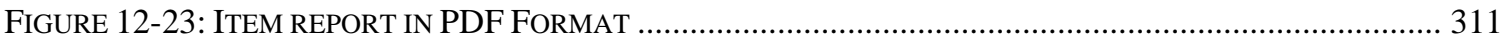

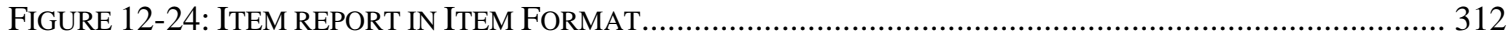




\section{LIST OF TABLES}

TABLE 2-1: OfFICER INFORMATION TABLE .............................................................................................. 17

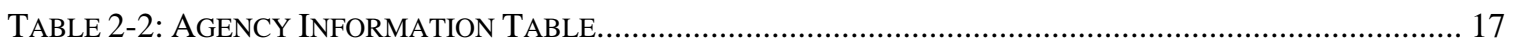

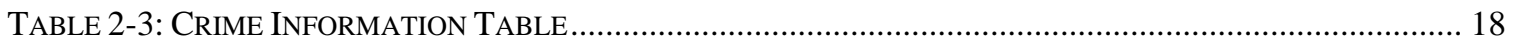

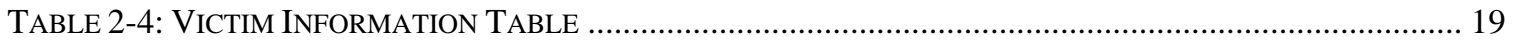

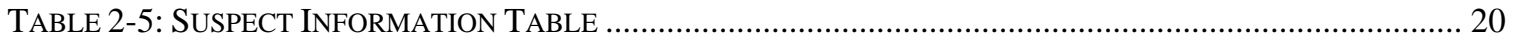

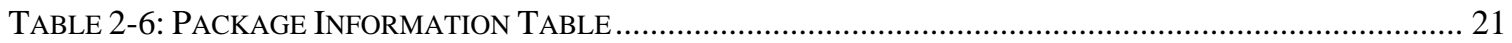

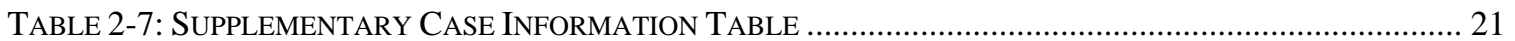

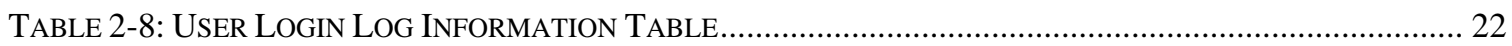

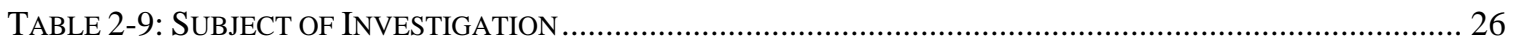

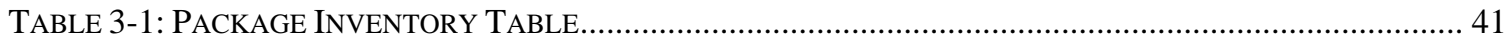

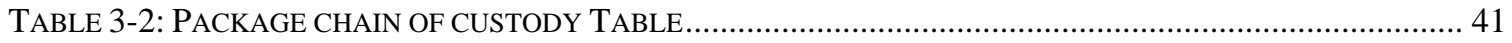

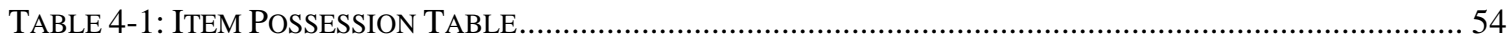

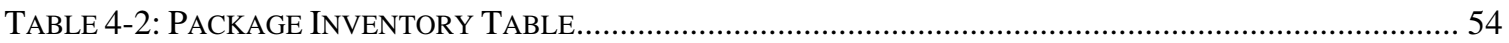

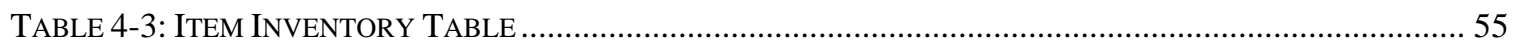

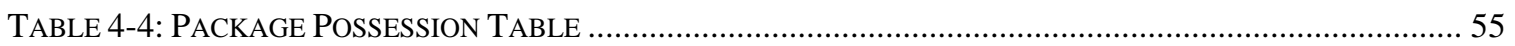

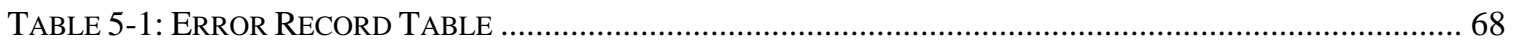

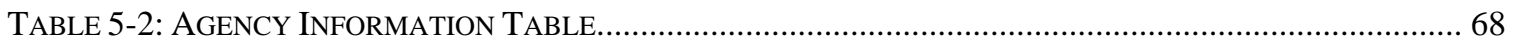

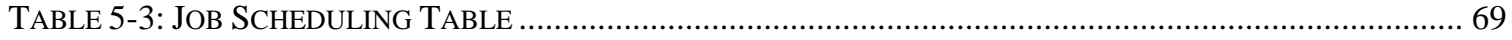

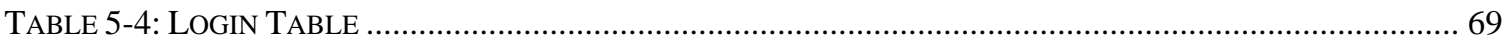

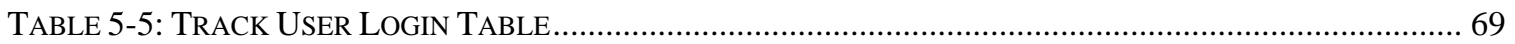

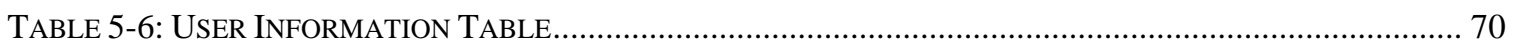

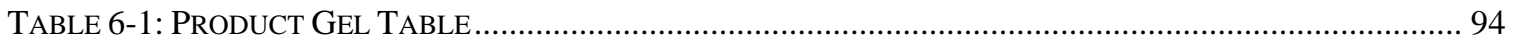

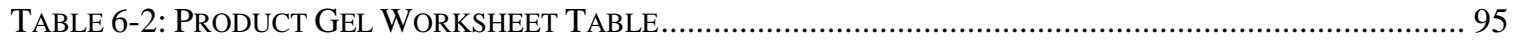

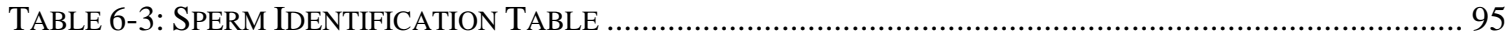

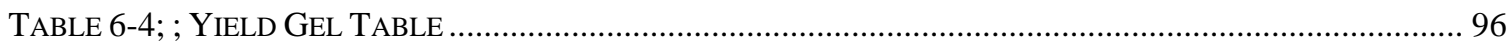

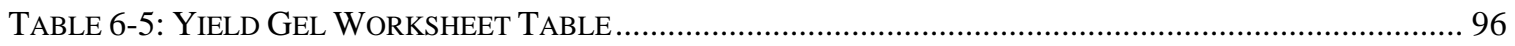

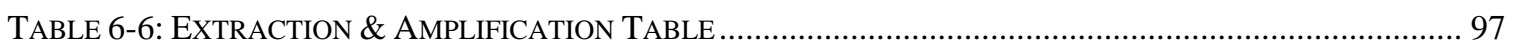

TABLE 6-7: EXTRACTION \& AMPLIFICATION WORKSHEET TABLE .............................................................. 97

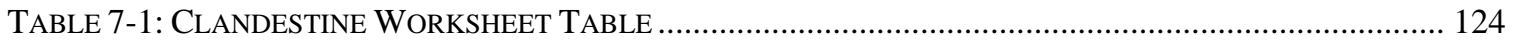

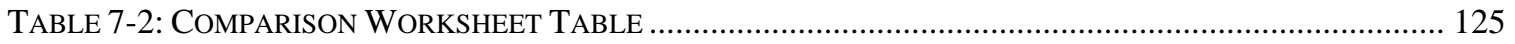

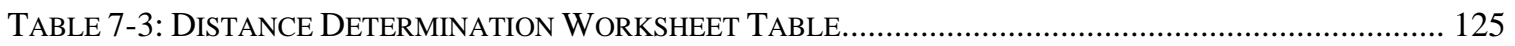

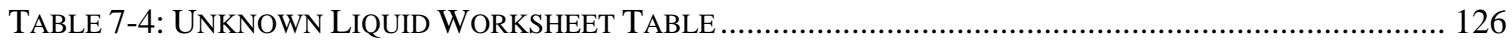

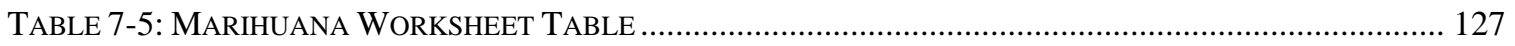

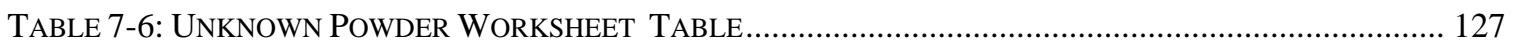

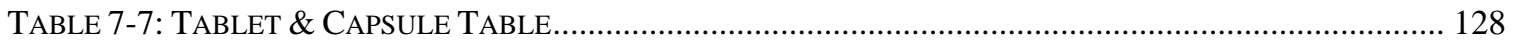

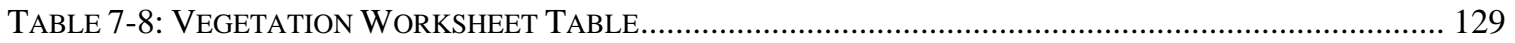

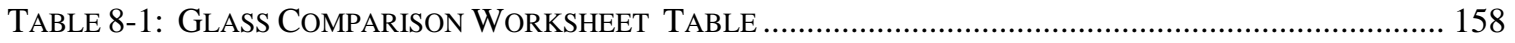

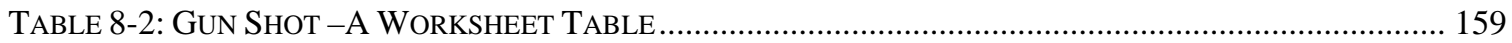

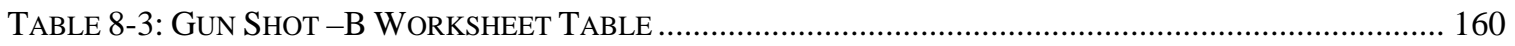

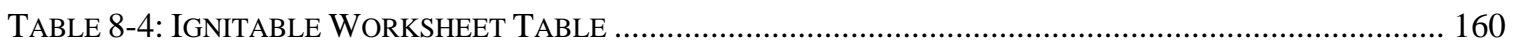

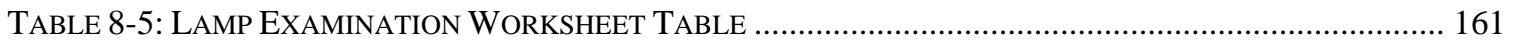

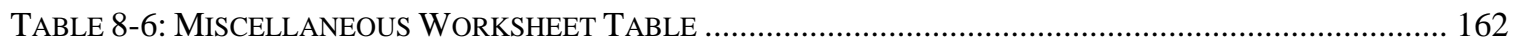

TABLE 8-7: PAINT COMPARISON ANALYSIS WORKSHEET TABLE ................................................................ 163

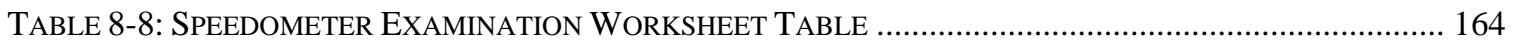

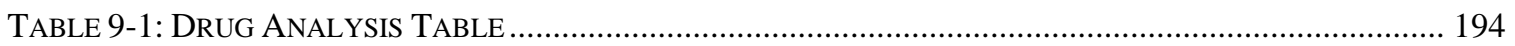

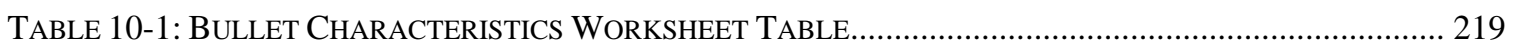

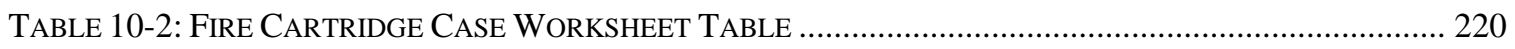

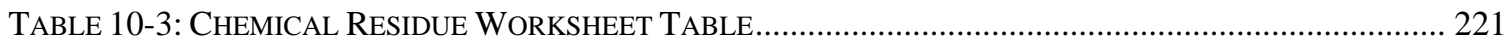

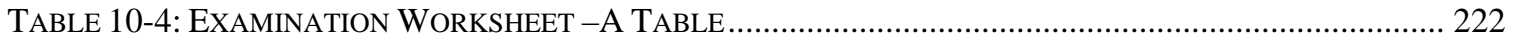

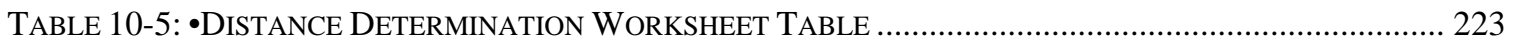

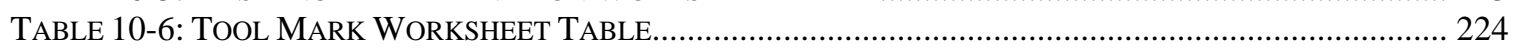

TABLE 10-7: VISUAL MiCRO RESIDUE EXAMINATION WORKSHEET TABLE ................................................... 225

TABLE 11-1: ACE ANALYSIS \& COMPARISON FOR INKED FINGER PRINTS WORKSHEET TABLE.................. 258 
TABLE 11-2: AFIS \& IAFIS COMPARISON REFERENCE WORKSHEET TABLE................................................ 259

TABLE 11-3: ANALYSIS OF LATENT PRINTS WORKSHEET TABLE................................................................ 260

TABLE 11-4: CoMPARISON, EVALUATION, \& VERIFICATION WORKSHEET TABLE ....................................... 261

TABLE 11-5: ANALYSIS Of EXEMPLAR PRINTS WORKSHEET TABLE ……................................................ 261

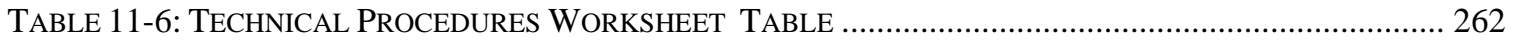

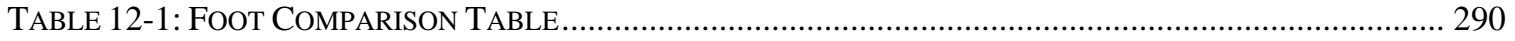

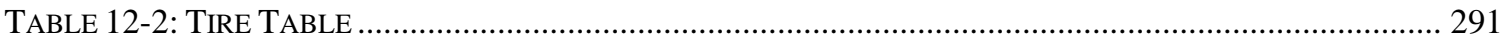

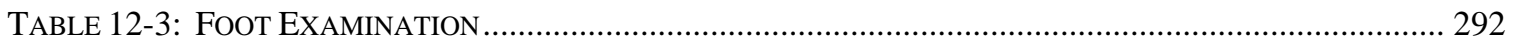




\section{LIST OF ACRONYMS}

ACN

AFIS

ASP

CEP

CER

CODIS

CR

DC

FIMS

GC/MS

GSR

JIS

LCN

LEIF

LIMS

NIBIN

NIJ

ORI

PDA

PDQ

SQL

WVSPFL

WWW
Agency Case Number

Automated Fingerprint Identification Systems

Active Server Pages

Central Evidence Processing

Central Evidence Receiving

Combined DNA Index System

Case Reporting

Document Checklist

Forensic Information Management System

Gas Chromatograph/Mass Spectrometer

Gunshot Residue

Judicial Information Systems

Lab Case Number

Laboratory Evidence Inventory Form

Laboratory Information Management System

National Integrated Ballistics Information Network

National Institute of Justice

ORIginating agency codes

Personal Digital Assistant

Paint Database Query

Structured Query Language

West Virginia State Police Forensics Laboratory

World Wide Web 


\section{CHAPTER 1 \\ INTRODUCTION}

\subsection{Introduction to Forensics}

Forensics science is the application of physical sciences to law in the search for truth in civil, criminal, and social behavioral matters to the end that injustice shall not be done to any member of society [1]. Forensic identification is a scientific technique focused on documenting, collecting, and analyzing evidence [2]. Information obtained through the scientific techniques are organized, verified, and then presented to the judicial system for determining innocence or guilt of a suspect. Some of the functions of a forensic scientist include: analysis of physical evidence, providing expert testimony, and furnishing training in the proper recognition, collection, and preservation of physical evidence [3].

\subsection{Introduction to Information Management System}

Information management has been defined as the organization-wide capability of creating, maintaining, retrieving, and making immediately available the right information, in the right place, at the right time, in hands of the right people, at the lowest cost, in the best media, for use in decision making [4]. An information management system deals with inputting, collating and organizing data that should provide selective information and reports to the management in order to monitor and control the project, organization, resources, activities and results [5].

The rapid evolution of computer technology is expanding man's ability to convert data into useful information and to utilize the information to solve complex problems. Information management systems are becoming of ever-greater interest in progressive and dynamic organizations. The need to obtain, access conveniently, quickly, and economically makes it imperative to devise procedures for the creation, management and utilization of databases [6]. 


\subsection{Web based Information Management}

Recent advances in web technology have made the focus of the information technology field to move towards development for the World Wide Web (WWW). More users have access to web information, and information providers are able to store information of various types on the web easily. The web has now become one of the most important medium for information interchange. Web based systems maximize information sharing and enable comprehensive management of data [7].

Information systems using the web technology are prevalent throughout the world. Applications that have emerged on intranets and internets using web technologies can be referred to as web base information management systems [8]. However, a web based information management system should be distinguished from a normal web application; a web page simply displays information to the user. A web based information management system can be defined as a system that not only provides information users but also proactively interacts with them to help in their task [9].

Web based information management systems take coordination, communication, and collaboration to a new level. One of the key assets of a web based information management systems is its ability to make large volume of data and information accessible to users in a wide variety of circumstances [10]. A web accessible device is all that is needed to view the information. Traditional information management systems focus on querying, reporting, and analyzing data related to business transactions. Web based information systems go beyond this functionality by integrating different media for knowledge representation thereby supporting not only the creation, integration, analysis, and distribution of structured information, but also the storage and transfer of knowledge. While web based systems do not conceptually differ from respective traditional systems, web-based information systems allow for more advanced forms of information management than traditional information systems [11].

Advancements in web technology have made it possible to move documentation and data management away from paper based systems. New web programming languages take advantage of high-end database technology to deliver the promise of a paperless society. A web based information management system manages and stores data in an 
electronic format. Any type of record or documentation that is needed can be completed online through the web browser. Some of the benefits of operating a web-based system are [12]:

- Significant cost savings. It has been estimated that 80 to $90 \%$ of costs are associated with searching paper documents after they are created.

- Time savings as much as 15 to $30 \%$, because web-based systems, due to their ability to easily search for documents. It eliminates tedious monitoring and freeing employees to work on more important tasks.

- Maintaining electronic documents are easy and require less storage space.

- Reduced costs of print, copy, distribute and update paper based forms.

- Ability to collaborate and view real-time documents reduces chances of duplication.

- Worldwide access through the Internet assures that users are connecting to the system from any where at anytime.

\subsection{Other Published Work}

Developing a forensic information management system is a relatively new topic, and the use of web technology in for information management is even newer. An exhaustive literature review only produced two relevant works.

The first is an information technology system that is in the process of development by the Nation Institute of Justice (NIJ). The system once implemented would provide inter-regional information sharing among law enforcement agencies. The system provides access to information within a jurisdiction and provides the capability to query information sources from other agencies with appropriate privacy and security [13].

The second work was also under an NIJ contract. A Forensic Information Management System (FIMS 1.0) was developed for the West Virginia State Police Forensic Laboratory (WVSPFL), Charleston, WV. FIMS 1.0 focused on the biochemistry unit of WVSPFL [14]. The three phases of the biochemistry unit: a) Case Reporting, b) Evidence Processing and c) Evidence Amplification were implemented on the Microsoft Windows platform. Programming was done in Microsoft Visual Basic. The current work 
aims to expand FIMS 1.0 to web environment and implement the system for all the functional units of the laboratory.

\subsection{Problem Statement}

A review of previously published work indicates that although the notion of forensic information management system has exploded, there are several concerns with existing software. These concerns include that the software is developed for windows platform and focus is on only one of the laboratory unit. There exists an unfulfilled need for a simple, streamlined, tool for information management.

It is proposed to develop a system with the use of web-based technology for management of forensic information. The goal of the project is to streamline the various processes at WVSPFL and to implement FIMS 3.0; a web based Forensic Information Management System for case submission, central evidence receiving, processing and all the units of the WVSPFL. Such a system will:

- Reduce handwritten paperwork.

- Reduce case processing time.

- Improve data reliability.

- Electronic chain of custody management.

- Enable printing of reports from remote sites.

- Provide a uniform approach to case reporting.

- Reduce case backlog.

- Eliminate duplication of work.

- Enable easy access to the system from anywhere at anytime.

- Result in case submission from remote sites.

- Result in checking of case status from remote sites.

- Result in improved communication between the various law enforcement agencies and the forensics laboratories. 


\subsection{FIMS System Architecture Design}

One of the key elements of any application design is the system architecture. The system architecture defines how pieces of the application interact with each other, and what functionality each piece is responsible for performing. There are three main style of application architecture with each style being characterized through the number of layers between the user and the data. Each layer generally runs on a different system or in a different process space on the same system. The three types of application architecture are single-tier (or monolithic), two-tier, and n-tier, where n can be three or more.

With three-tier applications, the business rules are removed from the client and are executed on a system in between the user interface and the data storage system. The client application provides user interface for the system. The business rules server ensures that all of the business processing is done correctly. It serves as an intermediary between the client and the data storage. In this type of application, the client does not access the data storage system directly. This type of system allows for any part of the system to be modified without having to change the other two parts. Since the parts of the application communicate through interfaces, then as long as the interface remains the same, the internal workings can be changed without affecting the rest of the system [15].

- Client Tier: The user interface or presentation layer. Through this topmost layer, the user can input data, view the results of requests, and interact with the underlying system. On the Web, the browser performs these user interface functions. In non-Web-based applications, the client tier is a stand-alone, compiled front-end application.

- Middle Tier: Components that encapsulate an organization's business logic. These processing rules closely mimic everyday business tasks, and can be singletask-oriented, or part of a more elaborate series of tasks in a business workflow. In a Web application, the middle tier might consist of components registered as 
part of a transactional application or instantiated by a script in Active Server Pages (ASP).

- Third Tier: A database management system (DBMS) such as a Microsoft ${ }^{\circledR}$ SQL Server ${ }^{\mathrm{TM}}$ database, an unstructured data store such as Microsoft ${ }^{\circledR}$ Exchange, or a transaction-processing mechanism such as Transaction Services or Message Queuing. A single application can enlist the services of one or more of these data providers.

Separating the application into layers isolates each major area of functionality. The presentation is independent of the business logic, which is separate from the data. Designing applications in this way has its tradeoffs; it requires a little more analysis and design at the start, but greatly reduces maintenance costs and increases functional flexibility. Other gains from this approach include [15]:

- Internet ready: Develop solutions that fully exploit the application platform's flexibility and the Internet's global reach and on-demand communication capabilities.

- Faster time to market: Develop and deploy solutions rapidly without requiring developer reeducation or a paradigm shift in how software is built. Expose services and functionality through the underlying "plumbing" to reduce the amount of code development.

- True interoperability: Build interoperability into all tiers so functionality can be added to existing systems. Adhere to open protocols and standards so other vendor solutions can be integrated.

- Reduced complexity: Integrate key services directly into the operating system and expose them in a unified way through the components. Reduce the need for information technology (IT) professionals to function as system integrators so they can focus on solving the business problem.

- Language, tool, and hardware independence: Provide a language-neutral component model so developers can use task-appropriate tools. Build on the PC 
model of computing, wherein customers can deploy solutions on widely available hardware.

- Lower the total cost of ownership: Develop applications that are easy to deploy, manage, and change over time.

\subsection{Introduction to FIMS Application}

FIMS (Forensic information management system) is complete Web-based forensic data entry and reporting system deployed on the West Virginia State Police Headquarter intranet. FIMS application integrates the multiple years' laboratory case information data from multiple laboratories and agencies all over the state of West Virginia into one database. FIMS also provides online, integrated reports generation, problem tracking, and trouble reporting capability.

All forensic laboratory and agency personnel have the ability to browse the FIMS data from their respective units. User credentials are gathered from the username and password supplied on the login page FIMS application URL (http://fims.rsa.wvu.edu/default.aspx). 


\section{Understanding the FIMS Work Area}

This section describes the modules available in the FIMS application. The details of each module are described below.

\section{FIMS homepage}

By default, the initial screen of FIMS is the home page screen as shown in Figure 1.1. The screen shows the different modules of the system. User can click on the rectangular sections to navigate to login screen of that unit.

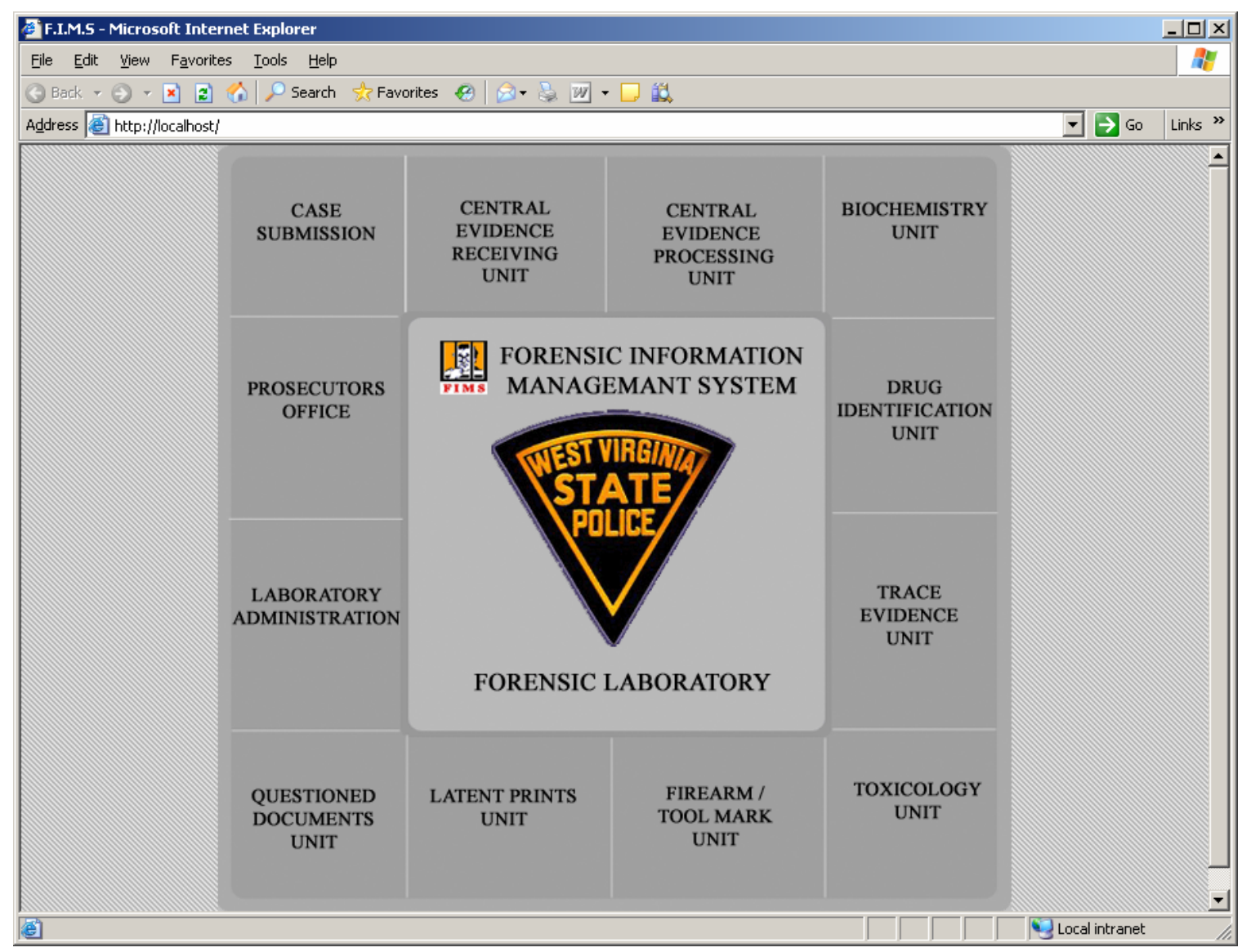

Figure 1.1: FIMS home page 


\section{Unit homepage}

The FIMS unit login screen assist in verifying user credentials. Each user must be enrolled with the system before accessing work area.

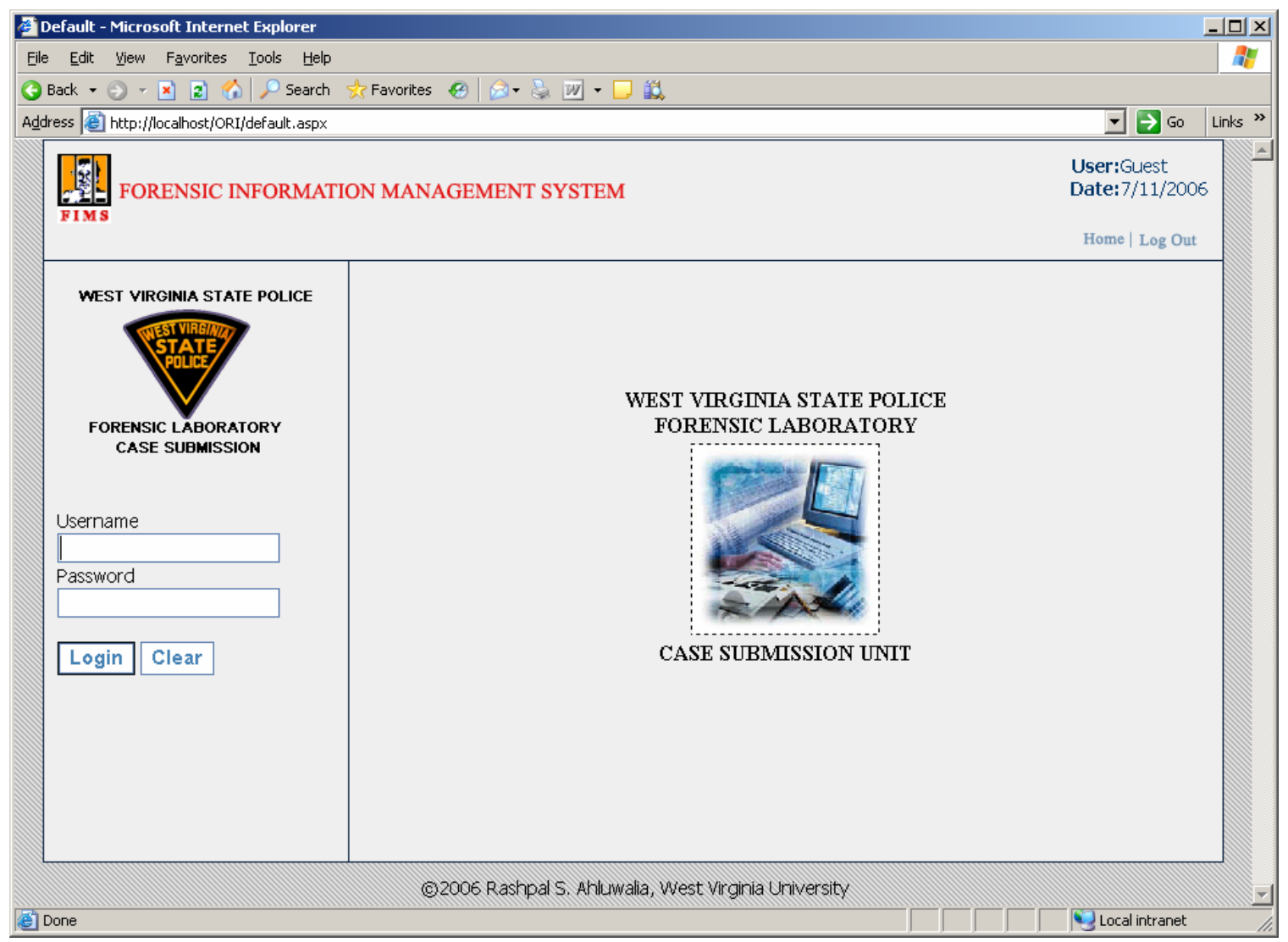

Figure 1.2: Unit home page

The header region enables the user to navigate to FIMS home page or end the current session. It also displays user's full name and current date on right hand upper corner. The side navigation bar shows the unit name, input box for username and password. After verifying user credentials content are updated according to the unit. For example, Case submission module users will see open new case and open existing case options after successful login. Main work area is the space in which all of the user action results are displayed. For example, if a user hits open new case hyperlink, the new case form will be displayed in this region. 


\section{CHAPTER 2 \\ CASE SUBMISSION}

\subsection{Existing Case Submission Process}

Case submission is the first step in evidence examination. The Originating (ORI) agency submits a case to the forensic laboratory via the Case Report (CR) or the Case Submission Form. The CR form is sent to the forensic laboratory along with the items of evidence either by personal delivery or by postal mail. Authorized individuals prepare the Case Submission Form (WVSP-53) when submitting items of evidence. Typically, the Case Submission Form is a self-carbon form that is to be prepared by the investigator. The original (white) and two copies (pink and canary) are submitted to the lab. The submitting officer retains a copy (goldenrod). The forensic laboratory returns a receipt copy (canary) to the submitting officer at the time a report is issued. The items of evidence received at the laboratory for examination are accepted only upon completion of the CR form. [16]

Case submission involves a series of methods to collect the evidence from the crime scene and to retrieve maximum possible information from it. At present, there are over 1000 Originating (ORI) agencies that send items for testing to the WVSPFL. The case submission process is shown in the Figure 2.1.

The case submission form has eight sections, which can be categorized into two major groups: Reporter Information group and Investigation Report group. The reporter information consists of agency information, reporting officer's personal information such as name, address, and phone number. This phase also includes the agency case number. The investigation report phase contains sections that include crime description, victim information, suspect information, evidence items and supplementary case information submitted to the forensics lab. Figure 2.2 shows the sections in the case reporting process [16]. 


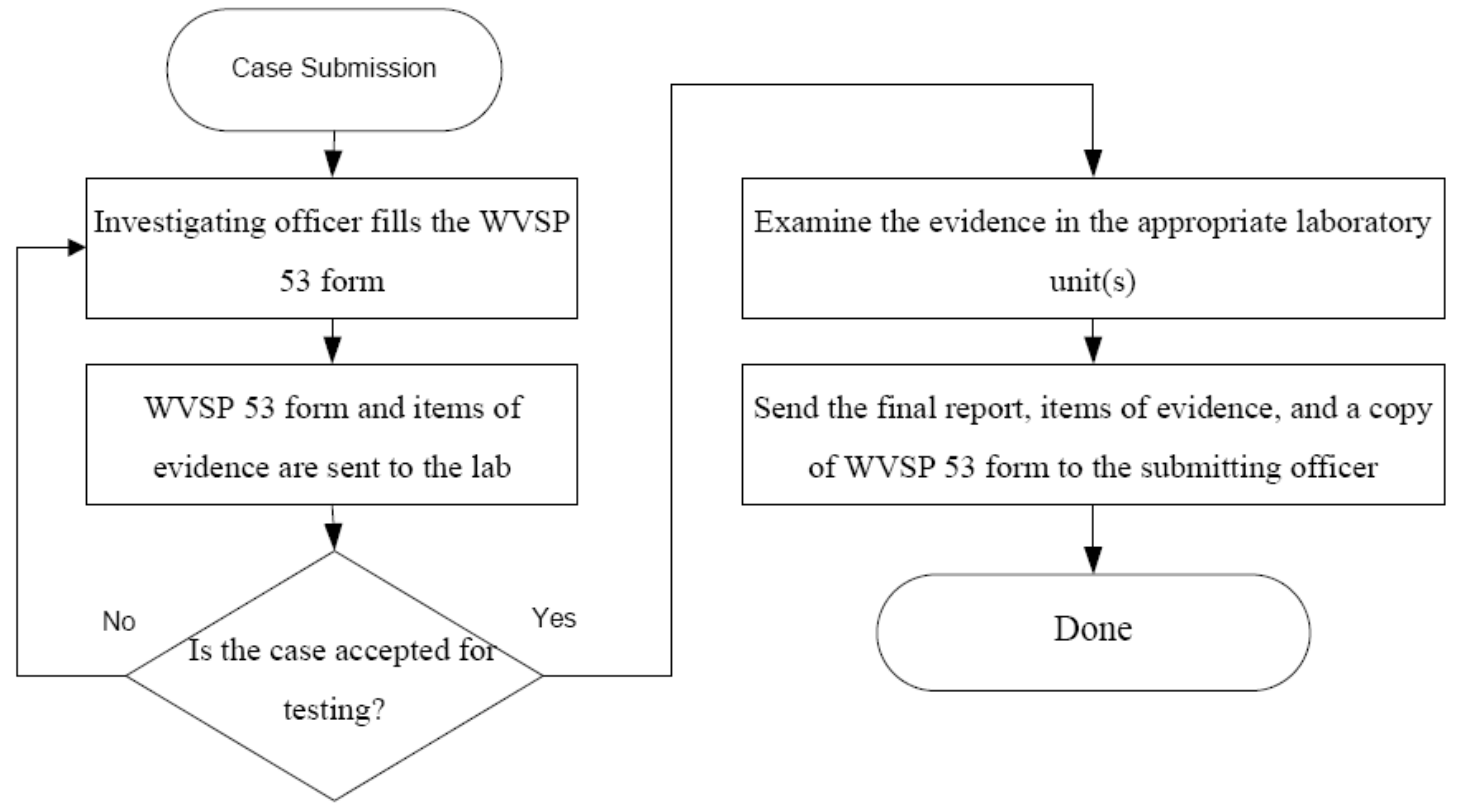

Figure 2.1: Existing Case Submission Process

A sample case submission form is shown in Figure 2.3. The top portion of the case submission form contains the reporter information. The next part of the CR form is the investigation report. The CR form also has a supplementary case information section, which is used when a case is submitted to the drug identification unit.

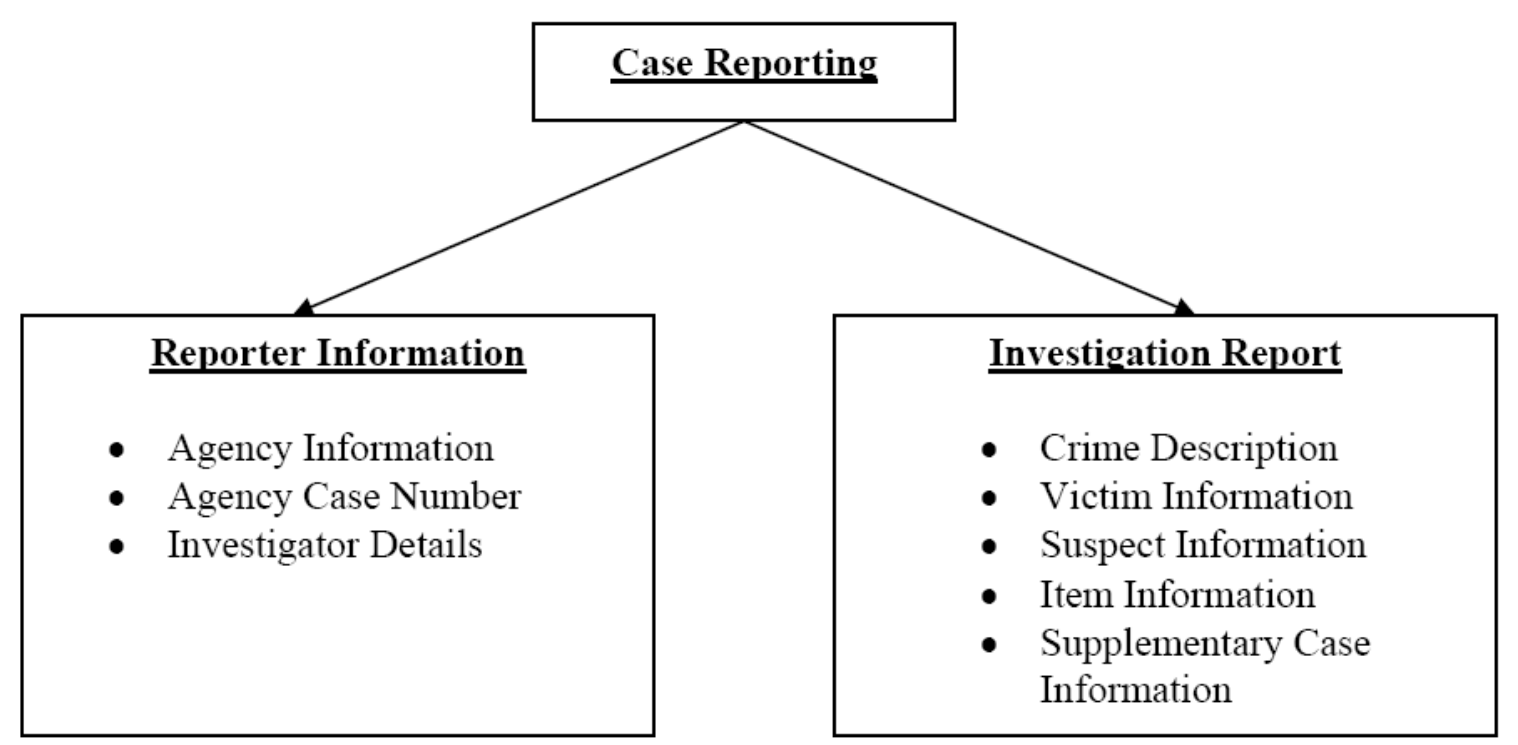

Figure 2.2: Case Reporting 


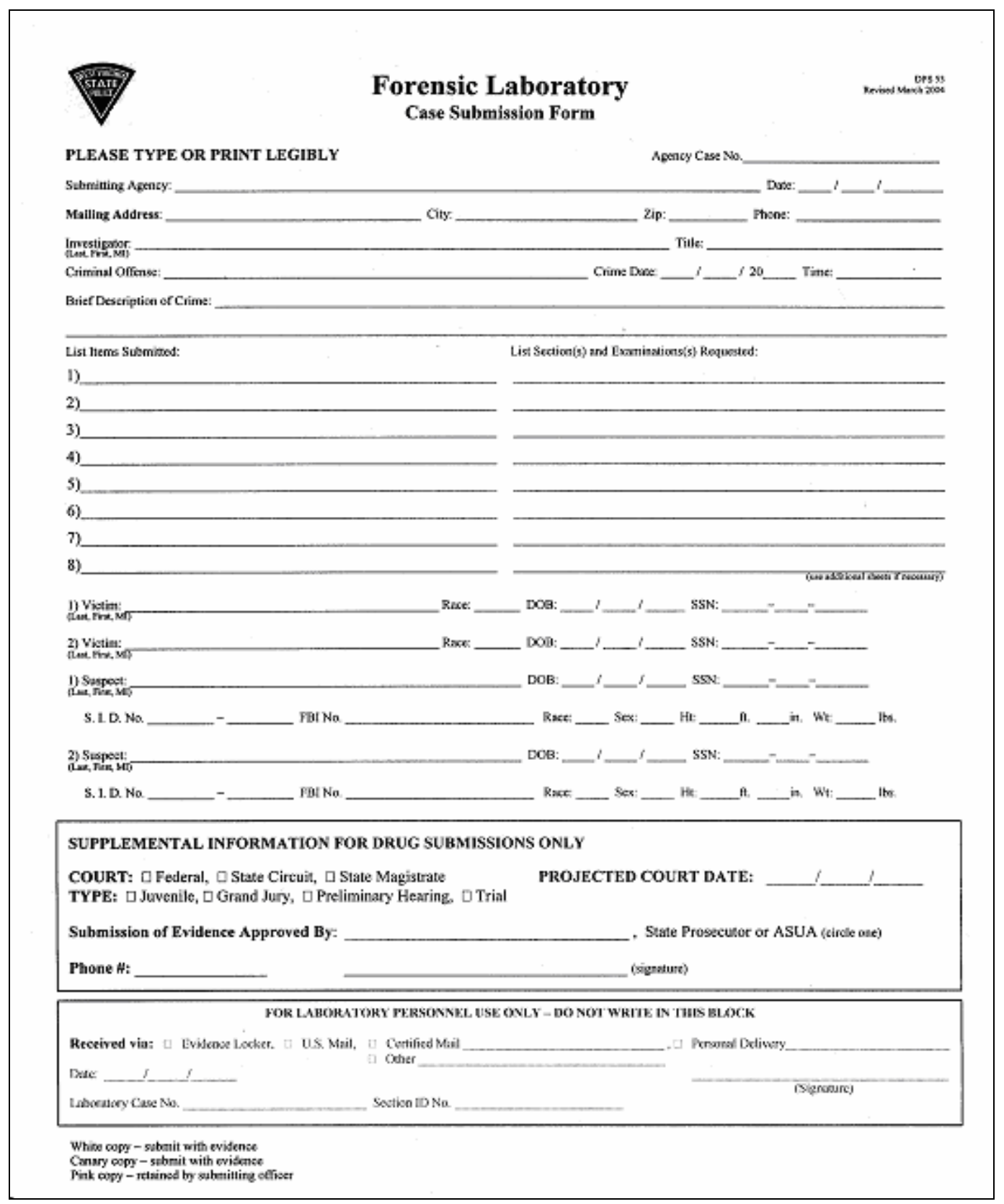

Figure 2.3: WVSP-53 Case Submission Paper Form 


\subsection{Re-engineering of the Case Submission Process}

The re-engineered case submission process is shown in Figure 2.4. ORI is responsible for filling WVSP-53 case submission form and sending it to the laboratory with the evidence package for evidence analysis. The ORI sends evidence package to laboratory via mail or personal delivery.

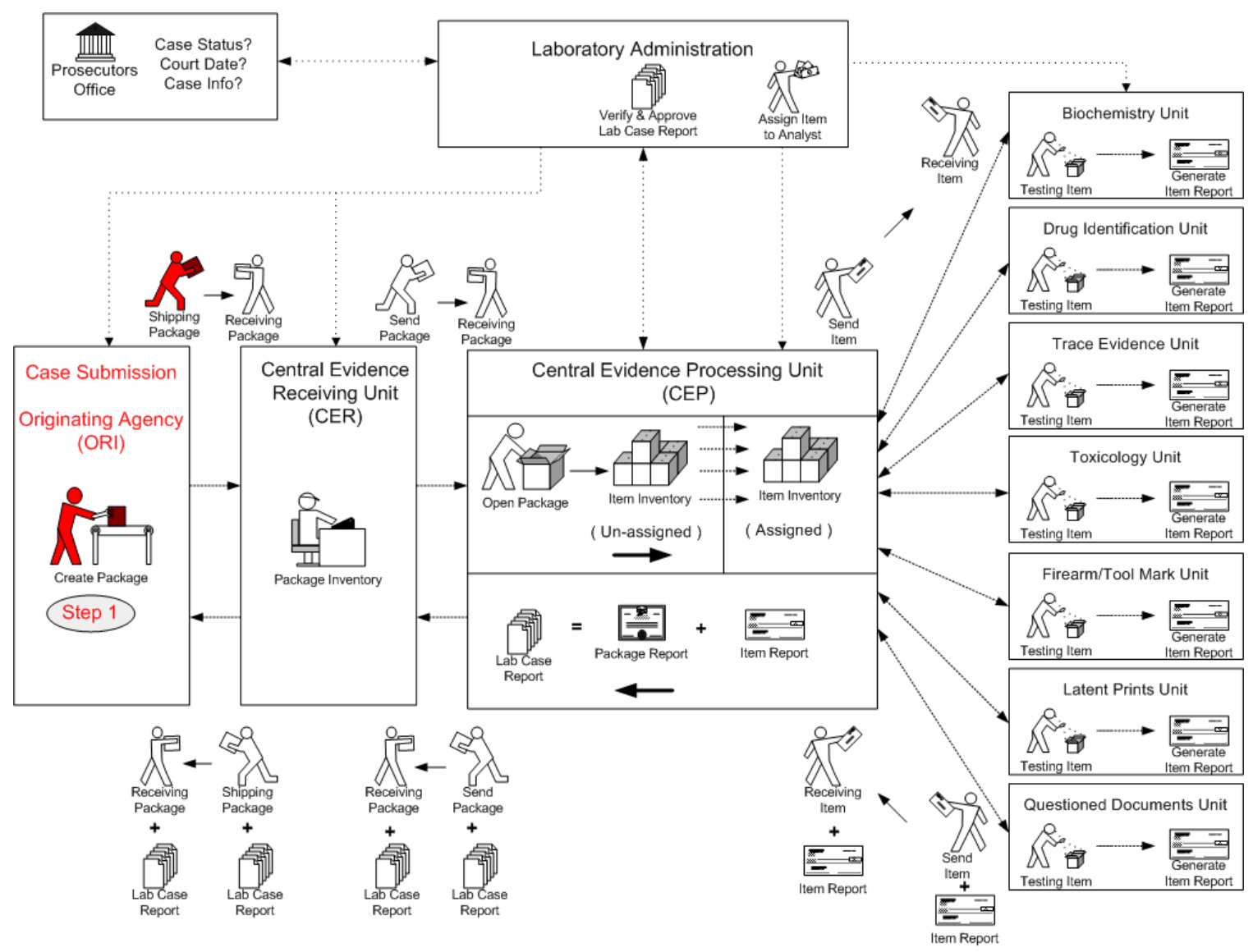

Figure 2.4: Step 1: Case Submission 
Figure 2.5 shows the evidence and information flow from CER to ORI module. The ORI receives evidence package and final approved case report back from CER via mail or personal delivery. This is the commencing step of the process.

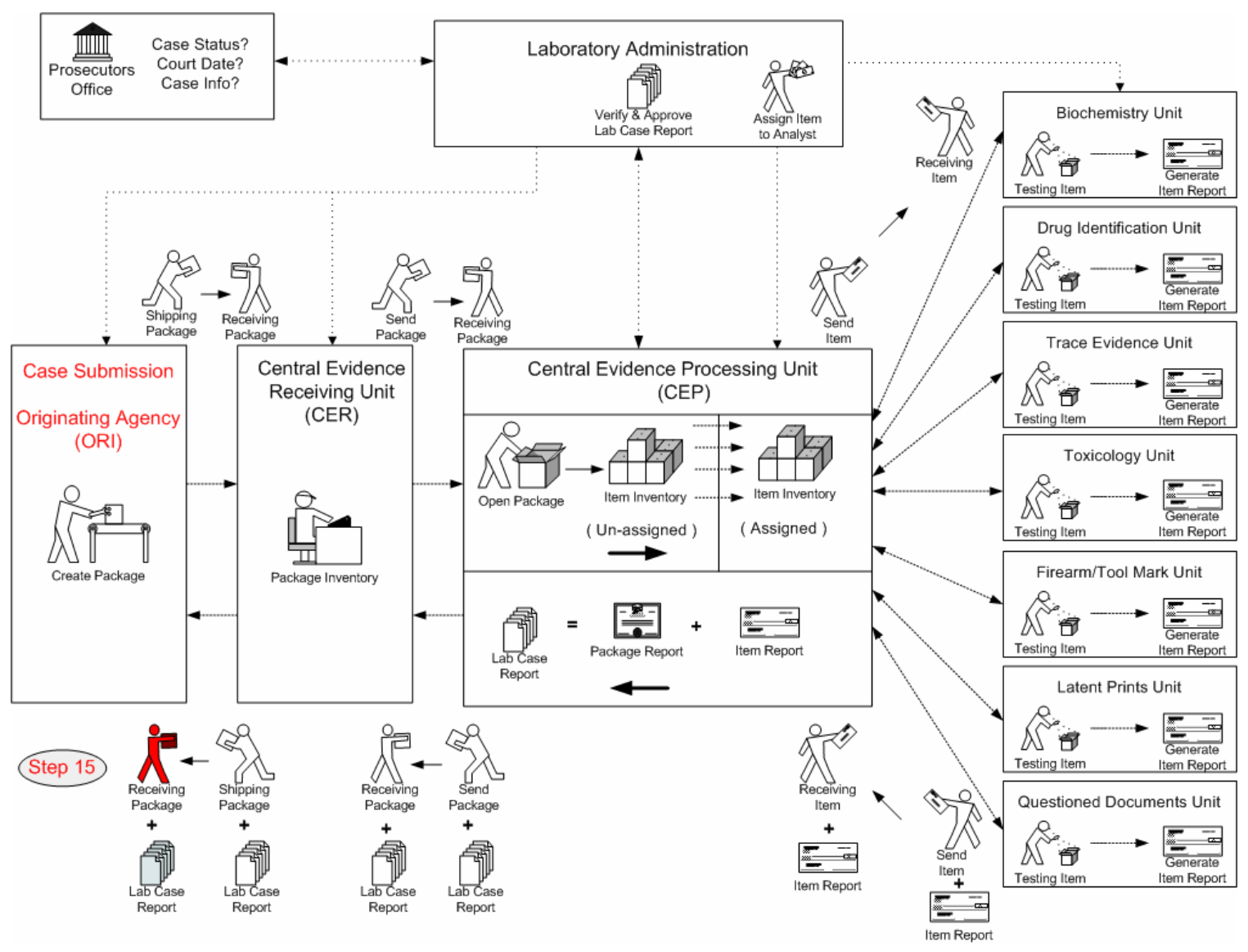

Figure 2.5: Step 15 Receive Package \& Final Case Report 


\subsubsection{Case Submission Entity Relationship Diagram}

Case submission data table are connected with each other through relations. Figure 2.6 shows relational diagram between different case submission data tables.

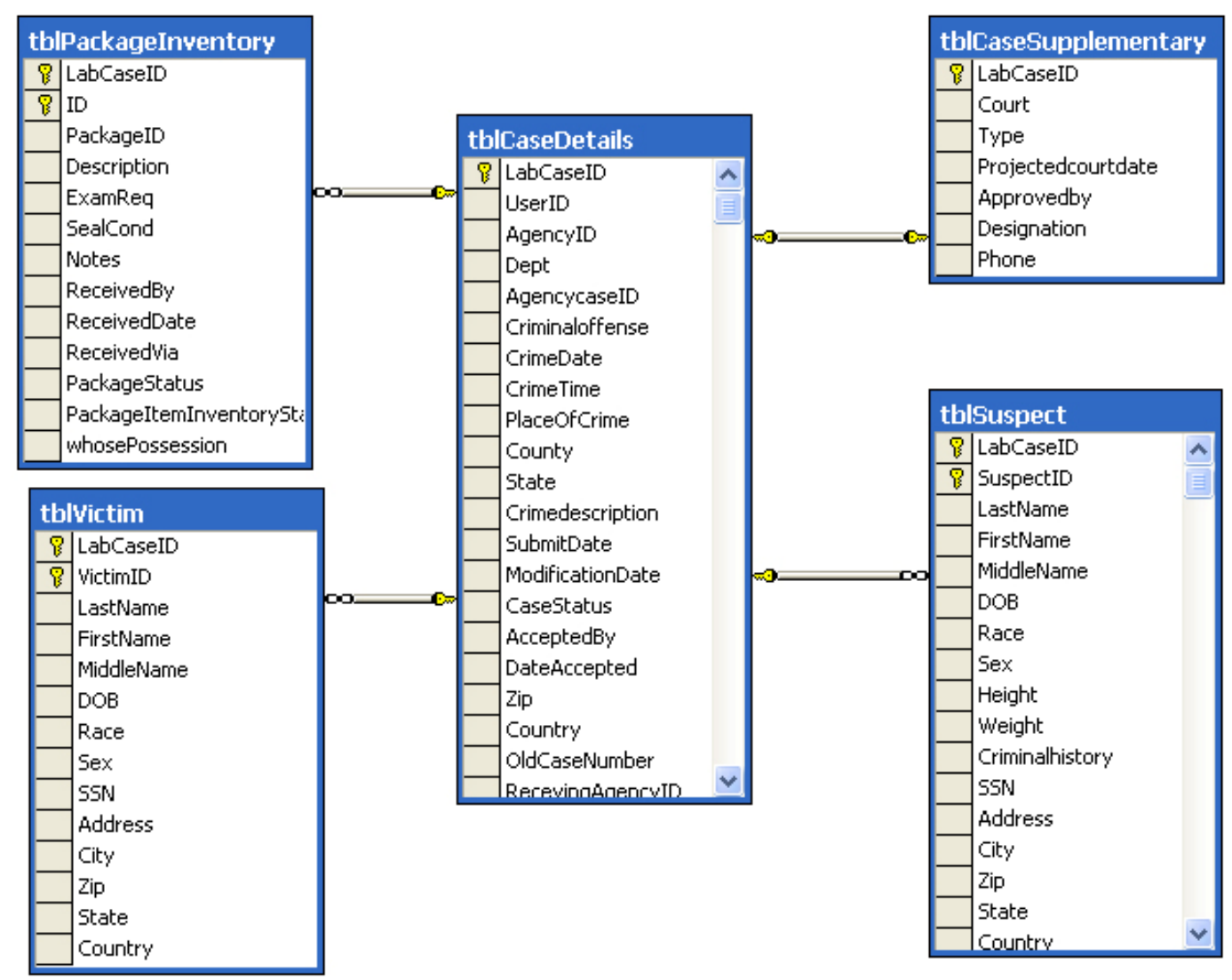

Figure 2.6: Case Submission Data Tables 


\subsubsection{Case Submission Database Tables}

Each section of the case submission form has a separate database table. The case submission module stores its data in the following tables:

- Lab Case ID Information (tbllabtest)

- Officer Information (tblUserInfo)

- Crime Information (tblCaseLoginForm)

- Supplementary Case Information (tblCaseSupplementary)

- Suspect Information (tblSuspect)

- Victim Information (tblVictim)

- Package Information (tblEvidence)

- Agency Information (tblAgencyInfo)

- User Login Tracking Information (tblTrackUserLogin) 
The various fields of the officer information table are shown in Table 2-1. Field LCID stores the unique lab case number. Fields LastName, FirstName, MiddleName, and Title store investigating officers last name, first name, middle name, and title respectively.

Table 2-1: Officer Information Table

\begin{tabular}{|c|c|}
\hline & UserInfo \\
\hline 8 & UserID \\
\hline & AgencyID \\
\hline & Dept \\
\hline & LName \\
\hline & FName \\
\hline & MName \\
\hline & Title \\
\hline & SSNumber \\
\hline & JobTitle \\
\hline & Address \\
\hline & City \\
\hline & State \\
\hline & Zipcode \\
\hline & Phone \\
\hline & Race \\
\hline & Sex \\
\hline & DOB \\
\hline & HireDate \\
\hline & TitleDate \\
\hline
\end{tabular}

The agency information table contains agency name, agency ID, street address with county, state, and zip code of the agency and contact person's name and also phone number of the agency as shown in Table 2-2.

Table 2-2: Agency Information Table

\begin{tabular}{|l|l|}
\hline tblagencyInfo \\
\hline AgencyID \\
\hline & AgencyName \\
\hline & Street \\
\hline & City \\
\hline & County \\
\hline & State \\
\hline & Zipcode \\
\hline & PhoneNumber \\
\hline & Notes \\
\hline & ContactPerson \\
\hline & AgencyTypeID \\
\hline
\end{tabular}


The various fields of the crime information table are shown in Table 2-3. Field LCID stores the unique lab case number. The other fields of the table are criminal offense, crime date, time of crime, street address with county and zip code and also brief description of crime.

Table 2-3: Crime Information Table

\begin{tabular}{|l|l|}
\hline tblCaseDetails \\
\hline & LabCaseID \\
UserID \\
AgencyID \\
Dept \\
\hline AgencycaseID \\
Criminaloffense \\
\hline CrimeDate \\
\hline CrimeTime \\
\hline PlaceofCrime \\
\hline County \\
\hline State \\
\hline & Crimedescription \\
\hline SubmitDate \\
\hline & ModificationDate \\
\hline & CaseStatus \\
\hline AcceptedBy \\
DateAccepted \\
\\
Zip \\
\hline & Country \\
\hline & OldCaseNumber \\
\hline & RecevingAgencyID \\
\hline \hline
\end{tabular}


The victim information table contains LCID, victim ID, victim name, date of birth, race, sex, social security number, victim address including city and zip code as shown in Table 2-4.

Table 2-4: Victim Information Table

\begin{tabular}{|l|l|}
\hline tblVictim \\
\hline B & LabCaseID \\
\hline F & VictimID \\
\hline & LastName \\
\hline & FirstName \\
\hline & MiddleName \\
\hline & DoB \\
\hline & Race \\
\hline & Sex \\
\hline SSN \\
\hline \\
Address \\
\hline City \\
\hline & Zip \\
\hline & State \\
\hline & Country \\
\hline
\end{tabular}


The suspect information table contains LCID, suspect ID, first name, middle name, last name, date of birth, race, sex, social security number, height, weight, address including city and zip code as shown in Table 2-5.

Table 2-5: Suspect Information Table

\begin{tabular}{|l|l|}
\hline tblSuspect \\
\hline Z LabCaseID \\
\hline SuspectiD \\
\hline & LastName \\
\hline & FirstName \\
MiddleName \\
DoB \\
\hline Race \\
\hline Sex \\
\hline Height \\
Weight \\
Criminalhistory \\
\hline SSN \\
\hline Address \\
\hline City \\
\hline Zip \\
\hline State \\
\hline Country \\
\hline
\end{tabular}


The Package information table contains LCID, Evidence ID, description of the package and the examinations desired for the item as shown in Table 2-67.

Table 2-6: Package Information Table

\begin{tabular}{|l|l|}
\hline tblPackageInventory \\
\hline i & LabCaseID \\
\hline | & ID \\
\hline & PackageID \\
\hline & Description \\
\hline & ExamReq \\
\hline & SealCond \\
\hline & Notes \\
\hline & ReceivedBy \\
\hline & ReceivedDate \\
\hline & ReceivedVia \\
\hline & PackageStatus \\
\hline & PackageItemInventoryst: \\
\hline & whosePossession \\
\hline
\end{tabular}

The supplementary information table is shown in Table 2-7. It contains the supplementary information for the drug submission cases. The main fields of the table include court, type, court date, and information of the person approving submission.

Table 2-7: Supplementary Case Information Table

\begin{tabular}{|l|l|}
\hline tblCaseSupplementary \\
\hline B & LabCaseID \\
\hline & Court \\
\hline & Type \\
\hline & Projectedcourtdate \\
\hline & Approvedby \\
\hline & Designation \\
\hline & Phone \\
\hline
\end{tabular}


The user login log information table contains user name, agency ID, unit name, date and time of login, and IP address of the system from which the user logged on to the FIMS case submission module as shown in Table 2-8

Table 2-8: User Login Log Information Table

\section{tblTrackUserLogin}

(8) SerialiD

UserID

AgencyID

Dept

UserMachineIP

Datelogged 


\subsubsection{Working with the Case Submission Module}

The law enforcement agencies can complete the CR form online through the FIMS case submission module. The electronic version of the CR form is similar to the paper based form. It has the following major sections: 1) Lab case ID, 2) Officer information, 3) Crime information, 4) Victim information, 5) Suspect information, 6) Item information, 7) Supplementary information, and 8) Agency information. The case submission module can be accessed via the Internet through any browser at the web address: http://fims.rsa.wvu.edu/ORI/Default.aspx or by clicking on the case submission link in the main page of FIMS. Figure 2.7 shows the login page for the case submission module. Each law enforcement agency in the state of West Virginia has a unique user name and password. For security purposes, the date and time of each login, IP address from which the system was logged, agency name and ID are recorded in the database.

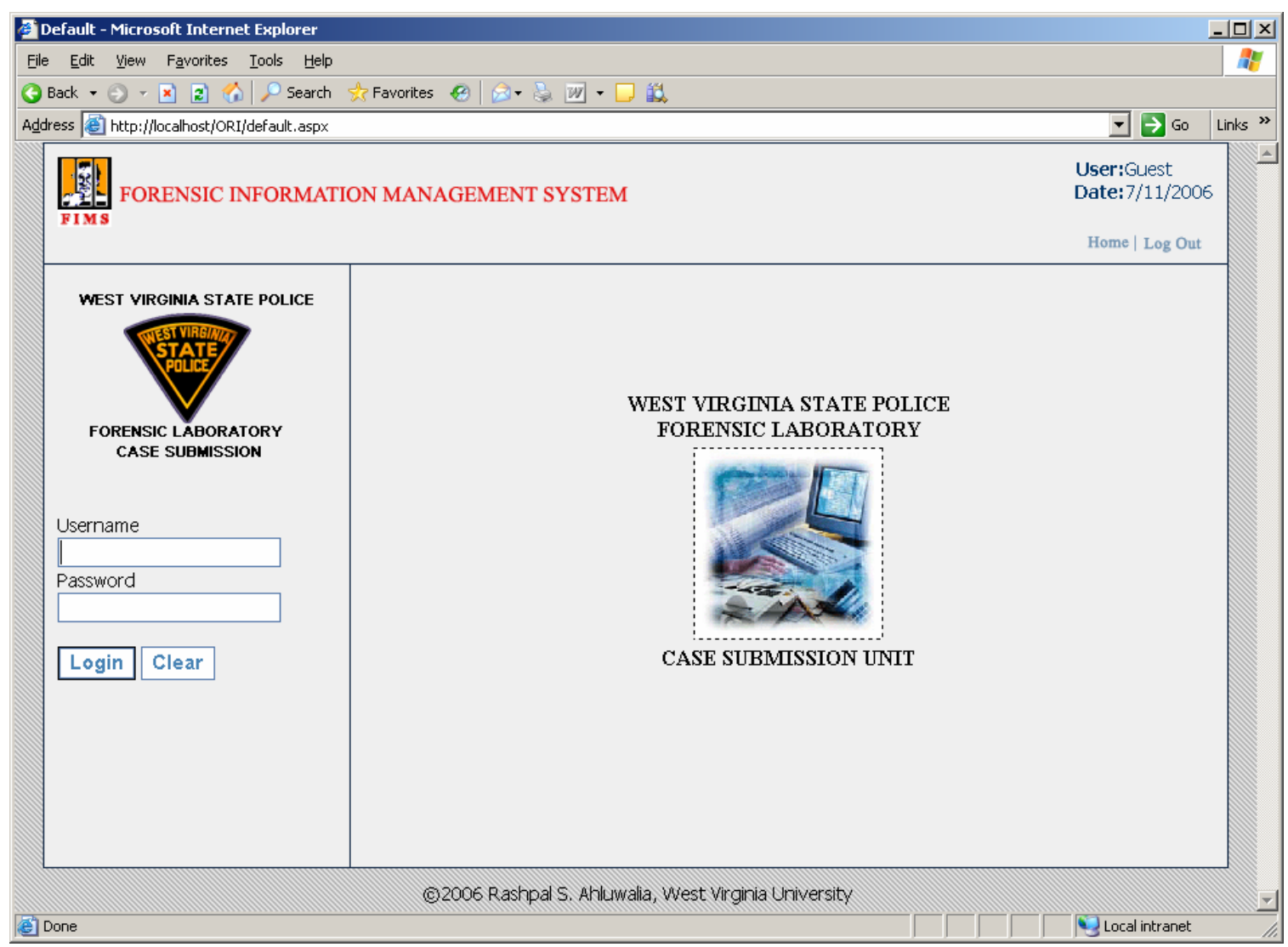

Figure 2.7: Case Submission Module Login Page 
After a user is authenticated, the main page of case submission module is opened on the left navigation bar as shown in Figure 2.8. The page has the following navigation links:

- New case - To open a new lab case

- Existing case - To Add/Modify an already submitted case

\section{Reporting new case}

When the user selects the link to open a new case, a unique LabCaseID (LCID) is generated for the case. LCID is a ten-digit number with the first four digits corresponding to the year of case submission and the remaining six digits representing the case number submitted on the year. For example the fifth case submitted in the year 2006 would have the LCID \# 2006000005. The user is redirected from the main page to the officer and crime information page.

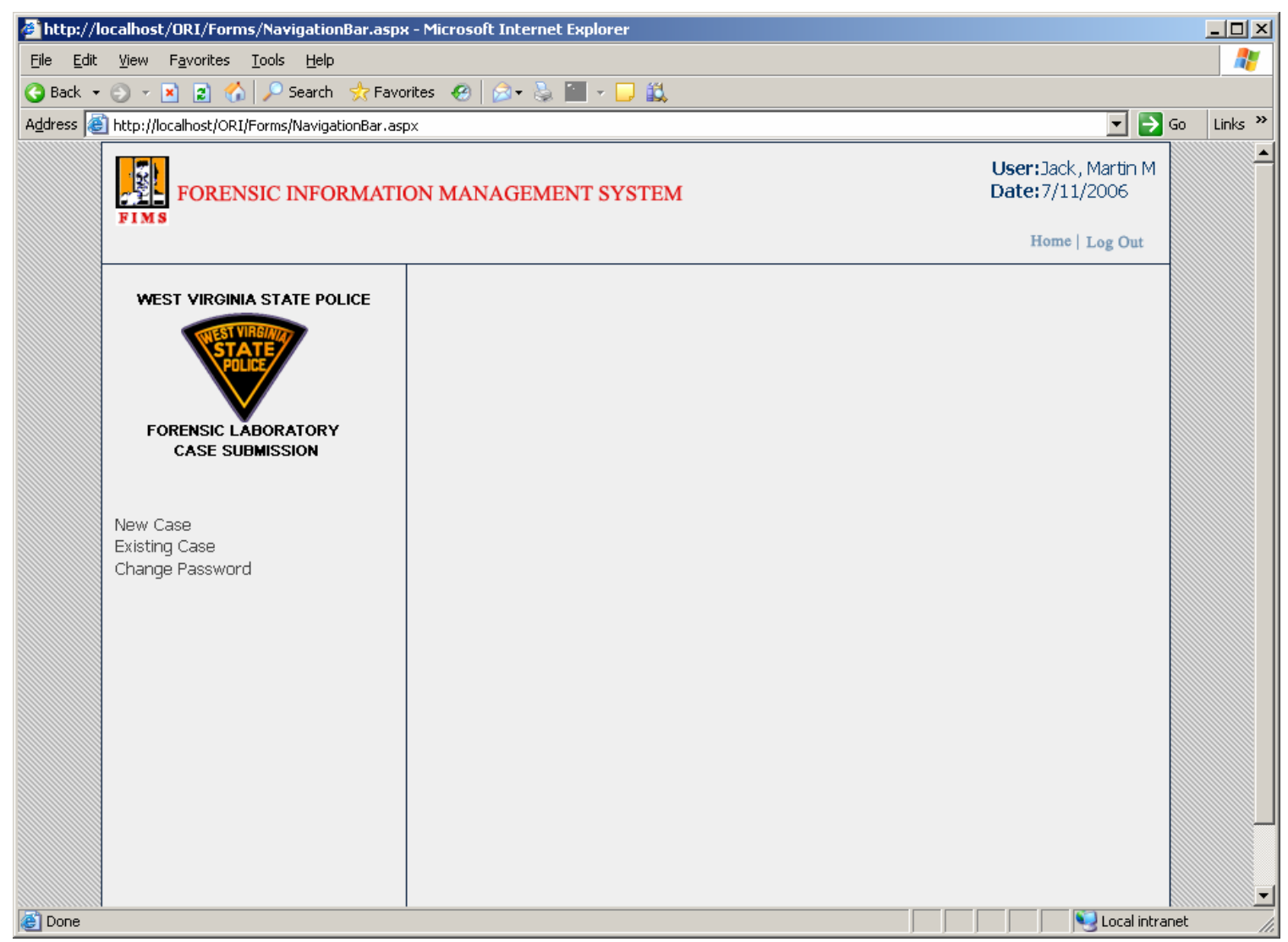

Figure 2.8: Case Submission module Main Page 


\section{Open Existing case}

List of the cases submitted in the past six months with their submission date and WVSP-53 CSF status is shown in Figure 2.9. There are two calendar popup controls on the page, which enables the user to open cases submitted during a different time period. After selecting the lab case and clicking on the "View Case" hyperlink, the officer and crime information page opens in the main screen as shown in Figure 2.9.

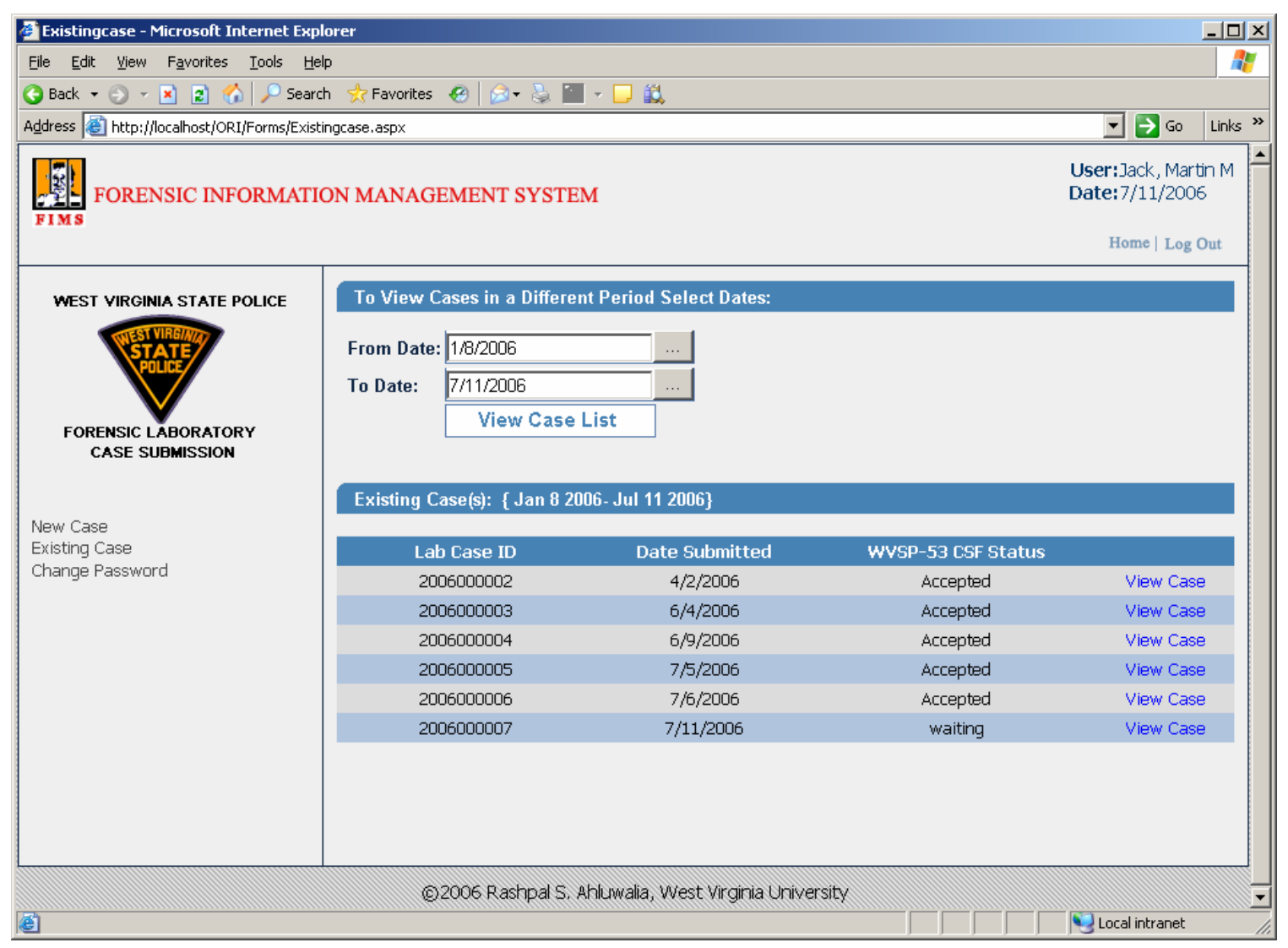

Figure 2.9: Existing Case Opening Page 


\section{Filling officer and crime information}

Case submitting officer's personal information and agency name is pulled from the database. The subject of investigation for the case can be selected from the dropdown list. Table 2.10 shows the choices that are available for the user to select the subject of investigation.

Table 2-9: Subject of Investigation

\begin{tabular}{|l|l|l|l|}
\hline Abduction & Illegal Wire Tap & Alcohol Content & Incest \\
\hline Arson & Indecent Exposure & Assault & Internal Investigation \\
\hline Assault/Battery & Intimidating & Automobile Accident & Joyriding \\
\hline Battery & Kidnapping & Bestiality & Killing of Animals \\
\hline Boating Accident & Larceny & Bombing & Larceny-Grand \\
\hline Brandishing & $\begin{array}{l}\text { Leaving the Scene of an } \\
\text { Accident }\end{array}$ & Breaking \& Entering & Littering \\
\hline Burglary & Lottery Examination & Check Fraud & $\begin{array}{l}\text { Mail } \\
\text { Fraud/Larceny/Tampering }\end{array}$ \\
\hline Child Abuse & Missing Person & Child Neglect & Money Laundering \\
\hline Civil Right Violation & $\begin{array}{l}\text { Motor Vehicle } \\
\text { Violation }\end{array}$ & Computer Crimes & $\begin{array}{l}\text { Negligent } \\
\text { Homicide/Shooting }\end{array}$ \\
\hline $\begin{array}{l}\text { Contributing to the } \\
\text { Delinquency of a Minor }\end{array}$ & $\begin{array}{l}\text { Obliterated Serial } \\
\text { Number }\end{array}$ & Counterfeiting & Obstructing \\
\hline Credit Card Fraud & $\begin{array}{l}\text { Officer Involved } \\
\text { Shooting }\end{array}$ & Cruelty to Animals & Parole Violation \\
\hline Death & Perjury & Destruction of Property & Poisoning \\
\hline Domestic Disturbance & Proficiency & Drive By Shooting & Recovered Property \\
\hline Driving Violation & Robbery & Driving Under the & Robbery-Armed \\
\hline Influence & Embezzlement & Sex Crime \\
\hline $\begin{array}{l}\text { Erugs } \\
\text { Breaking }\end{array}$ & Robbery-Bank & Escape & Sexual Abuse \\
\hline Explosives & Sexual Assault & Extortion & Shoplifting \\
\hline False Reporting & Shooting & False Pretense & Stalking \\
\hline Firearms Violation & Smuggling & Fleeing & Suicide \\
\hline Forgery & Suspicious Activity & Forgery \& Uttering & Tampering \\
\hline Fraud & Tax Evasion & Gambling & Threats \\
\hline Game Law Violation & Theft & Gunshot Residue & Trespassing \\
\hline Harassment & Unlawful Entry & Hate Crime & Uttering \\
\hline Hit and Run & Vandalism & Identity & Wanton Endangerment \\
\hline Hunting Accident & Wiretapping & & \\
\hline Illegal Alcohol & Others & & \\
\hline & & Wounding \\
\hline
\end{tabular}




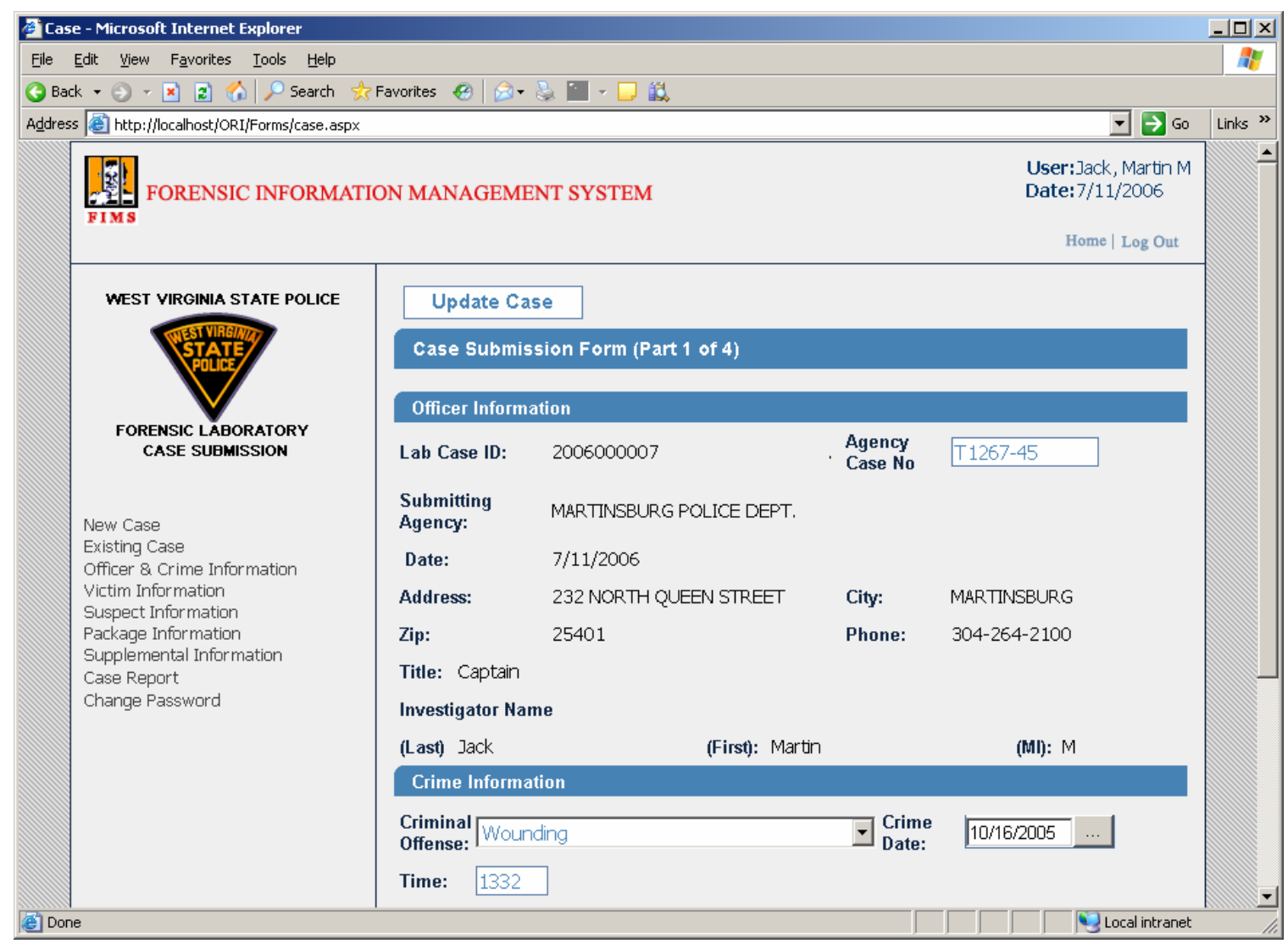

Figure 2.10: Officer and Crime Information Page

The links in navigation menu on the left side of the screen will now have the links:

- New Case - To open a new lab case

- $\quad$ Existing Case - To add/modify an already submitted case

- Officer \& Crime Information - To add/modify officer details and crime information

- Victim Information - To add/modify victim information

- Suspect Information - To add/modify suspect information

- Package Information - To add/modify Package information

- Supplemental Information - To add/modify supplemental case information for drug submissions

- Case Report - To view case submission report 


\section{Victim information}

The user can navigate through the pages at anytime by simply clicking on the respective links on the navigation bar. The victim information page has details regarding victim(s) of a particular case. Figure 2.11 shows the victim information page. A unique victim ID is created for each victim of a given case. The user has options to view a victim, add, edit or delete victim information. The details that can be entered for a victim are: name, date of birth, race, sex, SSN, street address with state, zip code, and country. Dropdown lists are provided for race, sex, state, and country.

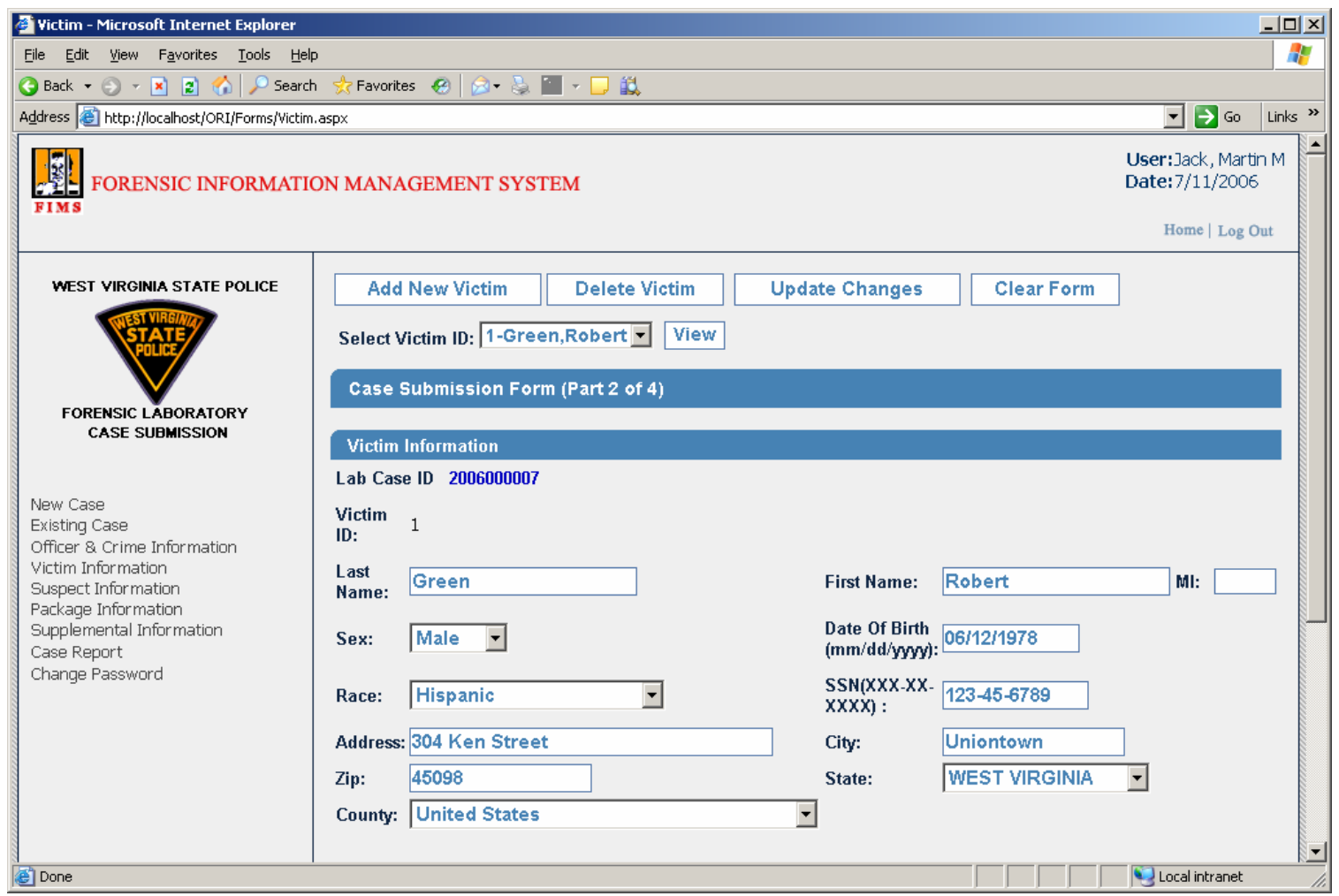

Figure 2.11: Victim Information Page

The choices for the State field in the victim information page include all US states in US along with N/A. 


\section{Suspect information}

The suspect information page has details regarding suspects of a particular case and resembles the victim information page with added details like suspect's criminal history, weight, height etc. as shown in Figure 2.12. A unique suspect id is created for each suspect in a given case. The user has options to view a suspect, add, edit, or delete suspect information similar to that of the victim information page.

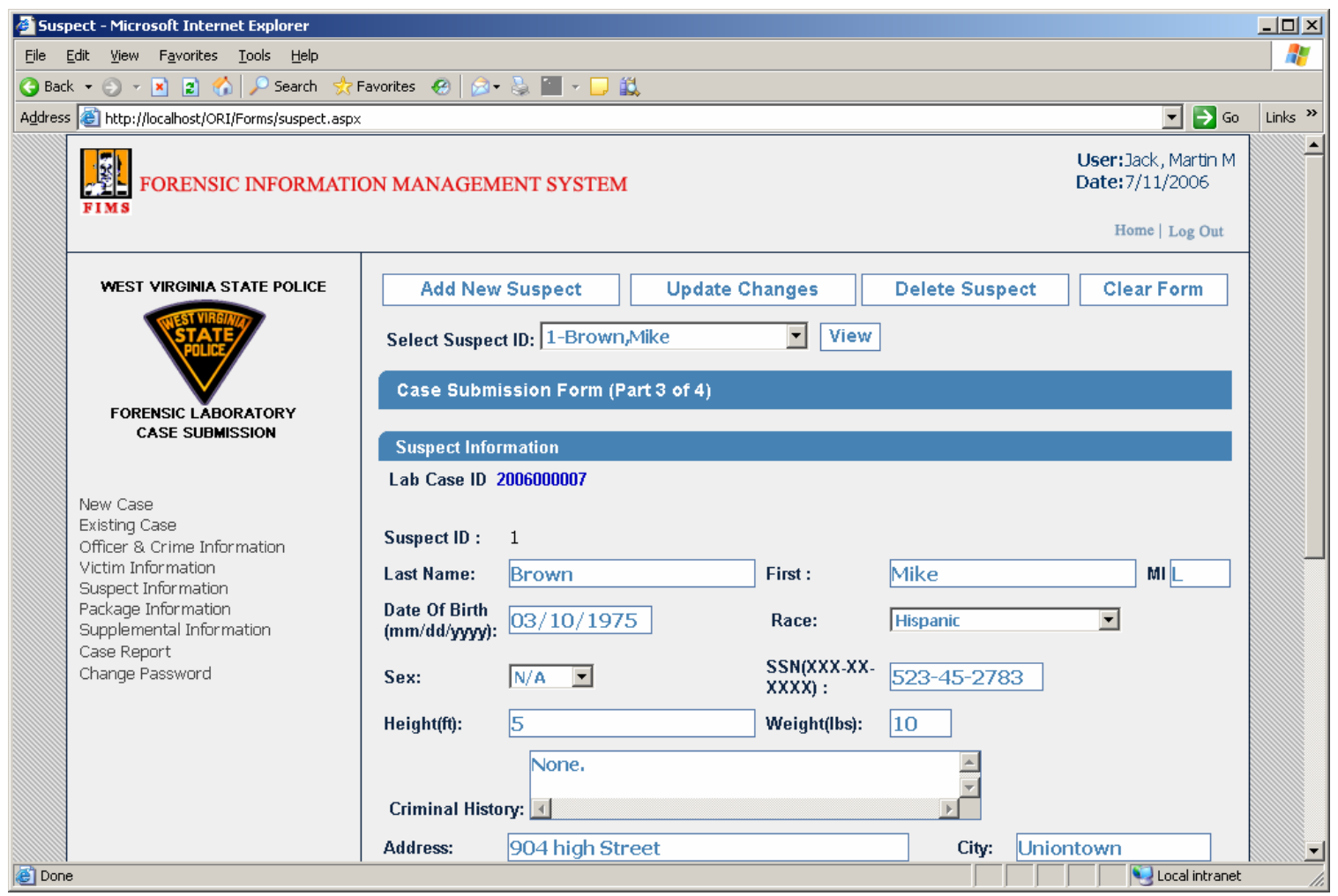

Figure 2.12: Suspect Information Page 


\section{Package information}

The package information page has details regarding the packages submitted to the laboratory for testing. The user can enter a brief description of the item and also the section/examinations required for the respective items. Similar to victim and suspect information pages, a unique id is created for each package submitted for a case. The user also has options to view a package, add, edit and delete package information. Figure 2.13 shows the package information page.

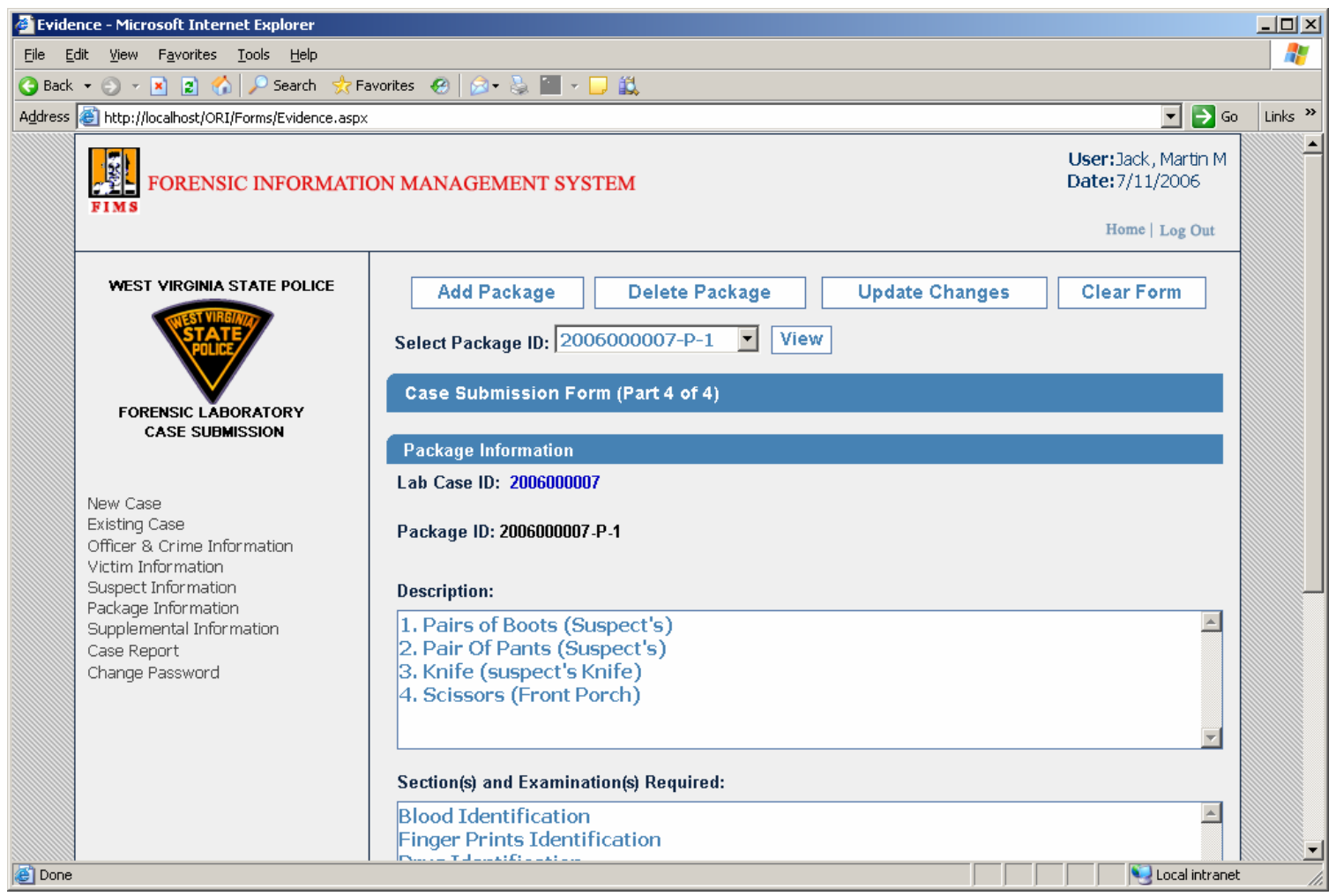

Figure 2.13: Package Information Page 


\section{Supplemental information}

The supplemental information page has details regarding the projected trial date and the court. It is filled only for drug submissions. Figure 2.14 shows the supplemental information page.

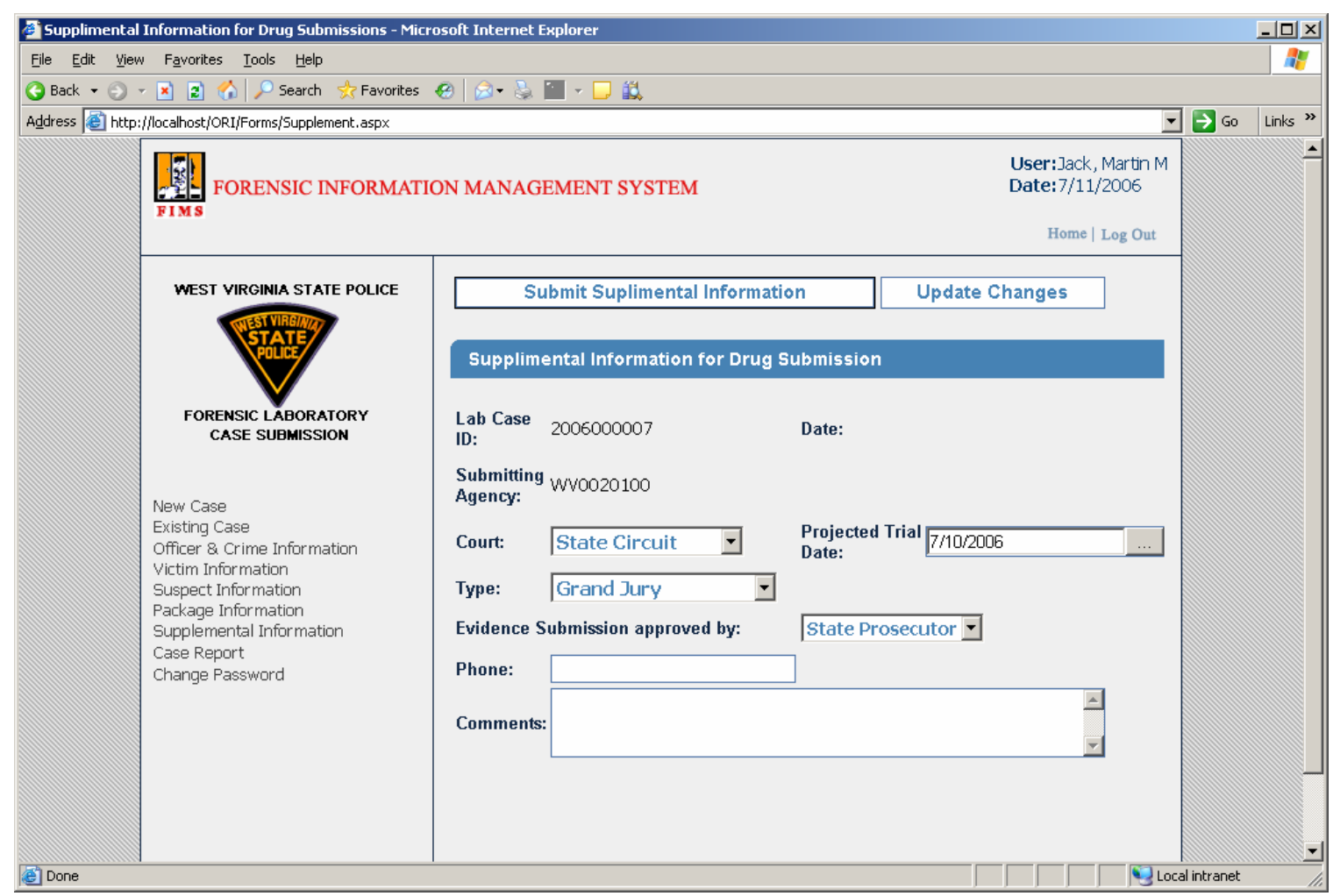

Figure 2.14: Supplemental Information Page 


\section{Printing and exporting DPS-53 form}

After completing all of the information for a case, the various law enforcement agencies can view the case submission report. The case submission report resembles the existing form and follows the same order of reporting. The user can also print the case report by the conventional print function in the browser. All of the forms are made userfriendly with dropdown lists wherever possible. The user can view different forms through a menu structure located on the top of the screen. Changes/additions to an already submitted case can be made easily by opening the respective case. The submitted information is stored in a secure database. An electronic version of the case submission report is shown in Figure 2.15.

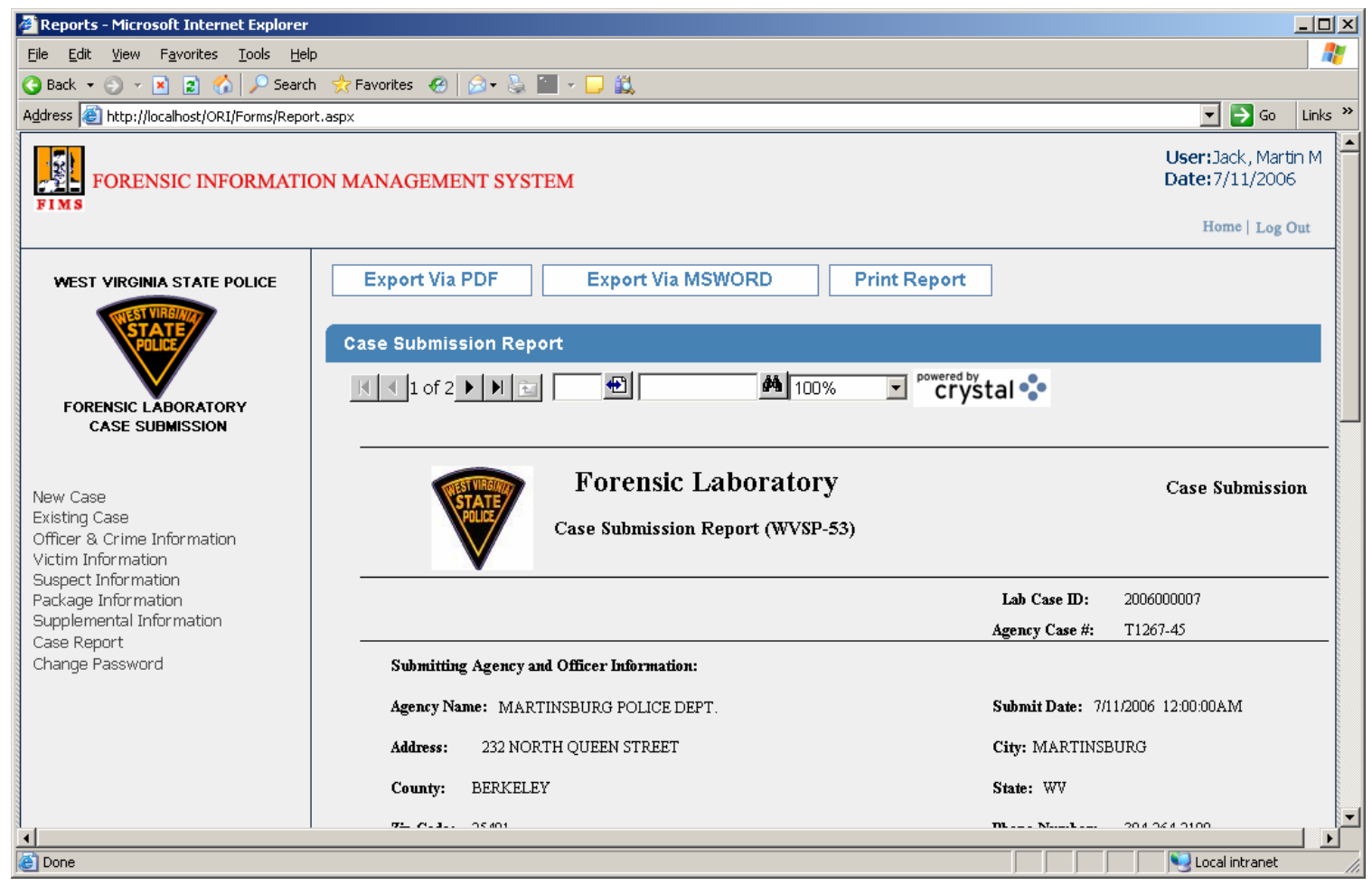

Figure 2.15: Case Report Page

By clicking on the Export via PDF / MS-Word button the case submission report can be exported in PDF/MS-Word format respectively. A Sample PDF report is shown in Figure 2.16. 


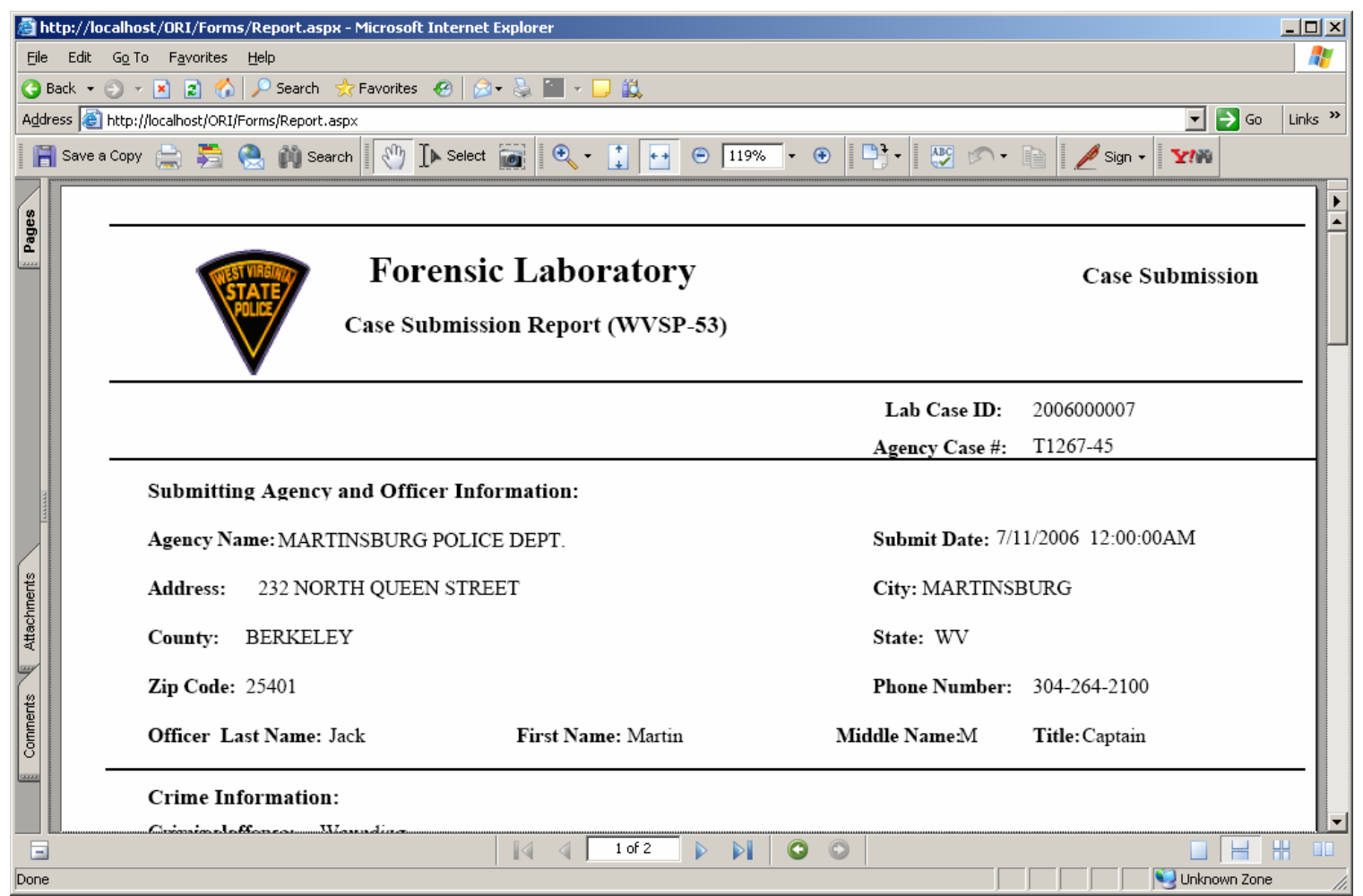

Figure 2.16: PDF Version of Case Submission Report

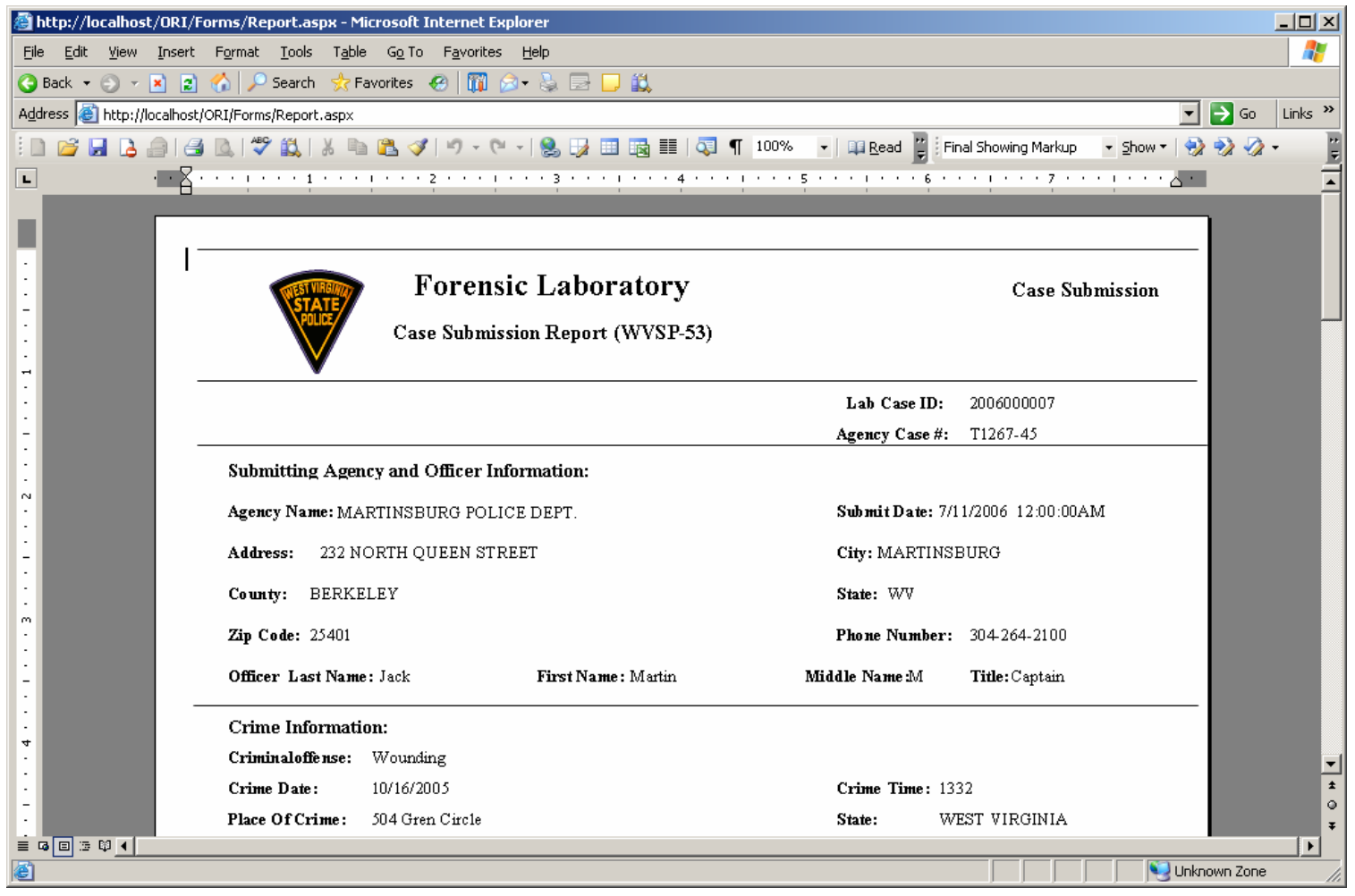

Figure 2.17: MS Word Version of Case Submission Report 


\section{Change User Password}

Users can change their password through this section by providing appropriate user credentials as shown in Figure 2-18.

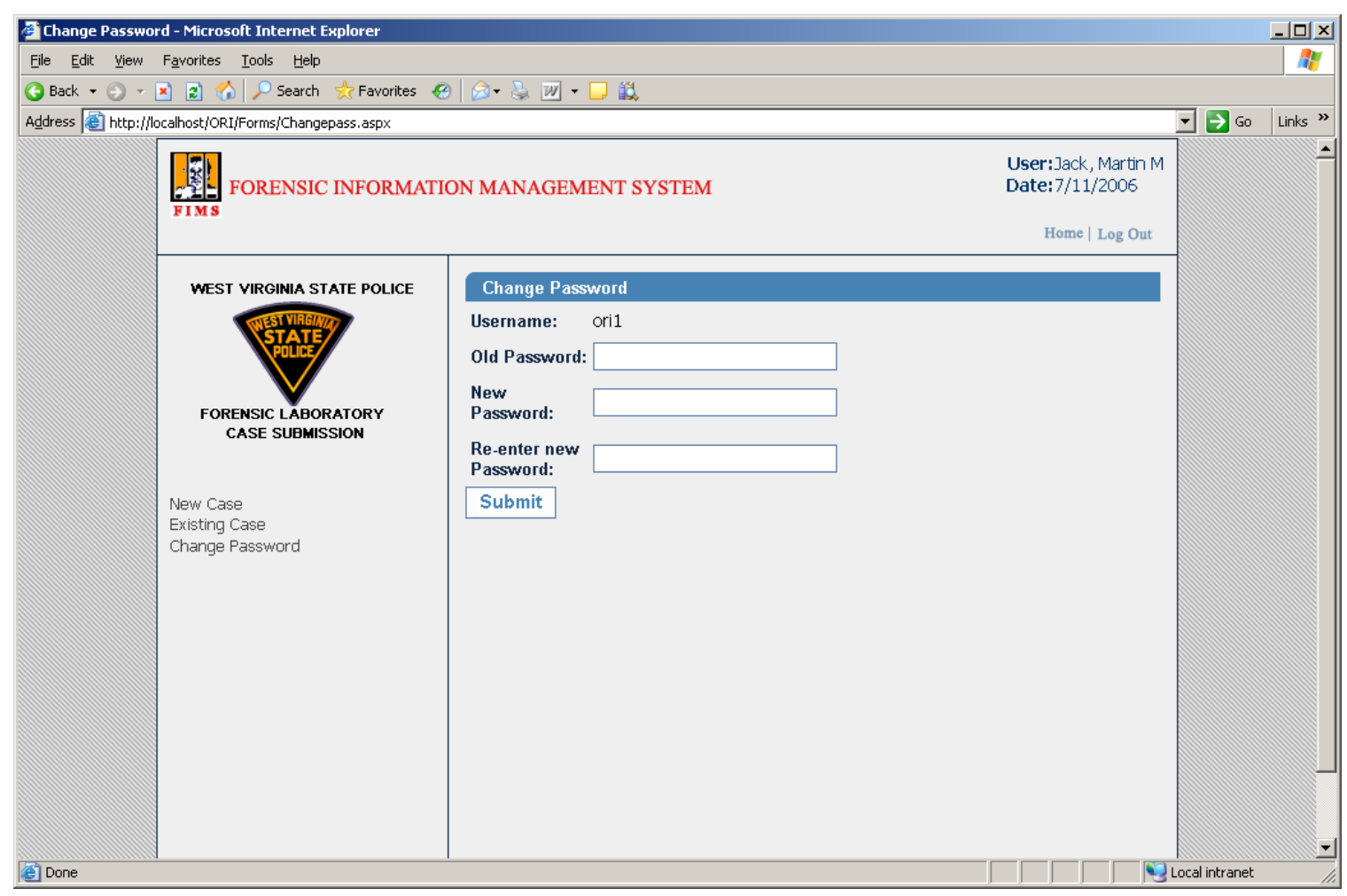

Figure 2.18: Change Password Section

\section{Exiting case submission}

User can terminate the current session by clicking on logout hyperlink on the top right corner of the page. 


\section{CHAPTER 3 \\ CENTRAL EVIDENCE RECEIVING}

\subsection{Existing Central Evidence Receiving Process}

The Central Evidence Receiving (CER) unit is responsible for receiving form DPS-53 and packages from ORI agencies. Hand written form DPS-53 is filled by ORI agency and then packages are sent via mail or personal delivery. CER can accept/deny form DPS-53. If accepted CER inventories packages received otherwise the respective agency is informed of the reason(s) for denial. Inventoried packages are then sent to CEP.

CER receives packages from the ORI agencies via mail or personal delivery. If the items within a package are for a single unit, CER sends the packages to the appropriate unit. The analyst in the unit then inventories the items within the packages using a Laboratory Evidence Inventory Form (LEIF). If the items are for multiple units, the packages are sent to processing. The Processing unit inventories the items in the packages using a LEIF based on CR and the items received. Figure 3.1 shows a LEIF form. After inventorying the items, the items are placed in bulk storage and placed in appropriate bags/containers depending upon its size. Each item is tagged and given an item number. Photographs of the packages are also taken. The processing unit then sends items to the appropriate unit(s) for testing. Processing unit completes a new LEIF form that details the items submitted to various units. The processing places completed LEIF form and CR form in an envelope and deliver it to the appropriate unit's mailbox. The analyst then collects the items and completes a LEIF form for the respective unit. The analyst initials the LEIF form and case submission form if items and other information match on both forms. The CER maintains inventory control via LEIF forms and performs inventory check every three months. If any of the items on the LEIF does not match/exists, then CER calls the respective units to verify. CER is also responsible for the chain-of-custody management. Chain of Custody is a process to maintain and document the chronological history of the evidence [16]. 
Laboratory Evidence Inventory Form

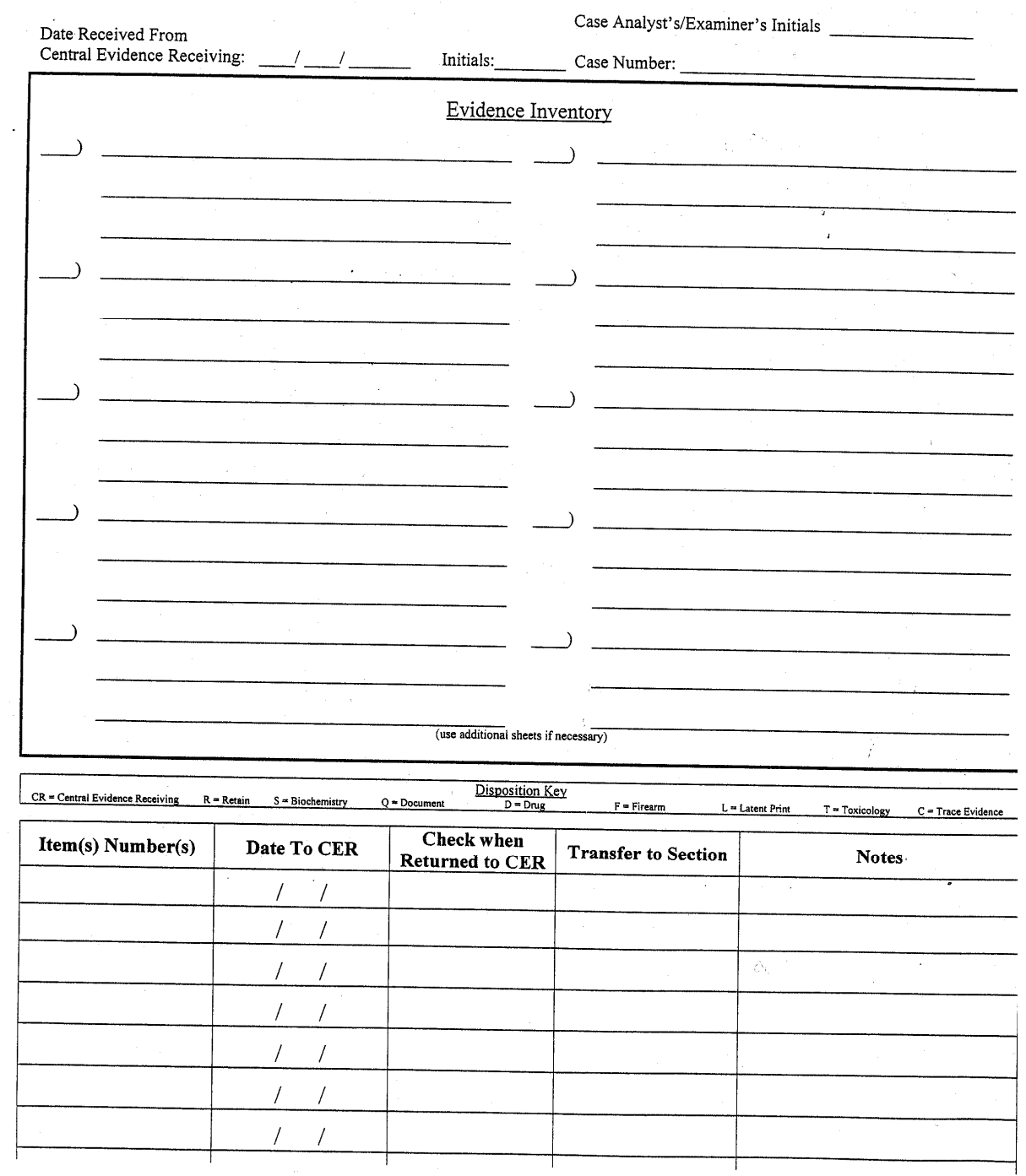

Figure 3.1: LEIF Paper Form

Chain-of-custody is essential for maintaining control of the evidence from the moment it is collected till it is accepted in court. CER maintains chain-of-custody form shown in Figure 3.2. This form remains with the evidence from the time it was received to its final disposition.

At each stage of evidence handling, hand written "hard copies" of all the records are maintained. After each application's paperwork is complete, it is entered through a computer software package that interfaces with an oracle-based Laboratory Information Management System (LIMS) server database, where the information is stored for future 
retrieval. Thereafter, any incident that reveals a correlation with past criminal activity may incite a search for particular case data in the LIMS database. The LIMS system is the primary source of data search. Furthermore, actual paper-based copies of case data are accumulated within the facility [16].

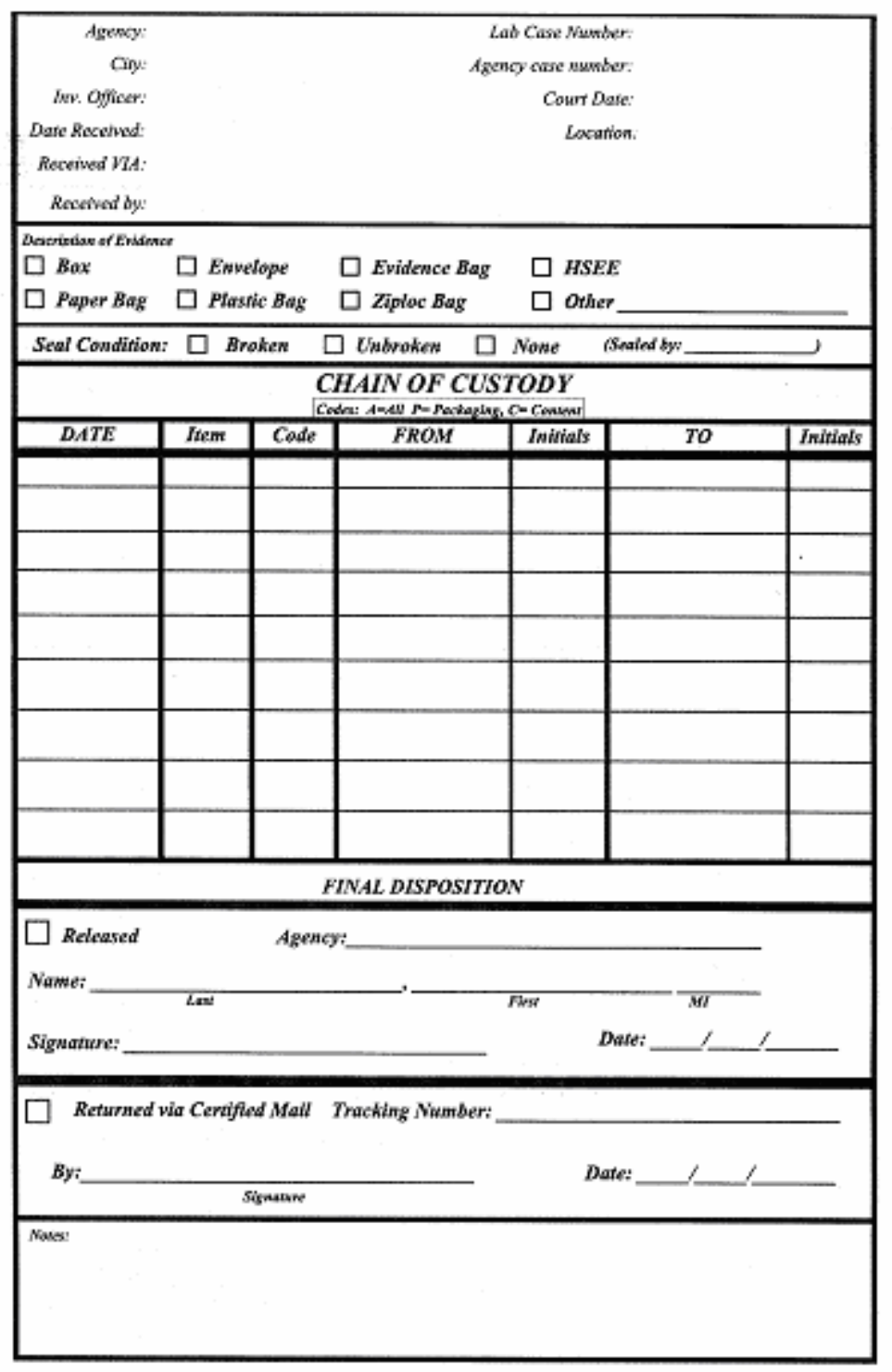

Figure 3.2: Chain of Custody paper form 


\subsection{Re-engineering the Central Evidence Receiving Process}

Figure 3.3 shows the evidence and information flow from ORI to CER module. The CER receives evidence package from ORI agencies via mail or personal delivery.

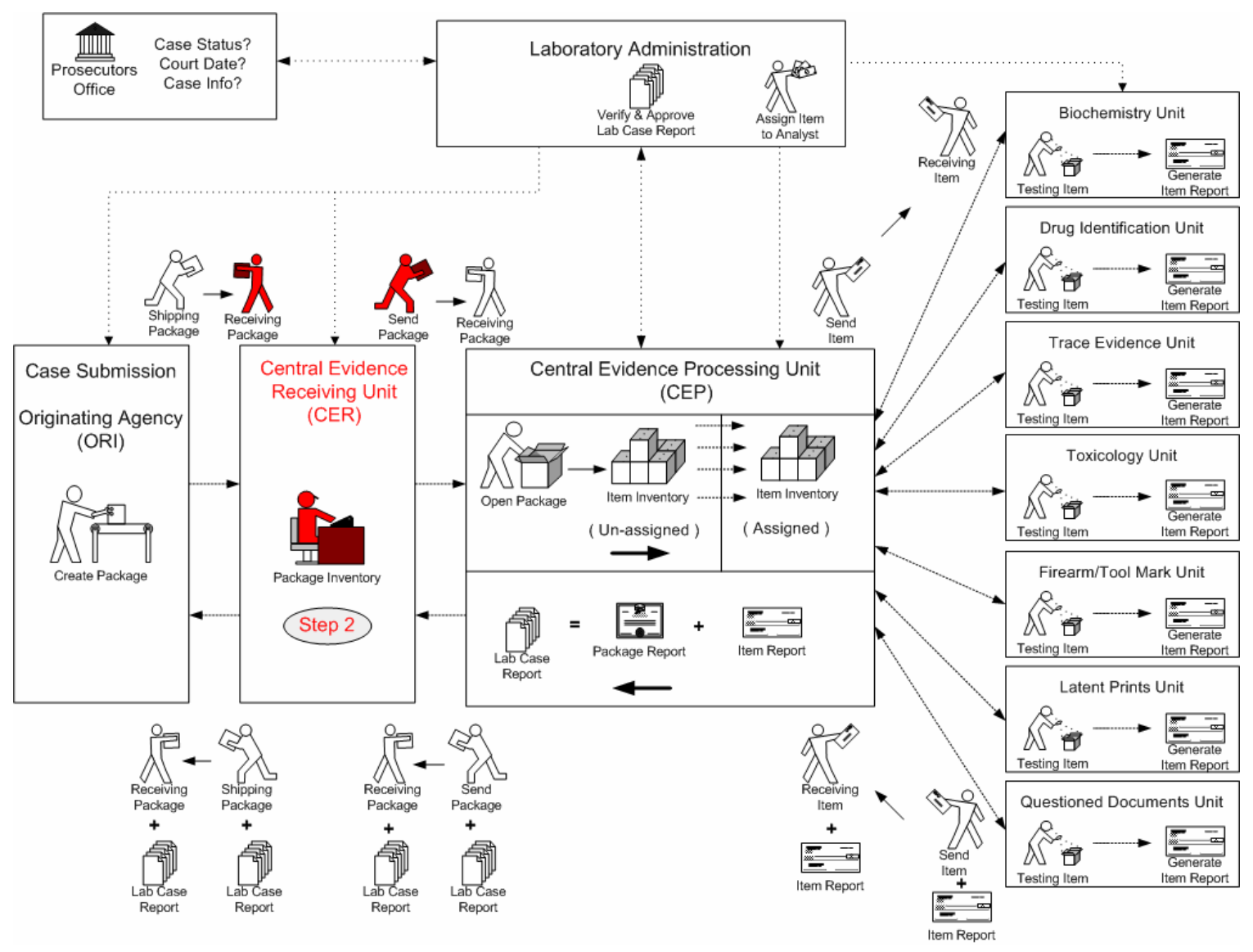

Figure 3.3 Information \& Evidence flow 
Figure 3.4 shows the evidence and information flow from Central Evidence Processing (CEP) to CER module. CER unit receives the evidence package and the final approved laboratory case report back from CEP unit and sends it back to the ORI agency.

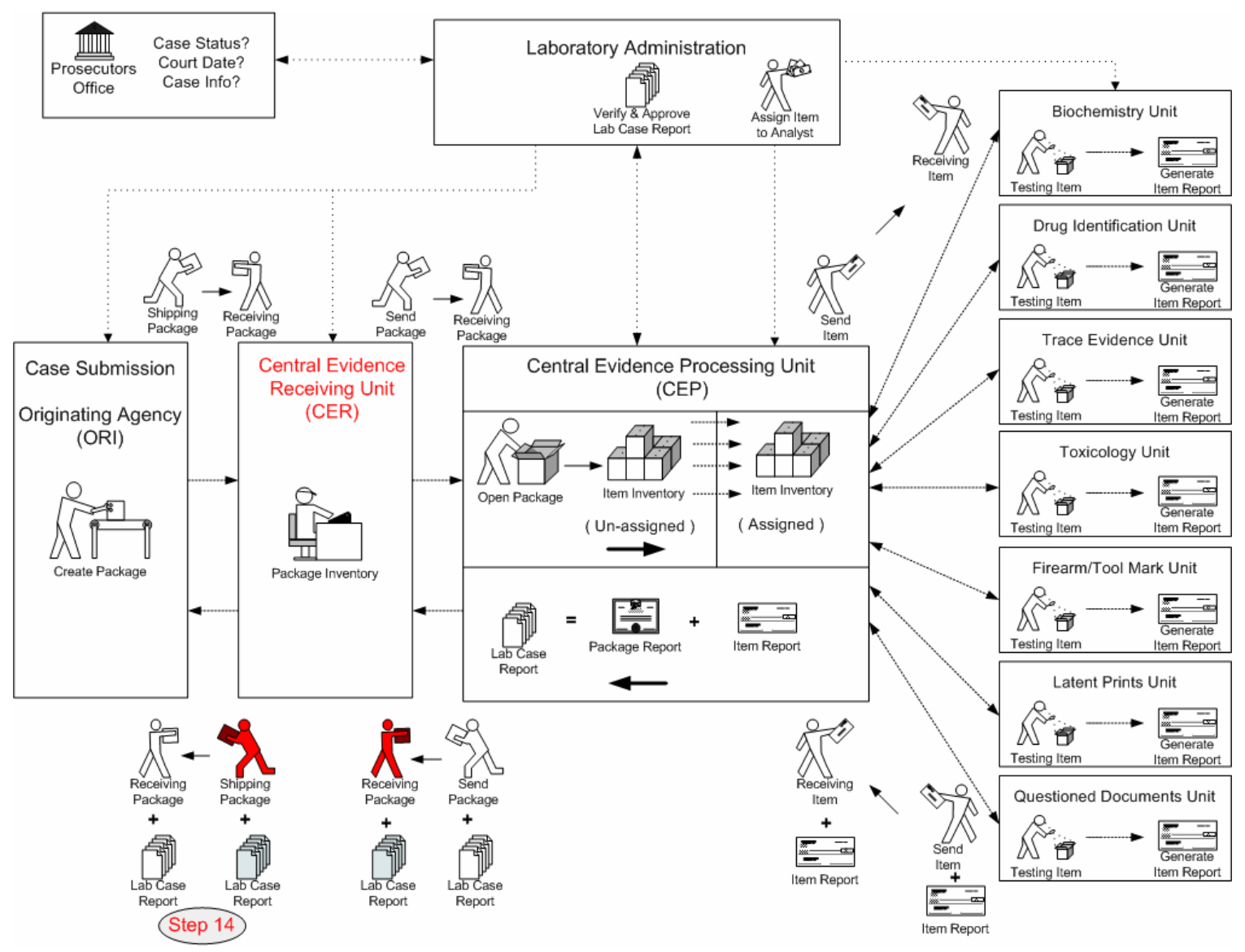

Figure 3.4: Receive Package\& Final Report from CEP 


\subsubsection{Central Evidence Receiving Entity Relationship Diagram}

CER data table are connected with each other through relations. Figure 3.5 shows relational diagram between CER data tables.

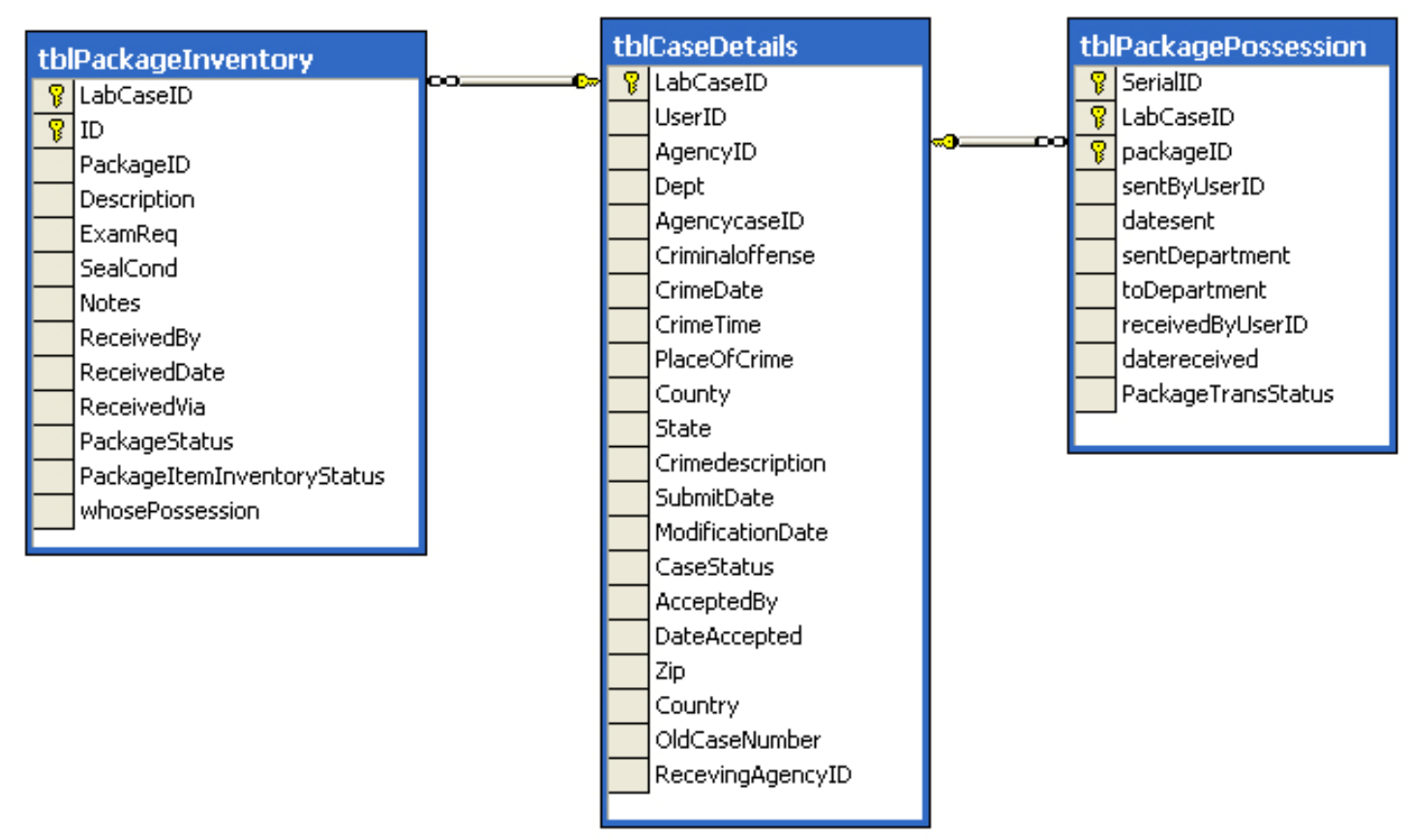

Figure 3.5: Entity Relationship Diagram 


\subsubsection{Central Evidence Receiving Database Tables}

The CER module stores its data in the following tables:

- Package Inventory Information (tblPackageInventory)

- Package Chain of Custody Information (tblPackagePossession)

The various fields of the Package Inventory information table are shown in Table

3-1. This table stores package inventory information. Package seal condition, notes, received by, date received, inventory status and custody.

\section{Table 3-1: Package Inventory Table}

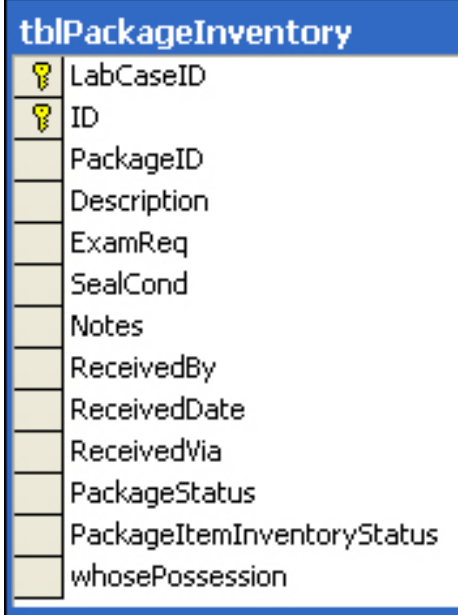

The various fields of the Package chain of custody information table are shown in Table 3-2. Package chain of custody is tracked by the information stored in tblPackagePossession. Package sent by, sent to, dept by, dept to with its respective date and time is stored.

Table 3-2: Package chain of custody Table

\begin{tabular}{|l|l|}
\hline tblPackagePossession \\
\hline B & SerialID \\
\hline E & LabCaseID \\
\hline E & packageID \\
\hline & sentEyUserID \\
\hline & datesent \\
\hline & sentDepartment \\
\hline & toDepartment \\
\hline & receivedByUserID \\
\hline & datereceived \\
\hline & PackageTransStatus \\
\hline
\end{tabular}




\subsubsection{Working with Central Evidence Receiving}

CER unit home page requires username and password to verify user credentials, as shown in the Figure 3.6. After verifying credentials, a user is directed to list of cases submitted by the ORI agencies.

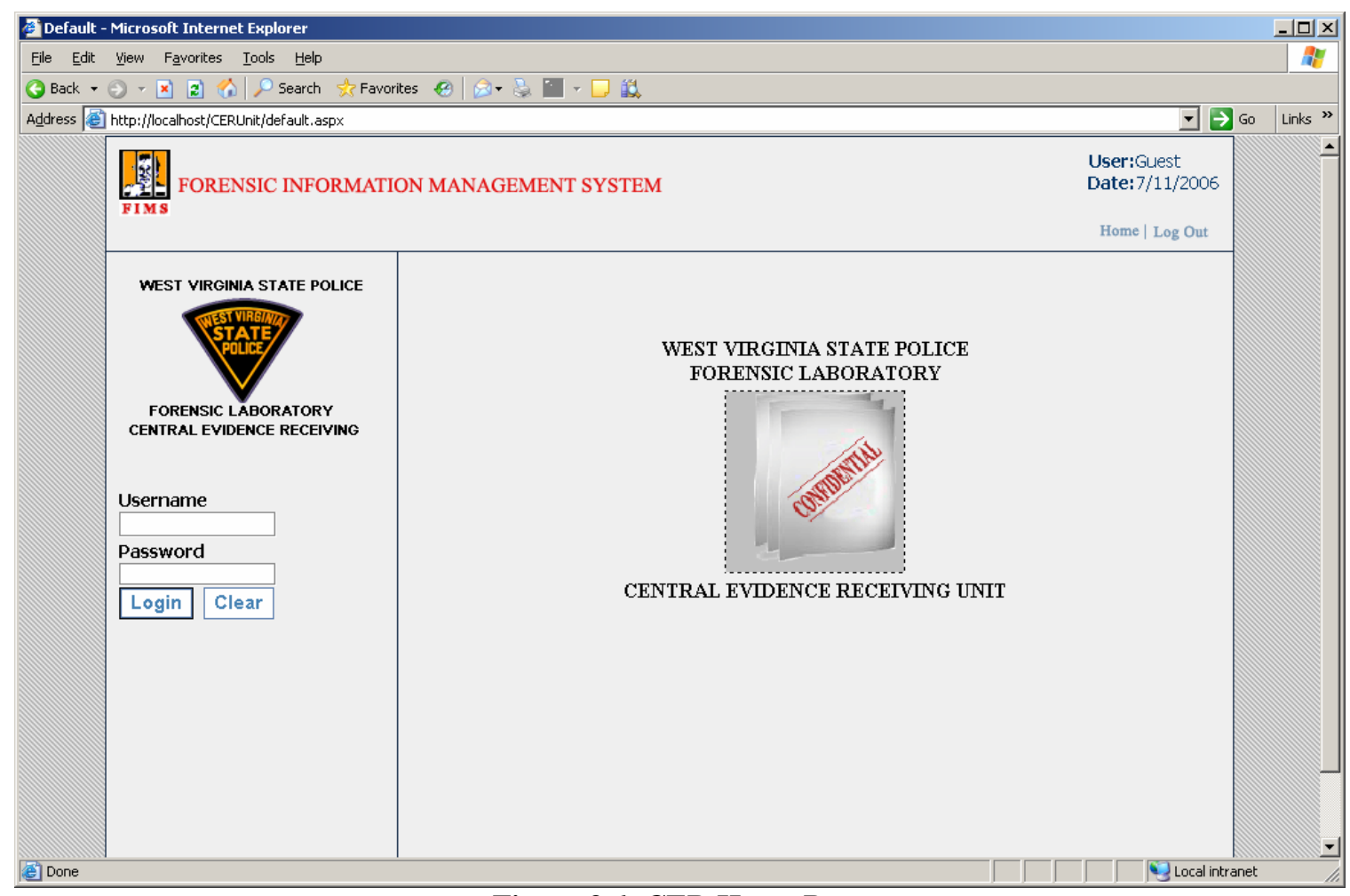

Figure 3.6: CER Home Page 
Figure 3.7 shows the list of cases submitted. Users can search for the desired case and view case information by clicking on view hyperlink.

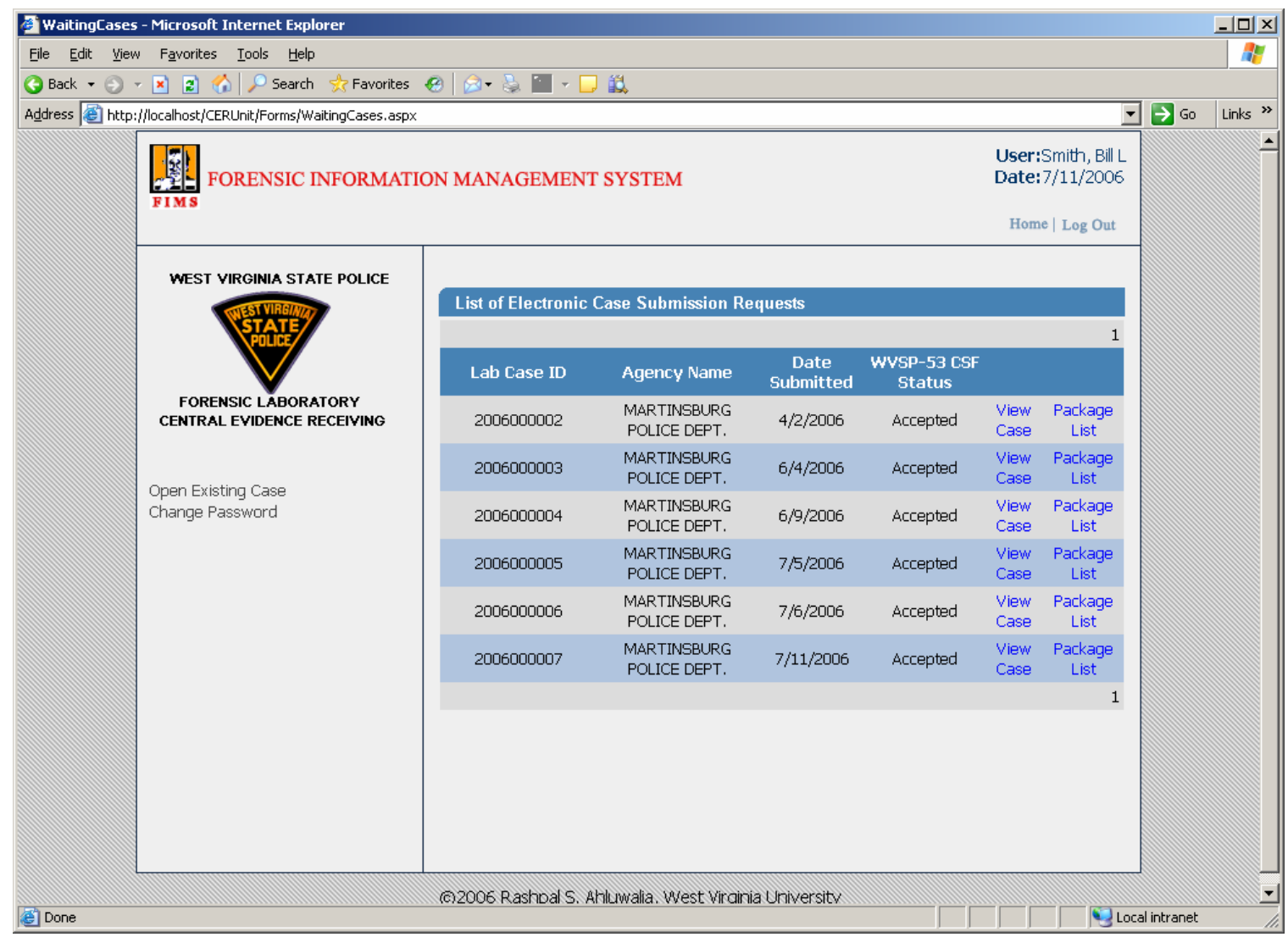

Figure 3.7: List of cases submitted 
Users can perform two kinds of operations from this section i.e. update case status and package inventory. This section is used to update the case status. Initially case status is set to "waiting" by default. Users can change the status to "accepted" or "denied" through this section, as shown in Figure 3.8.

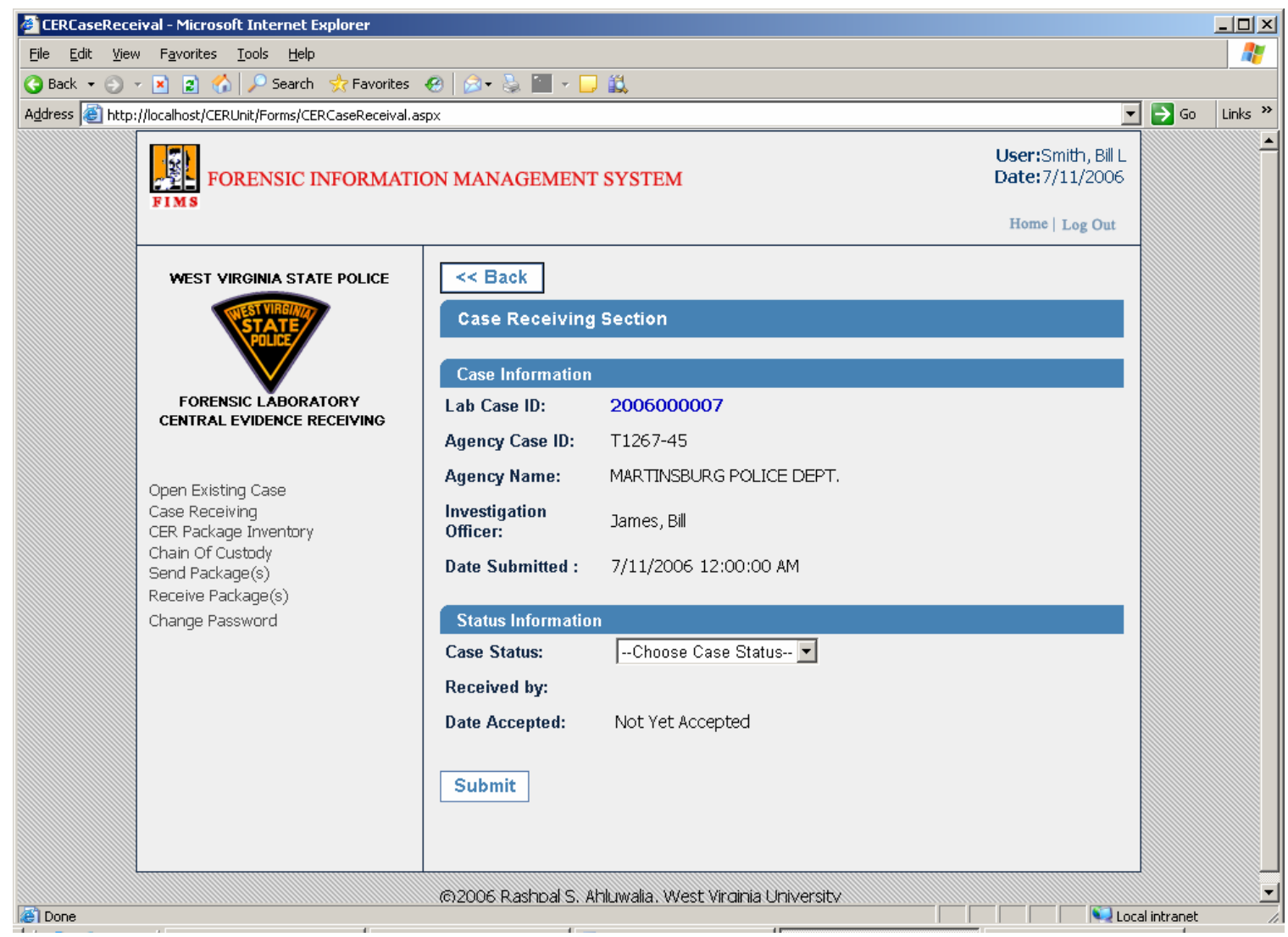

Figure 3.8: Case receiving form 


\section{Package Inventory}

Users can inventory packages received using this section. It displays the list of packages expected for that case and respective inventory status. Receive package section can be accessed by clicking on "Change Status" hyperlink as shown in Figure 3.9.

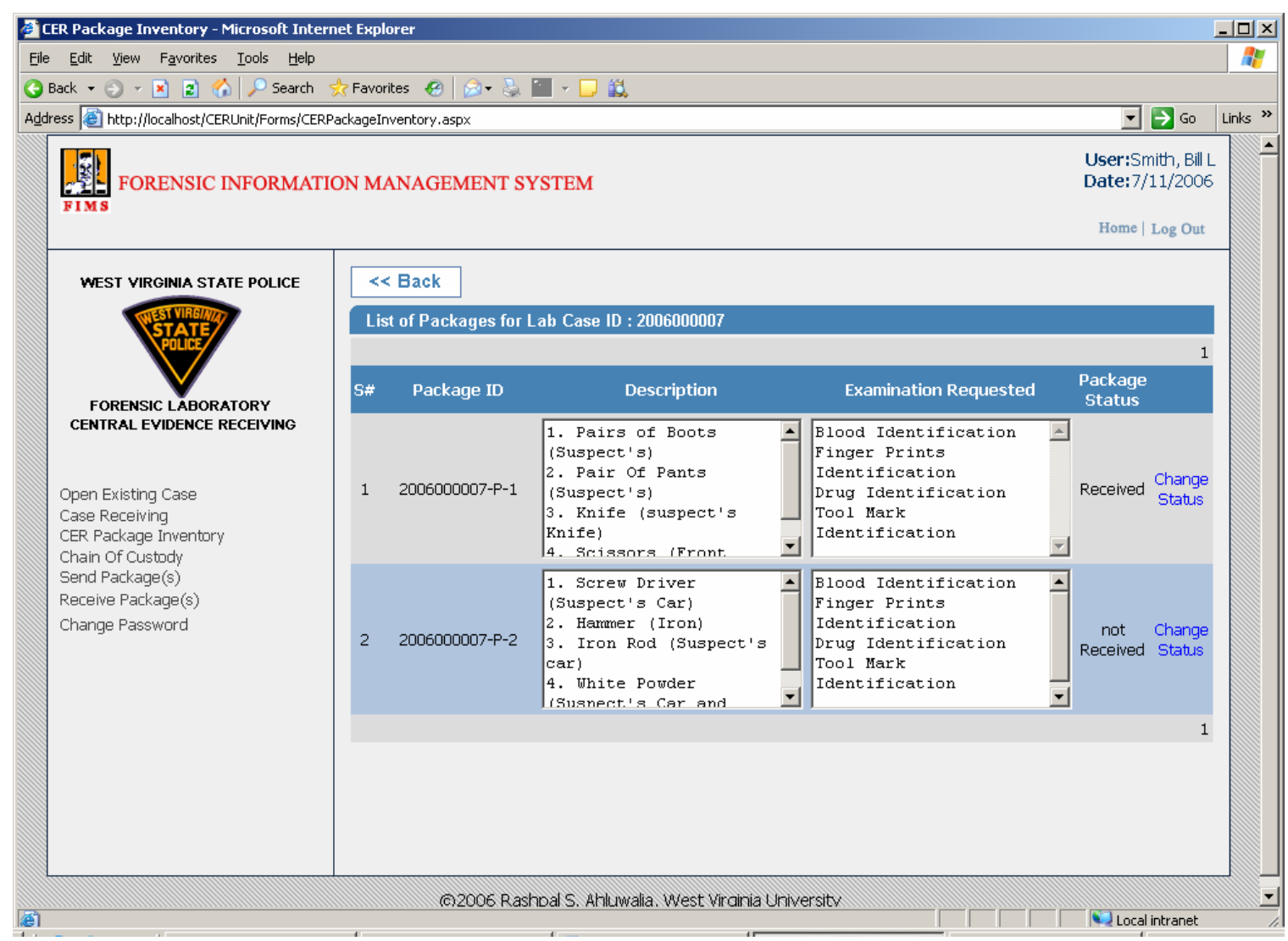

Figure 3.9: Package list 
This section enables users to inventory packages by changing their respective acceptance status. Received via, seal condition and package status dropdown list enable users to make appropriate choice. Additional notes can be attached with a package by filling notes text box on the bottom of the page, as shown in the Figure 3.10.

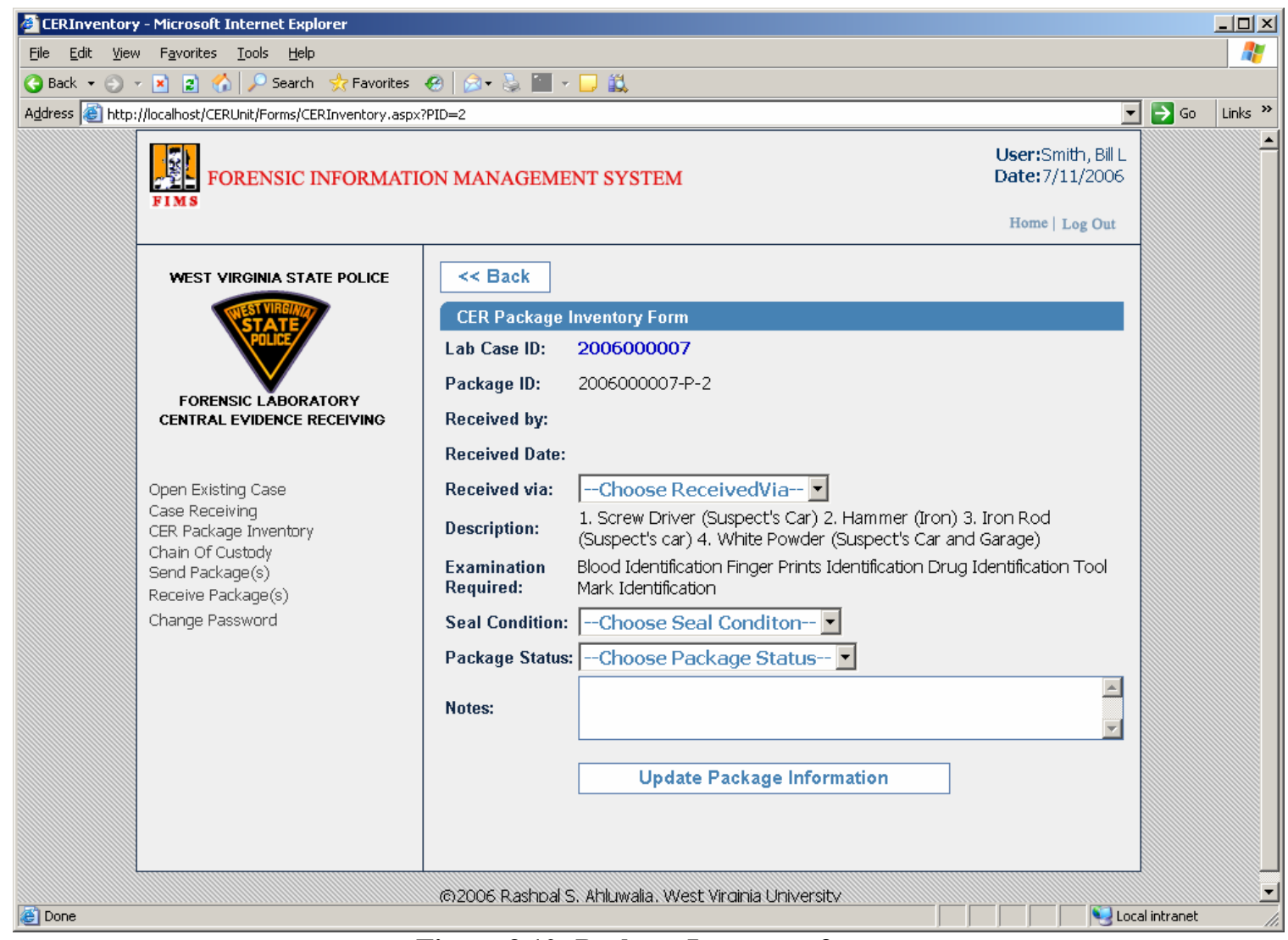

Figure 3.10: Package Inventory form 


\section{Chain of Custody}

Maintaining chain of custody of the evidence packages/items is integral part of the system. Users can view chain of custody of the packages by clicking on "Chain of Custody" hyperlink in the side navigation bar, as shown in Figure 3.11. Chain of custody tracks transfer of packages/items between units and associates time stamp with each transfer.

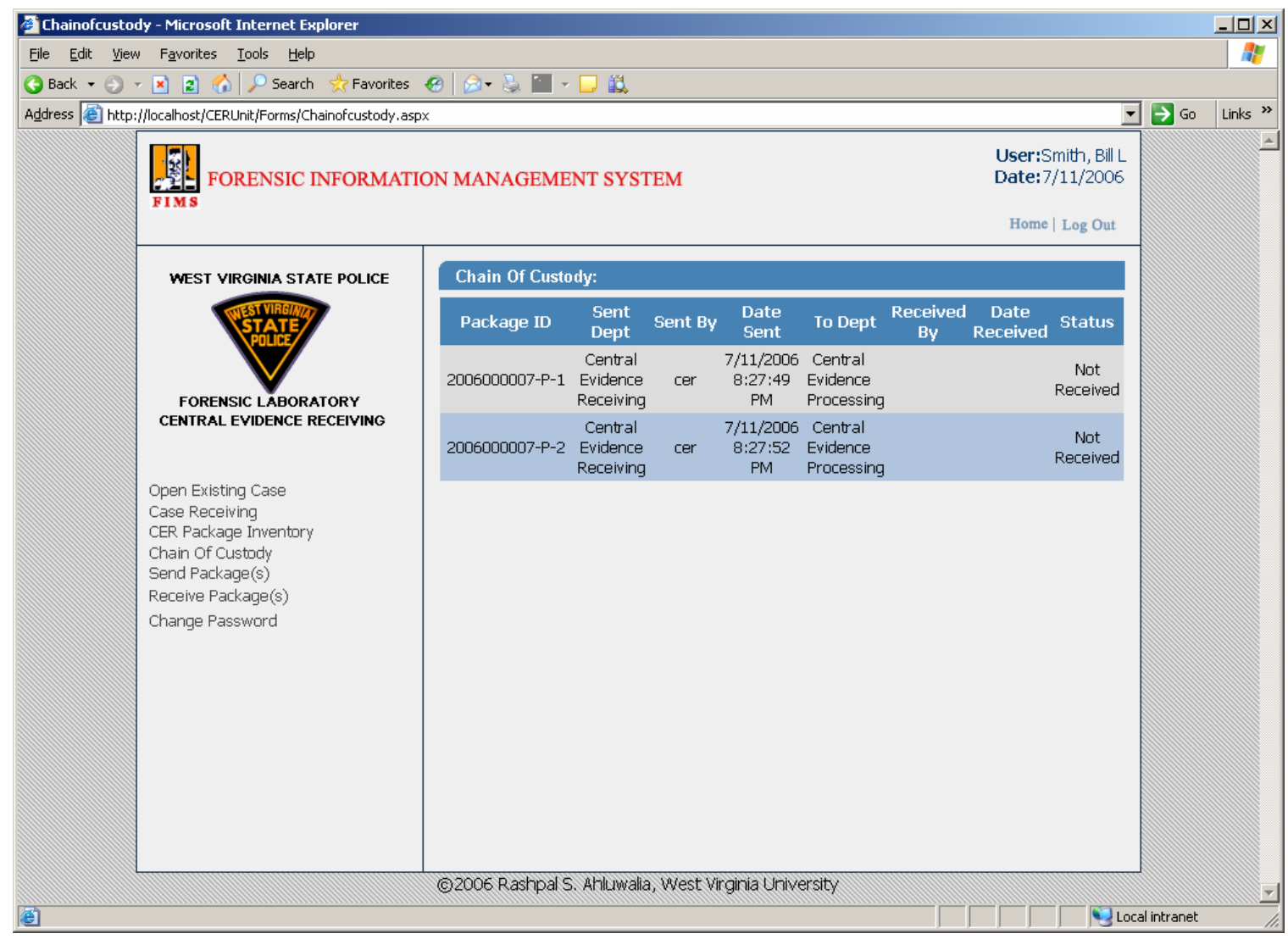

Figure 3.11: Chain Of Custody 


\section{Send Package}

Users can transfer packages from one unit to another by using the "Send Package" hyperlink. List of packages that are accepted is available through a dropdown list, as shown in Figure 3.12. Users can select one package at a time and click "Send" hyperlink to initiate the process of package transfer. It is a two step process, requesting package pickup by source unit and actual package pickup by the destination unit. This process is complete when the destination unit collects the package form the source unit.

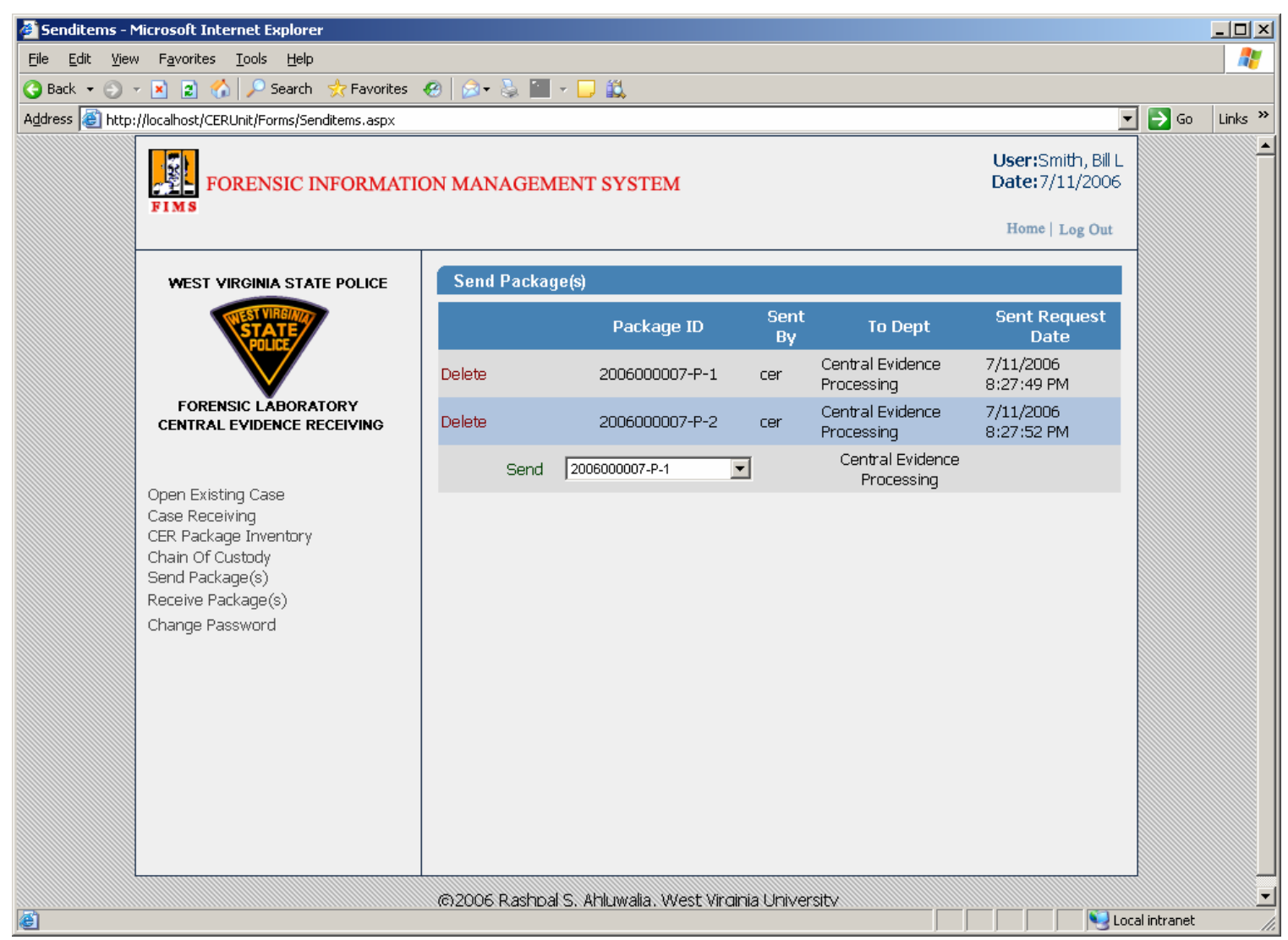

Figure 3.12: Send Package Form 


\section{Receive Package}

This is opposite of send package process. Users can collect package from the source unit through this section, as shown in Figure 3.13. Once user collects a package and click on "Receive" button the transfer process is complete and package custody information in the database is updated.

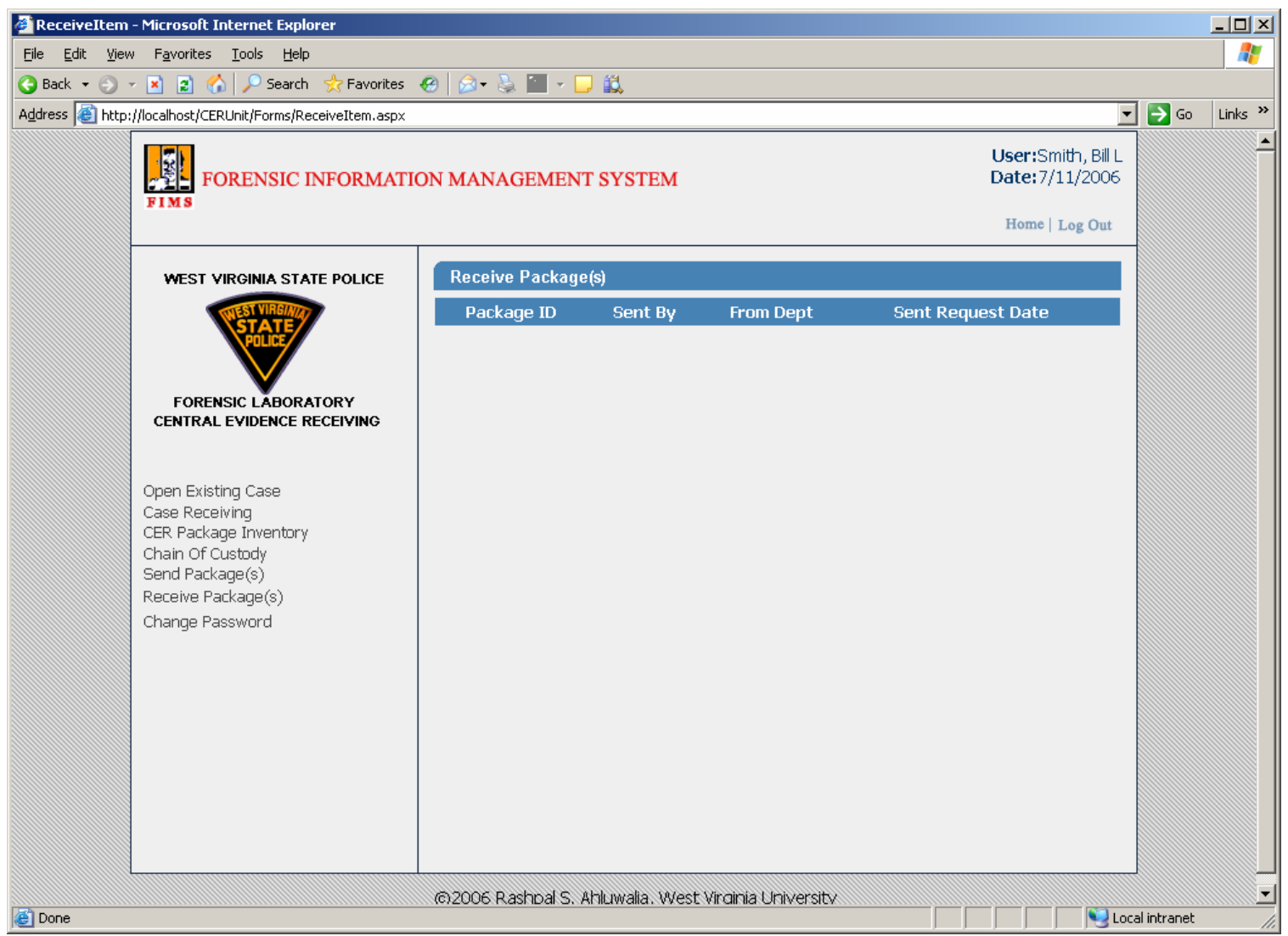

Figure 3.13: Receive Package Form 


\section{Change User Password}

User can change password through this section by providing appropriate user credentials as shown in Figure 3.14.

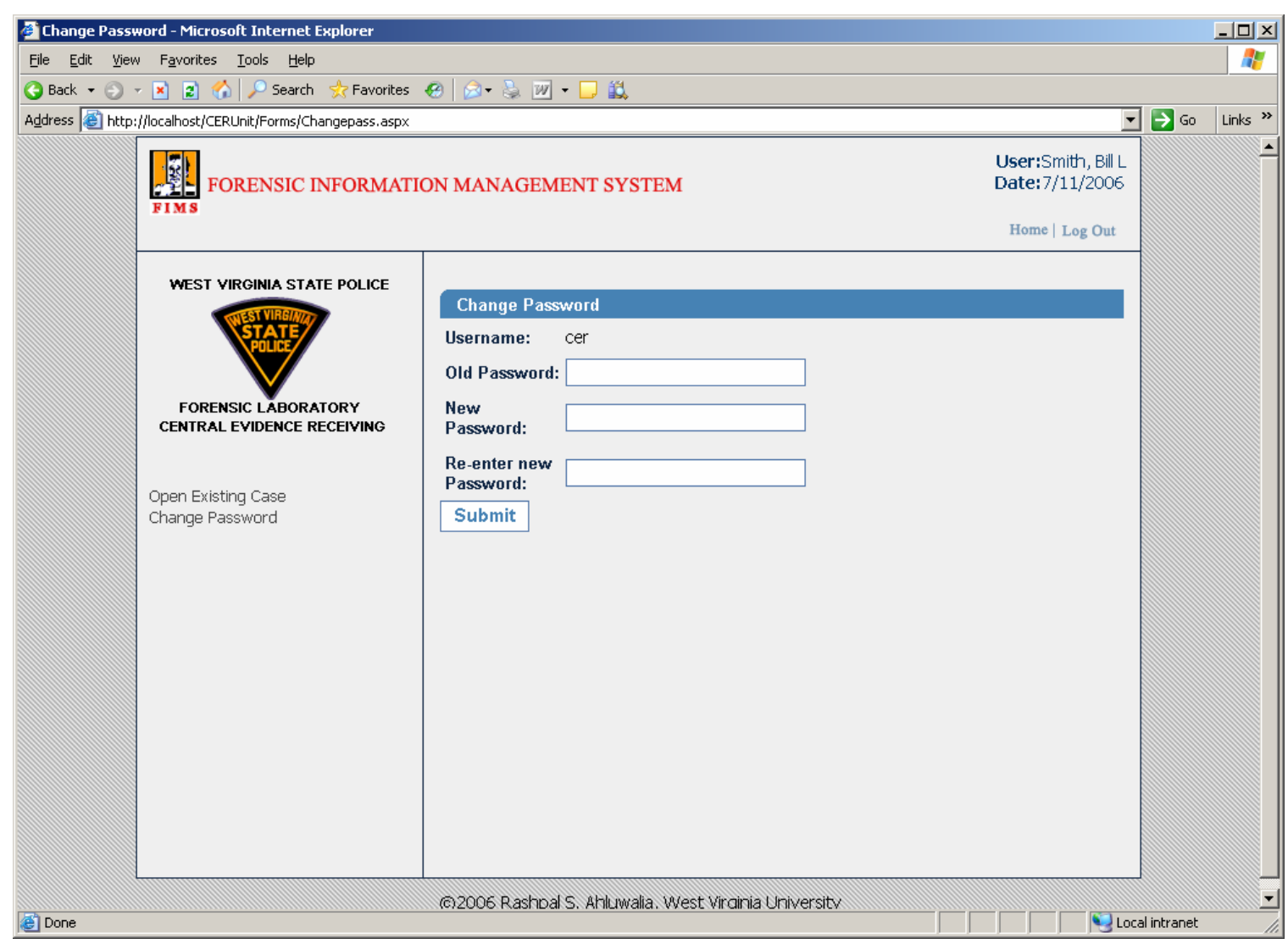

Figure 3.14: Change Password Section

\section{Exiting Central Evidence Receiving}

Users can terminate the current session by clicking on "Log Out" hyperlink on the top right corner of the page. 


\section{CHAPTER 4 \\ CENTRAL EVIDENCE PROCESSING}

\subsection{Central Evidence Processing Process}

Central Evidence Processing (CEP) unit was recently created by splitting the CER unit into two parts i.e. CER and CEP. An independent CEP unit enables better control of the existing evidence and information flow between different units. CEP receives packages from the CER unit for further processing. Evidence and information flow from CER to CEP module is show in Figure 4.1.

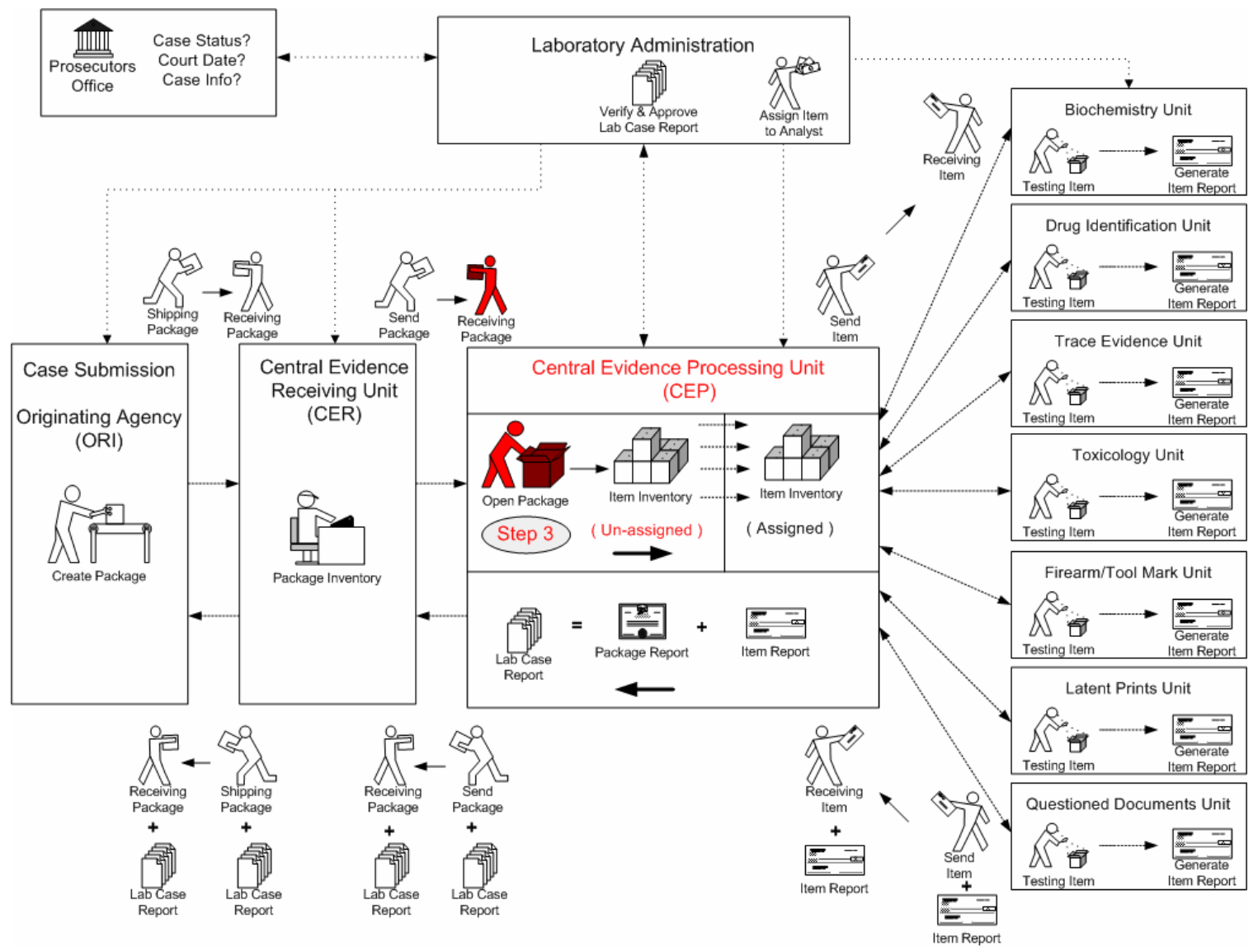

Figure 4.1: Step3-Evidence \& Information flow

CEP is responsible for item inventory and distribution of evidence items among different units. After receiving package from the CER unit, CEP unit is responsible for 
opening the package and inventory items. Item name and description entered by CEP overrides the name and description provided by the ORI officer.

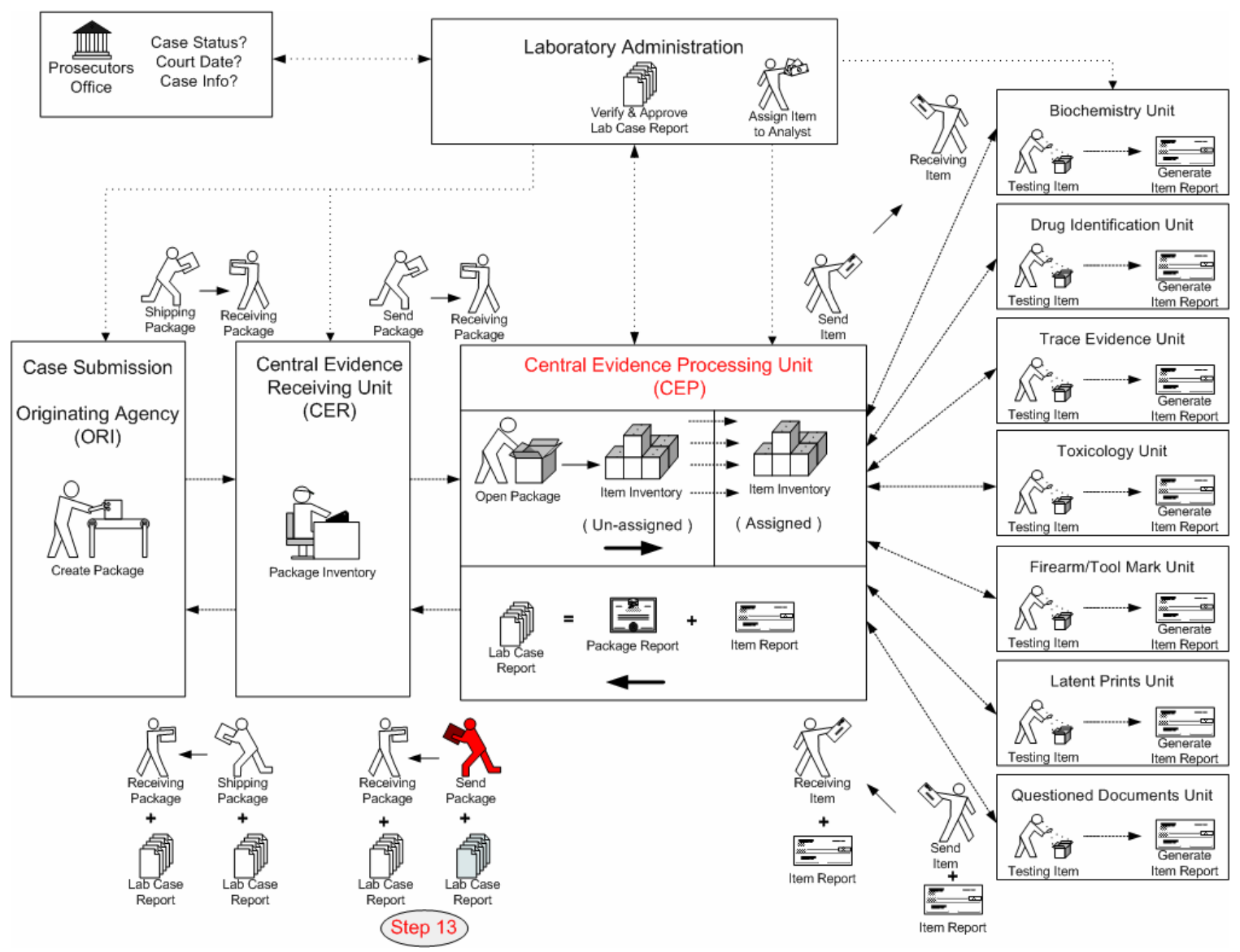

Figure 4.2: Step 13- Evidence \& Information flow

After completion of test and final item report generation, CEP is responsible for compiling package report. Compiled package report is then sent to the administration unit for final review and approval. 


\subsubsection{Central Evidence Processing Entity Relationship Diagram}

CEP data tables are connected with each other through relations as shown in the figure 4.3.

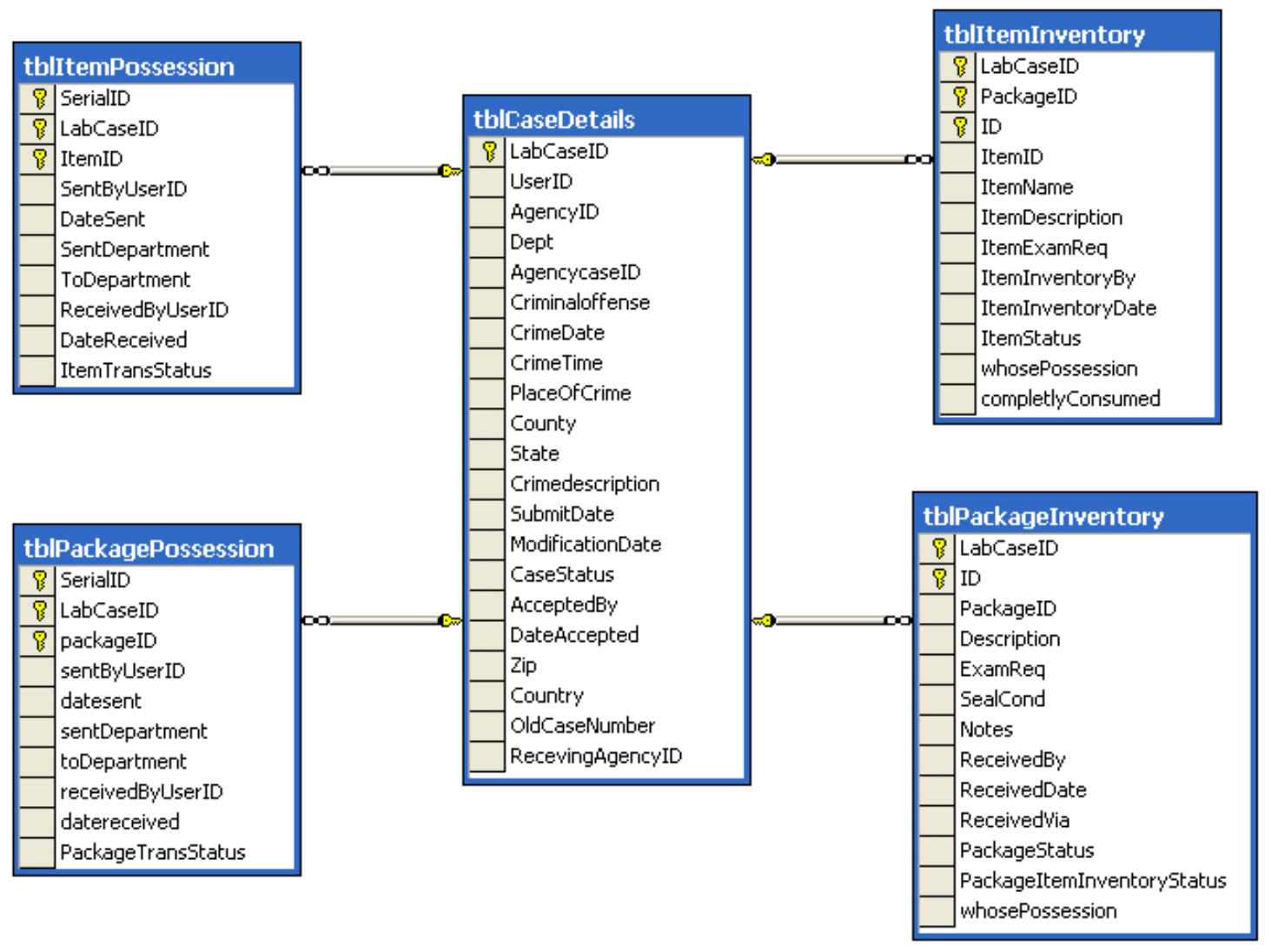

Figure 4.3: Entity relationship diagram 


\subsubsection{Central Evidence Processing Database Tables}

The CEP module stores its data in the following tables:

- Item possession

- Item inventory

- Package inventory

- Package possession

Item chain of custody information is maintained in item possession table as shown in Table 4-1.

Table 4-1: Item Possession Table

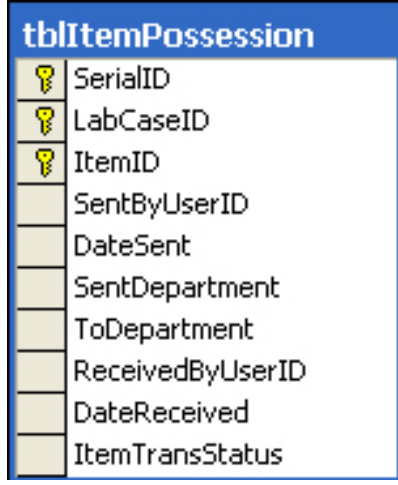

Package inventory is maintained in package inventory table as shown in Table 4-2

Table 4-2: Package Inventory Table

\begin{tabular}{|r|l|}
\hline tblPackageInventory \\
\hline i & LabCaseID \\
\hline & ID \\
\hline & DesckageID \\
& ExamReq \\
& SealCond \\
\hline & Notes \\
\hline & ReceivedBy \\
\hline & ReceivedDate \\
\hline & Receivedvia \\
\hline & PackageStatus \\
\hline & PackageItemInventoryStatus \\
& whosePossession
\end{tabular}


Item inventory data is maintained in item inventory table as shown in Table 4-3

Table 4-3: Item Inventory Table

\begin{tabular}{|r|l|}
\hline tblitemInventory \\
\hline ? & LabCaseID \\
\hline ID & PackageID \\
\hline & ItemID \\
& ItemName \\
& ItemDescription \\
& ItemExamReq \\
& ItemInventoryBy \\
& ItemInventoryDate \\
& ItemStatus \\
\hline & whosePossession \\
& completlyConsumed
\end{tabular}

Package possession data is maintained in package possession table as shown in Table 4-4

Table 4-4: Package Possession Table

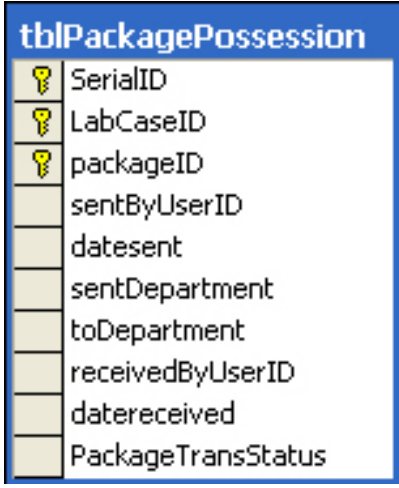




\subsubsection{Working with Central Evidence Processing}

CEP home page requires username and password to verify user credentials, as shown in the Figure 4.4. After verifying credentials, a user is directed to list of items assigned to him/her for further processing.

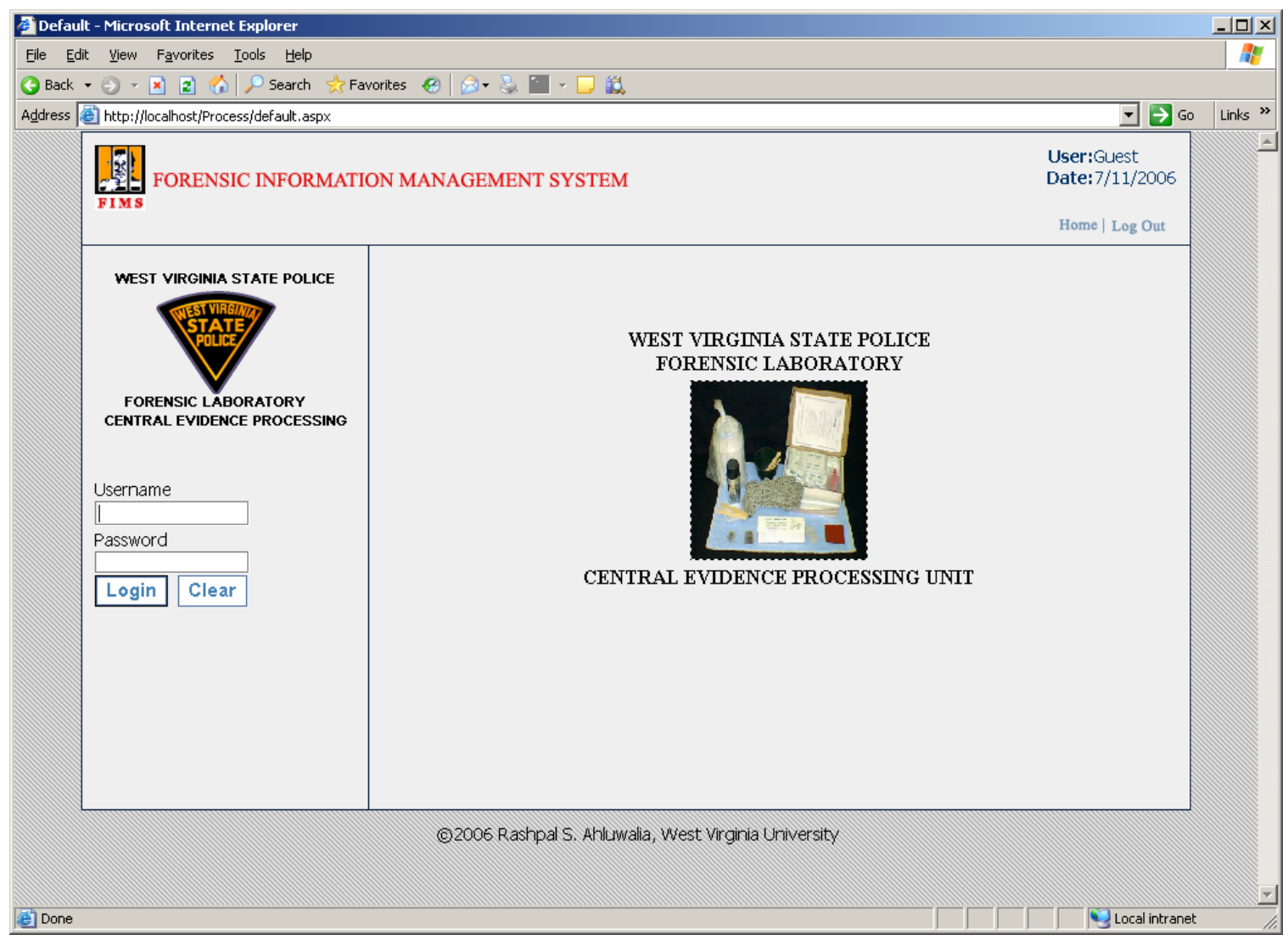

Figure 4.4: CEP Home Page 
Figure 4.5 shows the list of cases accepted by CER unit for further processing. CEP can view the list of packages for each case by clicking on "Package List" hyperlink. This section also enables users to check case status. They can also view case details by clicking on "Case Details" hyperlink. Once a case is selected, FIMS remembers the case selected and all other information related to that case can be accessed by navigating to different sections of the application.

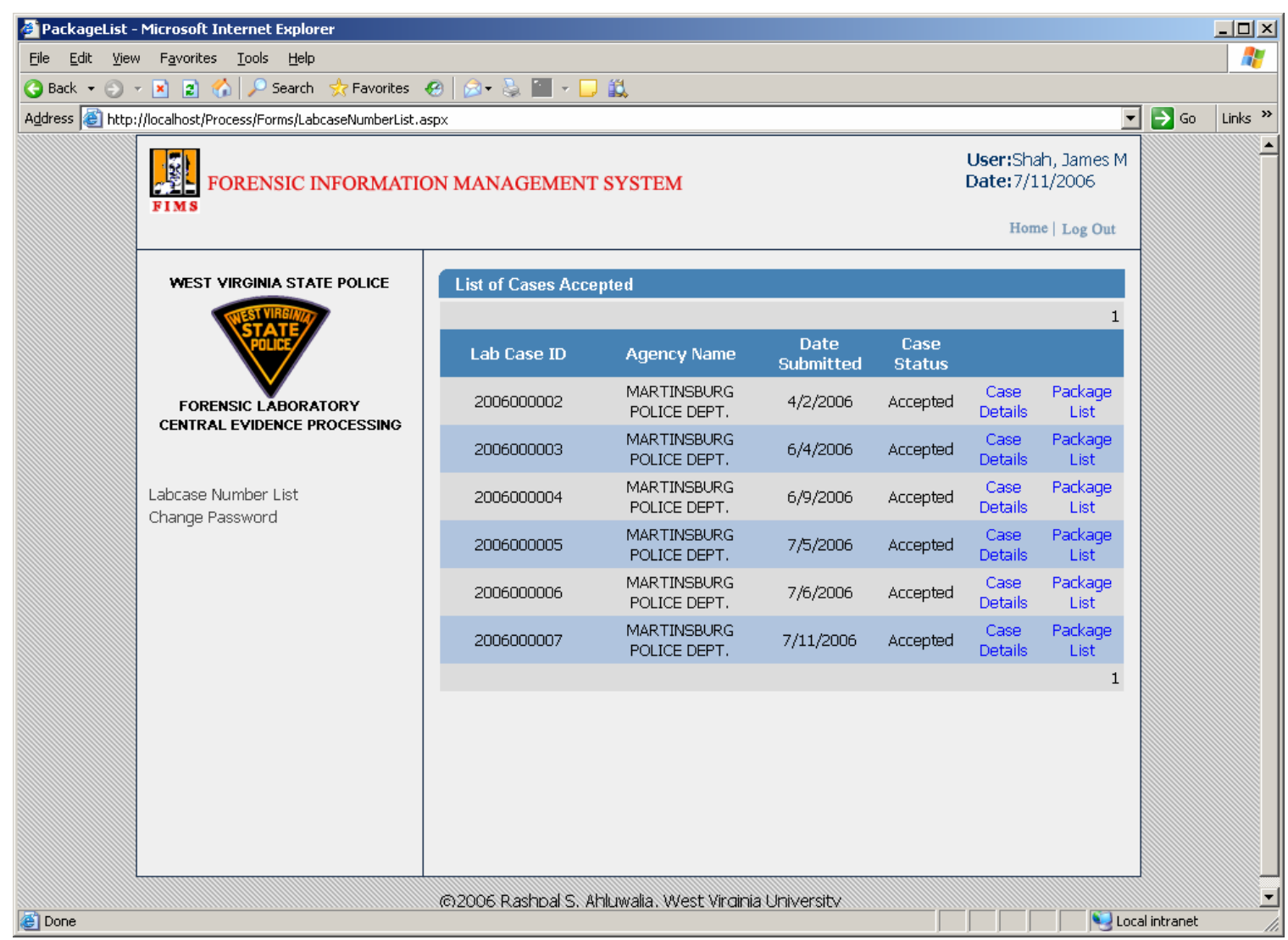

Figure 4.5: Accepted Case List 
Figure 4.6 shows case details, such as case status, received by, and date accepted. Users can search this section by clicking on "Case Details” hyperlink as shown in figure 4.6

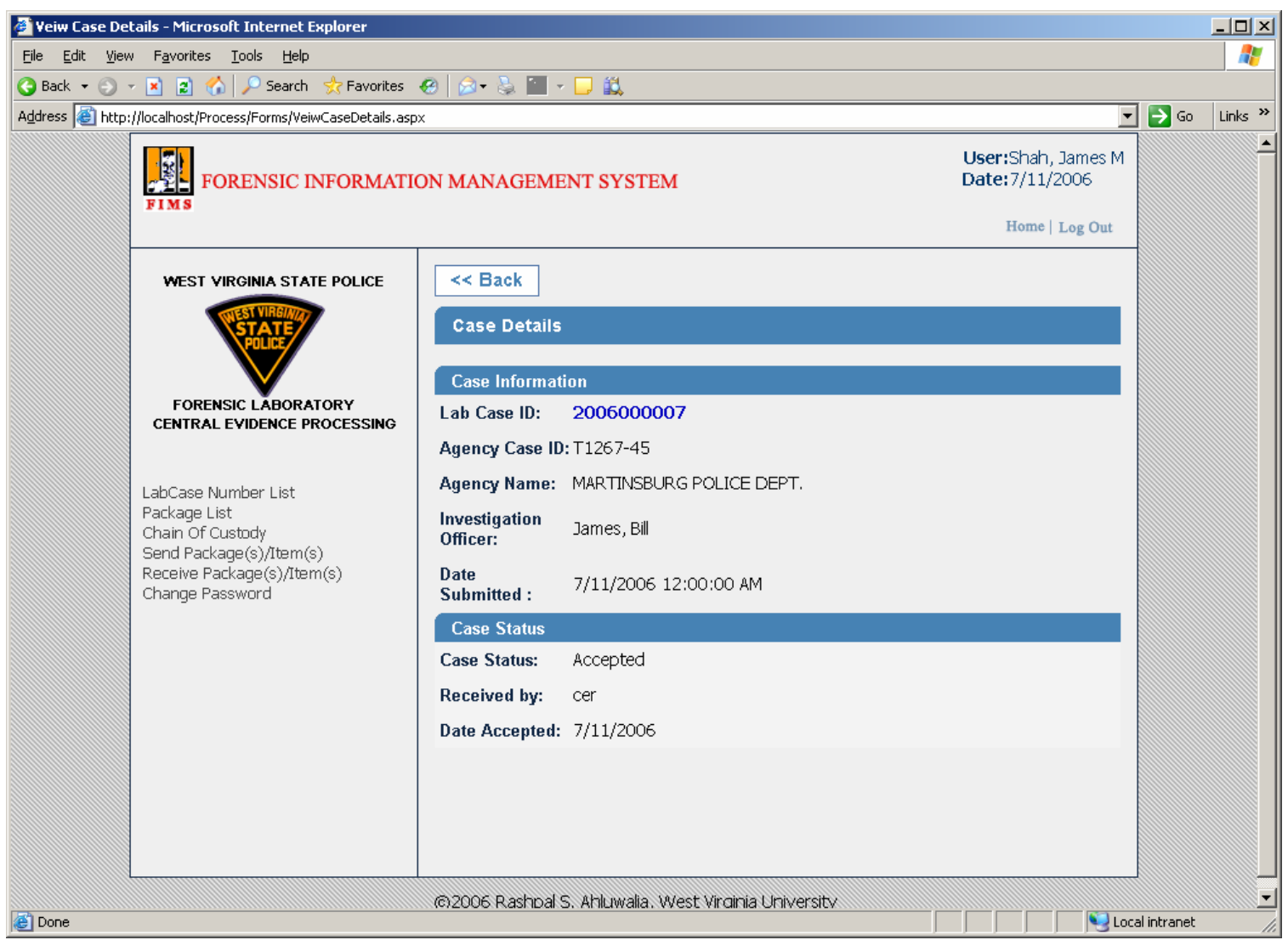

Figure 4.6: Case Details section 


\section{Package Item Inventory}

Figure 4.7 shows the list of packages with their description, examination requested, and item inventory status respectively. User can also check/alter item inventory status by clicking on "Inventory List" hyperlink.

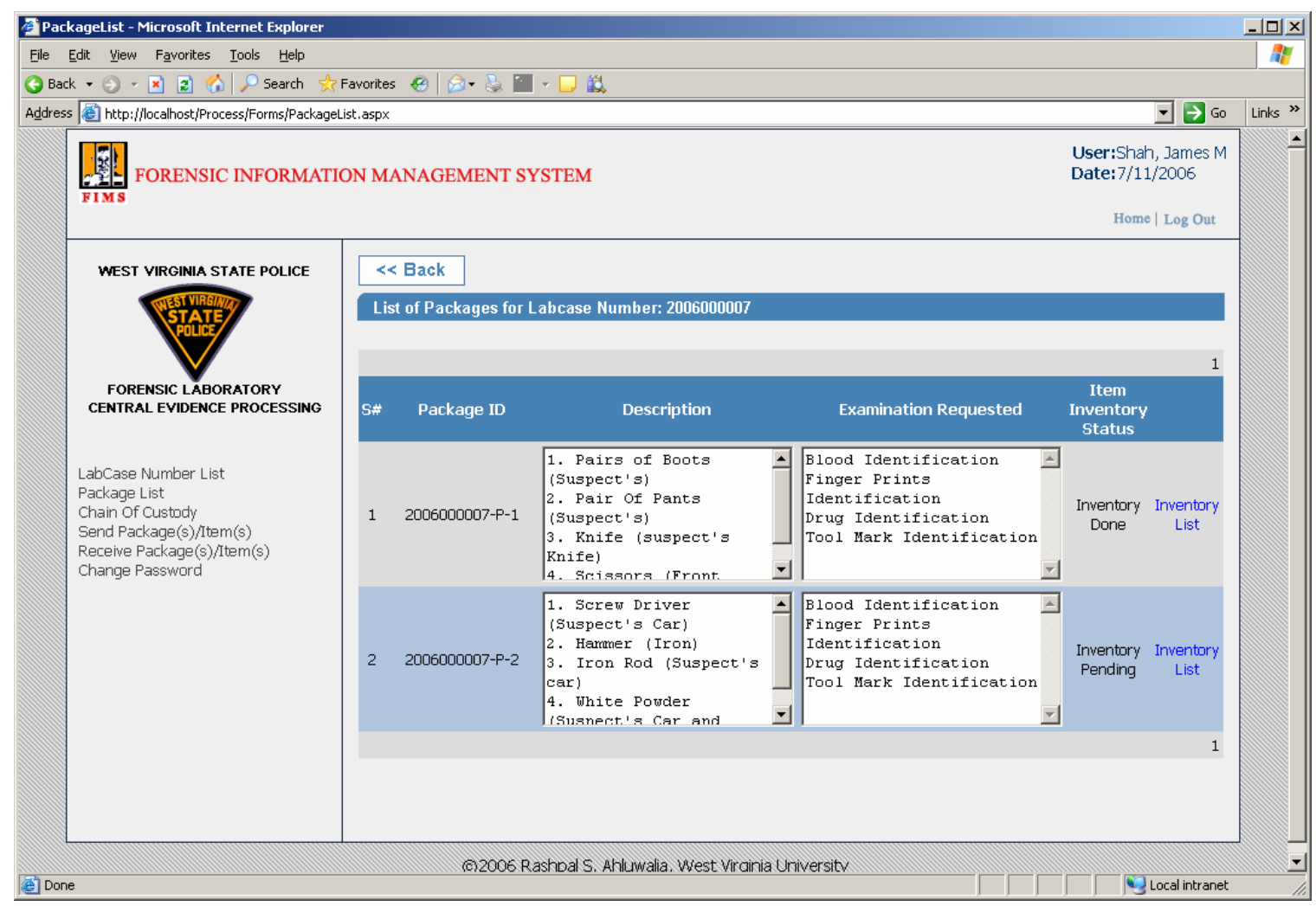

Figure 4.7: Package List Section 
After receiving package, CEP is responsible for filling laboratory item inventory form. Figure 4.8 shows the laboratory item inventory form. This section enables a user to add, update, or delete existing items. After inventorying all items in the package, CEP users need to click on "Item Inventory Complete" command button to finish the inventory process.

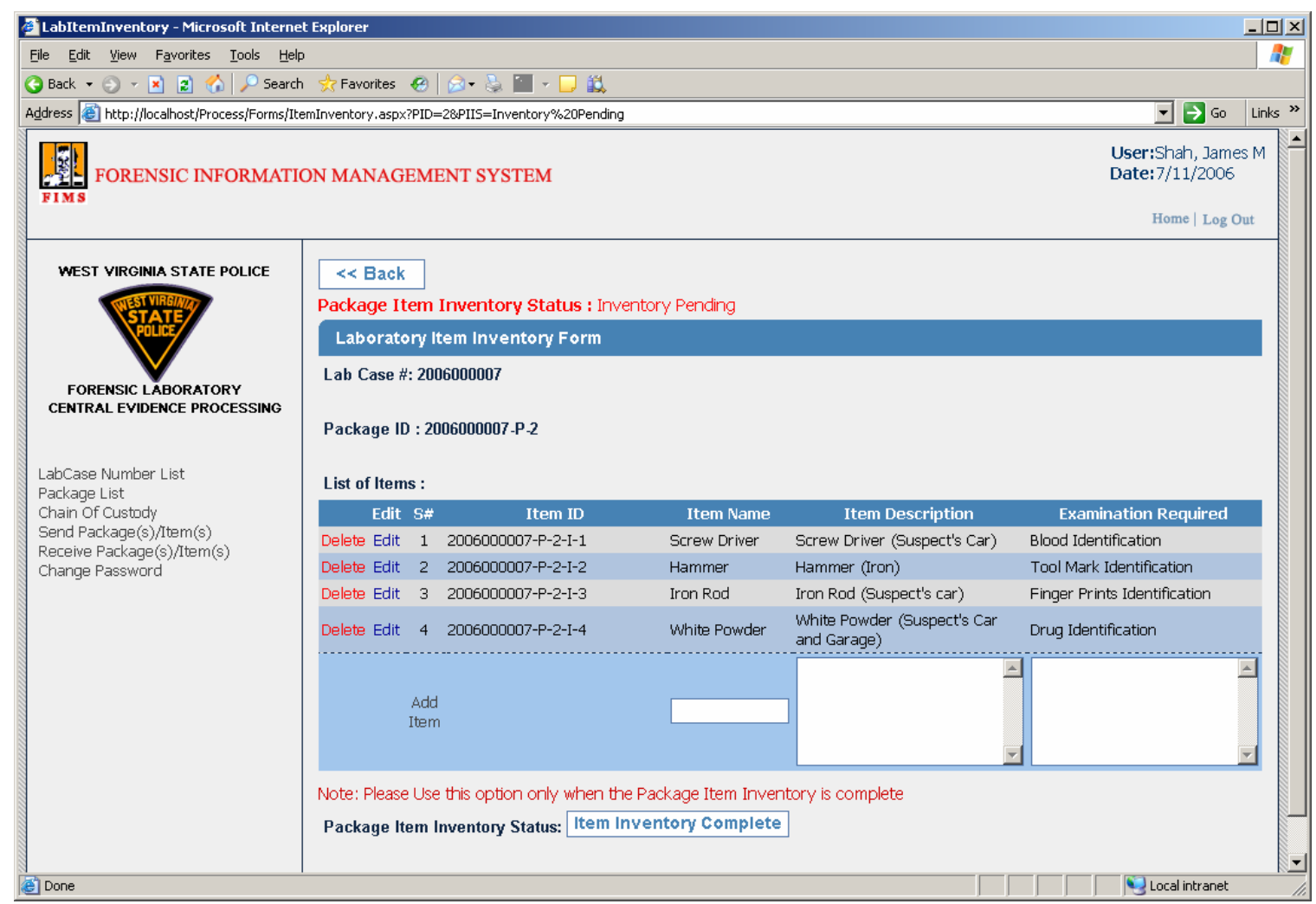

Figure 4.8: LEIF Form 


\section{Chain of Custody}

Package and item chain of custody information is shown in Figure 4.9. The top section shows the package chain of custody, and the bottom section shows the item chain of custody.

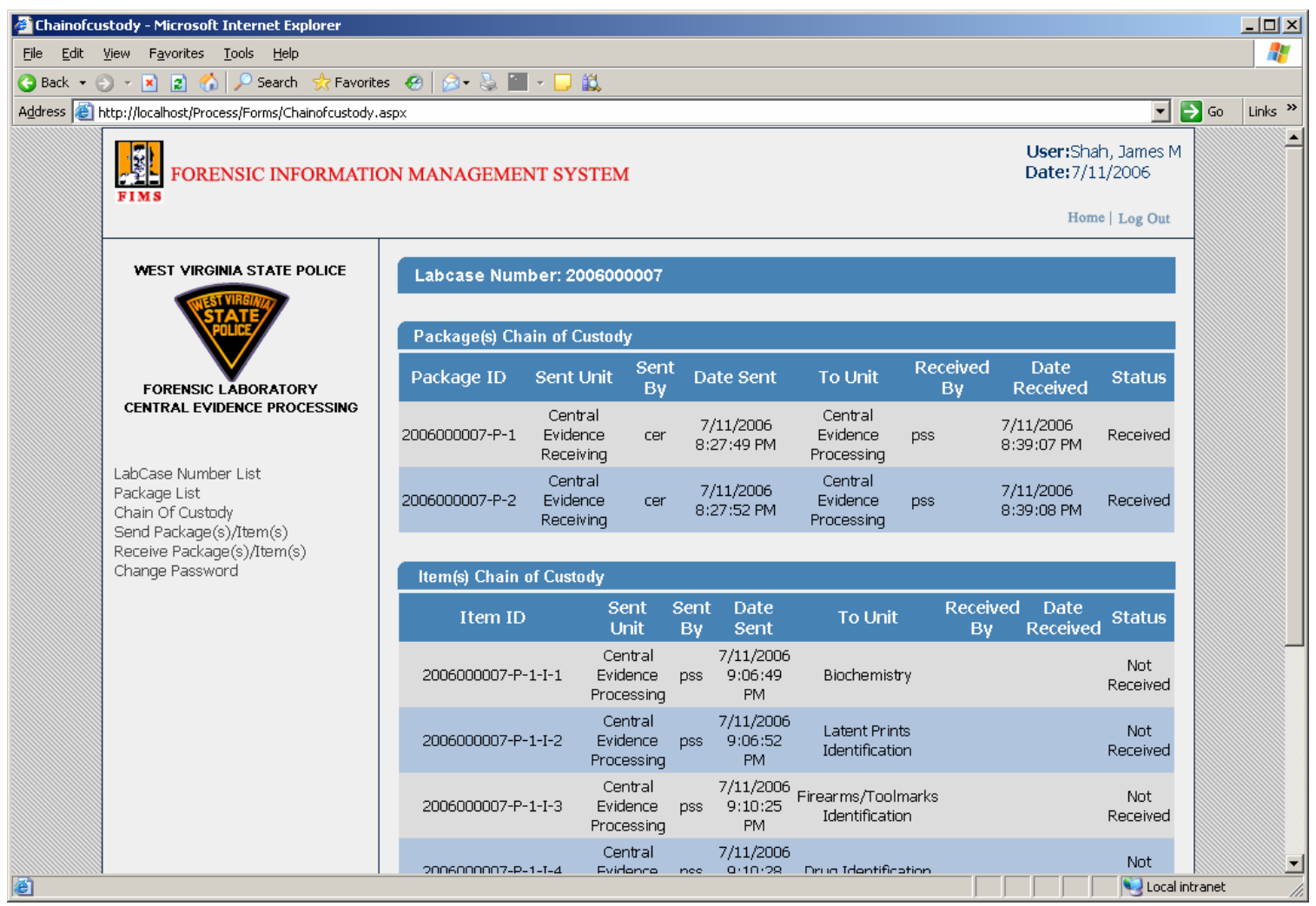

Figure 4.9: Chain of Custody Form 


\section{Send Package/Item}

This section describes how to send package/item from source to destination units. CEP user can send package by selecting the appropriate package number from the dropdown list under Send Package(s) section and then clicking on "Send" hyperlink as shown in Figure 4.10.

After item inventory completion, the CEP unit has custody of the items till they are assigned to an analyst. Laboratory administration unit is responsible for tracking and assigning items to the analysts in testing units. The CEP user can view the list of item assigned and then sends those items to their relevant unit through this section as shown in the Figure 4.10 .

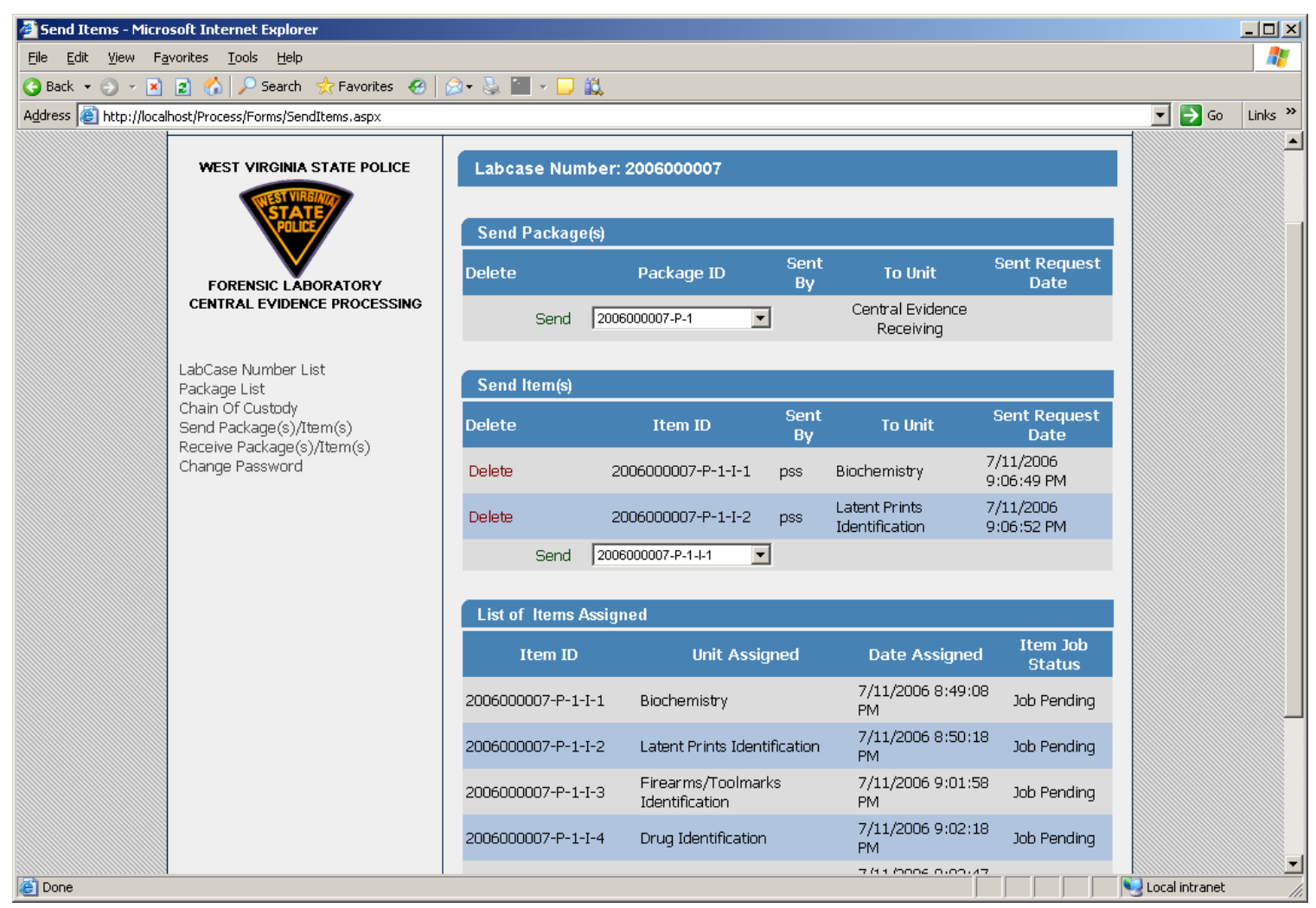

Figure 4.10: Send Package/Item Section 


\section{Receive Package/Item}

Once an item is sent by a source unit, then the destination unit is responsible for receiving it by clicking on "Receive" command button under receive package/item section as shown in Figure 4.11. This completes the handshaking protocol of item/package transfer.

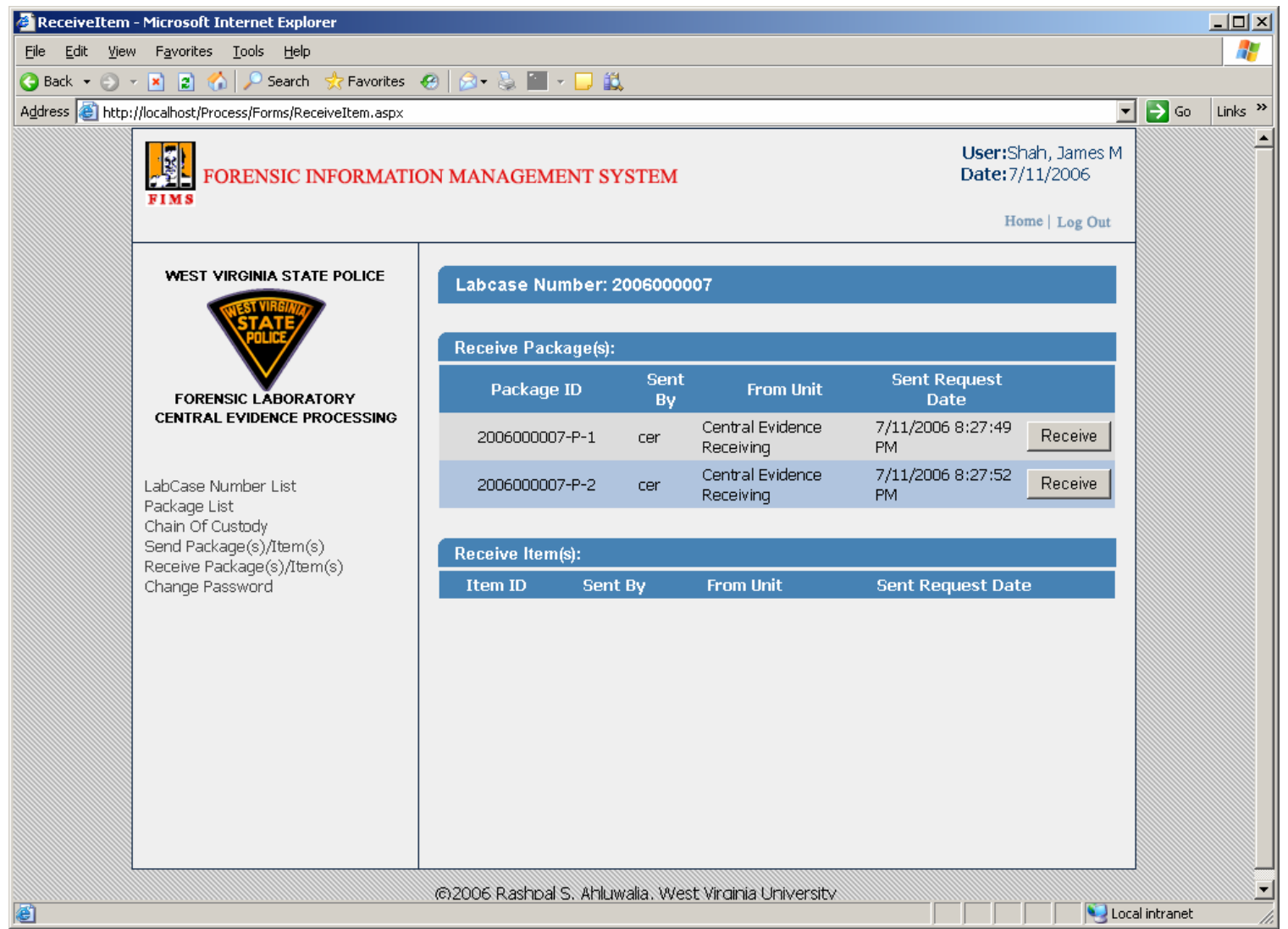

Figure 4.11: Receive Package/Item Section 


\section{Change User Password}

User can change password through this section by providing appropriate user credentials as shown in Figure 4.12.

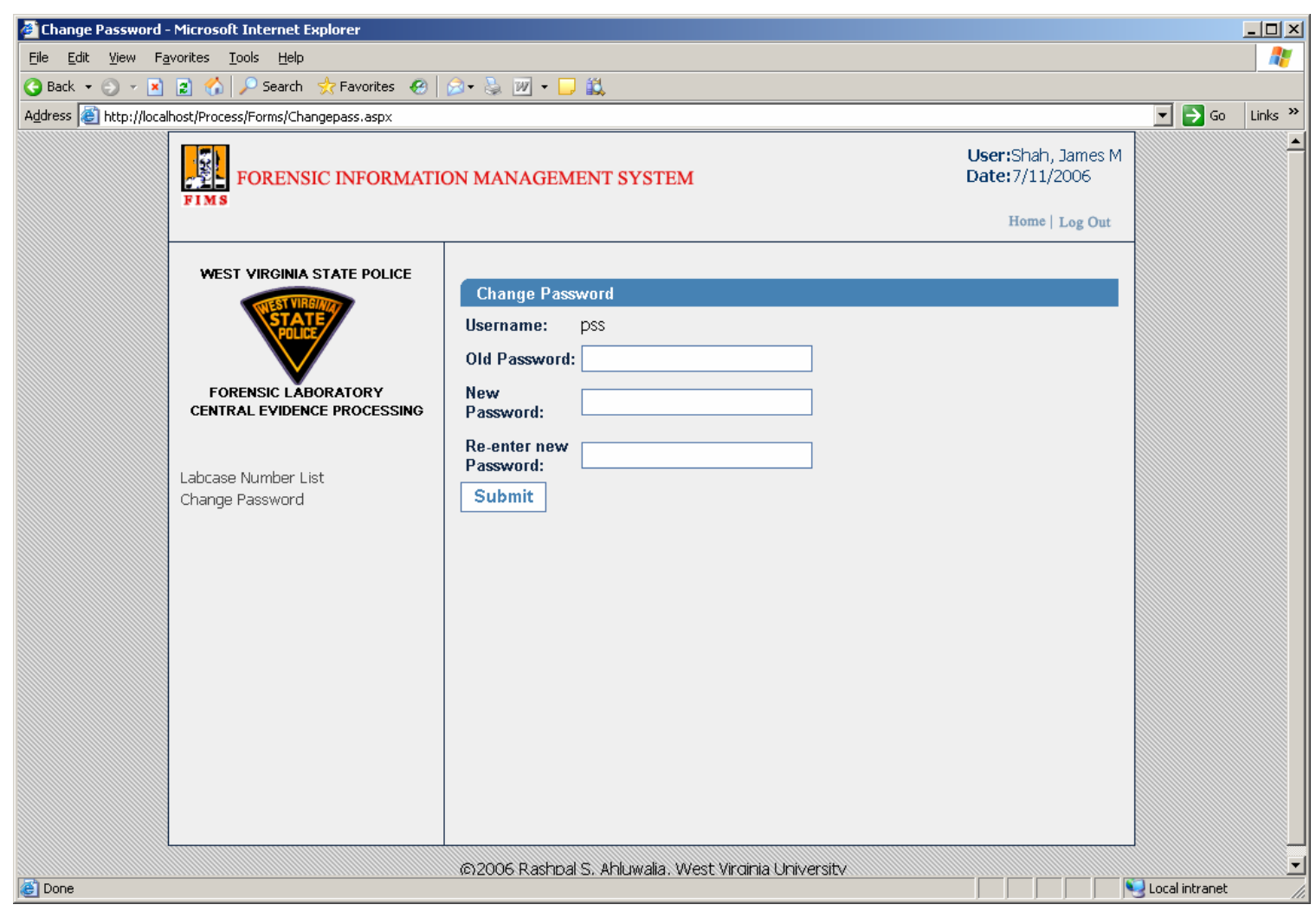

Figure 4.12: Change Password Section

\section{Exiting Central Evidence Processing}

Users can terminate the current session by clicking on "Log Out" hyperlink on the top right corner of the page. 


\section{CHAPTER 5 \\ LABORATORY ADMINISTRATION}

\subsection{Laboratory Administration Process}

The Administration module is responsible for meeting the many administrative, managerial, and technology needs of the laboratory. The primary goal of the laboratory administration is to provide a quality, operational work environment that supports the necessary work of forensic personnel. The section also serves as liaison to state, local, and federal agencies. Reviews and evaluates analyses and examinations performed in the laboratory. It is responsible for coordinating interagency and interdepartmental activities involving laboratory operations. It also oversees the preparation and maintenance of laboratory records and reports. It instructs law enforcement personnel in the proper laboratory procedures of identifying, handling, and examining of physical evidence. It assists in the planning of laboratory facility, equipment, staffing, and training to meet the day to day needs of the laboratory.

FIMS laboratory administration module is responsible for assigning the inventoried items to analyst in testing unit. Laboratory administration information flow diagram is shown in Figure 5.1. It is also responsible for verifying and approving the final laboratory case report, as shown in Figure 5.2. 


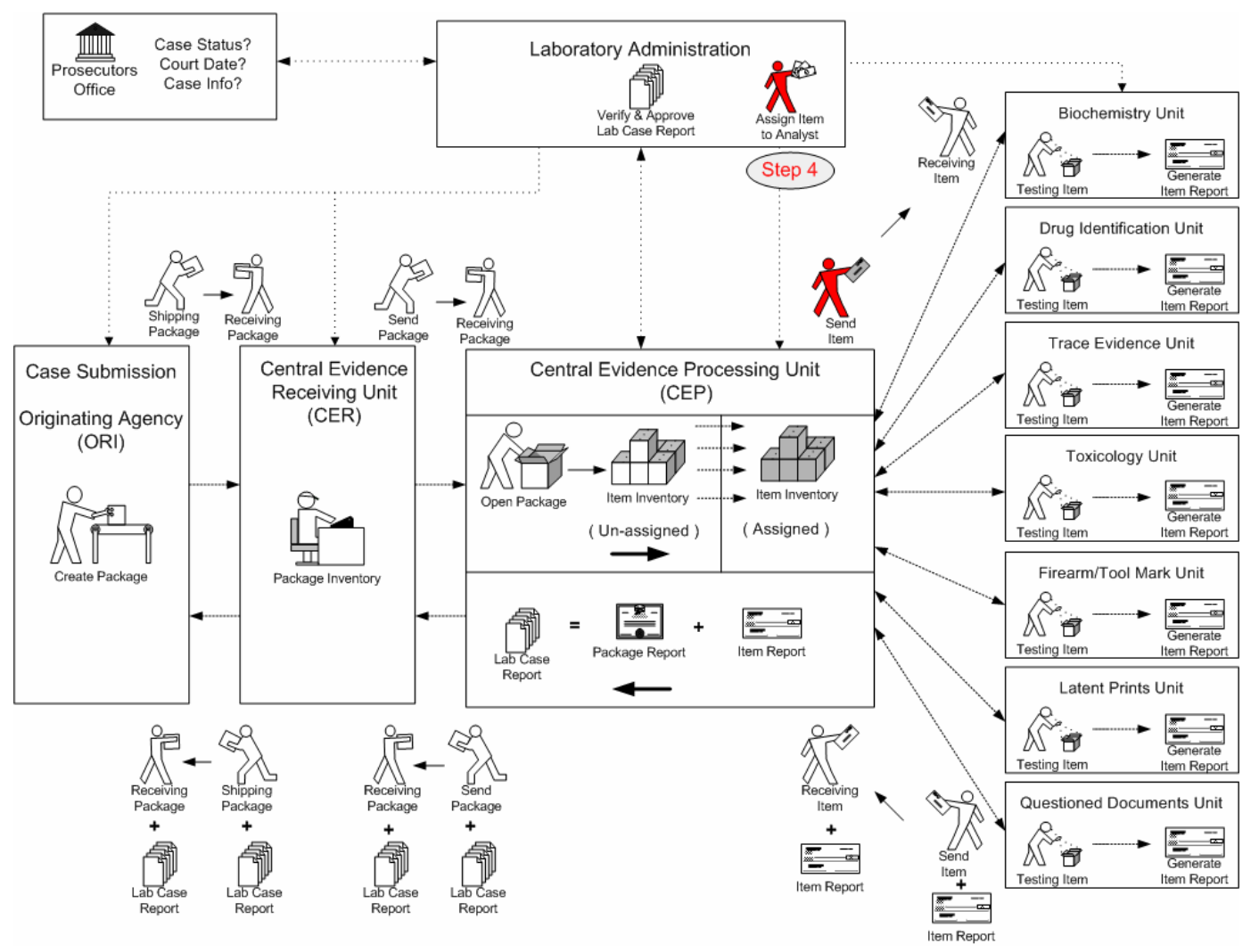

Figure 5.1: Step 4- Information flow diagram

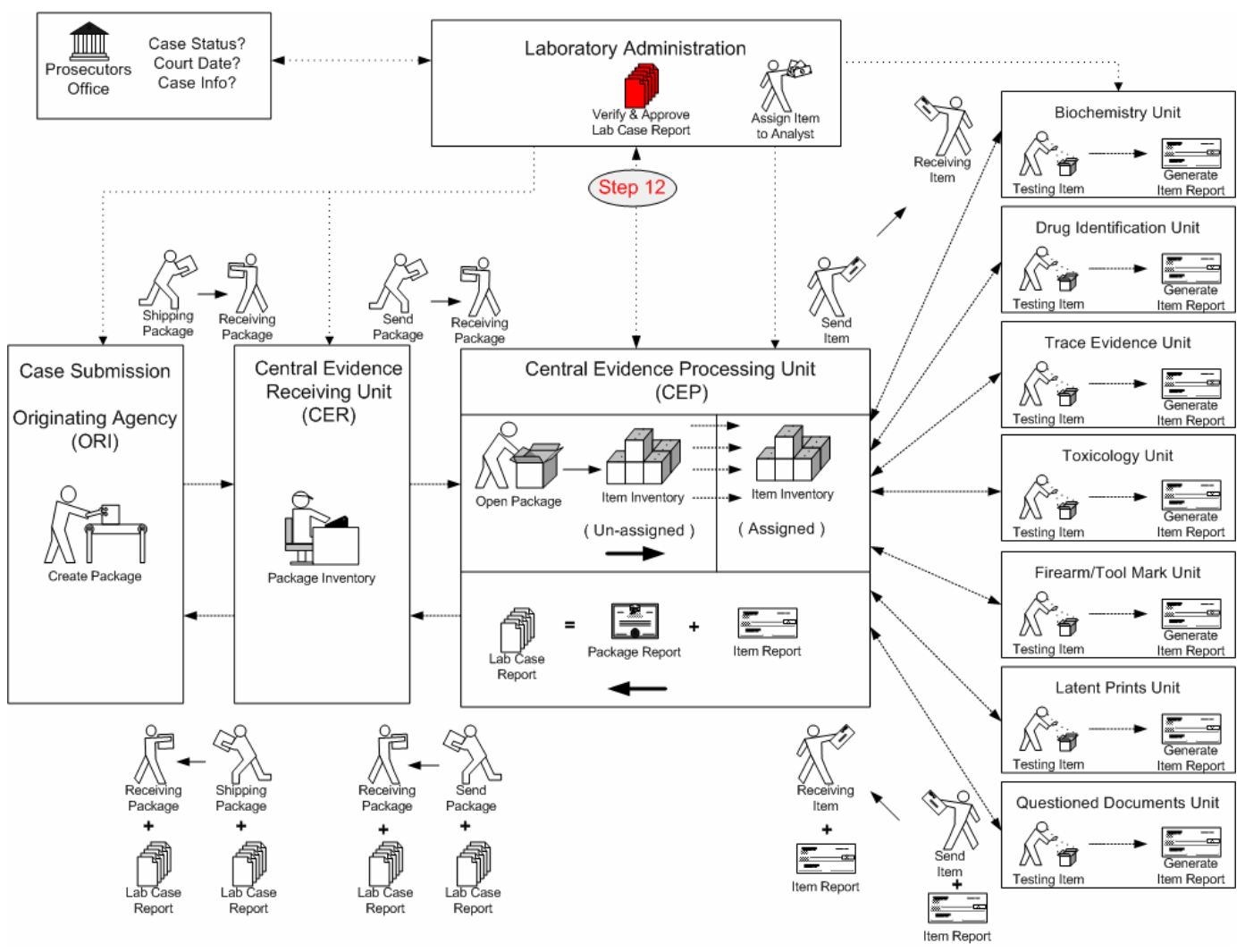

Figure 5.2: Step 12- Information flow diagram 


\subsubsection{Laboratory Administration Entity Relationship Diagram}

Laboratory administration data tables are connected with each other through relations as shown in the figure 5.3 .

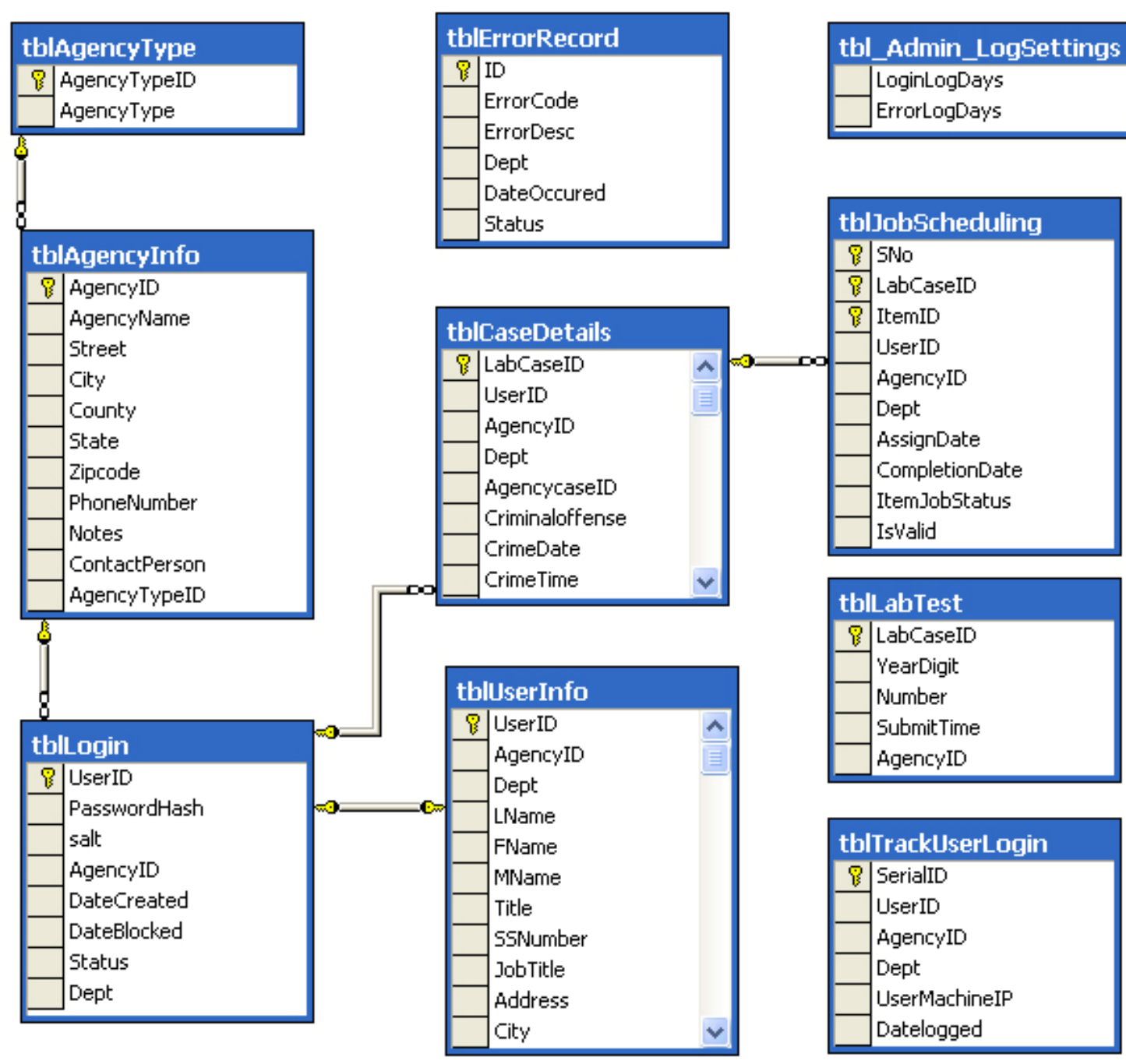

Figure 5.3: Laboratory administration data tables 


\subsubsection{Laboratory Administration Database Tables}

The laboratory administration unit module stores its data in the following tables

- Error Record Table(tblErrorRecord)

- Agency Information Table (tblAgencyInfo)

- Job Scheduling Table (tblJobScheduling)

- Login Table(tblLogin)

- Track User Login Table (tblTrackUserLogin)

- User Information Table (tblUserInfo)

The various fields of the error record table are shown in Table 5-1. Error record data is stored in error record table (tblErrorRecord)

Table 5-1: Error Record Table

\begin{tabular}{|l|l|}
\hline tblErrorRecord \\
\hline B & ID \\
\hline & ErrorCode \\
\hline & ErrorDesc \\
\hline & Dept \\
\hline & DateOccured \\
\hline & Status \\
\hline
\end{tabular}

The various fields of the agency information table are shown in Table 5-2. agency data is stored in Agency Information table(tblAgencyInfo)

Table 5-2: Agency Information Table

\begin{tabular}{|l|l|}
\hline tblagencyInfo \\
\hline \\
\hline & AgencyID \\
AgencyName \\
Street \\
\hline City \\
\hline County \\
\hline State \\
\hline Zipcode \\
\hline \\
PhoneNumber \\
\hline \\
\hline \\
Notes \\
\hline \\
ContactPerson \\
\hline
\end{tabular}


The various fields of the job scheduling table are shown in Table 5-3. Job assigned data is stored in job scheduling table (tblJobScheduling)

Table 5-3: Job Scheduling Table

\begin{tabular}{|l|l|}
\hline tblobScheduling \\
\hline i & SNo \\
\hline i & LabCaseID \\
\hline itemID & Itemi \\
\hline & UserID \\
\hline & AgencyID \\
\hline & Dept \\
\hline & AssignDate \\
\hline & CompletionDate \\
\hline & ItemJobStatus \\
\hline & Isvalid \\
\hline
\end{tabular}

The various fields of the login table are shown in Table 5-4. Login data is stored in error record table (tblLogin)

\section{Table 5-4: Login Table}

\begin{tabular}{|l|l|}
\hline tblLogin \\
\hline | UserID \\
\hline & PasswordHash \\
\hline & salt \\
\hline & AgencyID \\
& DateCreated \\
& DateBlocked \\
& Status \\
\hline & Dept \\
\hline
\end{tabular}

The various fields of the track user login table are shown in Table 5-5. Tracking data is stored in track user login table (tblTrackUserLogin)

Table 5-5: Track User Login Table

\begin{tabular}{|l|l|}
\hline tblTrackUserLogin \\
\hline B & SerialID \\
\hline & UserID \\
\hline & AgencyID \\
\hline & Dept \\
\hline & UserMachineIP \\
\hline & Datelogged \\
\hline
\end{tabular}


The various fields of the user information table are shown in Table 5-6. User data is stored in user information table (tblUserInfo)

Table 5-6: User Information Table

\begin{tabular}{|c|c|}
\hline \multicolumn{2}{|c|}{ tblUserInfo } \\
\hline (8) & UserID \\
\hline & AgencyID \\
\hline & Dept \\
\hline & LName \\
\hline - & FName \\
\hline & MName \\
\hline & Title \\
\hline & SSNumber \\
\hline & JobTitle \\
\hline & Address \\
\hline & City \\
\hline & State \\
\hline & Zipcode \\
\hline & Phone \\
\hline & Race \\
\hline & Sex \\
\hline & $\mathrm{DOB}$ \\
\hline & HireDate \\
\hline & TitleDate \\
\hline
\end{tabular}




\subsubsection{Working with Laboratory Administration}

Laboratory administration unit home page requires username and password to verify administrator credentials, as shown in the Figure 5.4. After verifying credentials, administrator is directed to FIMS administration work area as shown in the Figure 5.5.

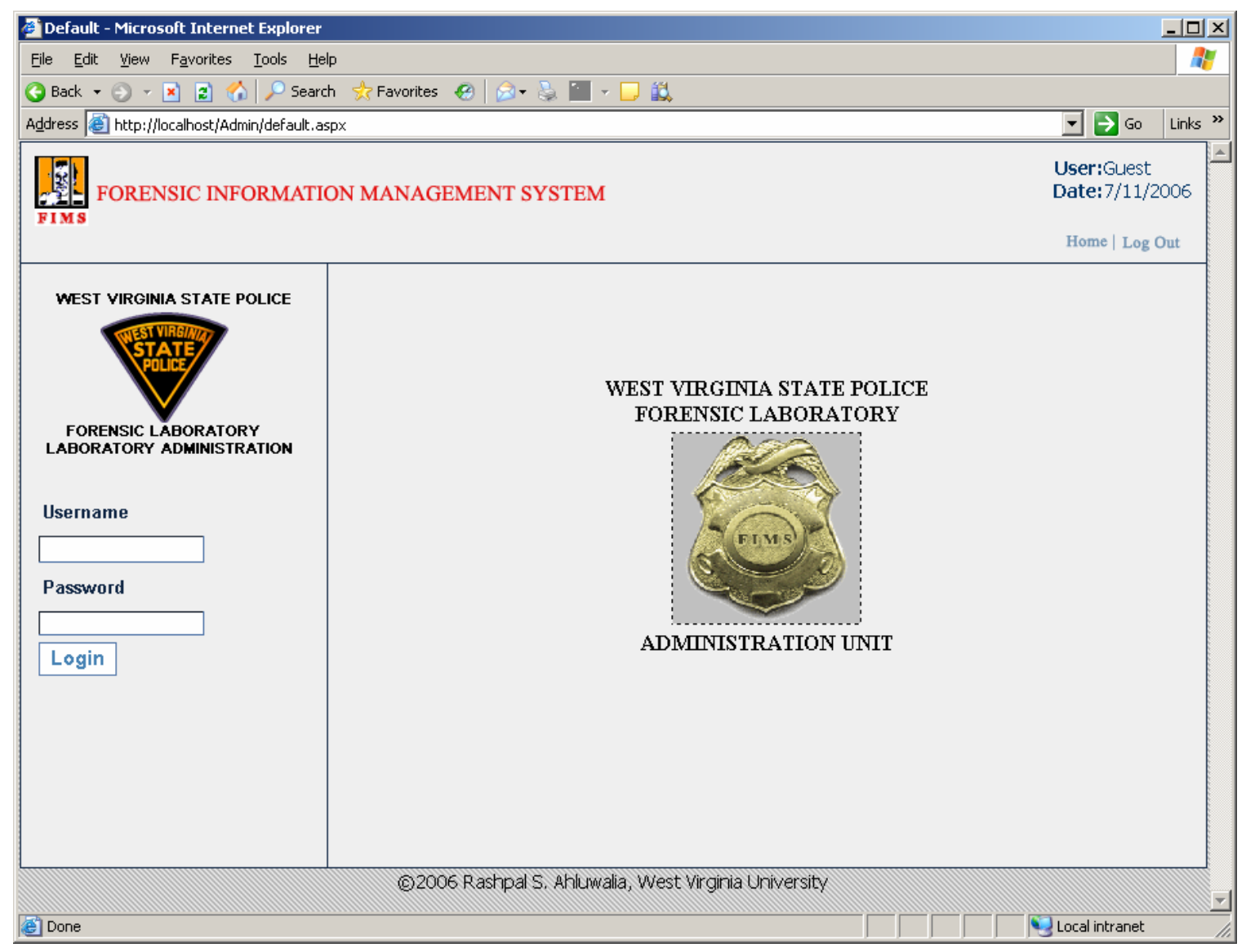

Figure 5.4: Laboratory administration login page 
FIMS laboratory administration work area enables administrator to handle a wide variety of administrative and operational issues in forensic laboratories. Side navigation bar allows navigating to the following sections:

- $\quad$ Laboratory Case Management

- User Management

- $\quad$ Agency Management

- $\quad$ Log Management

- $\quad$ System Settings

- $\quad$ System Statistics

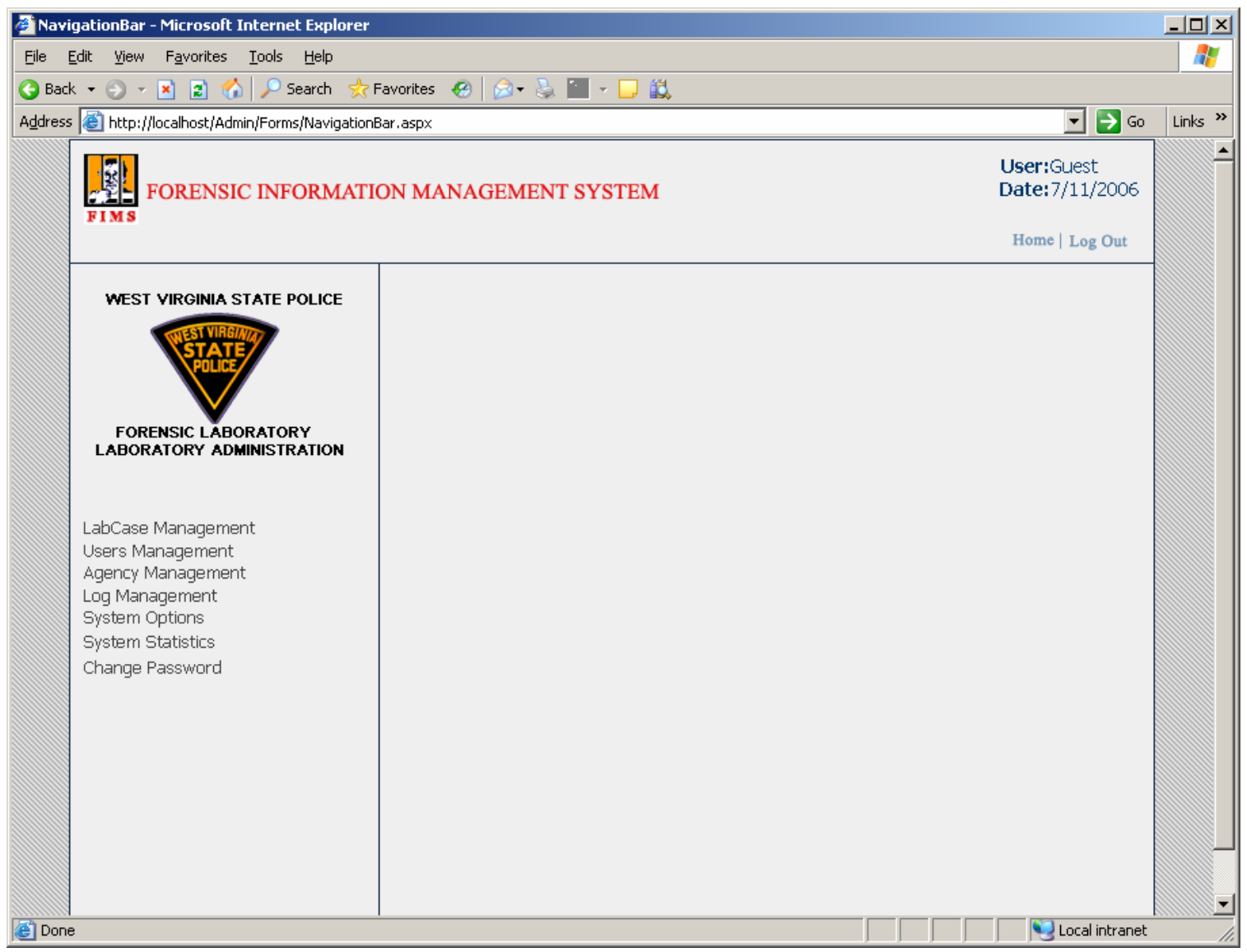

Figure 5.5: FIMS Administration Work Area 


\section{Laboratory Case Management}

FIMS laboratory case management module plays a critical role in managing the evidence handling and analysis process between CEP and testing units. List of cases accepted can be accessed by clicking on “LabCase Management” hyperlink, a shown in Figure 5.6. Administrator can access the list of packages belonging to a particular case by clicking on "Package List” hyperlink.

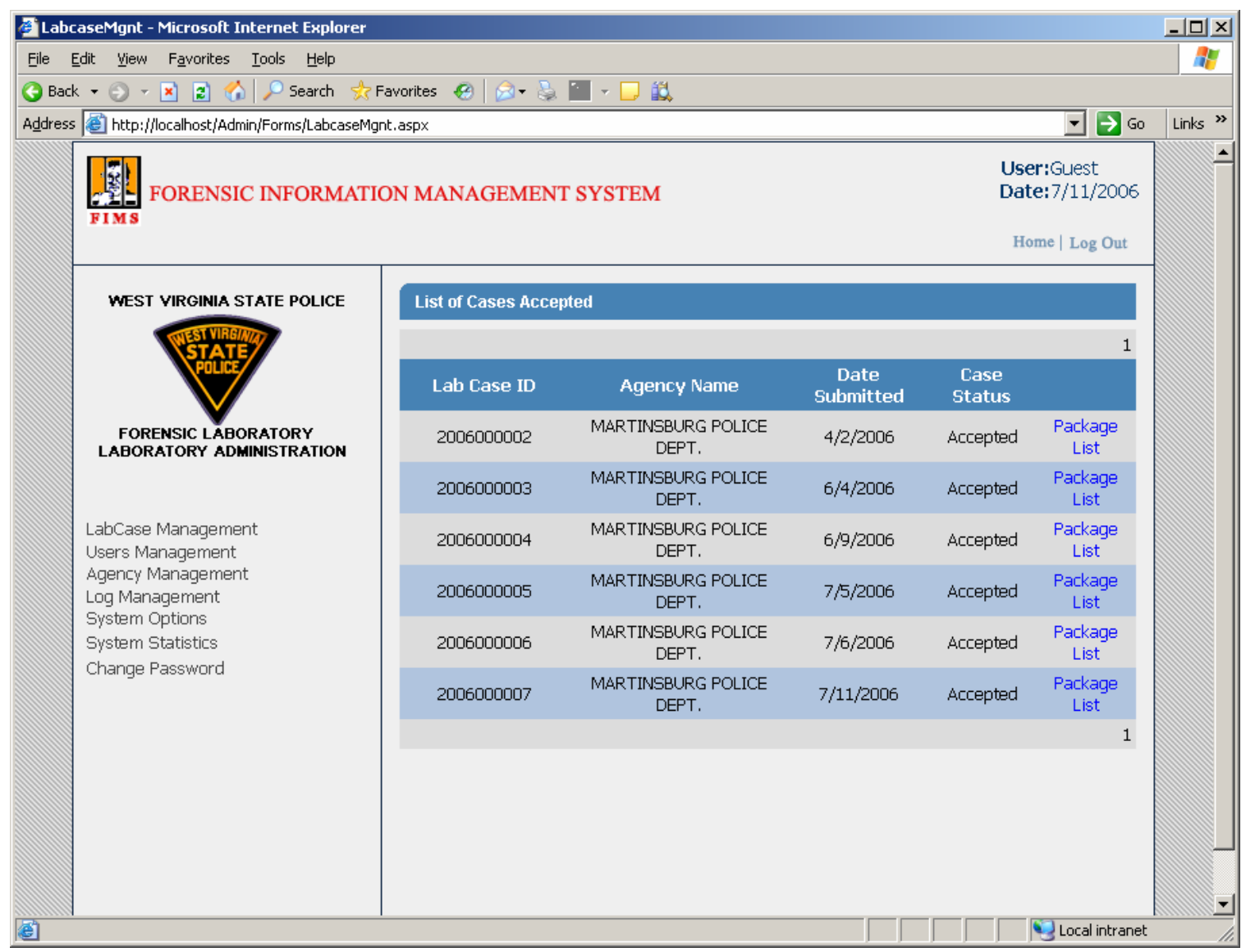

Figure 5.6: List of Accepted Cases 
This section lists all of the packages with their description, examination requested, package status and item inventory status, belonging to that particular case. Administrator can view the entire list of items in a package by clicking on "List" hyperlink as shown in Figure 5.7.

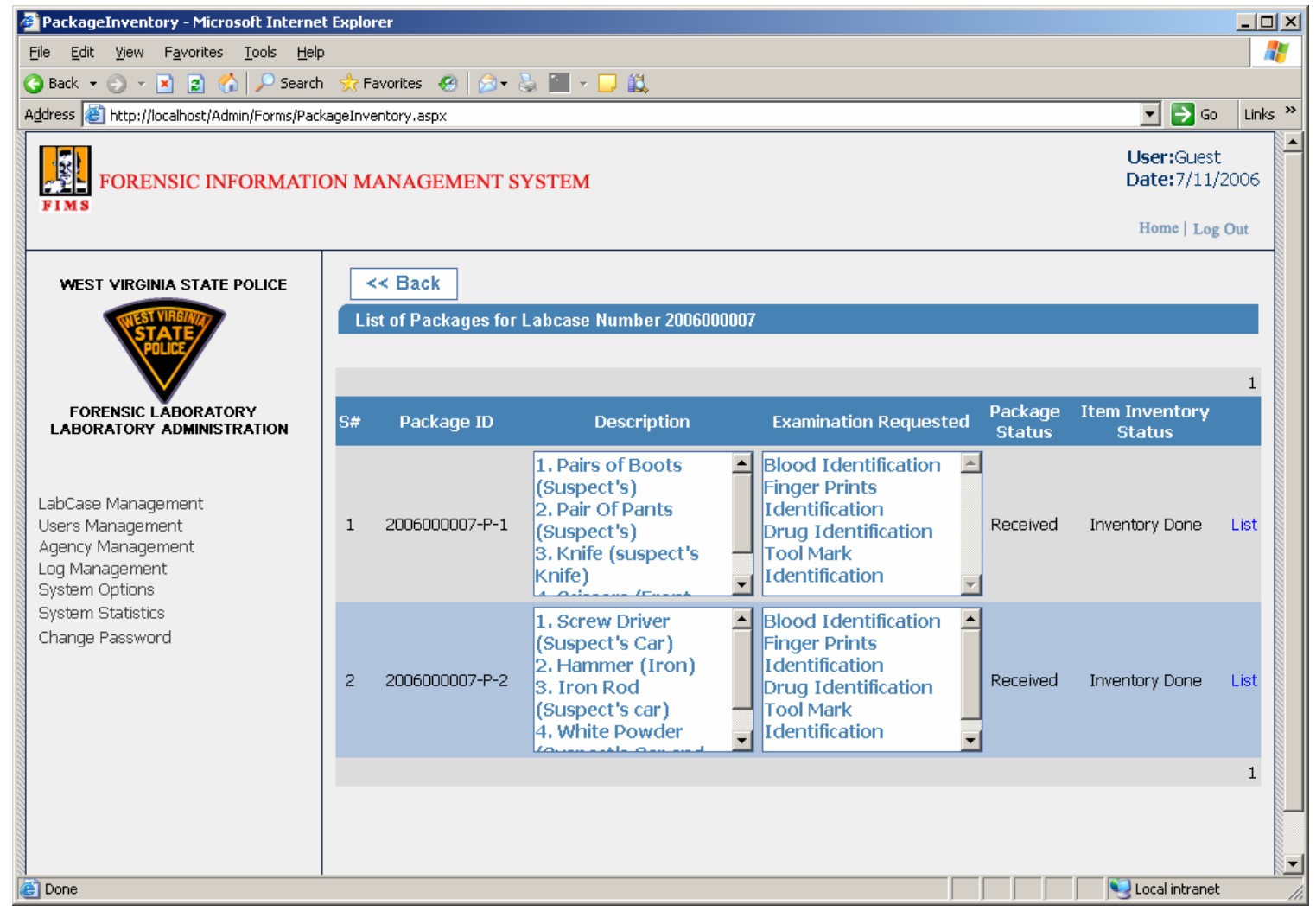

Figure 5.7: List of Packages 
This section enables administrator to add, update, or delete existing items. This section also enables an administrator to assign items to analyst with appropriate qualification and training in any of the testing units, by clicking on "Assign" hyperlink as shown in the Figure 5.8.

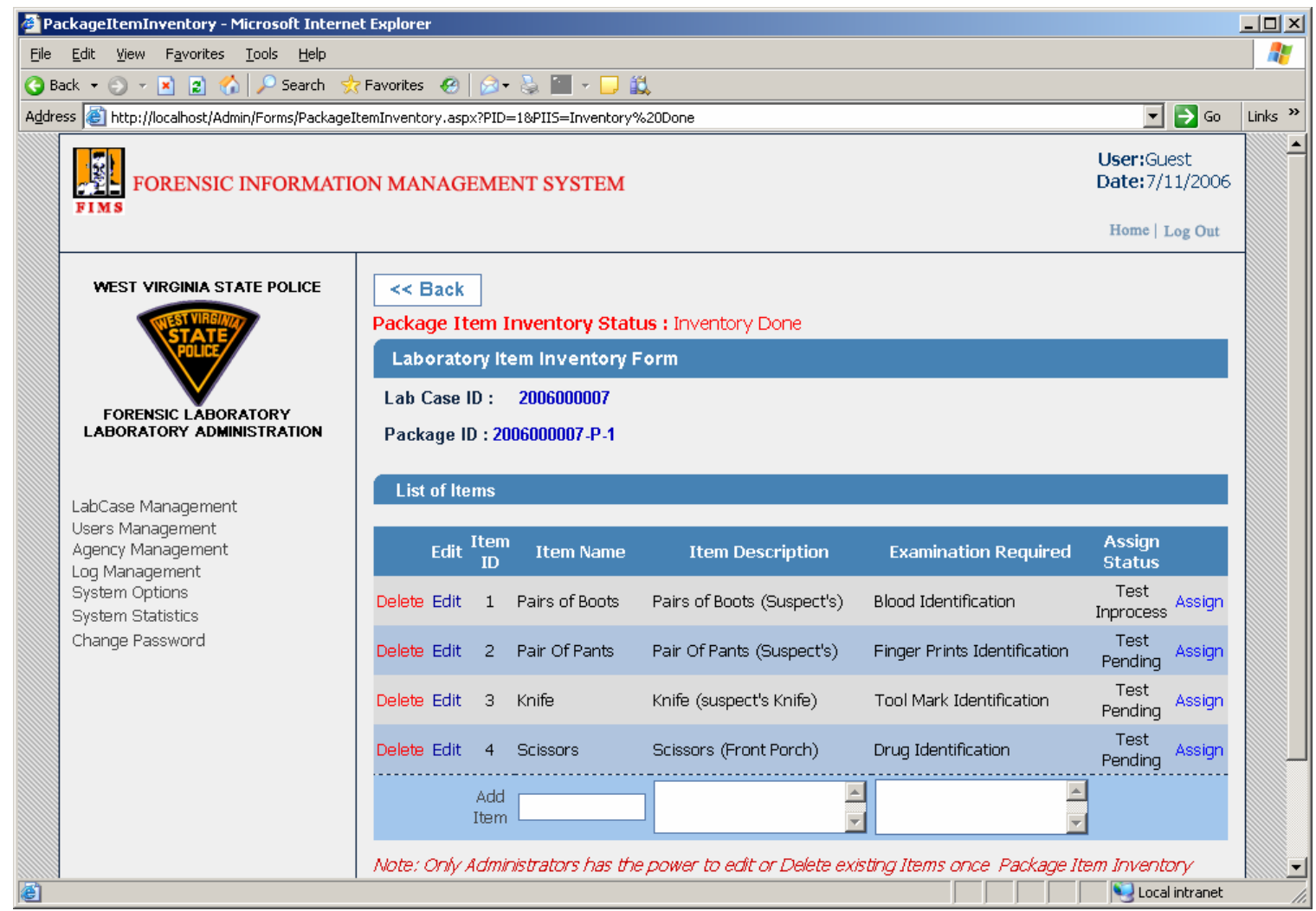

Figure 5.8: List of Items 
This section allows administrator to assign an item to analyst in any of the testing units, by choosing unit name from unit dropdown. When the selection in the unit dropdown is changed the list of analyst drop down is refilled with name and user ID of the available analysts in that unit. When an analyst is selected from the list, the total items pending for that analyst is displayed. This helps in load balancing among analysts in the same unit. The decision of choosing a analyst for testing an item is manual. After the analyst is selected, administrator is required to press “Assign Item" button to complete the process, as shown in Figure 5.9.

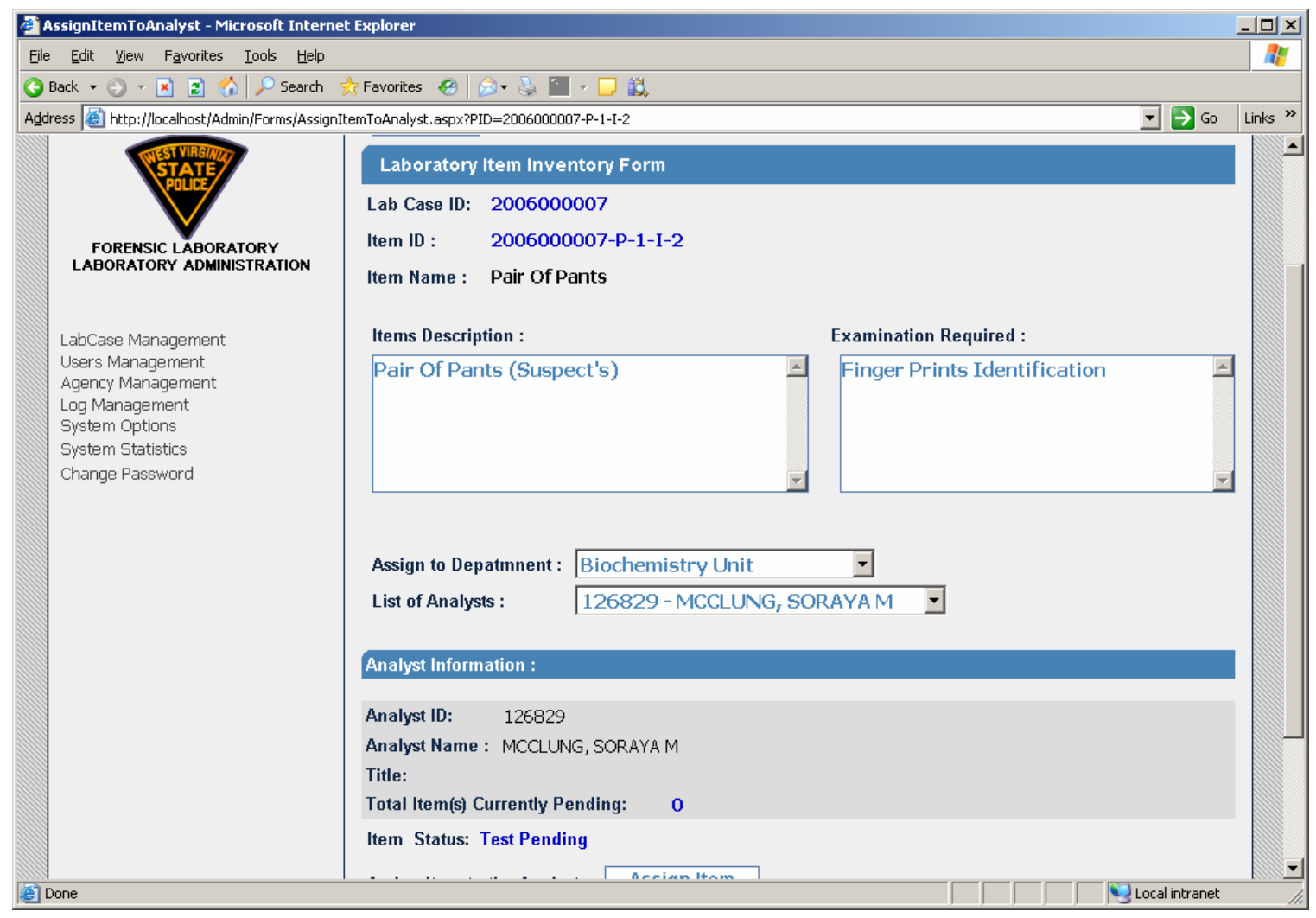

Figure 5.9: Assign Item Section 
The administrator can also cancel assigned item work order and reassign it. The items already assigned to a analyst can not be reassigned till the previous assigned work order is dismissed by clicking on "Cancel Item" command button as shown in Figure 5.10 .

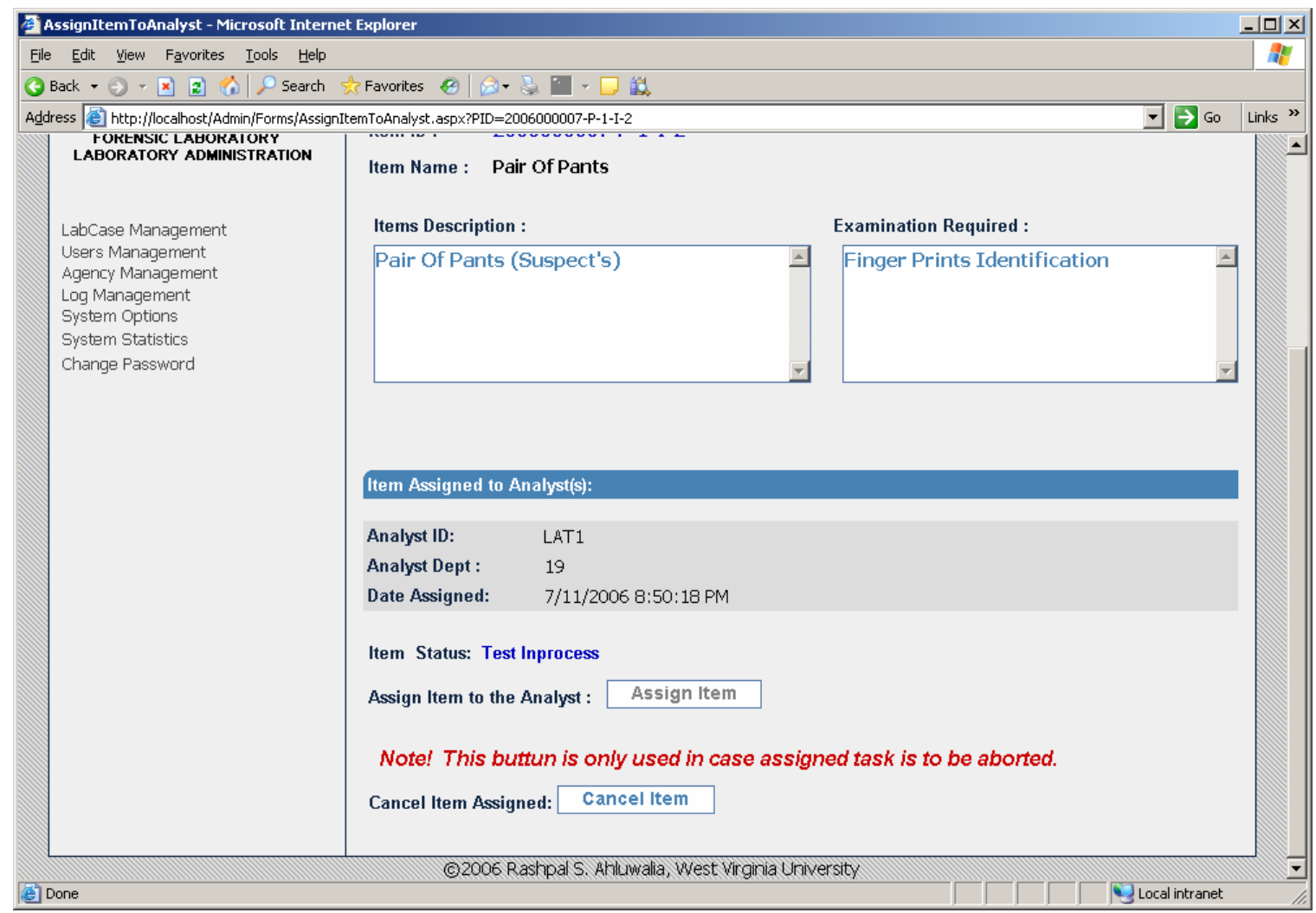

Figure 5.10: Cancel Item Option 


\section{User Management}

User management section enables administrator to view, add, delete, or disable FIMS users. User management section allows navigating to the following sections:

- Create User Account

- Existing Users Management

This section enables administrator to add new users to the FIMS, as shown in Figure 5.11 .

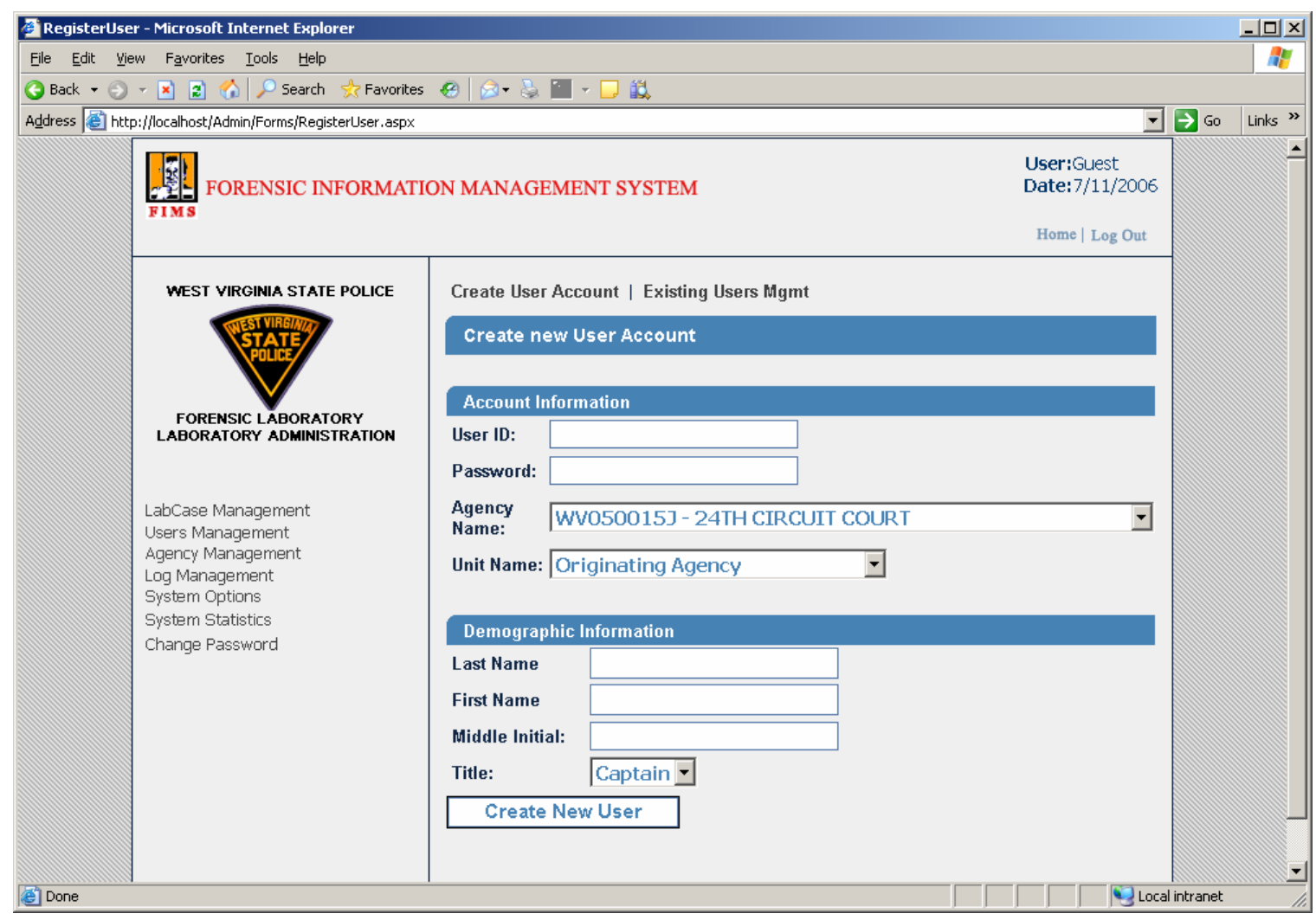

Figure 5.11: Add User Section 
Existing users section lists all the existing users in FIMS system. Agency dropdown allows administrator to choose agency and view all the users. Administrator can edit information, activate, or block a user. Blocking a user disables his/her, login and other rights granted, as shown in Figure 5.12.

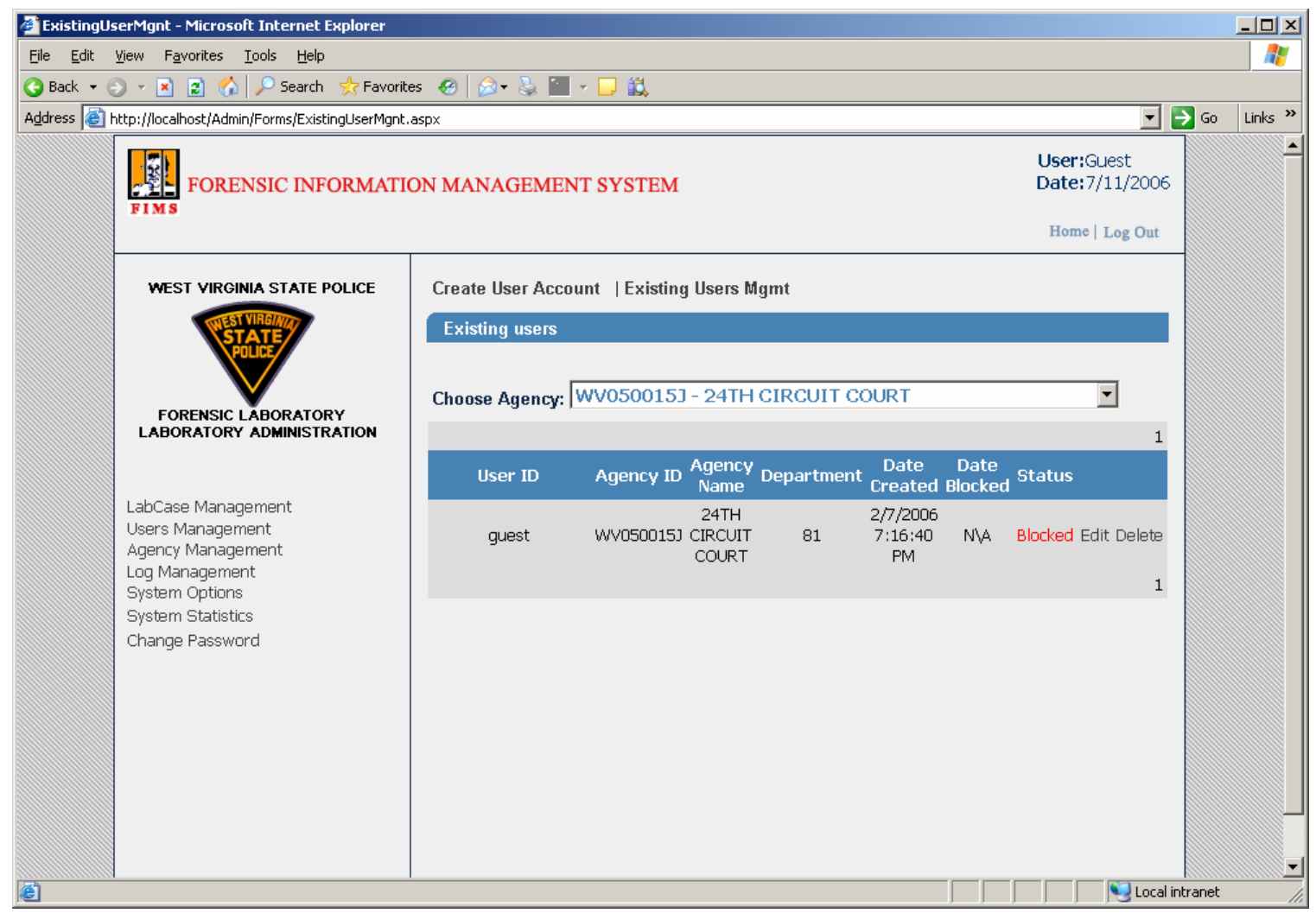

Figure 5.12: Existing User Management Section 


\section{Agency Management}

Agency management section enables administrator to view, add, delete, or disable existing agency. Agency management section allows navigating to the following sections:

- Add Agency

- Existing Agency Information Management

This section enables administrator to add new agency to the FIMS, as shown in Figure 5.13.

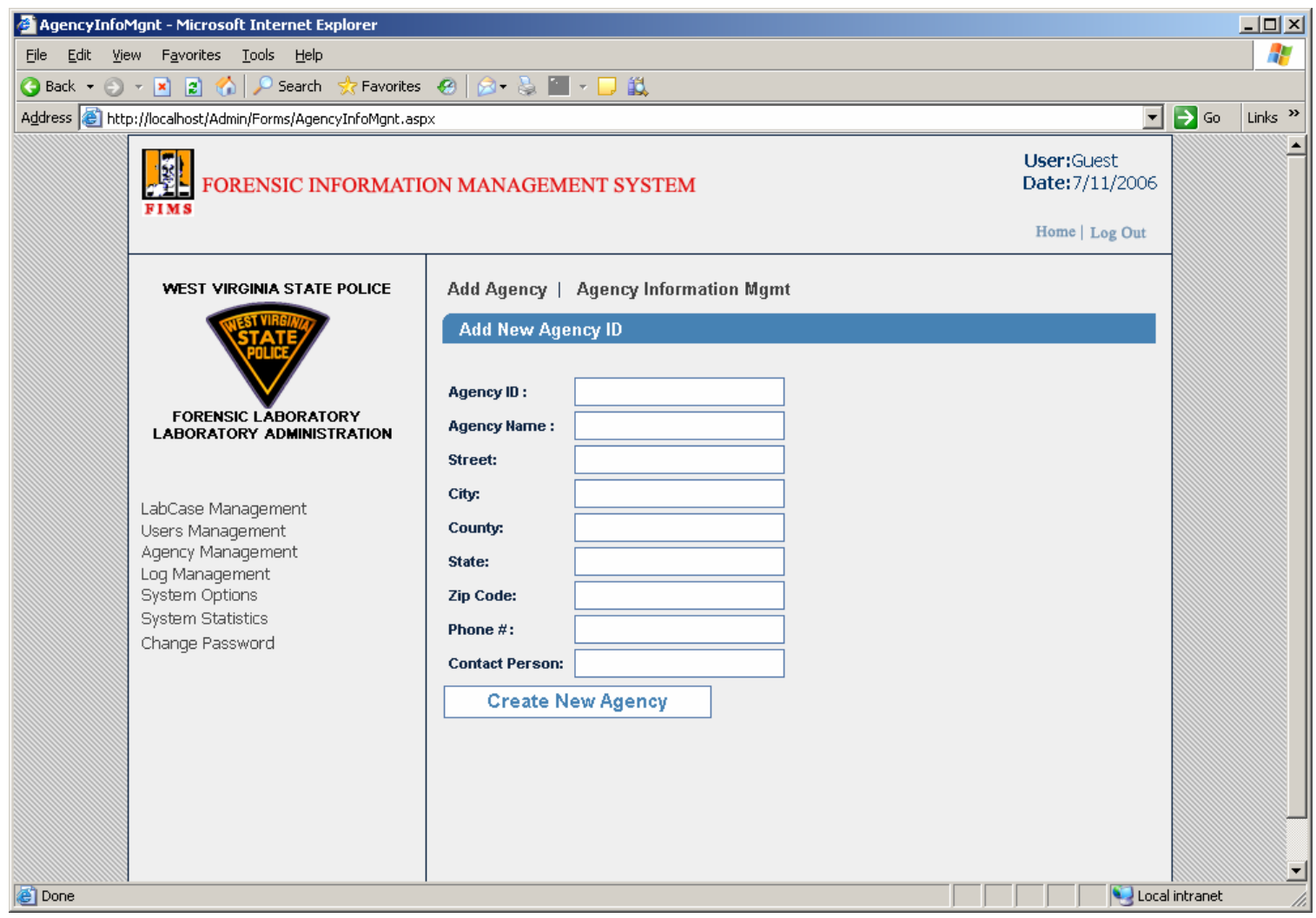

Figure 5.13: Add Agency Section 
Existing agency information section list all the existing agencies in FIMS system.

Administrator can delete and update agency information as shown in Figure 5.14.

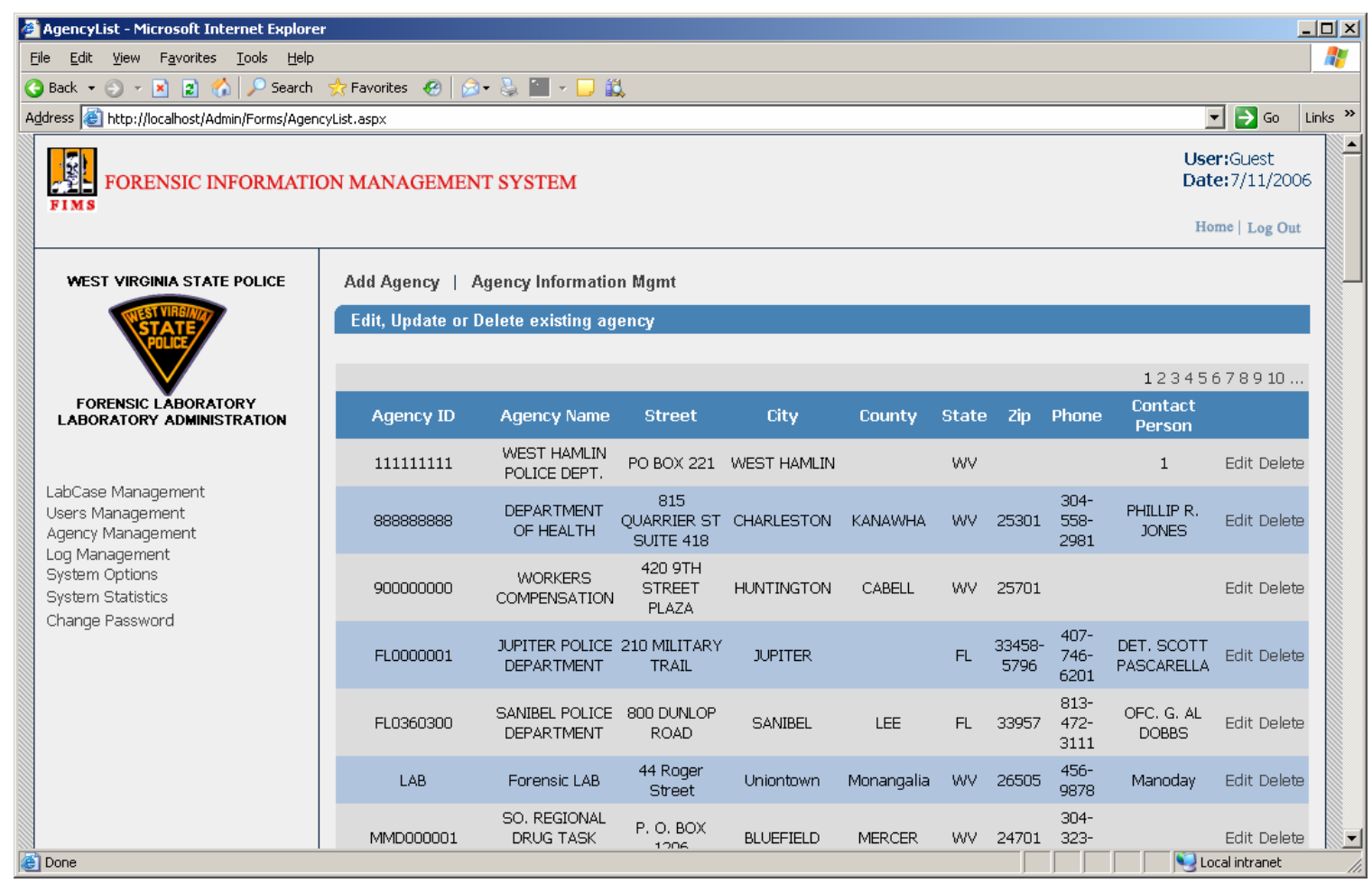

Figure 5.14: Existing Agency Information Management Section 


\section{Log management}

FIMS automatically tracks and records all errors occurred, as shown in Figure 5.15. Error handling procedures gracefully handles error and prevents application from crashing. It saves the entire stack trace information provided by the .Net framework in case of error. This helps in location the source of error and fixing it.

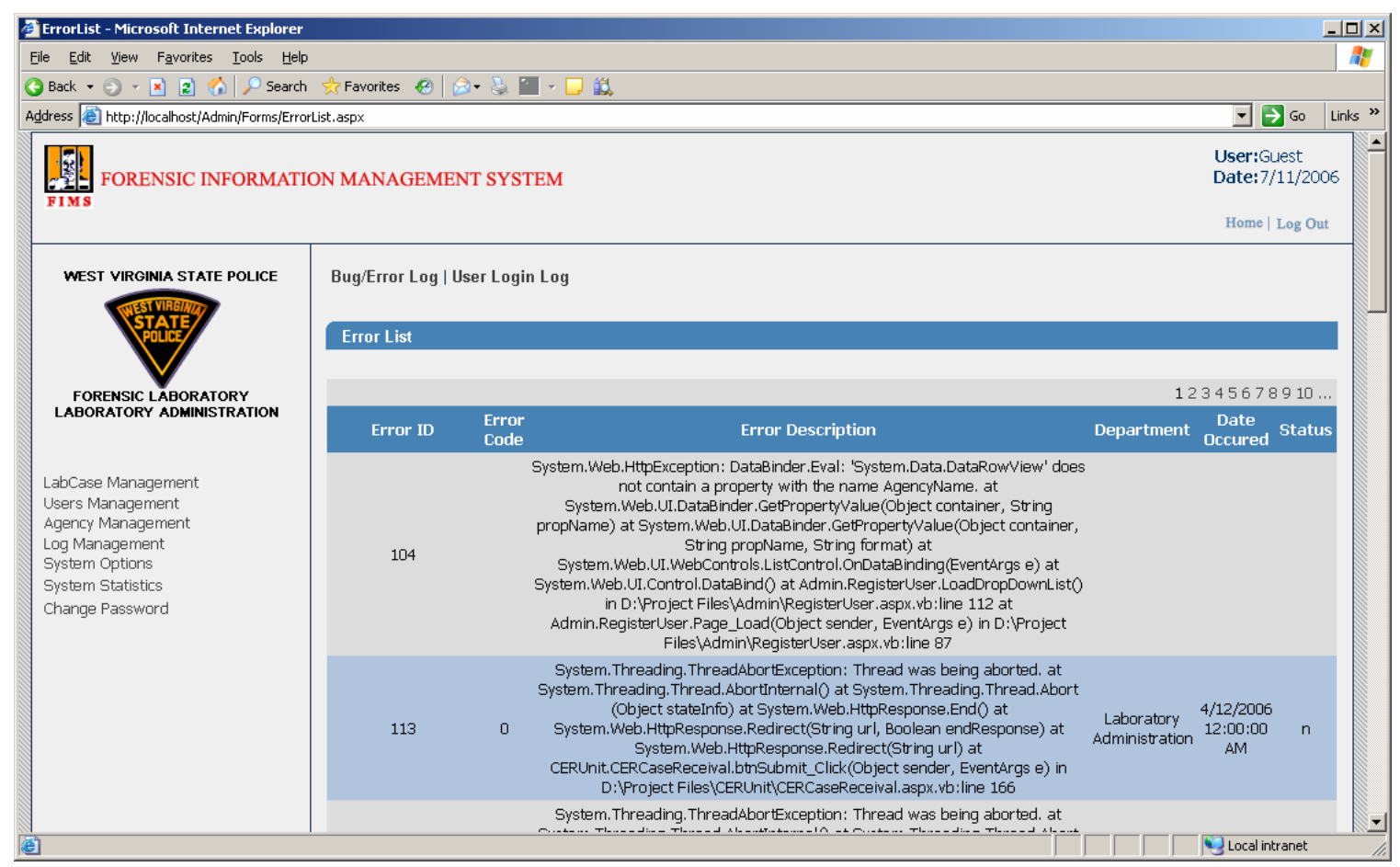

Figure 5.15: Error Management Section 
All user login are tracked by FIMS tracking procedures as shown in Figure 5.16. User ID, agency ID, unit, machine IP address and time of login are recorder for each login.

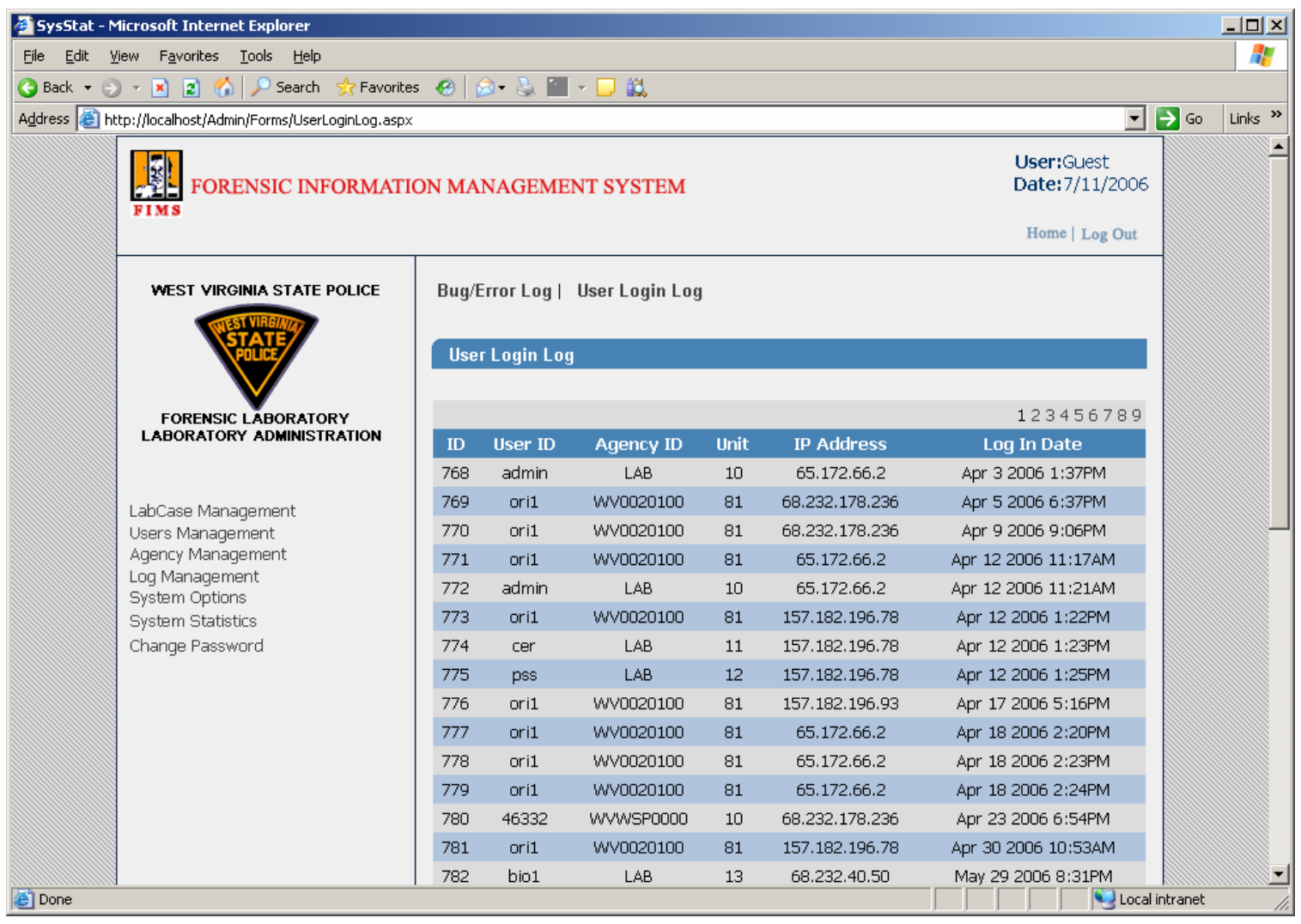

Figure 5.16: User Login Tracking Section 


\section{System Settings}

System settings section enables administrator to configure control parameters of FIMS, as shown in Figure 5.17. It allows navigating to the following sections:

- Change Error/Login Management Settings

- Dropdown List Options Management

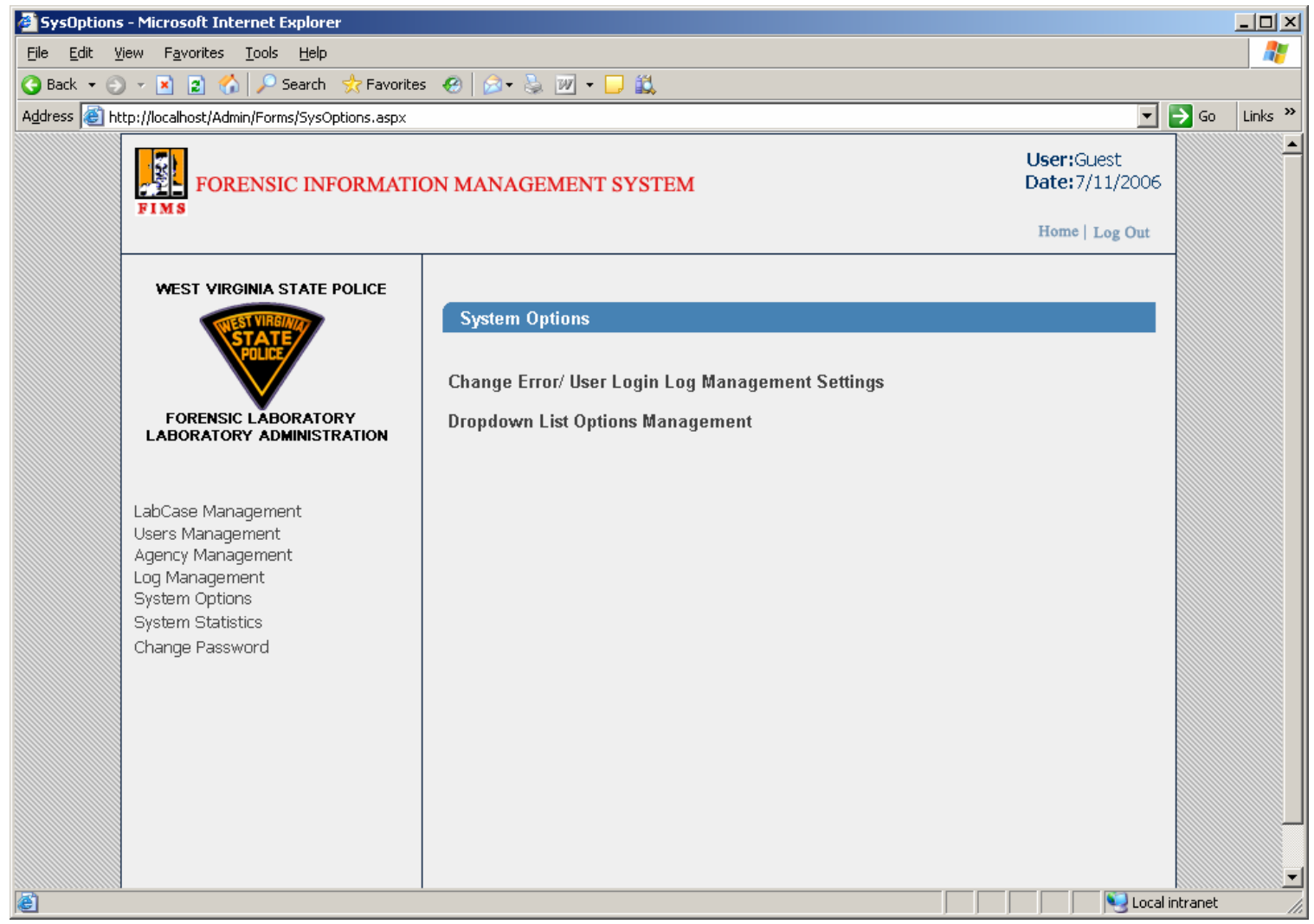

Figure 5.17: System Setting Section 
This section enables administrator to reset Error and Login list, as shown in Figure 5.18.

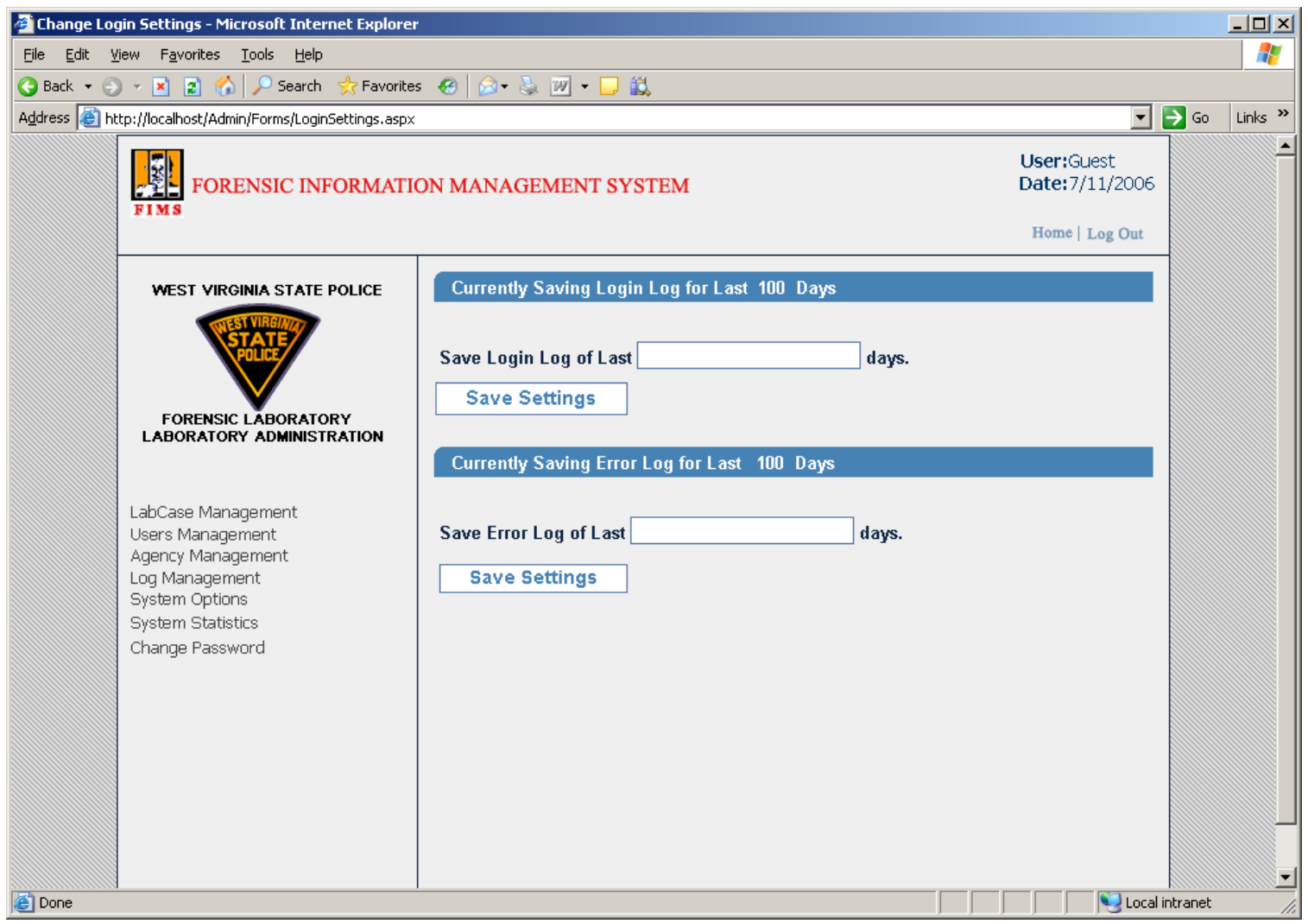

Figure 5.18: Error/Login parameters setting Section 
This section allows view and configures dropdown list values, as shown in Figure 5.19.

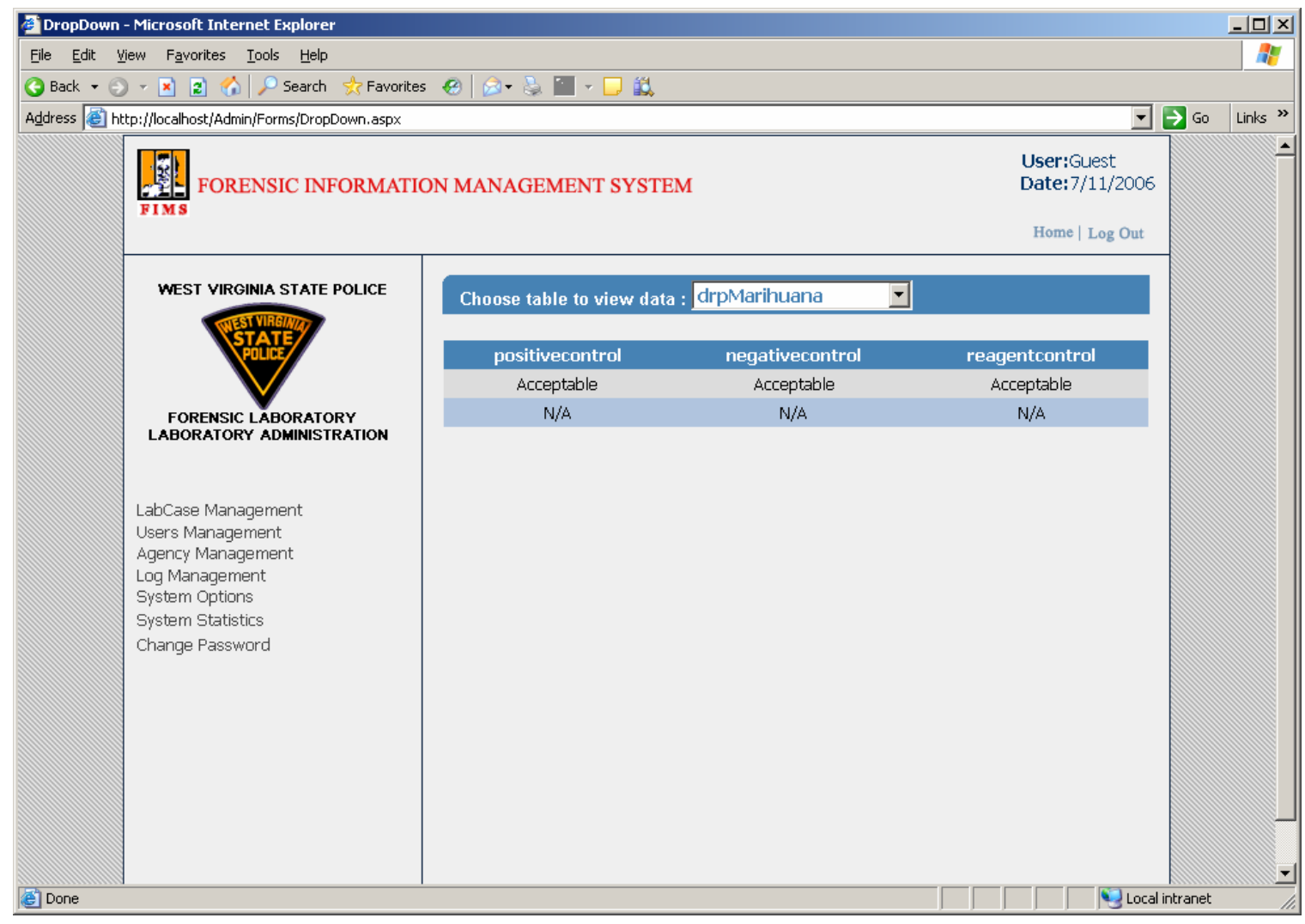

Figure 5.19: Dropdown List Options Section 


\section{System Statistics}

This section enables viewing all the cases by years as shown in figure 5.20. On completion this section enables administrator to generate case statistics.

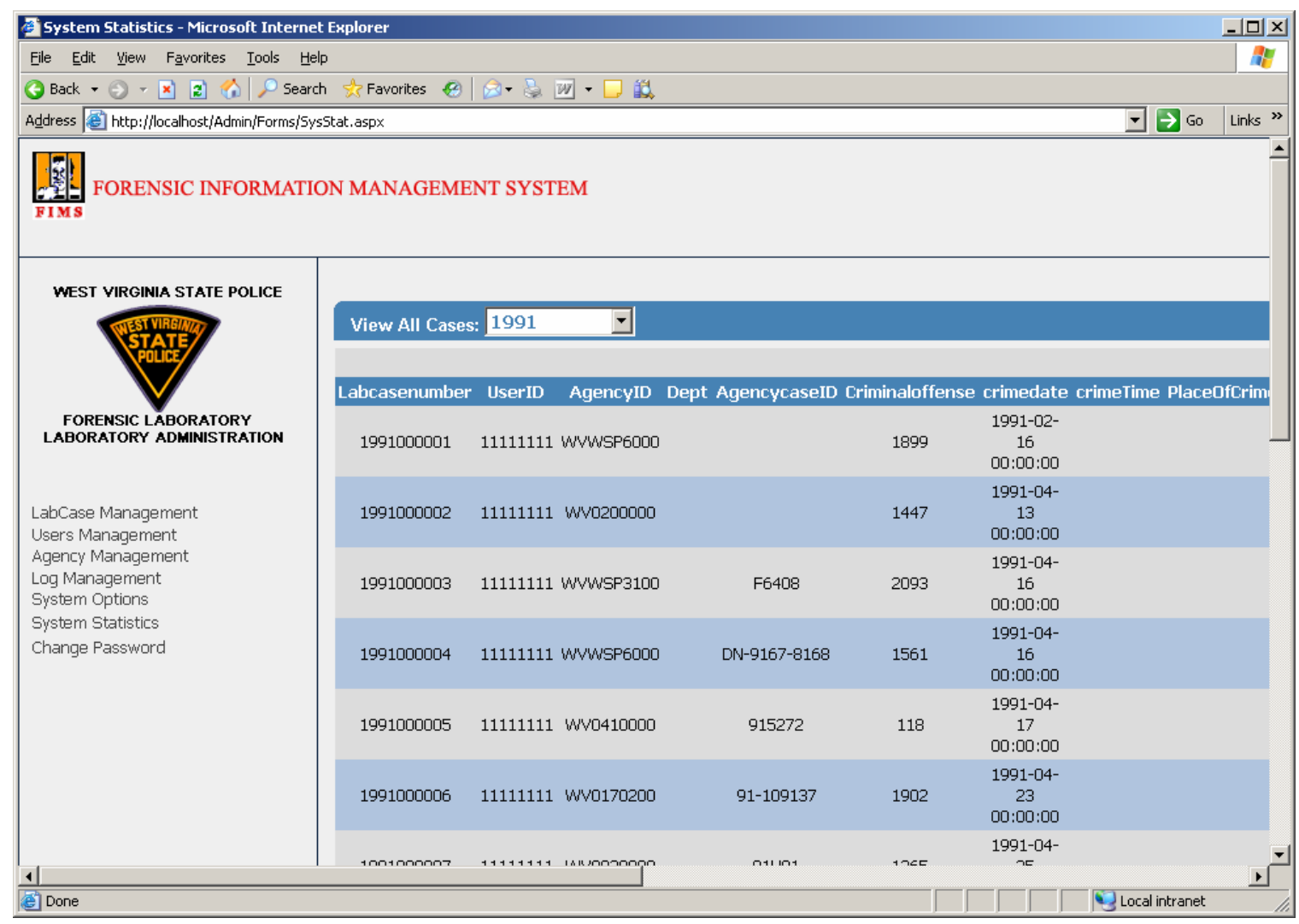

Figure 5.20: System Statistics Section 


\section{Change Password Section}

Administrator can change password through this section by providing appropriate user credentials as shown in Figure 5.21.

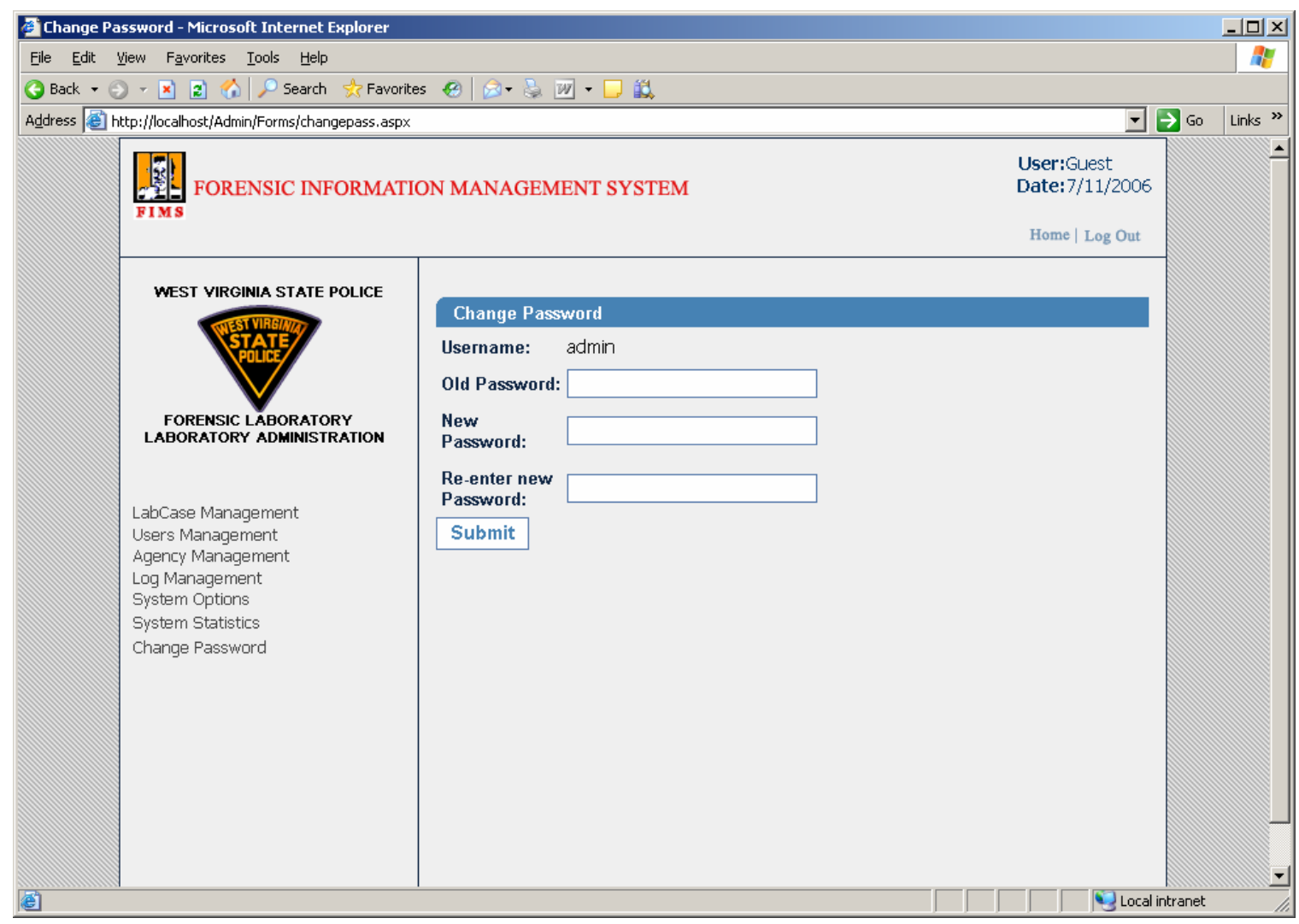

Figure 5.21: Change Password Section

\section{Exiting Laboratory Administration}

Administrator can terminate the current session by clicking on “Log Out” hyperlink on the top right corner of the page. 


\section{CHAPTER 6 \\ BIOCHEMISTRY}

\subsection{Existing Biochemistry Process}

The Biochemistry Unit of the laboratory receives and examines physical evidence for the presence of biological material. This unit performs DNA analysis on blood, semen, and other biological specimens. The sensitivity of DNA analysis allows a large variety of samples to be tested. Generally, any cellular material can be used as a source of DNA. In routine casework the techniques are so sensitive that DNA profiles have been obtained from blood stained clothing that had been washed, from gum that had been chewed, and from envelopes that have been licked. The Biochemistry unit manages the Convicted Offender DNA Identification System (CODIS) program for the State of West Virginia. All individuals convicted of violent crimes, sex offenses and most felonies in the State of West Virginia are required to provide the State Police with a DNA sample for analysis. After analysis, the DNA profile of the convicted offender is entered into a national database managed by the FBI. DNA profiles developed from evidence are compared to the convicted offender database. If a hit or match occurs as a result of a database search, laboratory personnel review the match to determine what additional action is required. If appropriate, the investigating agency is contacted with the name of the convicted offender whose profile matched the evidence [16].

The analyst in the biochemistry unit receives the LEIF form and chain of custody form along with the evidence items. The analyst prepares samples and performs the required test. The test results are documented on appropriate forms. The forms used in the Biochemistry unit are: Extraction \& Amplification (EA) worksheet, Product Gel (PG) worksheet, Yield Gel (YG) worksheet, and Sperm Identification (SI) worksheet. Once the examinations are completed, the evidence is returned to the CER and the secretary prepares a draft report. The draft report is then reviewed by the analyst/reviewer. After approval, the final report is sent to CER. Figure 6.1 shows the existing process for the biochemistry unit. 


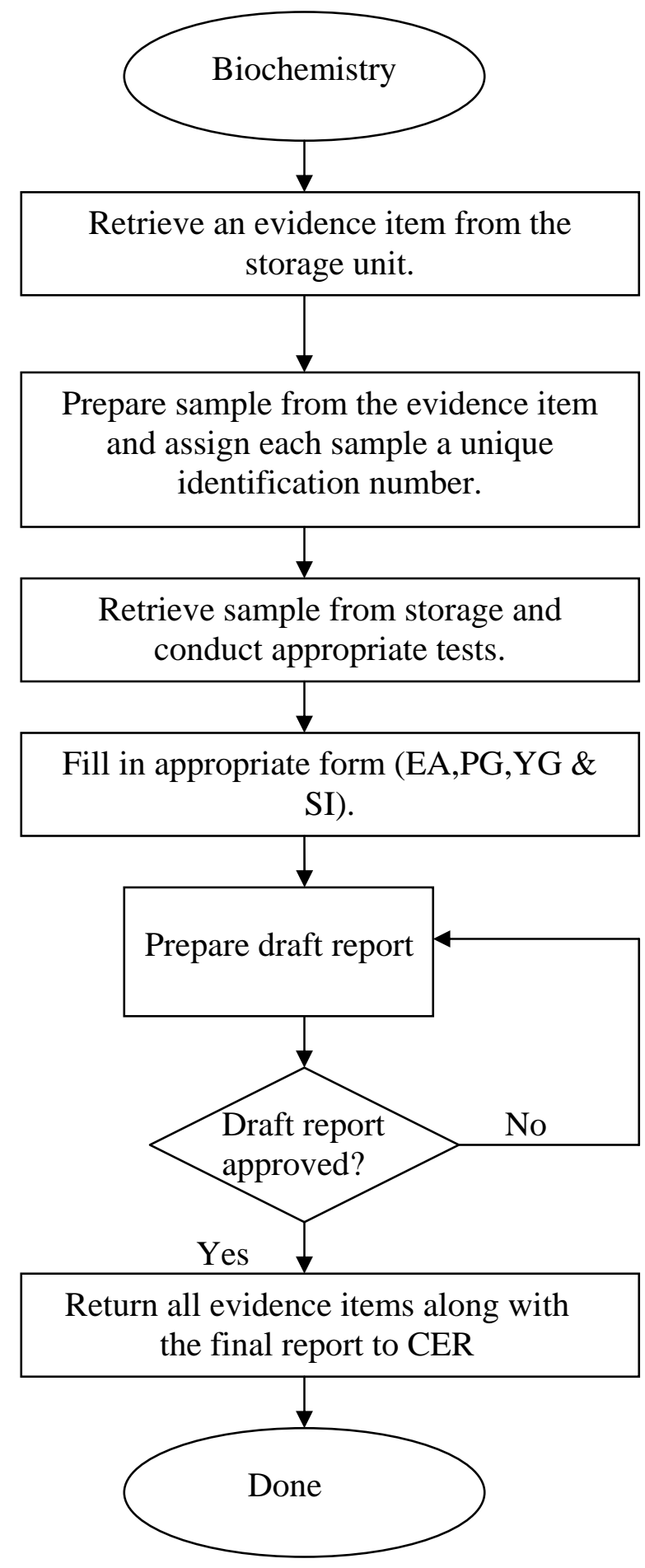

Figure 6.1: Existing Process for Biochemistry Unit 


\subsection{Re-engineering the Biochemistry Process}

The analyst in the biochemistry unit receives the items assigned for further processing and analysis from CEP. Received items are sub-divided into samples for conducting the requested tests. An individual item may be divided into many samples according to the requirement and complexity of the required test. A report for each sample is generated. An item report is compiled by combining sample reports. After the report is received, the reports along with the test items are sent back to CEP for further processing. Evidence and information flow diagram is shown in Figure 6.2.

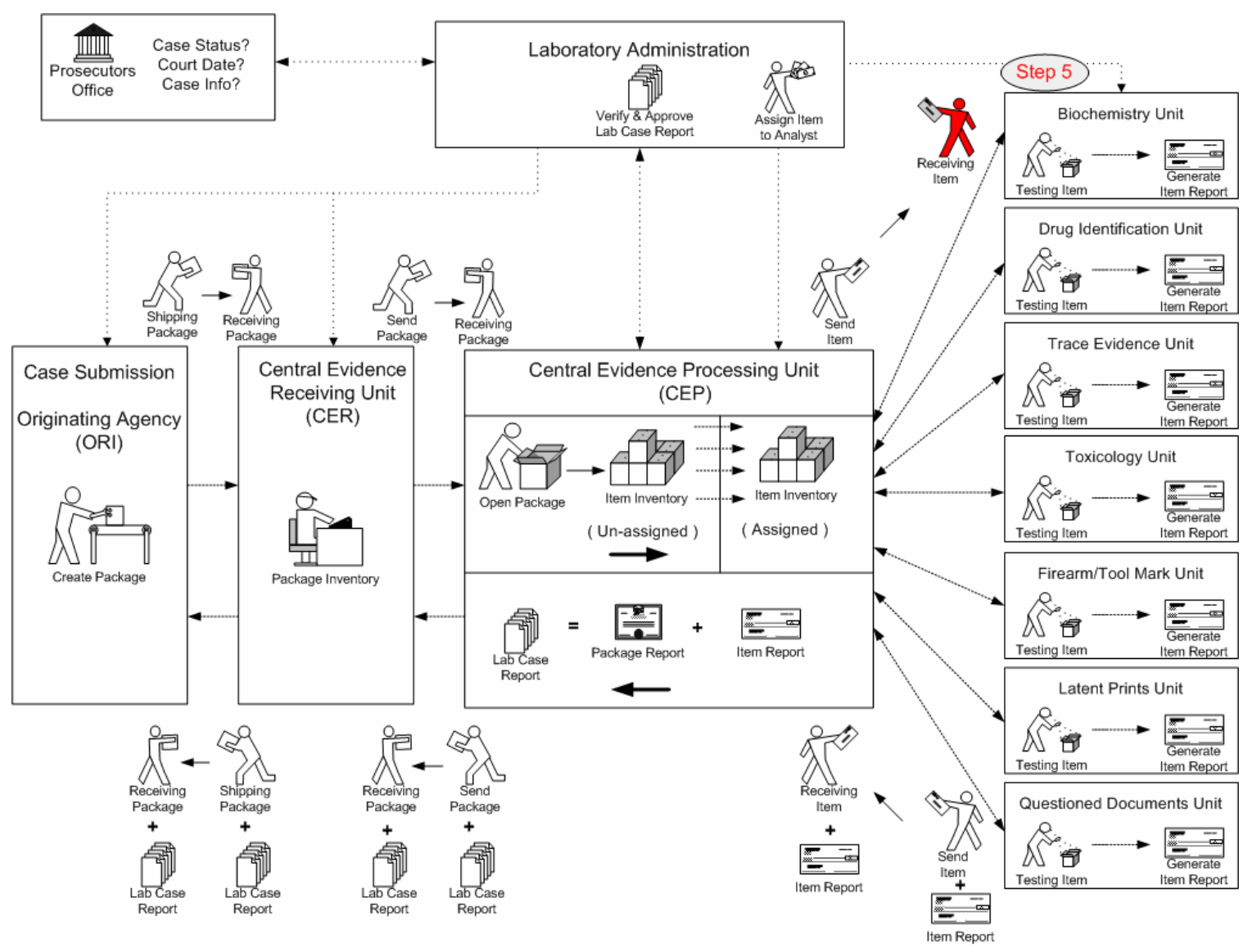

Figure 6.2: Evidence \& Information Flow 
Detail procedure followed by biochemistry unit for further processing and analysis is shown in Figure 6.3.

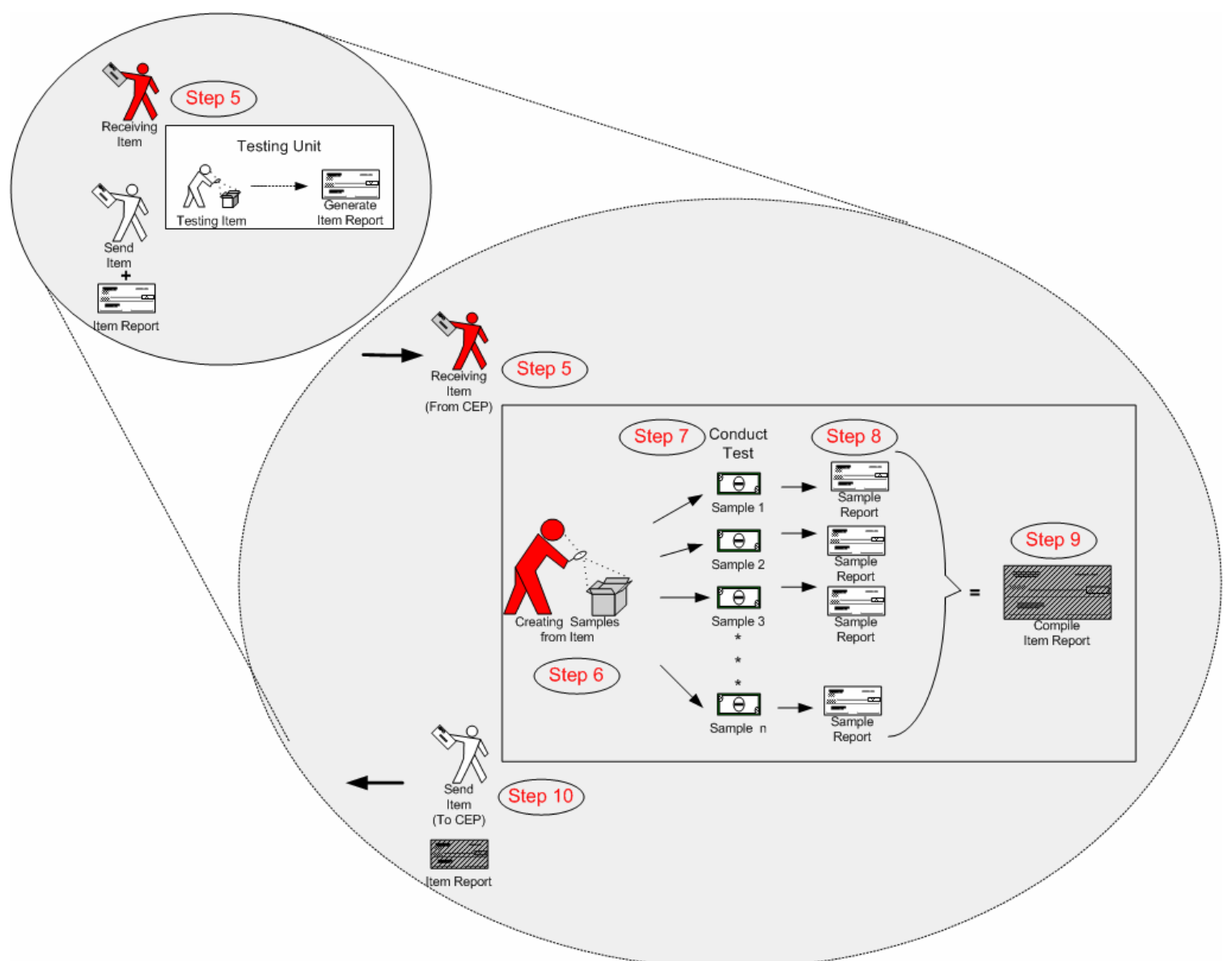

Figure 6.3: Evidence Item Processing \& Report Generation 


\subsubsection{Biochemistry Entity Relationship Diagram}

Biochemistry data tables are connected with each other through relations as shown in the figure 6.4

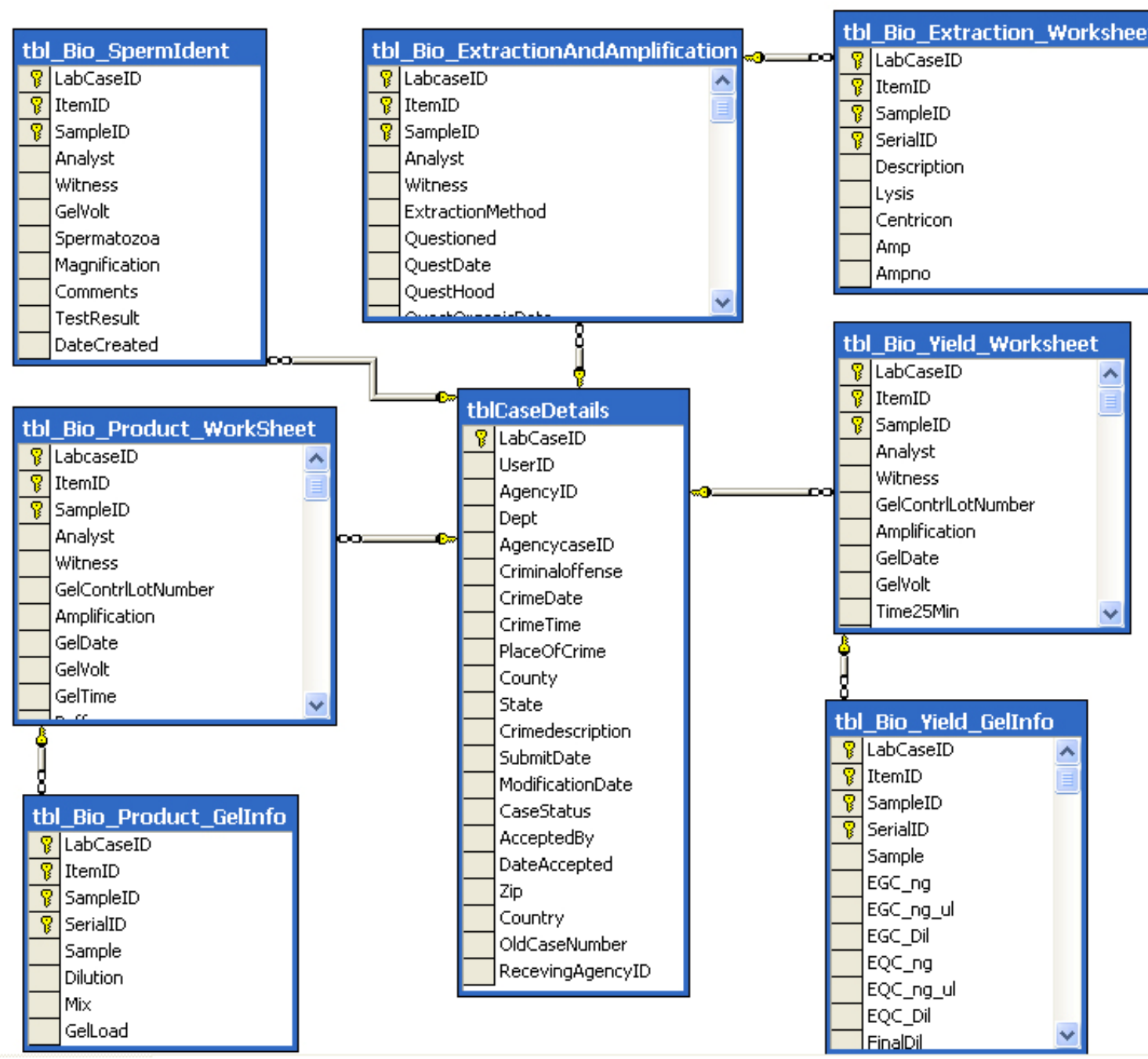

Figure 6.4: Entity Relationship Diagram 


\subsubsection{Biochemistry Database Tables}

The biochemistry unit module stores its data in the following tables

- Product Gel (tbl_Bio_Product_GelInfo \& tbl_Bio_Product_Worksheet)

- Sperm Identification (tbl_Bio_SpermIdent)

- Yield Gel (tbl_Bio_Yield_GelInfo)

- Extraction and Amplification (tbl_Bio_Exctration_Worksheet \& ExctractionAndAmplification)

The various fields of the Product gel table are shown in Table 6-1. Product gel worksheet data is stored in Product gel Information (tbl_Bio_Product_GelInfo) and Product gel worksheet (tbl_Bio_Product_Worksheet) tables.

\section{Table 6-1: Product Gel Table}

\begin{tabular}{|l|l|}
\hline tbl_Bio_Product_WorkSheet \\
\hline i & LabcaseID \\
\hline i & ItemID \\
\hline O & SampleID \\
\hline & Analyst \\
\hline & Witness \\
\hline & GelContriLotNumber \\
\hline & Amplification \\
\hline & GelDate \\
\hline & Gelvolt \\
\hline & GelTime \\
\hline & Buffer \\
\hline & Agarose_Nuisieve \\
\hline & Agarose_GTG \\
\hline & SampleVolume \\
\hline & Comments \\
\hline & TestResult \\
\hline & DateCreated
\end{tabular}


Table 6-2: Product Gel Worksheet Table

\begin{tabular}{|l|l|}
\hline tbl_Bio_Product_GelInfo \\
\hline P & LabCaseID \\
\hline i & ItemID \\
\hline ? & SampleID \\
\hline O & Serialid \\
\hline & Sample \\
\hline & Dilution \\
\hline & Mix \\
\hline & Gelload \\
\hline
\end{tabular}

The various fields of the sperm identification table are shown in Table 6-3. Sperm identification worksheet data is stored and maintained in this data table

Table 6-3: Sperm Identification Table

\begin{tabular}{|l|l|}
\hline tbl_Bio_SpermIdent \\
\hline B & LabCaseID \\
\hline B & ItemID \\
\hline & SampleID \\
\hline & Analyst \\
\hline & Witness \\
\hline & Gelvolt \\
\hline & Spermatozoa \\
\hline & Magnification \\
\hline & Comments \\
\hline & TestResult \\
\hline & DateCreated \\
\hline
\end{tabular}


The various fields of the yield gel worksheet table are shown in Table 6-4. Yield gel worksheet data is stored and maintained in this data table

\begin{tabular}{|c|c|}
\hline \multicolumn{2}{|c|}{ tbl_Bio_Yield_GelInfo } \\
\hline$\%$ & LabCaseID \\
\hline 9 & ItemID \\
\hline 8 & SampleID \\
\hline 8 & SerialID \\
\hline & Sample \\
\hline & EGC_ng \\
\hline & EGC_ng_ul \\
\hline & EGC_Dil \\
\hline & EQC_ng \\
\hline & EQC_ng_ul \\
\hline & EQC_Dil \\
\hline & FinalDil \\
\hline & AA_PowerPlex \\
\hline & AA_Other 1 \\
\hline & AA_other2 \\
\hline
\end{tabular}

Table 6-5: Yield Gel Worksheet Table

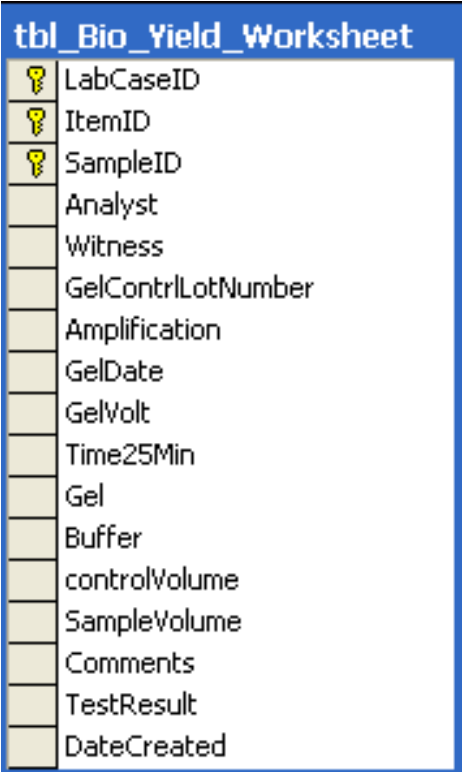


The various fields of the extraction and amplification table are shown in Table 6-6. Extraction and amplification worksheet data is stored in Extraction \& amplification and Product gel worksheet (tbl_Bio_Extraction_Worksheet) tables.

Table 6-6: Extraction \& Amplification Table

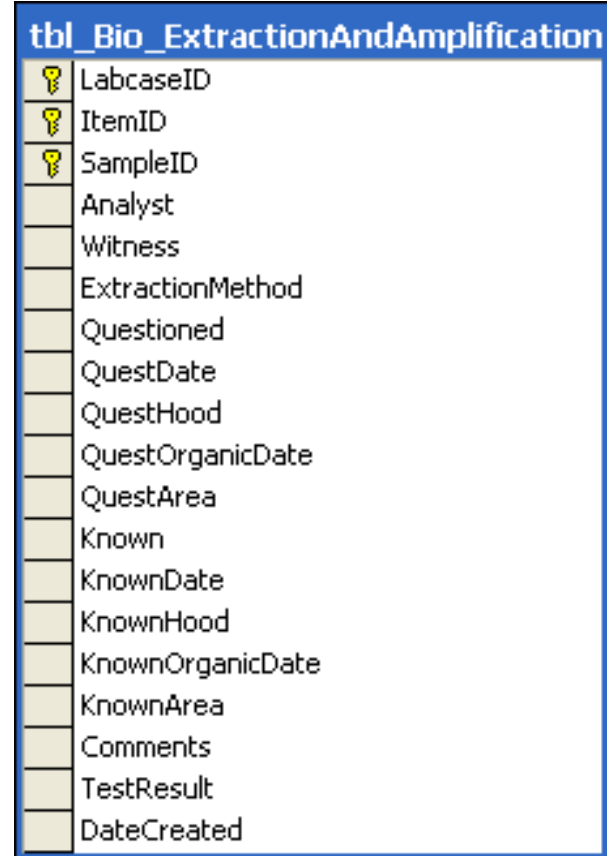

Table 6-7: Extraction \& Amplification Worksheet Table

\begin{tabular}{|c|l|}
\hline tbl_Bio_Extraction_Worksheet \\
\hline LabCaseID \\
\hline i & ItemID \\
\hline E & SampleID \\
\hline B & Serialid \\
\hline & Description \\
\hline & Lysis \\
\hline & Centricon \\
\hline & Amp \\
\hline & Ampno \\
\hline
\end{tabular}




\subsubsection{Working with Biochemistry}

Biochemistry home page requires username and password to verify user credentials, as shown in the Figure 6.5. After verifying credentials, a user is directed to list of items assigned to him/her for further processing.

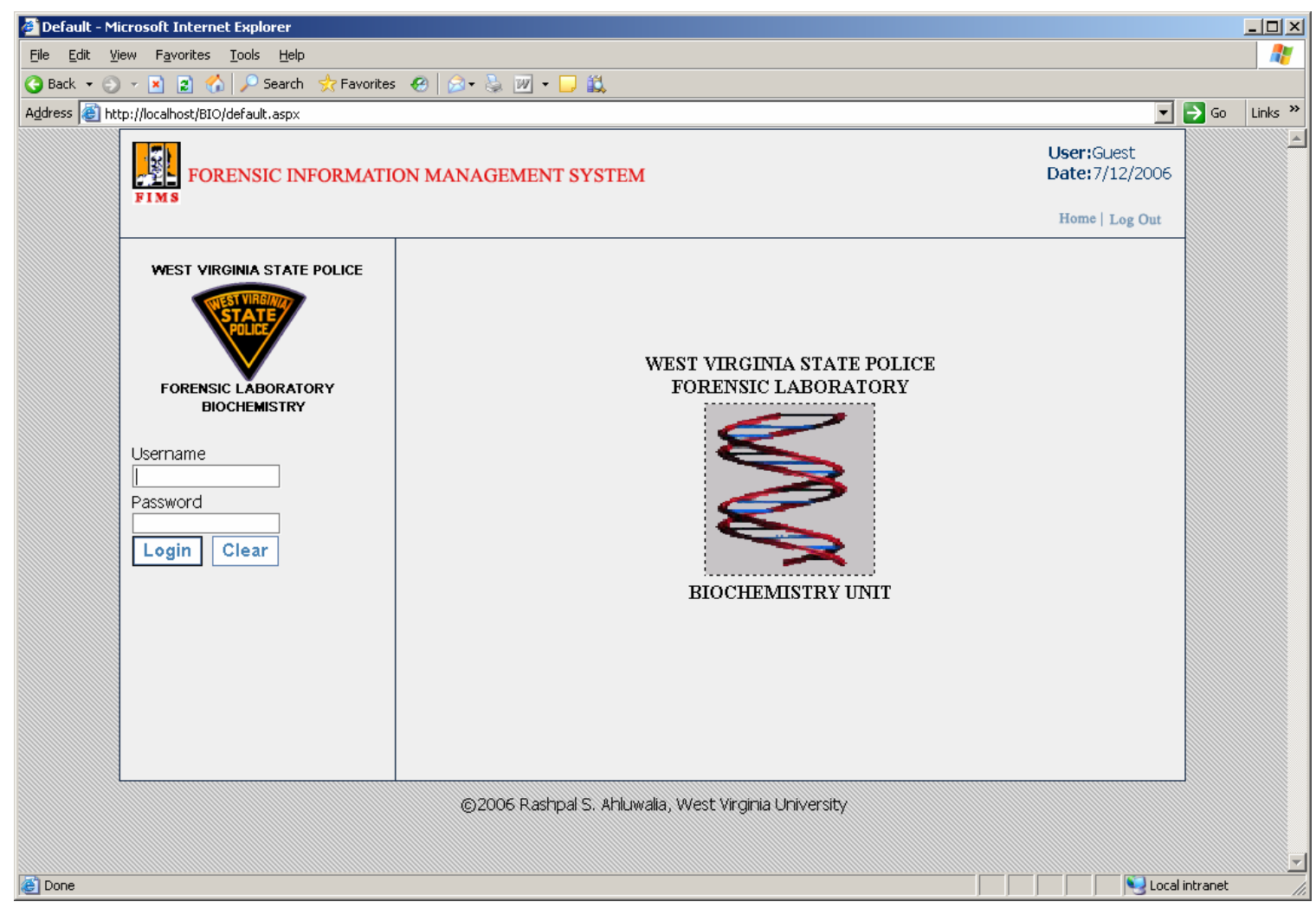

Figure 6.5: Biochemistry Home Page 


\section{View Items Assigned}

Figure 6.6 shows the list of items assigned to the analyst by the laboratory administration unit for further analysis. Initially job status for the item assigned is set to "Job Pending". The "View Details" hyperlink enables an analyst to view the details of the case. If the list of items assigned grows long i.e. more than 15 items, the record set is divided into multiple pages. Analysts can navigate between different pages by clicking on the header and footer navigation bar of the list.

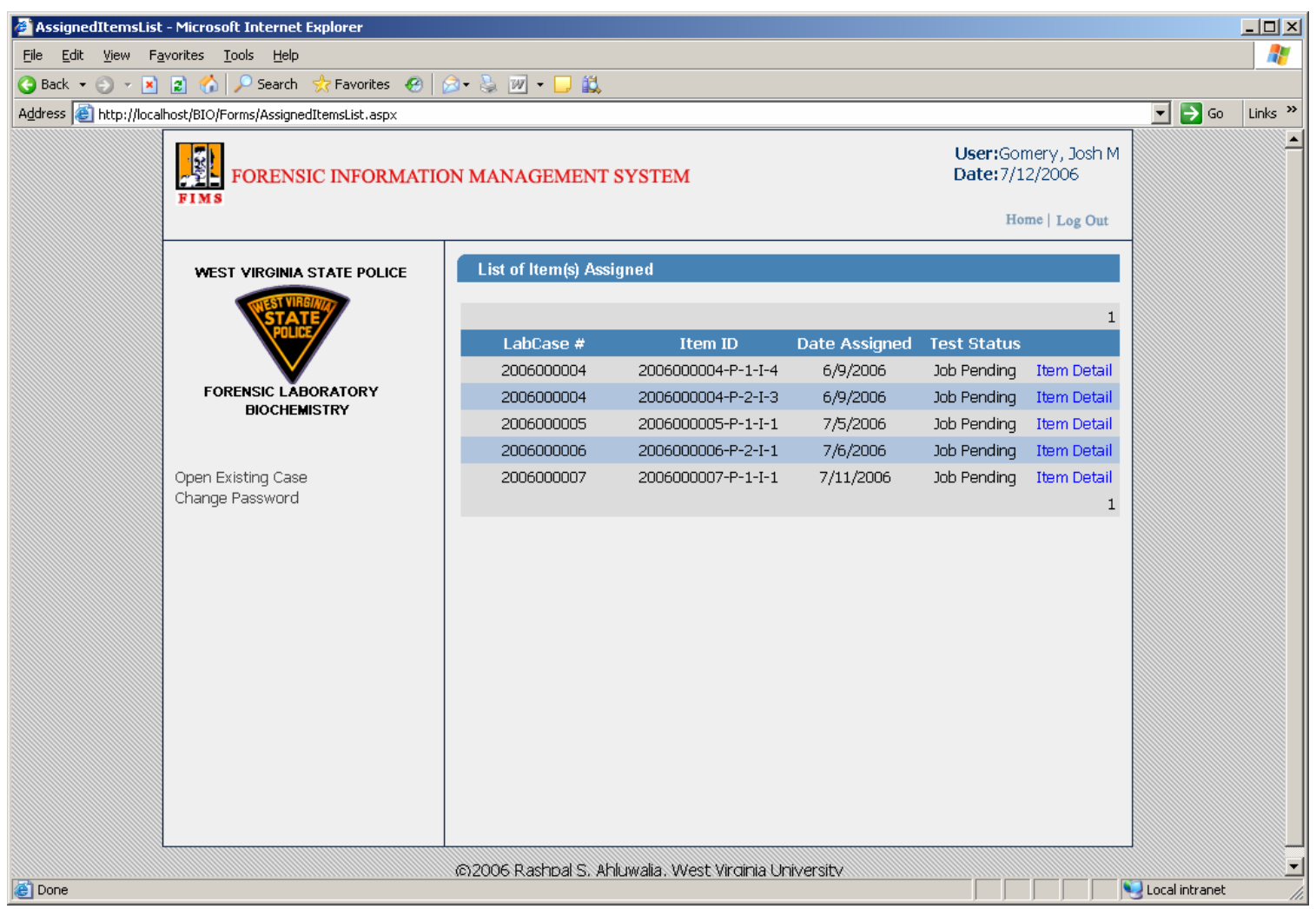

Figure 6.6: Assigned Item List 


\section{Item Picture Management}

This section describes the procedure of attaching pictures to an item. Analysts can perform two kinds of operation:

- View Pictures

- Upload Pictures

Analysts can access this section by clicking on the "View/Upload Item Photo" hyperlink in the item details section as shown inn Figure 6.9. View picture section list the pictures already attached to the item with their thumbnails as shown in Figure 6.7. Analysts can also view the full size image by clicking on the image thumbnail. Full size image opens in a new window, which can be closed after use.

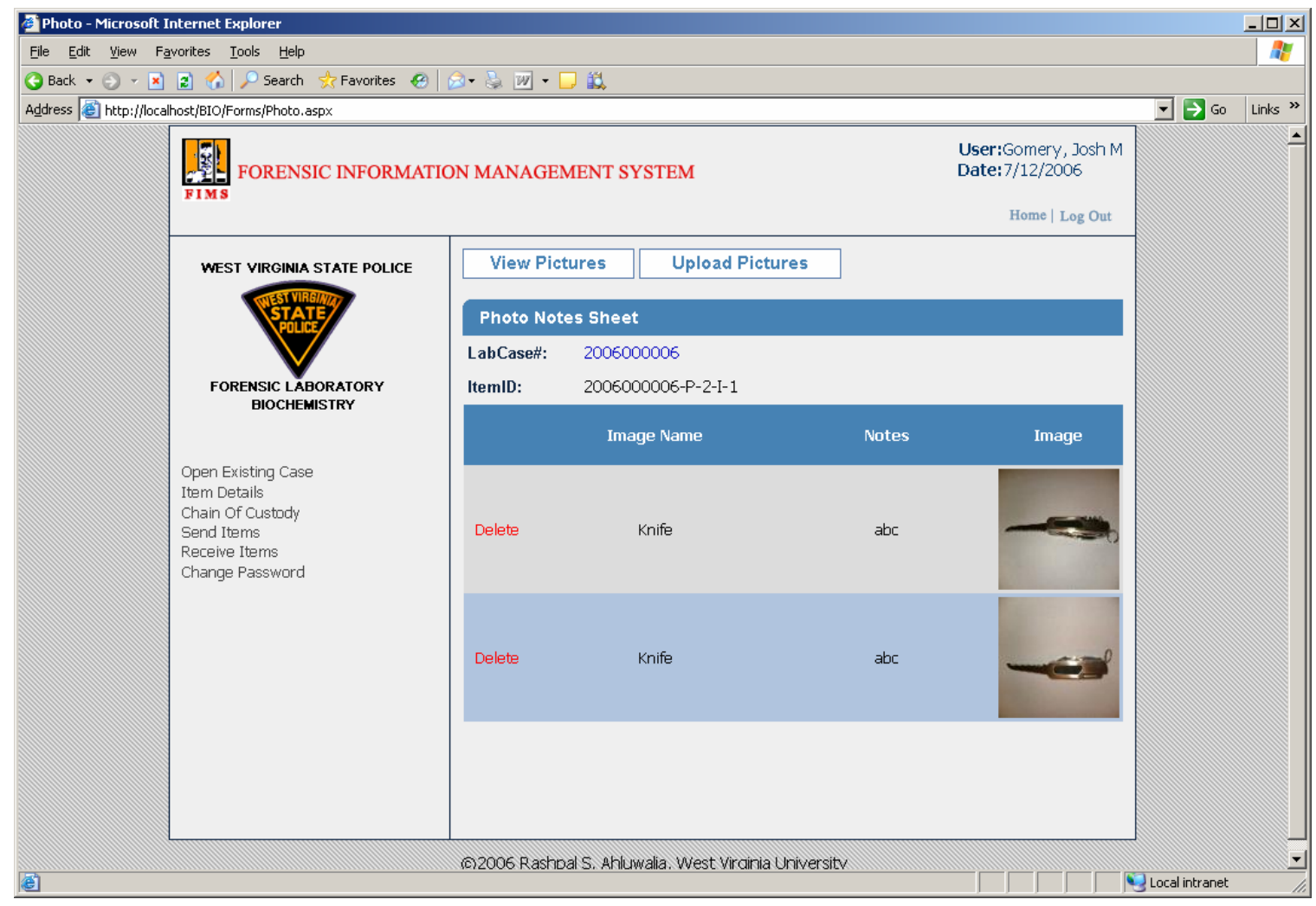

Figure 6.7: View Item Photo 
Analysts can upload any digital photo in JPG format directly into the database using this section as shown in Figure 6.8. To upload an image into database, analysts are required to browse for the image path, set other required fields and click on the "Upload the file” hyperlink. On successful upload a thumbnail appears on the bottom of the page with a success message.

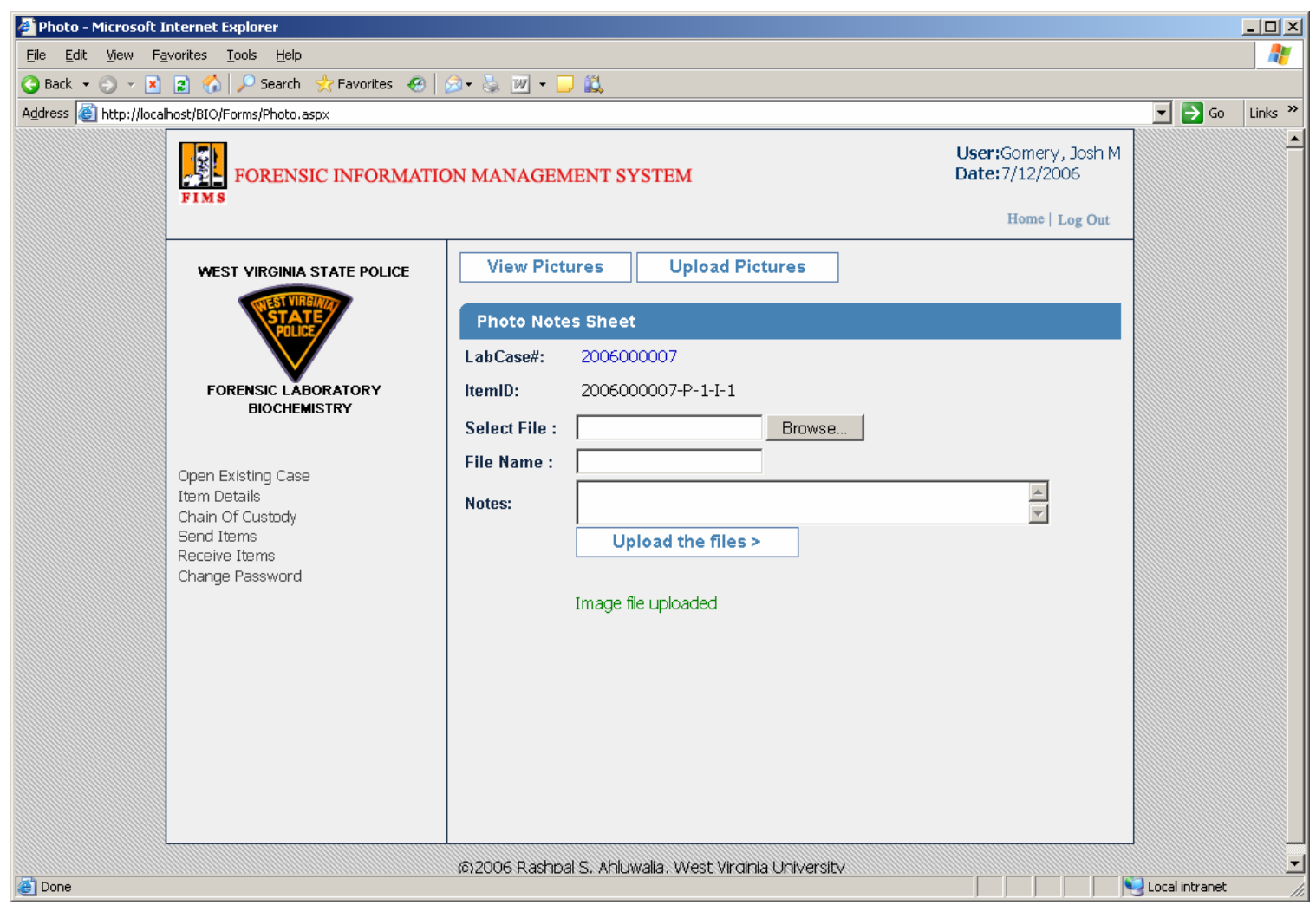

Figure 6.8: Upload Item Picture Section 


\section{Working with Samples}

Analysts receive items from the CEP unit for further analysis and processing. Once received items are sub-divided into samples for conducting requested tests. Each item is divided into one or more samples according to the requirement and complexity of the required test.

Analysts can create new, update or delete existing samples from the item details section as shown in Figure 6.9. To add a new sample analyst is required to provide description of sample and then click on "Add Sample” hyperlink. FIMS application automatically adds the time stamp to the item created. Initially, sample test status is set to "Test Pending" for the sample. Sample information can be updated by clicking on “Edit” hyperlink and existing sample can be deleted from the system by clicking on “Delete” hyperlink.

This section also enables analysts to view the final sample, sample photo, item, and item photo report. To access the individual sample/sample photo report analyst can click on "View Report" or "Photo Sheet" hyperlink. To view comprehensive item report analysts can click on "Item Report” or "Item Photo Report” hyperlink at the bottom of the page.

To complete the process for item testing, the analyst is required to click on "Item Test Complete” button, as shown in Figure 6.9. Once clicked, analyst will not be able to make any alterations to the existing item test report. 


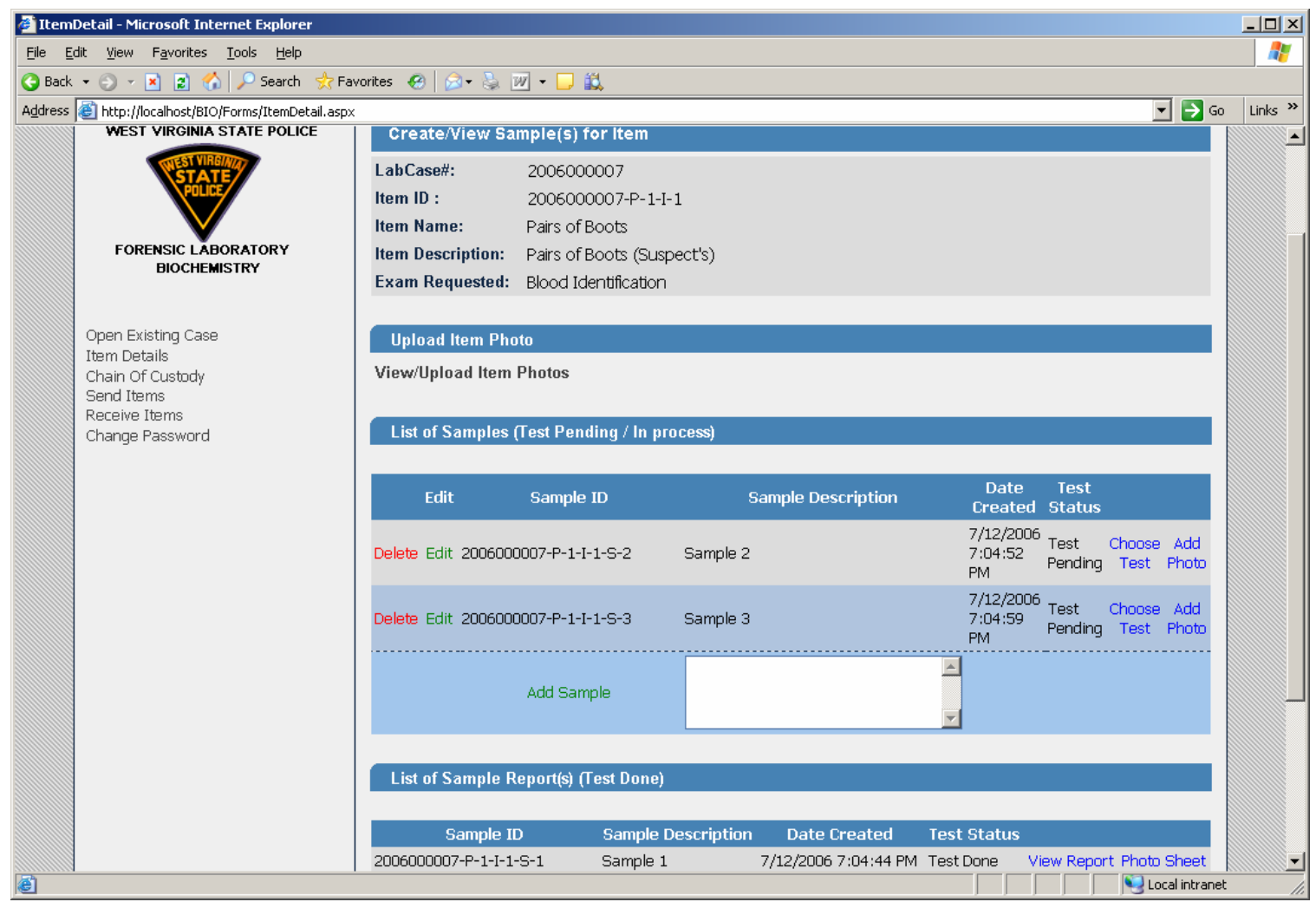

Figure 6.9: Item Detail Section 
Analysts can access this section by clicking on the "View/Upload Sample Photo" hyperlink in the item details section as shown in Figure 6.9. View picture section list the pictures already attached to the item with their thumbnails as shown in Figure 6.10. Analysts can also view the full size image by clicking on the image thumbnail. Full size image opens in a new window, which can be closed after use.

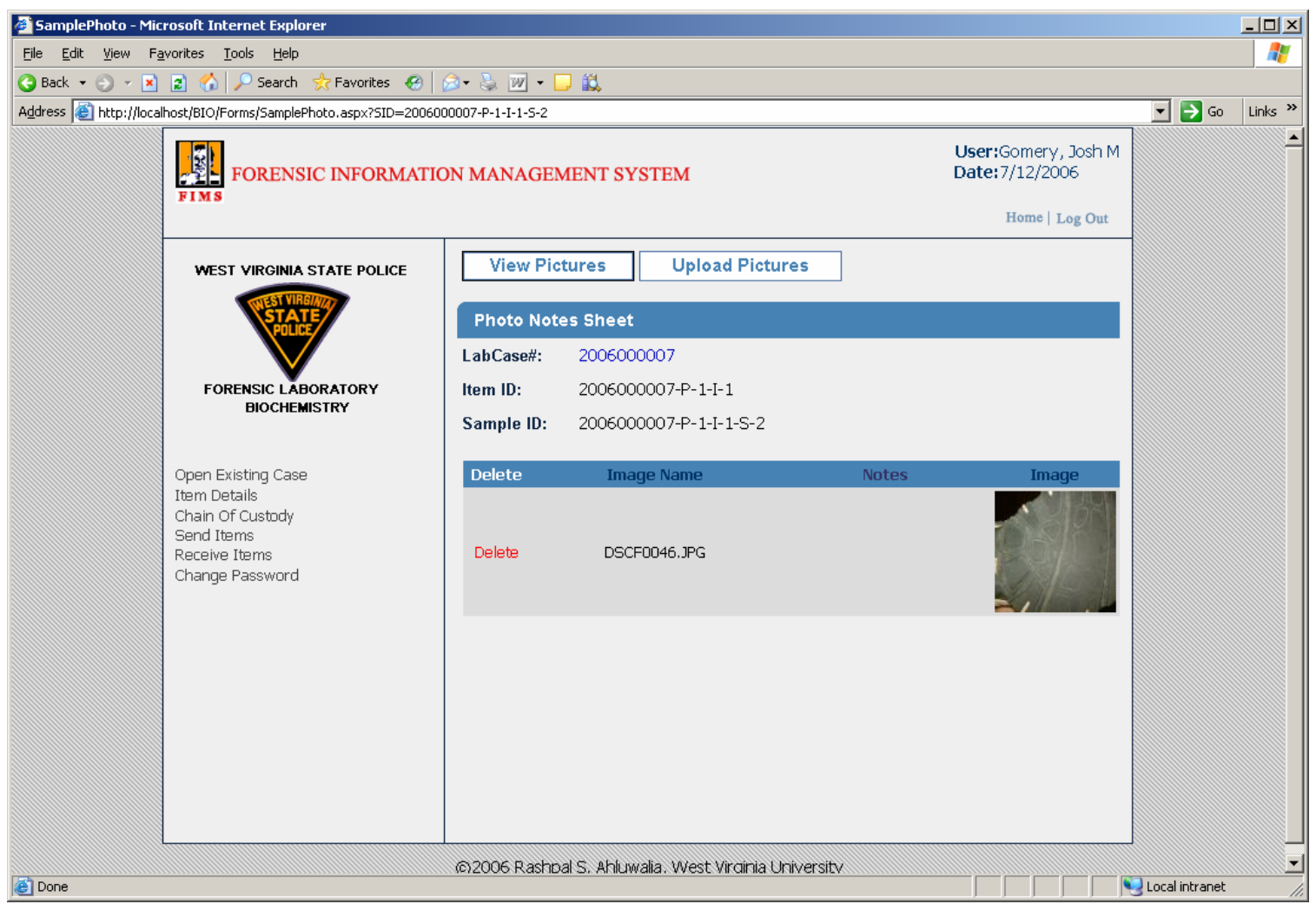

Figure 6.10: View Picture Section 
Analysts can upload any digital photo in JPG format directly into the database using this section as shown in Figure 6.11. To upload an image analysts are required to browse for the image path, set other required fields and click on the "Upload the file" hyperlink to upload the image into database. On successful upload a thumbnail appears on the bottom of the page with a success message.

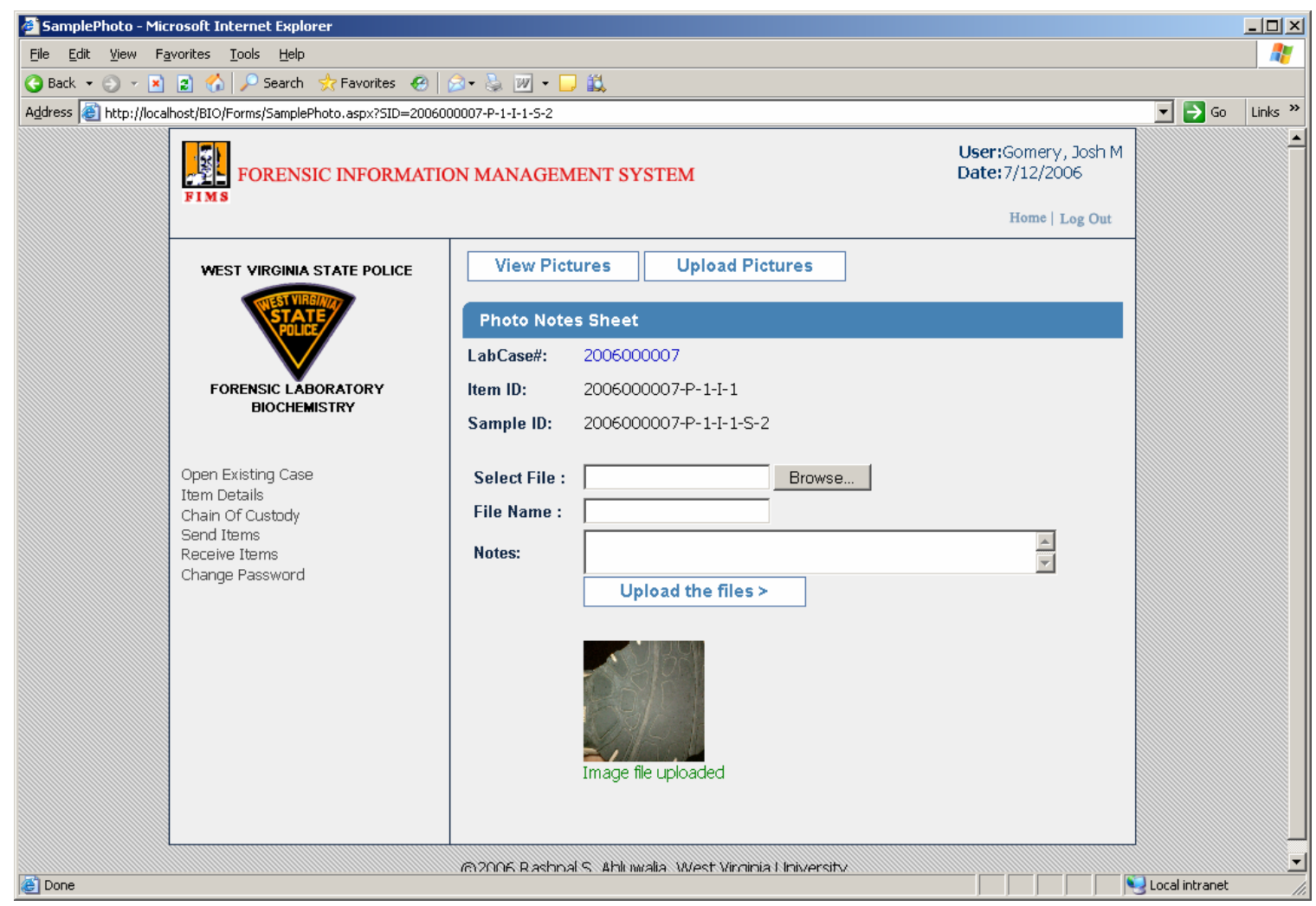

Figure 6.11: Upload Sample Picture Section 


\section{Choosing Test Worksheet}

After sample creation, analysts are required to decide what kinds of tests are required? Analysts choose type of test manually. Once the decision is made the analyst can see the list of tests available, and then click "Choose Test” hyperlink as shown in Figure 6.12. FIMS remembers the chosen sample ID during the process.

The worksheets for the biochemistry are:

- Extraction/Amplification

- Sperm Identification

- Product gel

- Yield gel

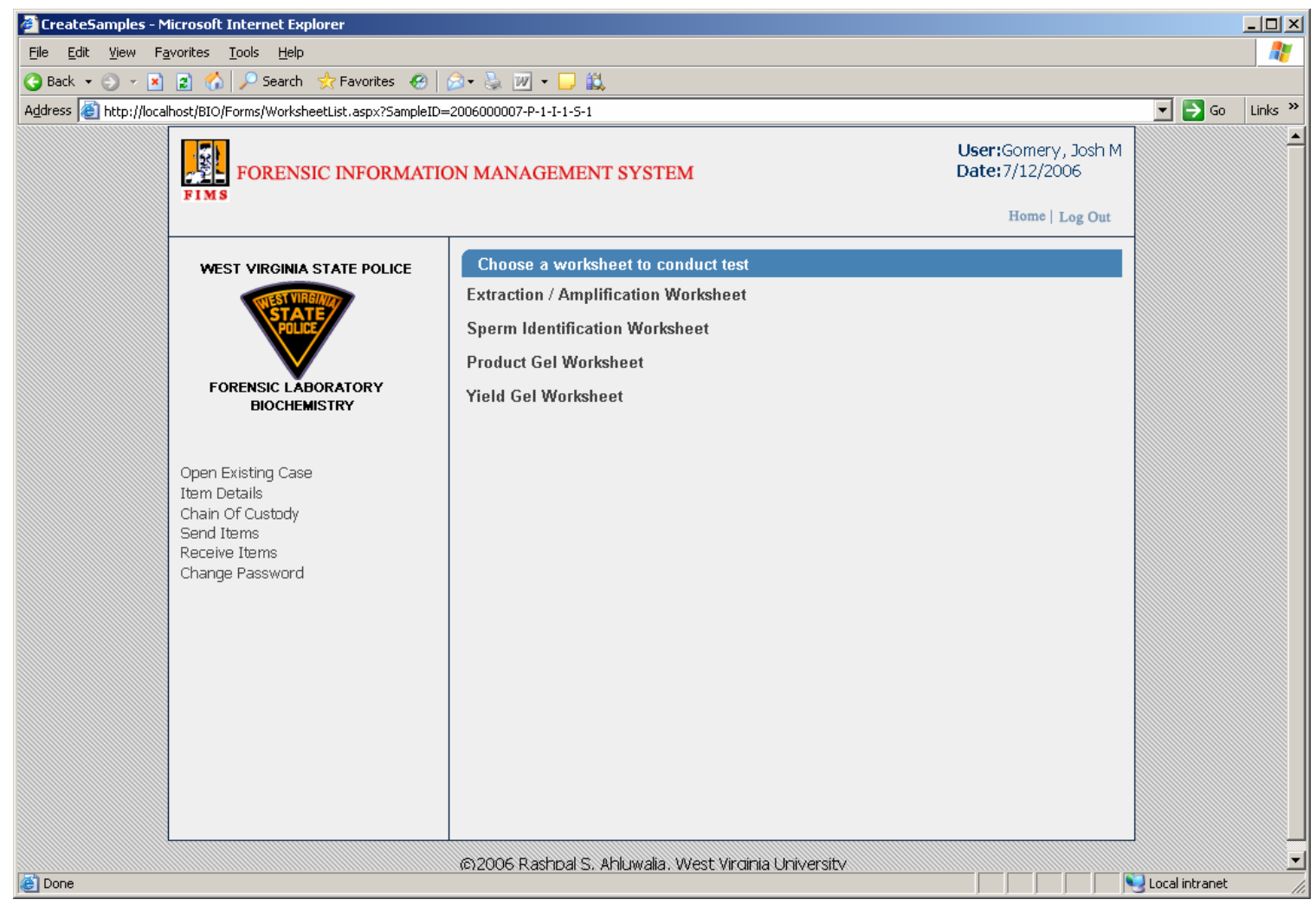

Figure 6.12: Choose Sample Test Section 


\section{Extract/Amplification Worksheet}

Extraction/Amplification worksheet is used for filling information pertaining to extraction/amplification test performed on the sample by the analyst. After filling the required data fields, analyst is required to click on "Save Results" button located on top of the page, as shown in Figure 6.13. After saving the worksheet data, analyst can finalize and close the worksheet by clicking on "Test Done" button. The analyst cannot make any alterations in the worksheet once the "Test Done" button is clicked.

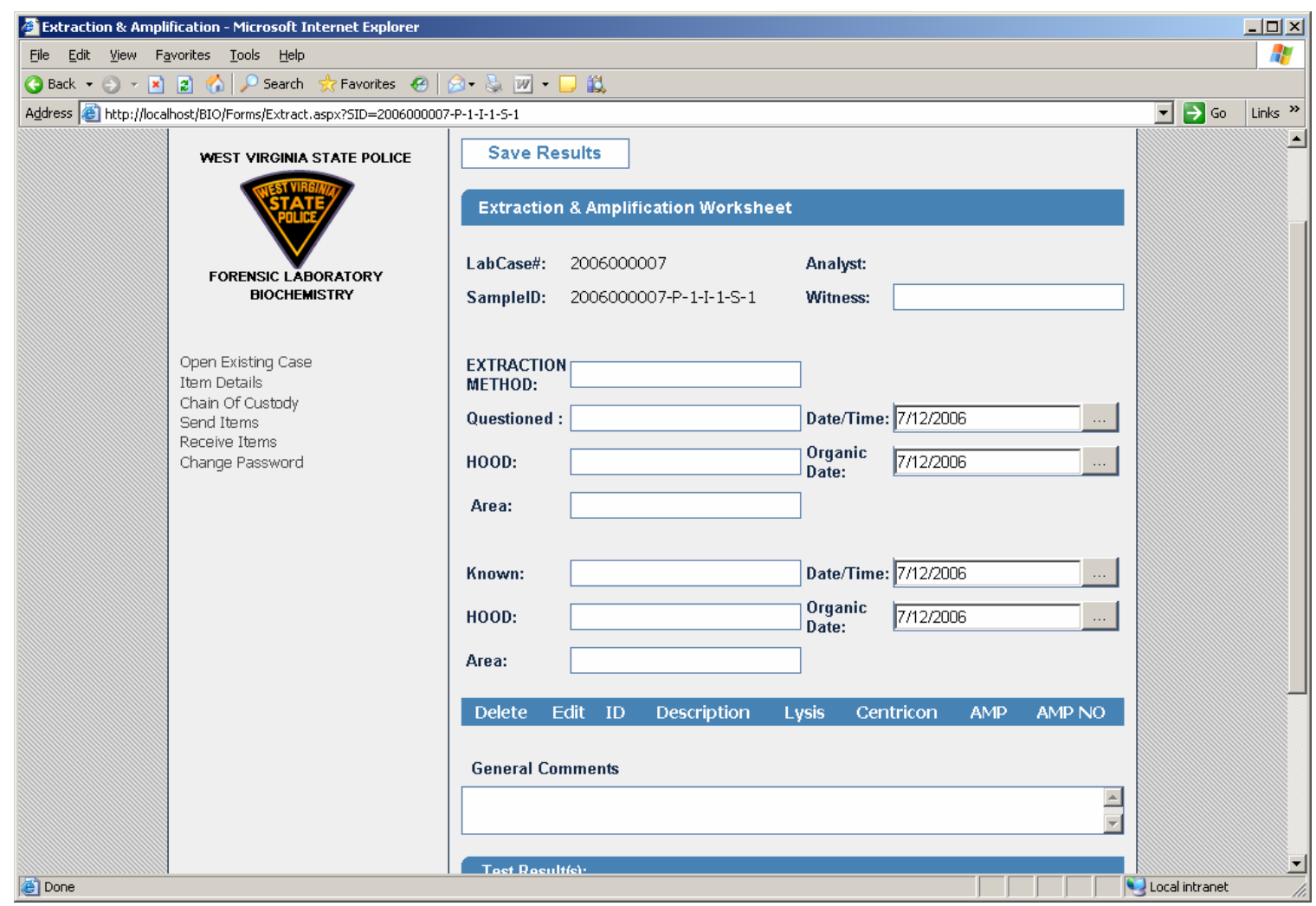

Figure 6.13: Extraction /Amplification worksheet 


\section{Sperm Identification Worksheet}

Sperm identification worksheet is used for filling information pertaining to sperm identification test performed on the sample by the analyst. After filling the required data fields, analyst is required to click on "Save Results" button located on top of the page, as shown in Figure 6.14. After saving the worksheet data, analyst can finalize and close the worksheet by clicking on "Test Done" button. The analyst cannot make any alterations in the worksheet once the "Test Done" button is clicked.

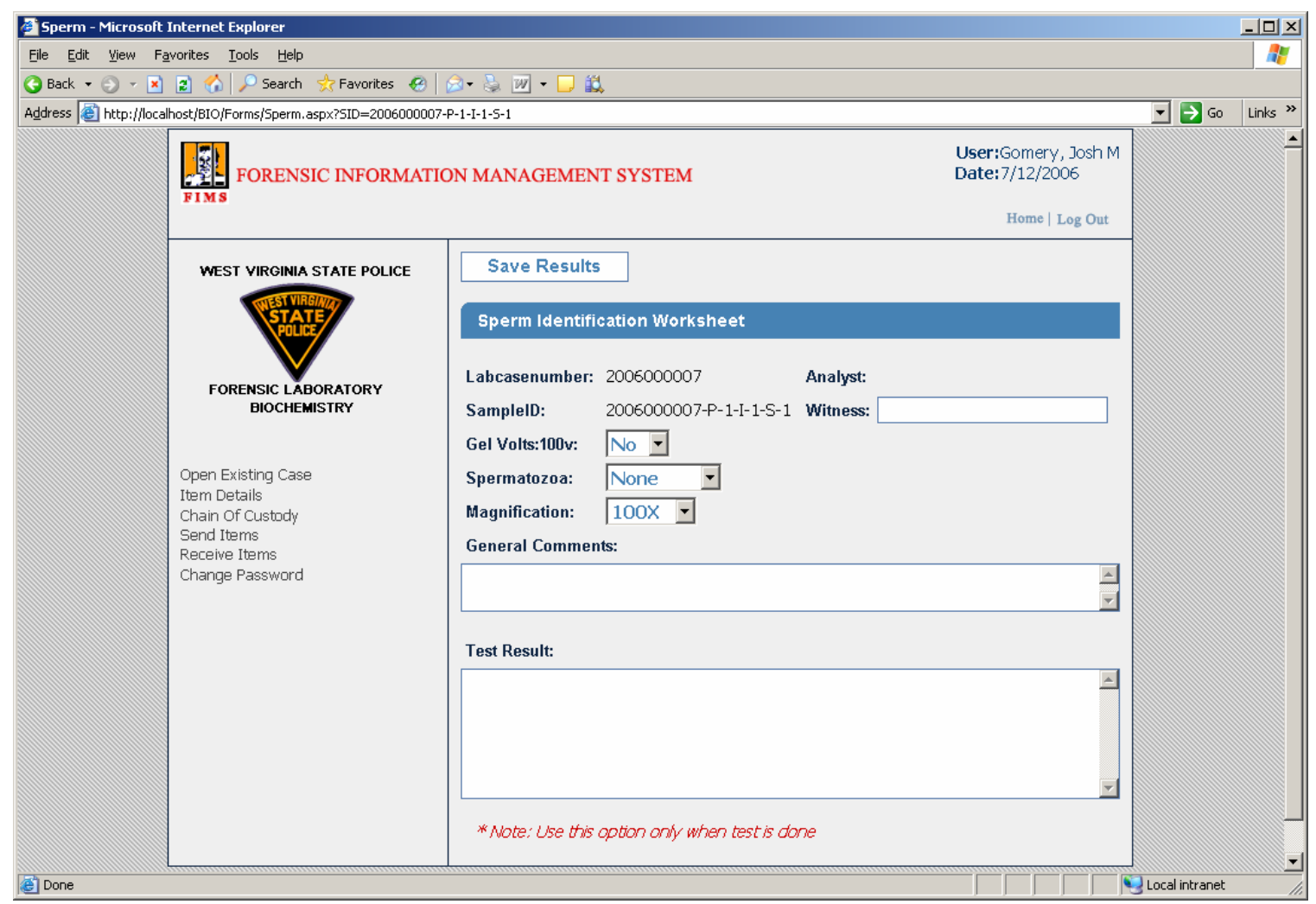

Figure 6.14: Sperm Identification Worksheet 


\section{Product Gel Worksheet}

Product gel worksheet is used for filling information pertaining to product get test performed on the sample by the analyst. After filling the required data fields, analyst is required to click on "Save Results" button located on top of the page, as shown in Figure 6.15. After saving the worksheet data, analyst can finalize and close the worksheet by clicking on "Test Done" button. The analyst cannot make any alterations in the worksheet once the “Test Done” button is clicked.

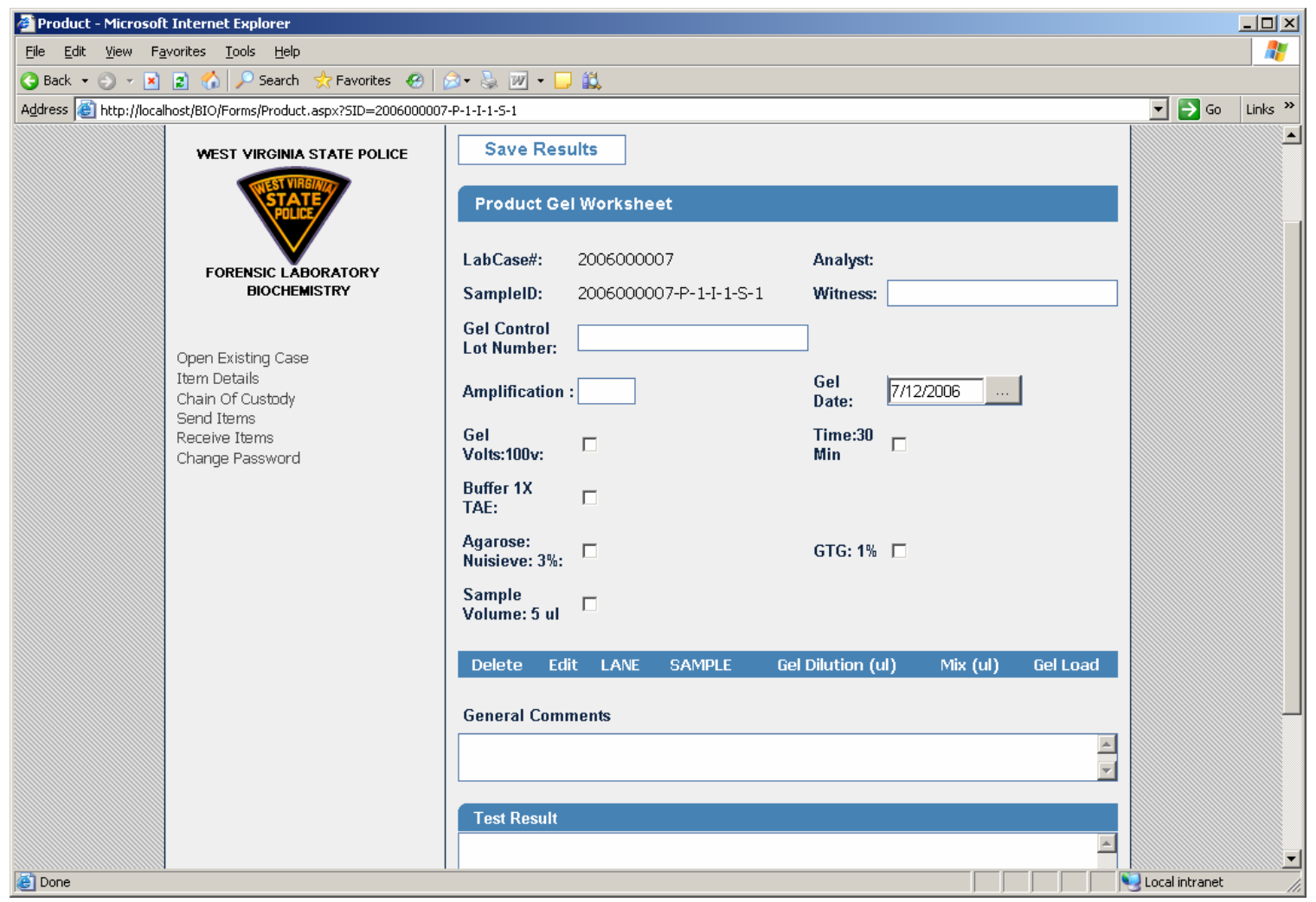

Figure 6.15: Product Gel Worksheet 


\section{Yield Gel Worksheet}

Yield gel worksheet is used for filling information pertaining to yield gel test performed on the sample by the analyst. After filling the required data fields, analyst is required to click on "Save Results" button located on top of the page, as shown in Figure 6.16. After saving the worksheet data, analyst can finalize and close the worksheet by clicking on "Test Done" button. The analyst cannot make any alterations in the worksheet once the "Test Done" button is clicked.

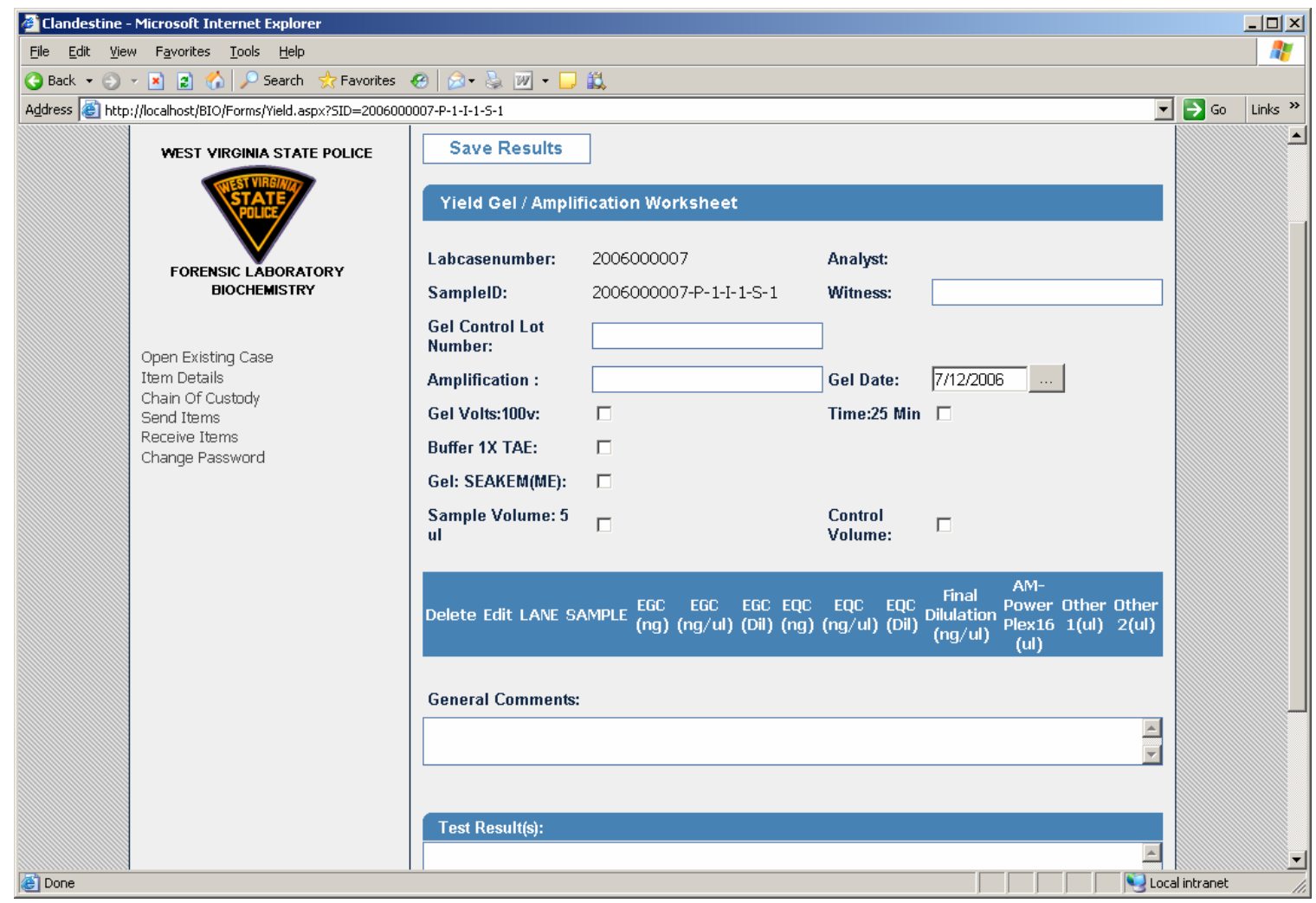

Figure 6.16: Yield Gel Worksheet 


\section{Receive Item}

Once an item is sent by CEP unit, Biochemistry unit analyst is responsible for receiving it by clicking on "Receive" button, under receive item section, as shown in Figure 6.17. This completes the handshaking protocol of item transfer between units.

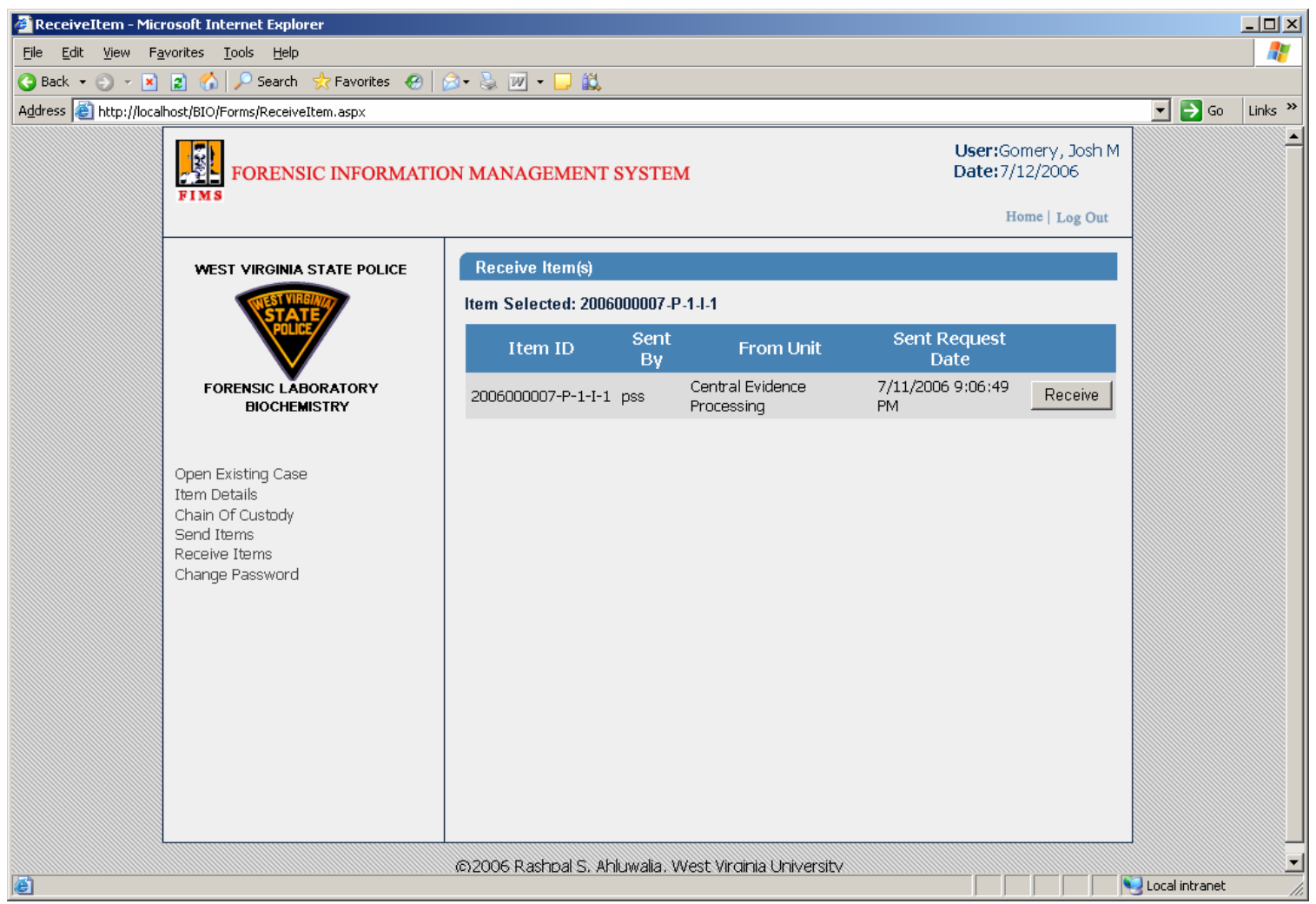

Figure 6.17: Receive Item Section 


\section{Send Item}

After completion of item testing, analyst is required to return the evidence item back to CEP unit. Figure 6.18 shows the section used to send an item back to the CEP unit. Analyst can use the drop down to select item ID and click on "Send" hyperlink, to send item back to CEP.

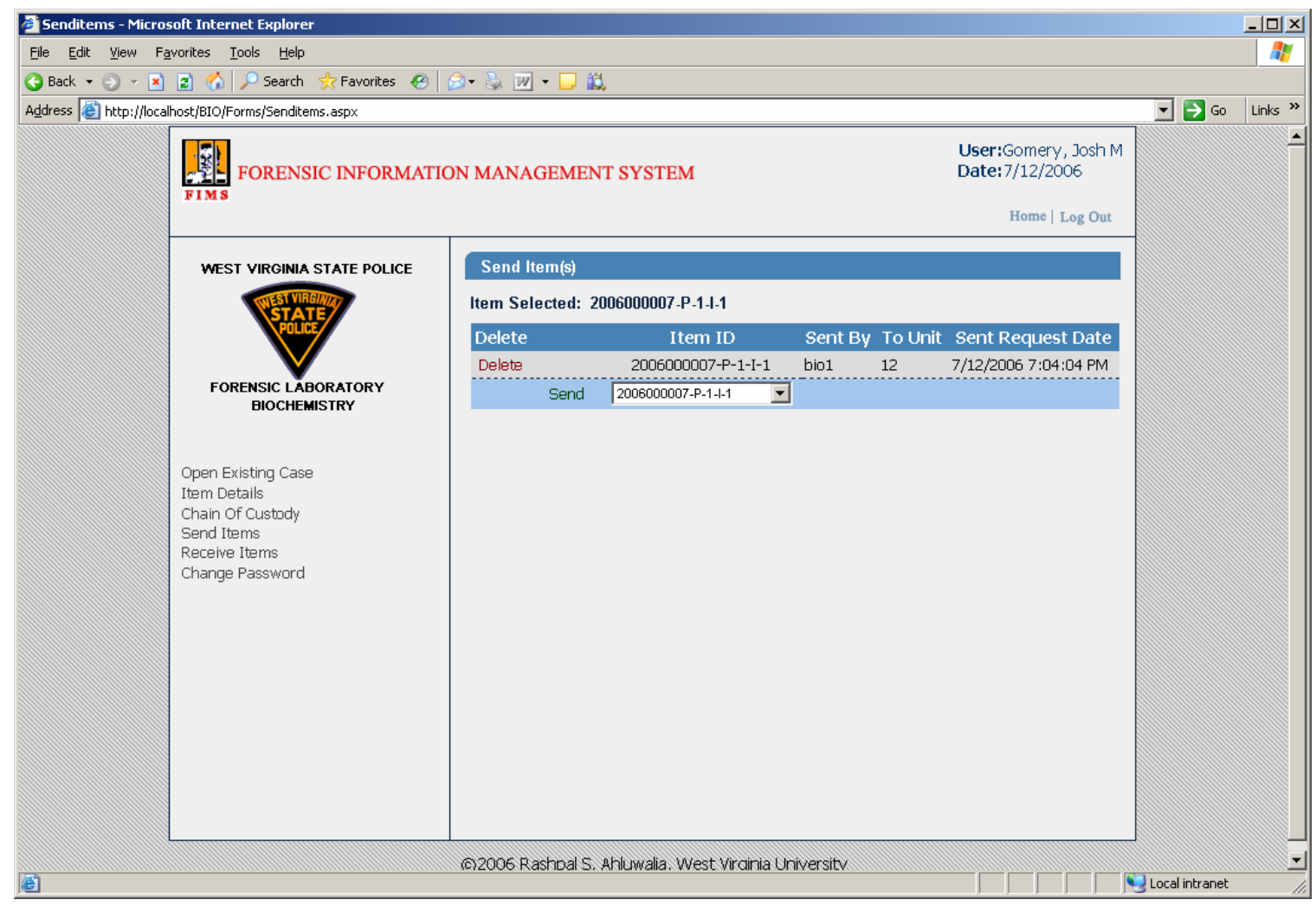

Figure 6.18: Send Item Section 


\section{Chain of Custody}

Item chain of custody information is shown in Figure 6.19. FIMS application records used ID, unit name, and time stamp for tracking item chain of custody.

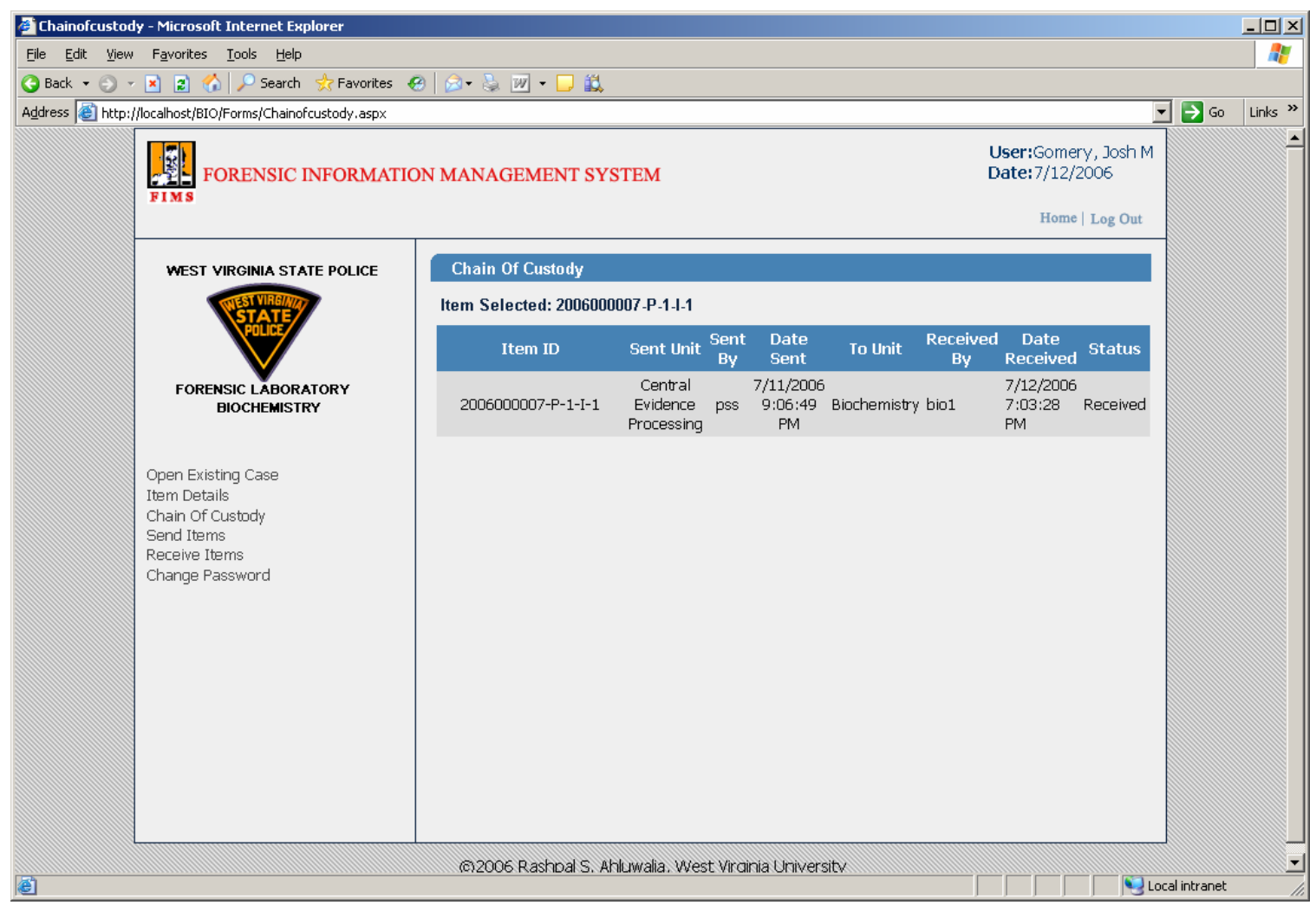

Figure 6.19: Item Chain Of Custody 


\section{Change User Password}

Analyst can change password through this section by providing appropriate credentials as shown in Figure 6.20.

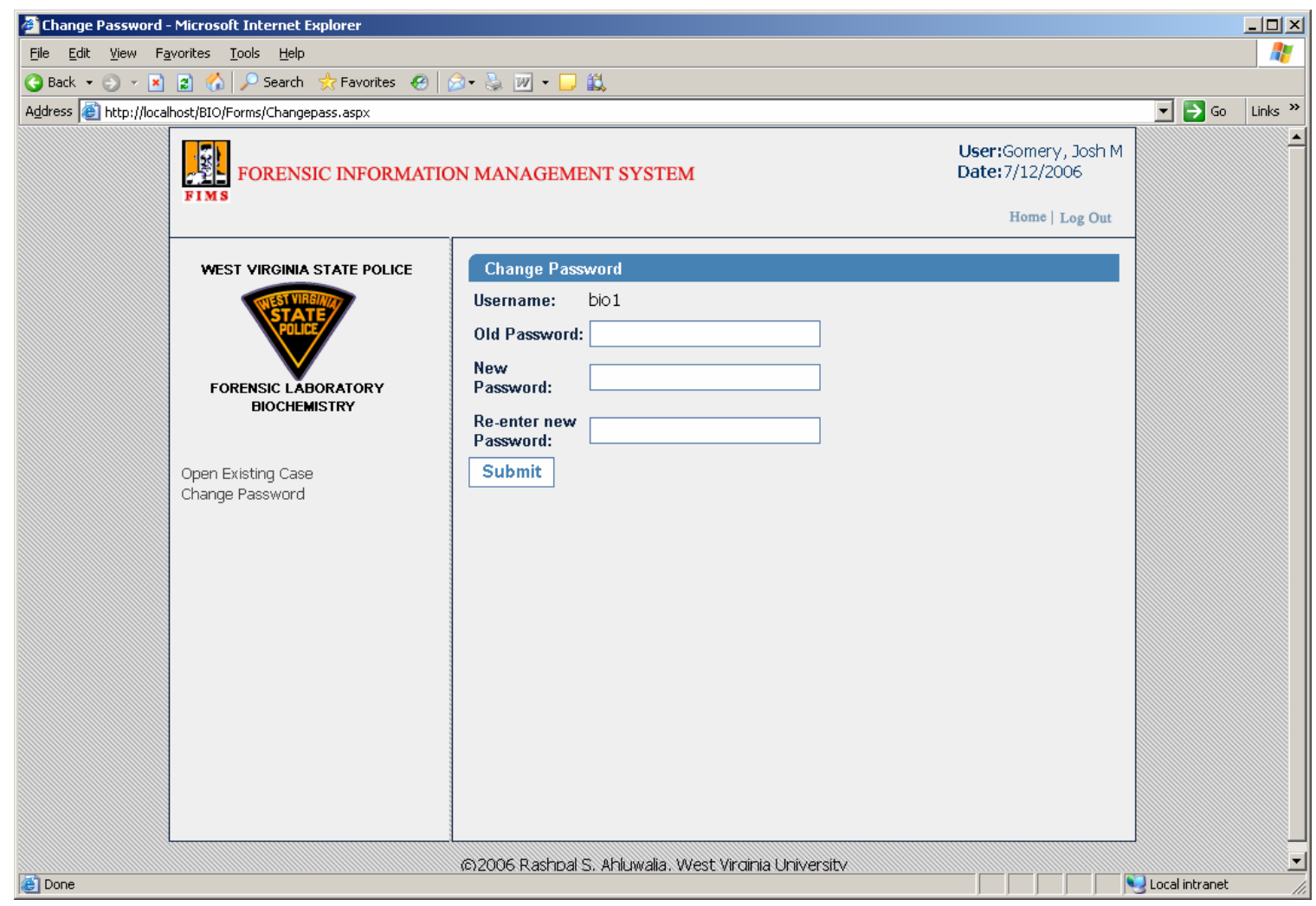

Figure 6.20: Change Password Section

\section{Exiting Biochemistry}

Users can terminate the current session by clicking on "Log Out" hyperlink on the top right corner of the page. 


\subsubsection{Generating Item and Sample Reports}

The analyst can generate the following types of reports

- Item Report

- Item Photo Report

- Sample Report

- Sample Photo Report

Figure 6.21 shows the item report, which is a cumulative report for all the samples. This section also facilitate analyst to export report via PDF/MS-Word format.

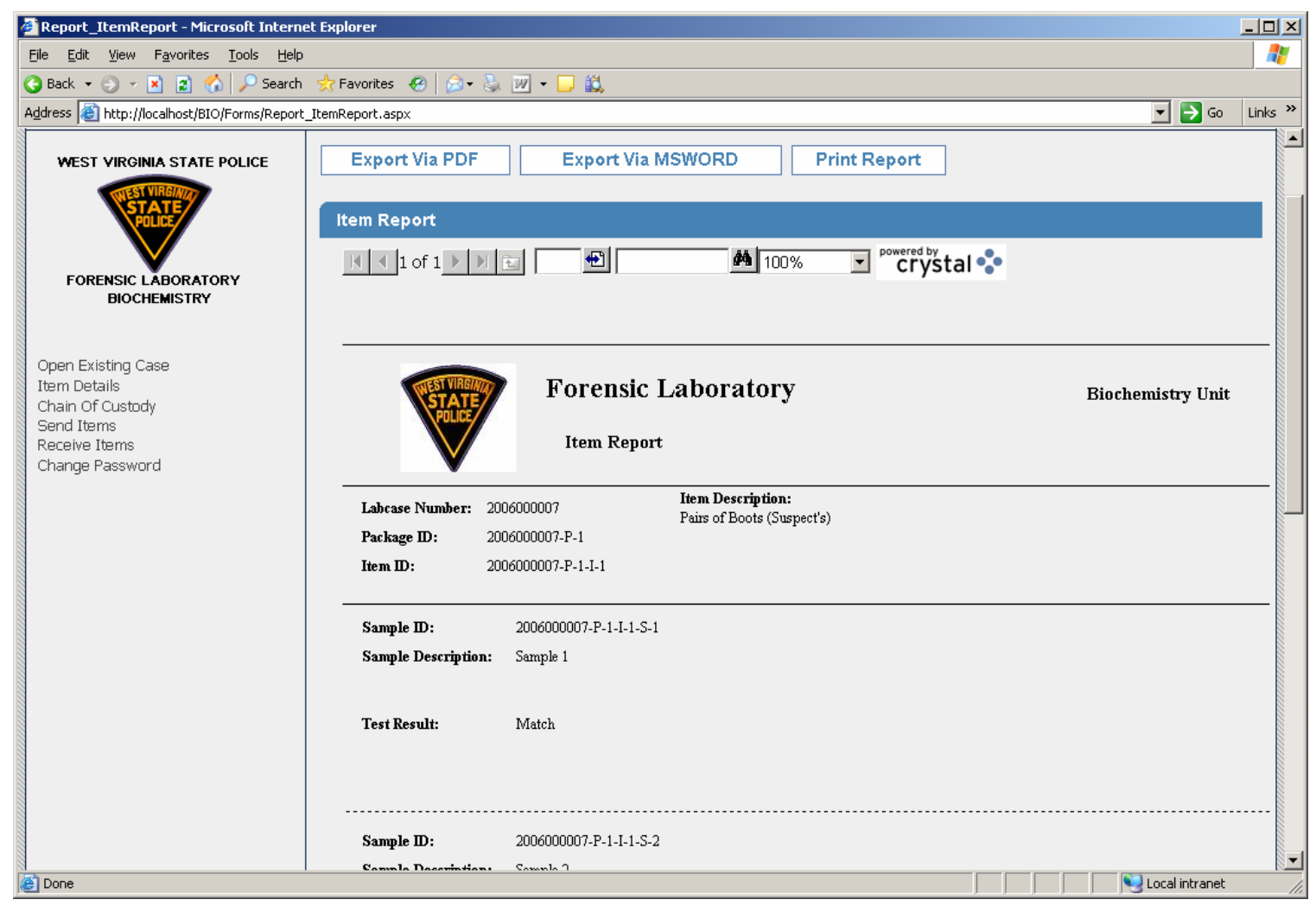

Figure 6.21: Item report 
Figure 6.22 shows the sample report for extraction and amplification test. This report can also be accessed via PDF format, as shown in Figure 6.23.

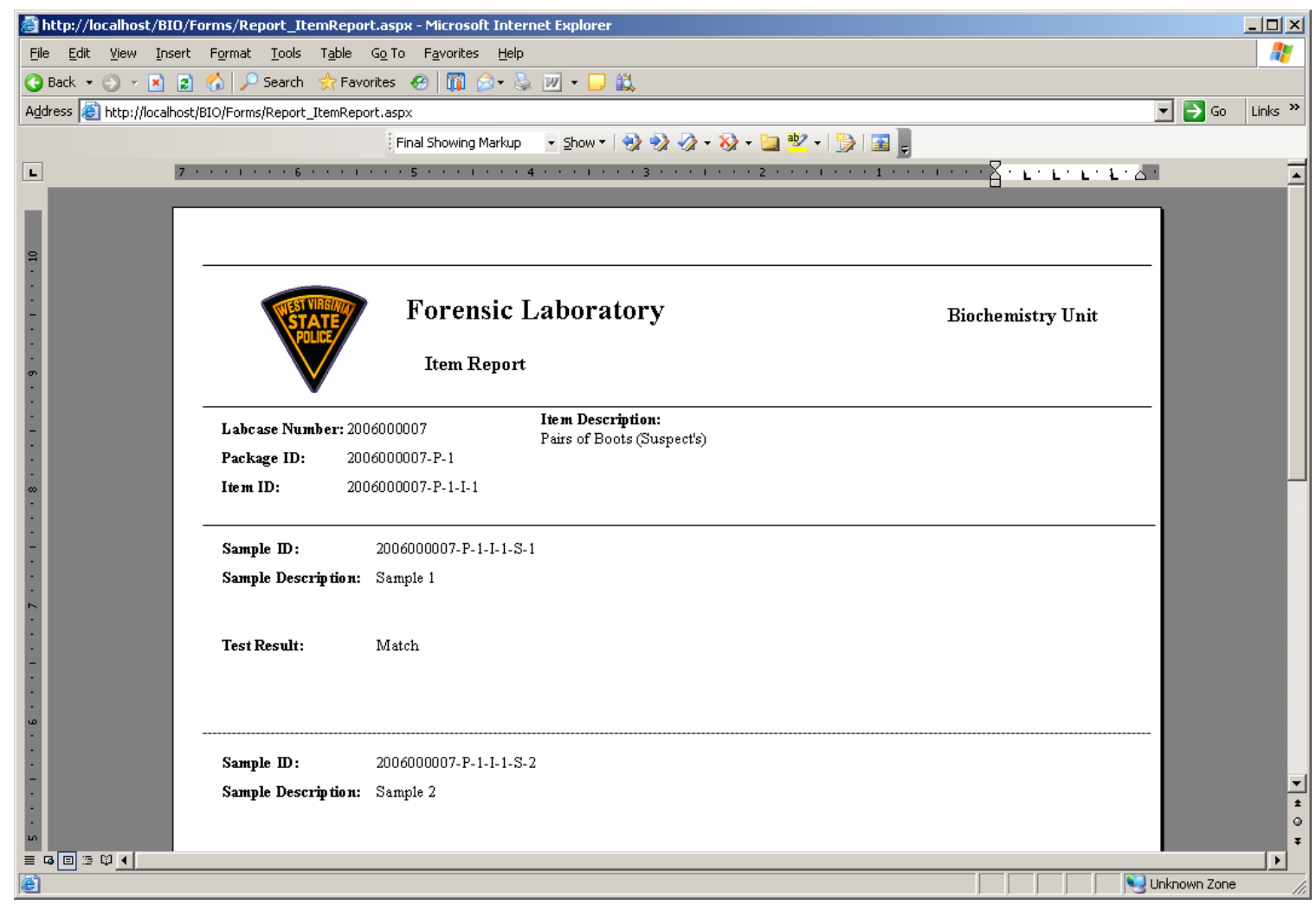

Figure 6.22: Sample Report in MS Word Format 


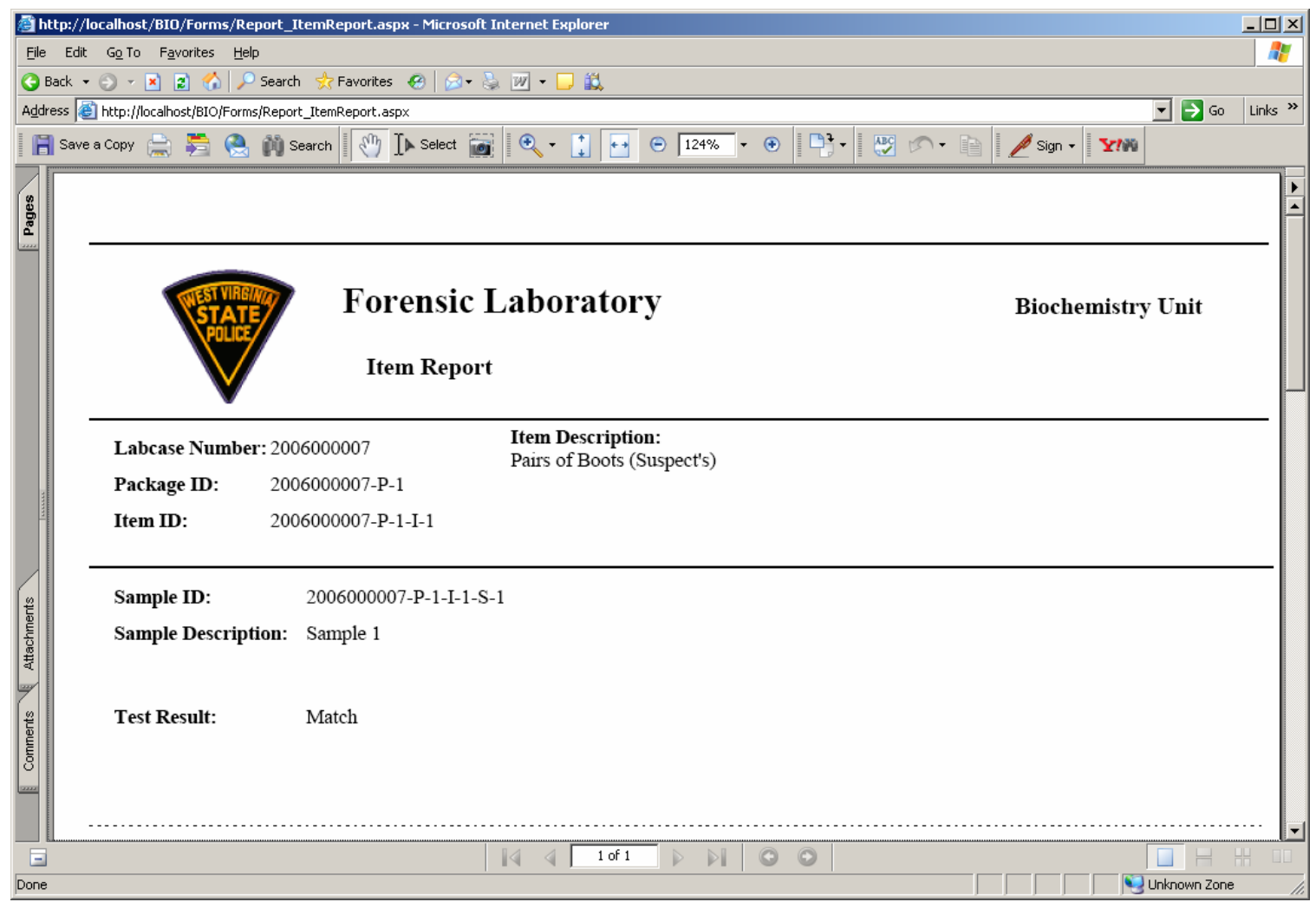

Figure 6.23: Sample Report in PDF Format

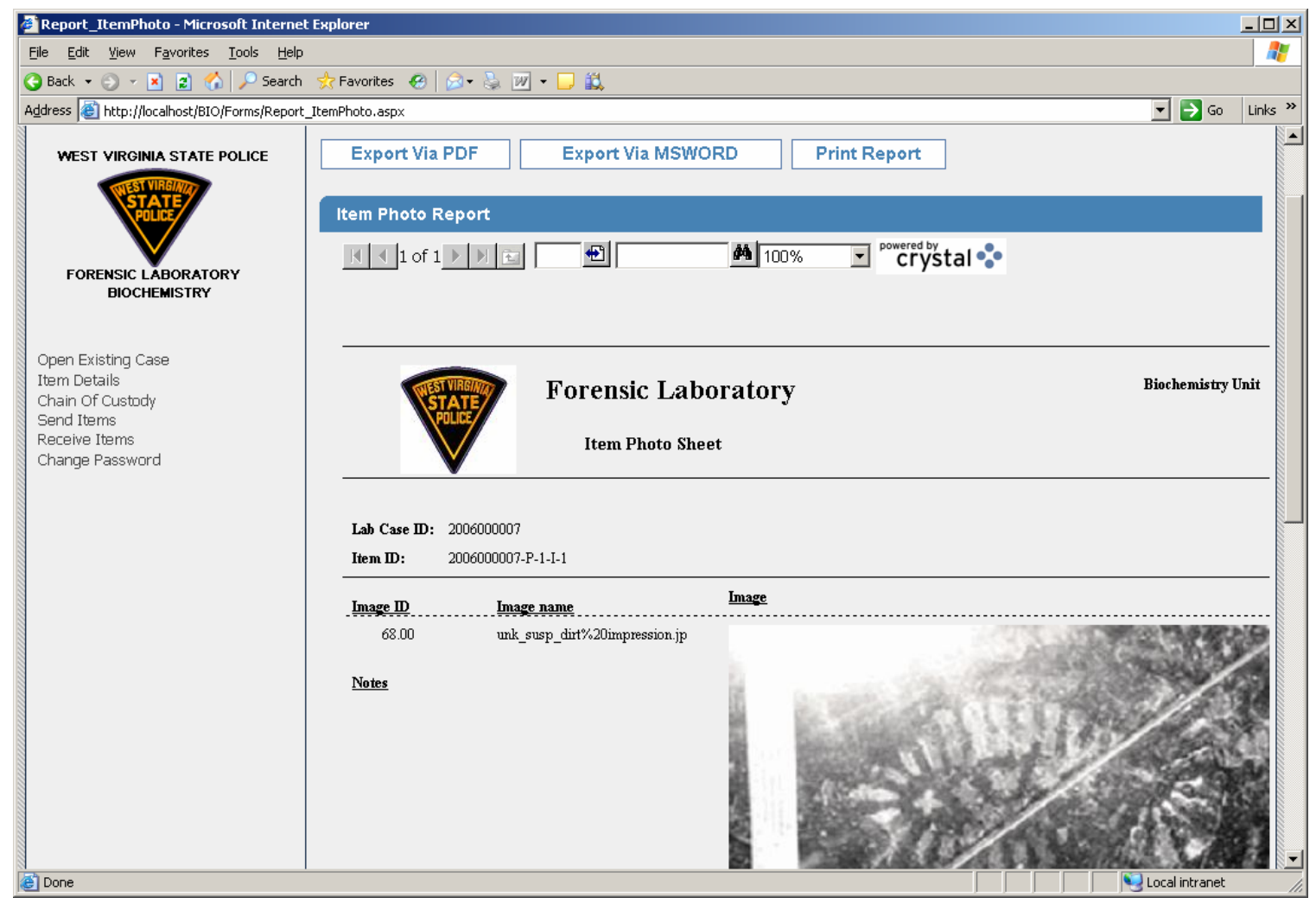

Figure 6.24: Item Photo Report 


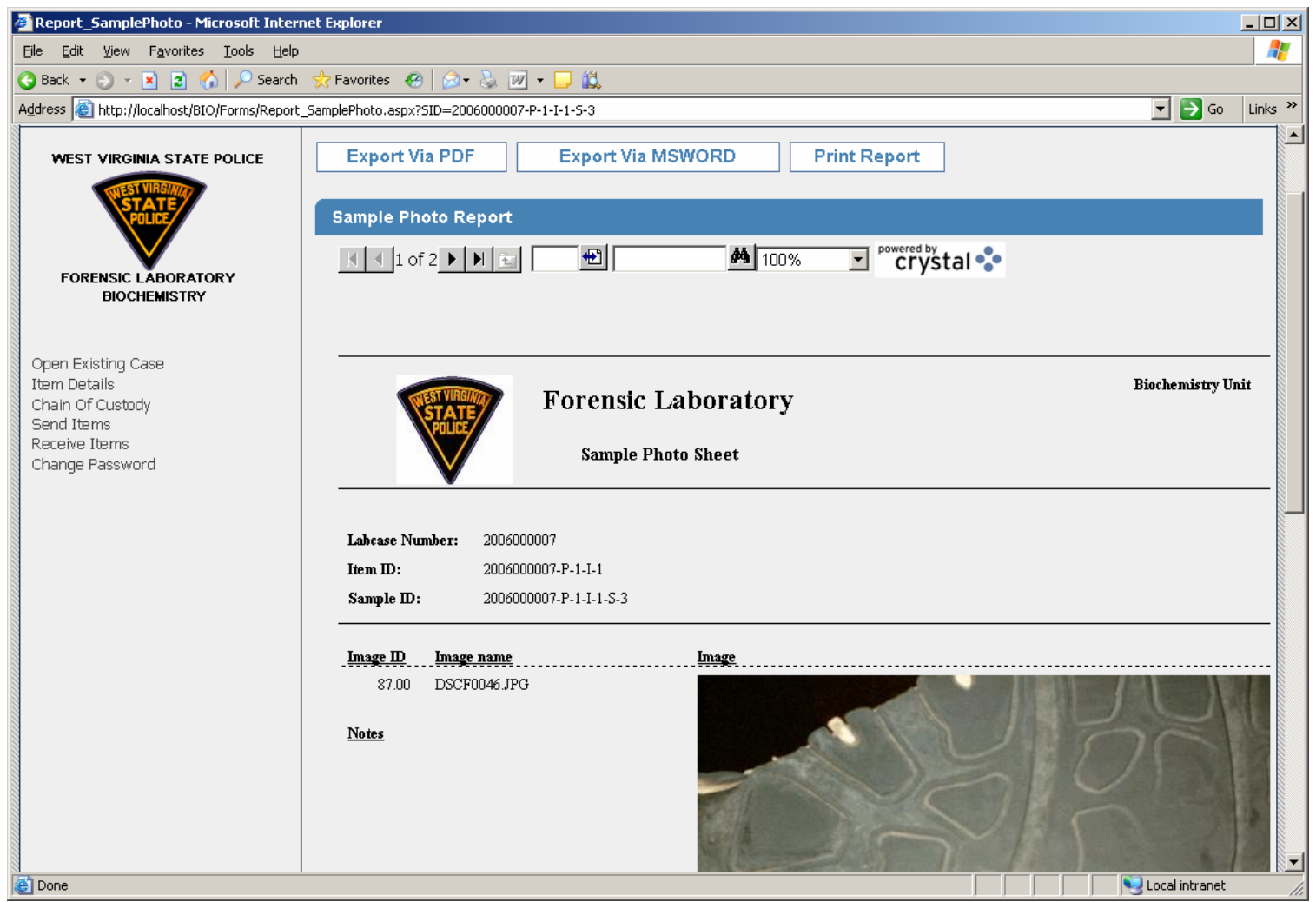

Figure 6.25: Sample Photo Report 


\section{CHAPTER 7 \\ DRUG IDENTIFICATION}

\subsection{Existing Drug Identification Process}

The purpose of the Drug Identification Unit is to analyze and identify any suspected controlled substances. Identification of controlled substances is necessary to prove violations of the Controlled Substances Act. Analysts in the drug identification unit receive the CR form, the LEIF form, and the evidence to be analyzed. A chain of custody form is also attached to the evidences. Drug evidence includes powders, liquids, tablets, capsules, and plant material samples suspected of being or containing controlled substances. The drug identification unit does not analyze blood, urine, or other pathological specimens for the presence of controlled substances. The forms/worksheets used by the Drug Identification unit are: Clandestine Test (CT), Marihuana Test (MT), Tablet \& Capsule Test (TC), Unknown Liquid Test (UL), Unknown Powder Test (UP), and Vegetation Test (VT). Once the examinations are completed, the evidence is returned to the CER and the secretary prepares a draft report. The draft report is then reviewed by the analyst/reviewer. After approval the final report is sent to CER. Figure 7.1 shows the existing process for the drug identification unit. 


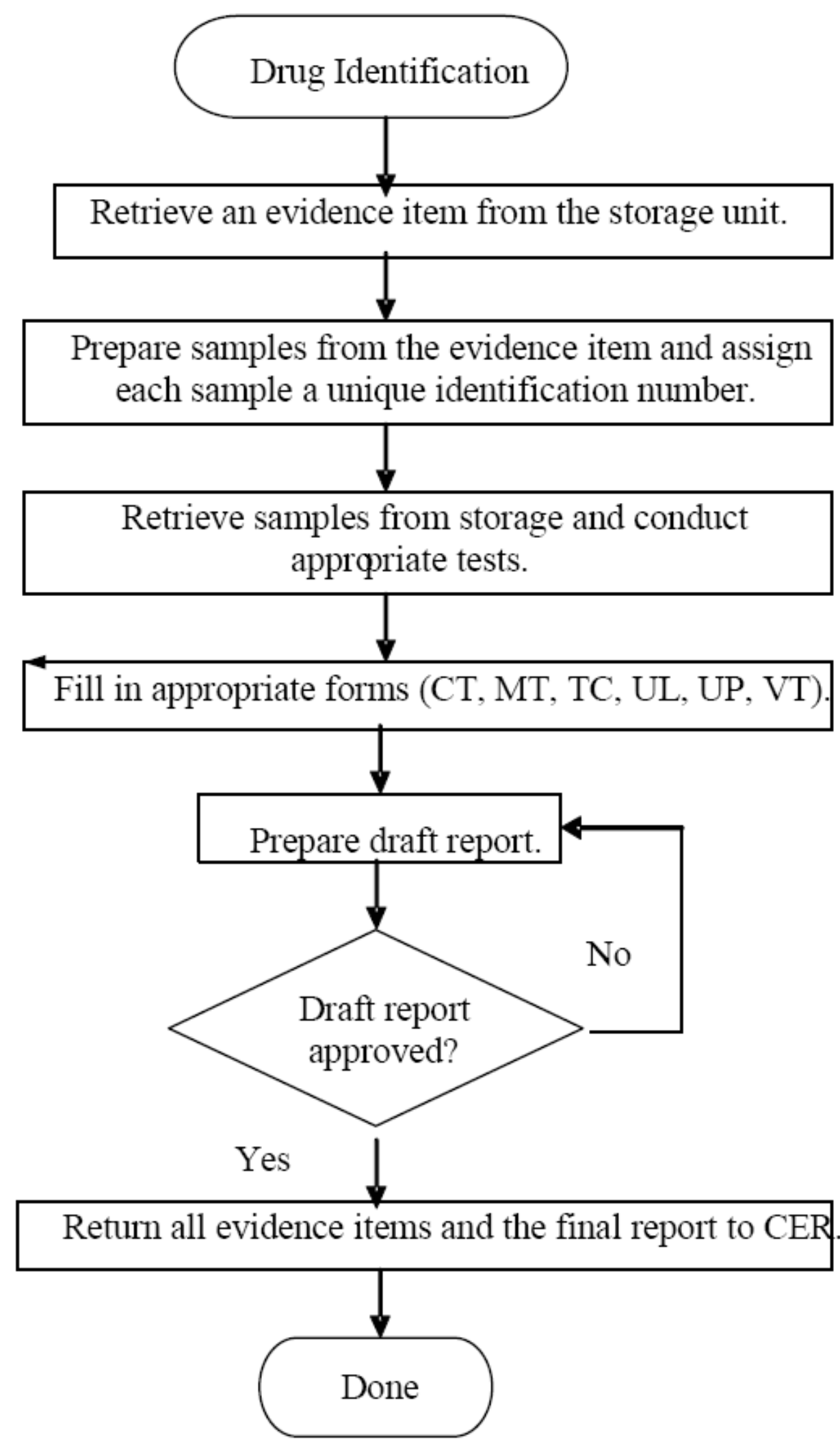

Figure 7.1: Existing Process for the Drug Identification Unit 


\subsection{Re-engineering the Drug Identification Process}

The analyst in the drug identification unit receives the items assigned for further processing and analysis from CEP. Received items are sub-divided into samples for conducting the requested tests. An individual item may be divided into many samples according to the requirement and complexity of the required test. A report for each sample is generated. An item report is compiled by combining sample reports. After the report is received, the reports along with the test items are sent back to CEP for further processing. The evidence and information flow diagram is shown in Figure 7.2.

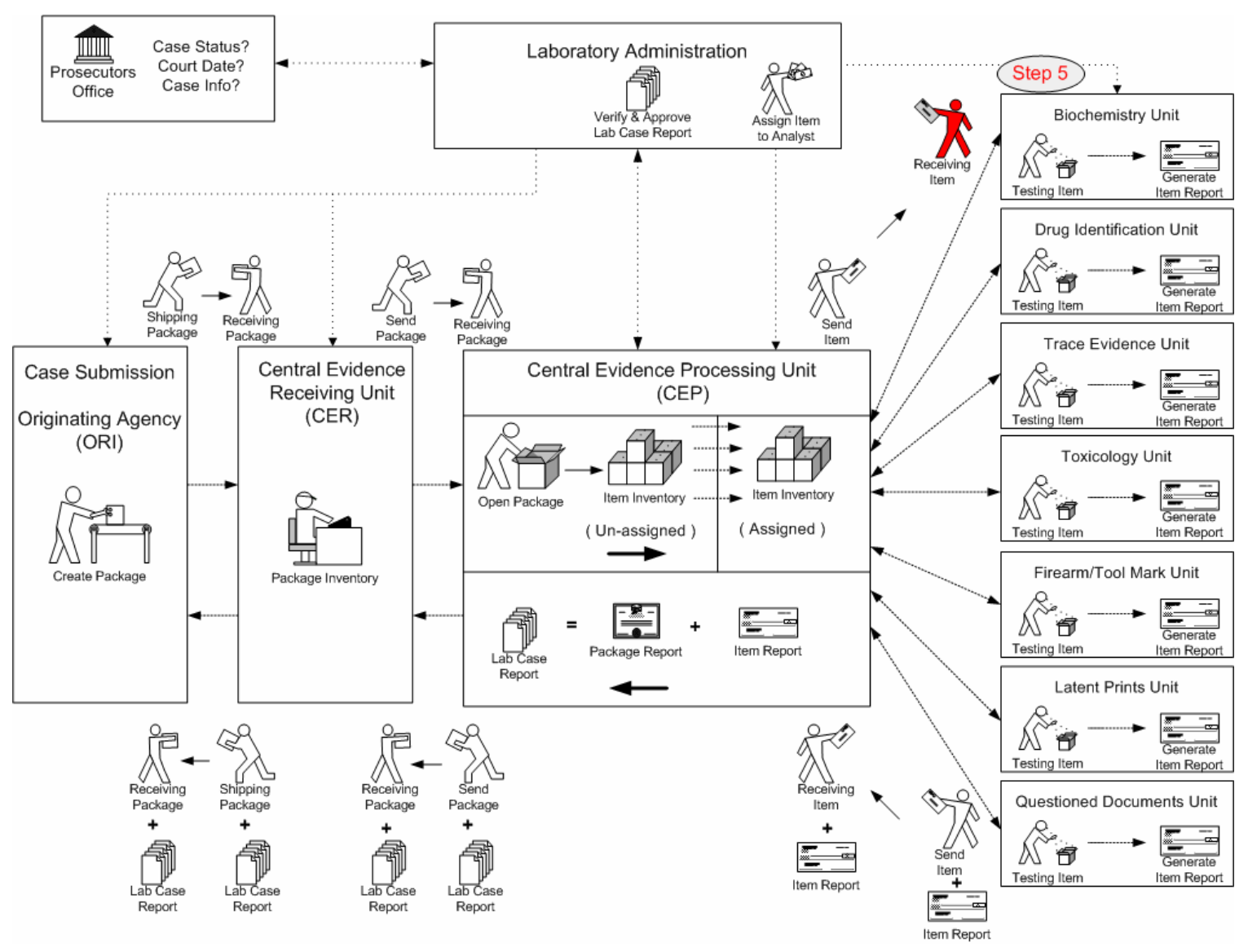

Figure 7.2: Step 5 Receive Assigned Items from CEP 
Detail procedure followed by drug identification unit for further processing and analysis is shown in Figure 7.3.

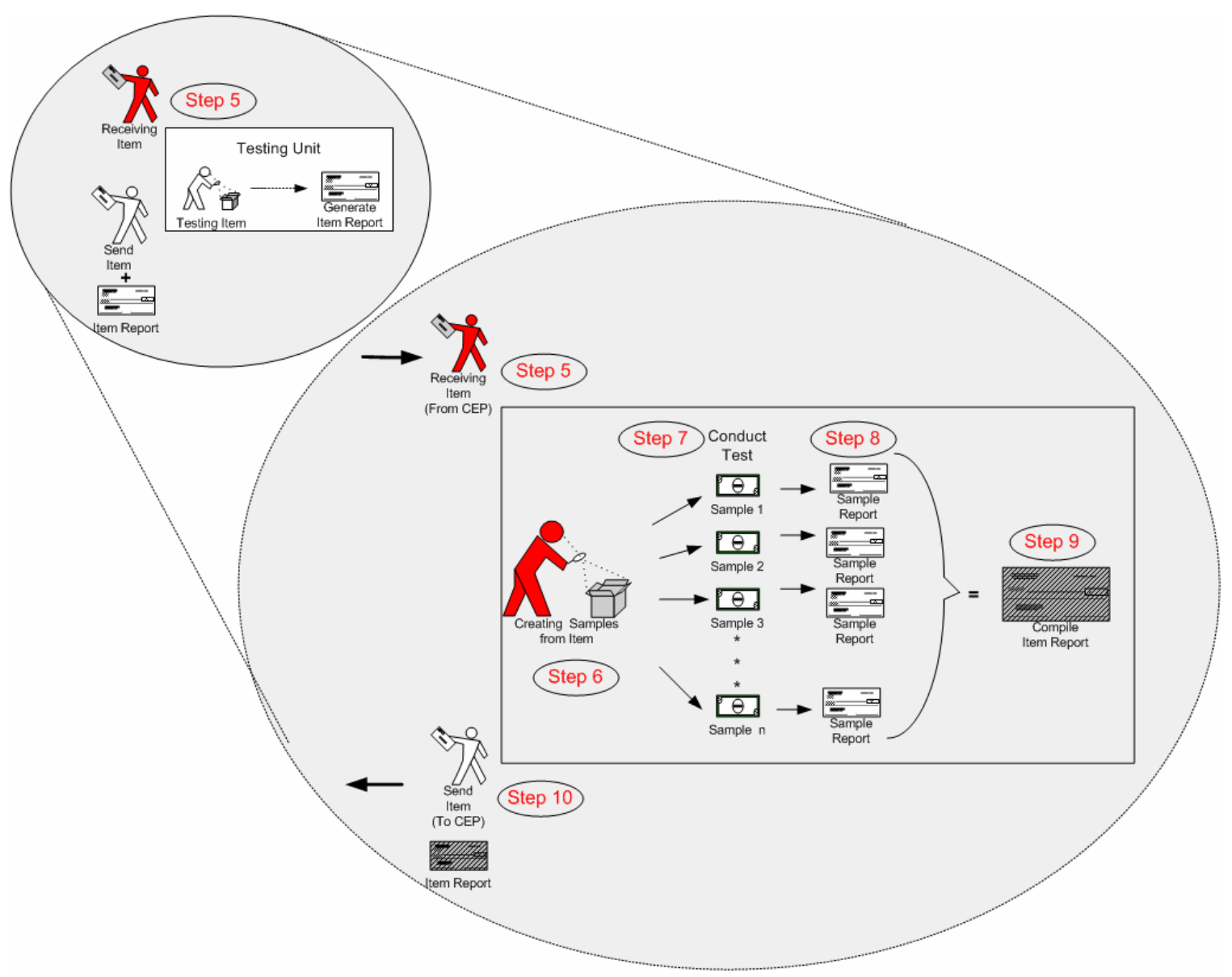

Figure 7.3: Detail Evidence Item Processing \& Report Generation Procedure 


\subsubsection{Drug Identification Entity Relationship Diagram}

Drug identification data tables are connected with each other through relations as shown in the figure 7.4.

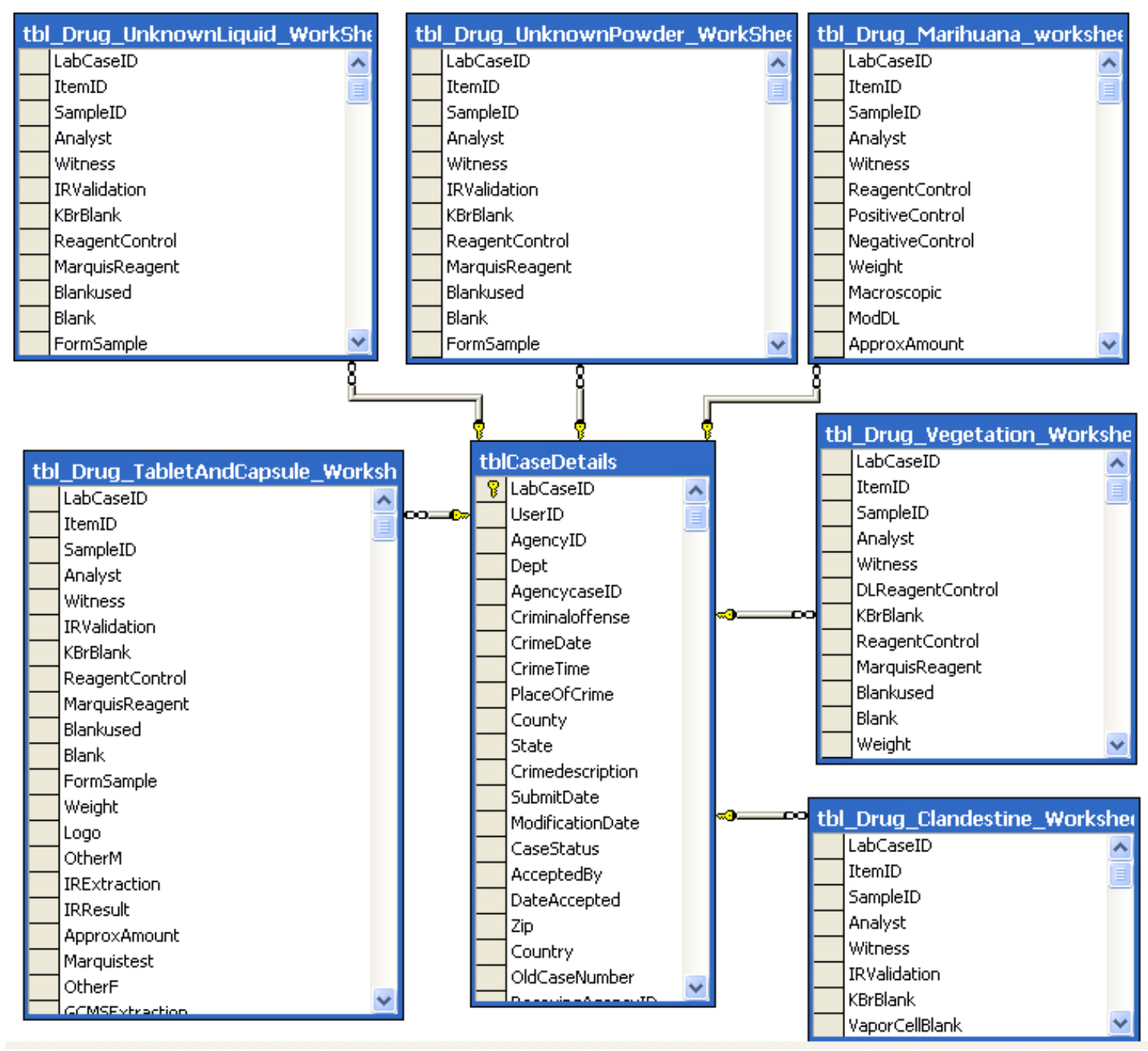

Figure 7.4: Entity Relationship Diagram 


\subsubsection{Drug Identification Data Tables}

The drug identification unit module stores its data in the following tables

- Clandestine(tbl_Drug_Clandestine_Worksheet)

- Marihuana (tbl_Drug_Marihuana_Worksheet)

- Unknown Powder (tbl_Drug_UnknownPowder_Worksheet)

- Tablet and Capsule (tbl_Drug_TabletAndCapsula_Worksheet)

- Unknown Liquid (tbl_Drug_UnknownLiquid_Worksheet)

- Vegetation(tbl_Drug_Vegetation_Worksheet)

The various fields of the clandestine worksheet table are shown in Table 7-1. Clandestine worksheet data is stored and maintained in this data table

Table 7-1: Clandestine Worksheet Table

\begin{tabular}{|l} 
tbl_Drug_Clandestine_Worksheet \\
\hline LabCaseID \\
\hline ItemID \\
\hline SampleID \\
\hline Analyst \\
\hline Witness \\
IRValidation \\
KBrilank \\
VaporCelliblank \\
MarquisReagent \\
Blankused \\
Blank \\
\hline FormSample \\
\hline phWalue \\
\hline Amountused \\
Marquis \\
OtherM \\
IRExtraction \\
IRResult \\
ApproxAmount \\
Flamerest
\end{tabular}


The various fields of the comparison worksheet table are shown in Table 7-2. Comparison worksheet data is stored and maintained in this data table

Table 7-2: Comparison Worksheet Table

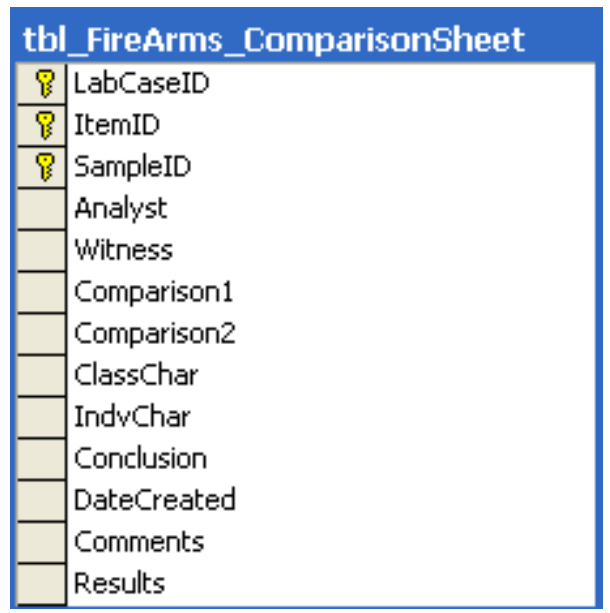

The various fields of the distance determination worksheet table are shown in Table 7-3. Distance determination worksheet data is stored and maintained in this data table

Table 7-3: Distance Determination Worksheet Table

\begin{tabular}{|l|}
\hline tbl_FireArms_DistDetermination \\
\hline LabCaseID \\
ItemID \\
\hline SampleID \\
IDLocation \\
Analyst \\
Witness \\
\hline GunManufacturer \\
SerialNumber \\
\hline GunModel \\
GunBilLength \\
\hline GunCaliber \\
\hline GunHdStampManuf \\
\hline GunProjcType \\
\hline GunProjcWt \\
\hline GunPowderType \\
CartDesignation \\
\hline IBISEntry \\
\hline EntryDate \\
ResonNoEntry \\
\hline IBISCorrelation \\
DateCreated \\
Comments \\
Results
\end{tabular}


The various fields of the unknown liquid worksheet table are shown in Table 7-4. Unknown liquid worksheet data is stored and maintained in this data table

Table 7-4: Unknown Liquid Worksheet Table

\begin{tabular}{|l|}
\hline tbl_Drug_UnknownLiquid_WorkSheet \\
\hline LabCaseID \\
ItemID \\
SampleID \\
Analyst \\
Witness \\
\hline IRValidation \\
KBrBlank \\
ReagentControl \\
MarquisReagent \\
\hline Blankused \\
\hline Blank \\
FormSample \\
phWalue \\
Amountused \\
Marquis \\
OtherM \\
IRExtraction \\
IRResult \\
ApproxAmount \\
Flametest \\
OtherF \\
GCMSExtraction \\
GCMSResult \\
datecompleted \\
Comments \\
TestResult \\
\hline
\end{tabular}


The various fields of marihuana worksheet table are shown in Table 7-5. Marihuana worksheet data is stored and maintained in this data table

Table 7-5: Marihuana Worksheet Table

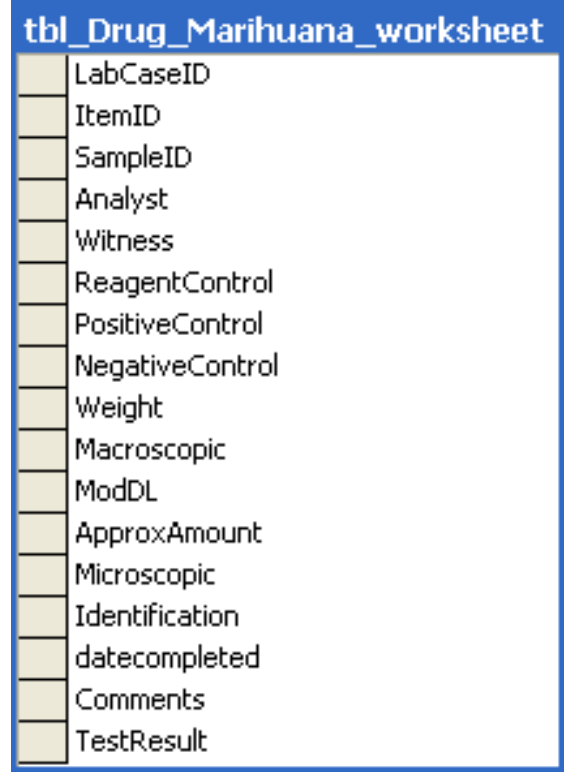

The various fields of the unknown powder worksheet table are shown in Table 76. Unknown powder worksheet data is stored and maintained in this data table

Table 7-6: Unknown Powder Worksheet Table

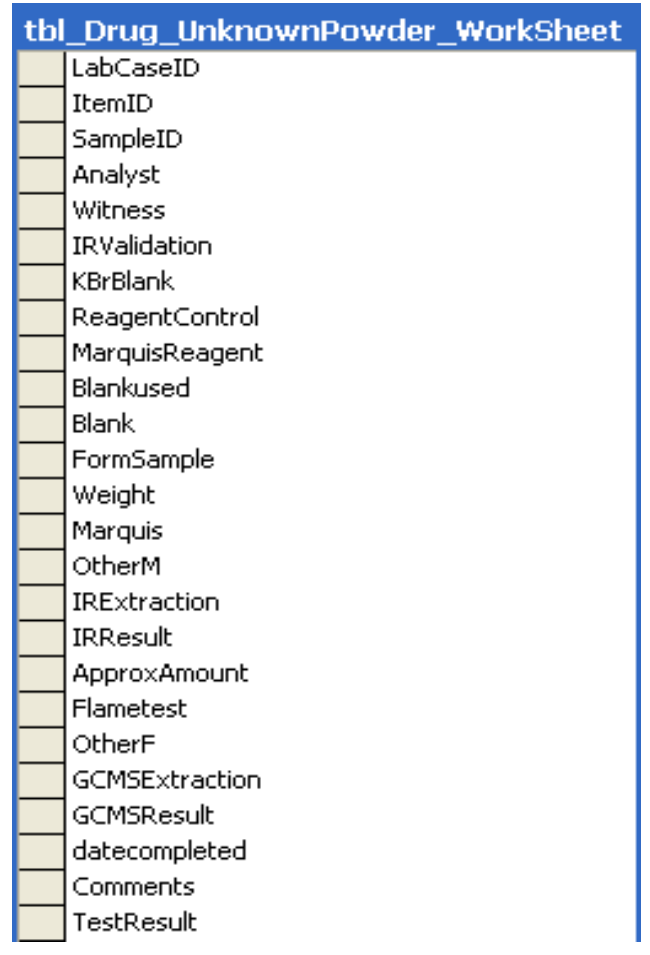


The various fields of the tablet and capsule worksheet table are shown in Table 7-

7. Tablet and capsule worksheet data is stored and maintained in this data table

Table 7-7: Tablet \& Capsule Table

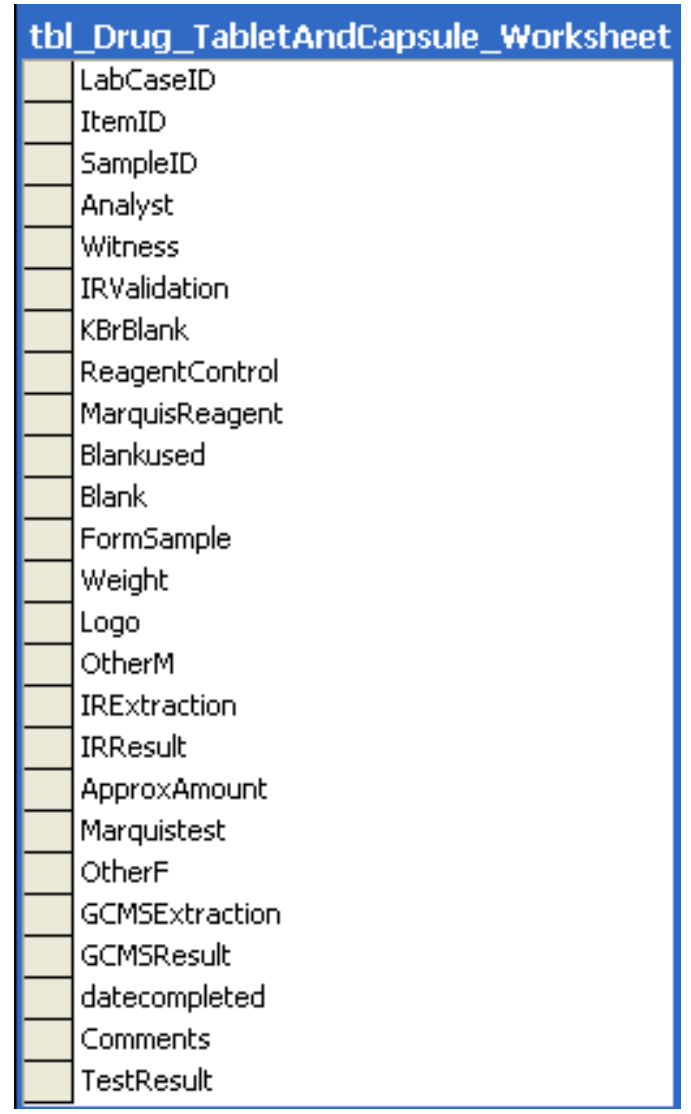


The various fields of the vegetation worksheet table are shown in Table 7-8. Vegetation worksheet data is stored and maintained in this data table

Table 7-8: Vegetation Worksheet Table

\begin{tabular}{|l|} 
tbl_Drug_Vegetation_Worksheet \\
\hline \\
LabCaseID \\
ItemID \\
SampleID \\
Analyst \\
Witness \\
DLReagentControl \\
KBrBlank \\
ReagentControl \\
MarquisReagent \\
Blankused \\
Blank \\
Weight \\
Macroscopic \\
ModDL \\
\hline ApproxAmount \\
Microscopic \\
TLC \\
\hline Other \\
\hline datecompleted \\
Comments \\
TestResult \\
\hline
\end{tabular}




\subsubsection{Working with Drug Identification}

Drug Identification home page requires username and password to verify user credentials, as shown in the Figure 7.5. After verifying credentials, a user is directed to list of items assigned to him/her for further processing.

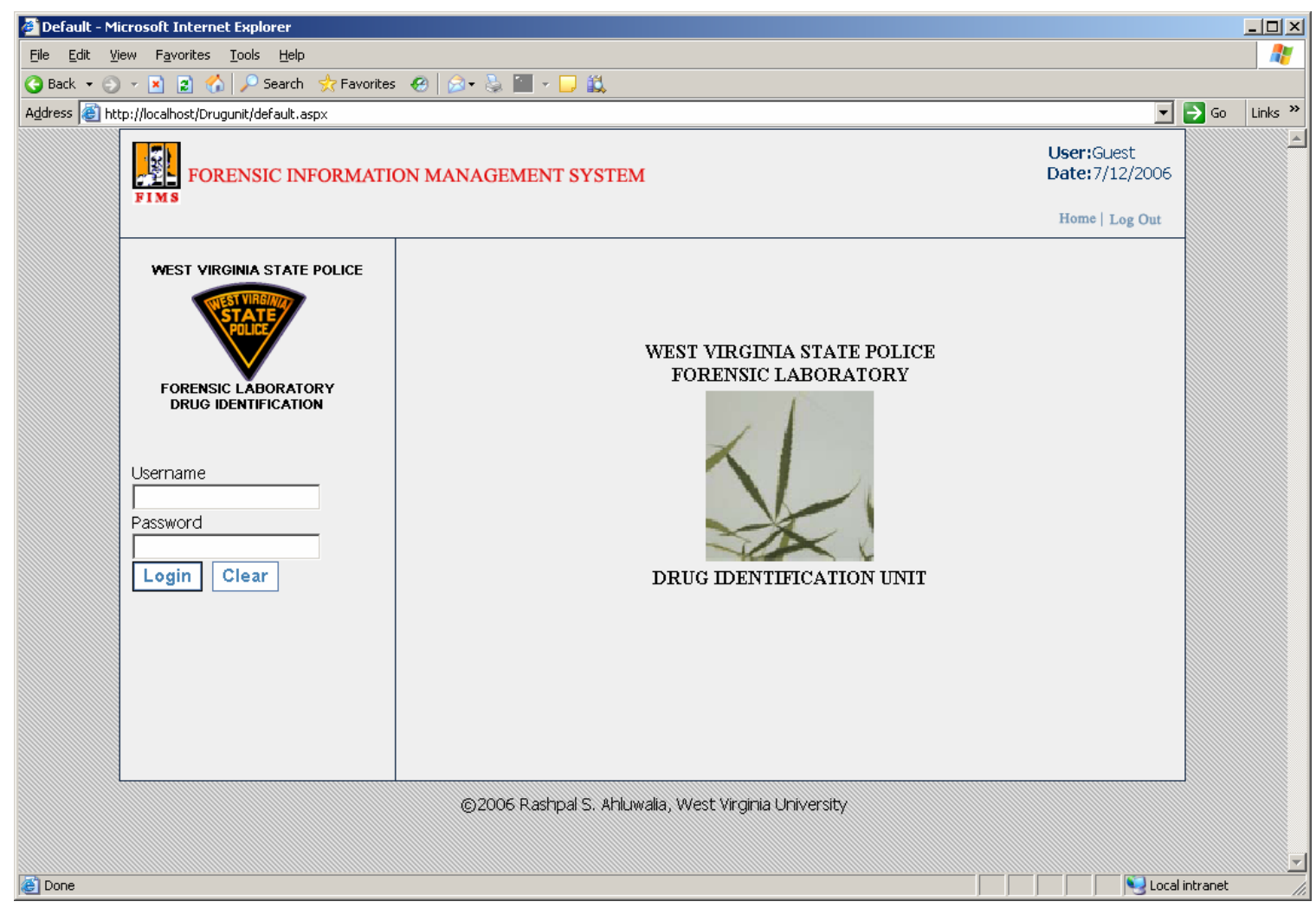

Figure 7.5: Drug Identification Home Page 


\section{View Items Assigned}

Figure 7.6 shows the list of items assigned to the analyst by the laboratory administration unit for further analysis. Initially, job status for the item assigned is set to "Job Pending". The "View Details" hyperlink enables an analyst to view the details of the case. If the list of items assigned grows to more than 15 items, the record set is divided into multiple pages. Analysts can navigate between different pages by clicking on the header and footer navigation bar of the list.

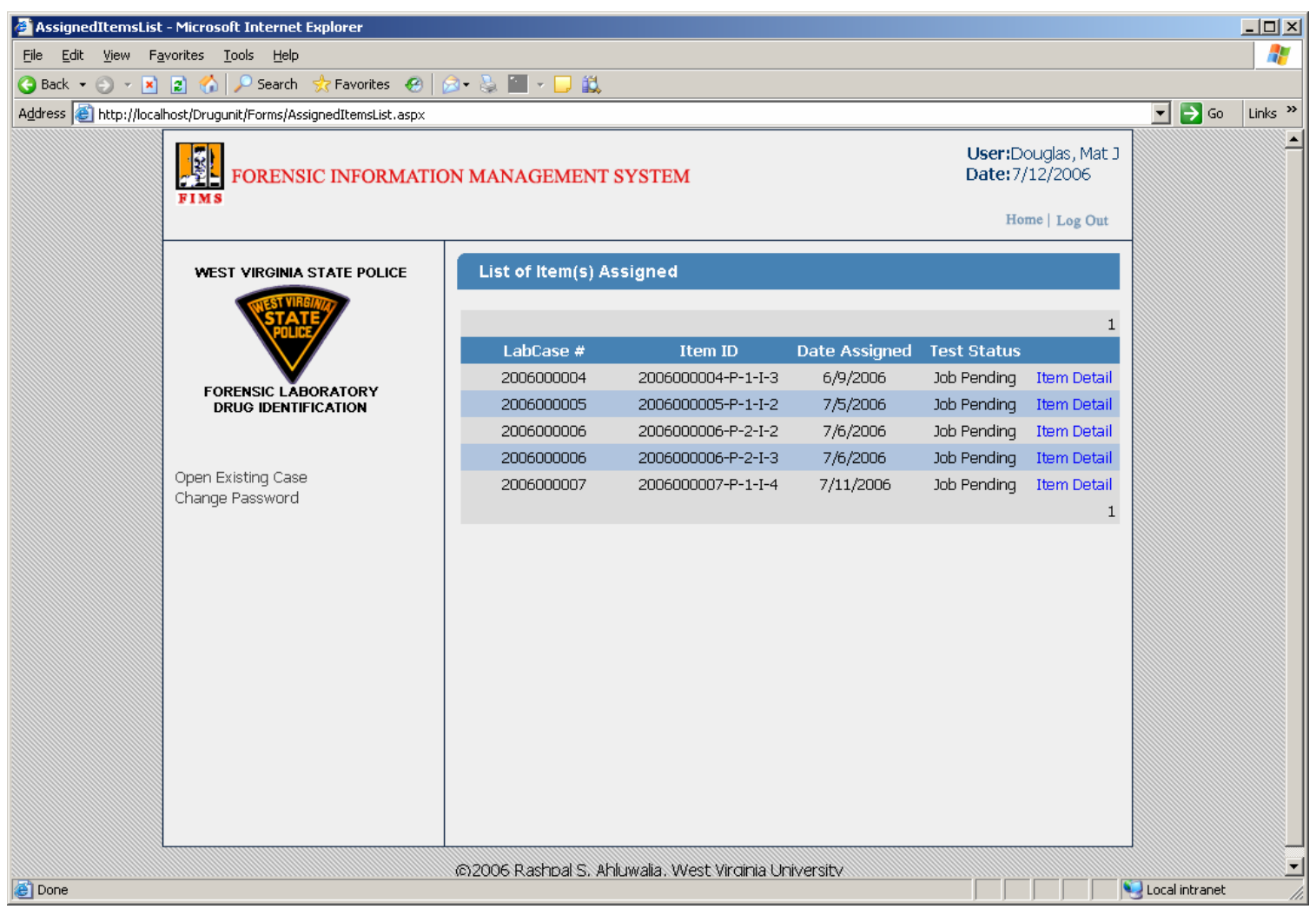

Figure 7.6: Assigned Item List 


\section{Item Picture Management}

This section describes the procedure of attaching pictures to an item. Analysts can perform two kinds of operation:

- View Pictures

- Upload Pictures

Analysts can access this section by clicking on the "View/Upload Item Photo" hyperlink in the item details section as shown inn Figure 7.9. View picture section list the pictures already attached to the item with their thumbnails as shown in Figure 7.7. Analysts can also view the full size image by clicking on the image thumbnail. Full size image opens in a new window, which can be closed after use.

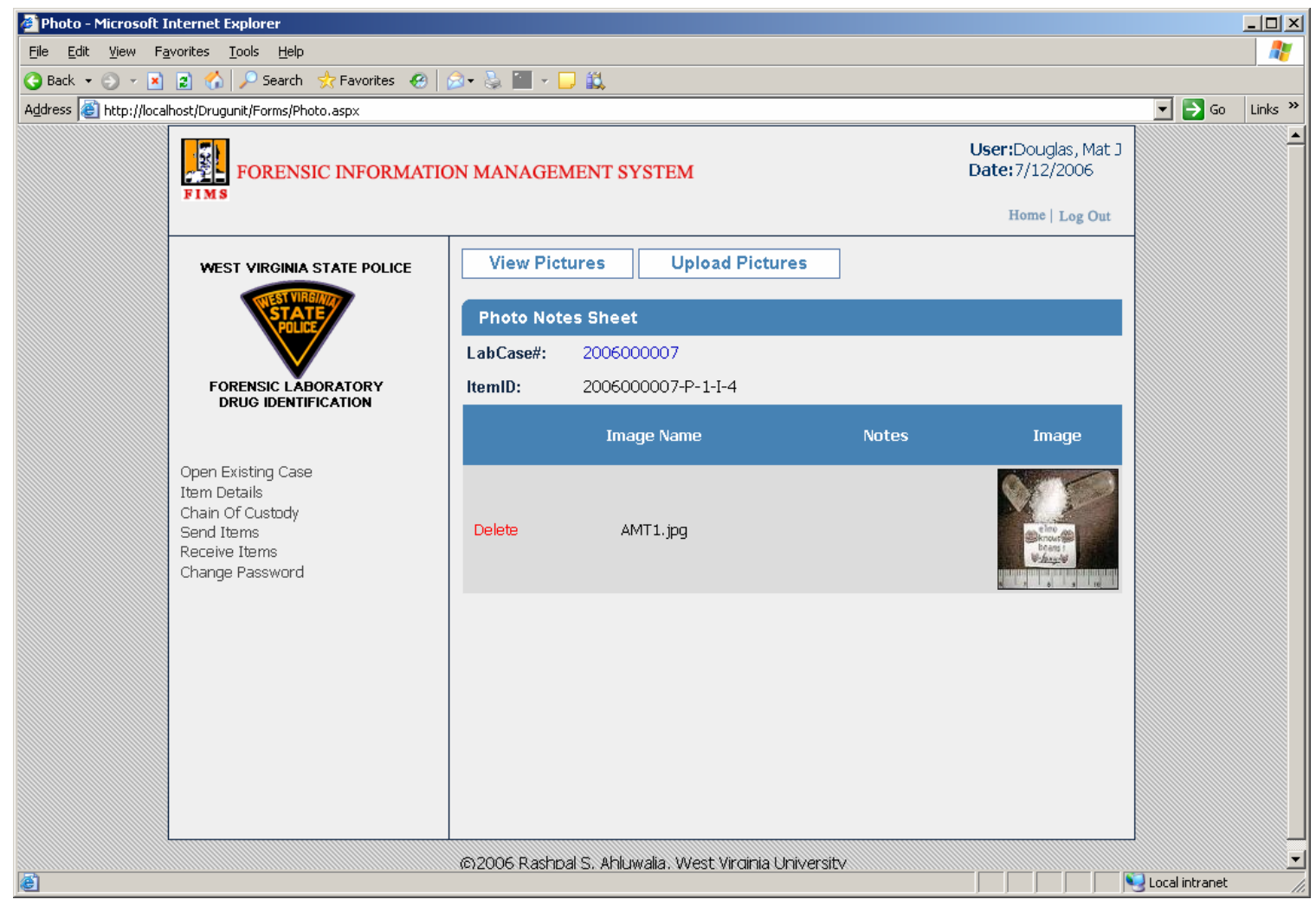

Figure 7.7: View Item Photo 
Analysts can upload any digital photo in JPG format directly into the database using this section as shown in Figure 7.8. To upload an image into database, analysts are required to browse for the image path, set other required fields and click on the "Upload the file” hyperlink. On successful upload a thumbnail appears on the bottom of the page with a success message.

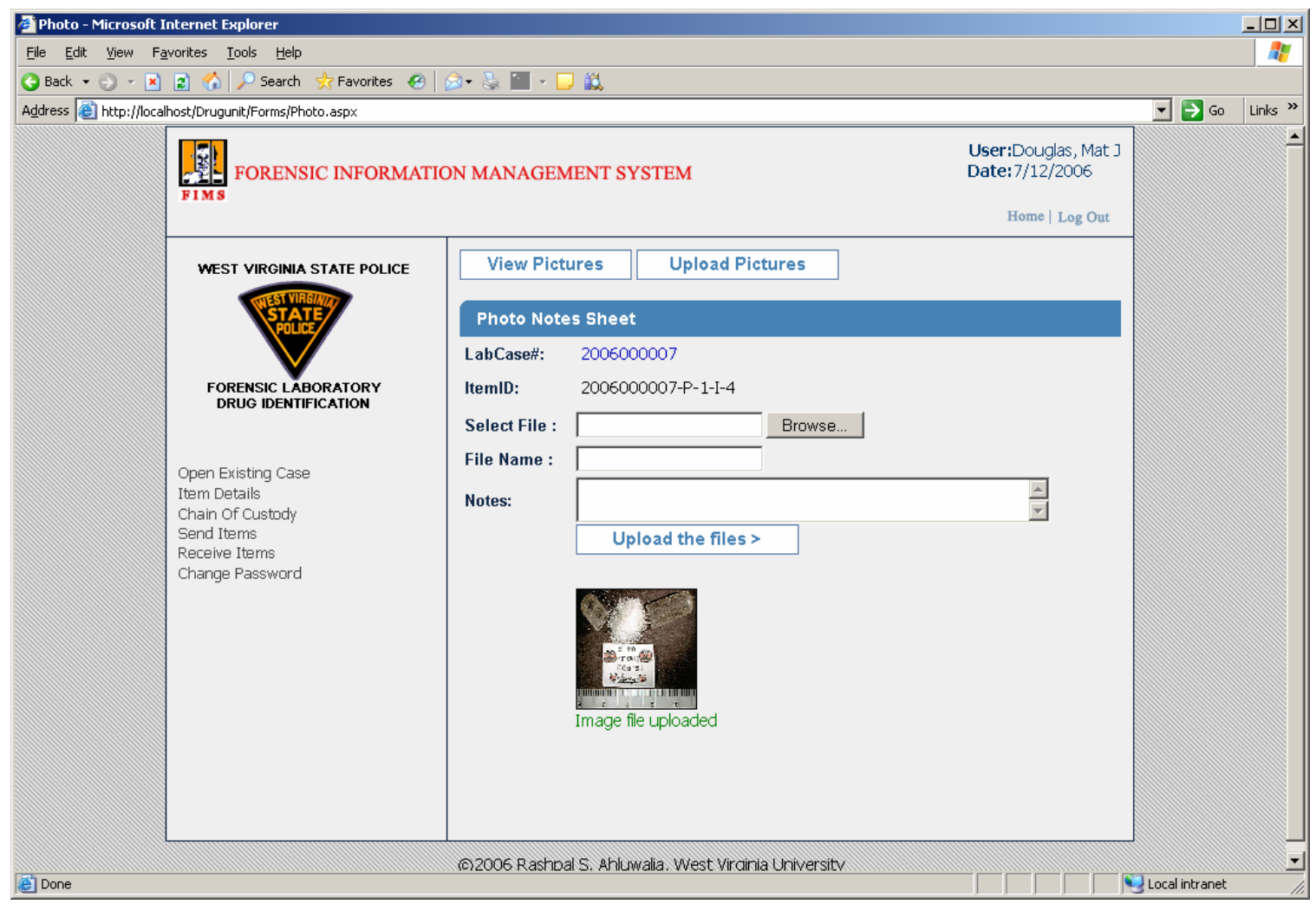

Figure 7.8: Upload Item Picture Section 


\section{Working with Samples}

Analysts receive items from the CEP unit for further analysis and processing. Once received items are sub-divided into samples for conducting requested tests. Each item is divided into one or more samples according to the requirement and complexity of the required test.

Analysts can create new, update or delete existing samples from the item details section as shown in Figure 7.9. To add a new sample, analyst is required to provide description of sample and then click on "Add Sample" hyperlink. FIMS application automatically adds the time stamp to the item created. Initially, sample test status is set to "Test Pending" for the sample. Sample information can be updated by clicking on "Edit" hyperlink and existing sample can be deleted from the system by clicking on "Delete" hyperlink.

This section also enables analysts to view the final sample, sample photo, item, and item photo report. To access the individual sample/sample photo report analyst can click on "View Report" or "Photo Sheet" hyperlink. To view comprehensive item report analysts can click on "Item Report" or "Item Photo Report" at the bottom of the page.

To complete the process for item testing, the analyst is required to click on "Item Test Complete” button, as shown in Figure 7.9. Once clicked, analyst will not be able to make any alterations to the existing item test report. 


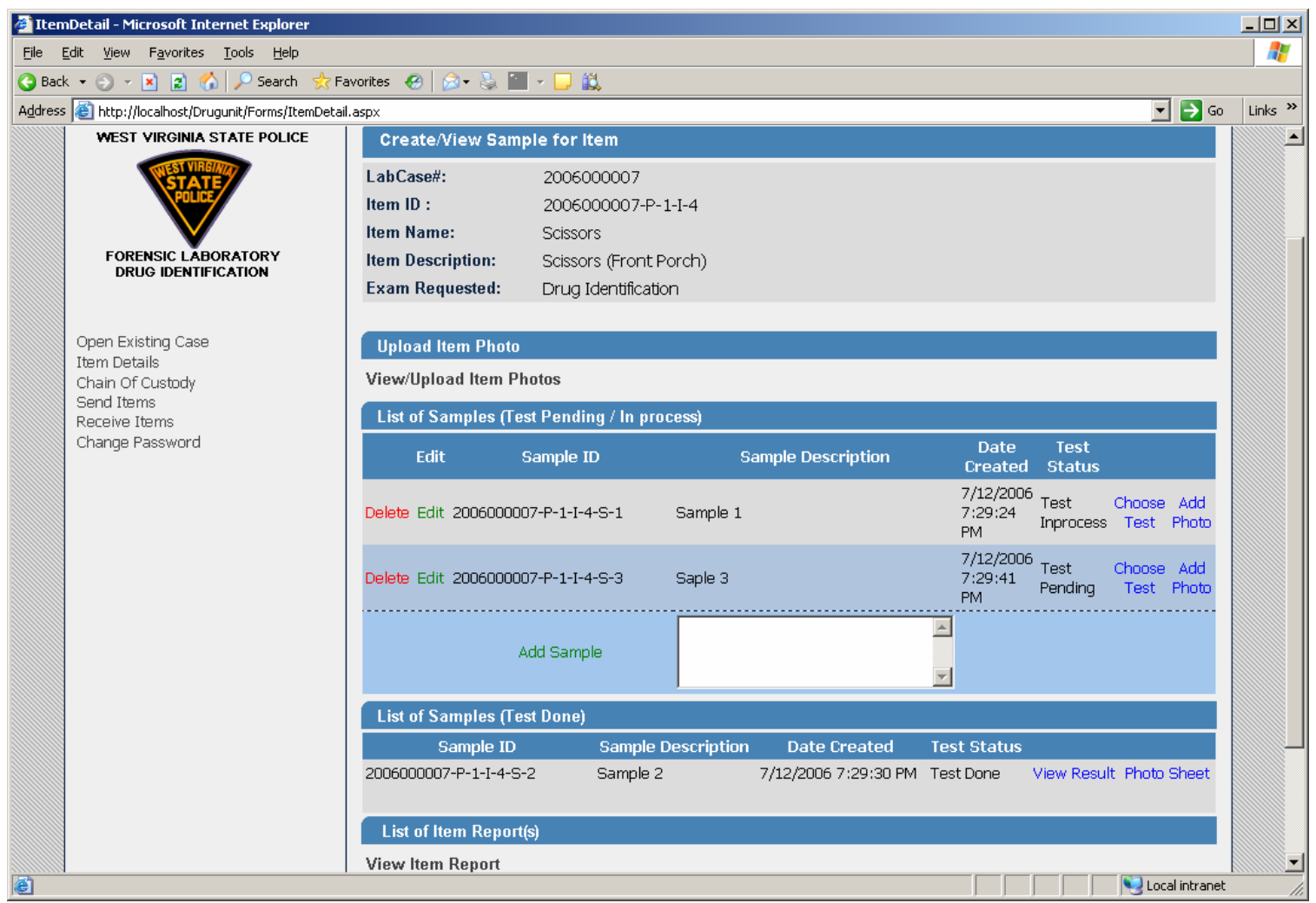

Figure 7.9: Item Detail Section 
Analysts can access this section by clicking on the "View/Upload Sample Photo" hyperlink in the item details section as shown inn Figure 7.9. View picture section list the pictures already attached to the item with their thumbnails as shown in Figure 7.10. Analysts can also view the full size image by clicking on the image thumbnail. Full size image opens in a new window, which can be closed after use.

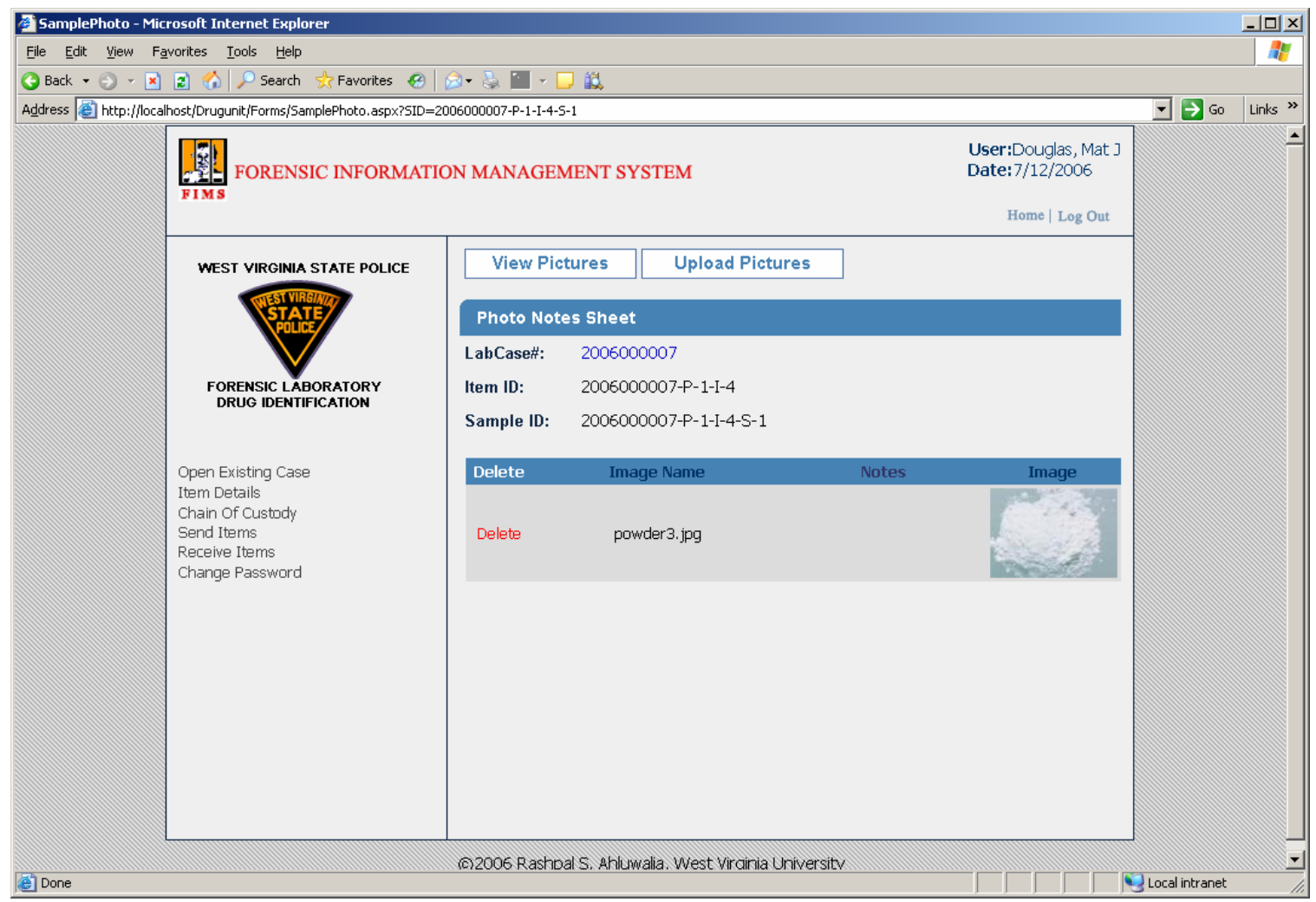

Figure 7.10: View Picture Section 
Analysts can upload any digital photo of JPG format directly into the database using this section as shown in Figure 7.11. To upload an image into database analysts are required to browse for the image path, set other required fields and click on the "Upload the file” hyperlink. On successful upload a thumbnail appears on the bottom of the page with a success message.

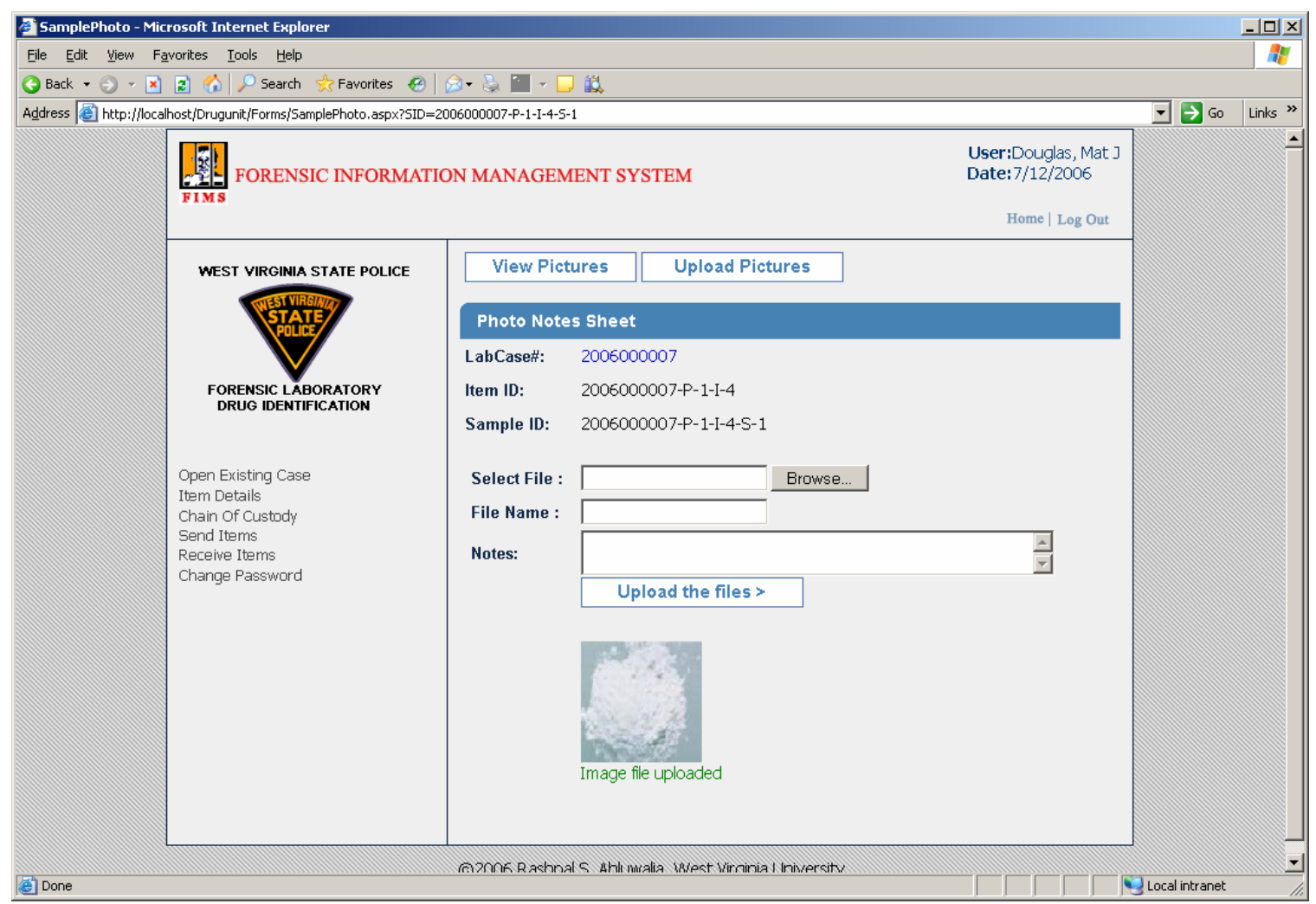

Figure 7.11: Upload Sample Picture Section 


\section{Choosing Test Worksheet}

After sample creation, analysts are required to decide what kinds of tests are required? Analysts choose type of test manually. Once the decision is made the analyst can see the list of tests available, and then click "Choose Test” hyperlink as shown in Figure 7.12. FIMS application remembers the chosen sample ID during the process.

The worksheets for the drug identification are:

- Clandestine

- Marihuana

- Tablet \& Capsule

- Unknown Powder

- Unknown Liquid

- Vegetation

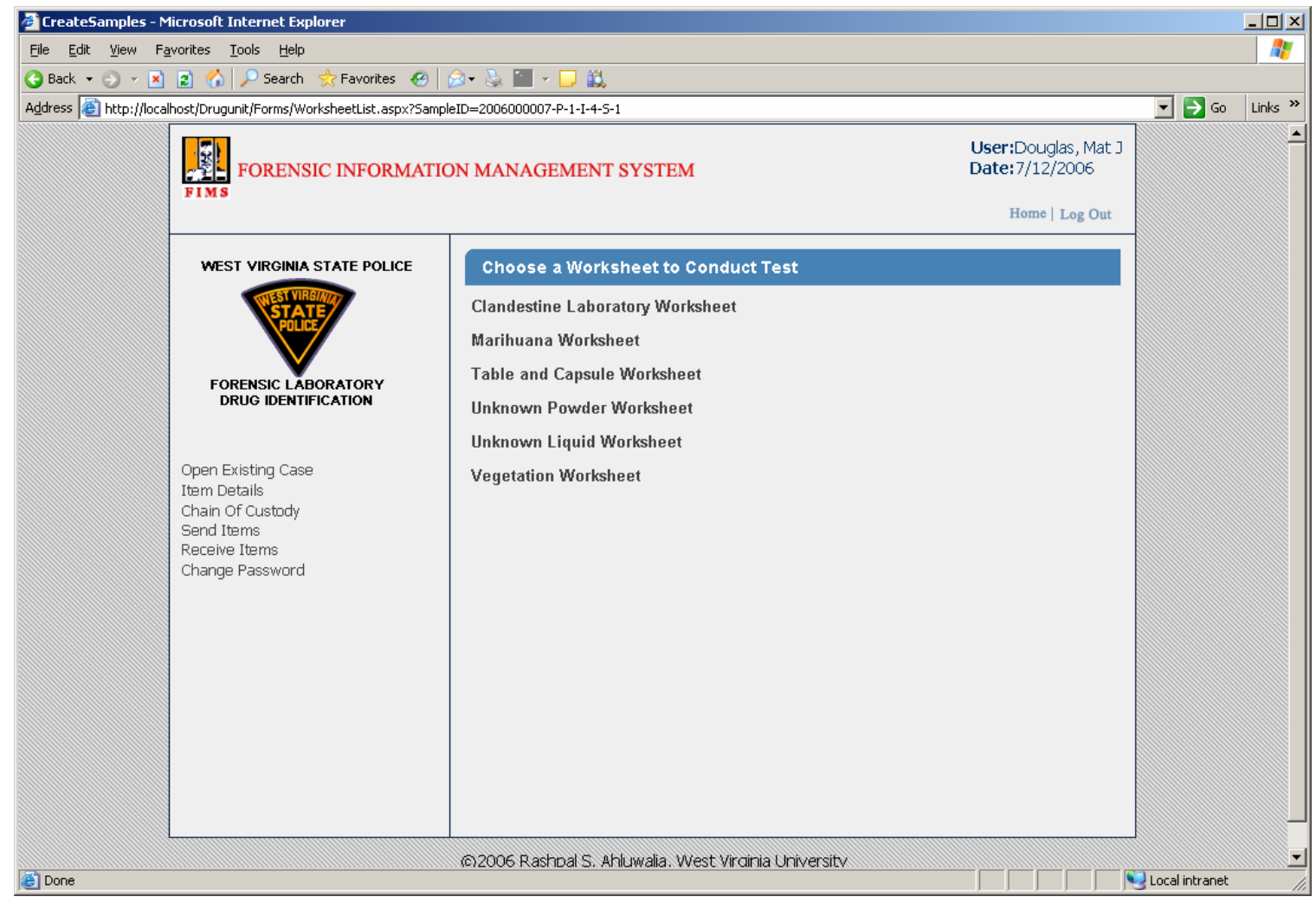

Figure 7.12: Choose Sample Test Section 


\section{Clandestine Laboratory Worksheet}

Clandestine Laboratory worksheet is used for filling information pertaining to clandestine test performed on the sample by the analyst. After filling the required data fields, analyst is required to click on "Save Results" button located on top of the page, as shown in Figure 7.13. After saving the worksheet data, analyst can finalize and close the worksheet by clicking on "Test Done" button. The analyst cannot make any alterations in the worksheet once the "Test Done" button is clicked.

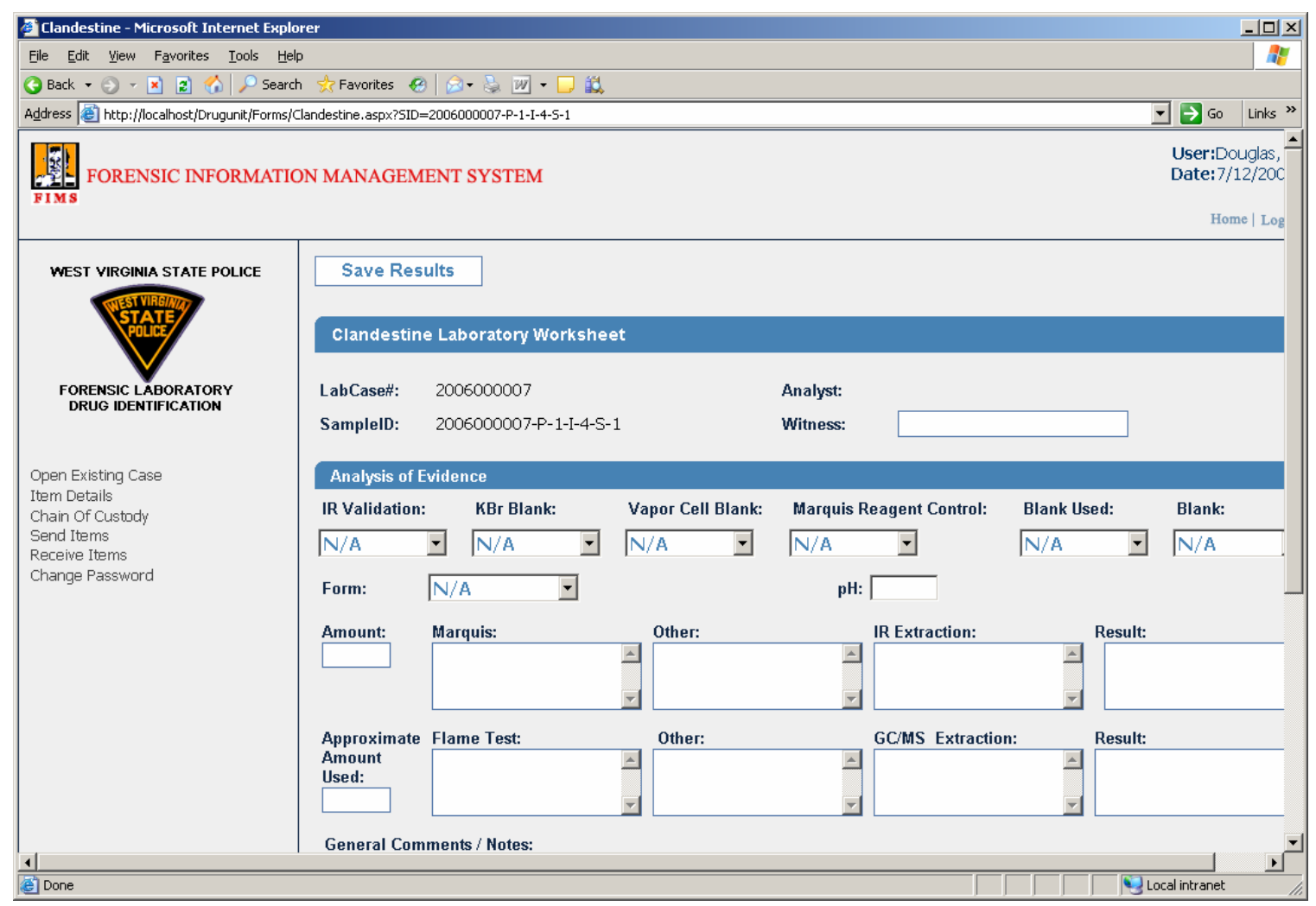

Figure 7.13: Clandestine Laboratory Worksheet 


\section{Marihuana Worksheet}

Marihuana worksheet is used for filling information pertaining sperm Marihuana test performed on the sample by the analyst. After filling the required data fields, analyst is required to click on "Save Results" button located on top of the page, as shown in Figure 7.14. After saving the worksheet data, analyst can finalize and close the worksheet by clicking on "Test Done" button. The analyst cannot make any alterations in the worksheet once the "Test Done" button is clicked.

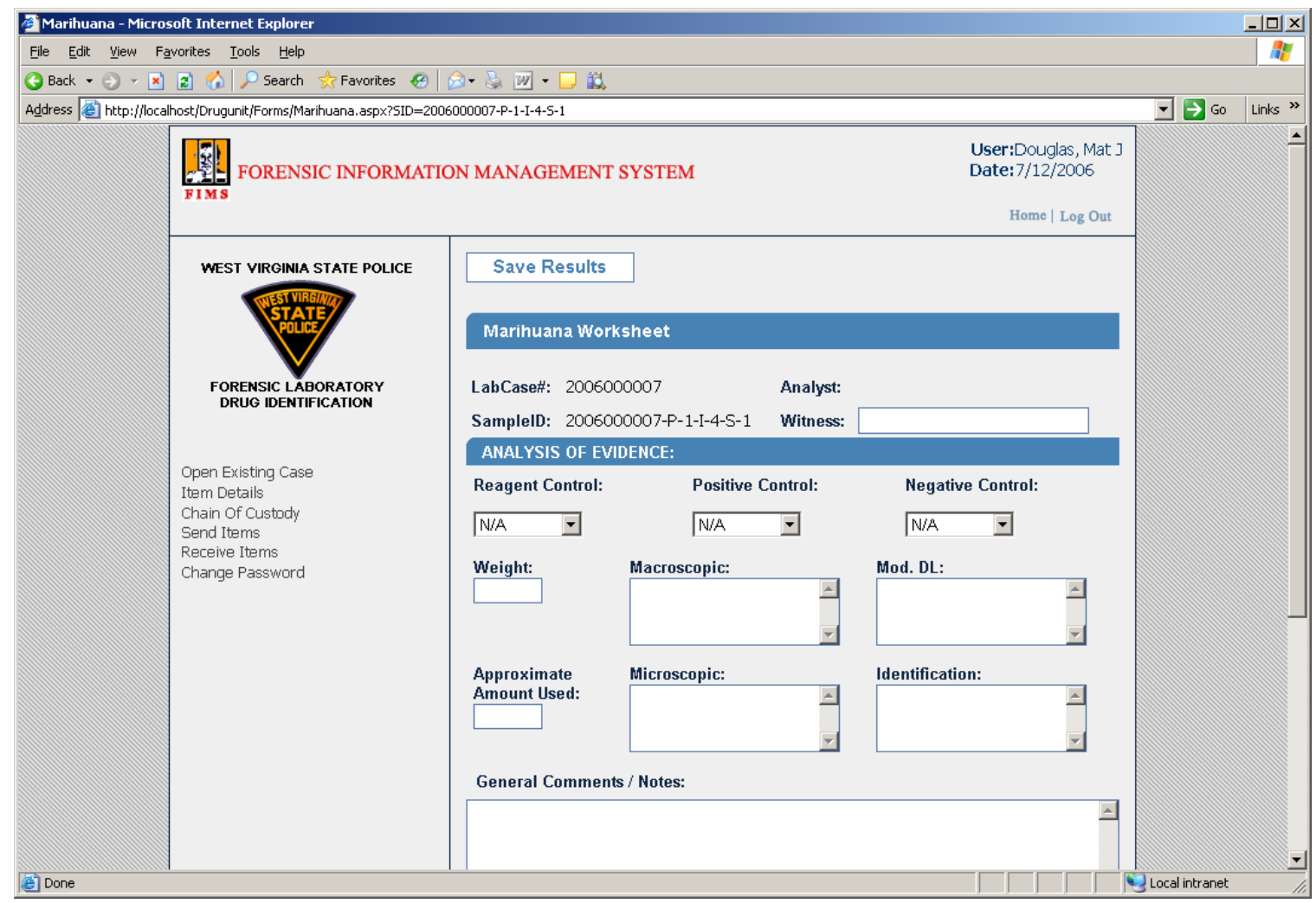

Figure 7.14: Sperm Identification Worksheet 


\section{Tablet \& Capsule Worksheet}

Tablet \& Capsule worksheet is used for filling information pertaining to tablet \& capsule test performed on the sample by the analyst. After filling the required data fields, analyst is required to click on "Save Results" button located on top of the page, as shown in Figure 7.15. After saving the worksheet data, analyst can finalize and close the worksheet by clicking on “Test Done” button. The analyst cannot make any alterations in the worksheet once the "Test Done” button is clicked.

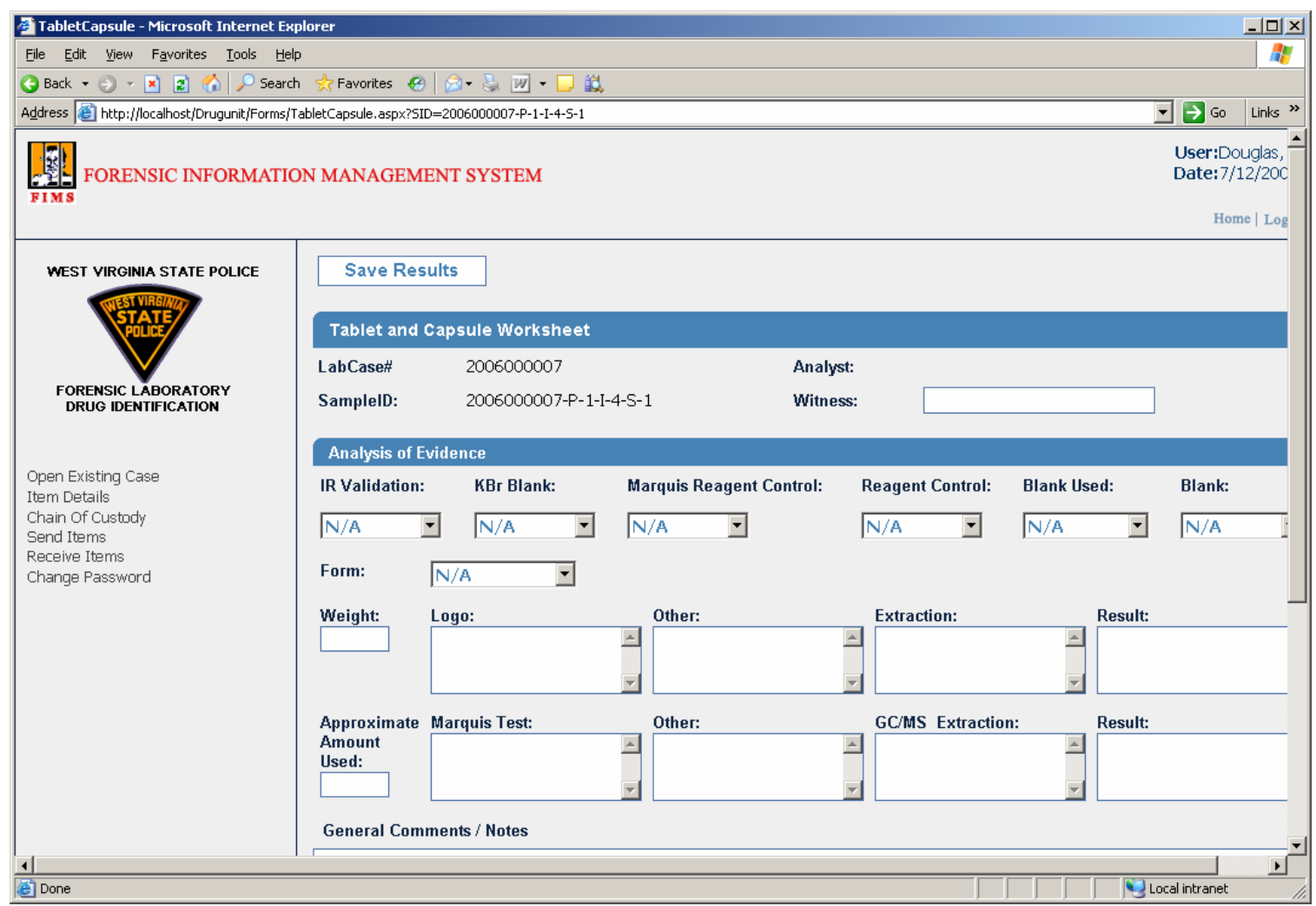

Figure 7.15: Tablet \& Capsule Worksheet 


\section{Unknown Powder Worksheet}

Unknown Powder worksheet is used for filling information pertaining Unknown Powder test performed on the sample by the analyst. After filling the required data fields, analyst is required to click on "Save Results" button located on top of the page, as shown in Figure 7.16. After saving the worksheet data, analyst can finalize and close the worksheet by clicking on “Test Done” button. The analyst cannot make any alterations in the worksheet once the "Test Done” button is clicked.

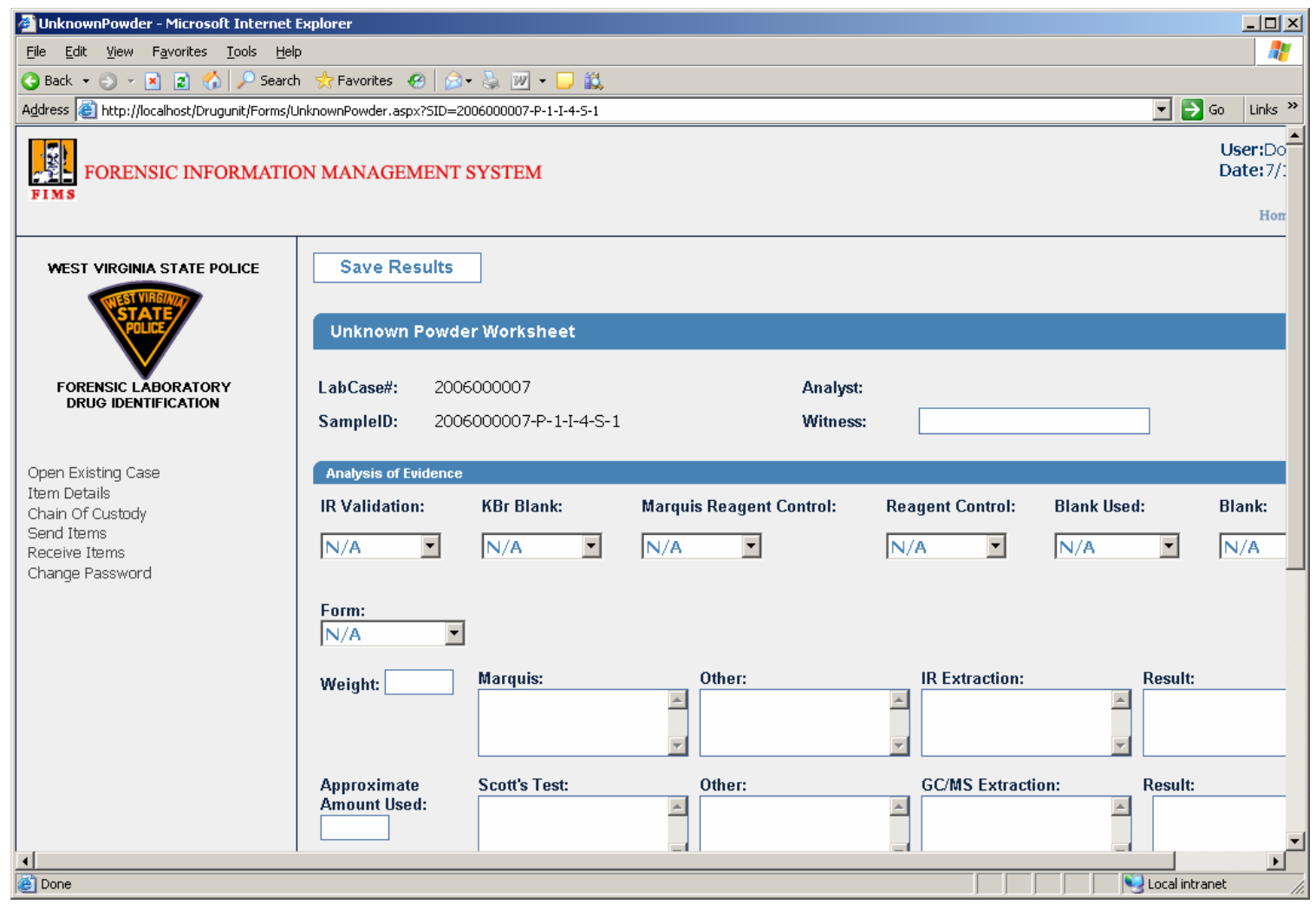

Figure 7.16: Unknown Powder Worksheet 


\section{Unknown Liquid Worksheet}

Unknown Liquid worksheet is used for filling information pertaining to unknown liquid test performed on the sample by the analyst. After filling the required data fields, analyst is required to click on "Save Results" button located on top of the page, as shown in Figure 7.17. After saving the worksheet data, analyst can finalize and close the worksheet by clicking on “Test Done” button. The analyst cannot make any alterations in the worksheet once the "Test Done" button is clicked.

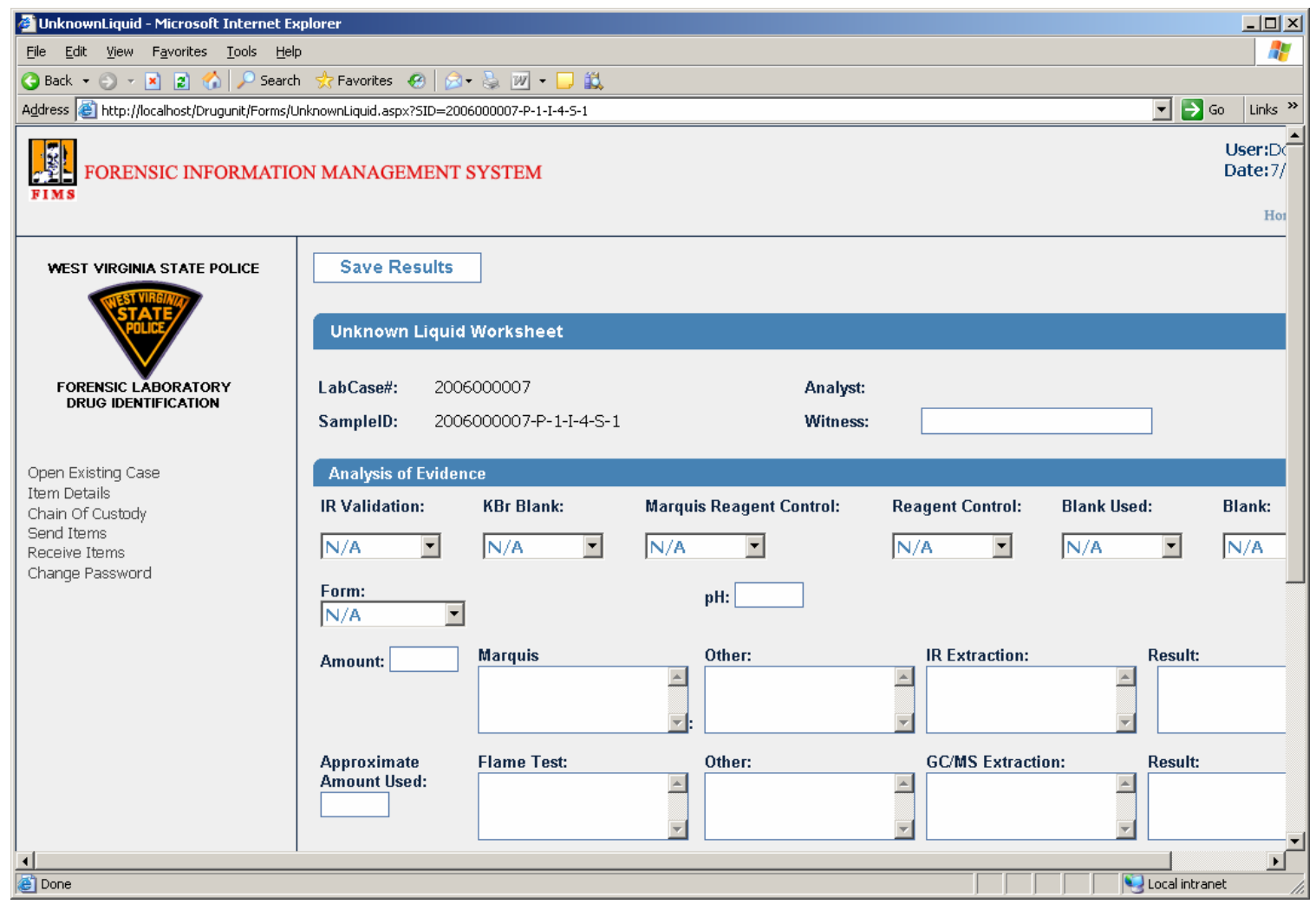

Figure 7.17: Unknown Liquid Worksheet 


\section{Vegetation Worksheet}

Vegetation worksheet is used for filling information pertaining to vegetation test performed on the sample by the analyst. After filling the required data fields, analyst is required to click on "Save Results" button located on top of the page, as shown in Figure 7.18. After saving the worksheet data, analyst can finalize and close the worksheet by clicking on "Test Done" button. The analyst cannot make any alterations in the worksheet once the “Test Done” button is clicked.

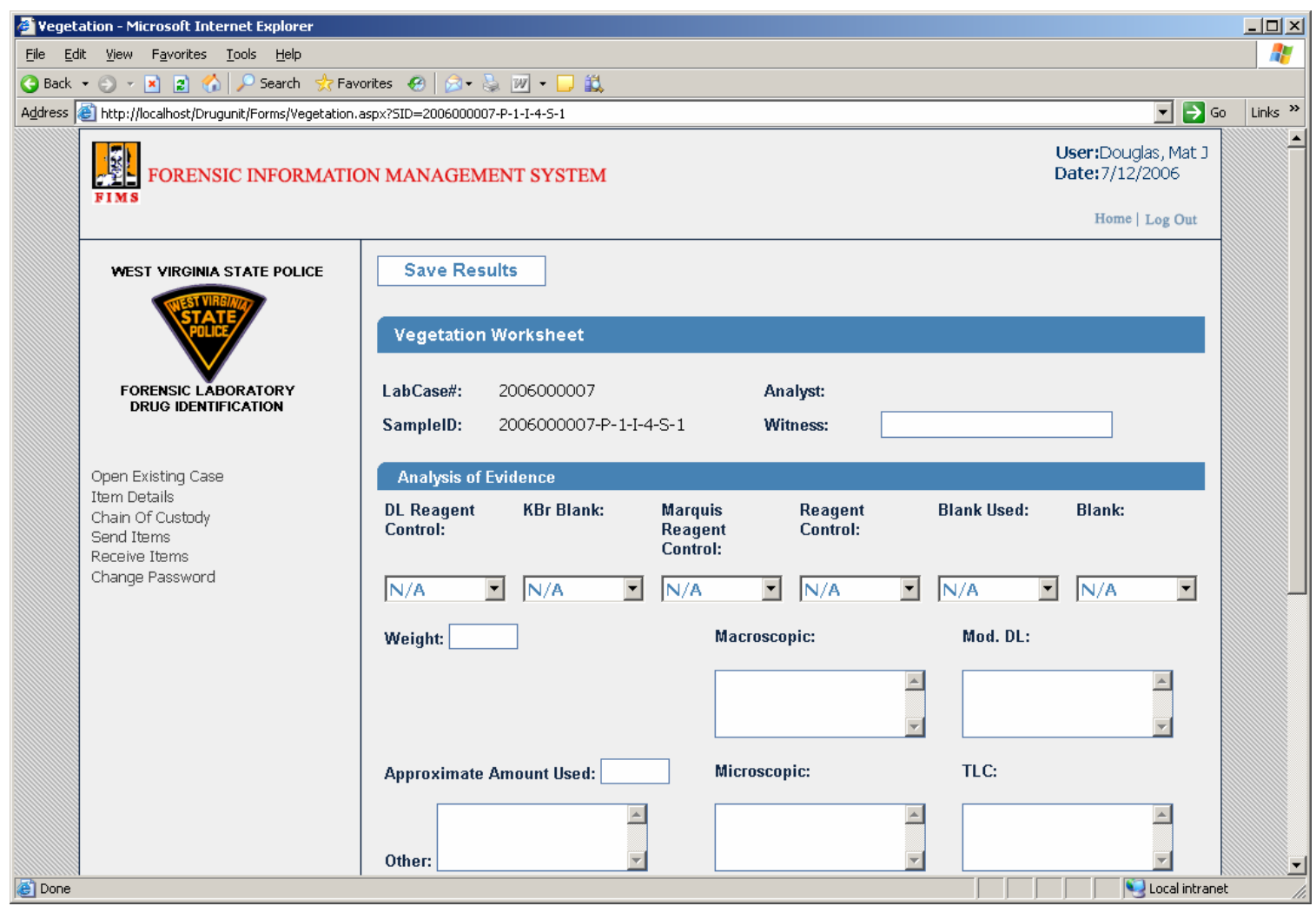

Figure 7.18: Vegetation Worksheet 


\section{Receive Item}

Once an item is sent by CEP unit, Drug identification unit analyst is responsible for receiving it by clicking on "Receive" button, under receive item section, as shown in Figure 7.19. This completes the handshaking process of item transfer between units.

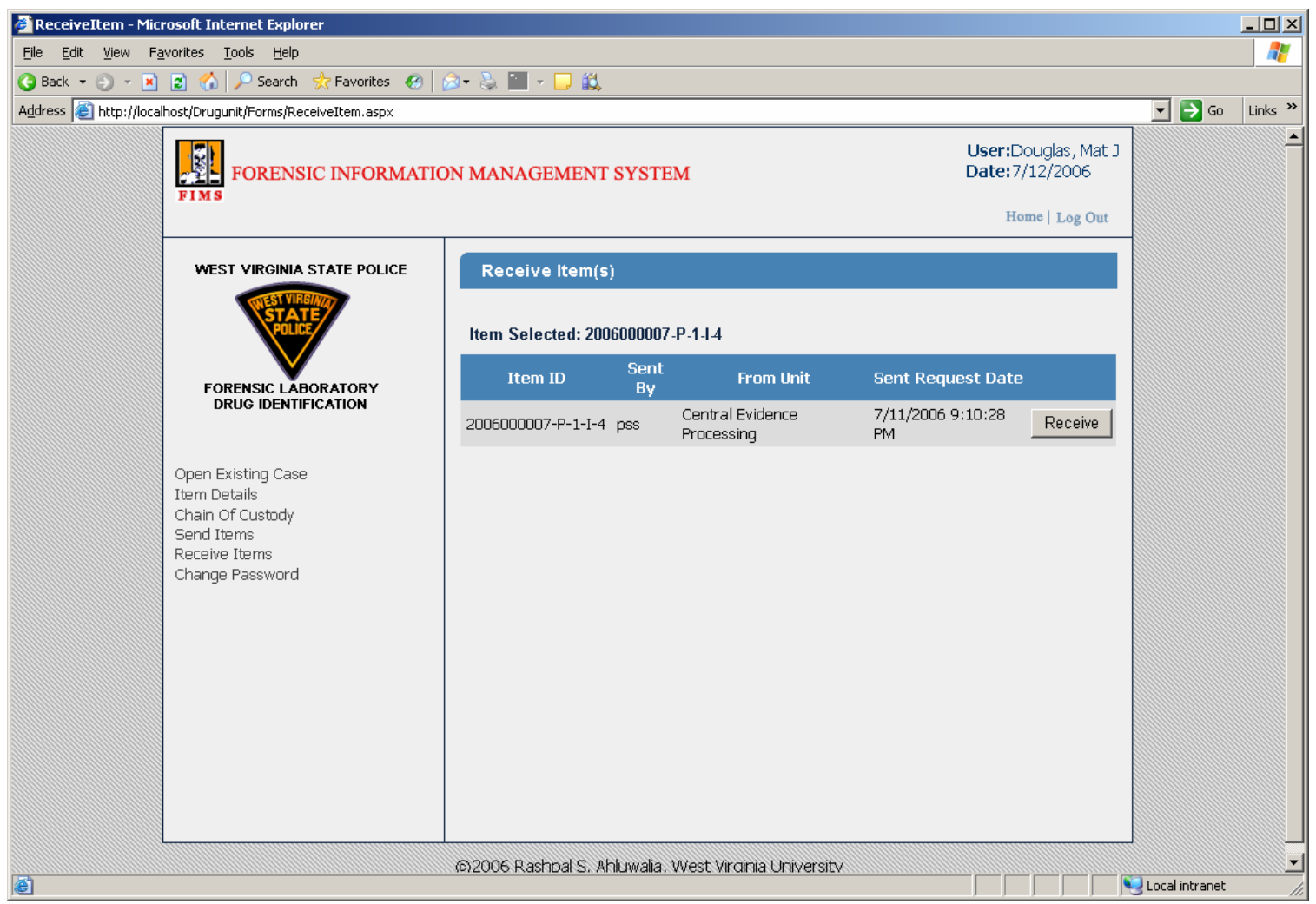

Figure 7.19: Receive Item Section 


\section{Send Item}

After completion of item testing, analyst is required to return the evidence item back to CEP unit. Figure 7.20 shows the section used to send an item back to the CEP unit. Analyst can use the drop down to select item ID and click on "Send" hyperlink, to send item back to CEP.

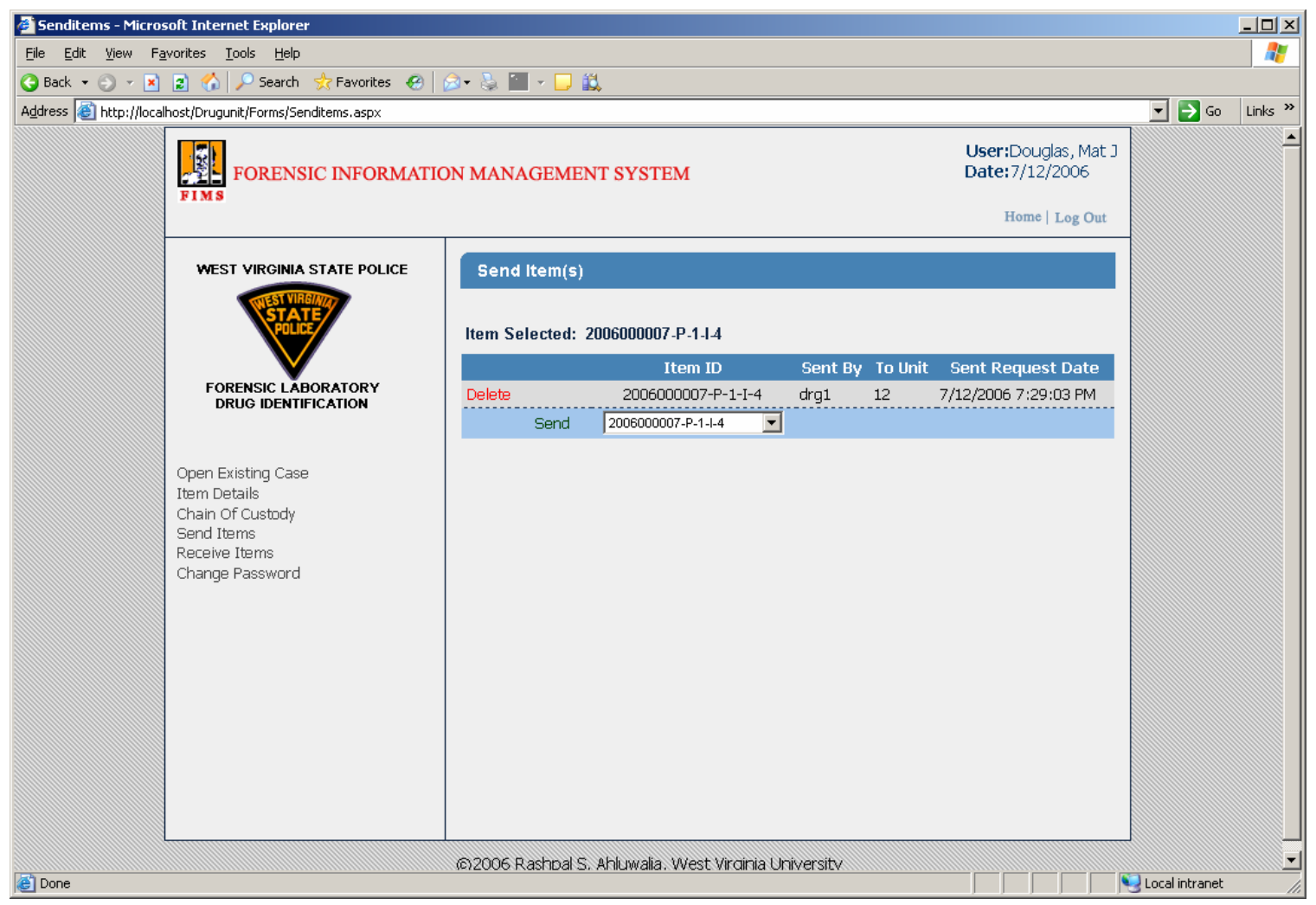

Figure 7.20: Send Item Section 


\section{Chain of Custody}

Item chain of custody information is shown in Figure 7.21. FIMS application records used ID, unit name, and time stamp for tracking item chain of custody.

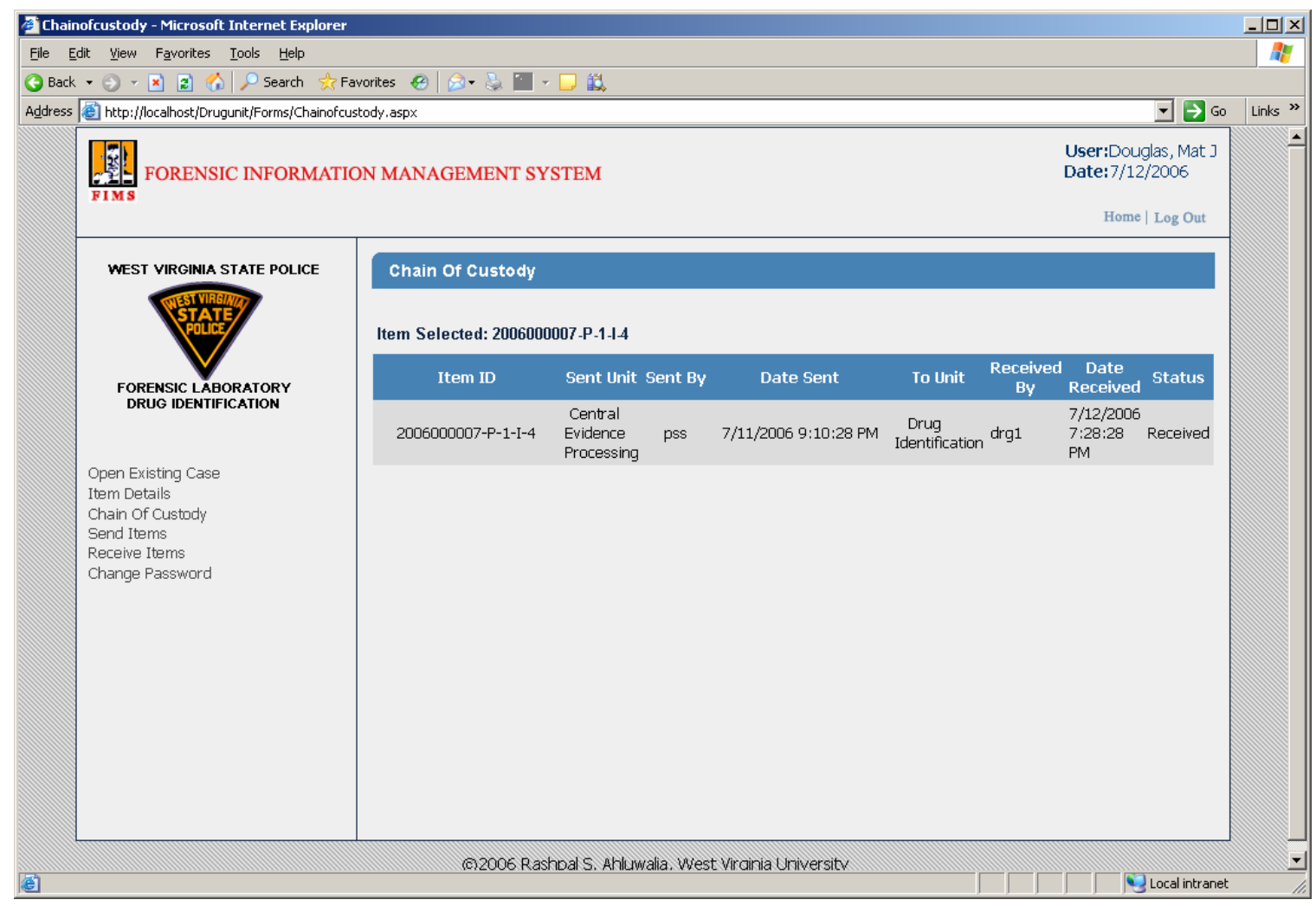

Figure 7.21: Item Chain Of Custody 


\section{Change User Password}

Analyst can change password through this section by providing appropriate credentials as shown in Figure 7.22.

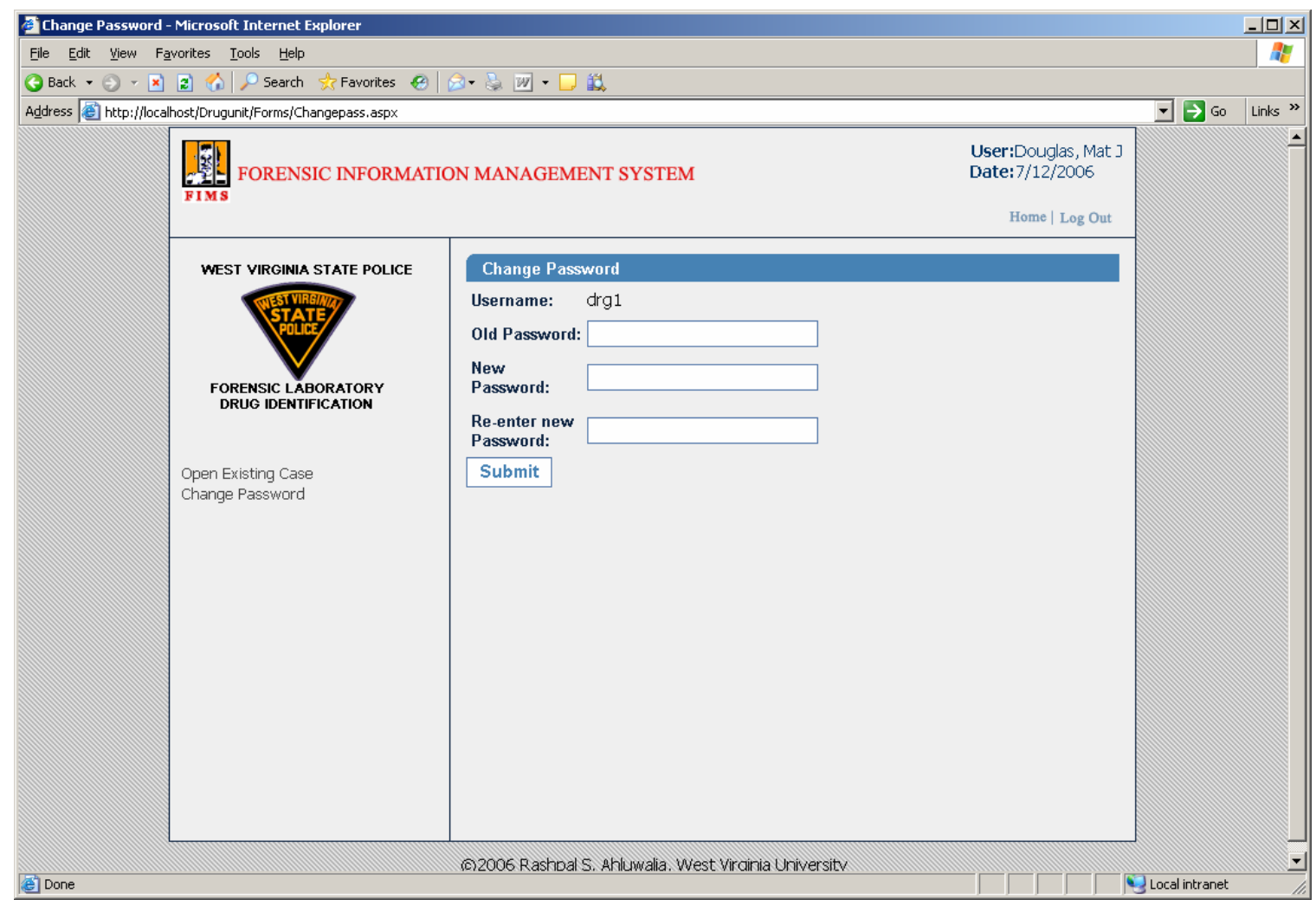

Figure 7.22: Change Password Section

\section{Exiting Drug Identification}

Users can terminate the current session by clicking on "Log Out" hyperlink on the top right corner of the page. 


\subsubsection{Generating Item and Sample Reports}

The analyst can generate the following types of reports

- Item Report

- Item Photo Report

- Sample Report

- Sample Photo Report

Figure 7.23 shows the item report, which is a cumulative report for all the samples. This section also facilitate analyst to export report via PDF/MS-Word format, as shown in Figure 7.25 and Figure 7.26

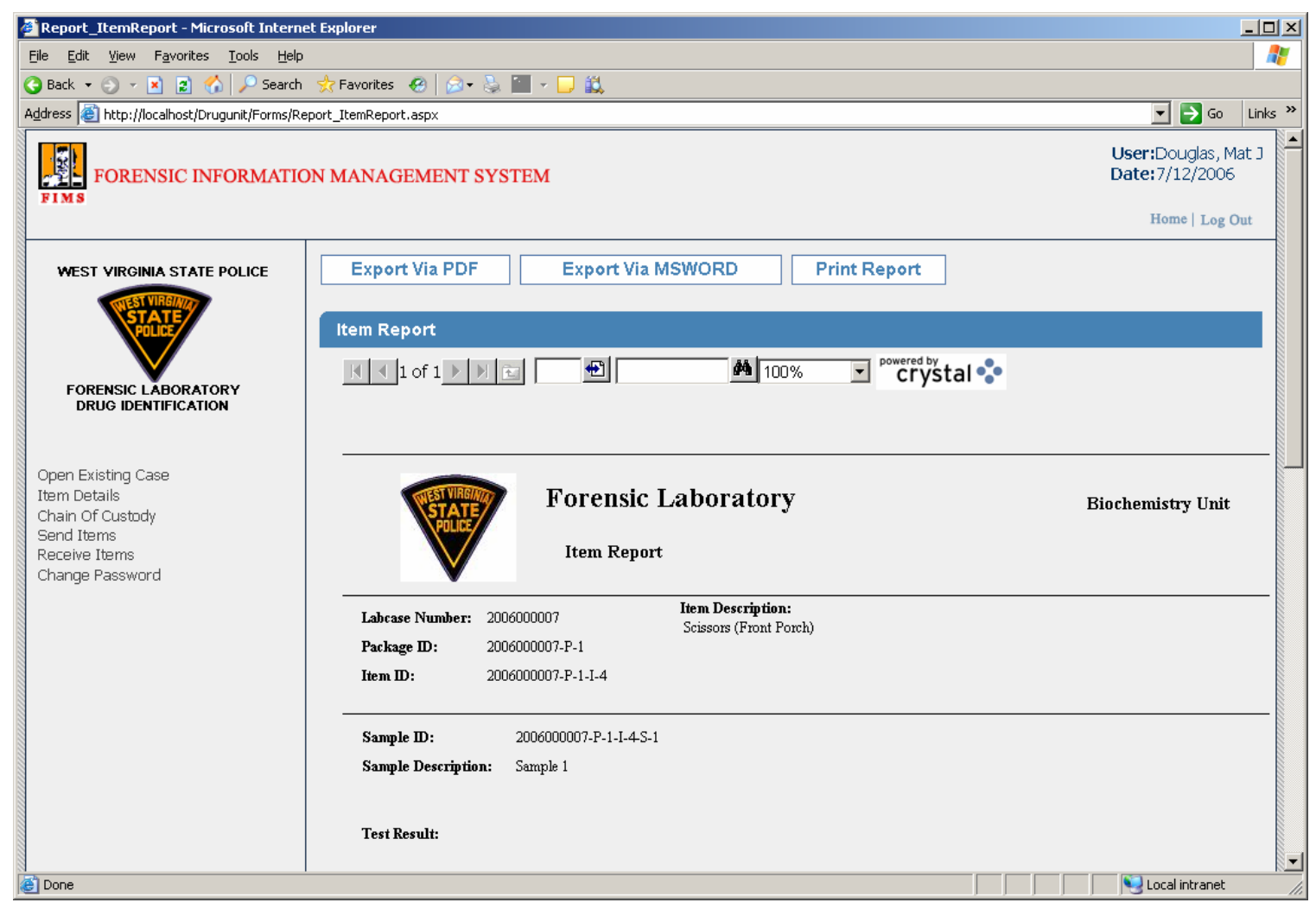

Figure 7.23: Item report 
Figure 7.24 shows the sample report for clandestine laboratory test. This report can also be accessed via PDF format, as shown in Figure 7.25.

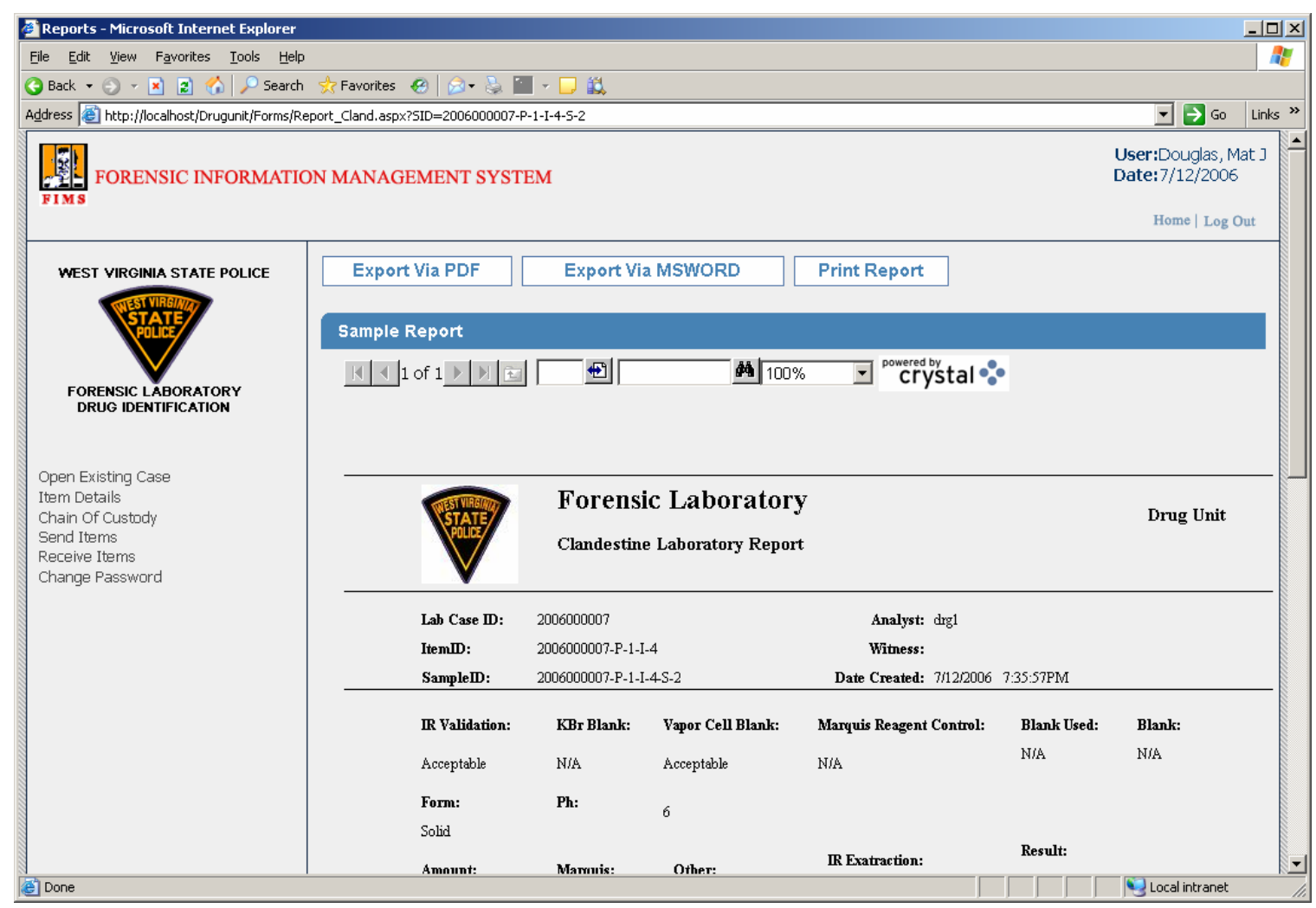

Figure 7.24: Sample Report 


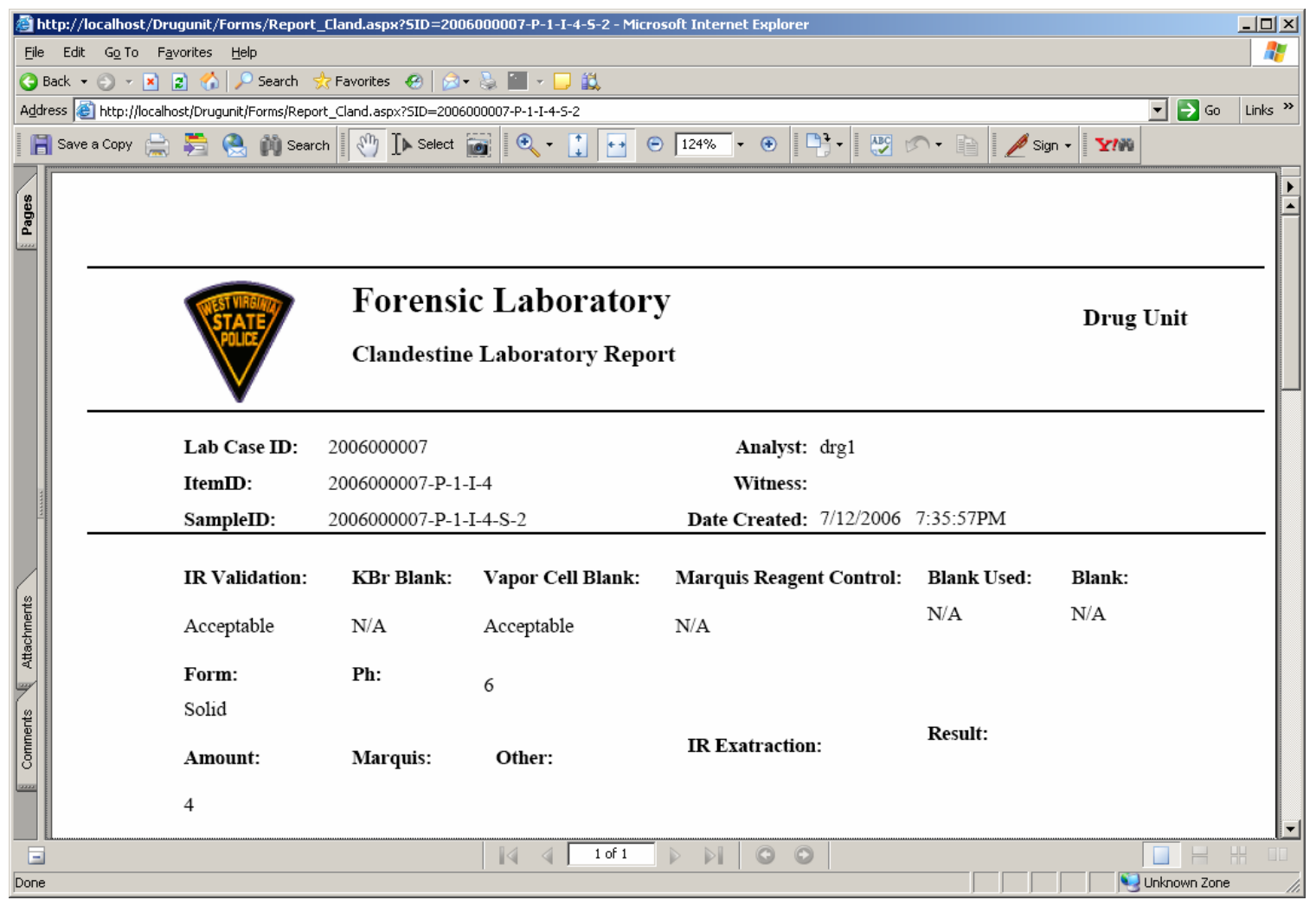

Figure 7.25: Item Report in PDF Format

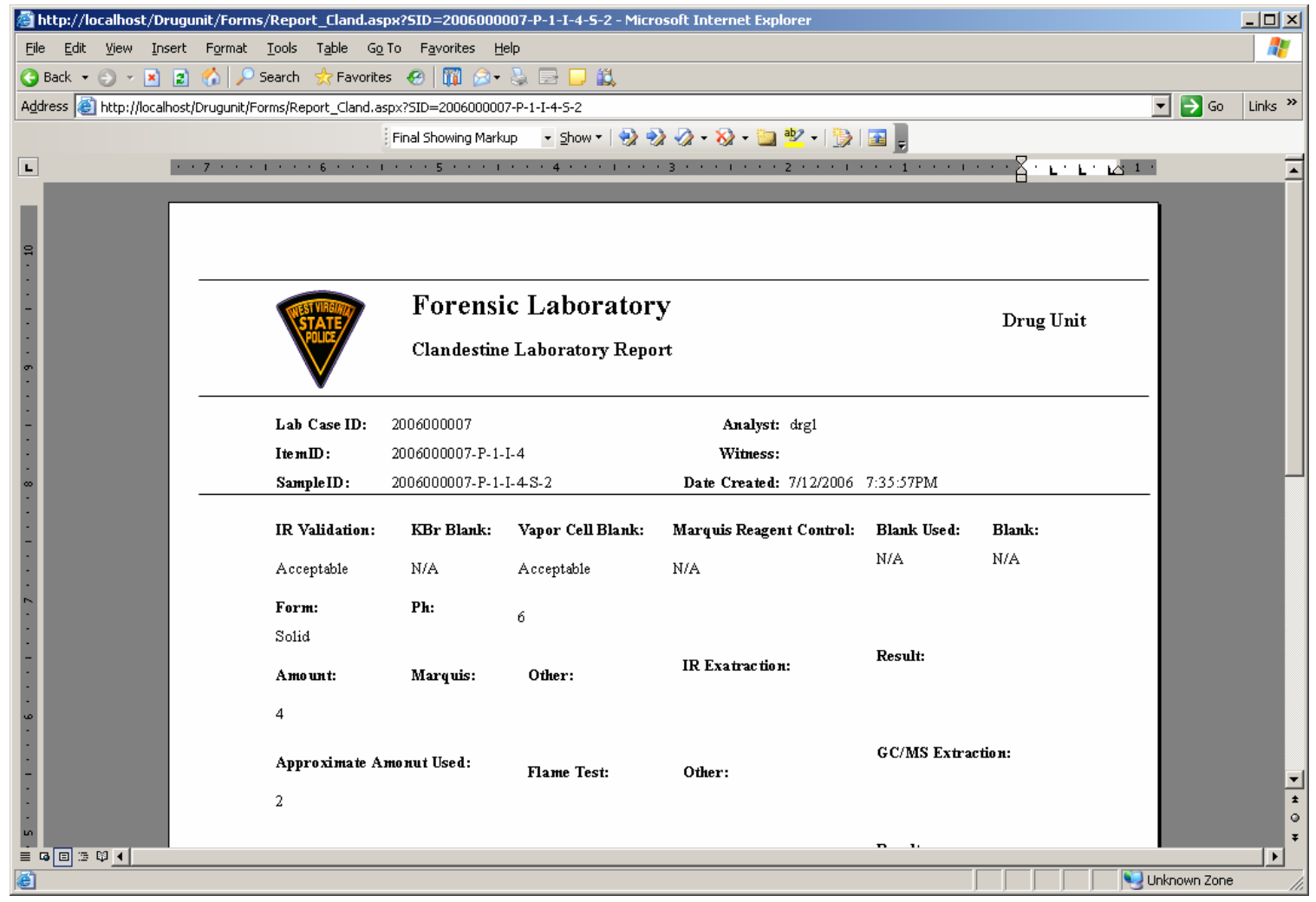

Figure 7.26: Item Report in MS Word Format 


\section{CHAPTER 8 \\ TRACE EVIDENCE}

\subsection{Existing Trace Evidence Process}

The Trace Evidence Unit is responsible for arson analysis, glass comparison/analysis, gunshot residue analysis, lamp analysis, paint comparison/analysis, speed determinations from speedometers, and various other types of analyses involving identifications, physical matches, and comparisons. The trace evidence unit has a valuable resource at its disposal, called the Paint Database Query (PDQ). This is a national and international database consisting of automotive paint data. For example, this database can be used to determine the make, model, and year of an automobile from a sample of paint found at the crime scene. The analyst in the trace evidence unit receives the LEIF form and chain of custody form along with the evidence items. Samples are prepared from the items received from the storage. After the tests are performed, appropriate forms are completed. The forms/worksheets used by this unit are: Paint Comparison (PC), Gun Shot Residue (GSR), Speedometer Examination (SE), Glass Comparison (GC), Ignitable Liquid (IL), and Miscellaneous Comparison (MC). Once the examinations are completed, evidence is returned to the CER and the secretary prepares a draft report. The draft report is reviewed by the analyst/reviewer. After approval, the final report is sent to CER. Figure 8.1 shows the existing process for the trace evidence unit 


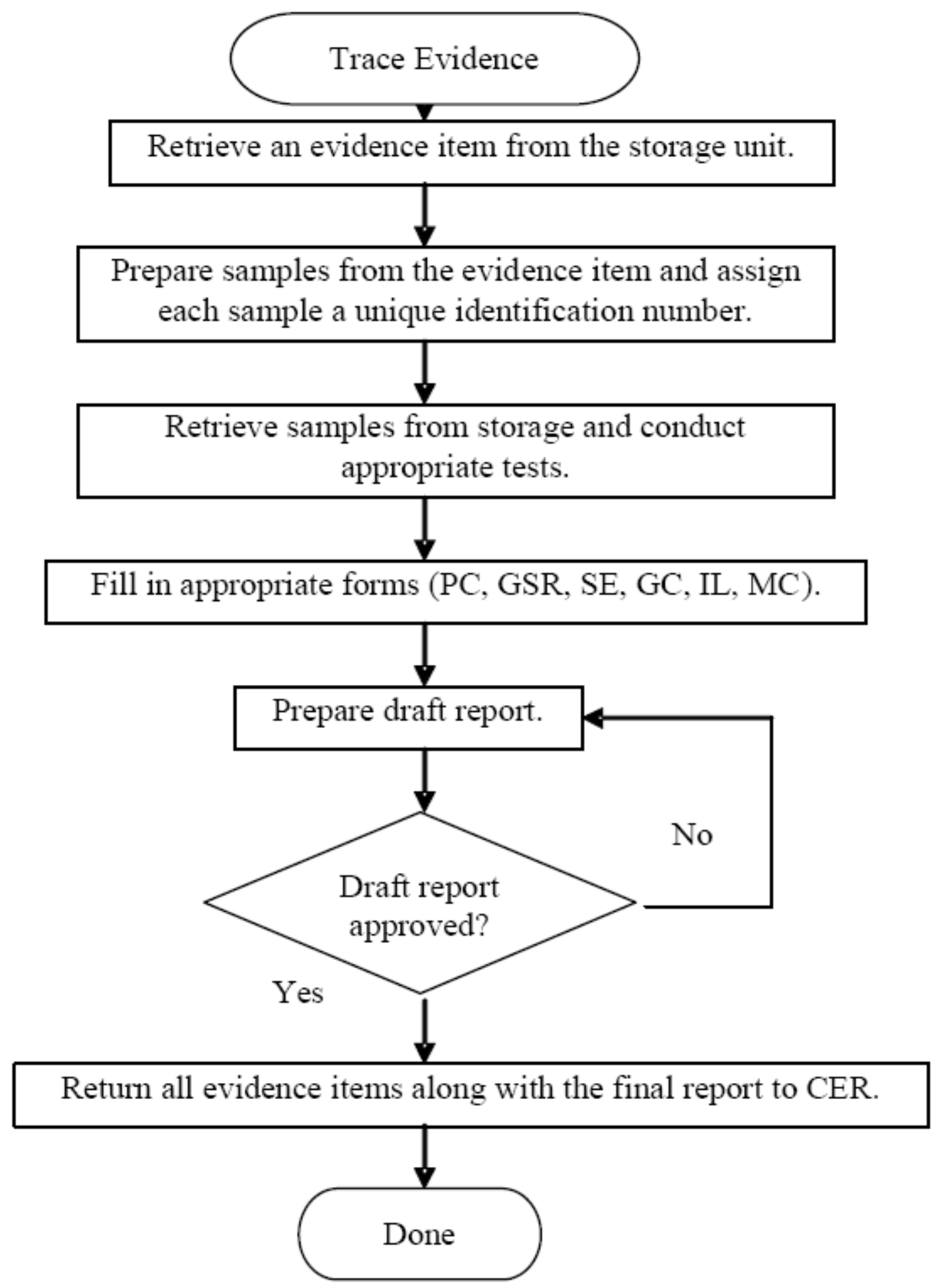

Figure 8.1: Existing Trace Evidence Process 


\subsection{Re-engineering the Trace Evidence Process}

The analyst in the trace evidence unit receives the items assigned for further processing and analysis from CEP. Received items are sub-divided into samples for conducting the requested tests. An individual item may be divided into many samples according to the requirement and complexity of the required test. A report for each sample is generated. An item report is compiled by combining sample reports. After the report is received, the reports along with the test items are sent back to CEP for further processing. The evidence and information flow diagram is shown in Figure 8.2.

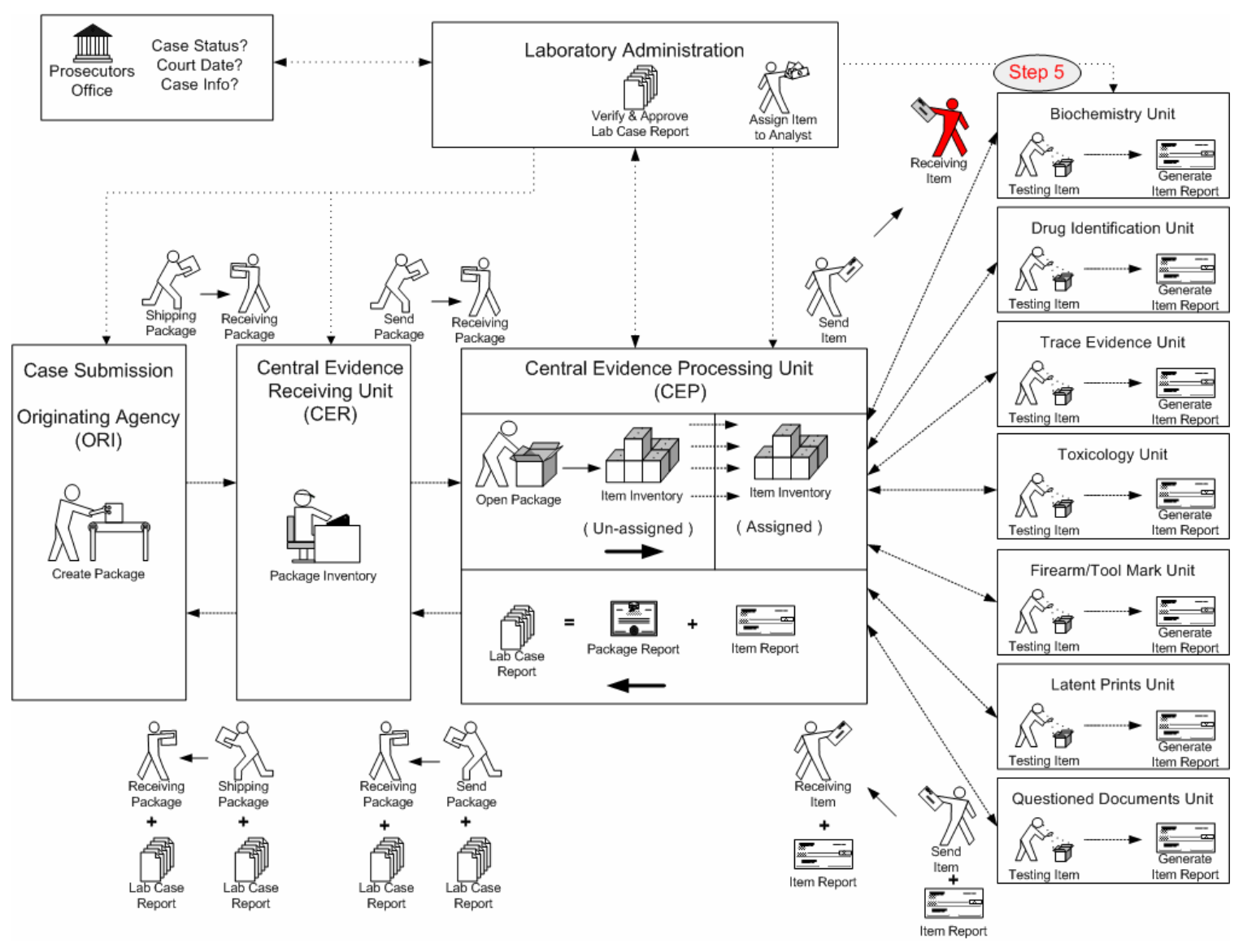

Figure 8.2: Step 5 Receive Assigned Items from CEP 
Detail procedure followed by trace evidence unit for further processing and analysis is shown in Figure 8.3.

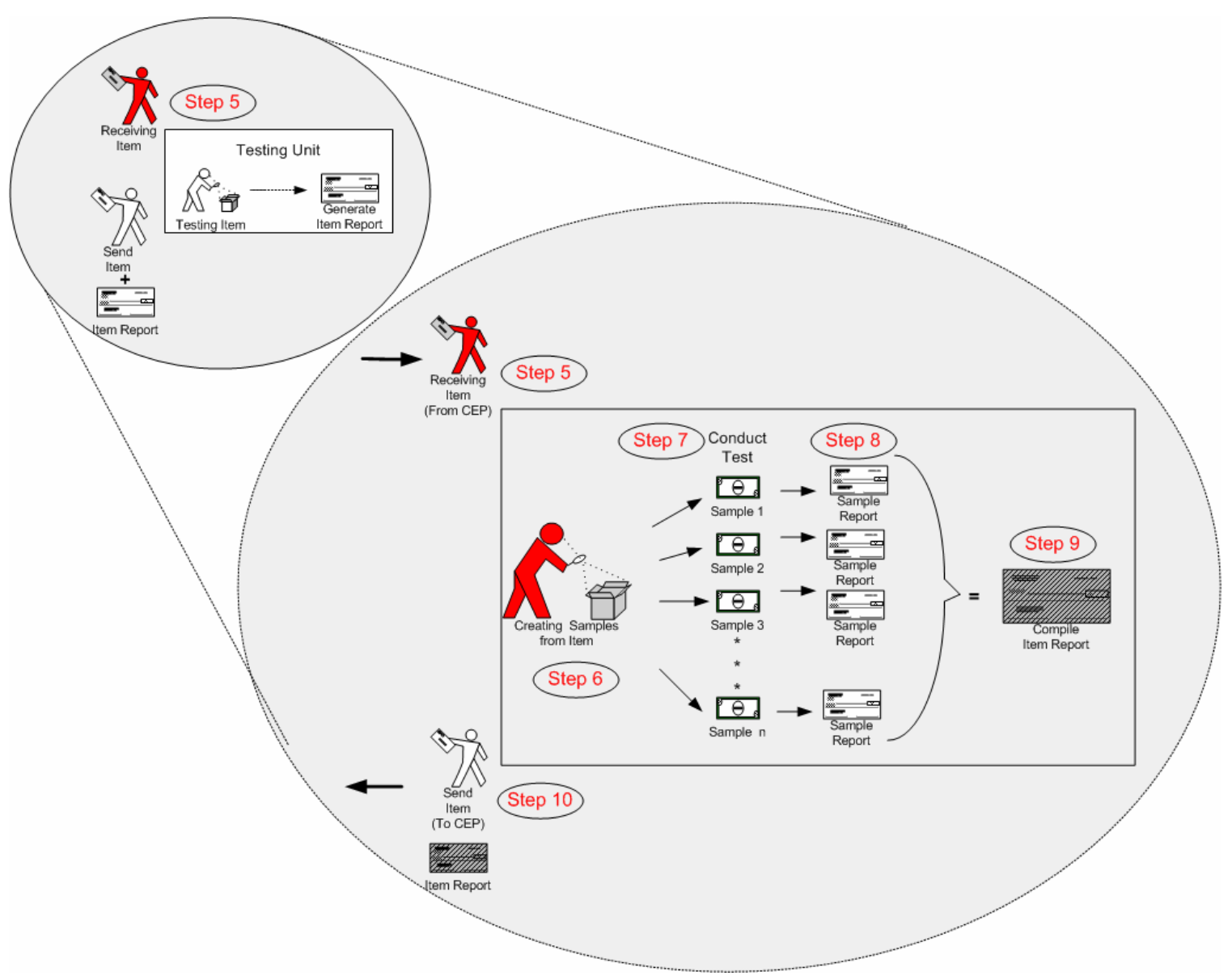

Figure 8.3: Detail Item Processing Procedure 


\subsubsection{Trace Evidence Entity Relationship Diagram}

CEP data tables are connected with each other through relations as shown in the figure 8.4.

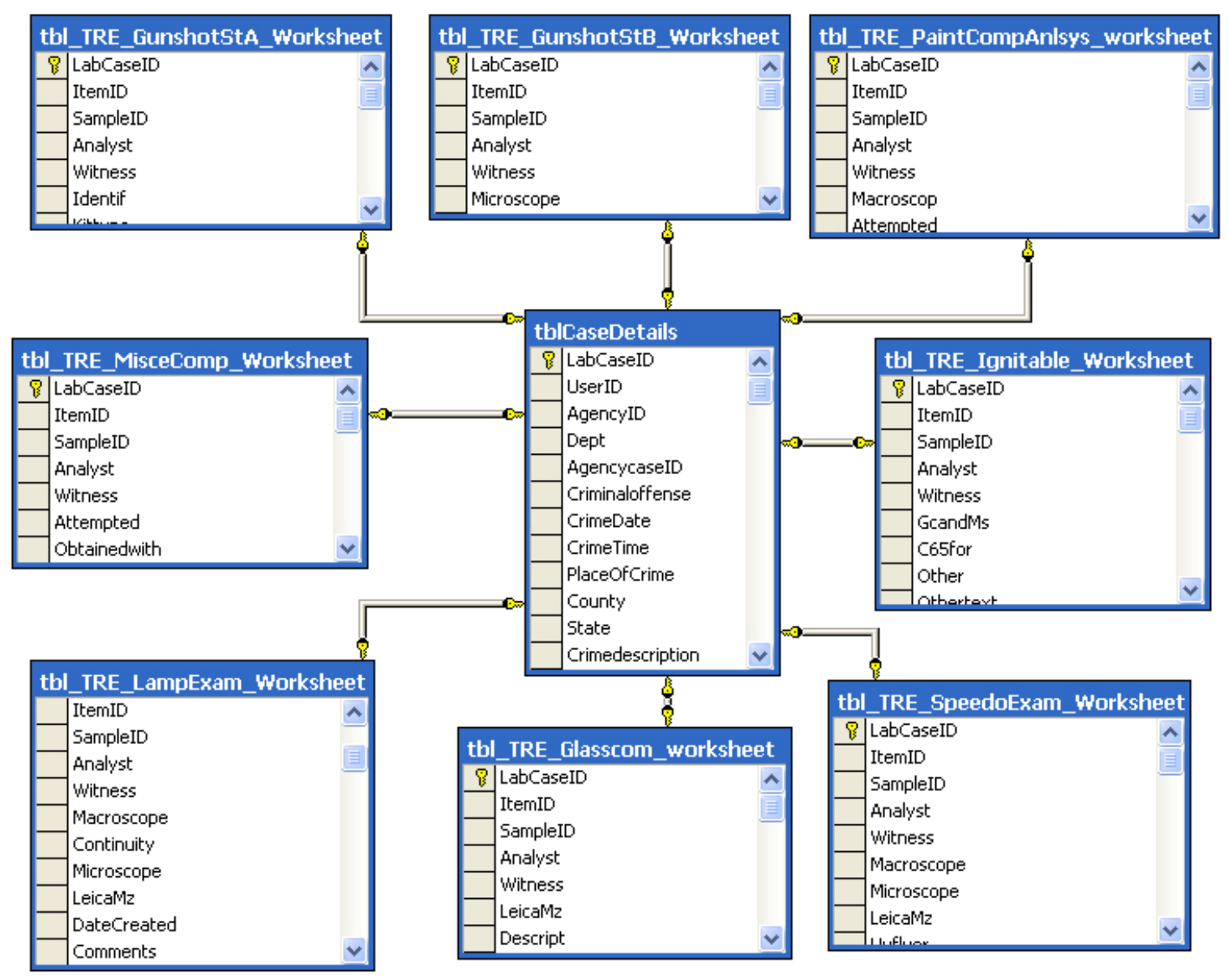

Figure 8.4: Entity Relationship Diagram 


\subsubsection{Trace Evidence Data Tables}

The trace evidence unit module stores its data in the following tables

- Glass Comparison Worksheet(tbl_TRE_Glasscom_worksheet)

- Gun shot worksheet-A(tbl_TRE_GunshotStA_worksheet)

- Gun shot Worksheet-B(tbl_TRE_GunshotStB_worksheet)

- Ignitable Worksheet(tbl_TRE_Ignitable_worksheet)

- Lamp Examination Worksheet(tbl_TRE_LampExam_worksheet)

- Miscellaneous Comparison Worksheet(tbl_TRE_MisceComp_worksheet)

- Paint Comparison Analysis worksheet(tbl_TRE_PaintCompAnlsys_worksheet)

- Speedometer Examination Worksheet(tbl_TRE_SpeedoExam_worksheet) 
The various fields of the glass comparison worksheet table are shown in Table 8-

1. Glass Comparison worksheet data is stored and maintained in this data table

Table 8-1: Glass Comparison Worksheet Table

\begin{tabular}{|l|l|}
\hline tbl_TRE_Glasscom_worksheet \\
\hline ItemiD \\
SampleID \\
Analyst \\
Witness \\
LeicaMz \\
Descript \\
Color \\
\hline Thinkness \\
\hline PhyMatch \\
\hline InsufSampletext \\
Attempted \\
Obtainedwith \\
Obtainedwithtext \\
Blackight \\
Longwave \\
Grim2 \\
\hline Oiltype \\
\hline Ri \\
\hline Notes \\
Wavelength \\
Mettler \\
Density \\
Camscantra \\
Elelments \\
Tescanoxford \\
EmtsPresnt \\
DateCreated \\
Comments \\
TestResult \\
\hline
\end{tabular}


The various fields of the gun shot -A worksheet table are shown in Table 8-2. Gun shot - A worksheet data is stored and maintained in this data table

Table 8-2: Gun Shot -A Worksheet Table

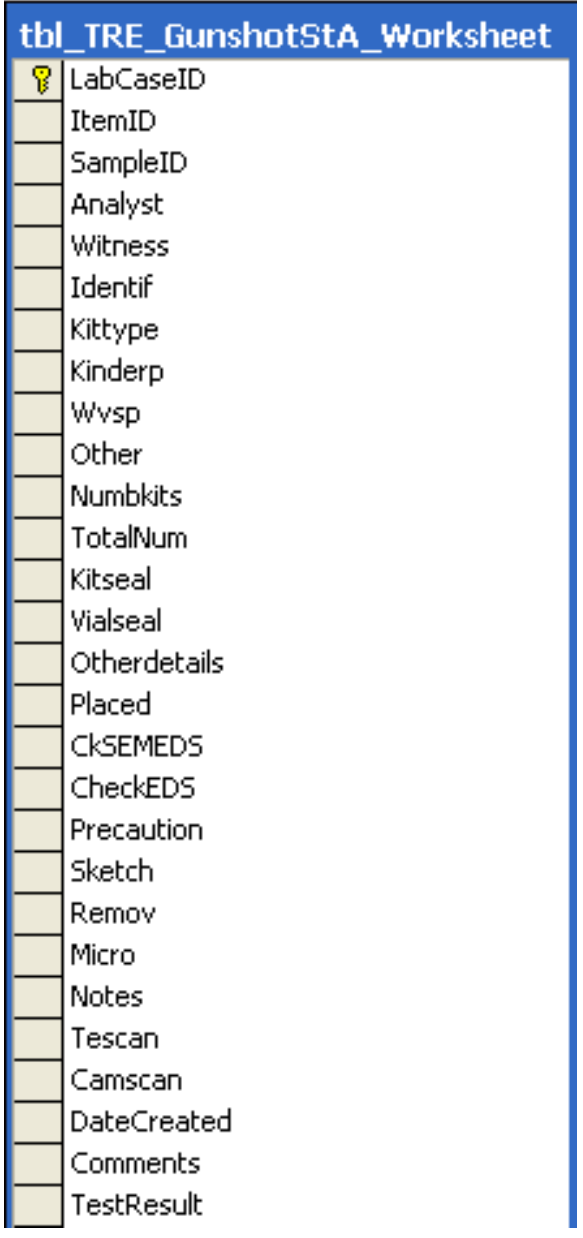


The various fields of the gun shot -B worksheet table are shown in Table 8-3.

Gun shot $-\mathrm{B}$ worksheet data is stored and maintained in this data table

Table 8-3: Gun Shot -B Worksheet Table

\begin{tabular}{|c|l|}
\hline tbl_TRE_GunshotStB_Worksheet \\
\hline & LabCaseID \\
\hline & ItemID \\
SampleID \\
Analyst \\
\hline Witness \\
\hline \\
Microscope \\
\hline Elelments \\
Tescanoxford \\
Camscantra \\
DateCreated \\
Comments \\
TestResult \\
\hline
\end{tabular}

The various fields of ignitable worksheet the worksheet table are shown in Table 8-4. Ignitable worksheet data is stored and maintained in this data table

Table 8-4: Ignitable Worksheet Table

\begin{tabular}{|l|l|}
\hline tbl_TRE_Ignitable_worksheet \\
\hline LabCaseID \\
ItemID \\
SampleID \\
Analyst \\
Witness \\
GcandMs \\
C65for \\
Other \\
Othertext \\
Coofor \\
Nadil \\
\hline Nadiltext \\
Container \\
Contents \\
Odor \\
Technique \\
Results \\
DateCreated \\
Comments \\
\hline TestResult \\
\hline
\end{tabular}


The various fields of the lamp examination worksheet table are shown in Table 8-

1. Lamp examination worksheet data is stored and maintained in this data table

Table 8-5: Lamp Examination Worksheet Table

\begin{tabular}{|l|l|}
\hline tbl_TRE_LampExam_Worksheet \\
\hline B LabCaseID \\
\hline & ItemID \\
SampleID \\
\hline Analyst \\
\hline Witness \\
\hline Macroscope \\
\hline \\
Continuity \\
Microscope \\
LeicaMz \\
DateCreated \\
Comments \\
TestResult \\
\hline
\end{tabular}


The various fields of the miscellaneous worksheet table are shown in Table 8-1. Miscellaneous worksheet data is stored and maintained in this data table

Table 8-6: Miscellaneous Worksheet Table

tbl_TRE_MisceComp_Worksheet

? LabCaseID

ItemID

SampleID

Analyst

Witness

Attempted

obtainedwith

Obtainedwithtext

PhysicInsuff

PhyInsufText

Macroscope

Microscope

LeicaMz

ChemInsufSam

ChemInsuftext

$\mathrm{Ph}$

Phtext

ChemTests

ChemTestText

Solubty

SolubtyText

Agilesnt

Pyroprob

Continum

OrganicText

Camscantra

TescanOxford

Inorganictext

DateCreated

Comments

TestResult 
The various fields of the paint comparison analysis worksheet table are shown in Table 8-1. Paint comparison analysis worksheet data is stored and maintained in this data table

Table 8-7: Paint Comparison Analysis Worksheet Table

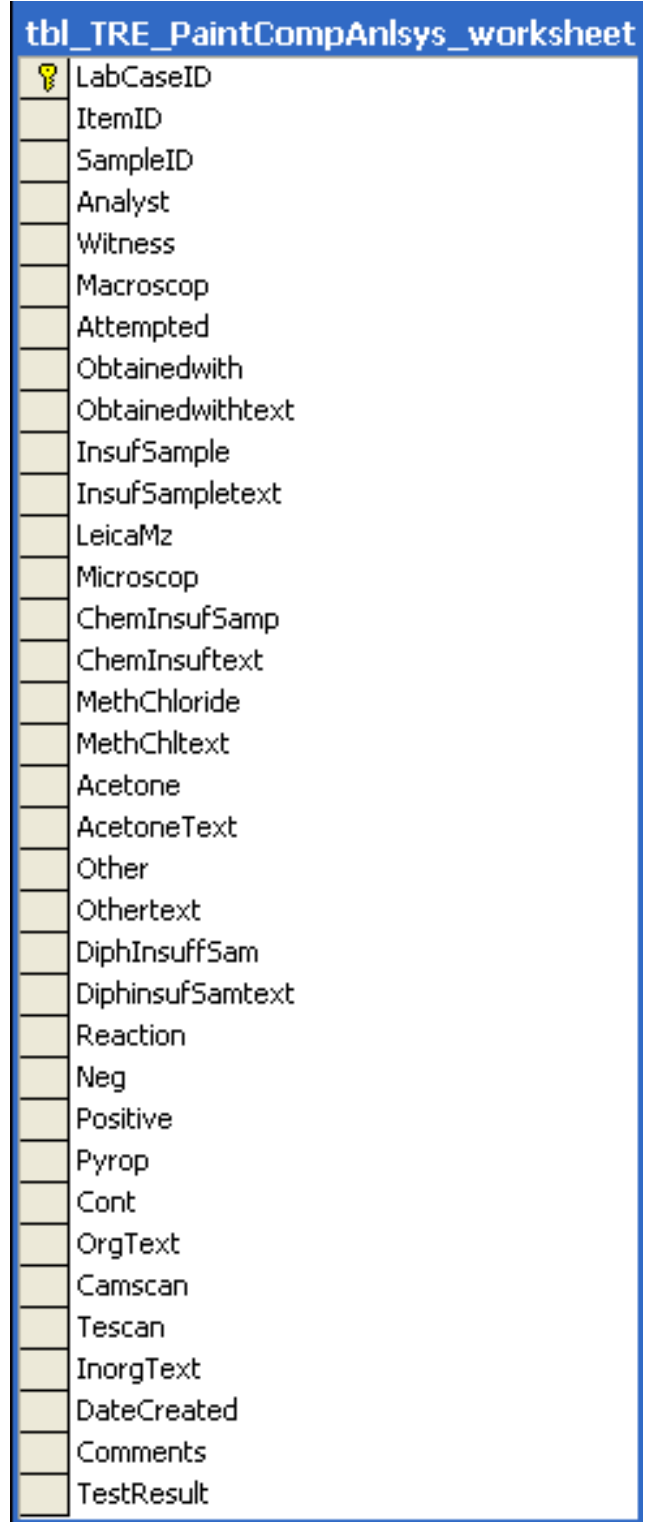


The various fields of the speedometer examination worksheet table are shown in Table 8-1. Speedometer examination worksheet data is stored and maintained in this data table

Table 8-8: Speedometer Examination Worksheet Table

\begin{tabular}{|l|l|}
\hline tbl_TRE_SpeedoExam_worksheet \\
\hline & LabCaseID \\
ItemID \\
SampleID \\
Analyst \\
Witness \\
Macroscope \\
Microscope \\
LeicaMz \\
Uvfluor \\
\hline BlackLight \\
AlterLight \\
Luma \\
DateCreated \\
Comments \\
TestResult \\
\hline
\end{tabular}




\subsubsection{Working with Trace Evidence}

Trace Evidence home page requires username and password to verify user credentials, as shown in the Figure 8.5. After verifying credentials, a user is directed to list of items assigned to him/her for further processing.

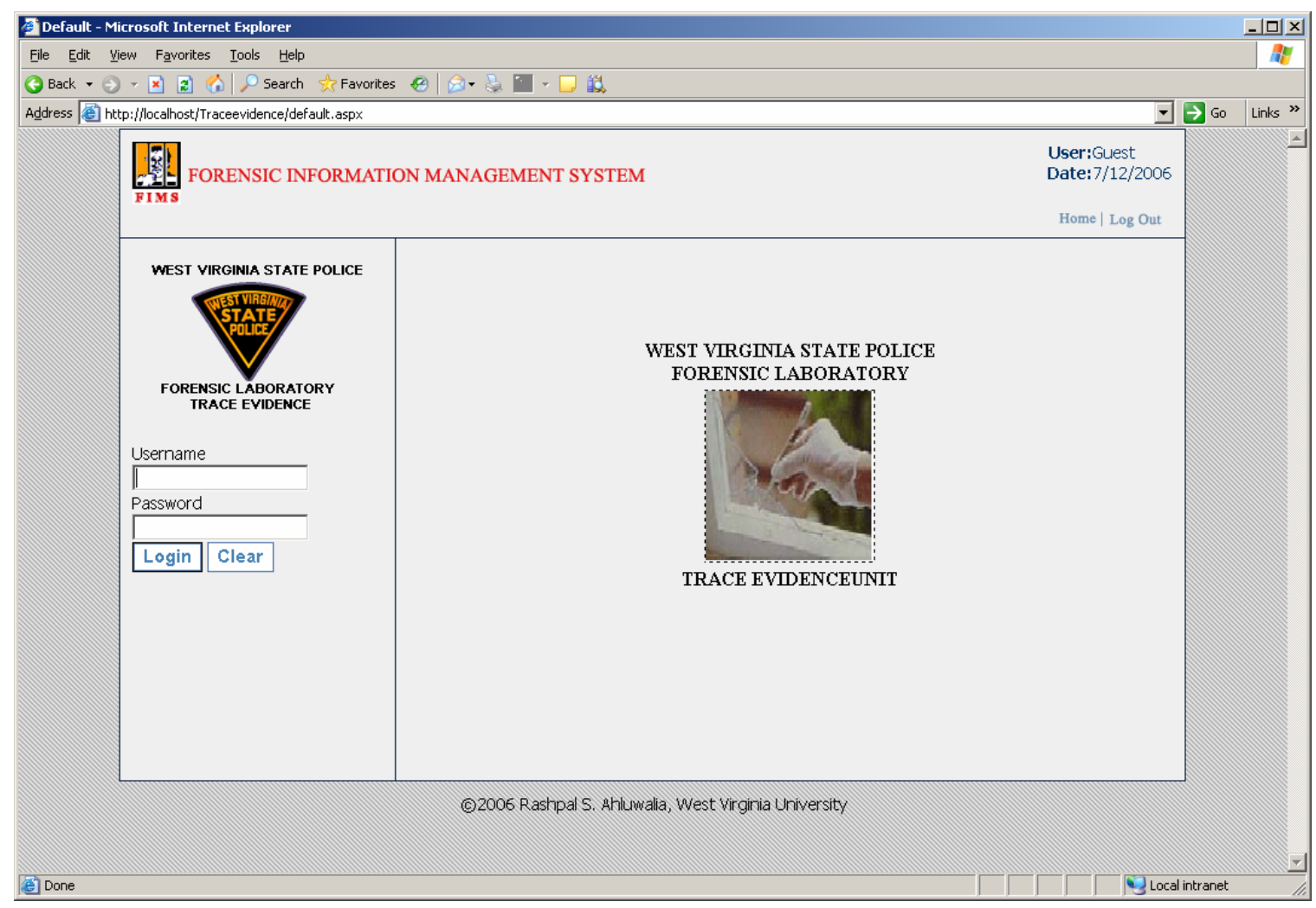

Figure 8.5: Trace Evidence Home Page 


\section{View Items Assigned}

Figure 8.6 shows the list of items assigned to the analyst by the laboratory administration unit for further analysis. Initially, job status for the item assigned is set to "Job Pending". The "View Details" hyperlink enables an analyst to view the details of the case. If the list of items assigned grows to more than 15 items, the record set is divided into multiple pages. Analysts can navigate between different pages by clicking on the header and footer navigation bar of the list.

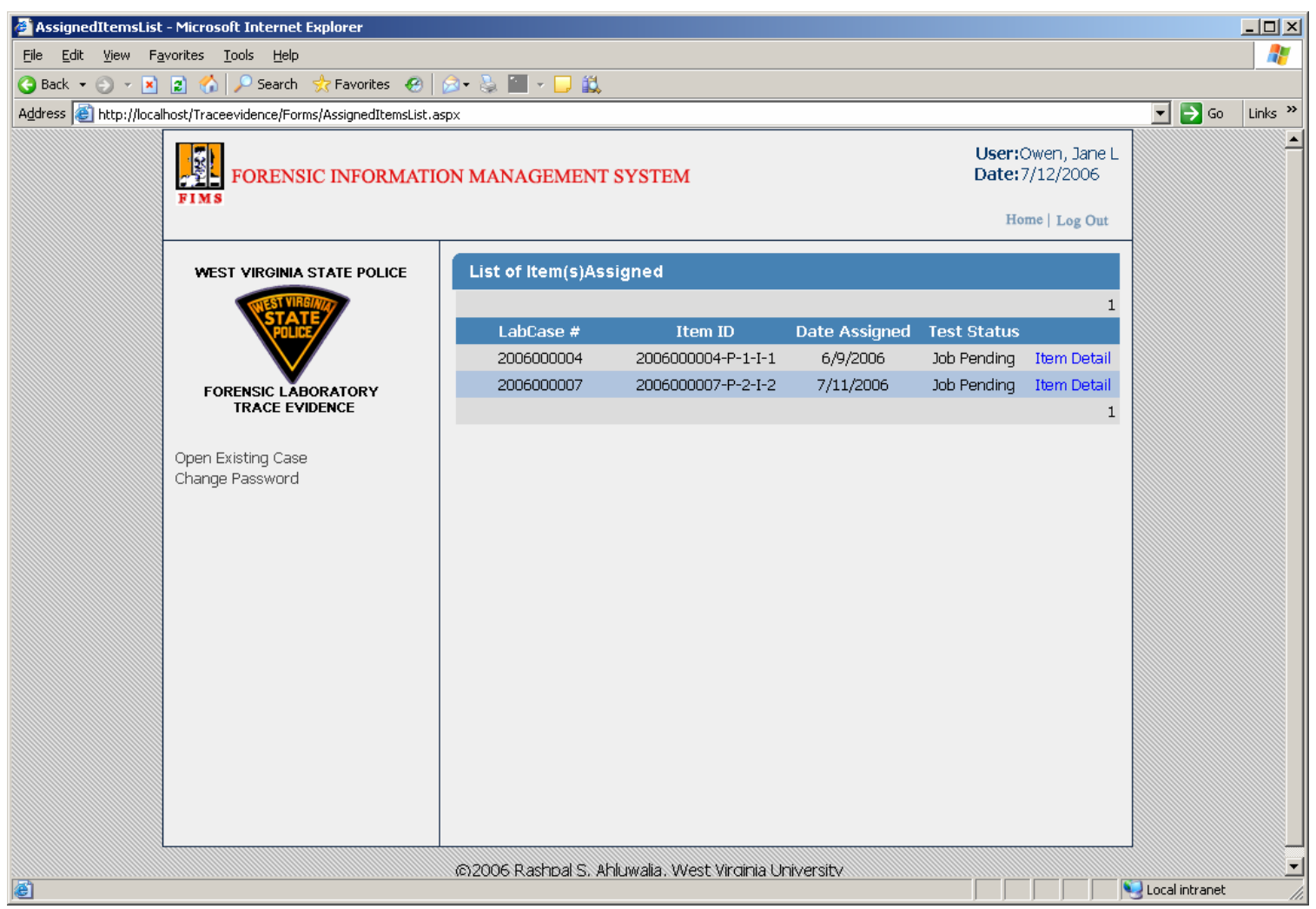

Figure 8.6: Assigned Item List 


\section{Item Picture Management}

This section describes the procedure of attaching pictures to an item. Analysts can perform two kinds of operation:

- View Pictures

- Upload Pictures

Analysts can access this section by clicking on the "View/Upload Item Photo" hyperlink in the item details section as shown inn Figure 8.9. View picture section list the pictures already attached to the item with their thumbnails as shown in Figure 8.7. Analysts can also view the full size image by clicking on the image thumbnail. Full size image opens in a new window, which can be closed after use.

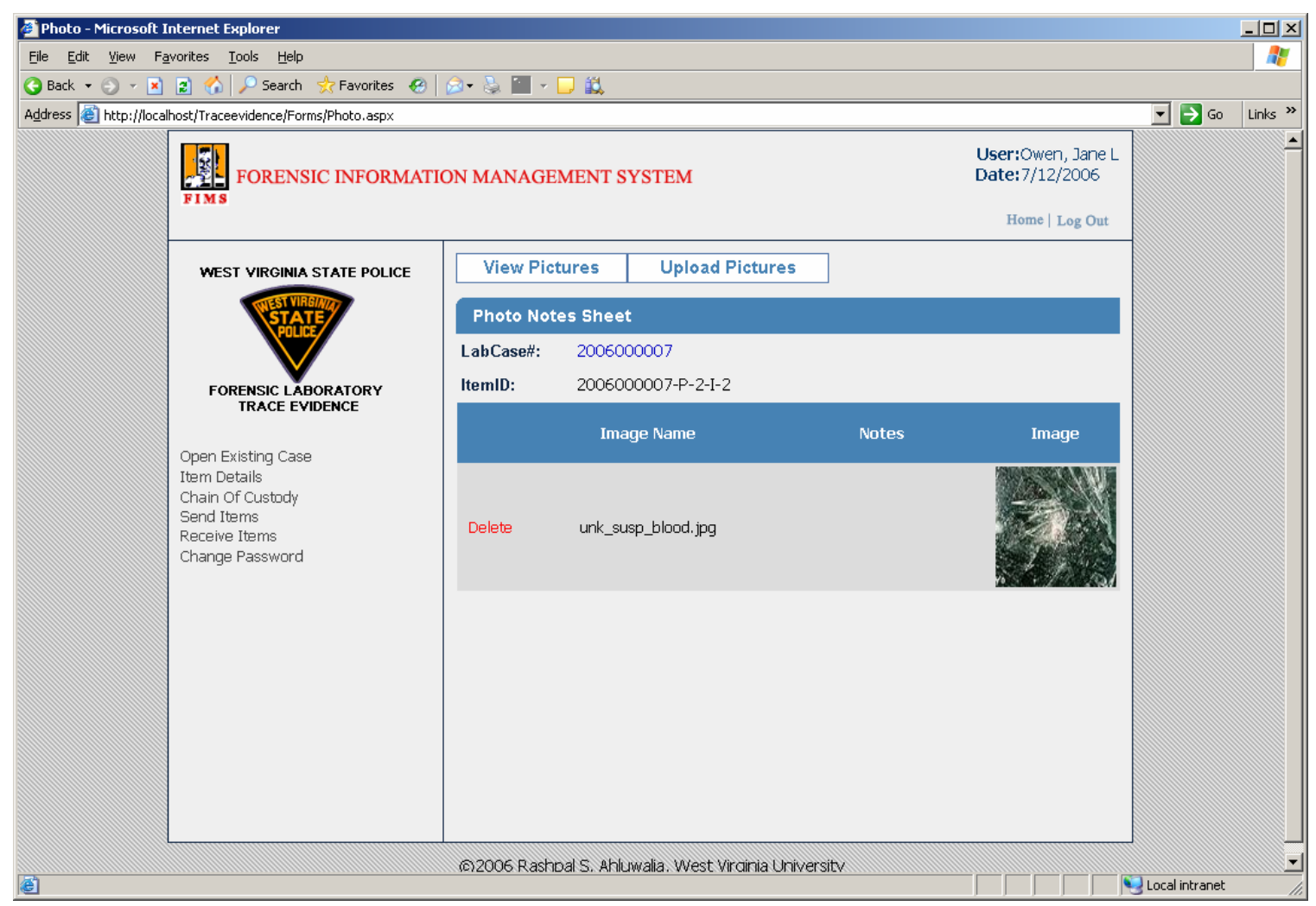

Figure 8.7: View Item Photo 
Analysts can upload any digital photo in JPG format directly into the database using this section as shown in Figure 8.8. To upload an image into database, analysts are required to browse for the image path, set other required fields and click on the "Upload the file” hyperlink. On successful upload a thumbnail appears on the bottom of the page with a success message.

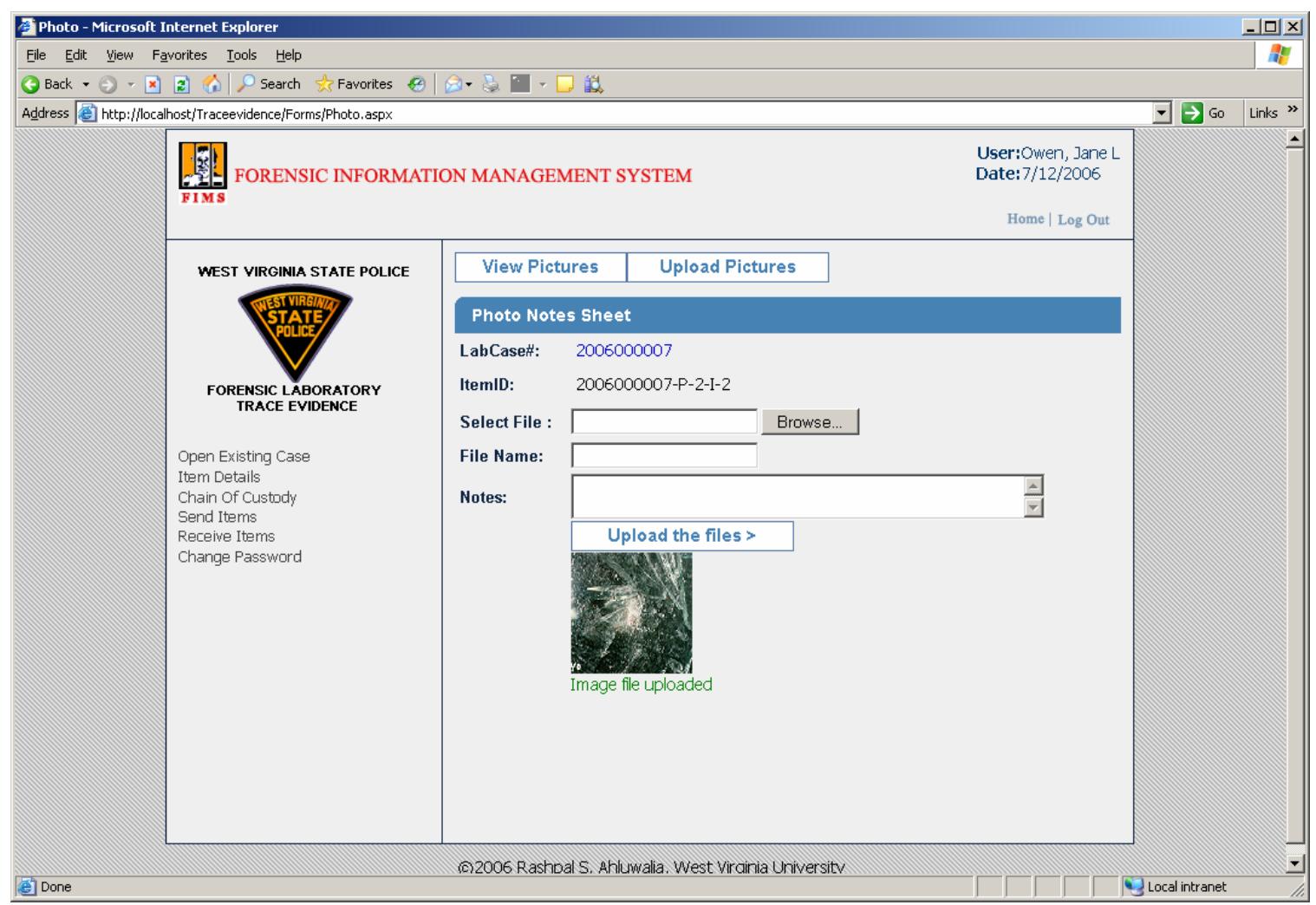

Figure 8.8: Upload Item Picture Section 


\section{Working with Samples}

Analysts receive items from the CEP unit for further analysis and processing. Once received items are sub-divided into samples for conducting requested tests. Each item is divided into one or more samples according to the requirement and complexity of the required test.

Analysts can create new, update or delete existing samples from the item details section as shown in Figure 8.9. To add a new sample analyst is required to provide description of sample and then click on "Add Sample” hyperlink. FIMS application automatically adds the time stamp to the item created. Initially, sample test status is set to "Test Pending" for the sample. Sample information can be updated by clicking on “Edit” hyperlink and existing sample can be deleted from the system by clicking on “Delete” hyperlink.

This section also enables analysts to view the final sample, sample photo, item, and item photo report. To access the individual sample/sample photo report analyst can click on "View Report" or "Photo Sheet" hyperlink. To view comprehensive item report analysts can click on "Item Report" or "Item Photo Report" at the bottom of the page. 
To complete the process for item testing, the analyst is required to click on "Item Test Complete" button, as shown in Figure 8.9. Once clicked, analyst will not be able to make any alterations to the existing item test report.

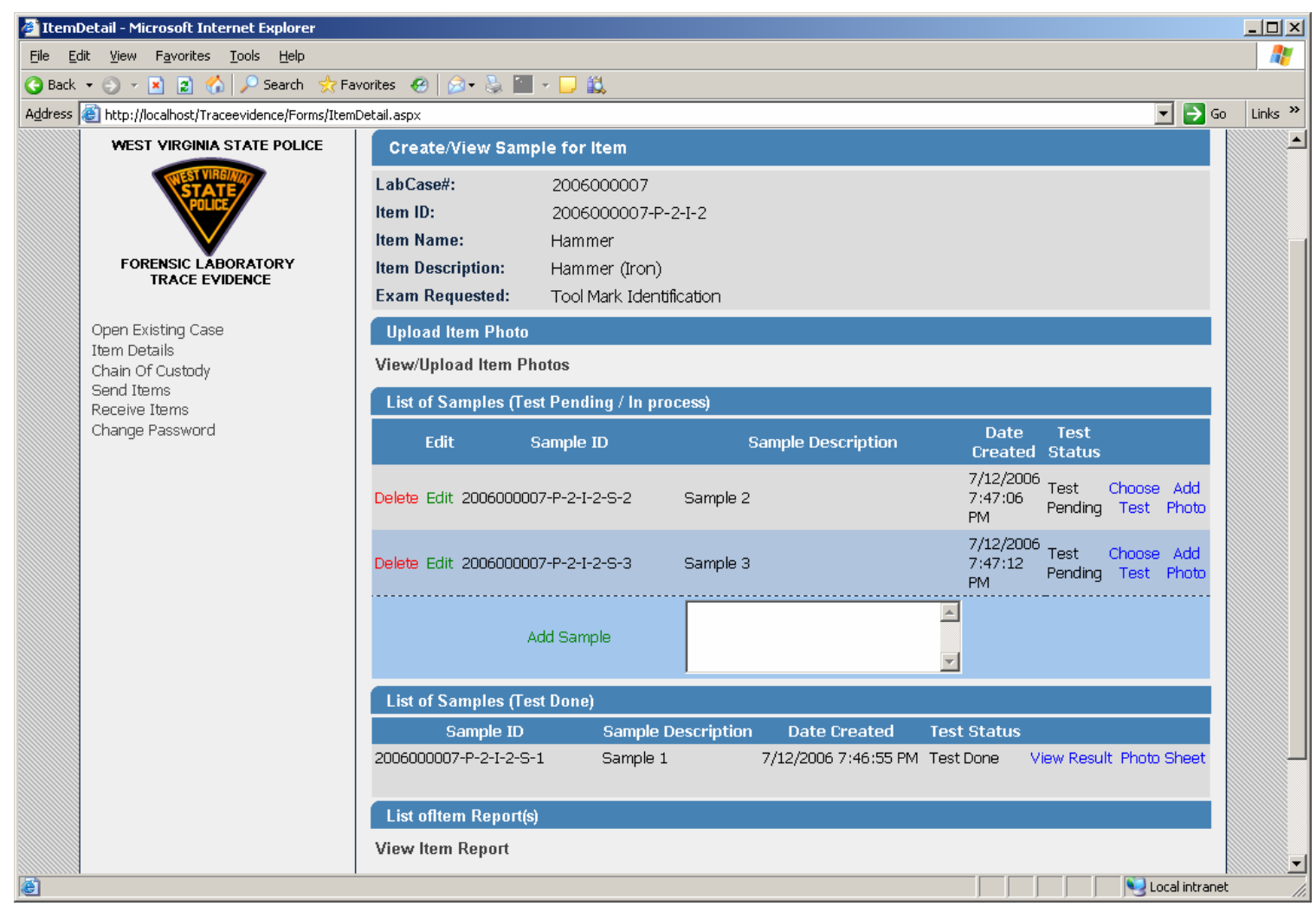

Figure 8.9: Item Detail Section 
Analysts can upload any digital photo in JPG format directly into the database using this section as shown in Figure 8.10 To upload an image analysts are required to browse for the image path, set other required fields and click on the "Upload the file" hyperlink to upload the image into database. On successful upload a thumbnail appears on the bottom of the page with a success message.

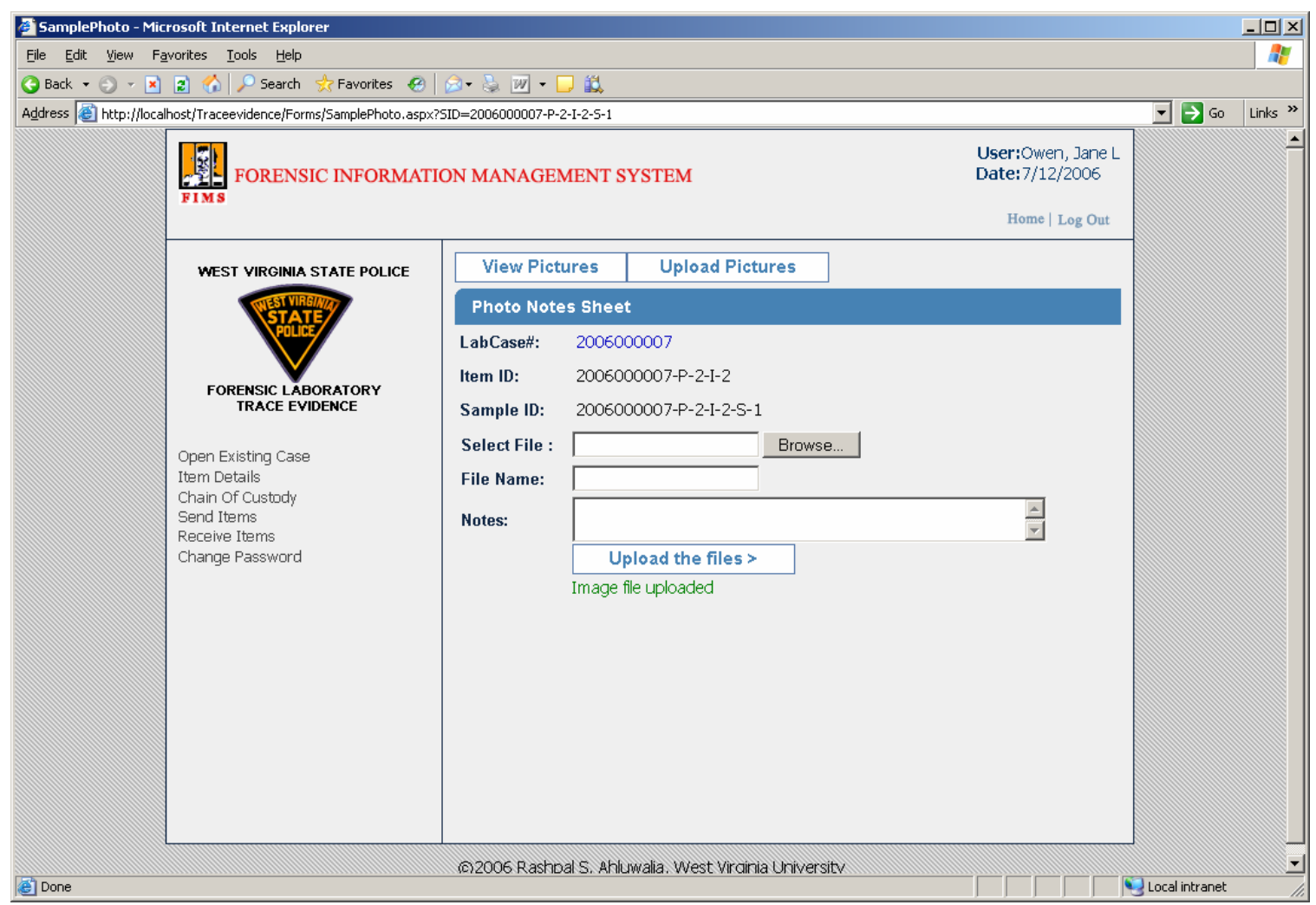

Figure 8.10: Upload Sample Picture Section 


\section{Choosing Test Worksheet}

After sample creation, analysts are required to decide what kinds of tests are required? Analysts are required to make decision of choosing type of test manually. Once the decision is made the analyst can see the list of tests available, and then click "Choose Test" hyperlink as shown in Figure 8.11. FIMS application remembers the chosen sample ID during the process.

The worksheet for trace evidence unit are:

- Speedometer examination

- Glass comparison

- Gunshot residue analysis

- Ignitable liquid

- Lamp examination

- Miscellaneous comparison

- Paint comparison and analysis

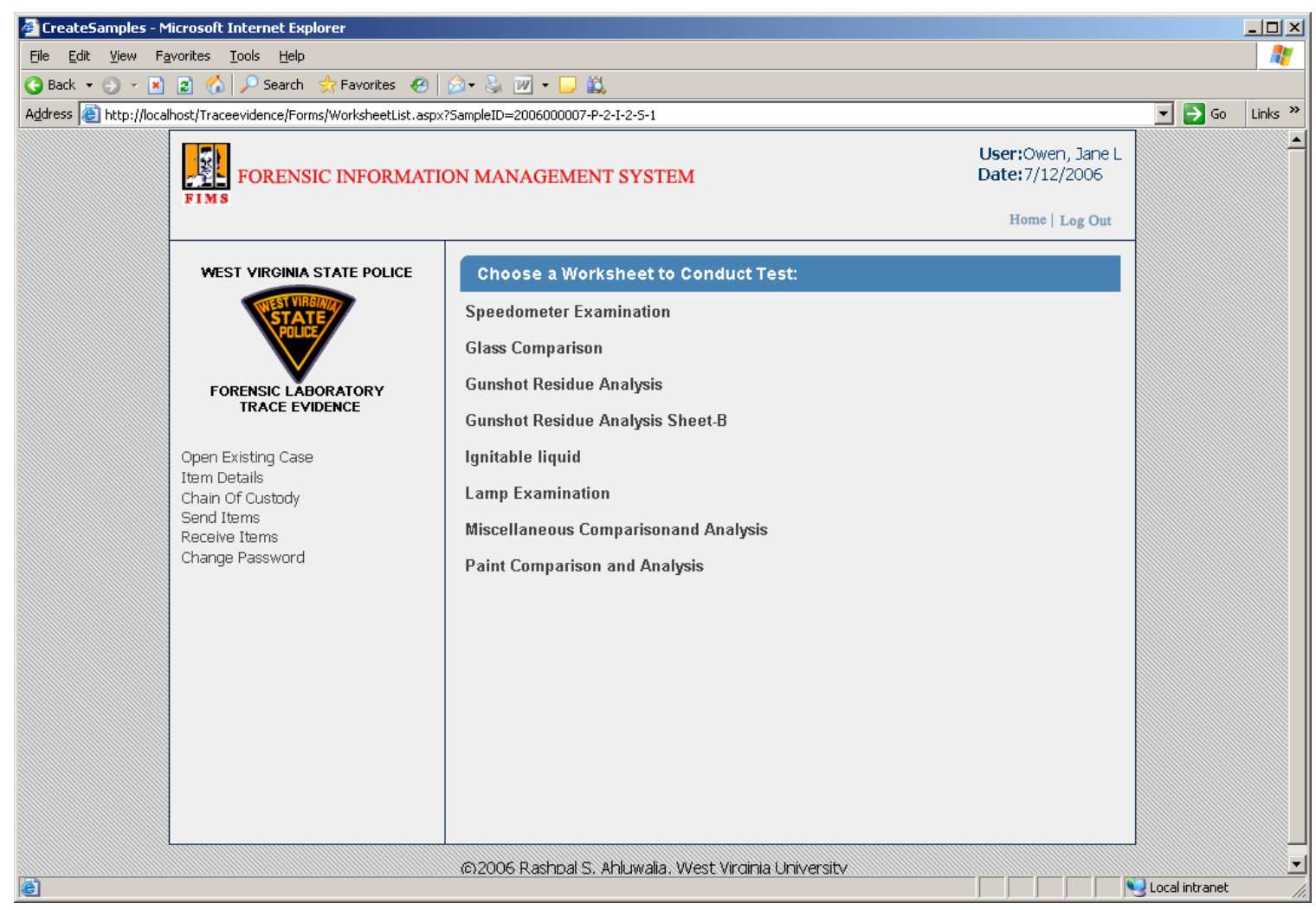

Figure 8.11: Choose Sample Test Section 


\section{Speedometer Examination Worksheet}

Speedometer Examination worksheet is used for filling information pertaining to speedometer examination test performed on the sample by the analyst. After filling the required data fields, analyst is required to click on "Save Results" button located on top of the page, as shown in Figure 8.12. After saving the worksheet data, analyst can finalize and close the worksheet by clicking on “Test Done” button. The analyst cannot make any alterations in the worksheet once the "Test Done" button is clicked.

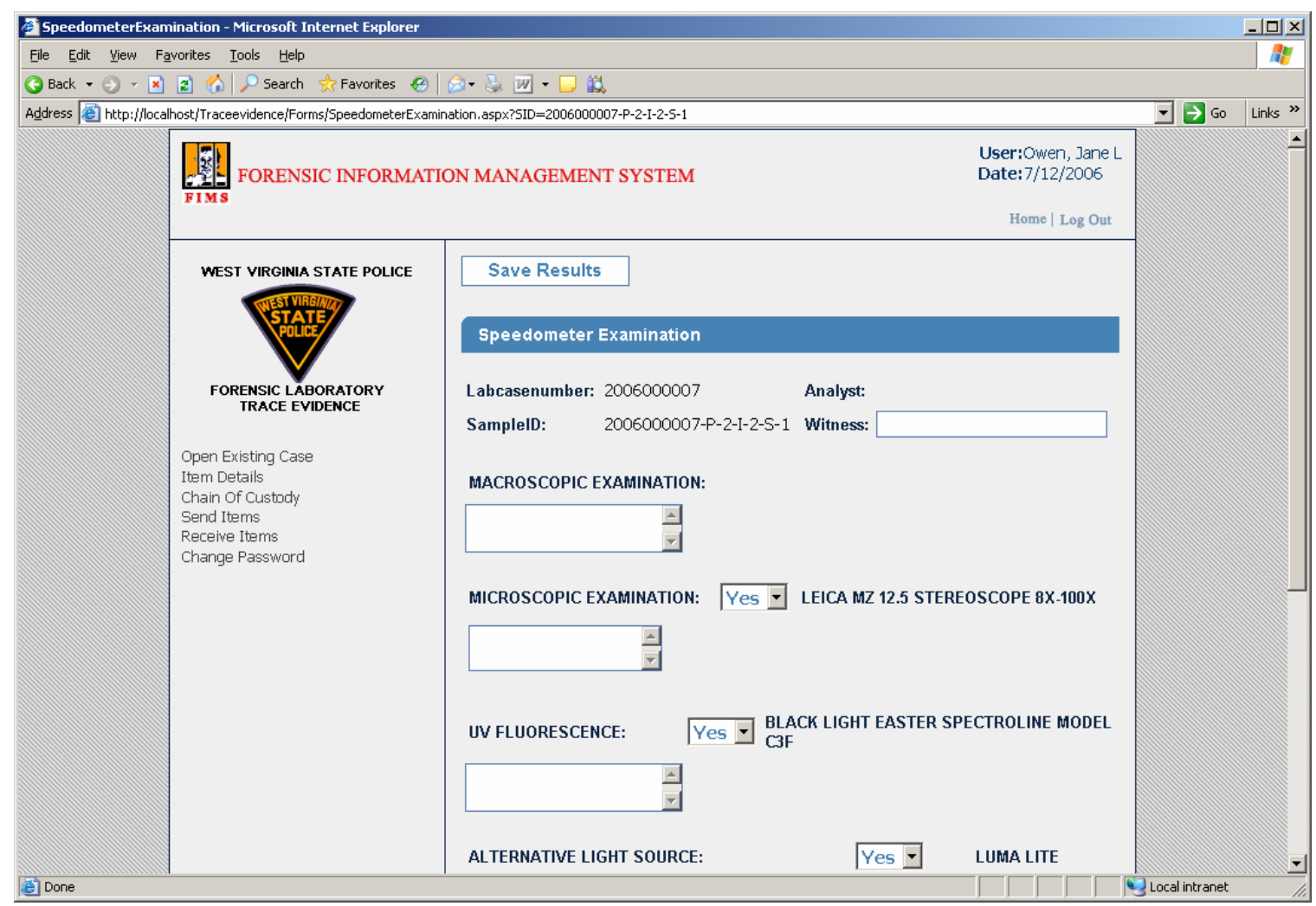

Figure 8.12: Speedometer Examination worksheet 


\section{Glass Comparison Worksheet}

Glass Comparison worksheet is used for filling information pertaining to Glass Comparison test performed on the sample by the analyst. After filling the required data fields, analyst is required to click on "Save Results" button located on top of the page, as shown in Figure 8.13. After saving the worksheet data, analyst can finalize and close the worksheet by clicking on "Test Done” button. The analyst cannot make any alterations in the worksheet once the "Test Done" button is clicked.

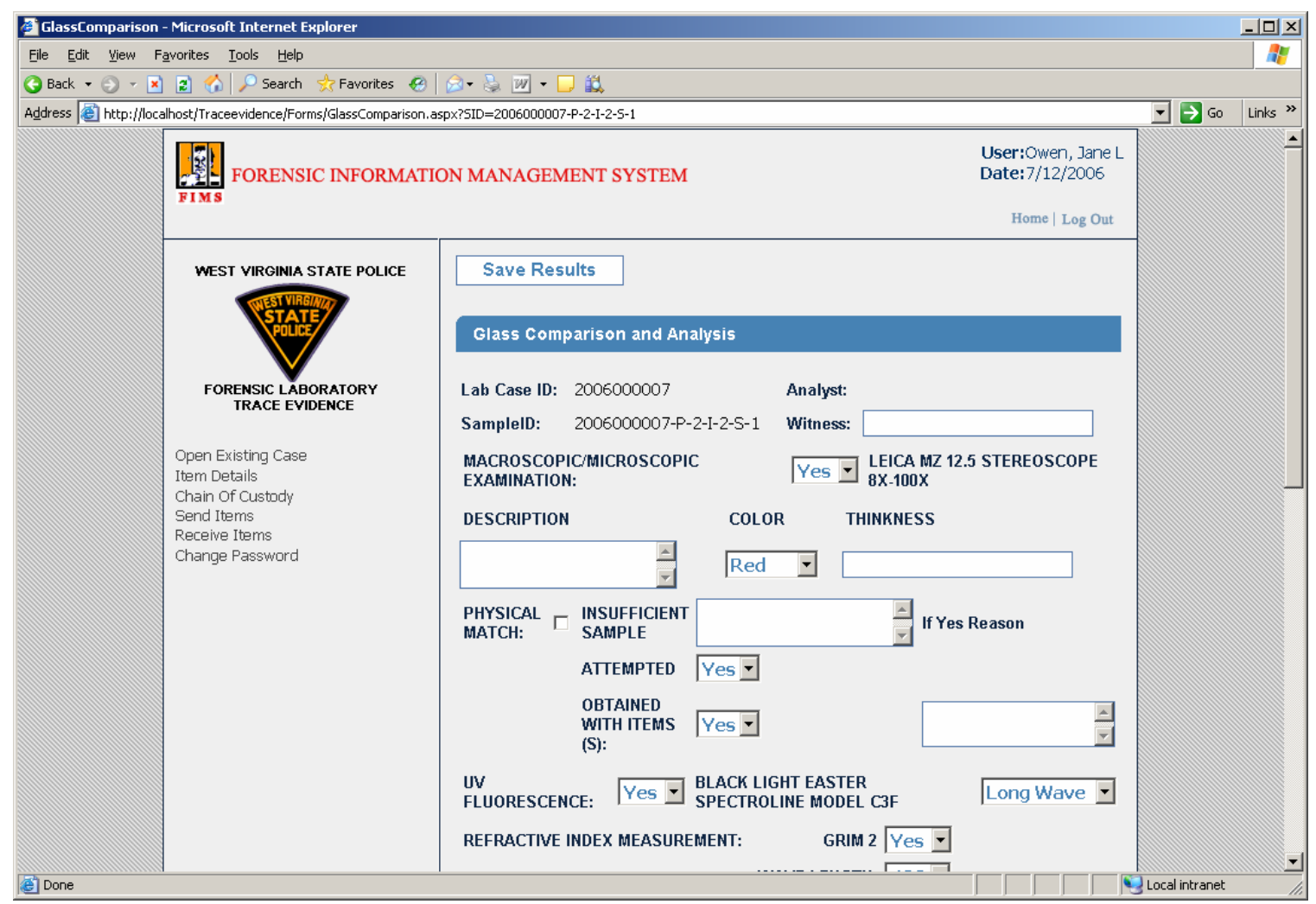

Figure 8.13: Glass Comparison Worksheet 


\section{Gun Shot Residue Worksheet}

Gun Shot Residue worksheet is used for filling information pertaining to gun shot residue test performed on the sample by the analyst. After filling the required data fields, analyst is required to click on "Save Results" button located on top of the page, as shown in Figure 8.14. After saving the worksheet data, analyst can finalize and close the worksheet by clicking on “Test Done” button. The analyst cannot make any alterations in the worksheet once the "Test Done” button is clicked.

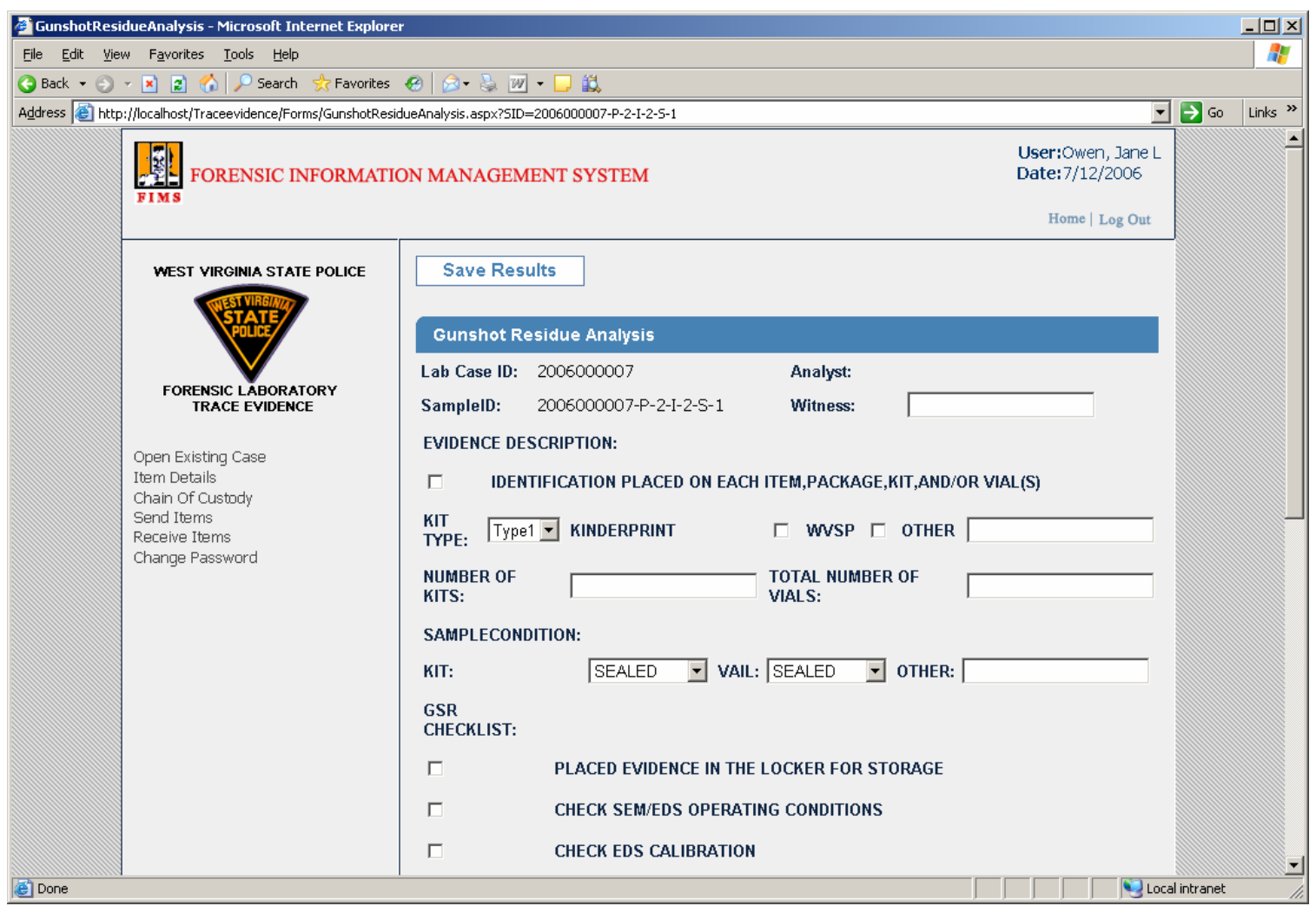

Figure 8.14: Gun Shot Residue Worksheet 


\section{Gun Shot Residue-B Worksheet}

Gun Shot Residue-B worksheet is used for filling information pertaining to gun shot residue-B test performed on the sample by the analyst. After filling the required data fields, analyst is required to click on "Save Results" button located on top of the page, as shown in Figure 8.15. After saving the worksheet data, analyst can finalize and close the worksheet by clicking on "Test Done" button. The analyst cannot make any alterations in the worksheet once the "Test Done" button is clicked.

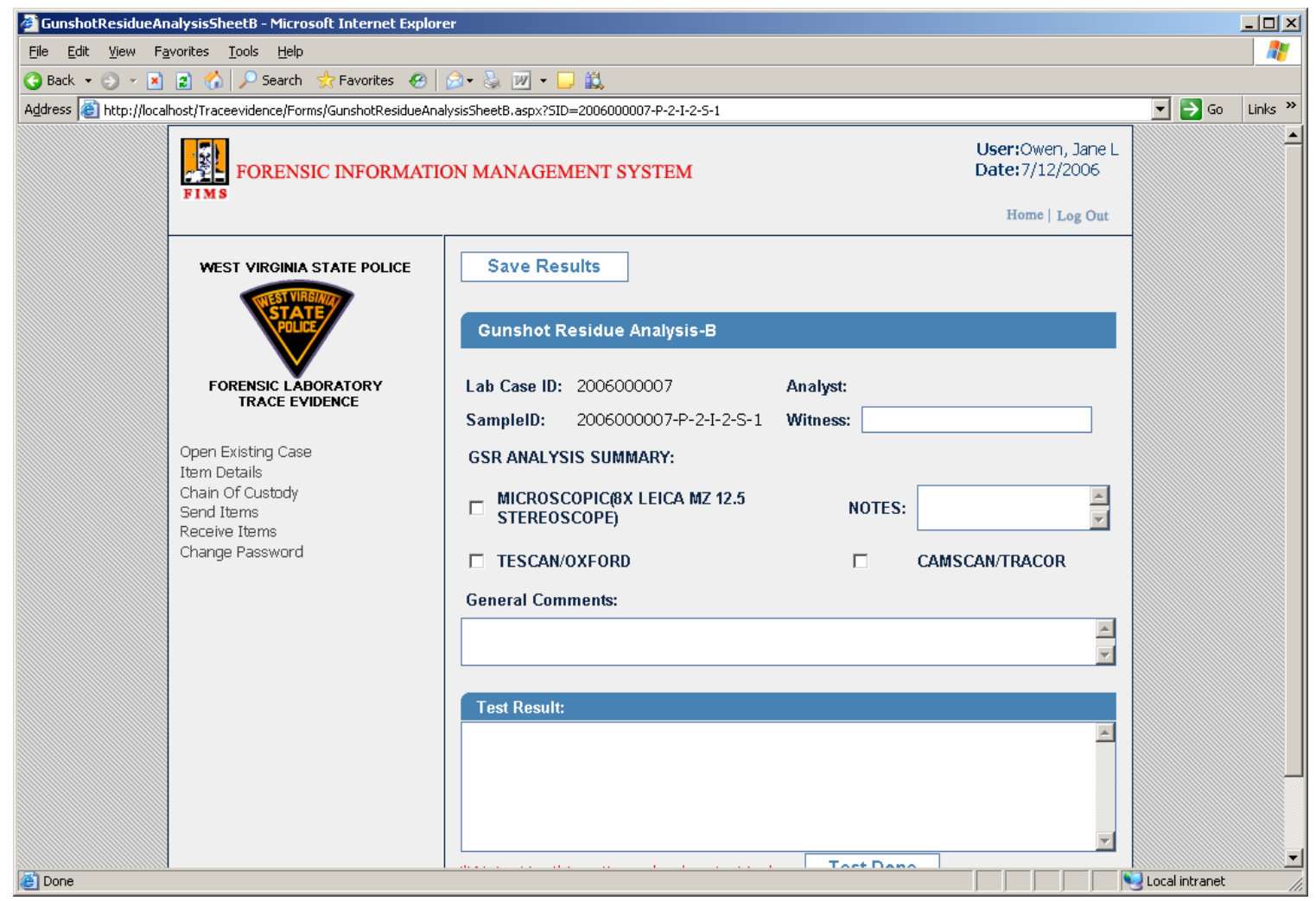

Figure 8.15: Gun Shot Residue-B Worksheet 


\section{Ignitable Liquid Worksheet}

Ignitable liquid worksheet is used for filling information pertaining to Ignitable liquid test performed on the sample by the analyst. After filling the required data fields, analyst is required to click on "Save Results" button located on top of the page, as shown in Figure 8.16. After saving the worksheet data, analyst can finalize and close the worksheet by clicking on “Test Done” button. The analyst cannot make any alterations in the worksheet once the "Test Done” button is clicked.

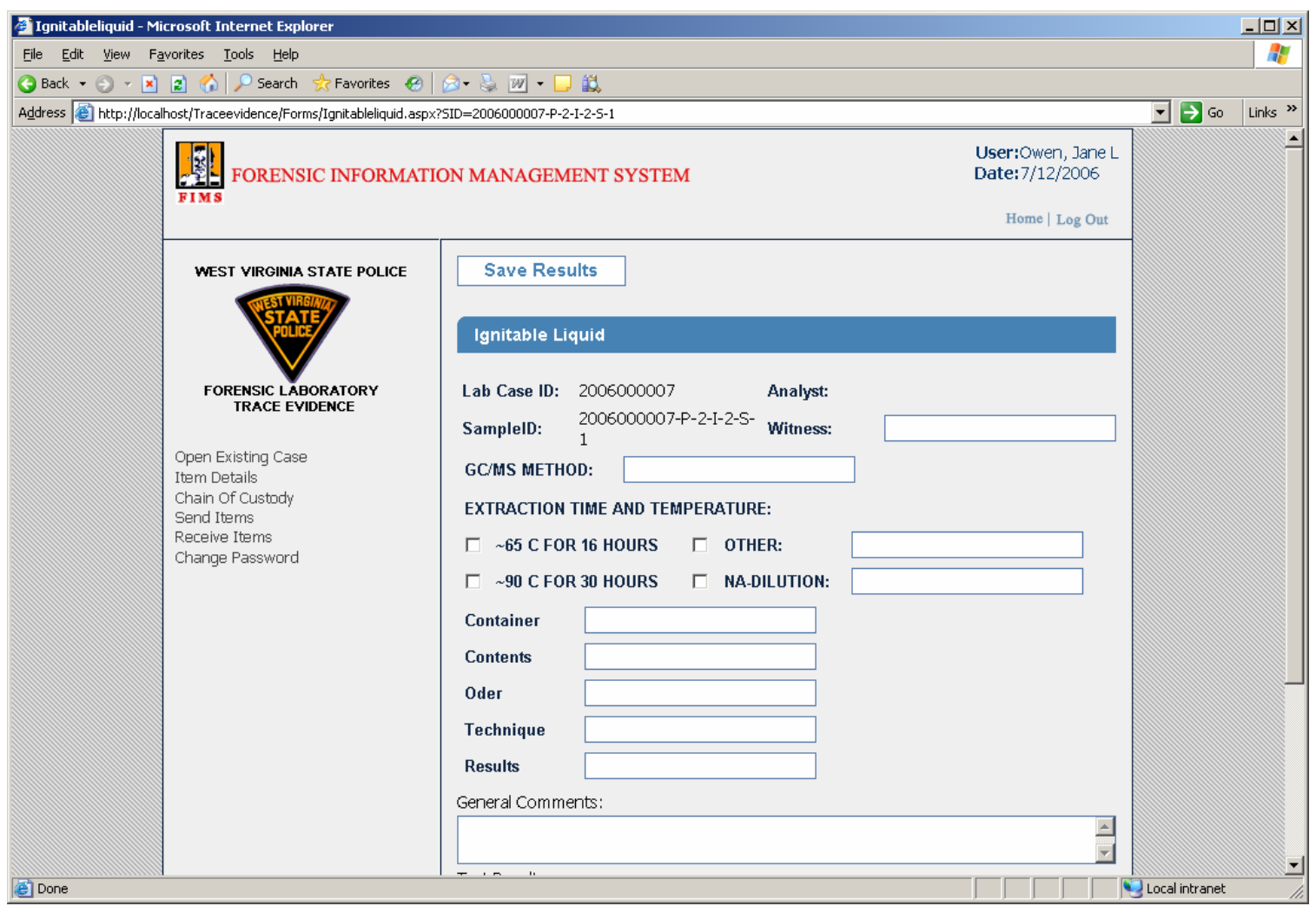

Figure 8.16: Ignitable Liquid Worksheet 


\section{Lamp Examination Worksheet}

Lamp examination worksheet is used for filling information pertaining to Lamp examination test performed on the sample by the analyst. After filling the required data fields, analyst is required to click on "Save Results" button located on top of the page, as shown in Figure 8.17. After saving the worksheet data, analyst can finalize and close the worksheet by clicking on "Test Done" button. The analyst cannot make any alterations in the worksheet once the "Test Done" button is clicked.

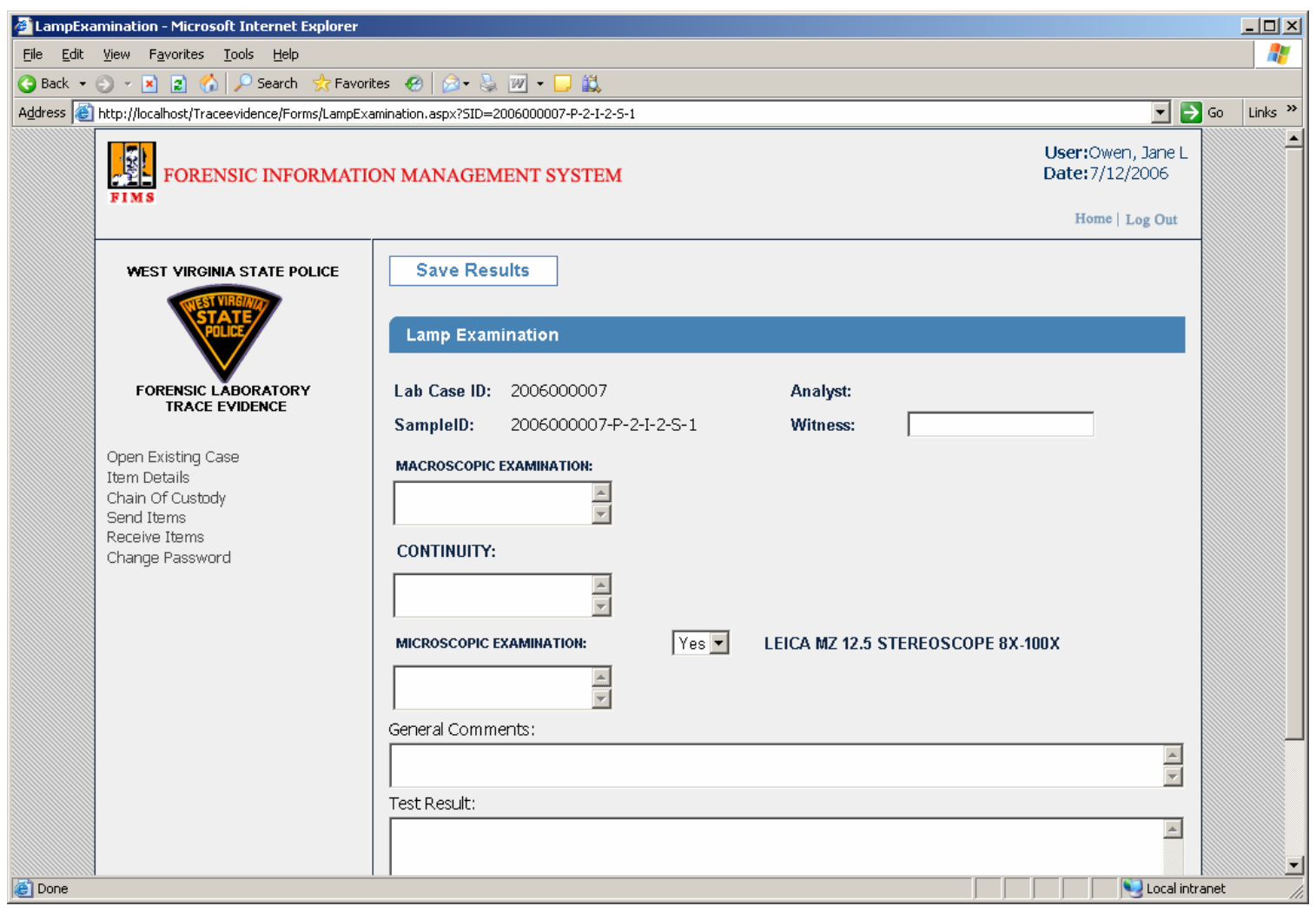

Figure 8.17: Lamp Examination worksheet 


\section{Miscellaneous Comparison Worksheet}

Miscellaneous comparison worksheet is used for filling information pertaining to miscellaneous comparison test performed on the sample by the analyst. After filling the required data fields, analyst is required to click on "Save Results" button located on top of the page, as shown in Figure 8.18. After saving the worksheet data, analyst can finalize and close the worksheet by clicking on “Test Done” button. The analyst cannot make any alterations in the worksheet once the "Test Done" button is clicked.

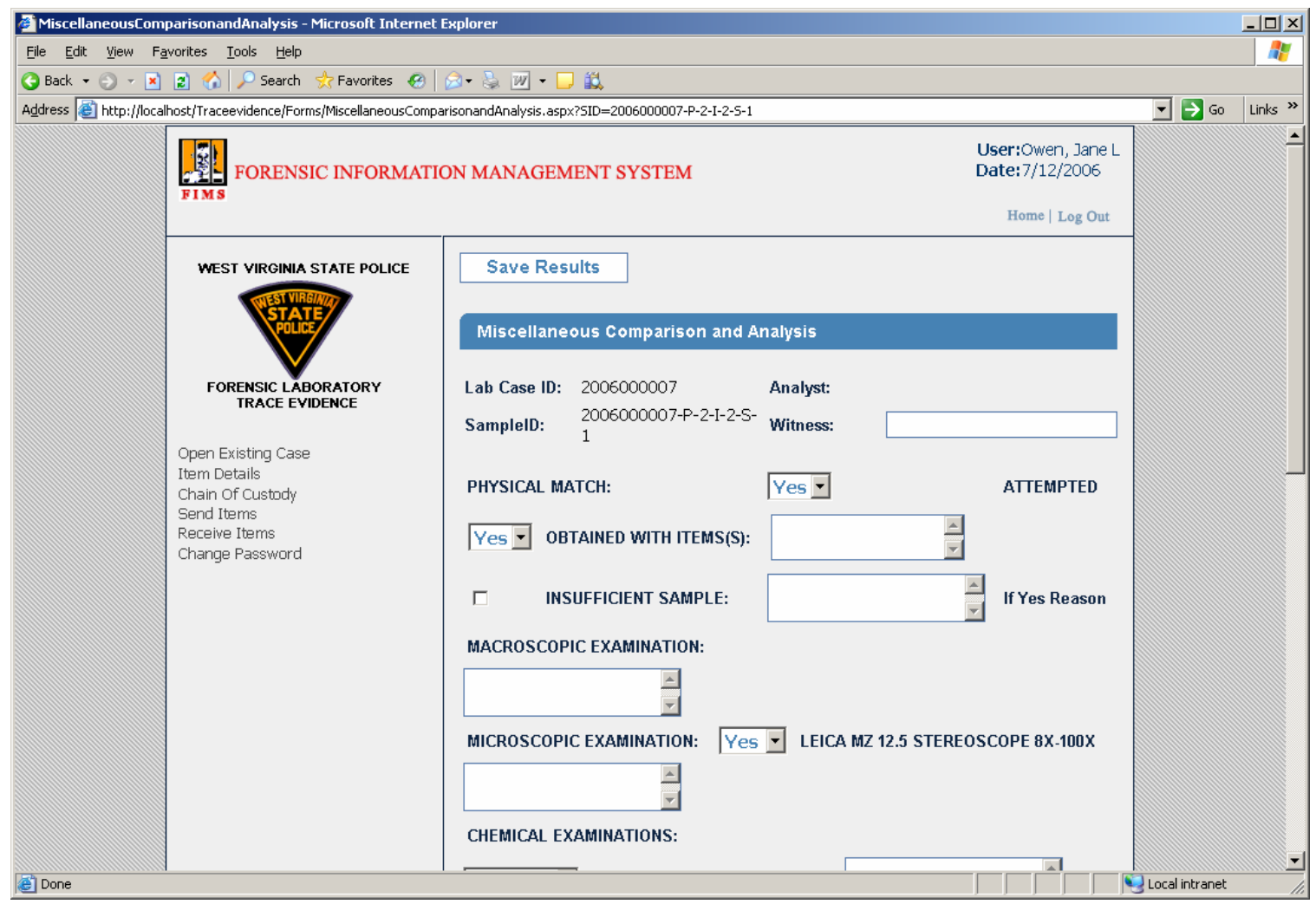

Figure 8.18: Miscellaneous Comparison Worksheet 


\section{Paint Comparison and Analysis Worksheet}

Paint comparison and analysis worksheet is used for filling information pertaining to paint comparison and analysis test performed on the sample by the analyst. After filling the required data fields, analyst is required to click on "Save Results" button located on top of the page, as shown in Figure 8.19. After saving the worksheet data, analyst can finalize and close the worksheet by clicking on "Test Done" button. The analyst cannot make any alterations in the worksheet once the "Test Done" button is clicked.

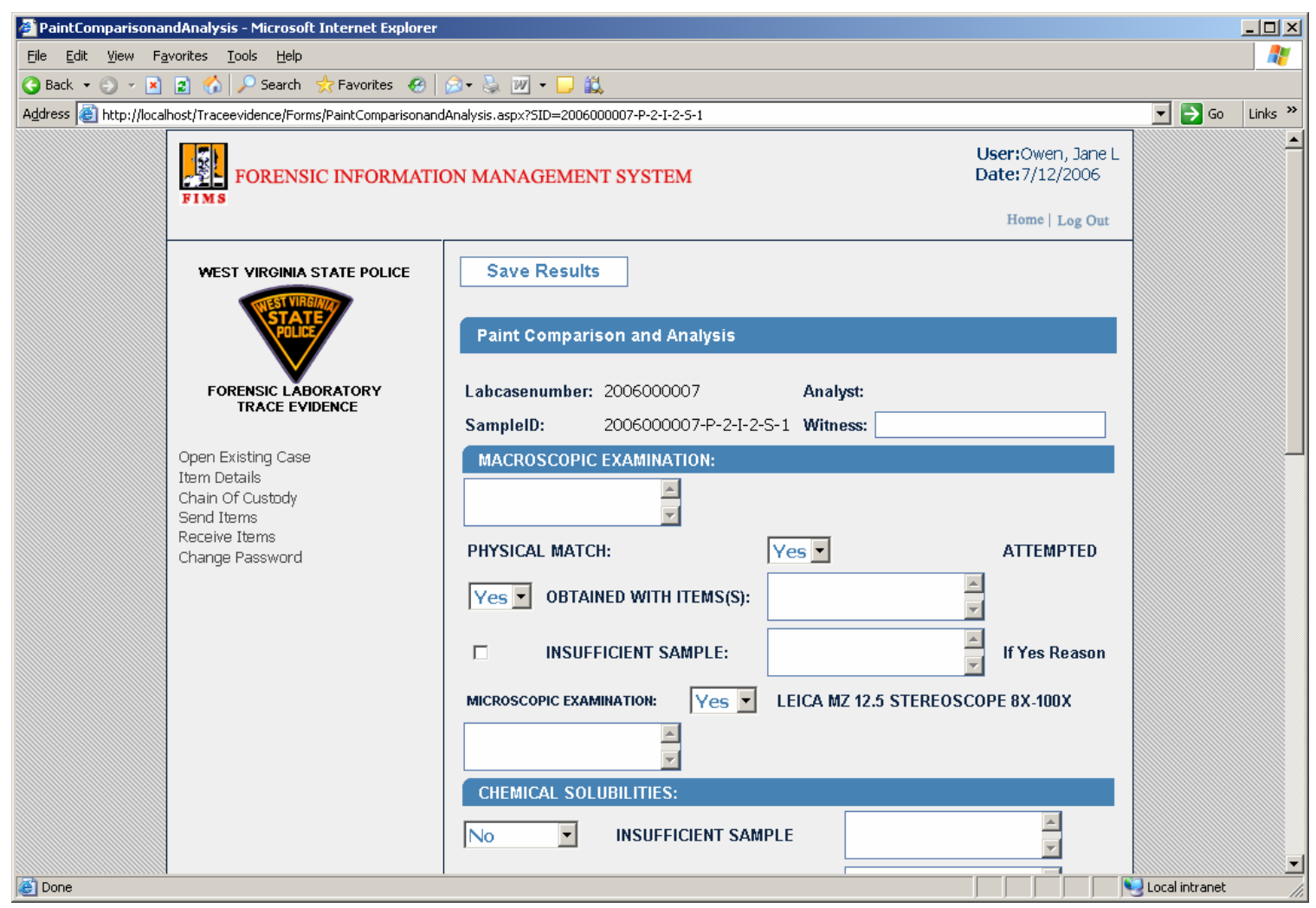

Figure 8.19: Paint comparison and analysis worksheet 


\section{Receive Item}

Once an item is sent by CEP unit, Trace Evidence unit analyst is responsible for receiving it by clicking on "Receive" button, under receive item section, as shown in Figure 8.20. This completes the handshaking process of item transfer between units.

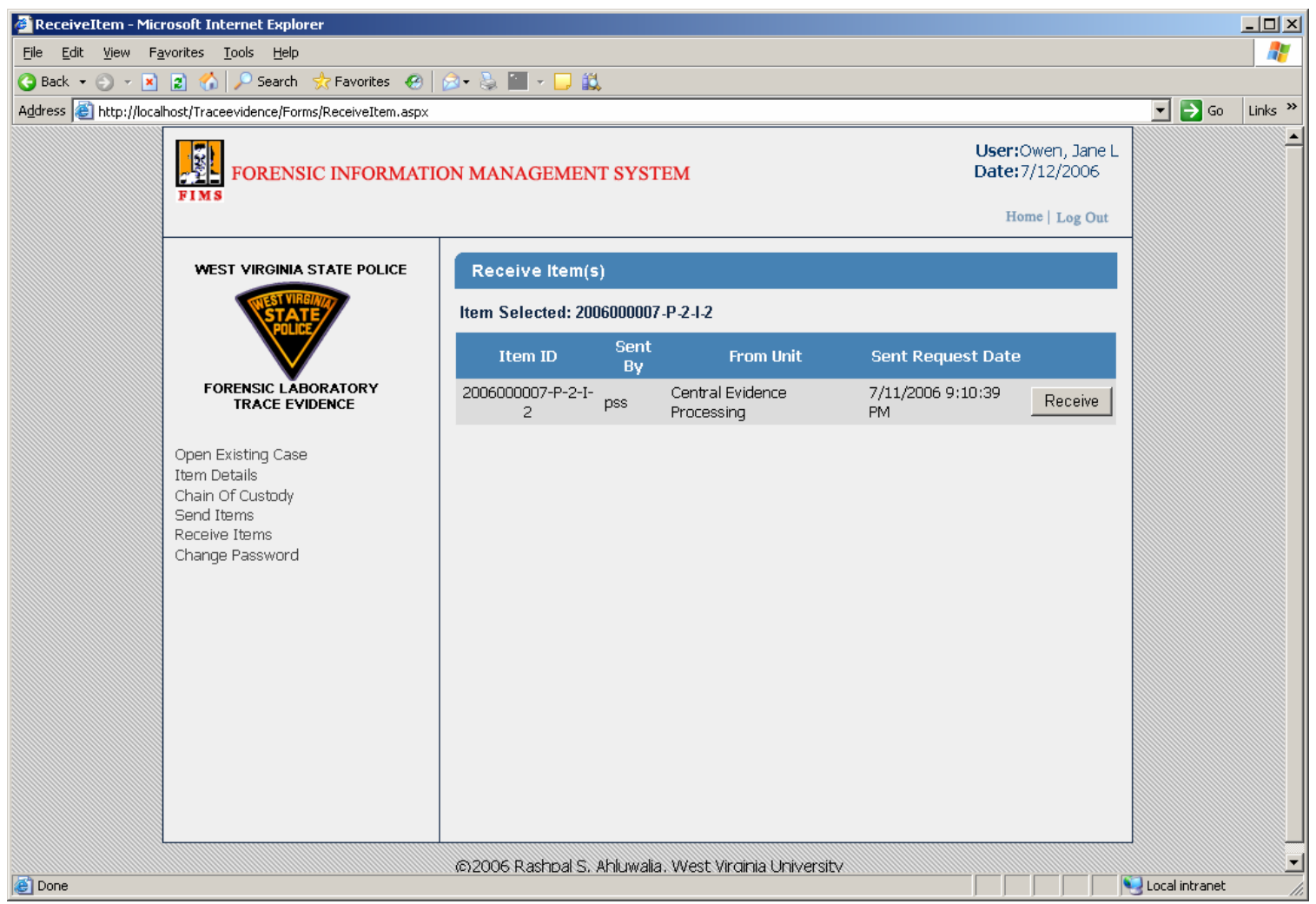

Figure 8.20: Receive Item Section 


\section{Send Item}

After completion of item testing, analyst is required to return the evidence item back to CEP unit. Figure 8.21 shows the section used to send an item back to the CEP unit. Analyst can use the drop down to select item ID and click on "Send" hyperlink, to send item back to CEP.

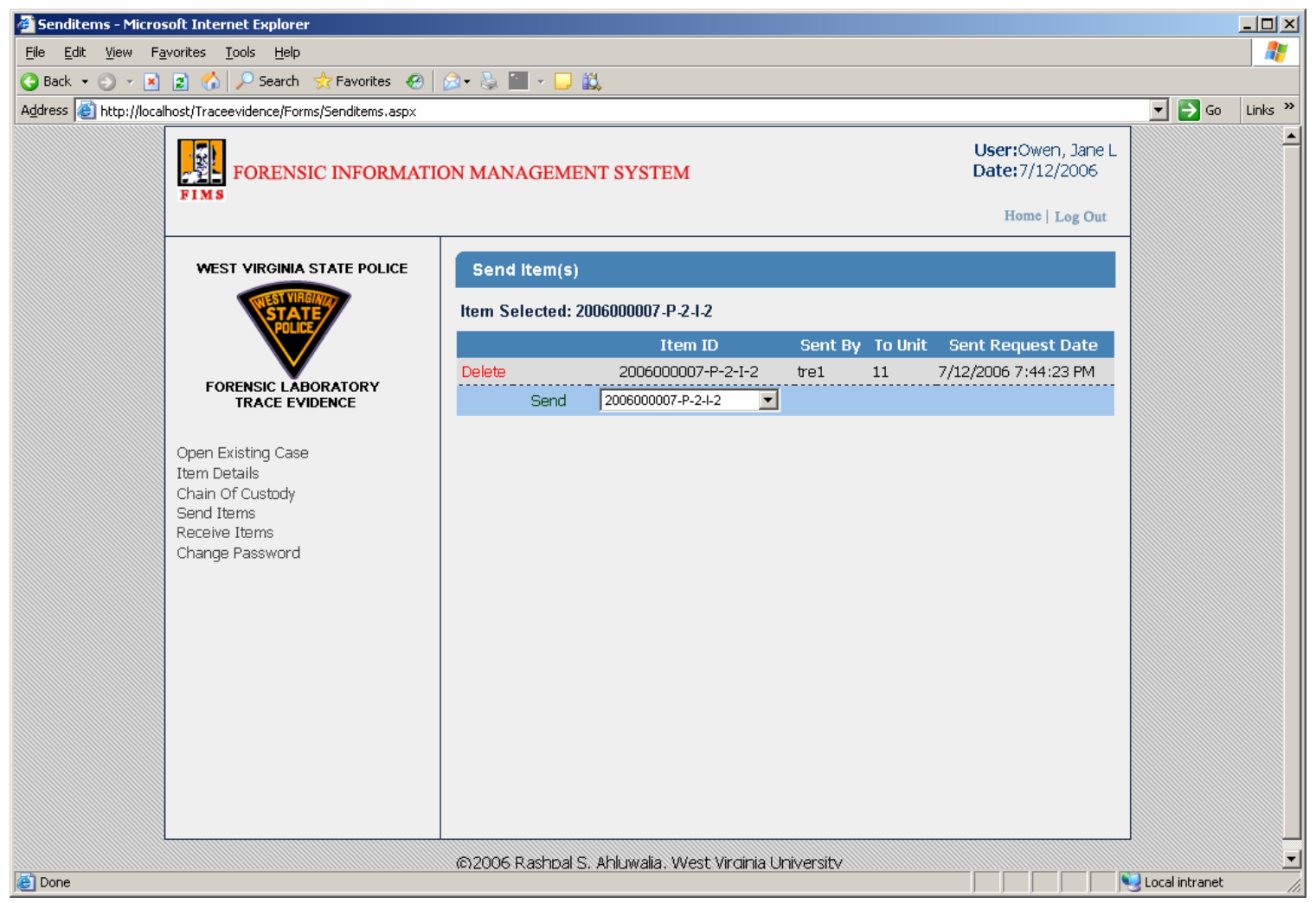

Figure 8.21: Send Item Section 


\section{Chain of Custody}

Item chain of custody information is shown in Figure 8.22. FIMS application records used ID, unit name, and time stamp for tracking item chain of custody.

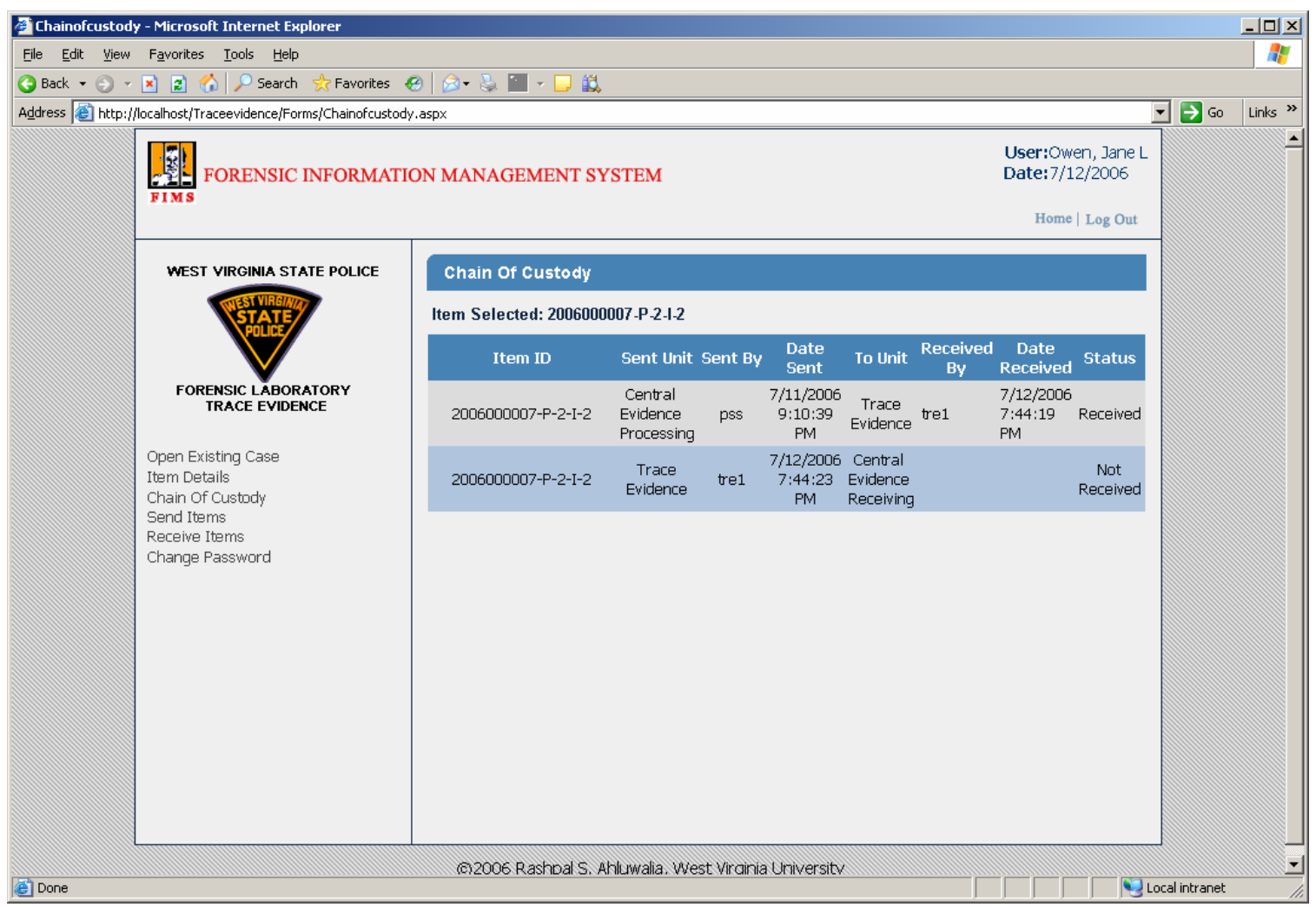

Figure 8.22: Item Chain Of Custody 


\section{Change User Password}

Analyst can change password through this section by providing appropriate credentials as shown in Figure 8.23.

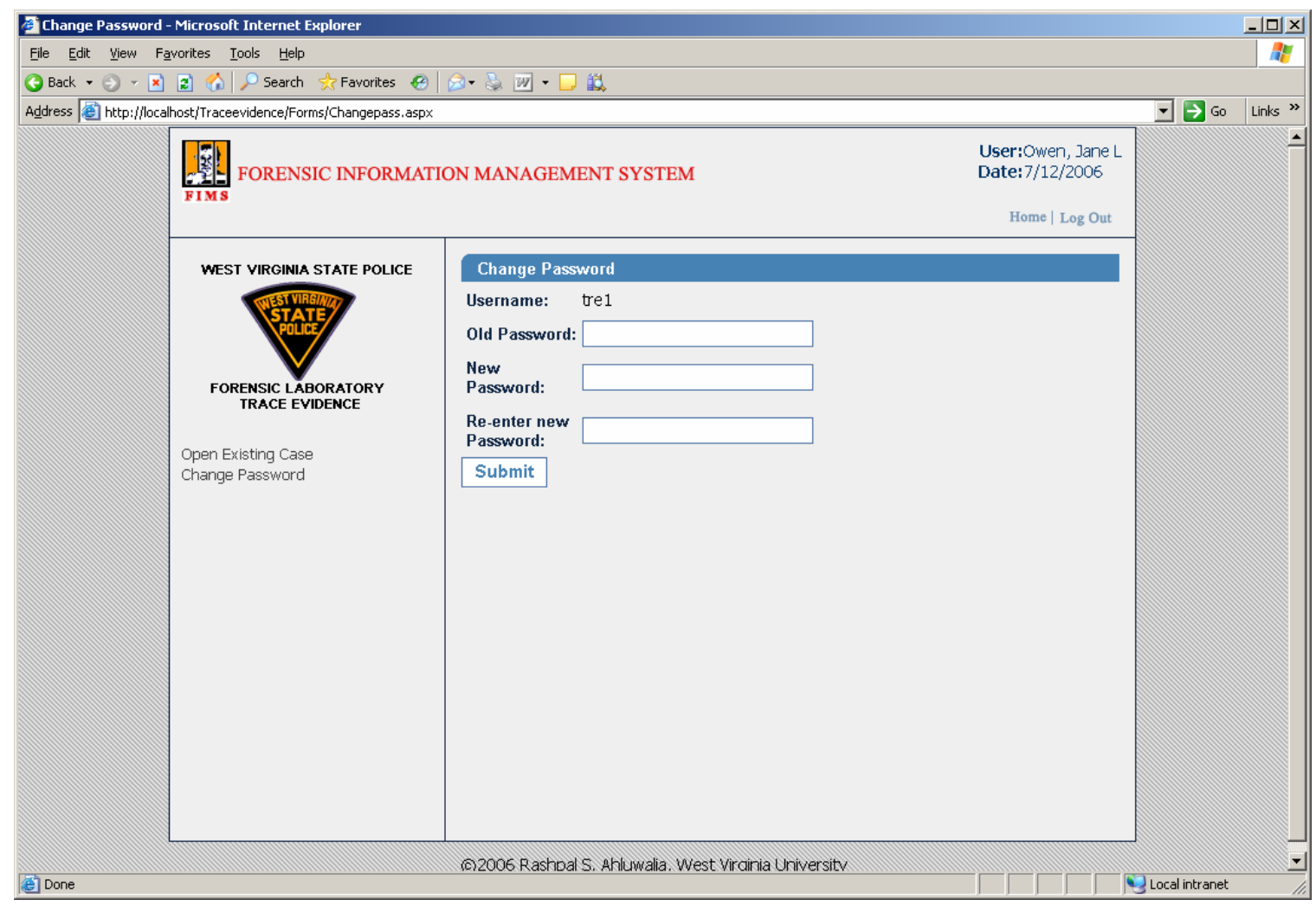

Figure 8.23: Change password section

\section{Exiting Trace Evidence}

Users can terminate the current session by clicking on "Log Out" hyperlink on the top right corner of the page. 


\subsubsection{Generating Item and Sample Reports}

The analyst can generate the following types of reports

- Item Report

- Item Photo Report

- Sample Report

- Sample Photo Report

Figure 8.24 shows the item report, which is a cumulative report for all the samples. This section also facilitate analyst to export report via PDF/MS-Word format as shown in Figure 8.26 and Figure 8.27.

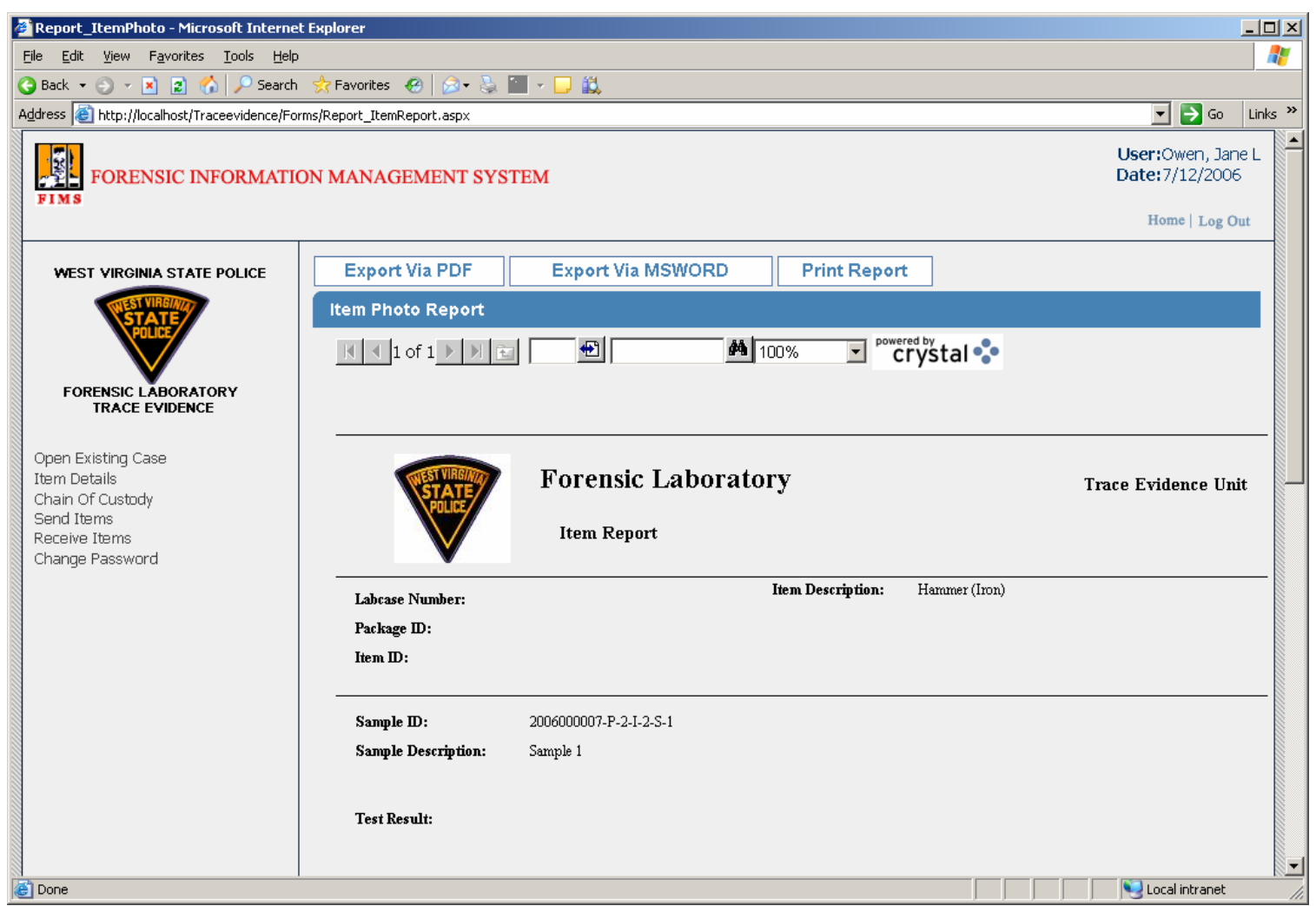

Figure 8.24: Item report 
Figure 8.25 shows the sample report. This section also facilitate analyst to photo report, as shown in Figure 8.26.

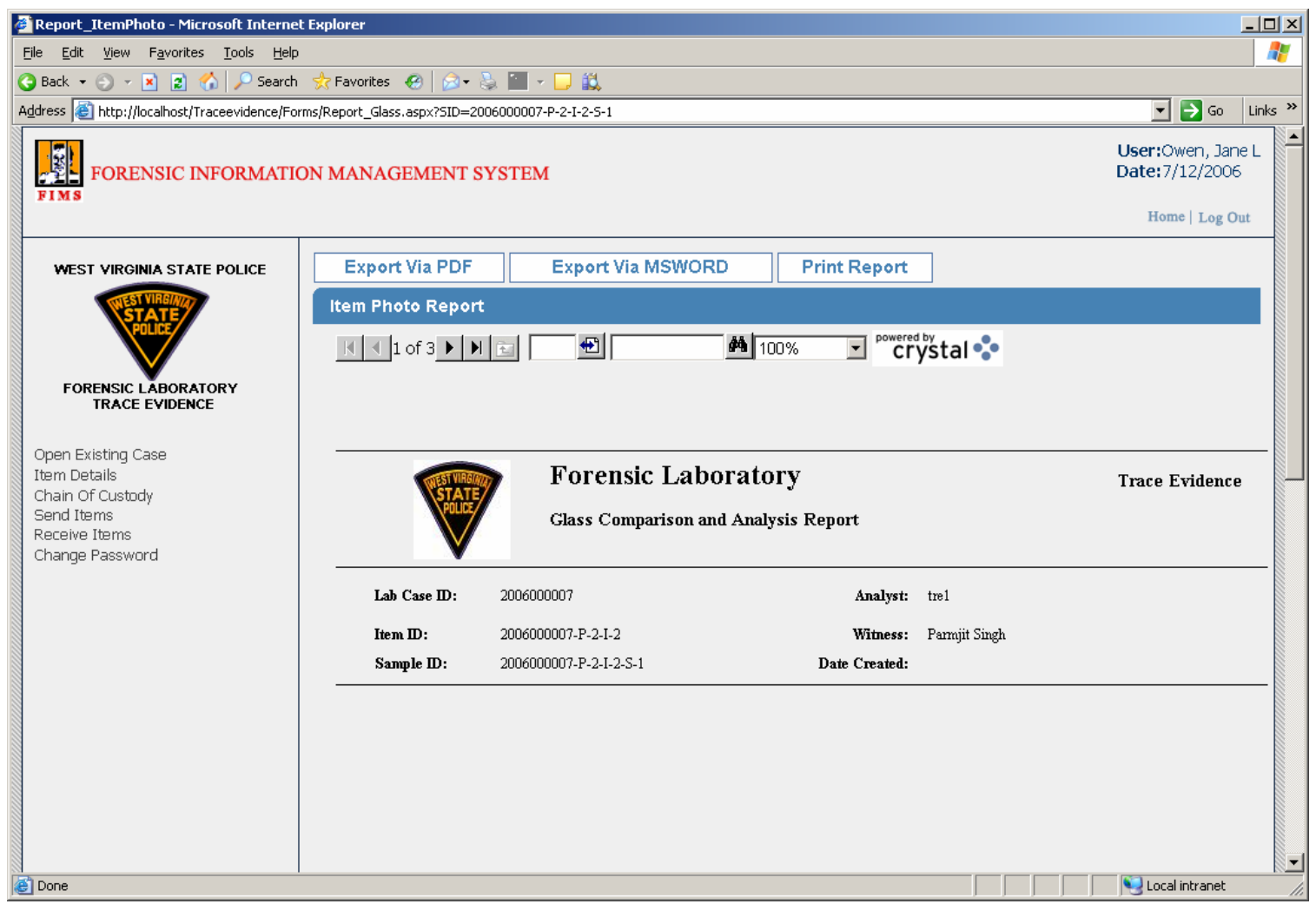

Figure 8.25: Sample Report 


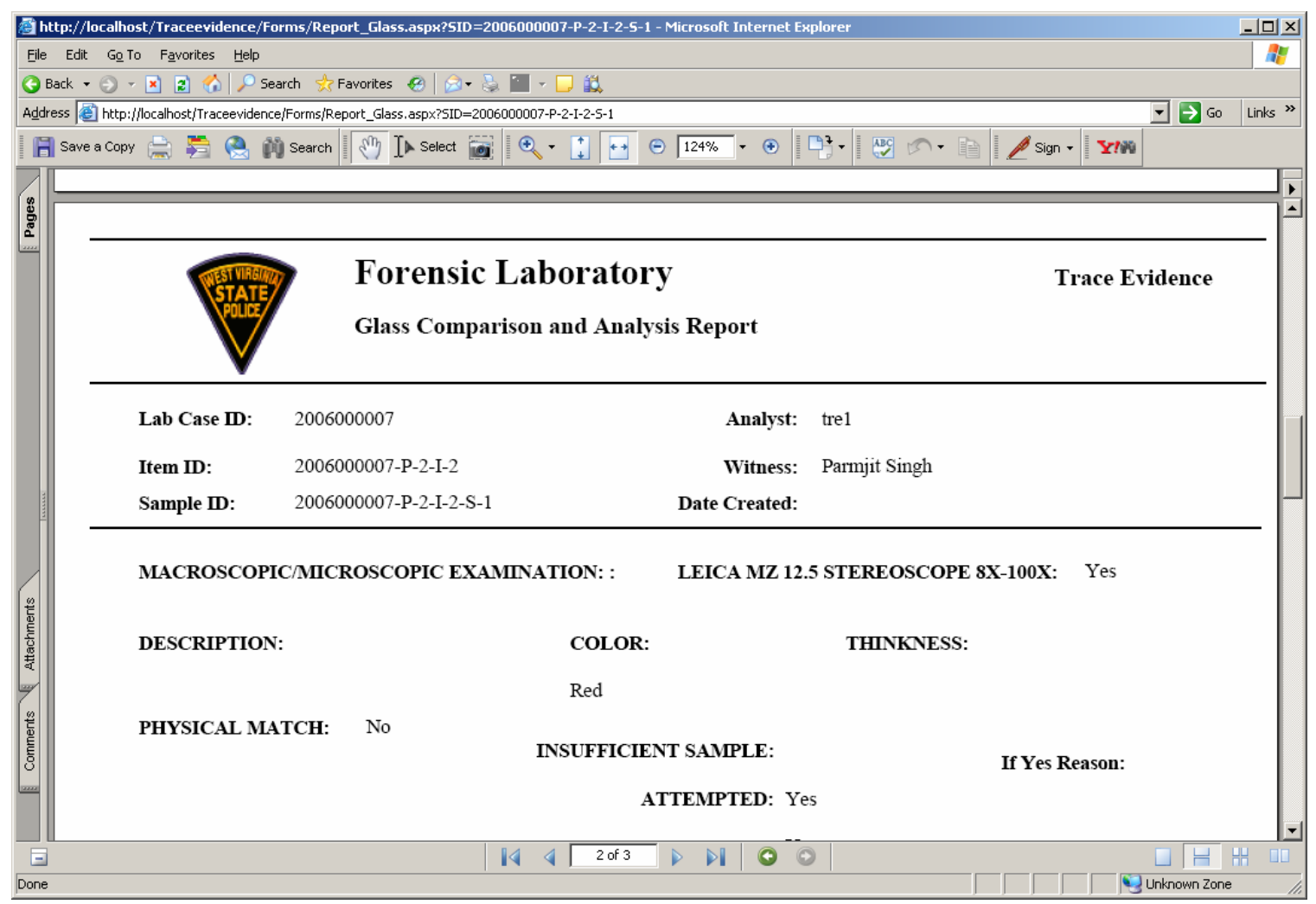

Figure 8.26: Item Report in PDF Format

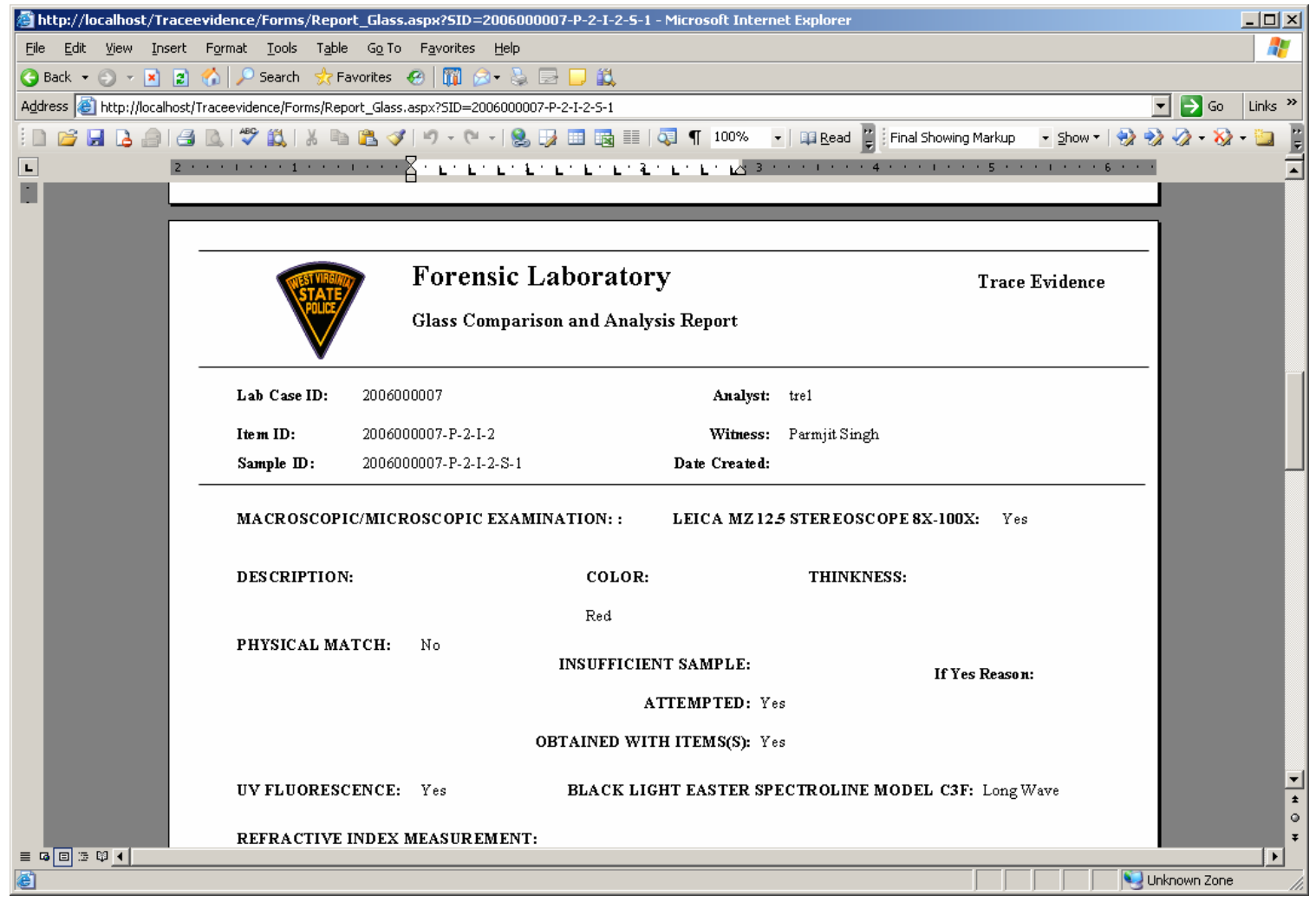

Figure 8.27: Item Report in MS Word Format 


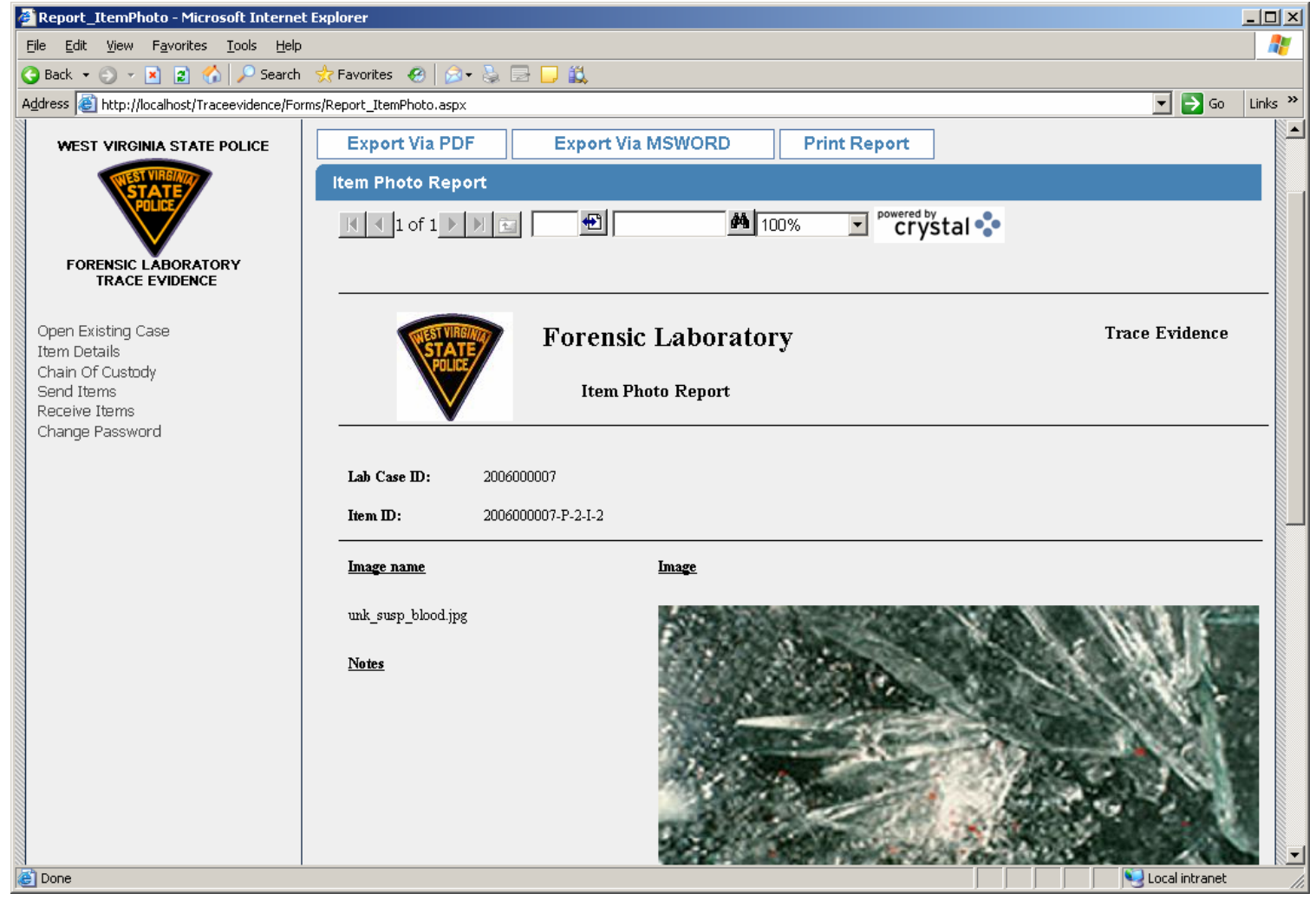

Figure 8.28: Item Photo Report 


\section{CHAPTER 9 TOXICOLOGY}

\subsection{Existing Toxicology Process}

The Toxicology Unit performs alcohol and drug analysis on blood and urine samples taken from subjects believed to be driving under the influence. The samples submitted to the lab are analyzed using gas chromatography for alcohol and gas chromatography/mass spectrometry for drugs. The toxicology unit performs drug screens on blood or urine specimens at the request of the arresting officer or at any time the blood alcohol level is below $0.10 \%$ blood alcohol by weight. The duties performed by the toxicology unit can be broken down into three primary areas: (1) Breath Alcohol, (2) Blood Alcohol, and (3) Drug Toxicology. The analyst in the toxicology unit receives the LEIF form and chain of custody form along with the evidence items. Samples are prepared from the items received from the storage. The analyst then performs the required tests and completes the appropriate forms. The forms/worksheets used by the toxicology unit are: Drug Analysis (DA), and Toxicology Worksheet (TW). Once the

examinations are performed, evidence is returned to the CER and the secretary prepares a draft report. The draft report is reviewed by the analyst/reviewer. After approval the final report is sent to CER. Figure 9.1 shows the existing process for the toxicology unit 


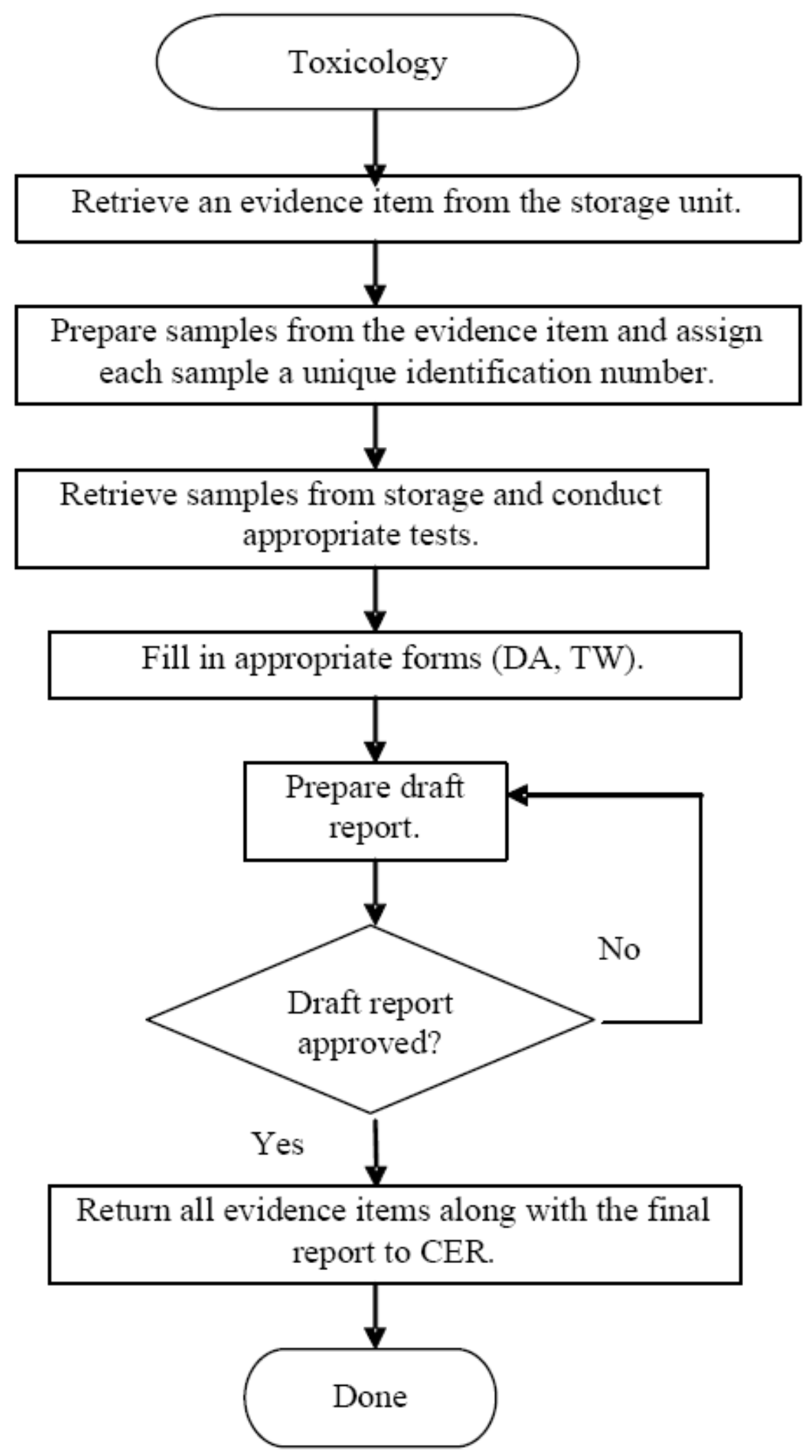

Figure 9.1: Existing Process for Toxicology Unit 


\subsection{Re-engineering the Toxicology Process}

The analyst in the toxicology unit receives the items assigned for further processing and analysis from CEP. Received items are sub-divided into samples for conducting the requested tests. An individual item may be divided into many samples according to the requirement and complexity of the required test. A report for each sample is generated. An item report is compiled by combining sample reports. After the report is received, the reports along with the test items are sent back to CEP for further processing. The evidence and information flow diagram is shown in Figure 9.2.

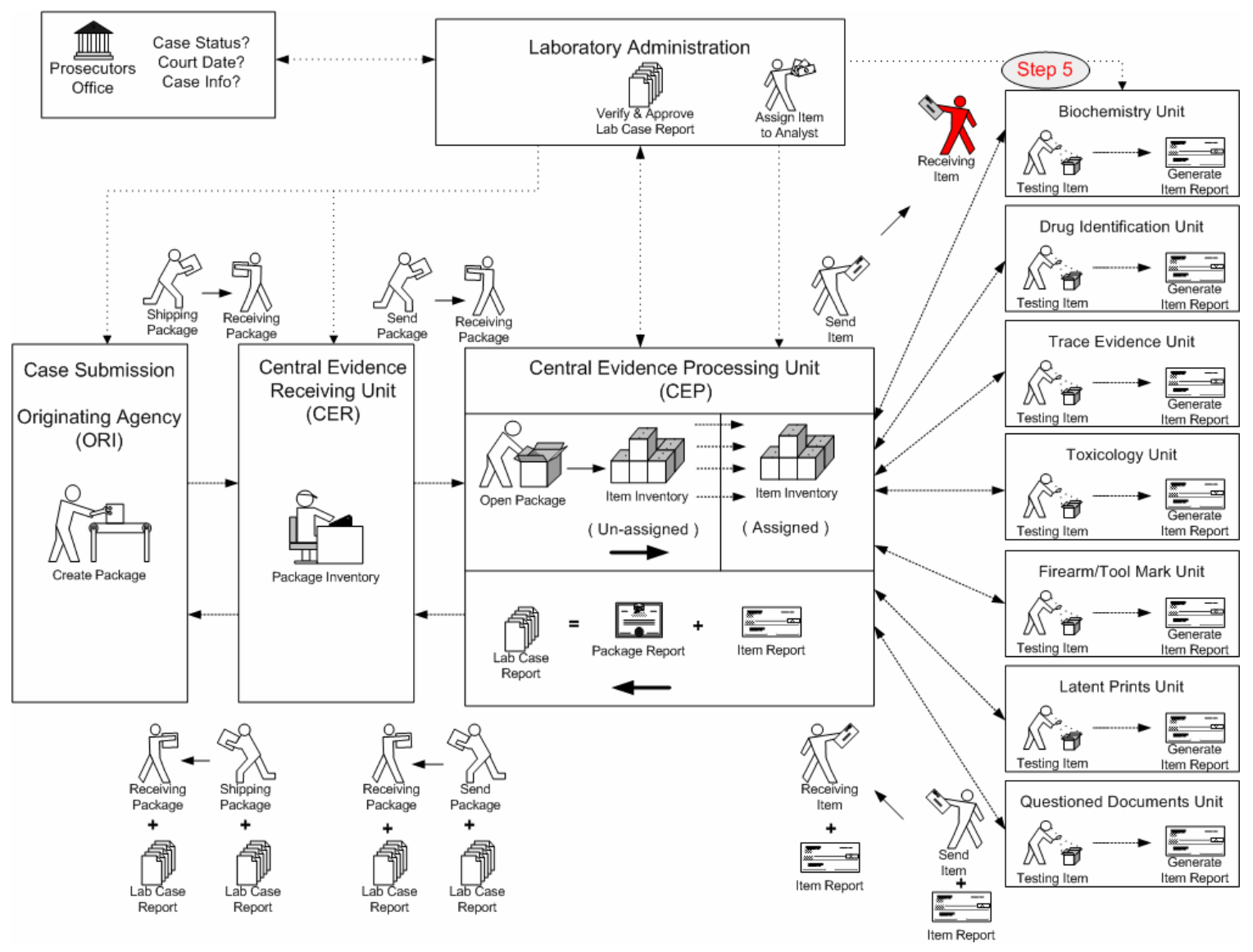

Figure 9.2: Step 5 Receive Assigned Items from CEP 
Detail procedure followed by toxicology unit for further processing and analysis is shown in Figure 9.3.

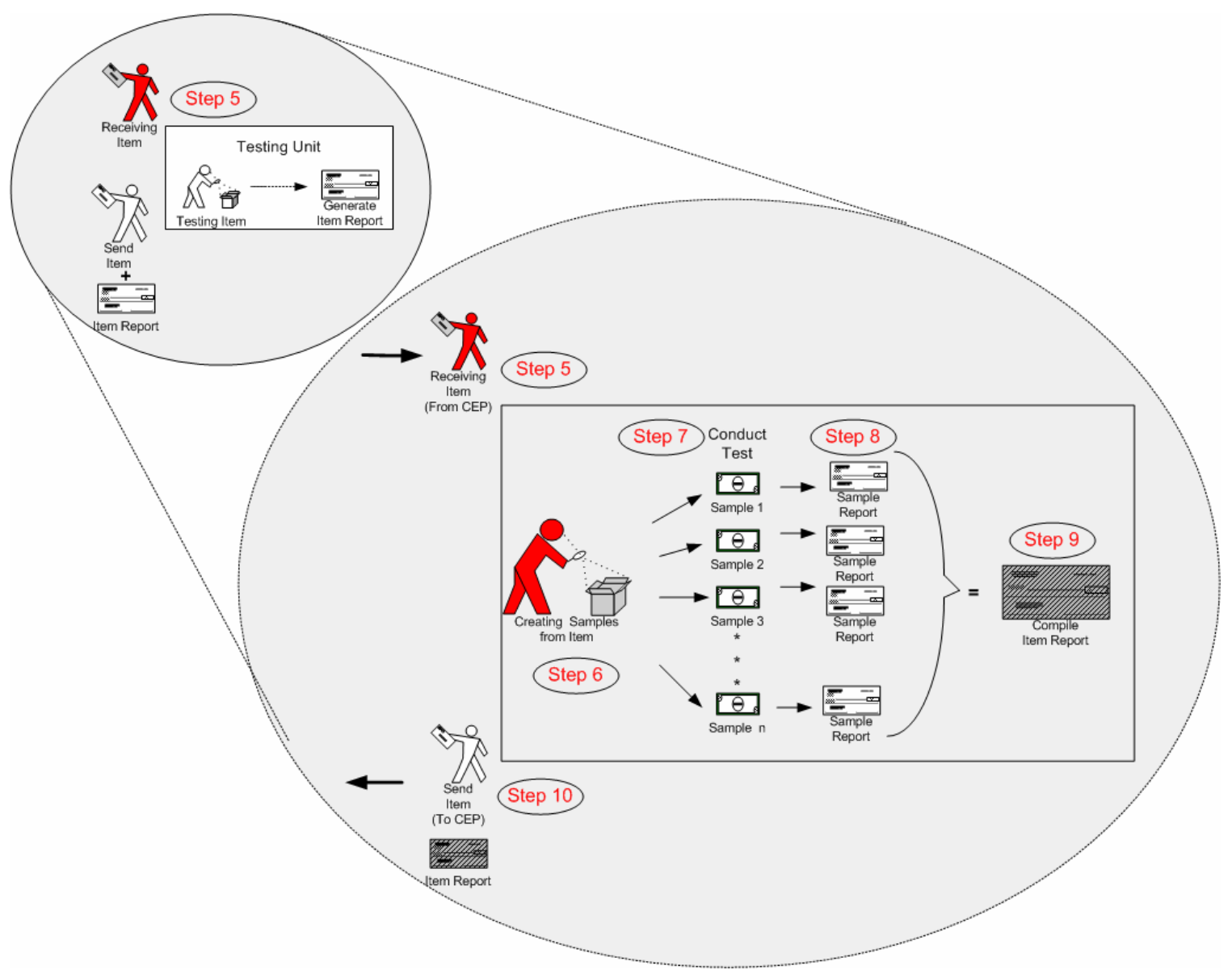

Figure 9.3: Detail Item Processing Procedure 


\subsubsection{Toxicology Entity Relationship Diagram}

Toxicology data tables are connected with each other through relations as shown in the figure 9.4 .

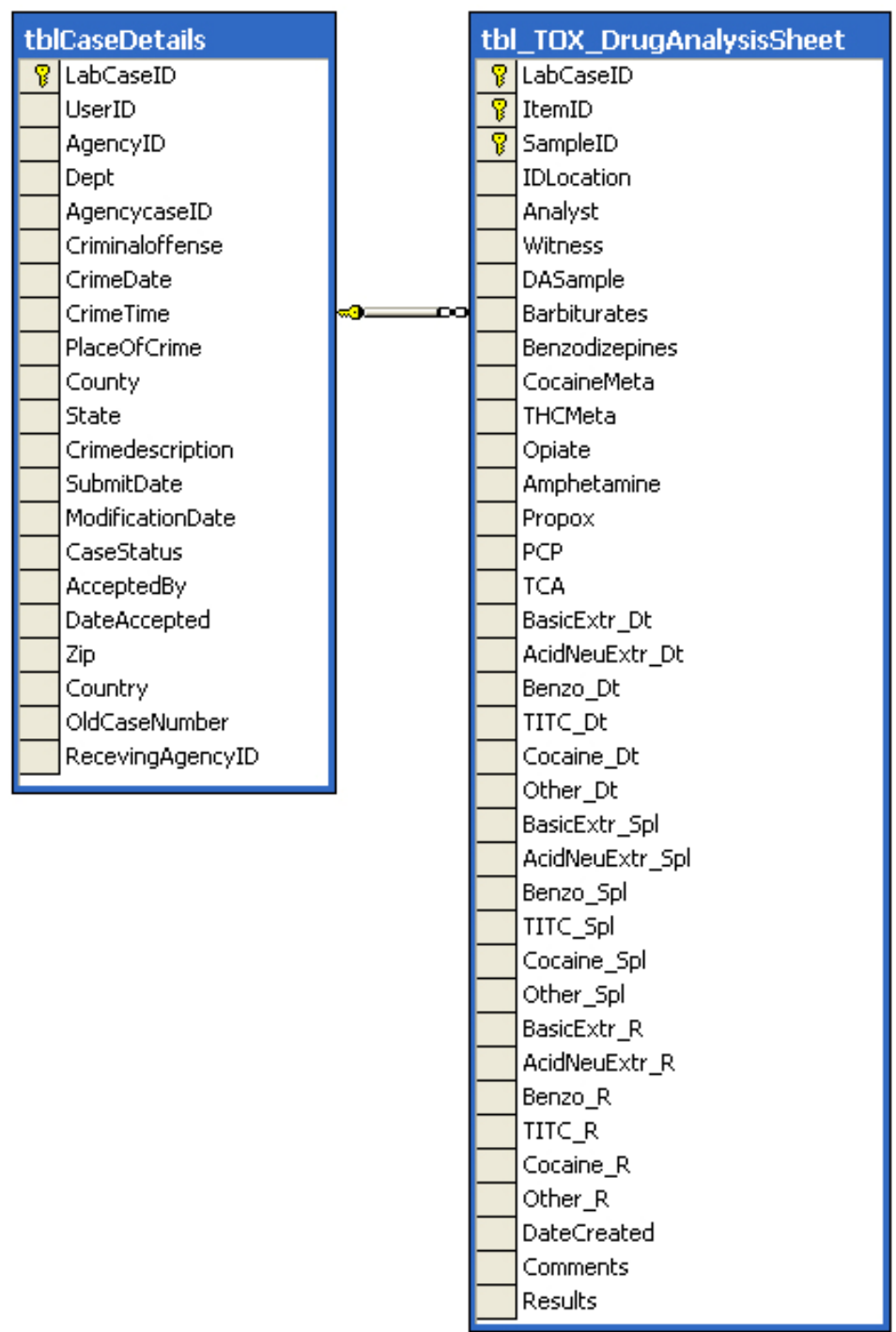

Figure 9.4: Entity Relationship Diagram 


\subsubsection{Toxicology Database Tables}

The Toxicology unit module stores its data in the following table:

- Drug analysis (tbl_Tox_DrugAnalysisSheet)

The various fields of the worksheet table are shown in Table 9-1. Worksheet data is stored and maintained in this data table

Table 9-1: Drug Analysis Table

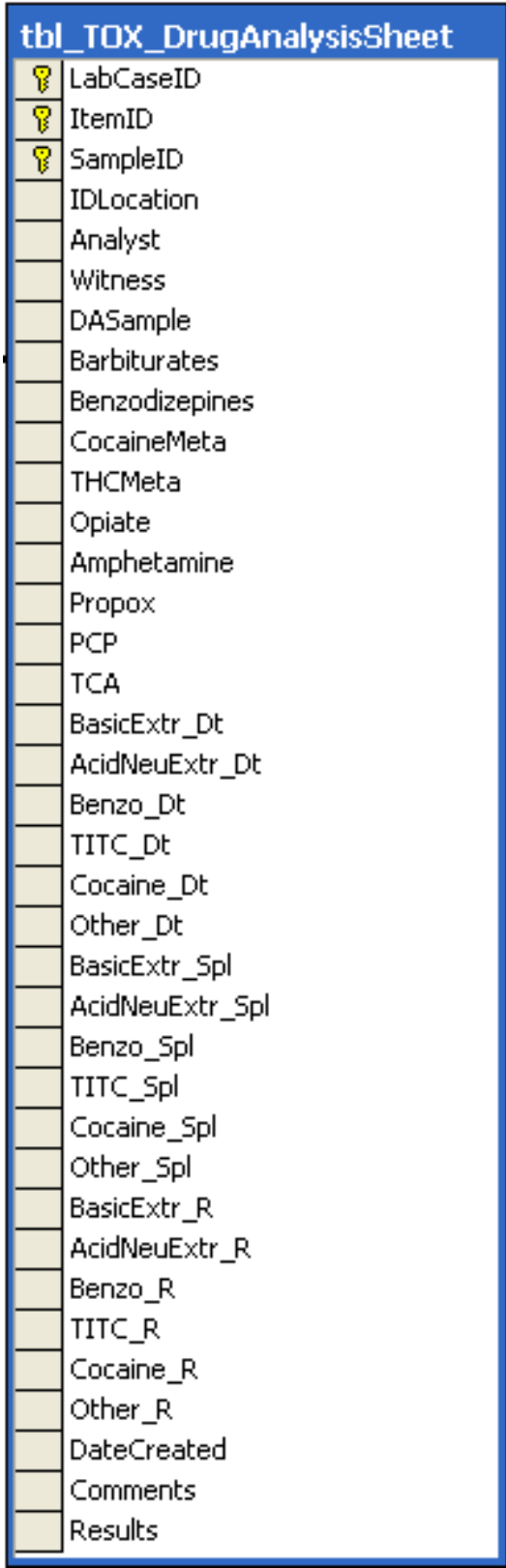




\subsubsection{Working with Toxicology}

Toxicology home page requires username and password to verify user credentials, as shown in the Figure 9.5. After verifying credentials, a user is directed to list of items assigned to him/her for further processing.

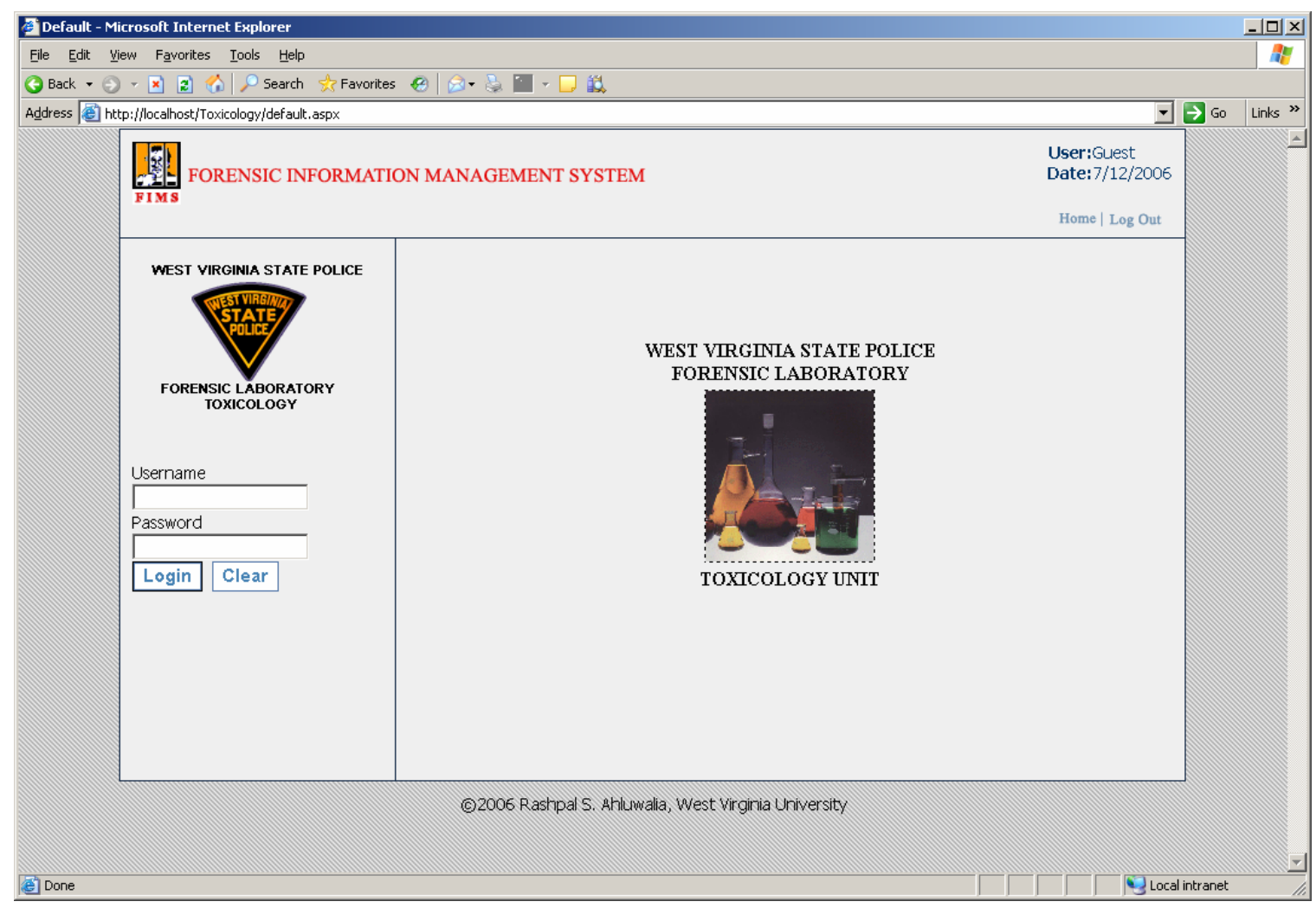

Figure 9.5: Toxicology Home Page 


\section{View Items Assigned}

Figure 9.6 shows the list of items assigned to the analyst by the laboratory administration unit for further analysis. Initially, job status for the item assigned is set to "Job Pending". The "View Details" hyperlink enables an analyst to view the details of the case. If the list of items assigned grows long i.e. more than 15 items, the record set is divided into multiple pages. Analysts can navigate between different pages by clicking on the header and footer navigation bar of the list.

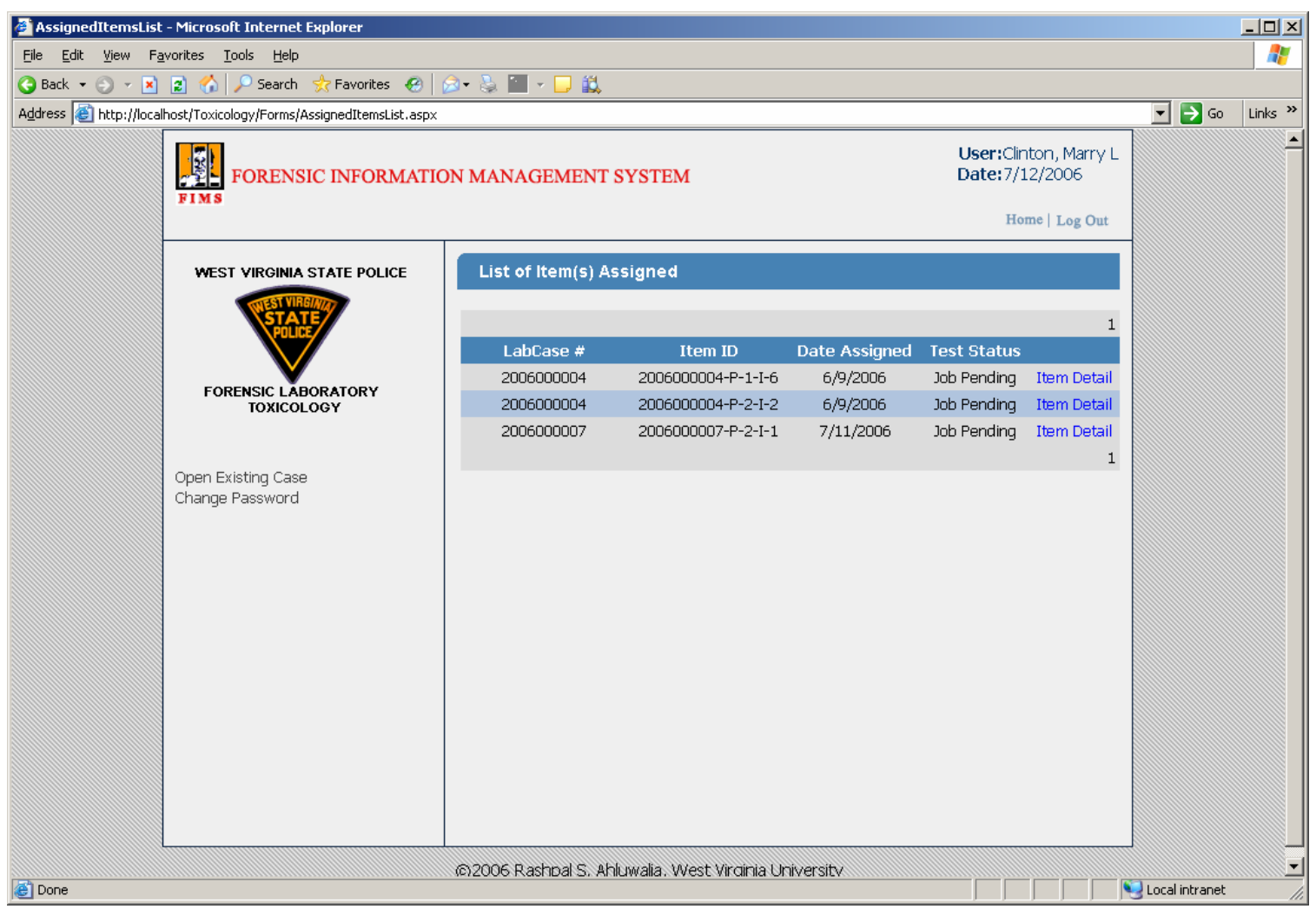

Figure 9.6: Assigned Item List 


\section{Item Picture Management}

This section describes the procedure of attaching pictures to an item. Analysts can perform two kinds of operation:

- View Pictures

- Upload Pictures

Analysts can access this section by clicking on the "View/Upload Item Photo" hyperlink in the item details section as shown inn Figure 9.9. View picture section list the pictures already attached to the item with their thumbnails. Analysts can also view the full size image by clicking on the image thumbnail. Full size image opens in a new window, which can be closed after use.

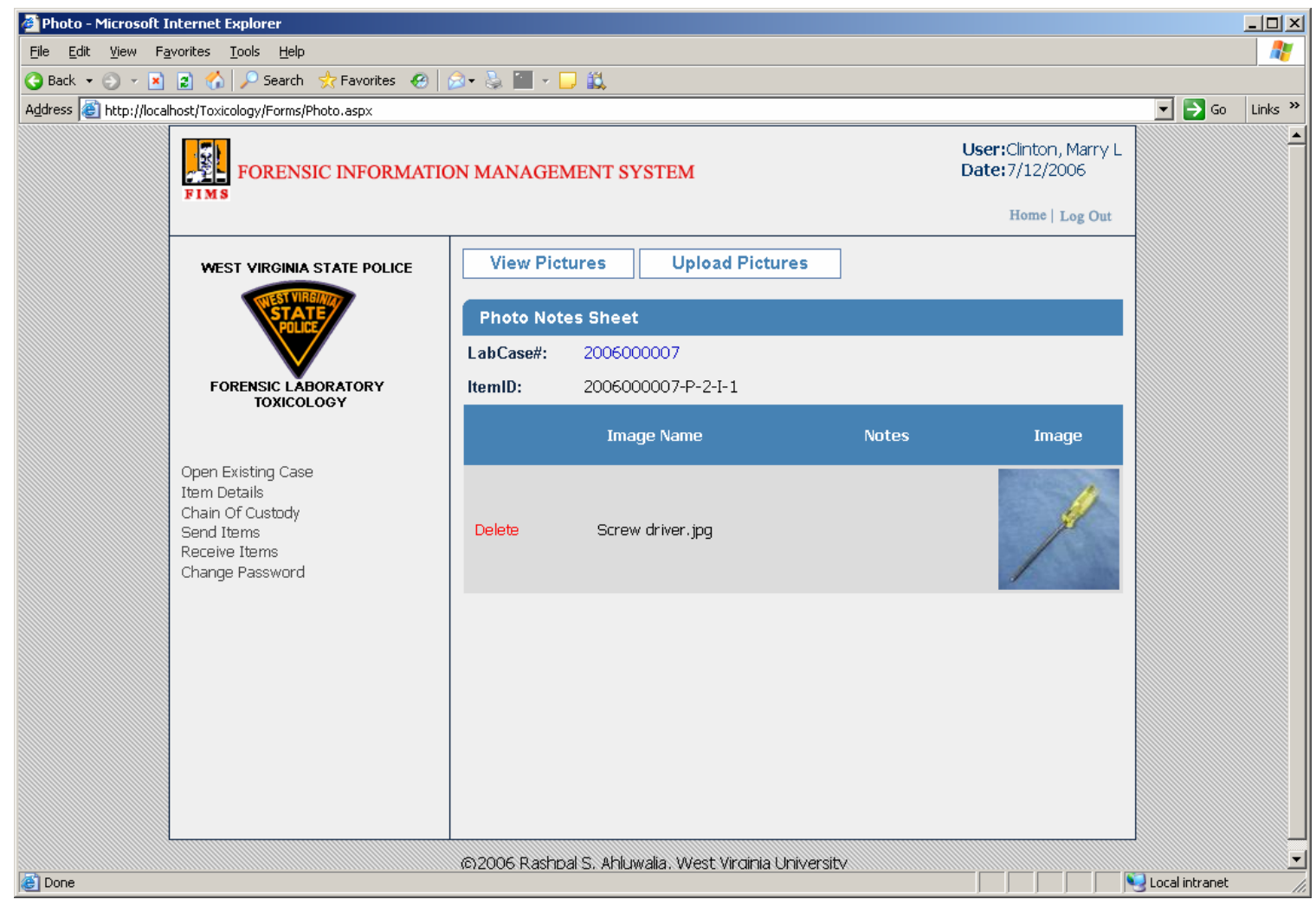

Figure 9.7: View Item Photo 
Analysts can upload any digital photo in JPG format directly into the database using this section as shown in Figure 9.8. To upload an image into database, analysts are required to browse for the image path, set other required fields and click on the "Upload the file” hyperlink. On successful upload a thumbnail appears on the bottom of the page with a success message.

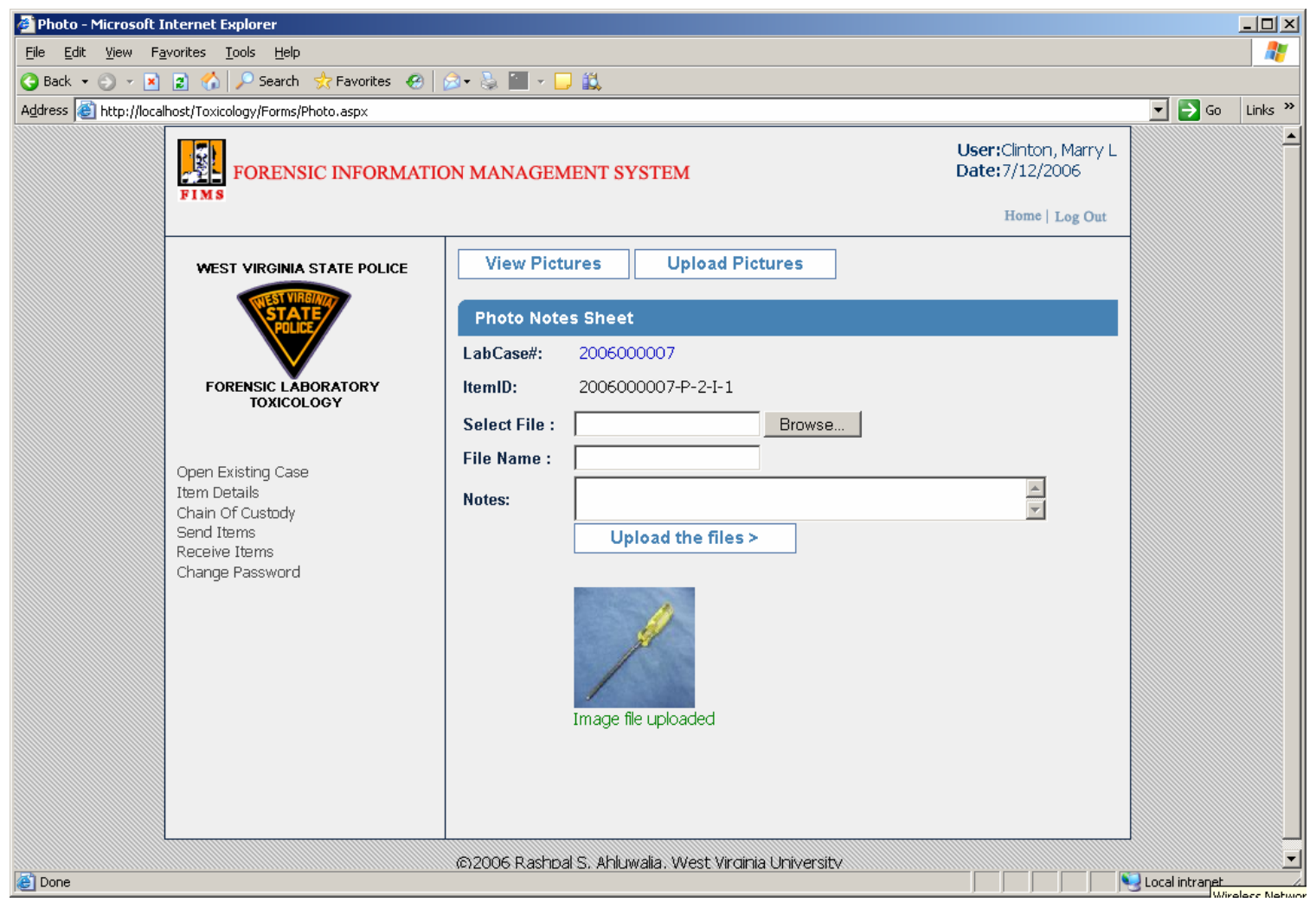

Figure 9.8: Upload Item Picture Section 


\section{Working with Samples}

Analysts receive items from the CEP unit for further analysis and processing. Once received items are sub-divided into samples for conducting requested tests. Each item is divided into one or more samples according to the requirement and complexity of the required test.

Analysts can create new, update or delete existing samples from the item details section as shown in Figure 9.9. To add a new sample analyst is required to provide description of sample and then click on "Add Sample” hyperlink. FIMS application automatically adds the time stamp to the item created. Initially, sample test status is set to "Test Pending" for the sample. Sample information can be updated by clicking on “Edit” hyperlink and existing sample can be deleted from the system by clicking on “Delete” hyperlink.

This section also enables analysts to view the final sample, sample photo, item, and item photo report. To access the individual sample/sample photo report analyst can click on "View Report" or "Photo Sheet" hyperlink. To view comprehensive item report analysts can click on "Item Report" or "Item Photo Report" at the bottom of the page. 
To complete the process for item testing, the analyst is required to click on "Item Test Complete" button, as shown in Figure 9.9. Once clicked, analyst will not be able to make any alterations to the existing item test report.

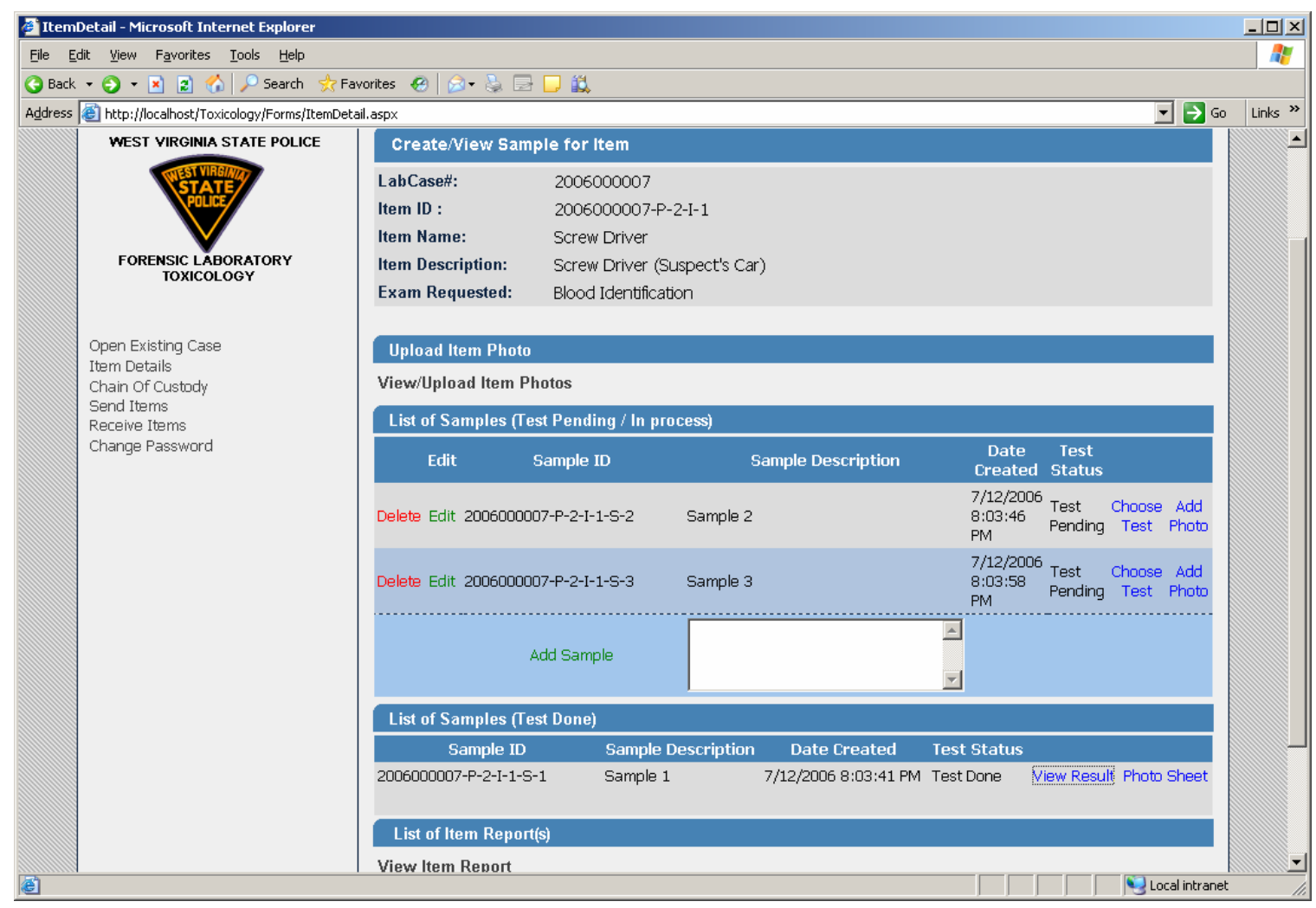

Figure 9.9: Item Detail Section 
Analysts can access this section by clicking on the "View/Upload Sample Photo" hyperlink in the item details section as shown inn Figure 9.9. View picture section list the pictures already attached to the item with their thumbnails as shown in Figure 9.10. Analysts can also view the full size image by clicking on the image thumbnail. Full size image opens in a new window, which can be closed after use.

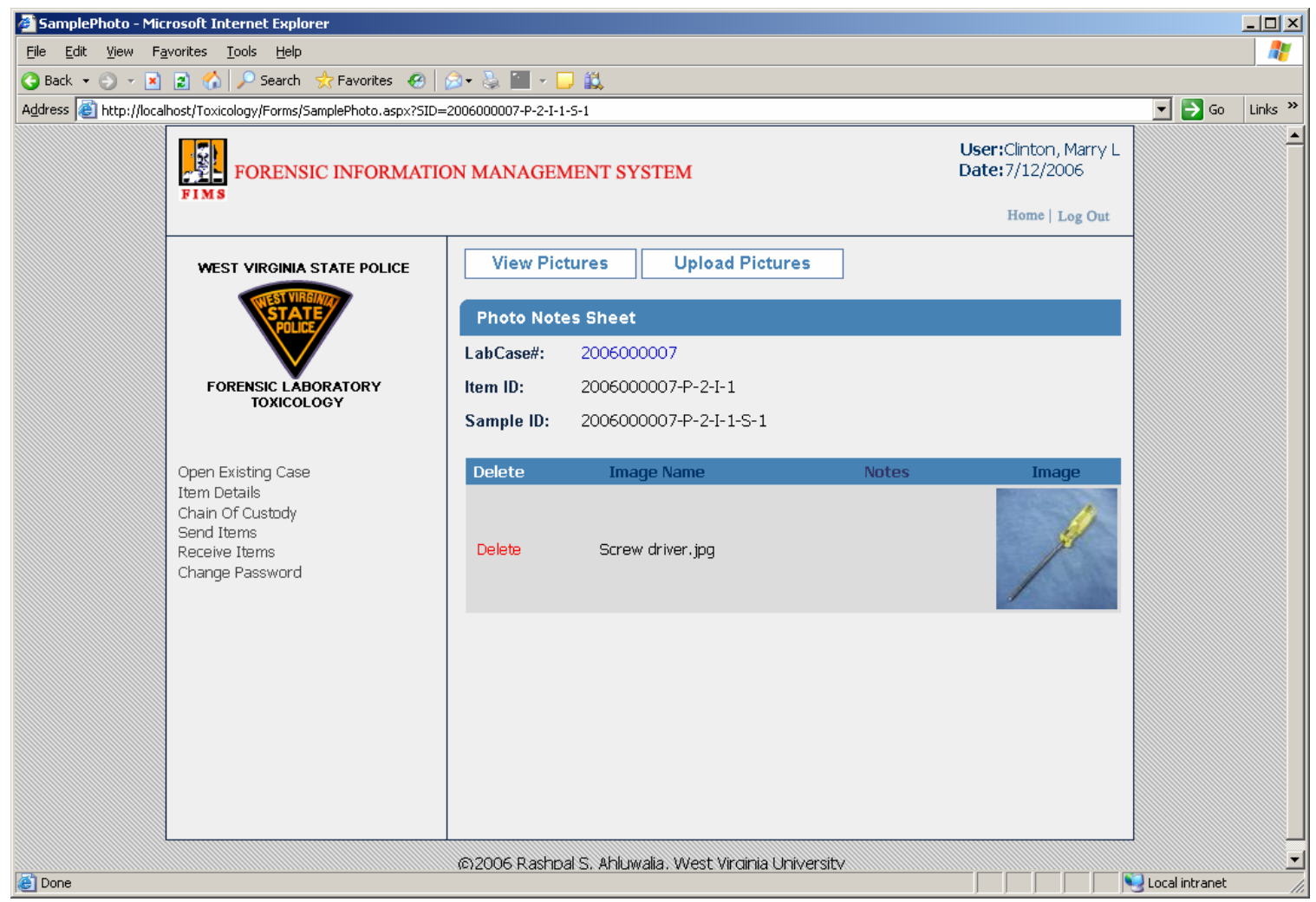

Figure 9.10: View Picture Section 
Analysts can upload any digital photo in JPG format directly into the database using this section as shown in Figure 9.11. To upload an image analysts are required to browse for the image path, set other required fields and click on the "Upload the file" hyperlink to upload the image into database. On successful upload a thumbnail appears on the bottom of the page with a success message.

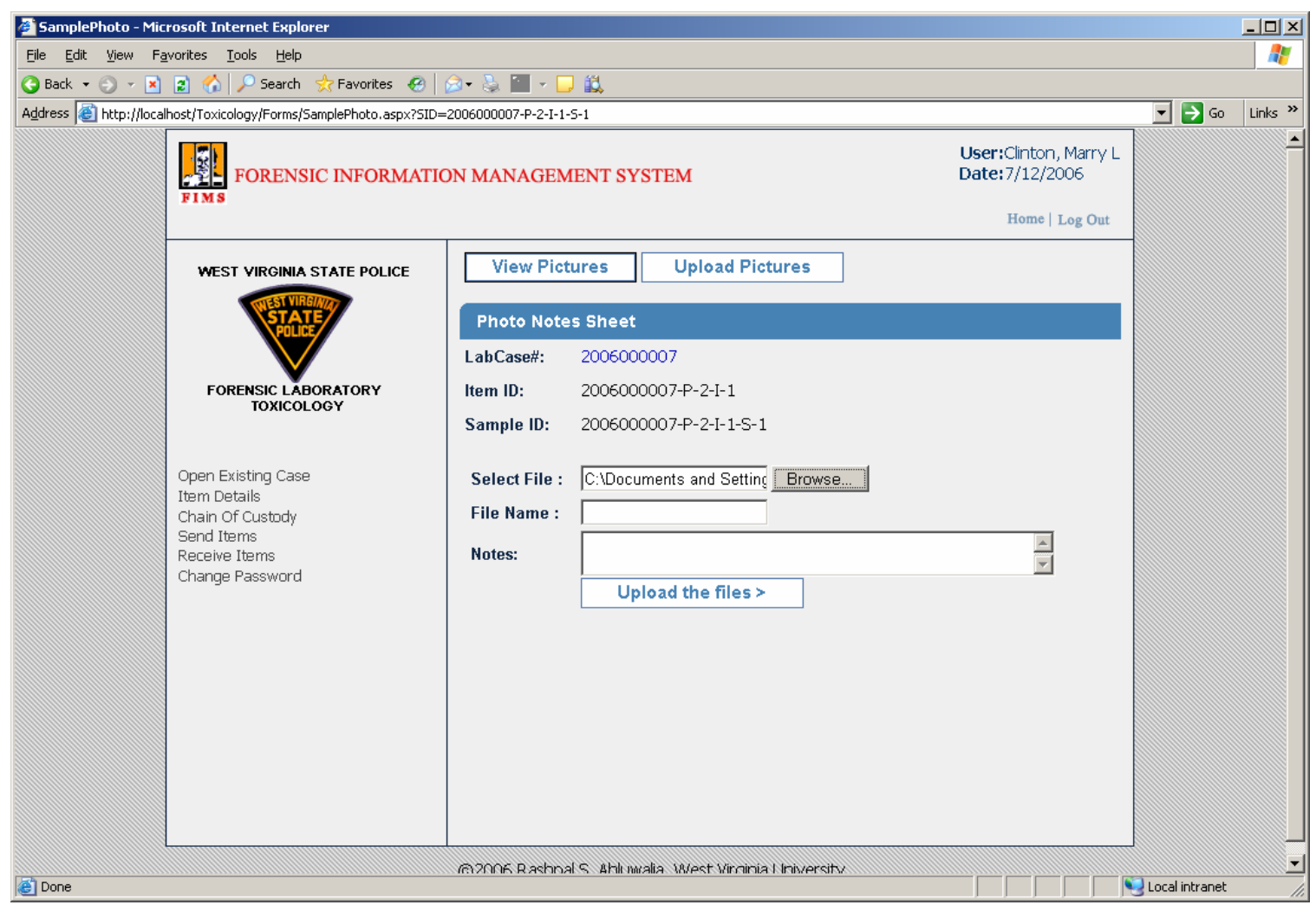

Figure 9.11: Upload Sample Picture Section 


\section{Choosing Test Worksheet}

After sample creation, analysts are required to decide what kinds of tests are required? Analysts are required to make decision of choosing type of test manually. One decision is made the analyst can see the list of tests available, and then click "Choose Test” hyperlink as shown in Figure 9.12. FIMS application remembers the chosen sample ID during the process.

The worksheet for the toxicology unit is:

- Drug Analysis

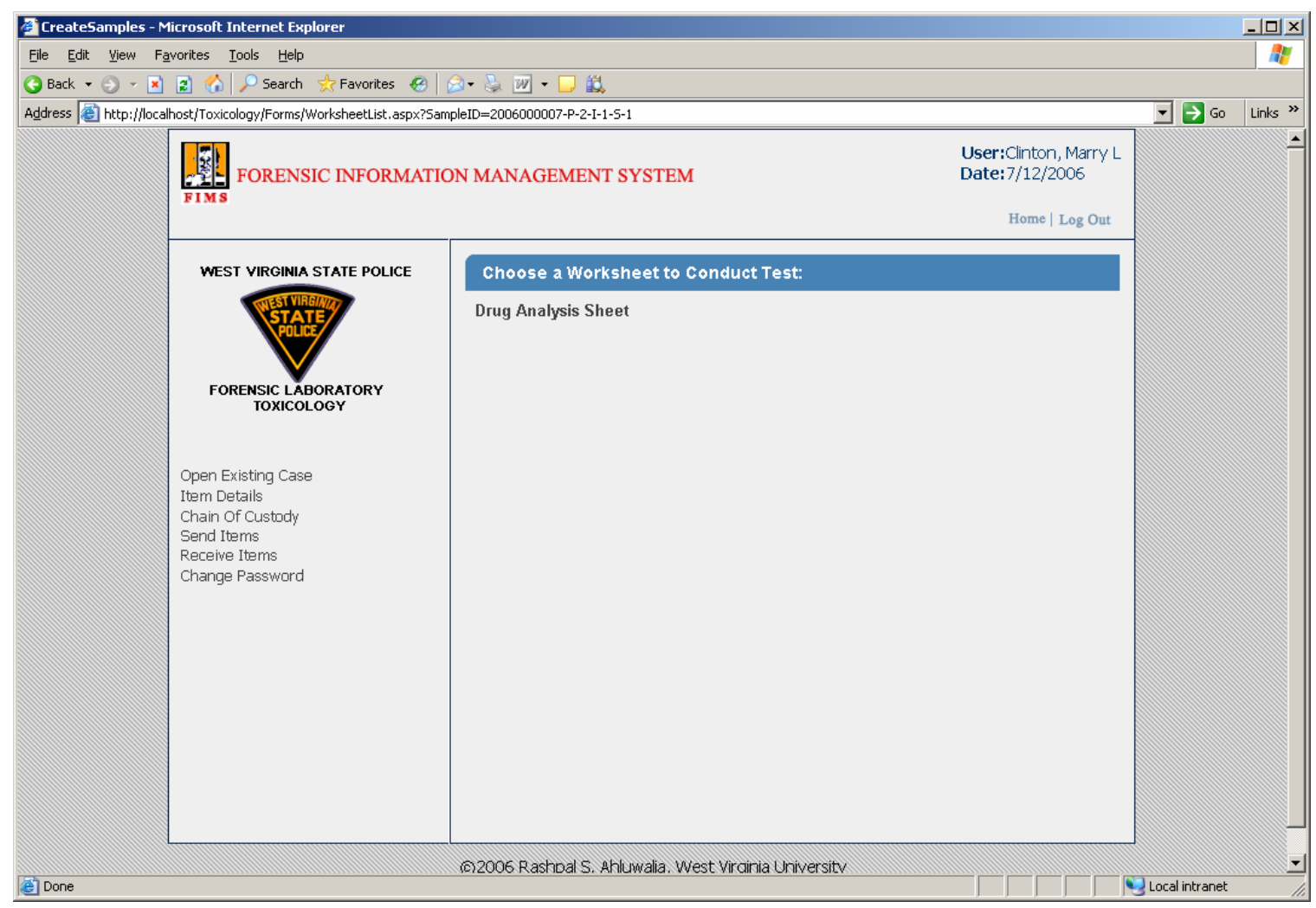

Figure 9.12: Choose Sample Test Section 


\section{Drug Analysis Worksheet}

Drug Analysis Worksheet is used for filling information pertaining Drug analysis test performed on the sample by the analyst. After filling the required data fields, analyst is required to click on "Save Results" button located on top of the page, as shown in Figure 9.13. After saving the worksheet data, analyst can finalize and close the worksheet by clicking on "Test Done" button. The analyst cannot make any alterations in the worksheet once the "Test Done" button is clicked.

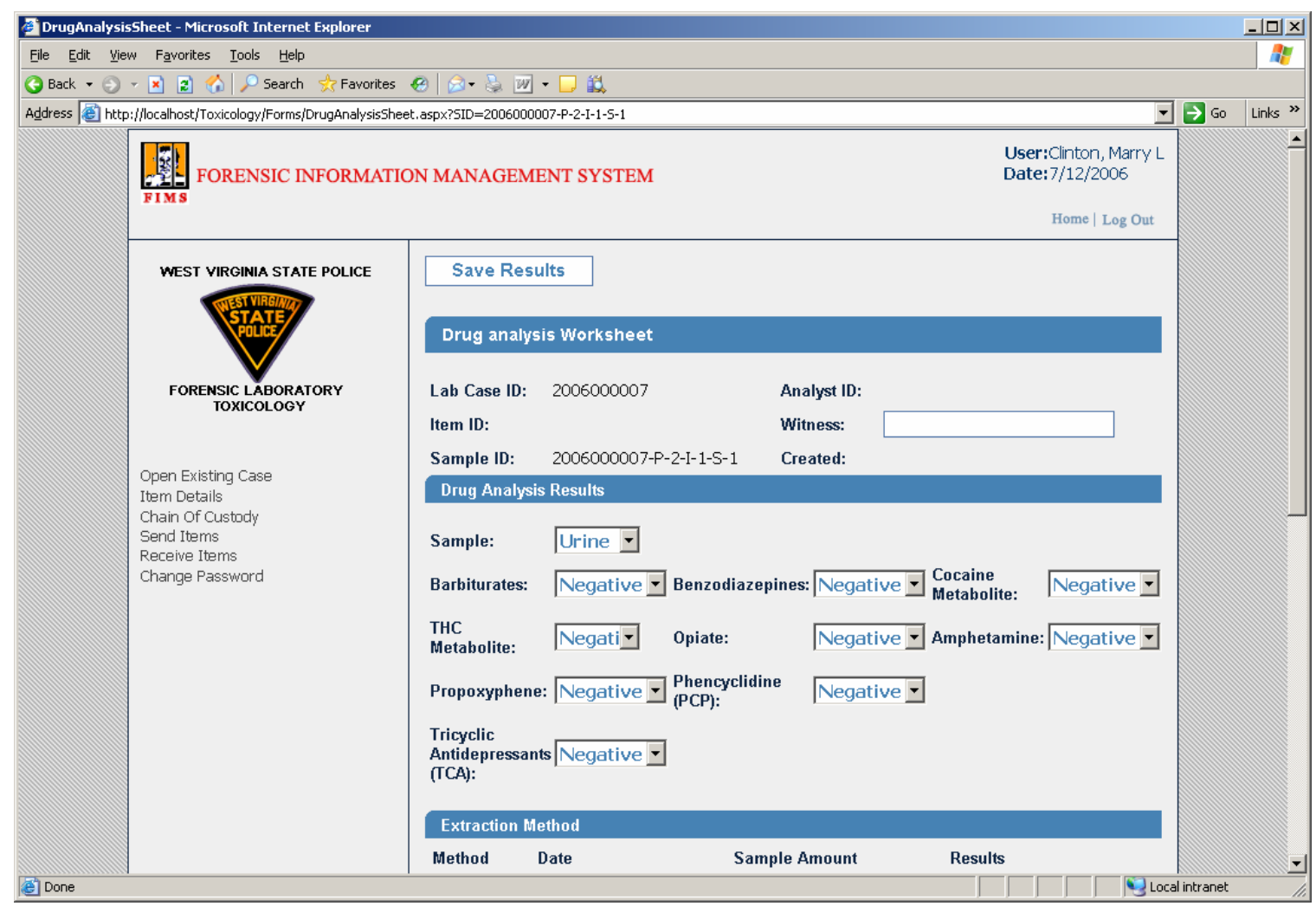

Figure 9.13: Drug Analysis Worksheet 


\section{Receive Item}

Once an item is sent by CEP unit, Toxicology unit analyst is responsible for receiving it by clicking on "Receive" button, under receive item section, as shown in Figure 9.14. This completes the handshaking process of item transfer between units.

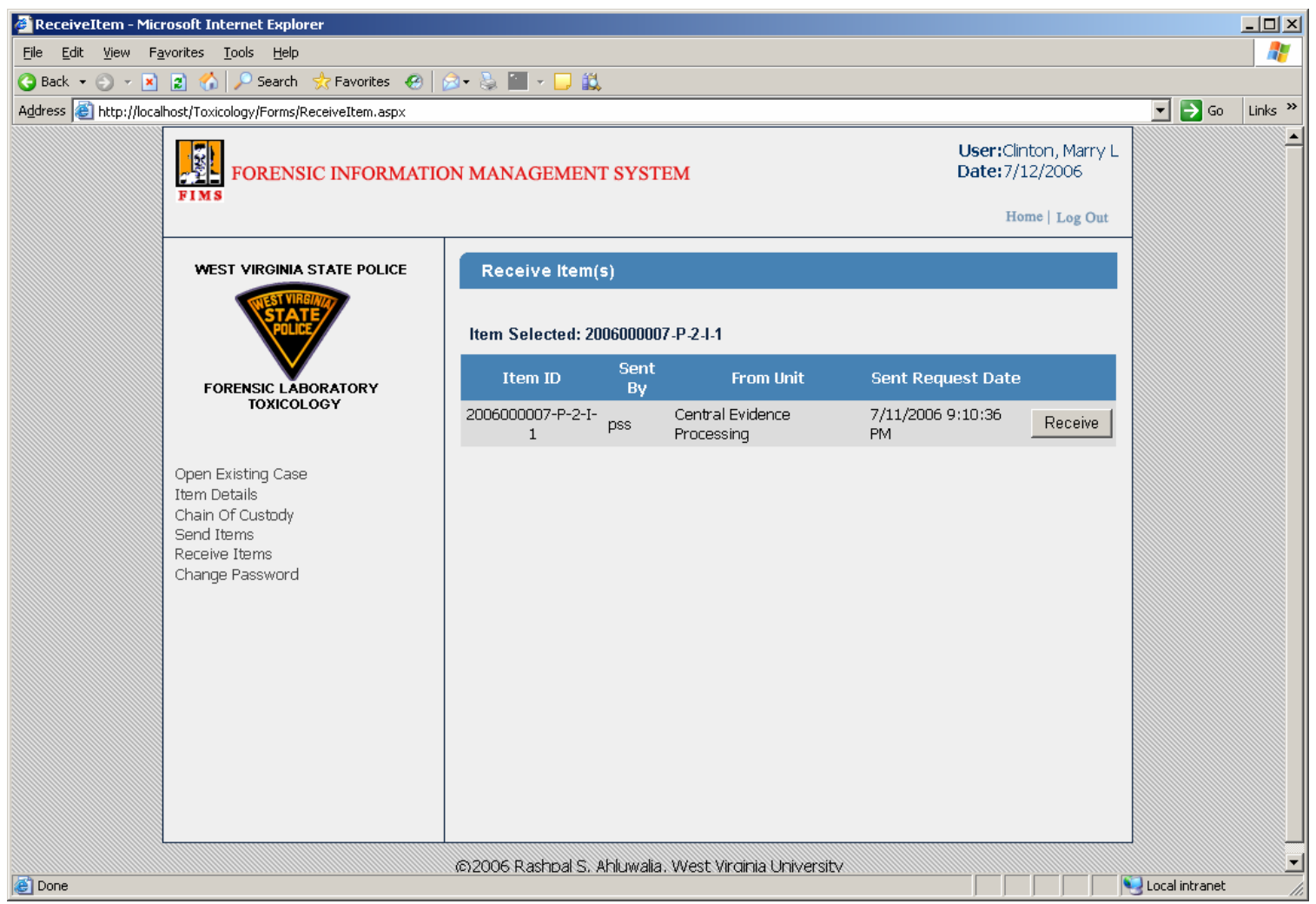

Figure 9.14: Receive Item Section 


\section{Send Item}

After completion of item testing, analyst is required to return the evidence item back to CEP unit. Figure 9.15 shows the section used to send an item back to the CEP unit. Analyst can use the drop down to select item ID and click on "Send" hyperlink, to send item back to CEP.

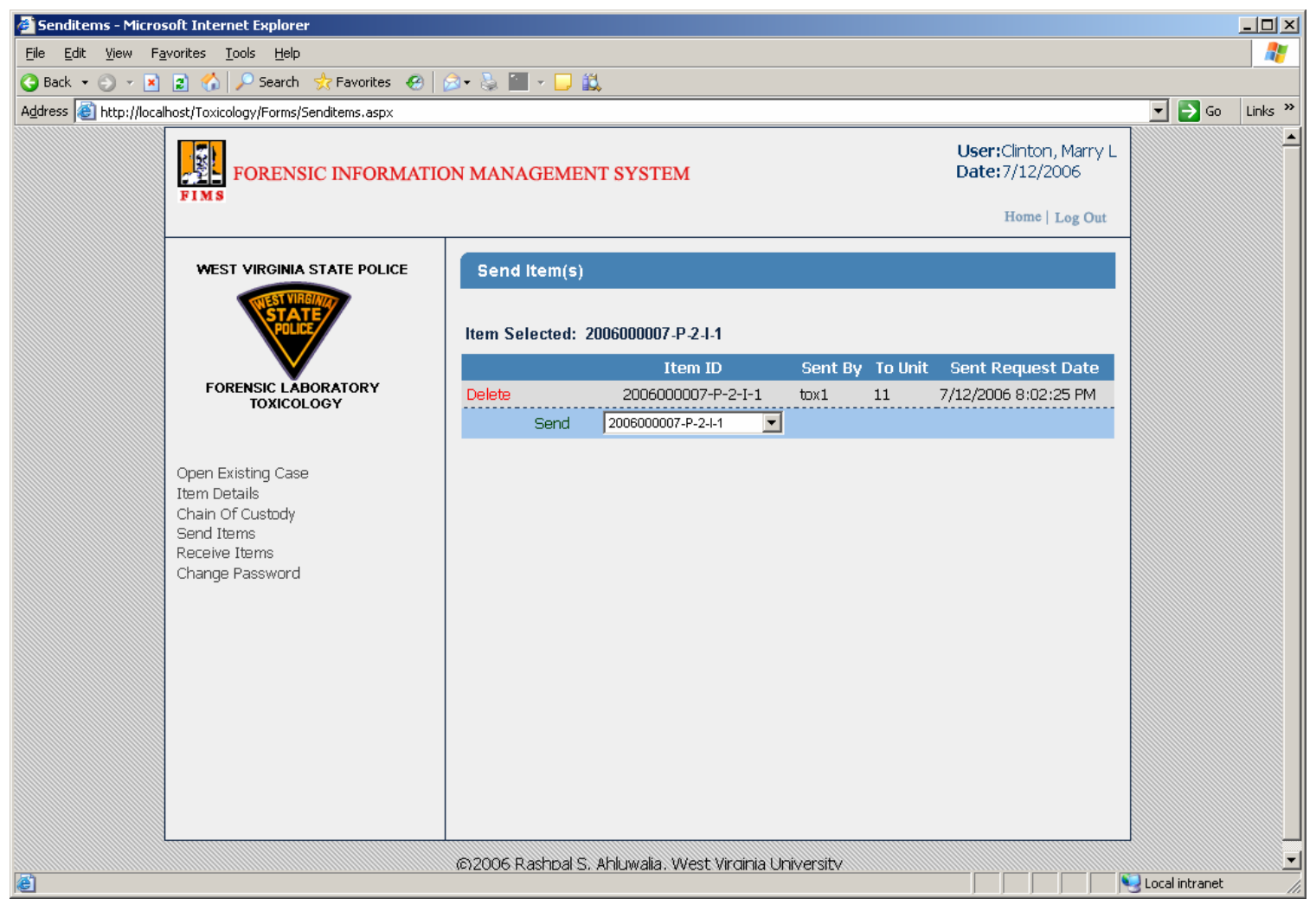

Figure 9.15: Send Item Section 


\section{Chain of Custody}

Item chain of custody information is shown in Figure 9.16. FIMS application records used ID, unit name, and time stamp for tracking item chain of custody.

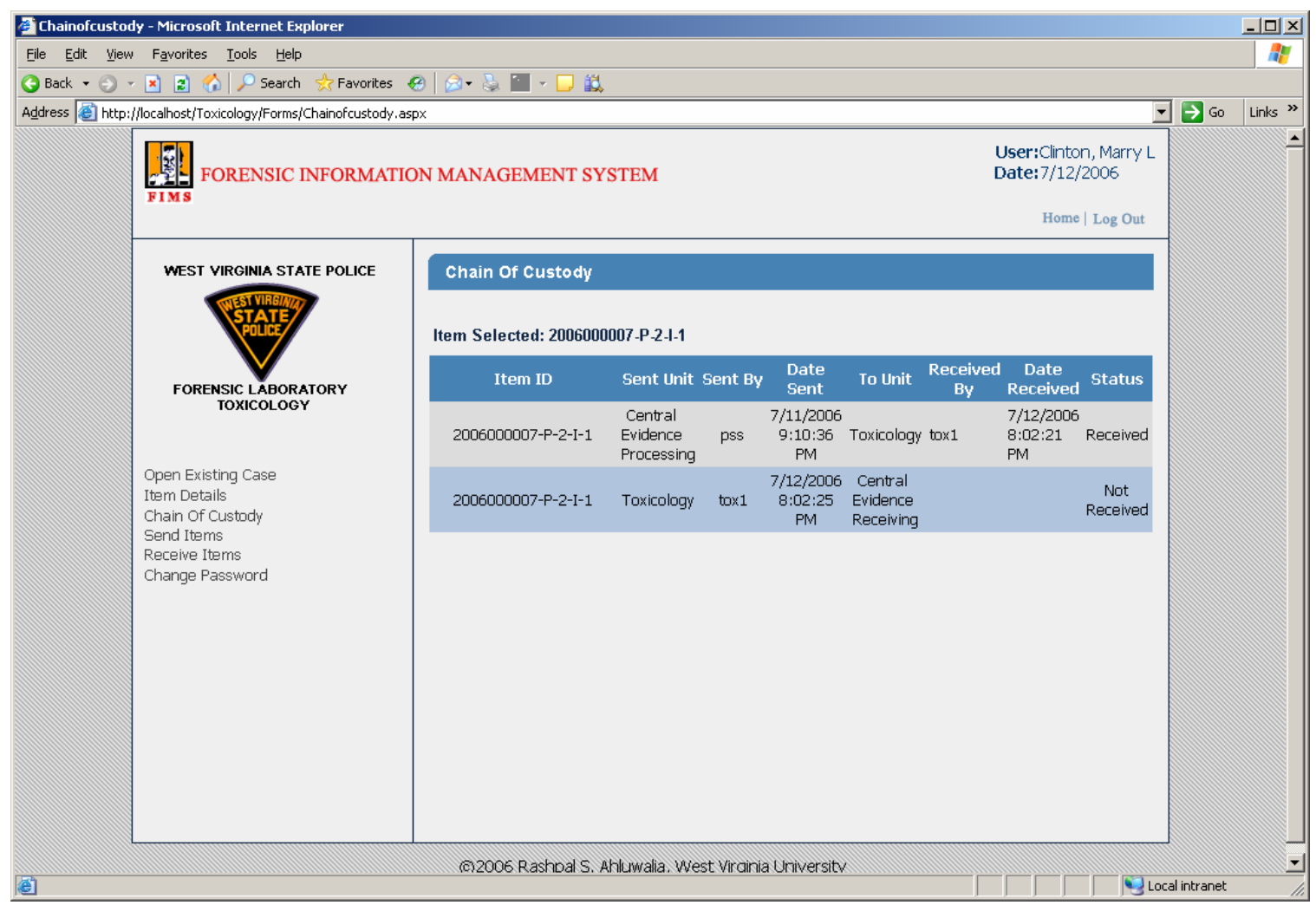

Figure 9.16: Item Chain Of Custody 


\section{Change User Password}

Analyst can change password through this section by providing appropriate credentials as shown in Figure 9.17.

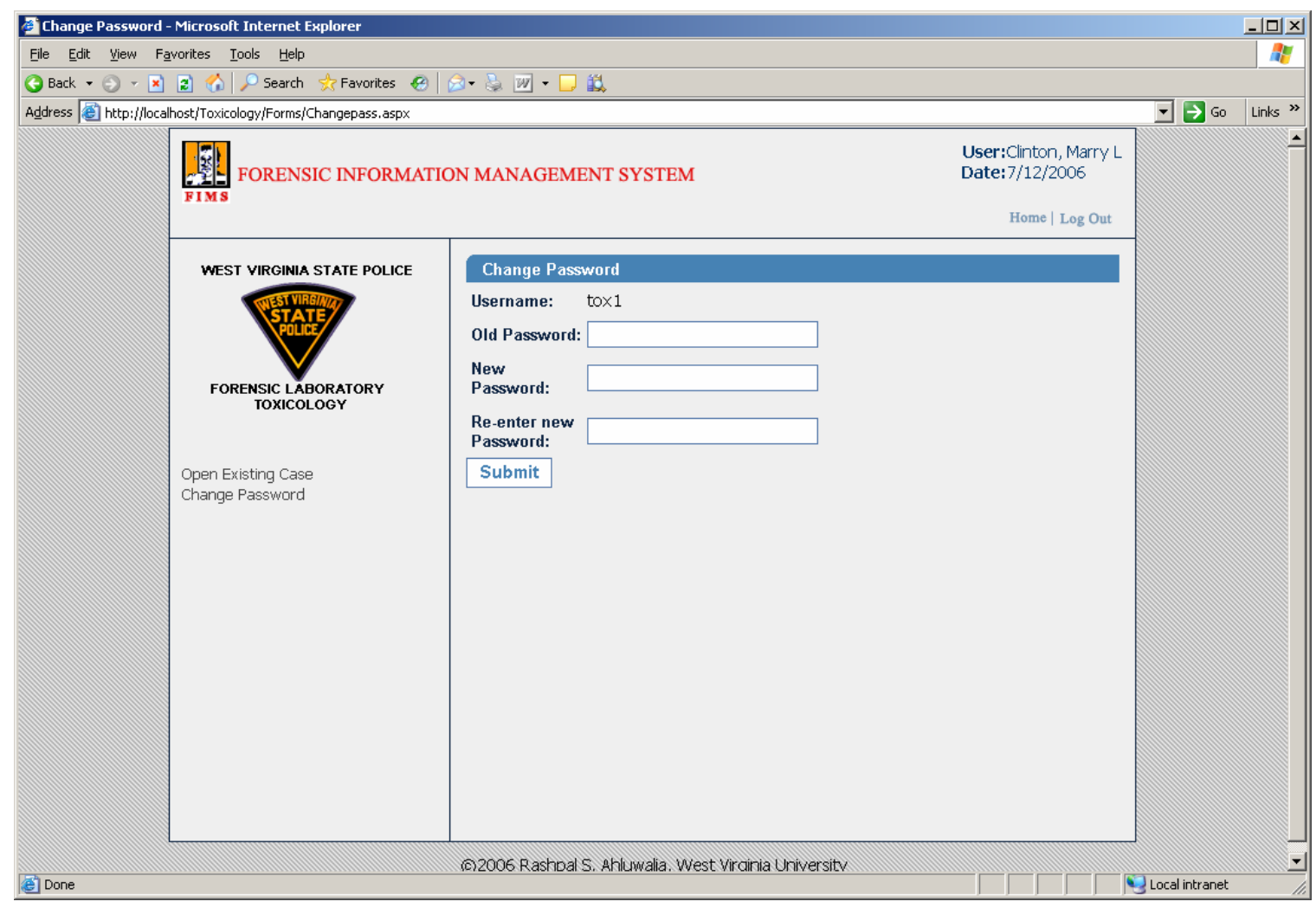

Figure 9.17: Change Password Section

\section{Exiting Toxicology}

Users can terminate the current session by clicking on "Log Out” hyperlink on the top right corner of the page. 


\subsubsection{Generating Item and Sample Reports}

The analyst can generate the following types of reports

- Item Report

- Item Photo Report

- Sample Report

- Sample Photo Report

Figure 9.18 shows the item report, which is a cumulative report for all the samples. This section also facilitate analyst to export report via PDF/MS-Word format, as shown in Figure 9.20 and Figure 9.21.

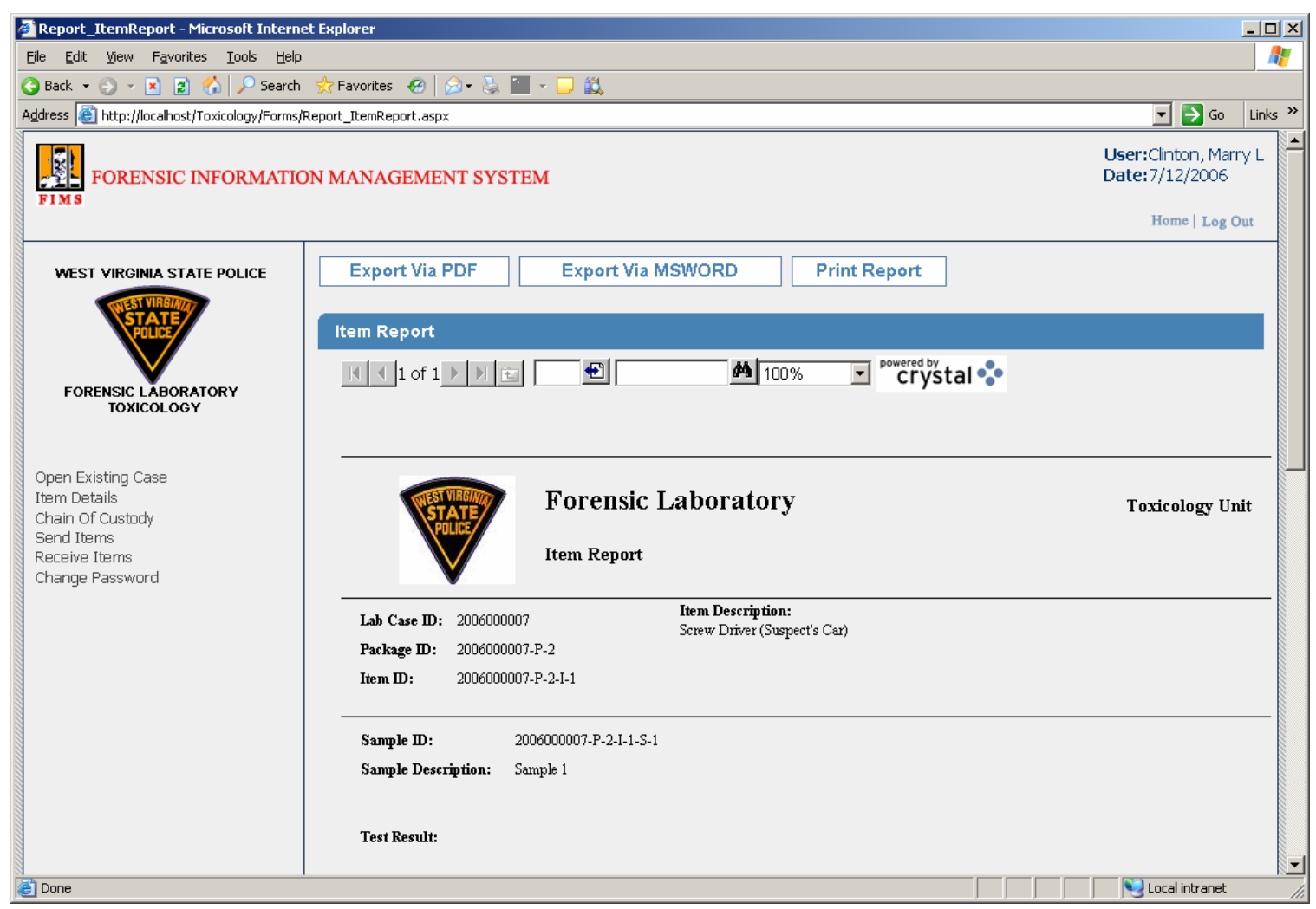

Figure 9.18: Item report 


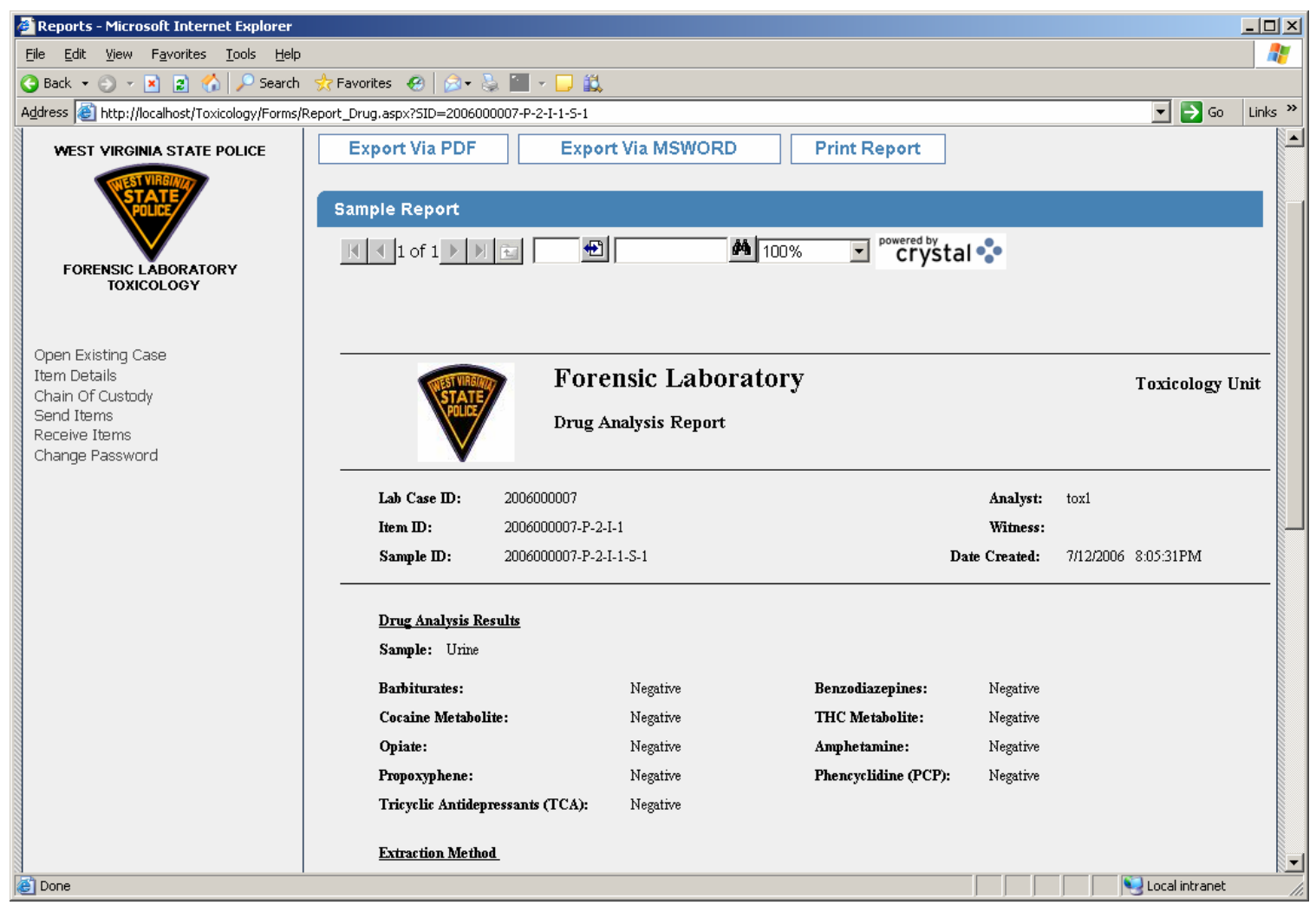

Figure 9.19: Sample Report 


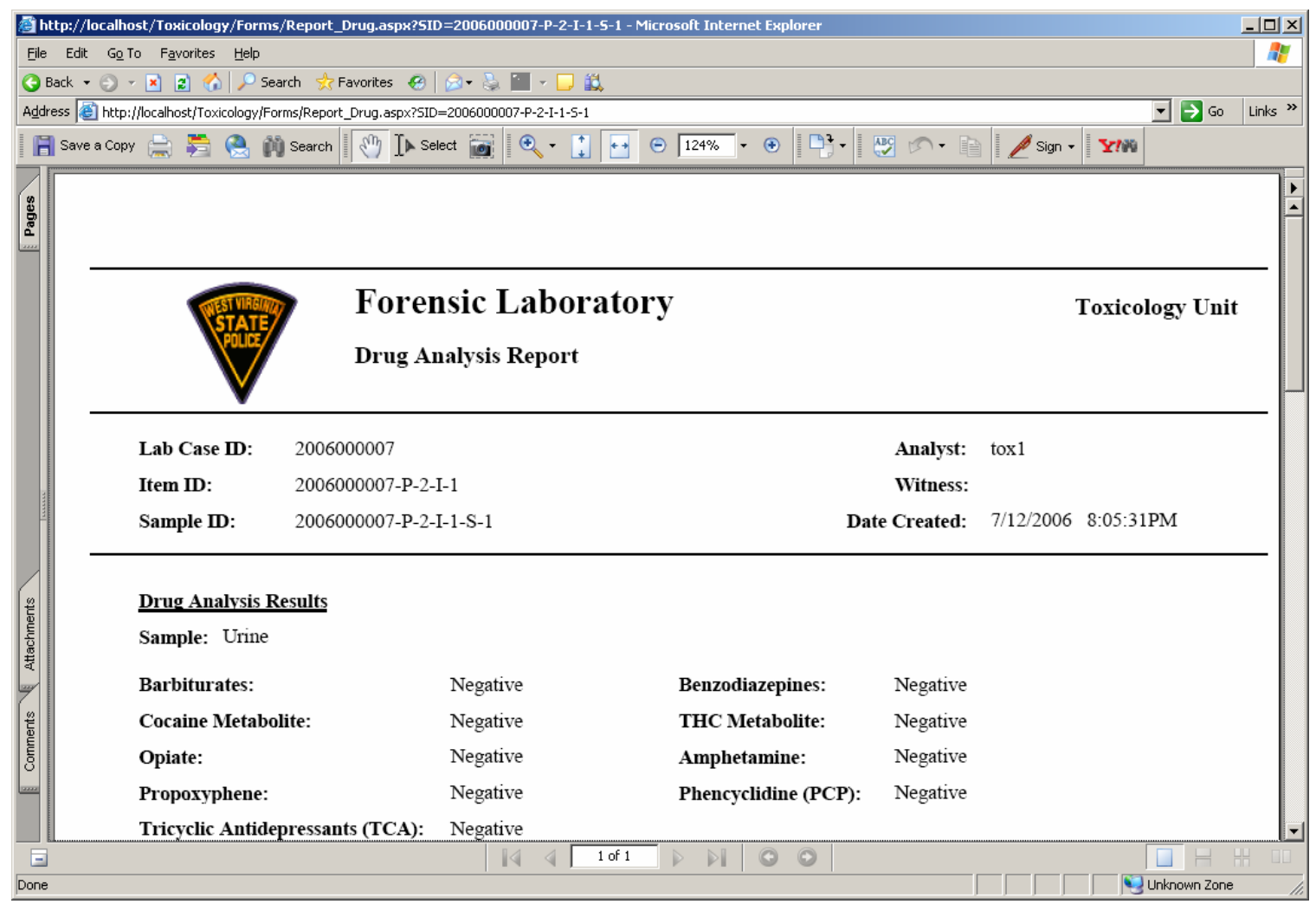

Figure 9.20: Sample Report in PDF Format 


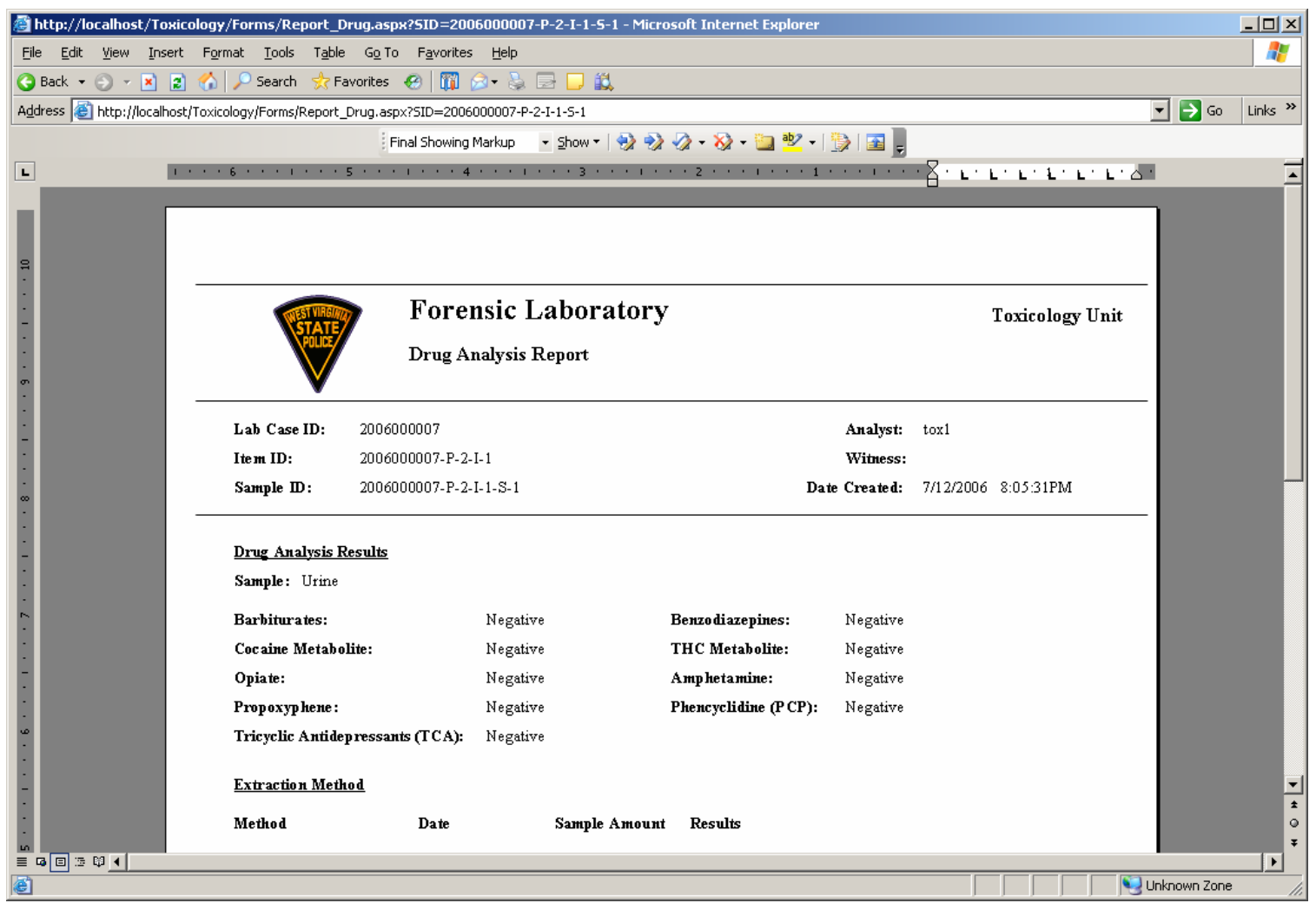

Figure 9.21: Sample Report in MS Word Format 


\section{CHAPTER 10 FIRE ARMS/TOOL MARKS}

\subsection{Existing Fire Arms/Tool Marks Process}

The Firearms/Tool Marks Unit analyzes firearms, fired cartridge cases, and fired bullets. By test firing the weapon and recovering the known fired bullets and cartridge cases, a comparison can be made with the evidence from the crime scene. The Firearms unit has a computerized digital imaging system that allows firearm evidence to be compared on screen, stored and printed. This unit manages the National Integrated Ballistics Information Network (NIBIN). NIBIN allows nationwide comparisons of fired cartridge cases. New technology allows for a computerized search of data to possibly link serial shooting cases that may have never been linked before. The analyst in the firearms unit receives the LEIF form and chain of custody form along with the evidence items. Samples are prepared and evidence is analyzed. After the tests are performed, appropriate forms are completed. The forms/worksheets used by this unit are: Worksheet A (WA), Worksheet B (WB), Fired Bullet (F), Fired Cartridge (FC), Tool/Tool Mark (TT), Chemical Residue (CE), and Distance Determination (DD). Once the examinations are performed, evidence is returned to the CER and the secretary prepares a draft report. The draft report is reviewed by the analyst/reviewer. After approval, the final report is sent to CER. Figure 10.1 shows the existing process for the firearms unit 


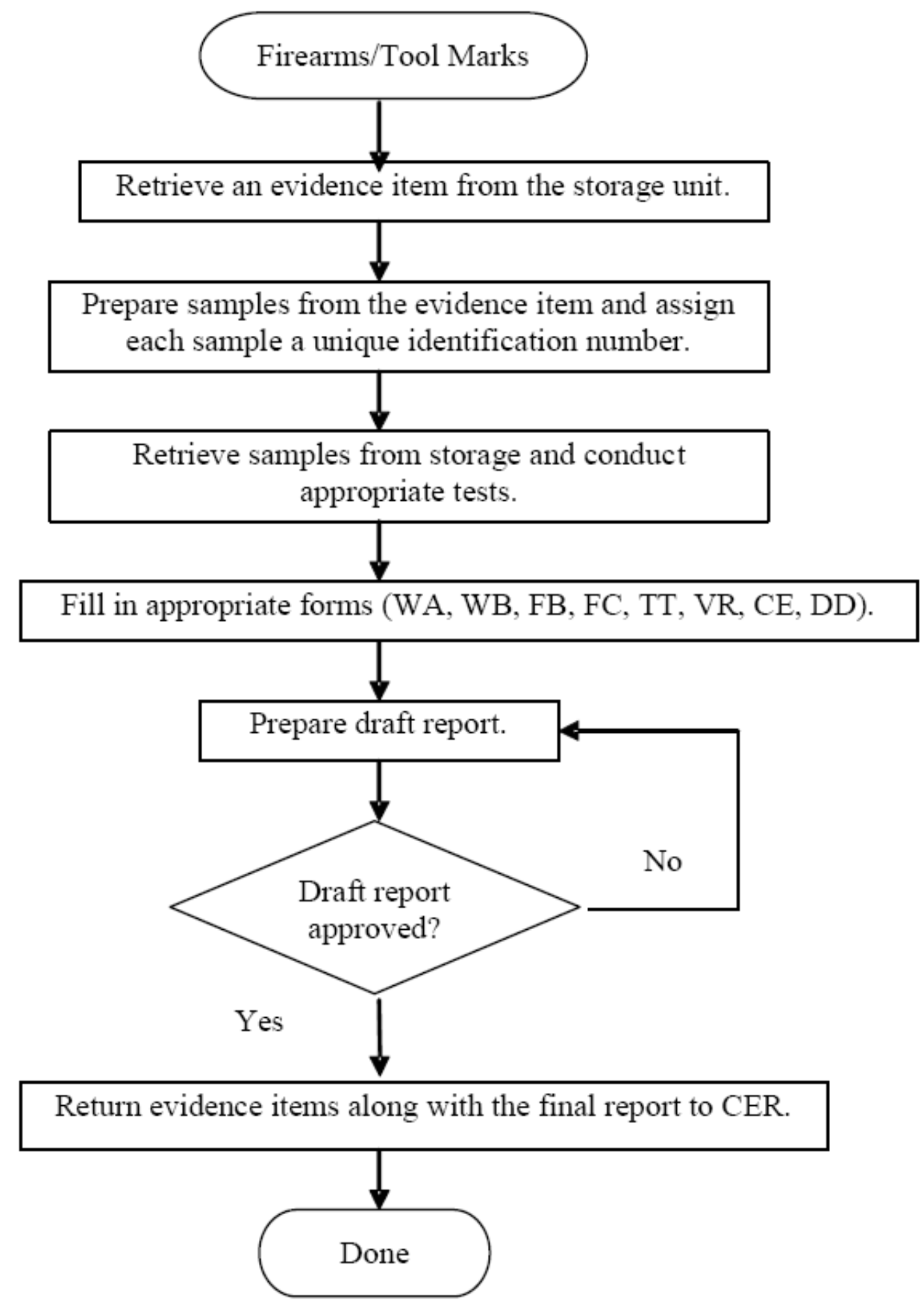

Figure 10.1: Existing Fire Arms Process 


\subsection{Re-engineering the Fire Arms/Tool Marks Process}

The analyst in the fire arms unit receives the items assigned for further processing and analysis from CEP. Received items are sub-divided into samples for conducting the requested tests. An individual item may be divided into many samples according to the requirement and complexity of the required test. A report for each sample is generated. An item report is compiled by combining sample reports. After the report is received, the reports along with the test items are sent back to CEP for further processing. The evidence and information flow diagram is shown in Figure 10.2.

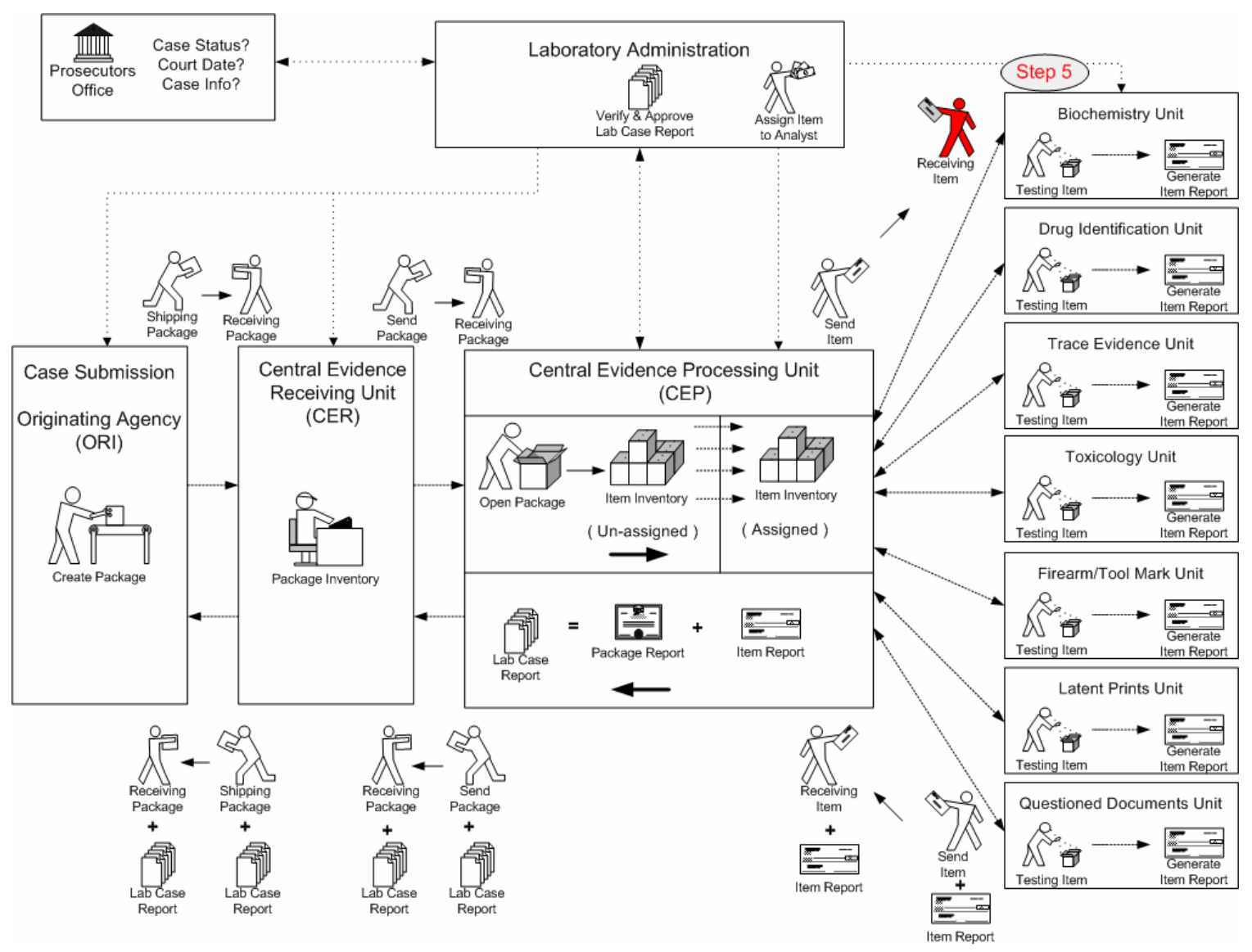

Figure 10.2: Step 5 Receive Assigned Items from CEP 
Detail procedure followed by fire arms/tool marks unit for further processing and analysis is shown in Figure 10.3.

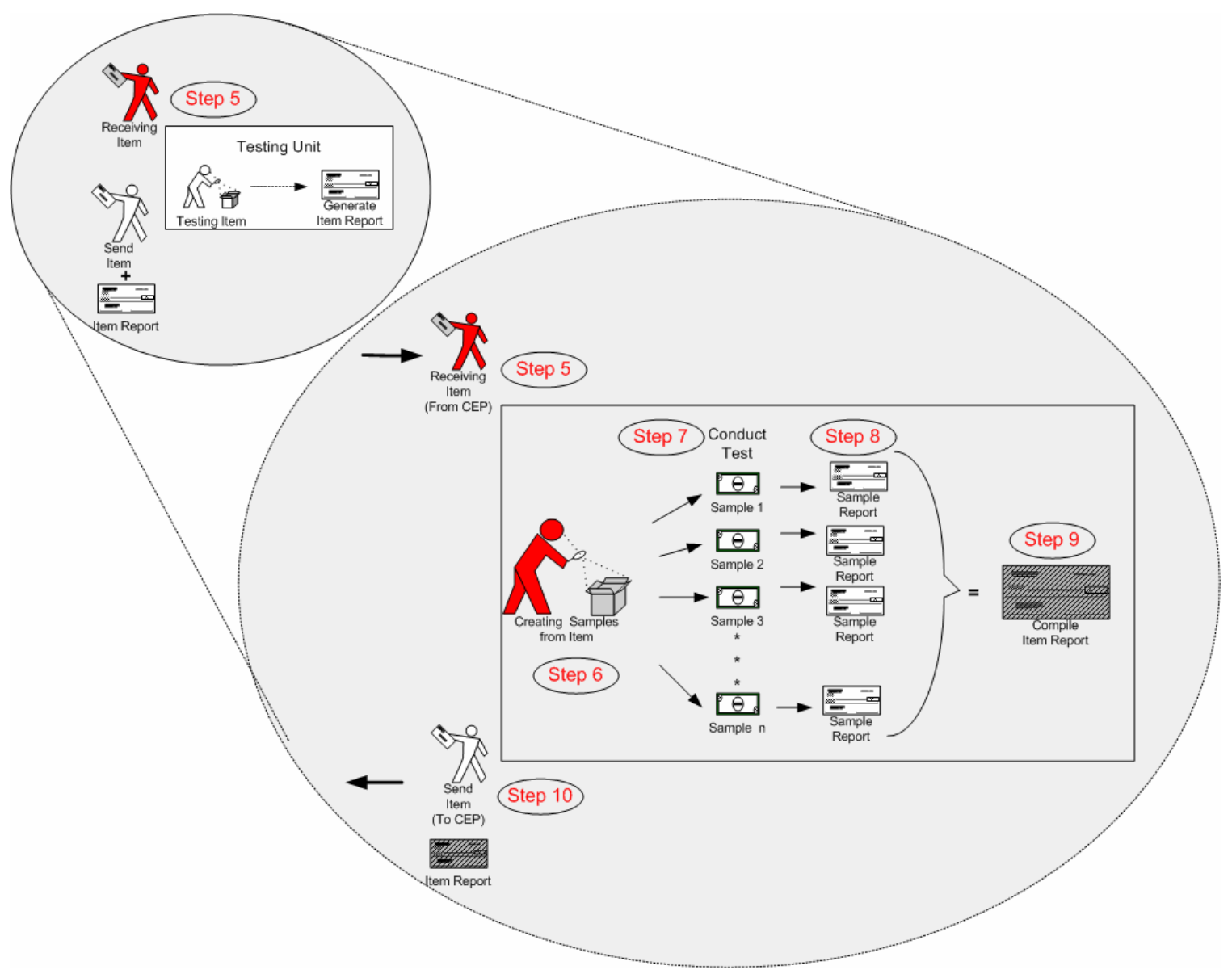

Figure 10.3: Detail Item Processing Procedure 


\subsubsection{Fire Arms/Tool Marks Entity Relationship Diagram}

Fire Arms/Tool Marks data tables are connected with each other through relations as shown in the figure 10.4 .

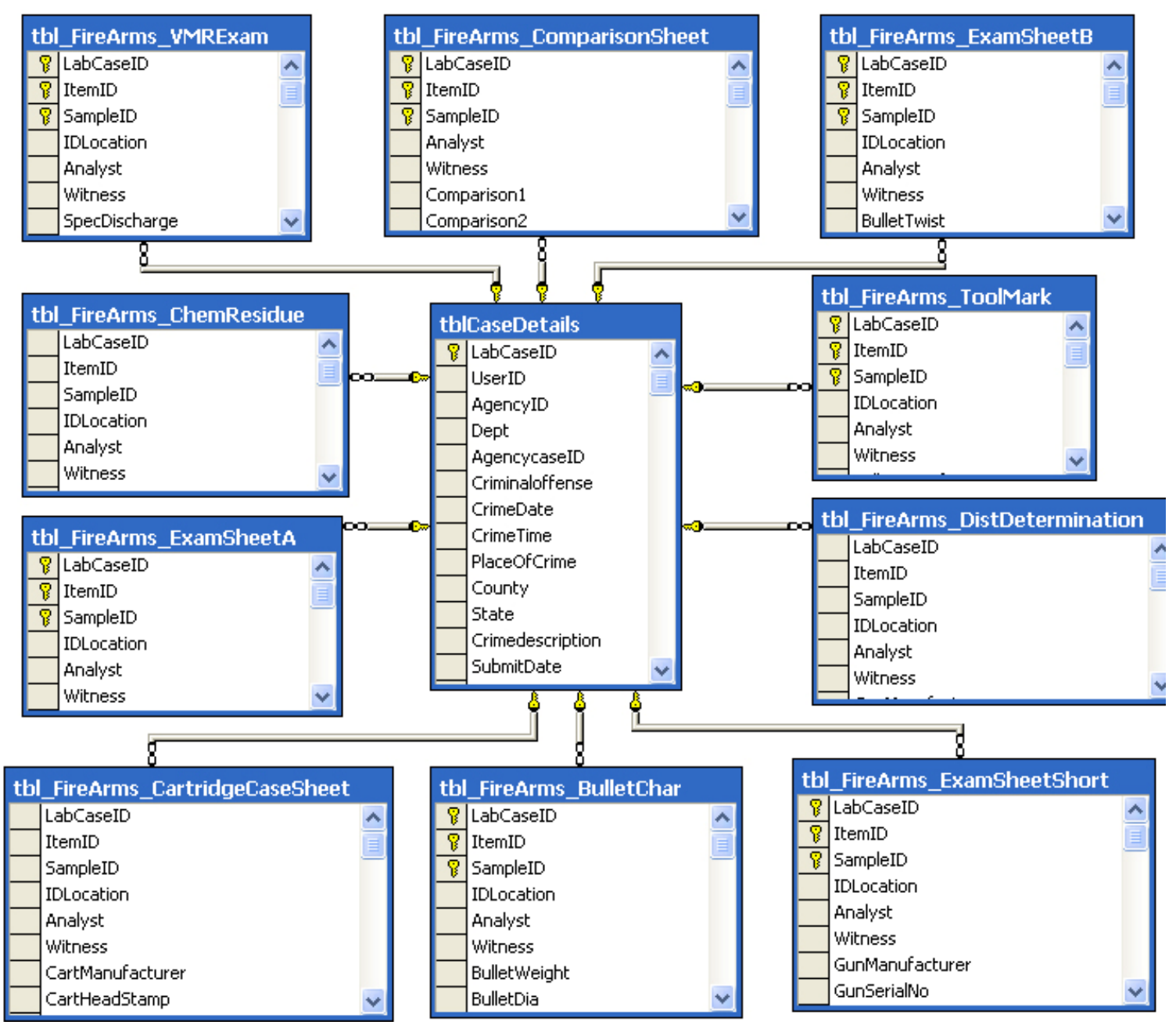

Figure 10.4: Entity Relationship diagram 


\subsubsection{Fire Arms Database Tables}

The Fire Arms unit module stores its data in the following tables

- Bullet Characteristics Worksheet

- Fire Cartridge Case Worksheet

- Chemical Residue Worksheet

- Comparison Examination Worksheet

- Distance Determination Worksheet

- Examination Worksheet - A

- Examination Worksheet - B

- Examination Short Form Worksheet

- Tool Mark Worksheet

- Visual Micro Residue Examination Worksheet 
The various fields of the bullet characteristics worksheet table are shown in Table 10-1. Bullet characteristics worksheet data is stored and maintained in this data table

Table 10-1: Bullet Characteristics Worksheet Table

\begin{tabular}{|c|c|}
\hline LabCaseID & \\
\hline ItemID & \\
\hline SampleID & \\
\hline IDLocation & \\
\hline Analyst & \\
\hline Witness & \\
\hline Bulletweight & \\
\hline BulletDia & \\
\hline BulletCaliber & \\
\hline BulletManufacturer & \\
\hline BulletComposition & \\
\hline BulletType & \\
\hline BulletMagnetic & \\
\hline BulletTwist & \\
\hline LGNumber & \\
\hline BallisticCondition & \\
\hline ForeignMaterial & \\
\hline Lwidth1 & \\
\hline Lwidth2 & \\
\hline Lwidth3 & \\
\hline Lwidth4 & \\
\hline Lwidth5 & \\
\hline LWidth6 & \\
\hline LWidth7 & \\
\hline LWidth8 & \\
\hline LWidthAvg & \\
\hline RWidth1 & \\
\hline Rwidth2 & \\
\hline Rwidth 3 & \\
\hline Rwidth4 & \\
\hline RWidth5 & \\
\hline RWidth6 & \\
\hline RWidth7 & \\
\hline RWidth8 & \\
\hline RWidthAvg & \\
\hline IBISEntry & \\
\hline EntryDate & \\
\hline ResonNoEntry & $v$ \\
\hline
\end{tabular}


The various fields of the fire cartridge case worksheet table are shown in Table 10-2. Fire cartridge case worksheet data is stored and maintained in this data table

Table 10-2: Fire Cartridge Case Worksheet Table

\begin{tabular}{|l|}
\hline tbl_FireArms_CartridgeCaseSheet \\
\hline LabCaseID \\
ItemID \\
SampleID \\
IDLocation \\
Analyst \\
Witness \\
CartManufacturer \\
CartHeadStamp \\
CartDesignation \\
CartFinish \\
CartType \\
CartDamage \\
\hline CartPrimerType \\
CartPrimerFinish \\
CartPrimerLacquer \\
CartModifications \\
CartForeignMat \\
CartBreechface \\
CartExtractor \\
CartEjector \\
CartChamber \\
CartFPinImpression \\
IBISEntry \\
EntryDate \\
ResonNoEntry \\
IBISCorrelation \\
DateCreated \\
Comments \\
Results \\
\hline
\end{tabular}


The various fields of the chemical residue worksheet table are shown in Table 10-

3. Chemical residue worksheet data is stored and maintained in this data table

Table 10-3: Chemical Residue Worksheet Table

tbl_FireArms_ChemResidue

LabCaseID

ItemID

SampleID

IDLocation

Analyst

Witness

SpecDesc

ChemTest

Reagent

MGTest

DTest

SRTest

MGResult

DResult

SRResult

DateCreated

Comments

Results 
The various fields of the examination worksheet - A table are shown in Table 10-4. Examination worksheet - A data is stored and maintained in this data table

Table 10-4: Examination Worksheet -A Table

\begin{tabular}{|c|c|c|}
\hline 8 & LabCaseID & 人 \\
\hline 8 & ItemID & \\
\hline 8 & SampleID & \\
\hline & IDLocation & \\
\hline & Analyst & \\
\hline & Witness & \\
\hline & SafetyPos & \\
\hline & HammerPos & \\
\hline & Safetyopn & \\
\hline & GunManufacturer & \\
\hline & GunSerialNo & \\
\hline & GunImporter & \\
\hline & GunType & \\
\hline & GunFinish & \\
\hline & GunMagCapacity & \\
\hline & GunMechanism & \\
\hline & GunBillength & \\
\hline & GunModel & \\
\hline & GunOverLength & \\
\hline & GunCaliber & \\
\hline & GunWt & \\
\hline & GunGripType & \\
\hline & ForeignMat & \\
\hline & TriggerPull & \\
\hline & ActionType 1 & \\
\hline & ActionType2 & \\
\hline & ActwtNum & \\
\hline & ActwtDeno & \\
\hline & Actwt1 & \\
\hline & Actwt2 & \\
\hline & Actwt3 & \\
\hline & BoreCondition & \\
\hline & PowderParticles & \\
\hline & SafetyRecall & \\
\hline & AmmuType & \\
\hline & TestFires 1 & \\
\hline & TestFires2 & \\
\hline & AmmuBrand1 & $v$ \\
\hline
\end{tabular}


The various fields of the examination worksheet table are shown in Table 105. Examination worksheet data is stored and maintained in this data table

Table 10-5: •Distance Determination Worksheet Table

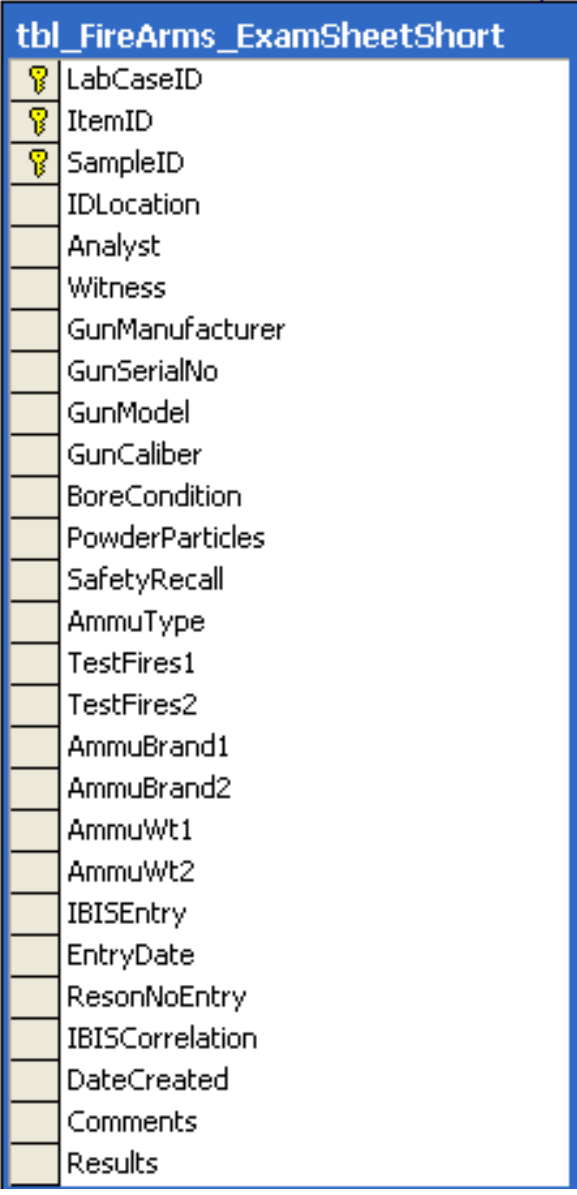


The various fields of the tool mark worksheet table are shown in Table 10-6. Tool mark worksheet data is stored and maintained in this data table

Table 10-6: Tool Mark Worksheet Table

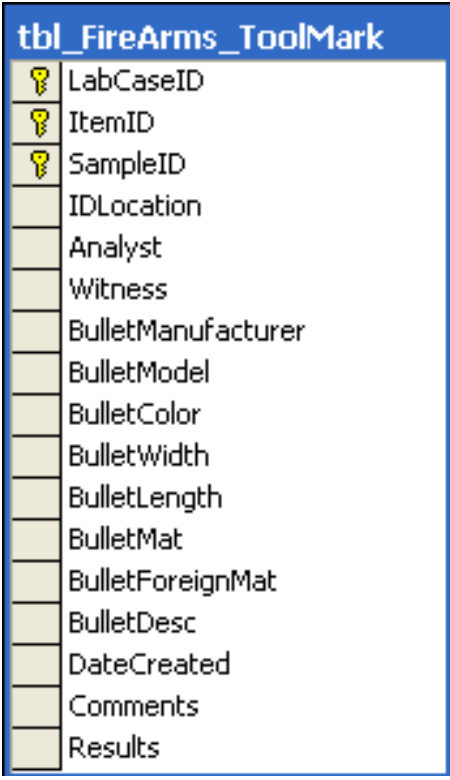


The various fields of the visual micro residue examination worksheet table are shown in Table 10-7. Visual micro residue examination worksheet data is stored and maintained in this data table

Table 10-7: Visual Micro Residue Examination Worksheet Table

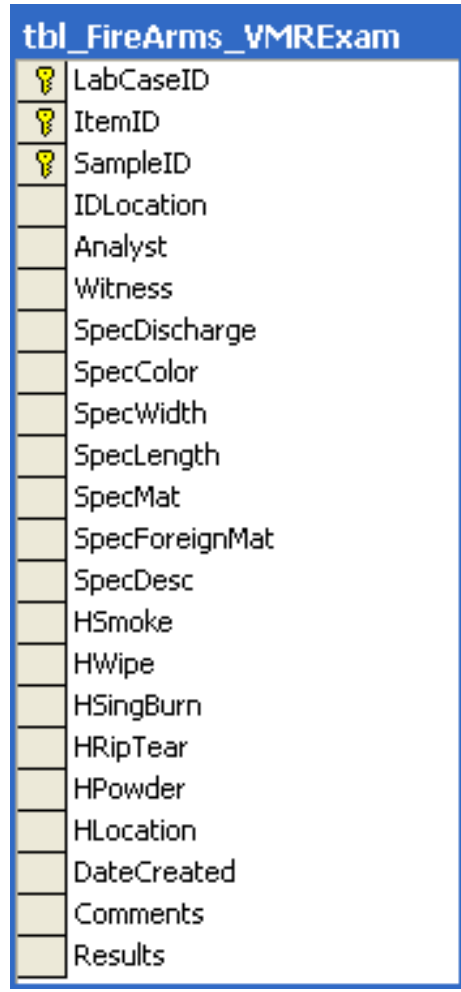




\subsubsection{Working with Fire Arms}

Fire Arms home page requires username and password to verify user credentials, as shown in the Figure 10.5. After verifying credentials, a user is directed to list of items assigned to him/her for further processing.

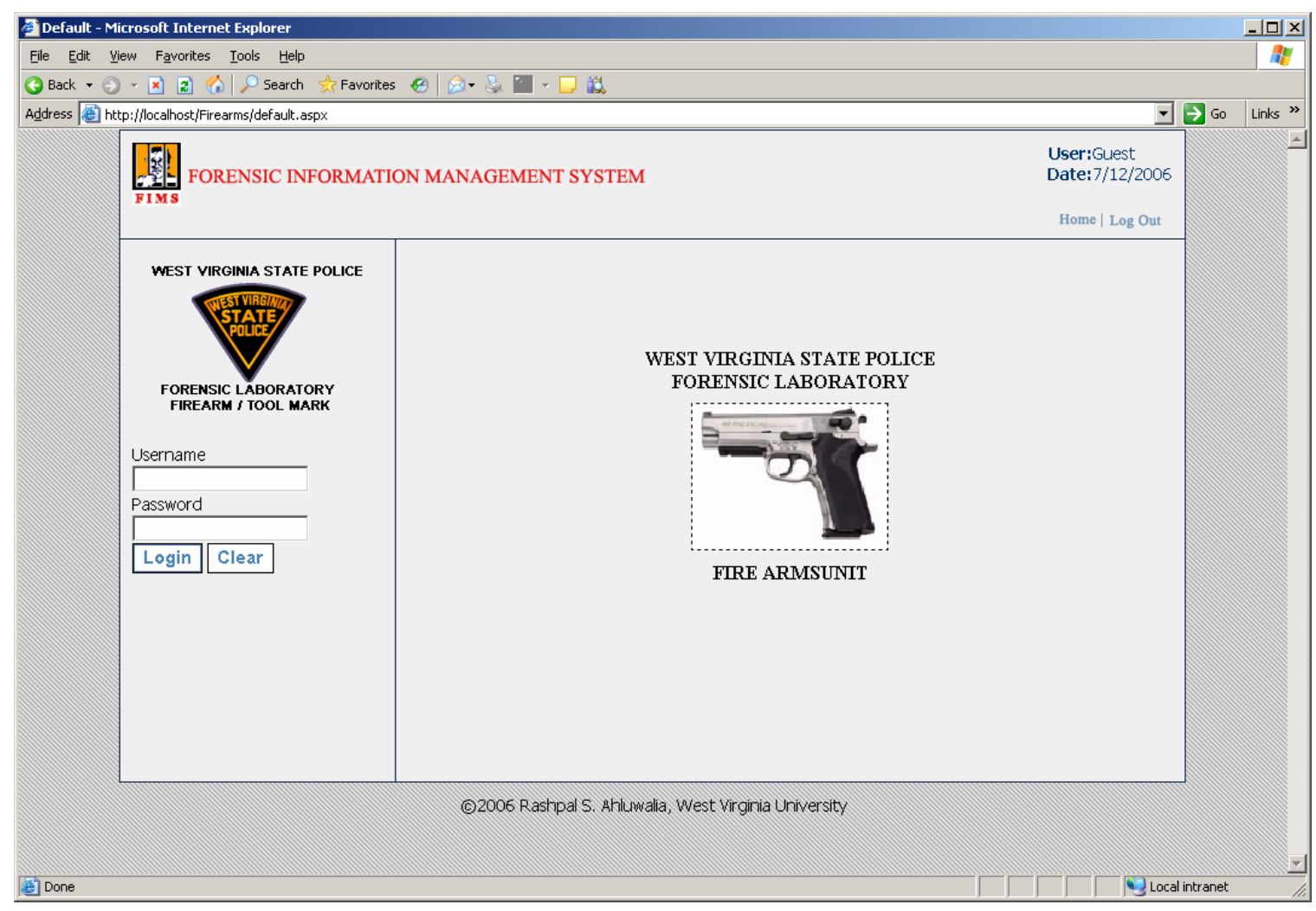

Figure 10.5: Fire Arms Home Page 


\section{View Items Assigned}

Figure 10.6 shows the list of items assigned to the analyst by the laboratory administration unit for further analysis. Initially, job status for the item assigned is set to "Job Pending". The "View Details" hyperlink enables an analyst to view the details of the case. If the list of items assigned grows long i.e. more than 15 items, the record set is divided into multiple pages. Analysts can navigate between different pages by clicking on the header and footer navigation bar of the list.

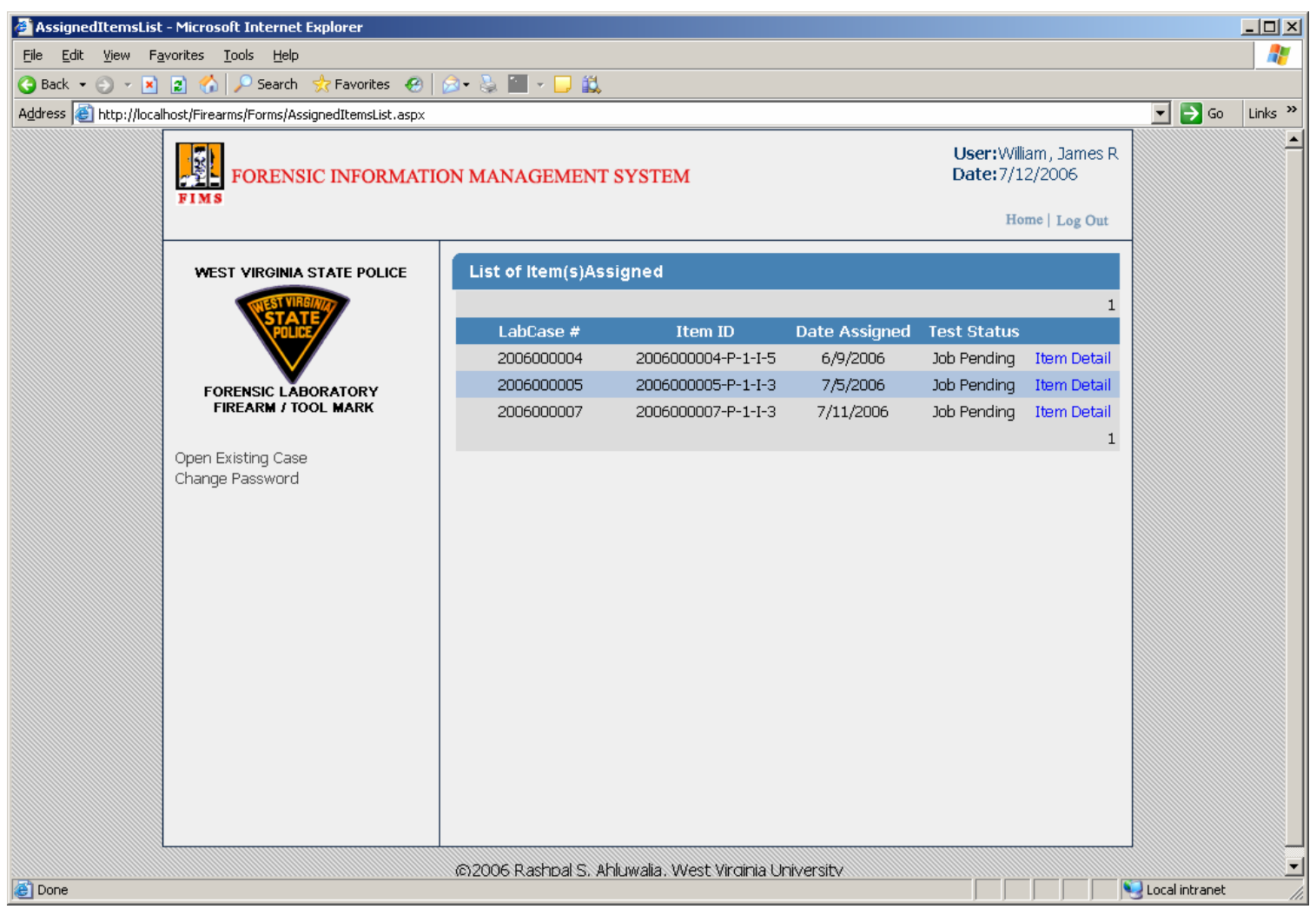

Figure 10.6: Assigned Item List 


\section{Item Picture Management}

This section describes the procedure of attaching pictures with an item. Analysts can perform two kinds of operation:

- View Pictures

- Upload Pictures

Analysts can access this section by clicking on the "View/Upload Item Photo" hyperlink in the item details section as shown in Figure 10.9. View picture section list the pictures already attached to the item with their thumbnails as shown in Figure 10.7. Analysts can also view the full size image by clicking on the image thumbnail. Full size image opens in a new window, which can be closed after use.

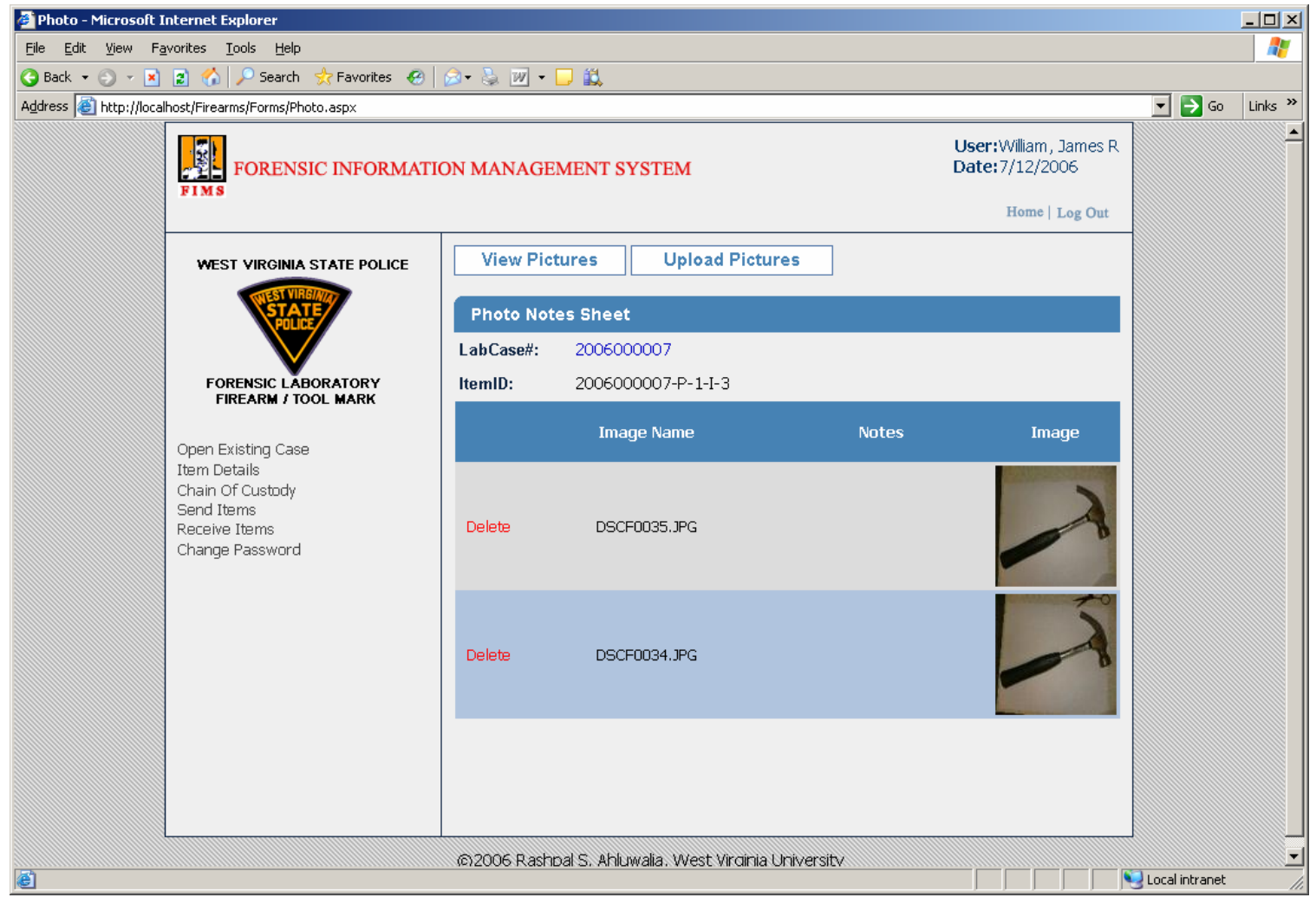

Figure 10.7: View Item Photo 
The analysts can upload any digital photo in JPG format directly into the database using this section as shown in Figure 10.8. To upload an image into database, analysts are required to browse for the image path, set other required fields and click on the "Upload the file" hyperlink. On successful upload a thumbnail appears on the bottom of the page with a success message.

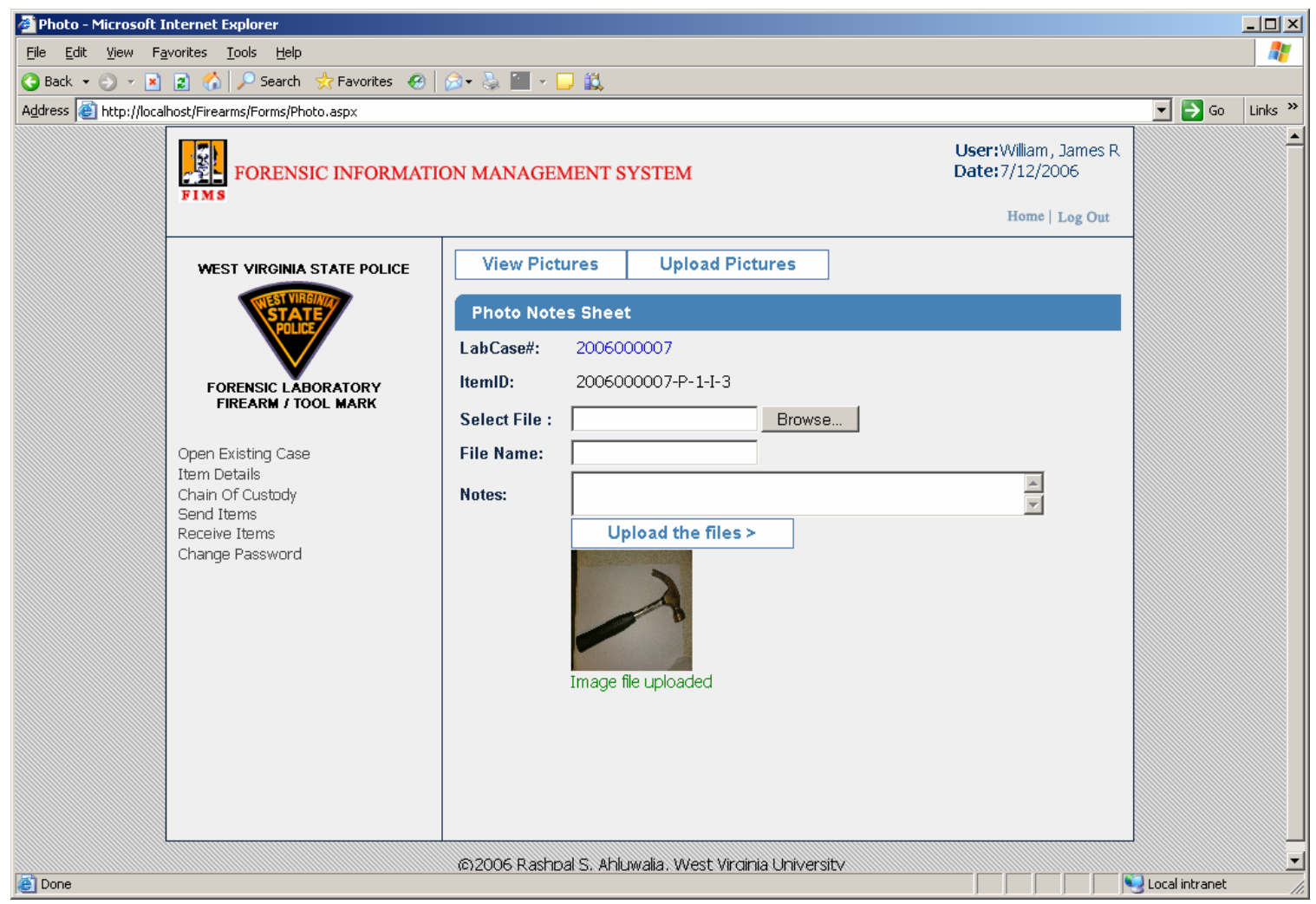

Figure 10.8: Upload Item Picture Section 


\section{Working with Samples}

Analysts receive items from the CEP unit for further analysis and processing. Once received items are sub-divided into samples for conducting requested tests. Each item can be divided into one or more samples according to the requirement and complexity of the required test.

Analysts can create new, update or delete existing samples from the item details section as shown in Figure 10.9. To add a new sample analyst is required to provide description of sample and then click on "Add Sample" hyperlink. FIMS application automatically adds the time stamp to the item created. Initially, sample test status is set to "Test Pending" for the sample. Sample information can be updated by clicking on “Edit” hyperlink and existing sample can be deleted from the system by clicking on "Delete" hyperlink.

This section also enables analysts to view the final sample, sample photo, item, and item photo report. To access the individual sample/sample photo report analyst can click on "View Report" or "Photo Sheet" hyperlink. To view comprehensive item report analysts can click on "Item Report” or "Item Photo Report” at the bottom of the page. 
To complete the process for item testing, the analyst is required to click on "Item Test Complete" button, as shown in Figure 10.9. Once clicked, analyst will not be able to make any alterations to the existing item test report.

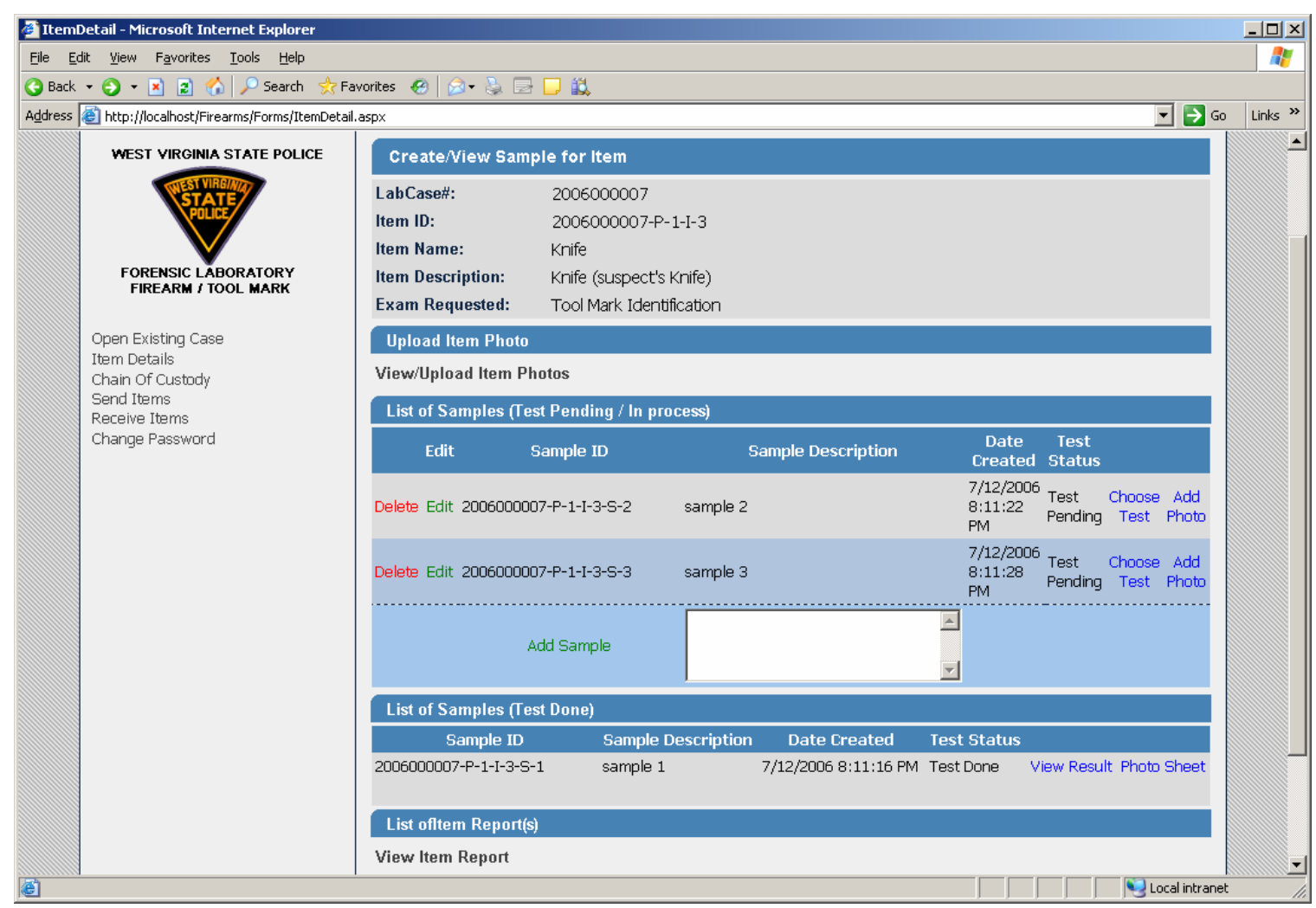

Figure 10.9: Item Detail Section 
Analysts can access this section by clicking on the "View/Upload Sample Photo" hyperlink in the item details section as shown in Figure 10.9. View picture section list the pictures already attached to the item with their thumbnails as shown in Figure 10.10. Analysts can also view the full size image by clicking on the image thumbnail. Full size image opens in a new window, which can be closed after use.

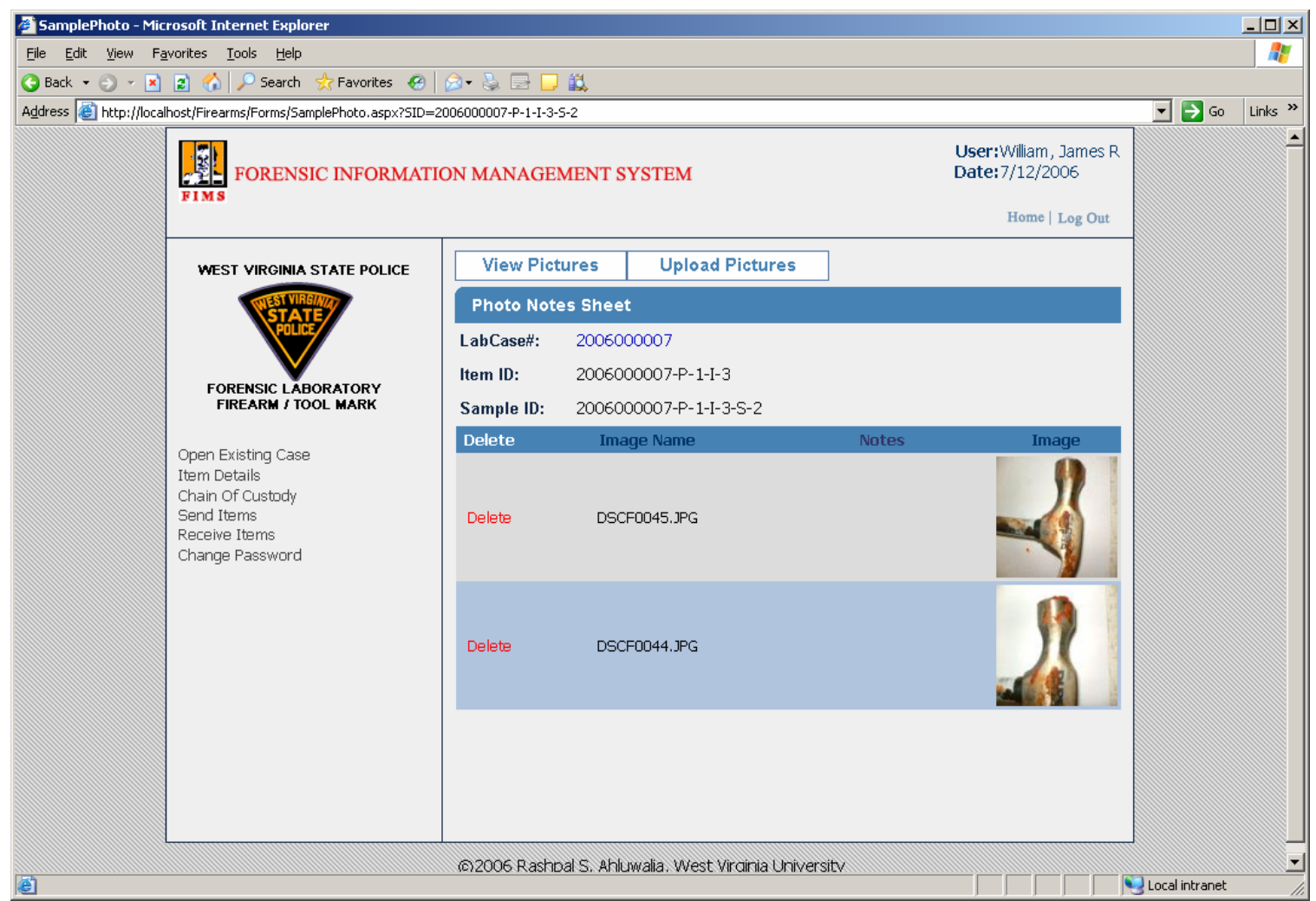

Figure 10.10: View Picture Section 
Analysts can upload any digital photo in JPG format directly into the database using this section as shown in Figure 10.11. To upload an image analysts are required to browse for the image path, set other required fields and click on the "Upload the file" hyperlink to upload the image into database. On successful upload a thumbnail appears on the bottom of the page with a success message.

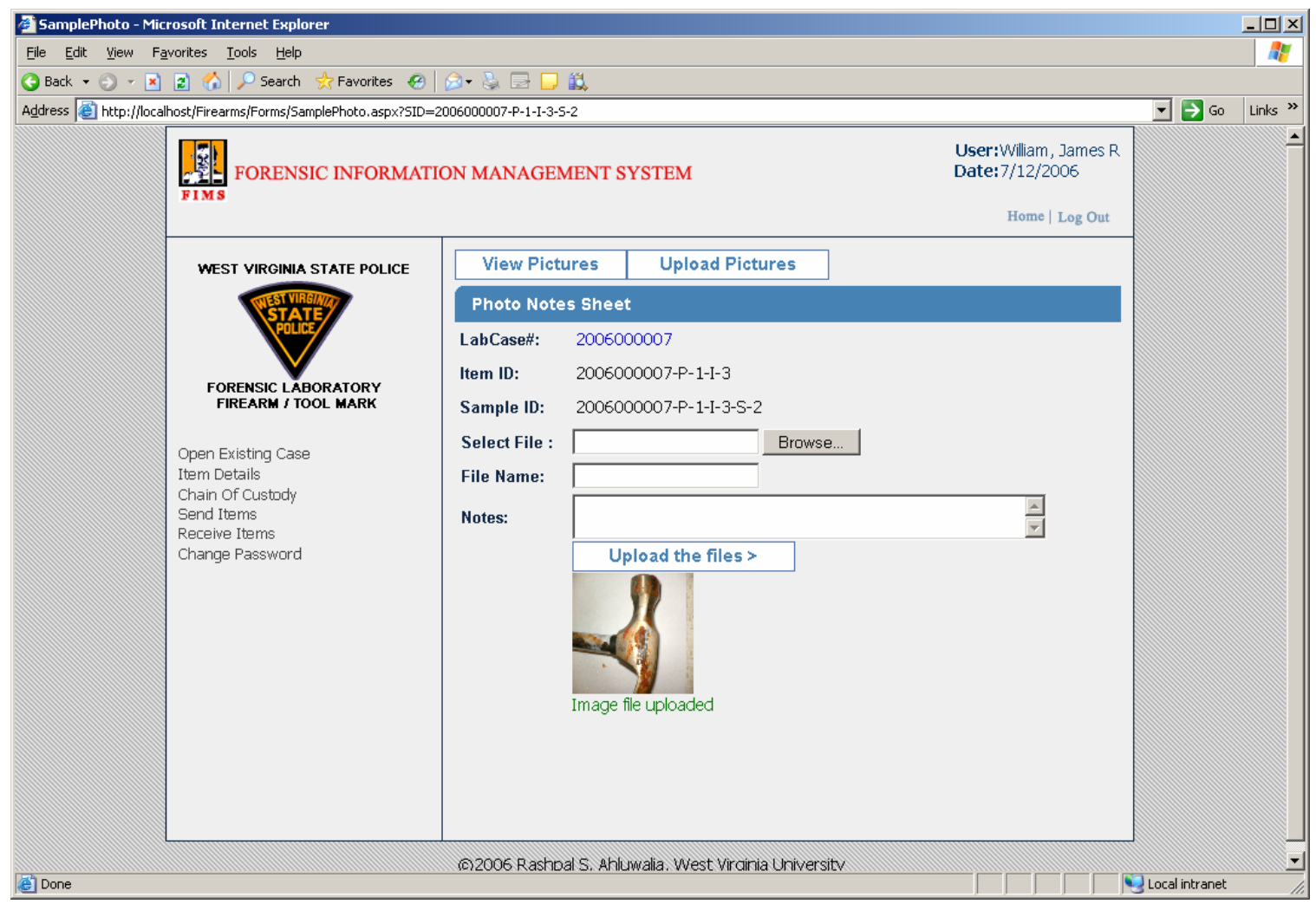

Figure 10.11: Upload Sample Picture Section 


\section{Choosing Test worksheet}

After sample creation, analysts are required to decide what kinds of tests are required? Analysts are required to make decision of choosing type of test manually. Once decision is made the analyst can see the list of tests available, and then click "Choose Test" hyperlink as shown in Figure 10.12. FIMS application remembers the chosen sample ID during the process.

The worksheet for the fire arms/tool marks are:

- Bullet Characteristics

- Fire Cartridge Case

- Chemical Residue

- Comparison Examination

- Distance Determination

- Examination sheet - A

- Examination sheet - B

- Examination Short Form

- Tool Mark Analysis

- Visual Micro Residue 


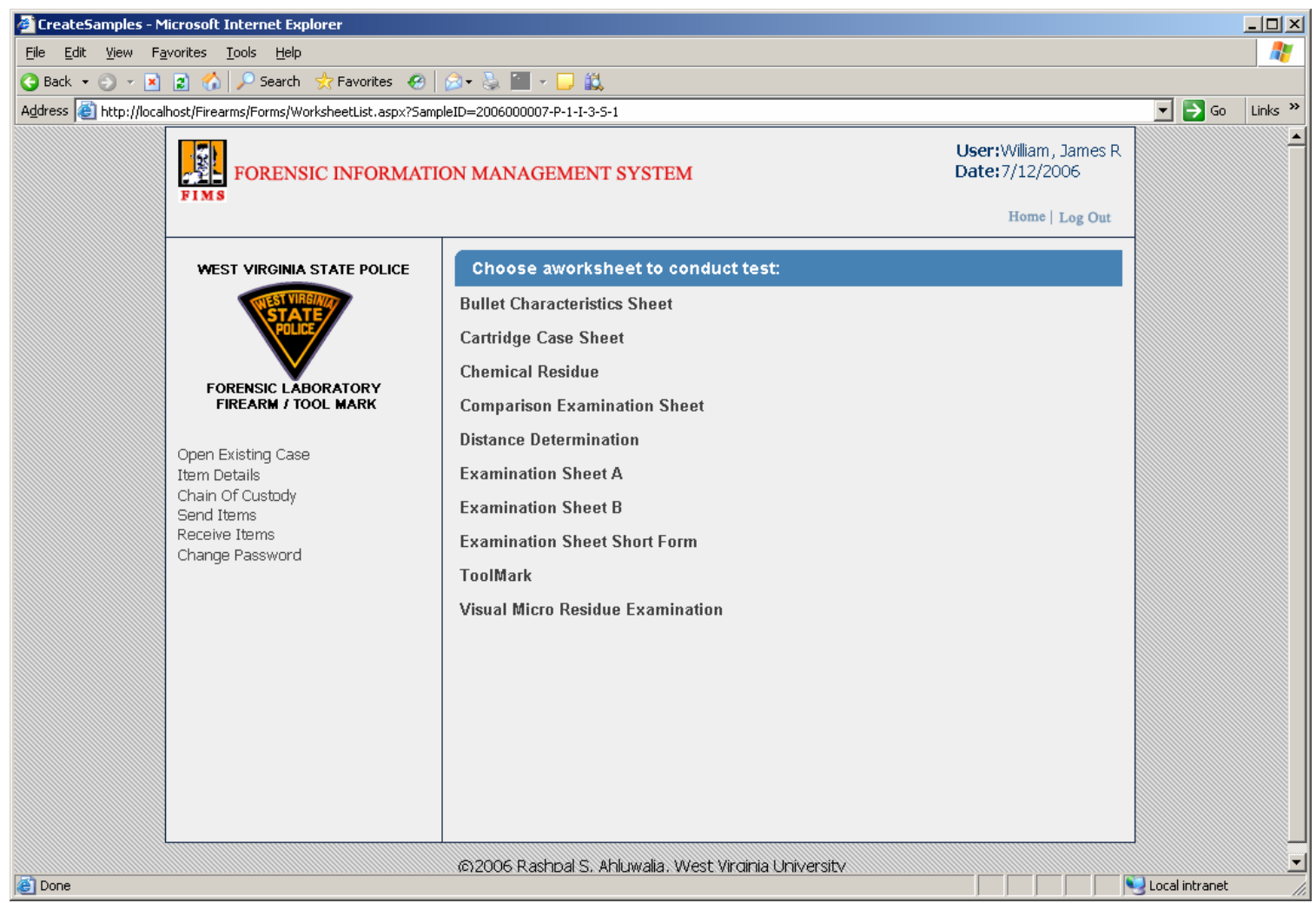

Figure 10.12: Choose Sample Test Section 


\section{Bullet Characteristics Worksheet}

Bullet characteristics worksheet is used for filling information pertaining to bullet characteristics test performed on the sample by the analyst. After filling the required data fields, analyst is required to click on "Save Results" button located on top of the page, as shown in Figure 10.13. After saving the worksheet data, analyst can finalize and close the worksheet by clicking on “Test Done” button. The analyst cannot make any alterations in the worksheet once the "Test Done" button is clicked.

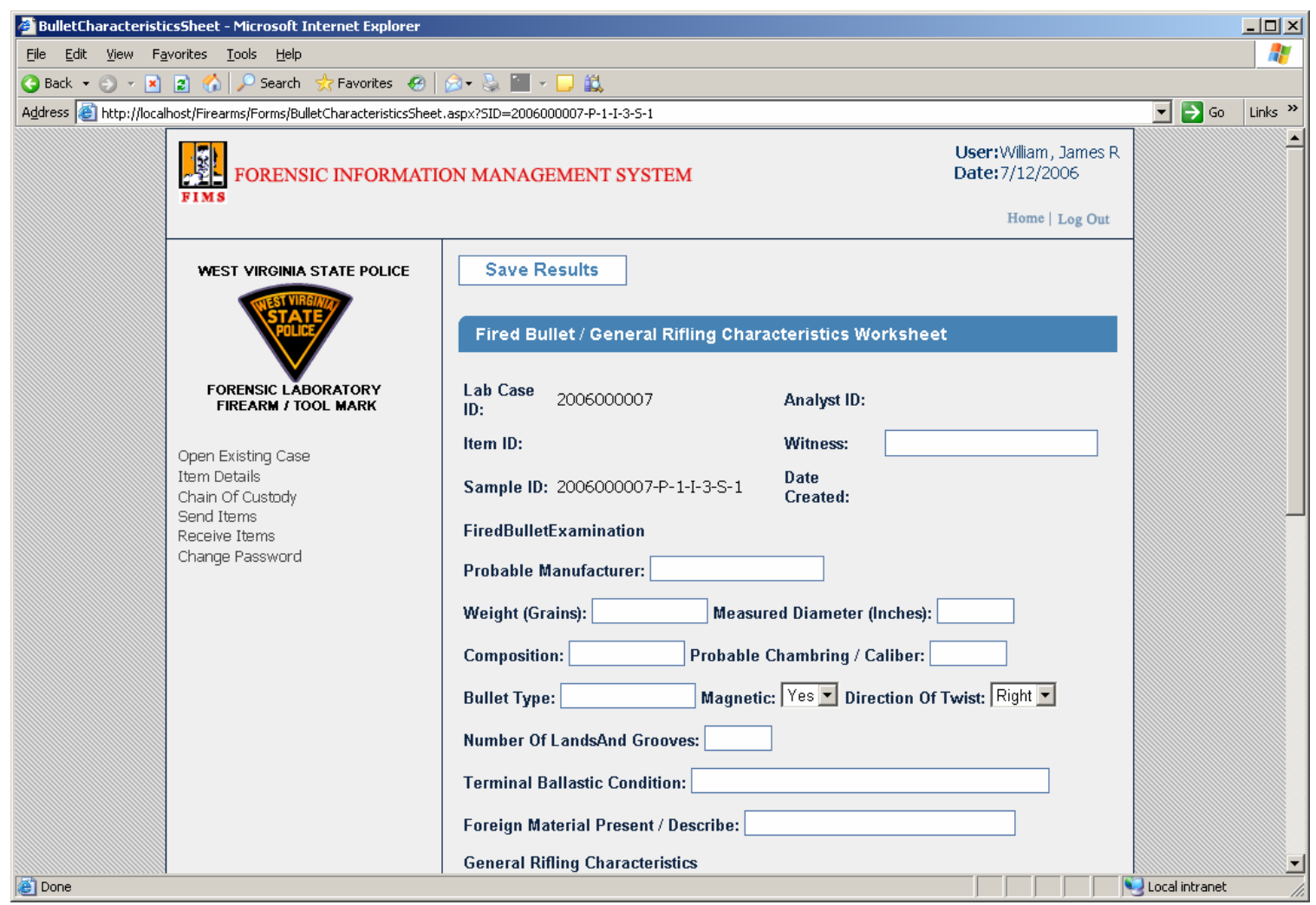

Figure 10.13: Bullet Characteristics Worksheet 


\section{Fire Cartridge Case Worksheet}

Fire cartridge case worksheet is used for filling information pertaining to fire cartridge case test performed on the sample by the analyst. After filling the required data fields, analyst is required to click on "Save Results" button located on top of the page, as shown in Figure 10.14. After saving the worksheet data, analyst can finalize and close the worksheet by clicking on "Test Done" button. The analyst cannot make any alterations in the worksheet once the "Test Done" button is clicked.

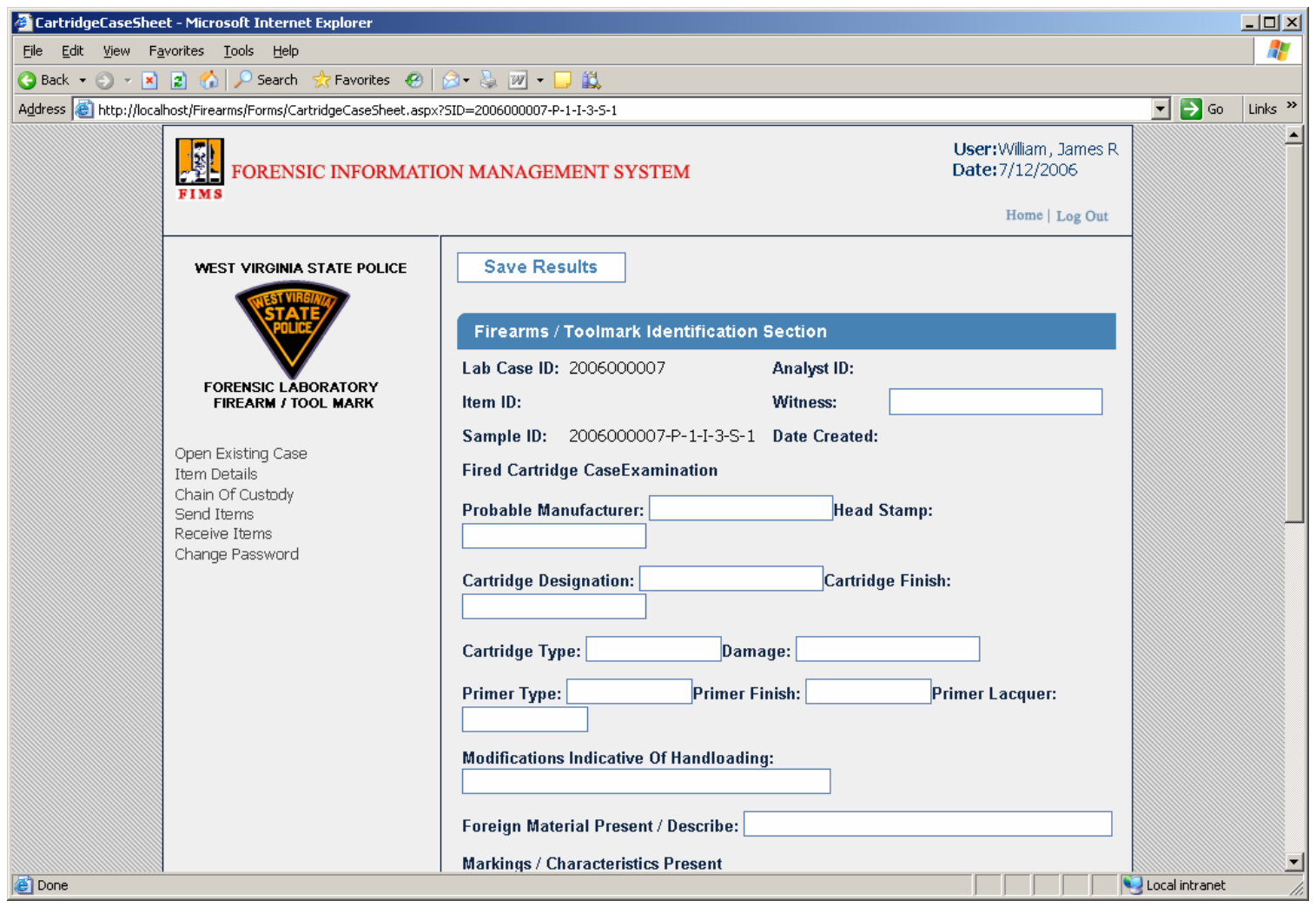

Figure 10.14: Fire Cartridge Case Worksheet 


\section{Chemical Residue Examination Worksheet}

Chemical residue examination worksheet is used for filling information pertaining chemical residue examination test performed on the sample by the analyst. After filling the required data fields, analyst is required to click on "Save Results" button located on top of the page, as shown in Figure 10.15. After saving the worksheet data, analyst can finalize and close the worksheet by clicking on "Test Done" button. The analyst cannot make any alterations in the worksheet once the "Test Done" button is clicked.

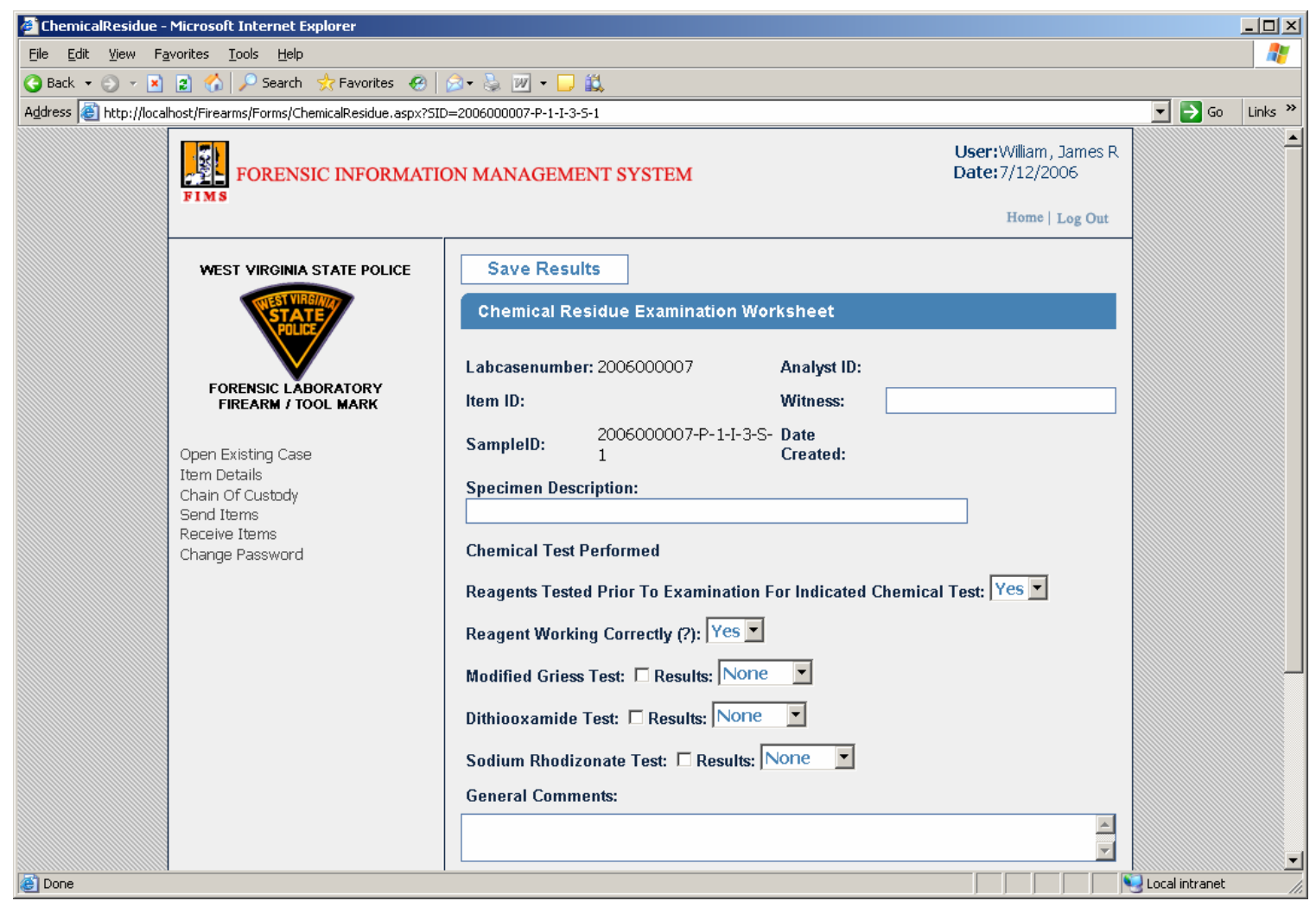

Figure 10.15: Chemical Residue Examination Worksheet 


\section{Comparison/Examination Worksheet}

Comparison/Examination worksheet is used for filling information pertaining to comparison/examination test performed on the sample by the analyst. After filling the required data fields, analyst is required to click on "Save Results" button located on top of the page, as shown in Figure 10.16. After saving the worksheet data, analyst can finalize and close the worksheet by clicking on “Test Done” button. The analyst cannot make any alterations in the worksheet once the "Test Done" button is clicked.

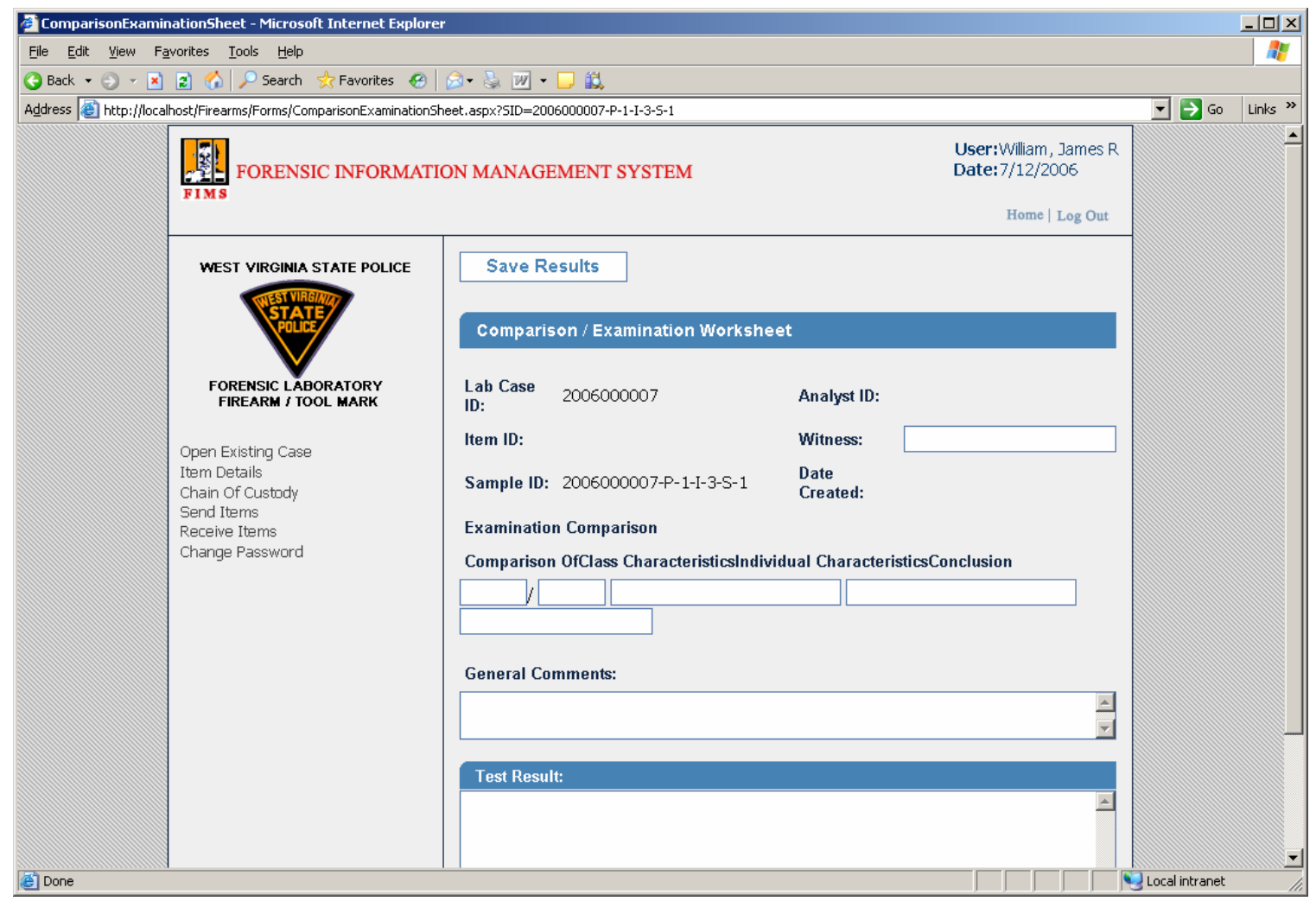

Figure 10.16: Comparison/Examination Worksheet 


\section{Distance Determination Worksheet}

Distance Determination Worksheet is used for filling information pertaining to distance determination test performed on the sample by the analyst. After filling the required data fields, analyst is required to click on "Save Results" button located on top of the page, as shown in Figure 10.17. After saving the worksheet data, analyst can finalize and close the worksheet by clicking on "Test Done" button. The analyst cannot make any alterations in the worksheet once the "Test Done" button is clicked.

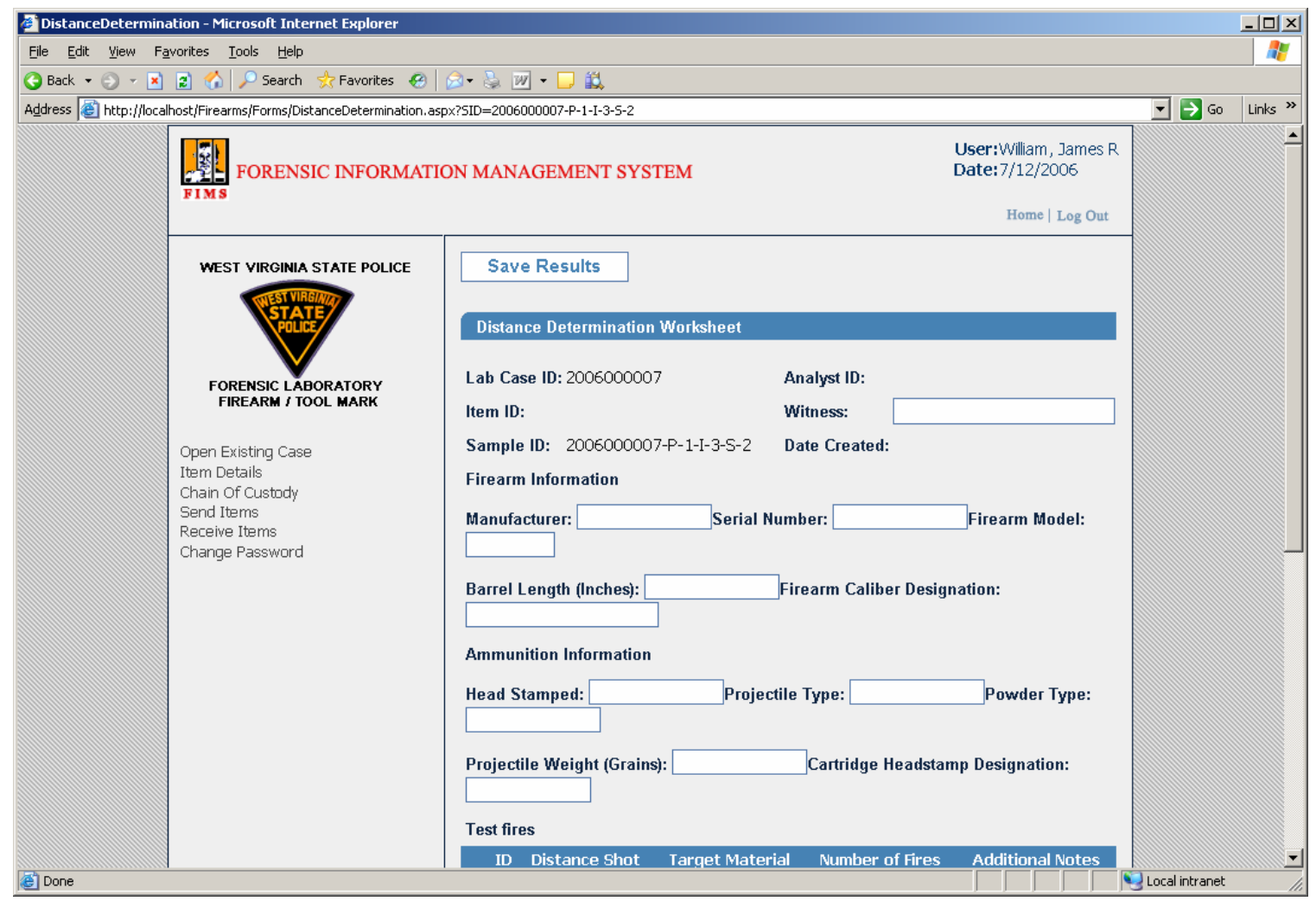

Figure 10.17: Distance Determination Worksheet 


\section{Firearm Examination Worksheet - A}

Firearm examination worksheet - A is used for filling information pertaining to firearm examination - A test performed on the sample by the analyst. After filling the required data fields, analyst is required to click on "Save Results" button located on top of the page, as shown in Figure 10.18. After saving the worksheet data, analyst can finalize and close the worksheet by clicking on "Test Done" button. The analyst cannot make any alterations in the worksheet once the "Test Done" button is clicked.

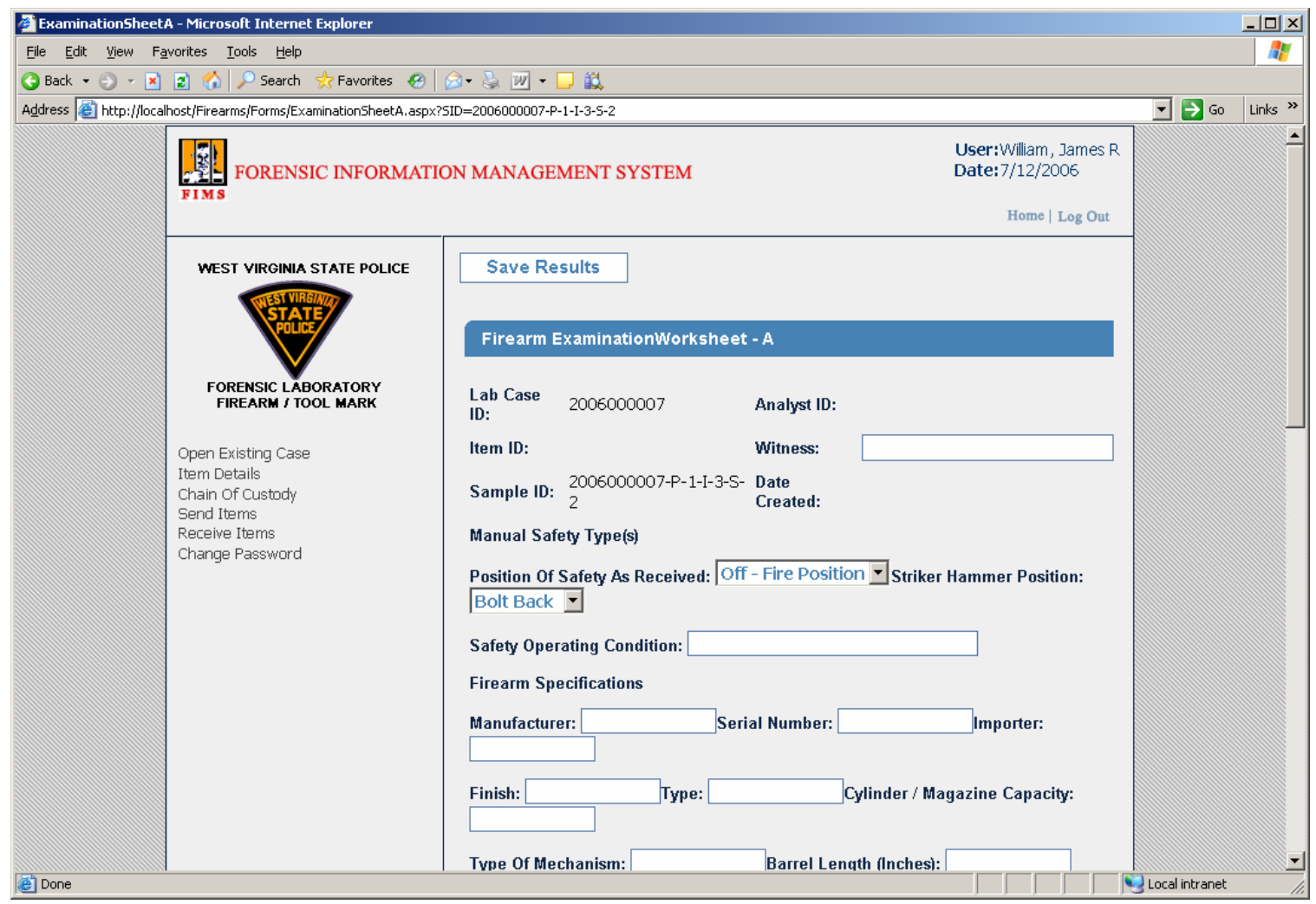

Figure 10.18 Firearm Examination Worksheet - A 


\section{Firearm Examination Worksheet - B}

Firearm examination worksheet - B is used for filling information pertaining fire examination worksheet - B test performed on the sample by the analyst. After filling the required data fields, analyst is required to click on "Save Results" button located on top of the page, as shown in Figure 10.19. After saving the worksheet data, analyst can finalize and close the worksheet by clicking on "Test Done” button. The analyst cannot make any alterations in the worksheet once the "Test Done" button is clicked.

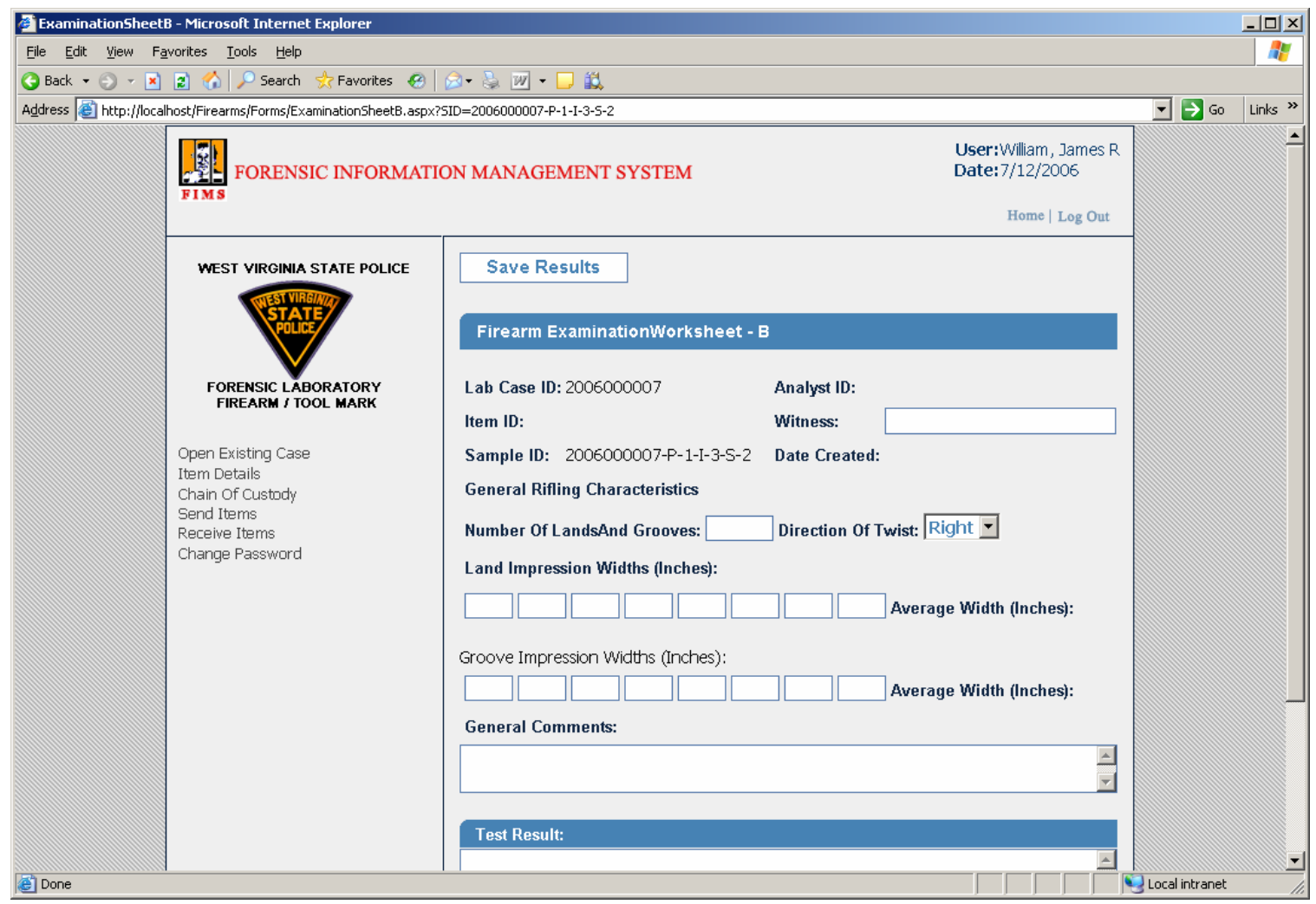

Figure 10.19: Examination sheet - B 


\section{Examination Sheet (Short Form) Worksheet}

Examination sheet (Short Form) worksheet is used for filling information pertaining to examination sheet (Short Form) worksheet test performed on the sample by the analyst. After filling the required data fields, analyst is required to click on "Save Results" button located on top of the page, as shown in Figure 10.20. After saving the worksheet data, analyst can finalize and close the worksheet by clicking on "Test Done" button. The analyst cannot make any alterations in the worksheet once the "Test Done" button is clicked.

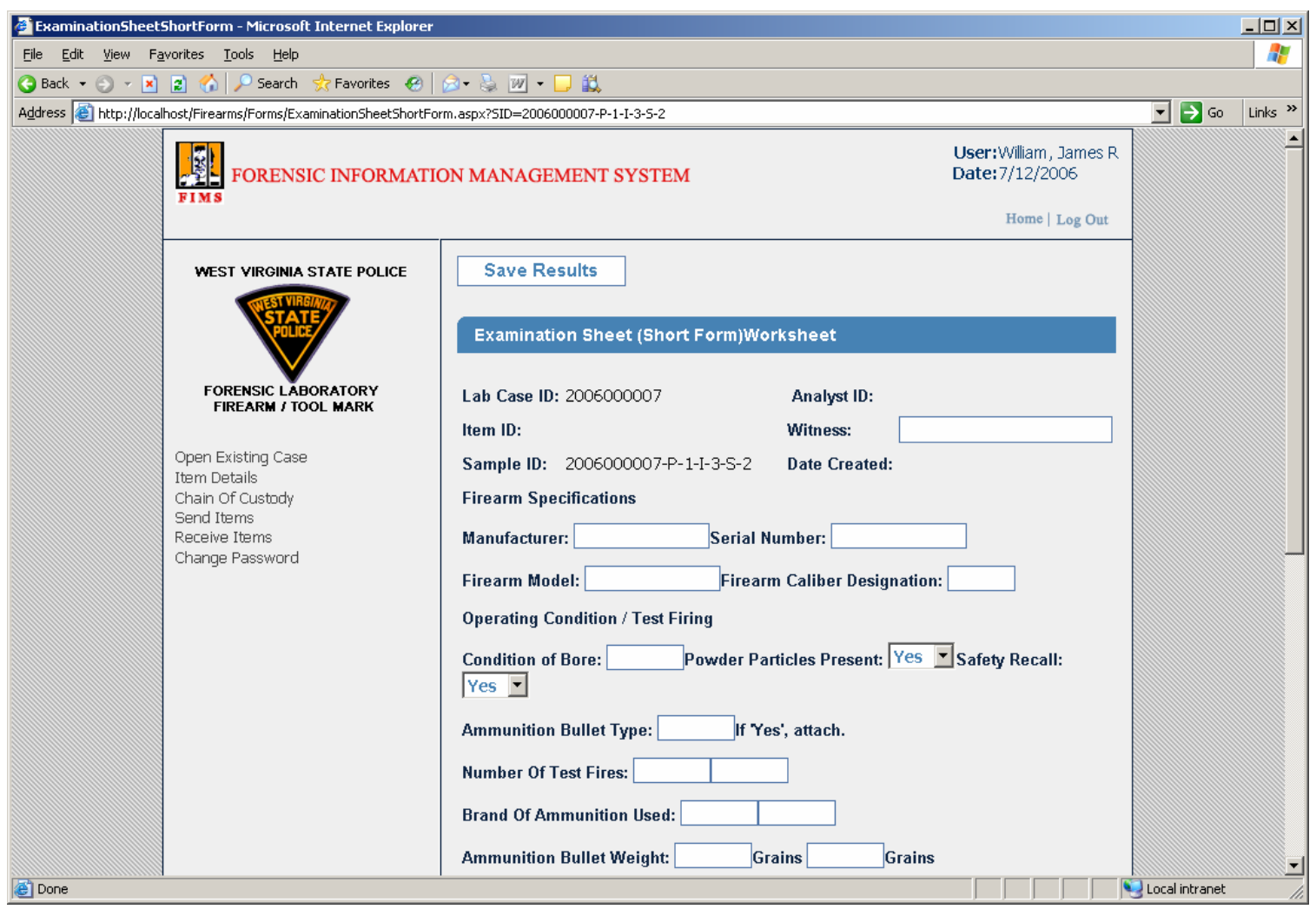

Figure 10.20: Examination sheet (Short Form) Worksheet 


\section{Tool Mark Worksheet}

Tool Mark Worksheet is used for filling information pertaining Tool Mark Worksheet test performed on the sample by the analyst. After filling the required data fields, analyst is required to click on "Save Results" button located on top of the page, as shown in Figure 10.21. After saving the worksheet data, analyst can finalize and close the worksheet by clicking on "Test Done" button. The analyst can not make any alterations in the worksheet once the "Test Done" button is clicked.

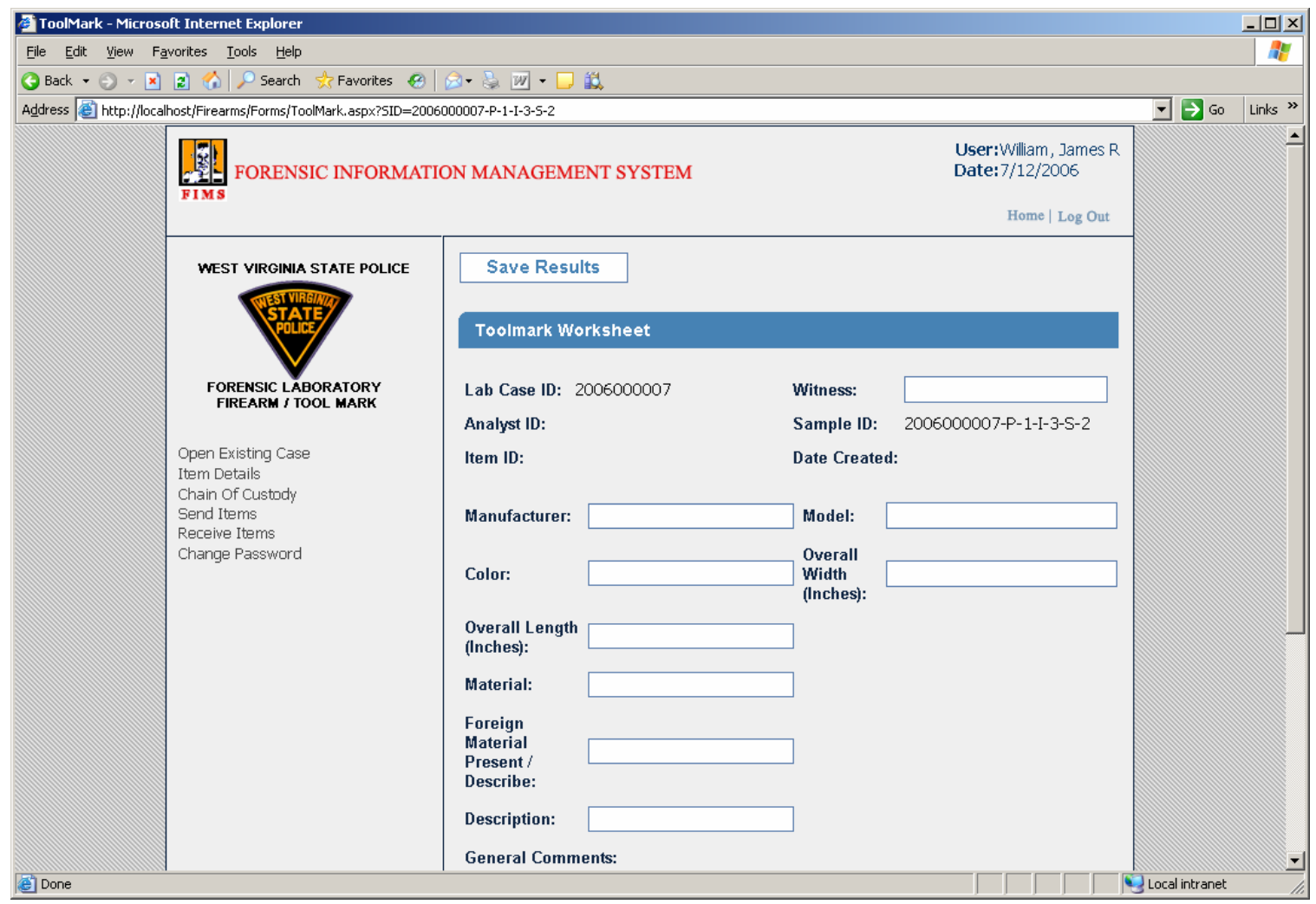

Figure 10.21: Tool Mark Worksheet 


\section{Visual/Microscopic Residue Examination Worksheet}

Visual/Microscopic residue examination worksheet is used for filling information pertaining visual/microscopic residue examination worksheet test performed on the sample by the analyst. After filling the required data fields, analyst is required to click on "Save Results" button located on top of the page, as shown in Figure 10.22. After saving the worksheet data, analyst can finalize and close the worksheet by clicking on "Test Done" button. The analyst cannot make any alterations in the worksheet once the "Test Done” button is clicked.

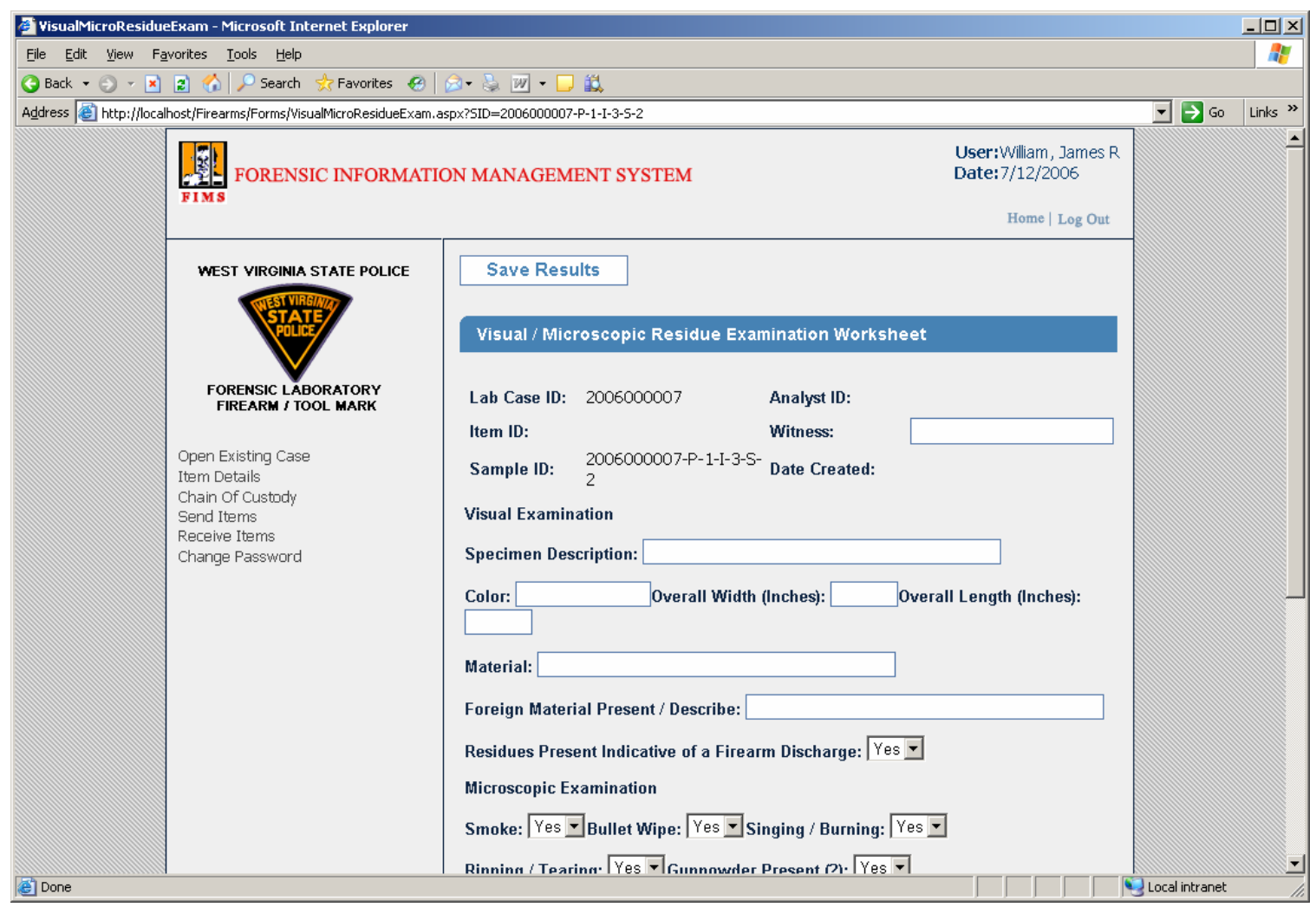

Figure 10.22: Visual/Microscopic Residue Examination Worksheet 


\section{Receive Item}

Once an item is sent by CEP unit, firearms unit analyst is responsible for receiving it by clicking on "Receive" button, under receive item section, as shown in Figure 10.23. This completes the handshaking process of item transfer between units.

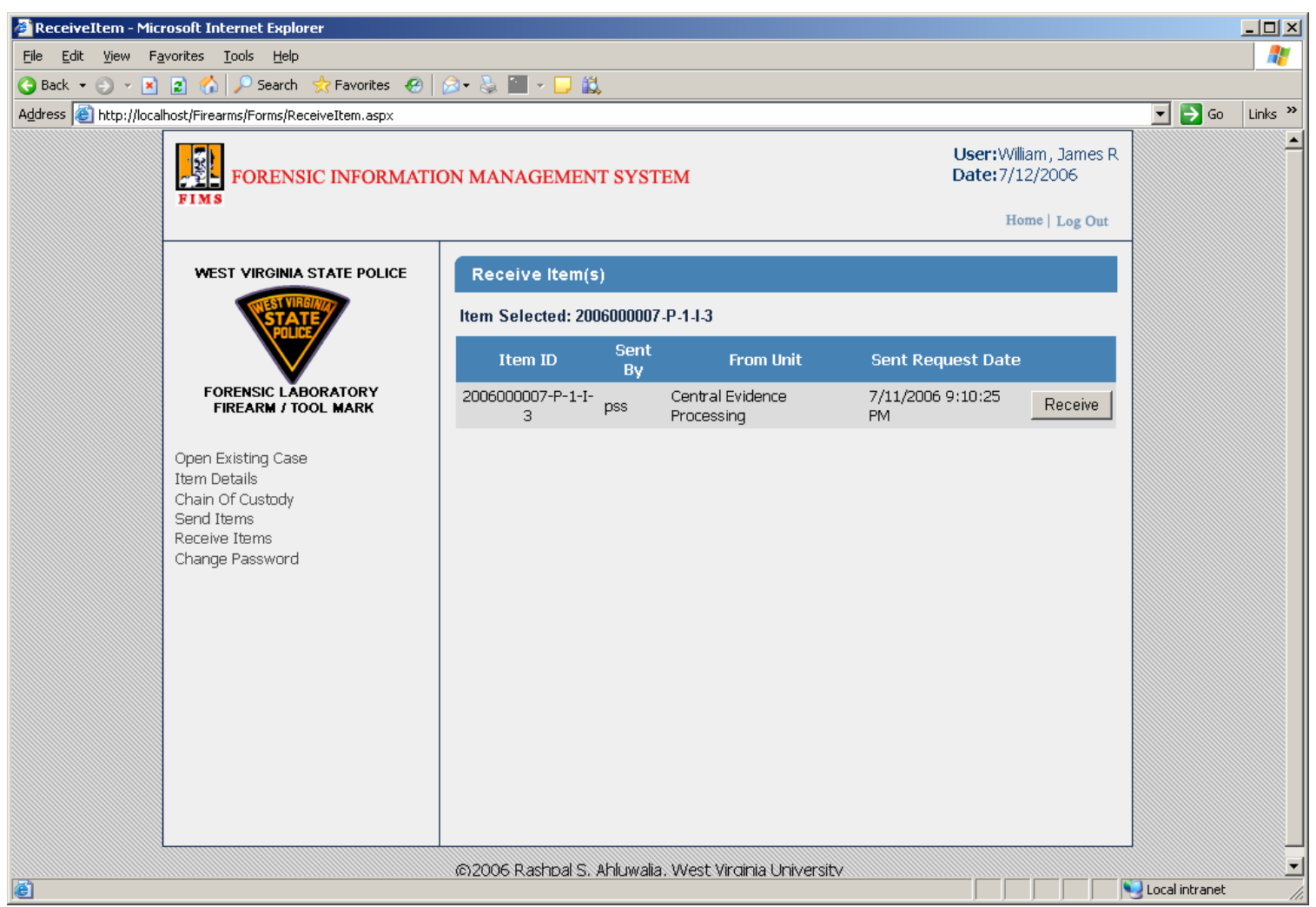

Figure 10.23: Receive Item Section 


\section{Send Item}

After completion of item testing, analyst is required to return the evidence item back to CEP unit. Figure 10.24 shows the section used to send an item back to the CEP unit. Analyst can use the drop down to select item ID and click on "Send" hyperlink, to send item back to CEP.

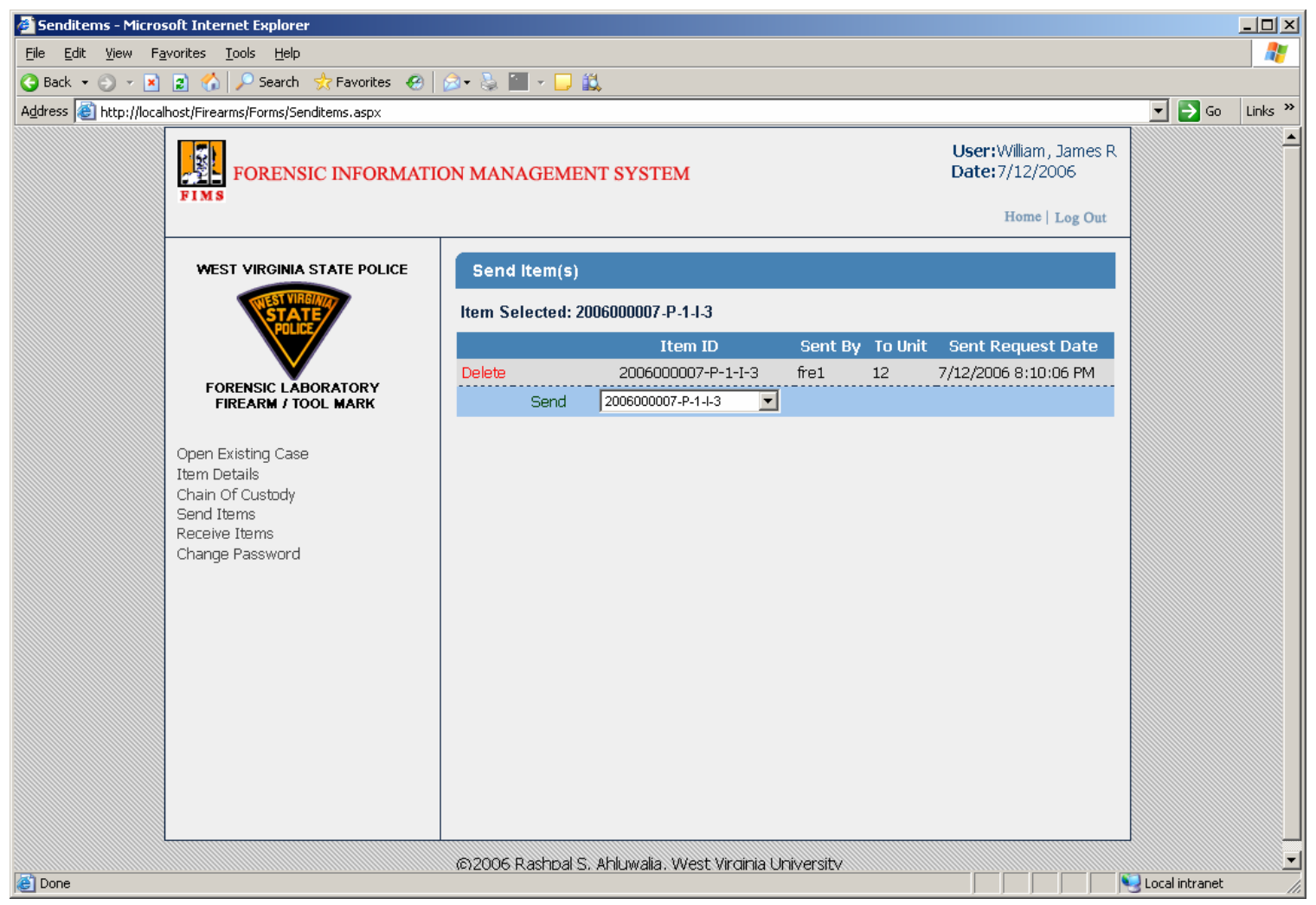

Figure 10.24: Send Item Section 


\section{Chain of Custody}

Item chain of custody information is shown in Figure 10.25. FIMS application records used ID, unit name, and time stamp for tracking item chain of custody.

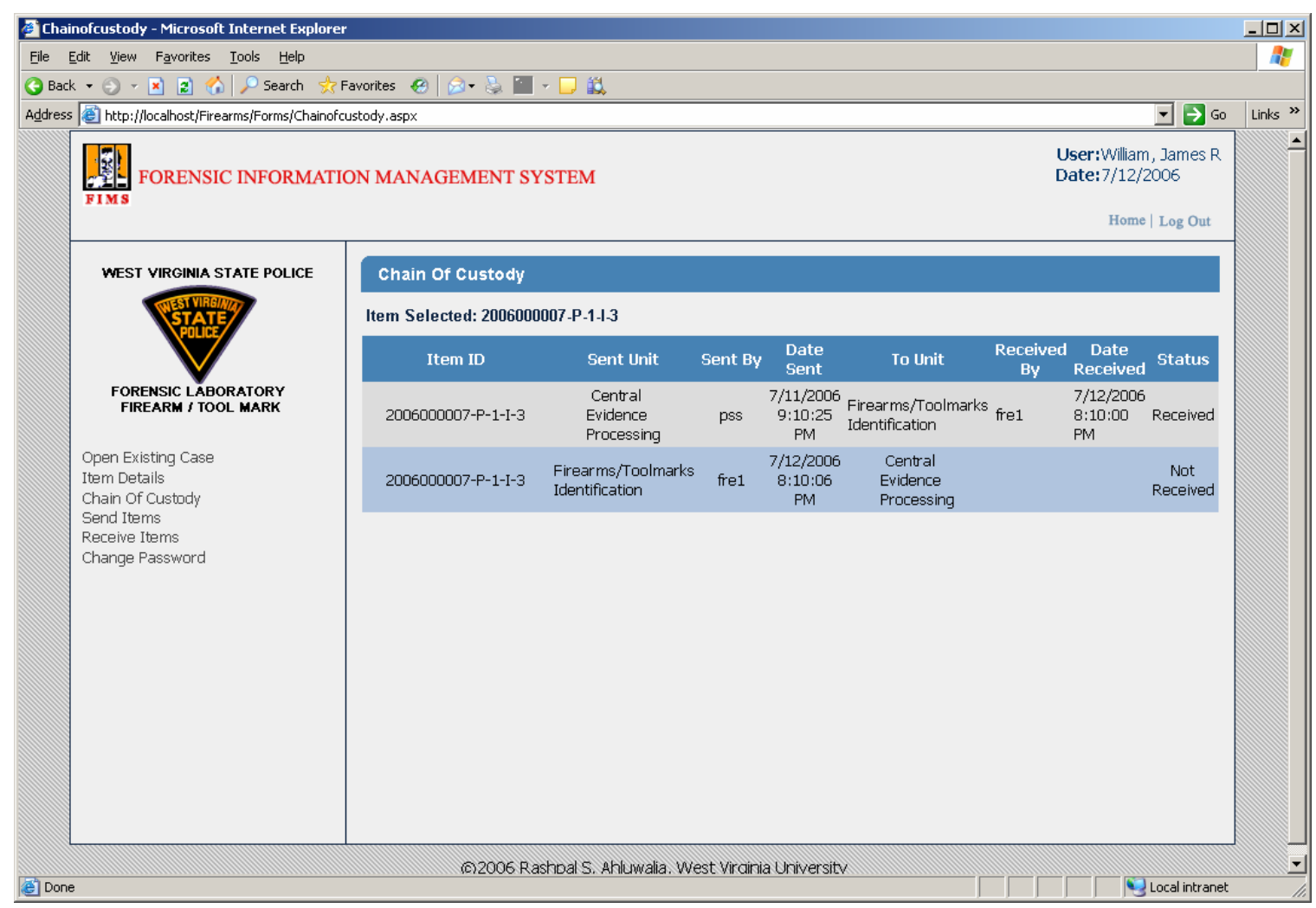

Figure 10.25: Item Chain Of Custody 


\section{Change User Password}

Analyst can change password through this section by providing appropriate credentials as shown in Figure 10.26

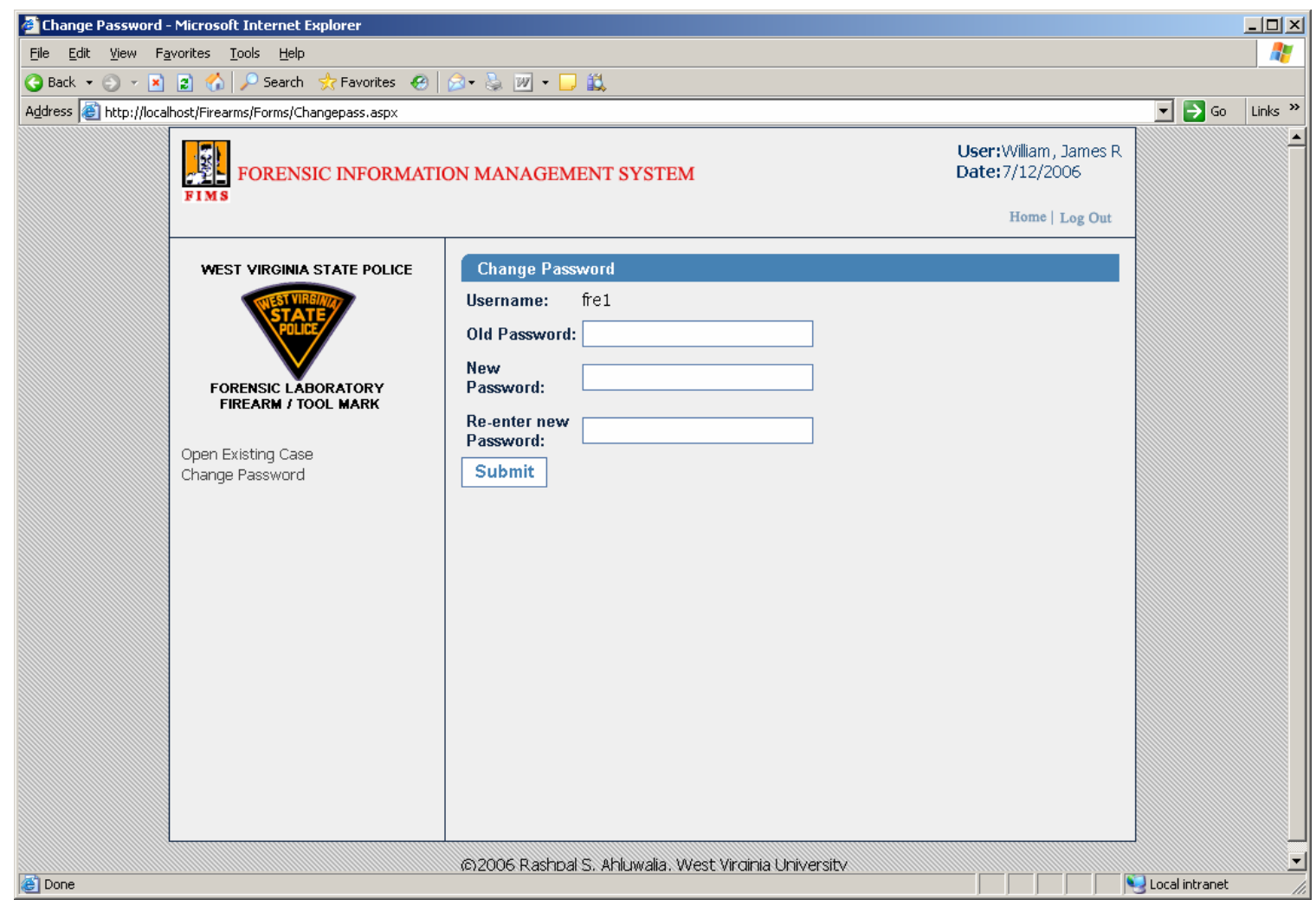

Figure 10.26: Change Password Section

\section{Exiting Fire Arms}

Users can terminate the current session by clicking on "Log Out” hyperlink on the top right corner of the page. 


\subsubsection{Generating Item and Sample Reports}

The analyst can generate the following types of reports

- Item Report

- Item Photo Report

- Sample Report

- Sample Photo Report

Figure 10.27 shows the item report, which is a cumulative report for all the samples. Figure 10.28 shows the sample report. This section also facilitate analyst to export report via PDF/MS-Word format, as shown in Figure 10.29 and Figure 10.30.

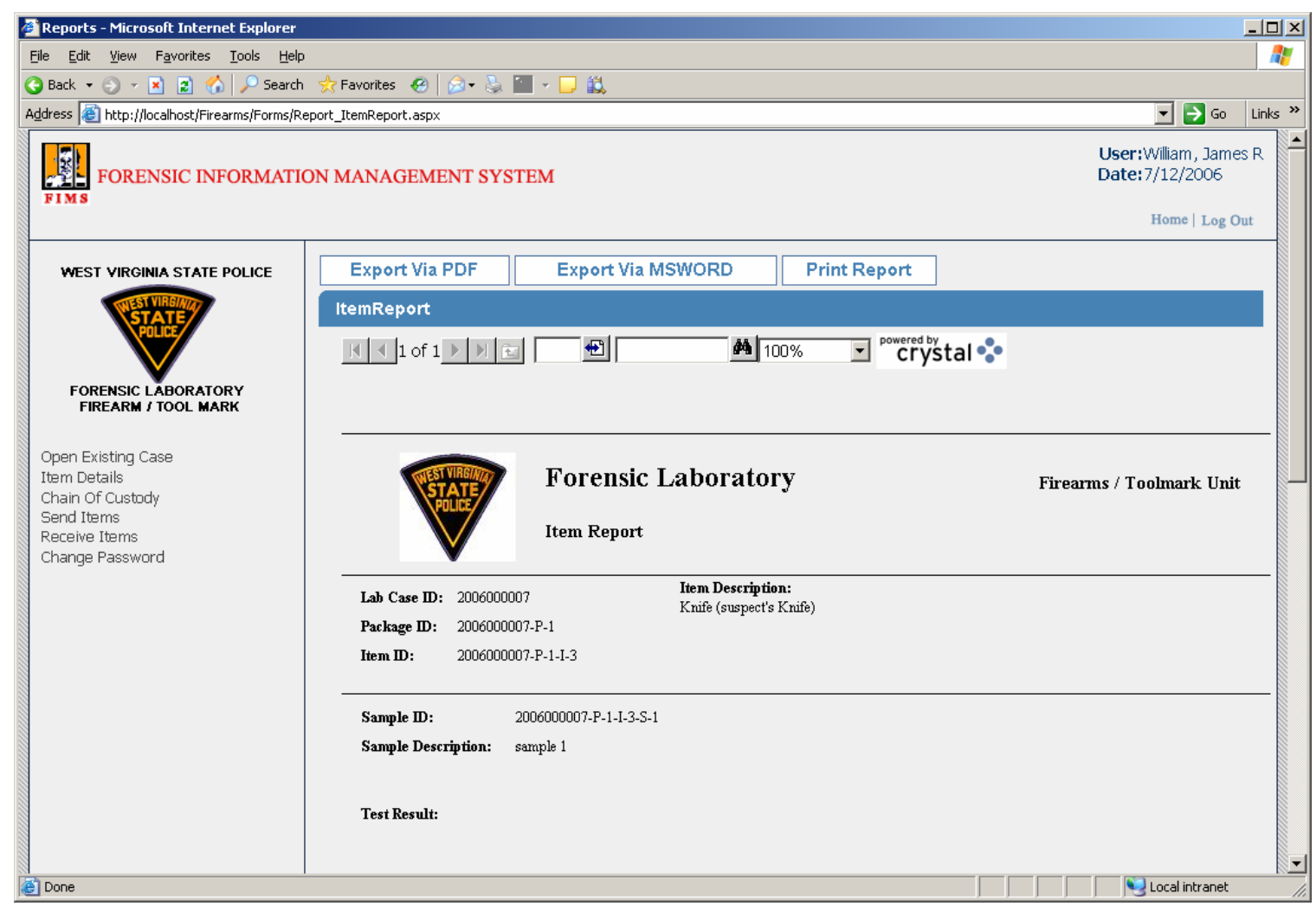

Figure 10.27: Item report 


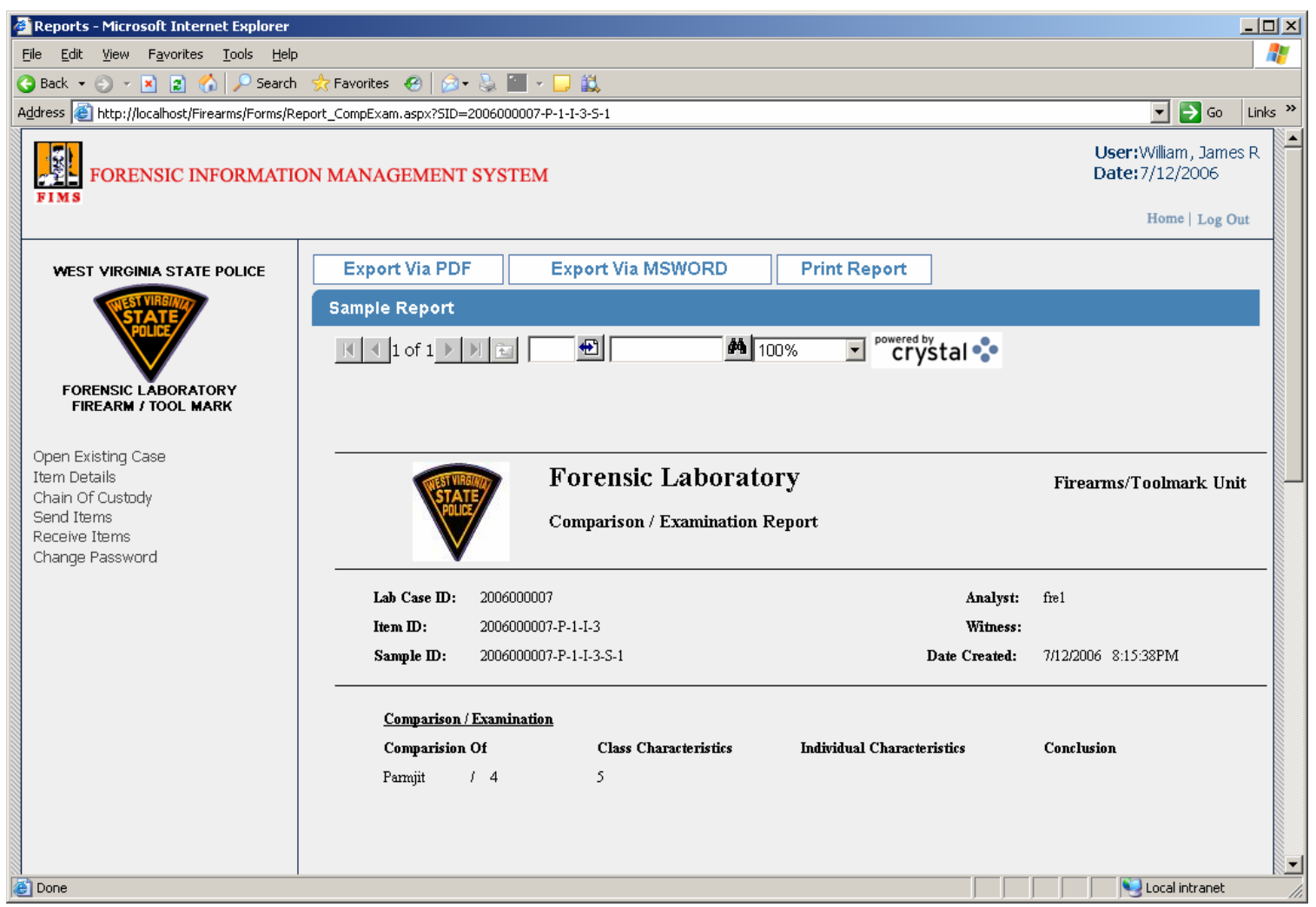

Figure 10.28: Sample Report 


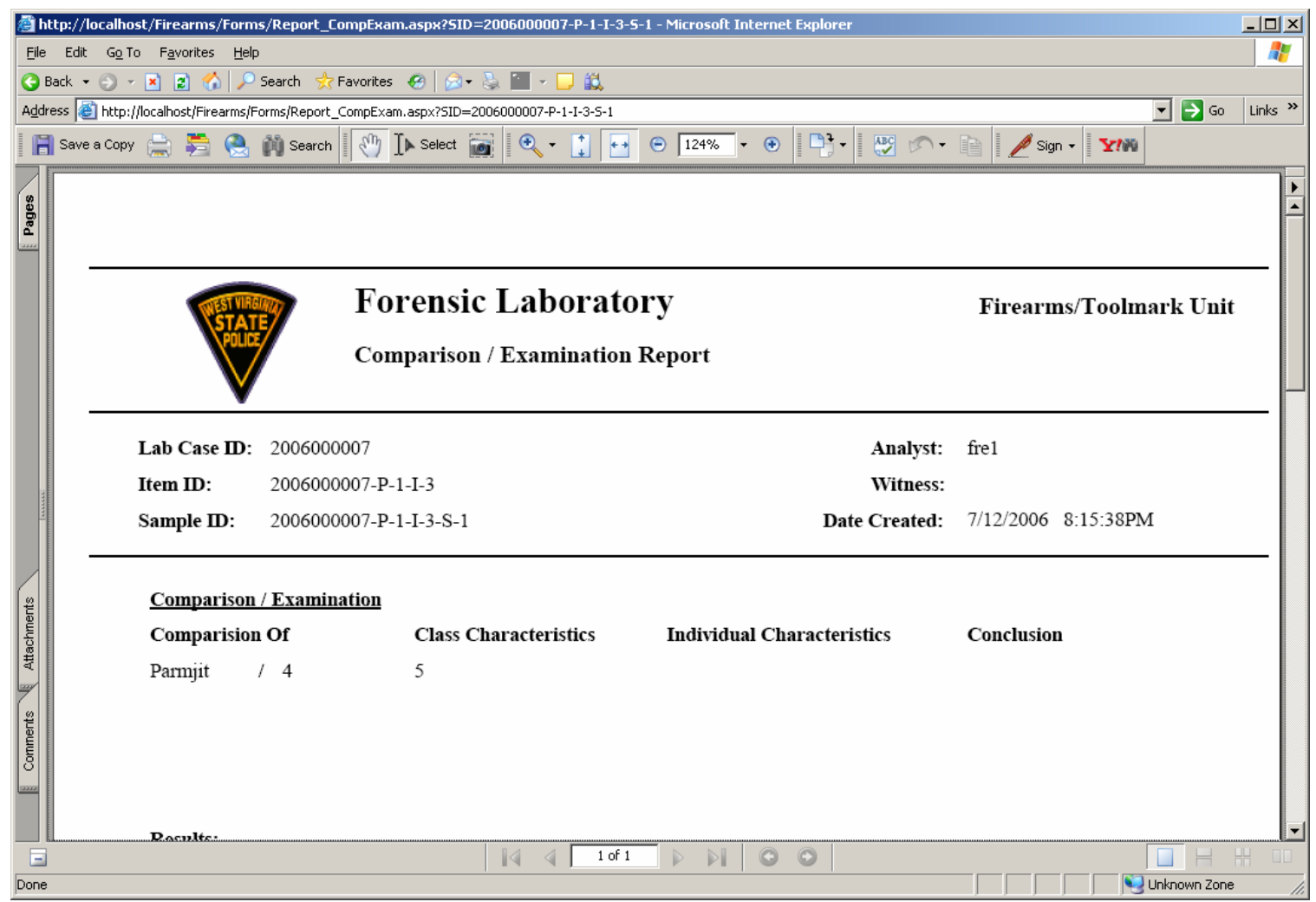

Figure 10.29: Sample Report in PDF Format

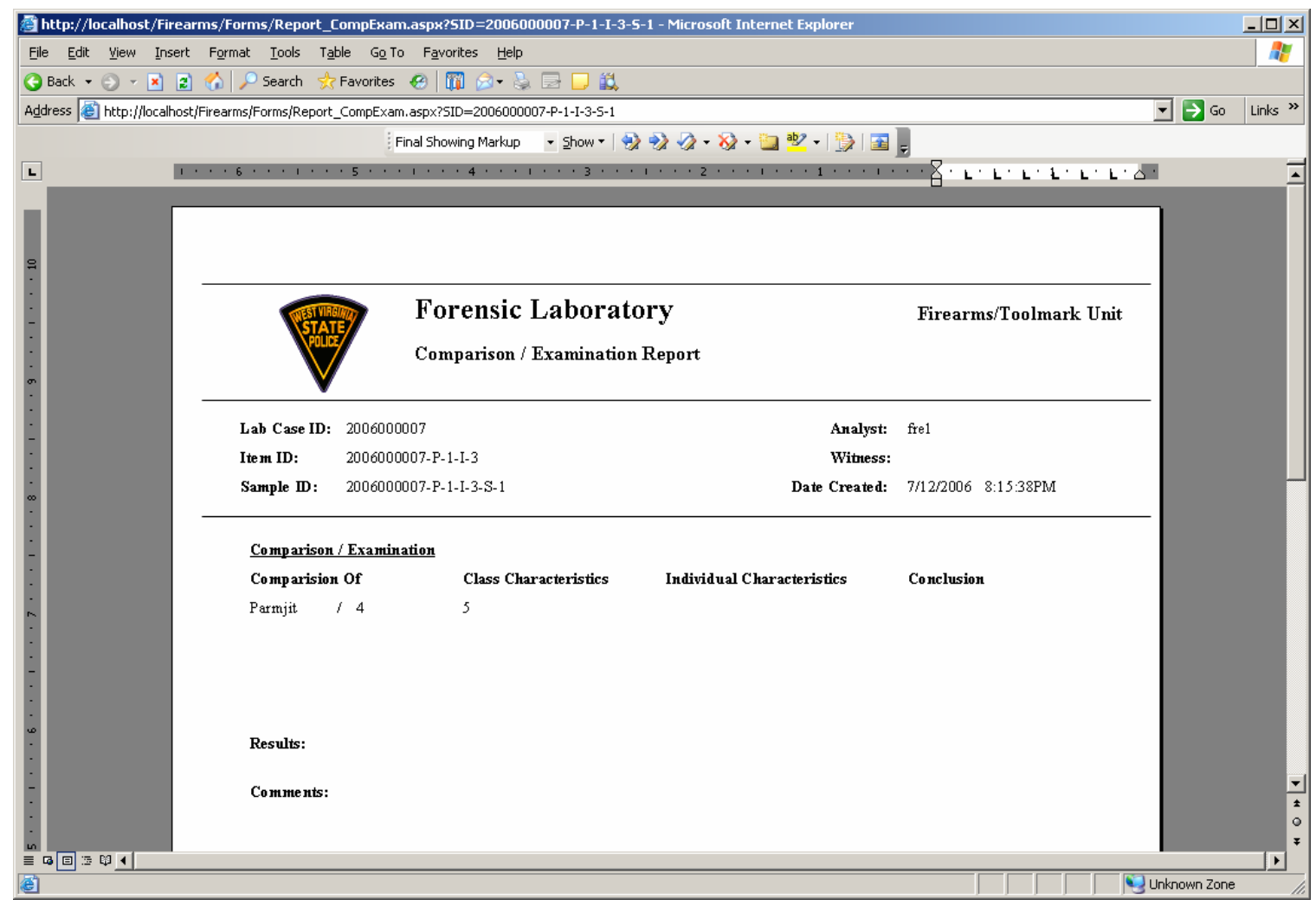

Figure 10.30: Item Report in MS Word Format 


\section{CHAPTER 11 \\ LATENT PRINTS}

\subsection{Existing Latent Prints Process}

A Latent Print Unit deals with hidden residue (perspiration, natural oils) left when the underside of the fingers and palms (or the underside of the bare toes and feet) come in contact with a surface. Because the impression is often invisible to the naked eye, it is called a latent or hidden print. If properly recovered, the latent print can lead the investigator to the perpetrator of the crime and later be used as evidence against that individual at a trial. The latent print evidence is put through a variety of physical and chemical processes designed to reveal the presence of latent impressions. The analyst in the latent prints unit receives the LEIF form and chain of custody form along with the evidence items. The analyst prepares samples and performs the required tests. After the tests are performed, appropriate forms are completed. The forms/worksheets used by this unit are: Analysis of Latent Prints (AL), Analysis of Exemplar Prints (AE), Comparison Evaluation \& Verification (CEV) worksheet, AFIS/IAFIS Launch (AI) worksheet, ACE worksheet (ACE), and Technical Procedures (TP) worksheet. Once the examinations are completed, the evidence is returned to the CER and the secretary prepares a draft report. The draft report is reviewed by the analyst/reviewer. After approval the final report is sent are returned to CER. Figure 11.1 shows the existing process for the latent prints unit. 


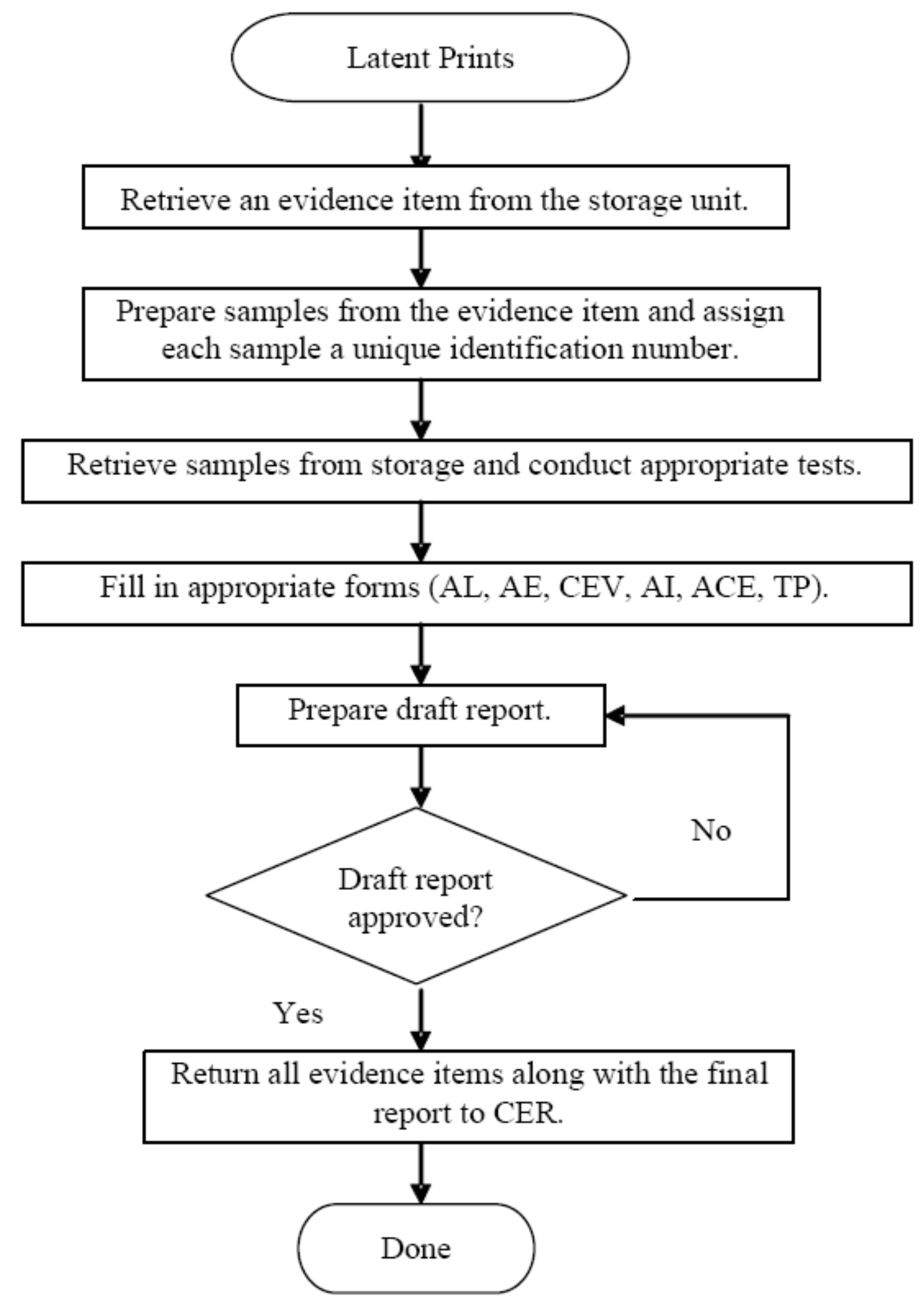

Figure 11.1: Existing Latent Prints Process 


\subsection{Re-engineering the Latent Prints Process}

The analyst in the latent prints unit receives the items assigned for further processing and analysis from CEP. Received items are sub-divided into samples for conducting the requested tests. An individual item may be divided into many samples according to the requirement and complexity of the required test. A report for each sample is generated. An item report is compiled by combining sample reports. After the report is received, the reports along with the test items are sent back to CEP for further processing. The evidence and information flow diagram is shown in Figure 11.2.

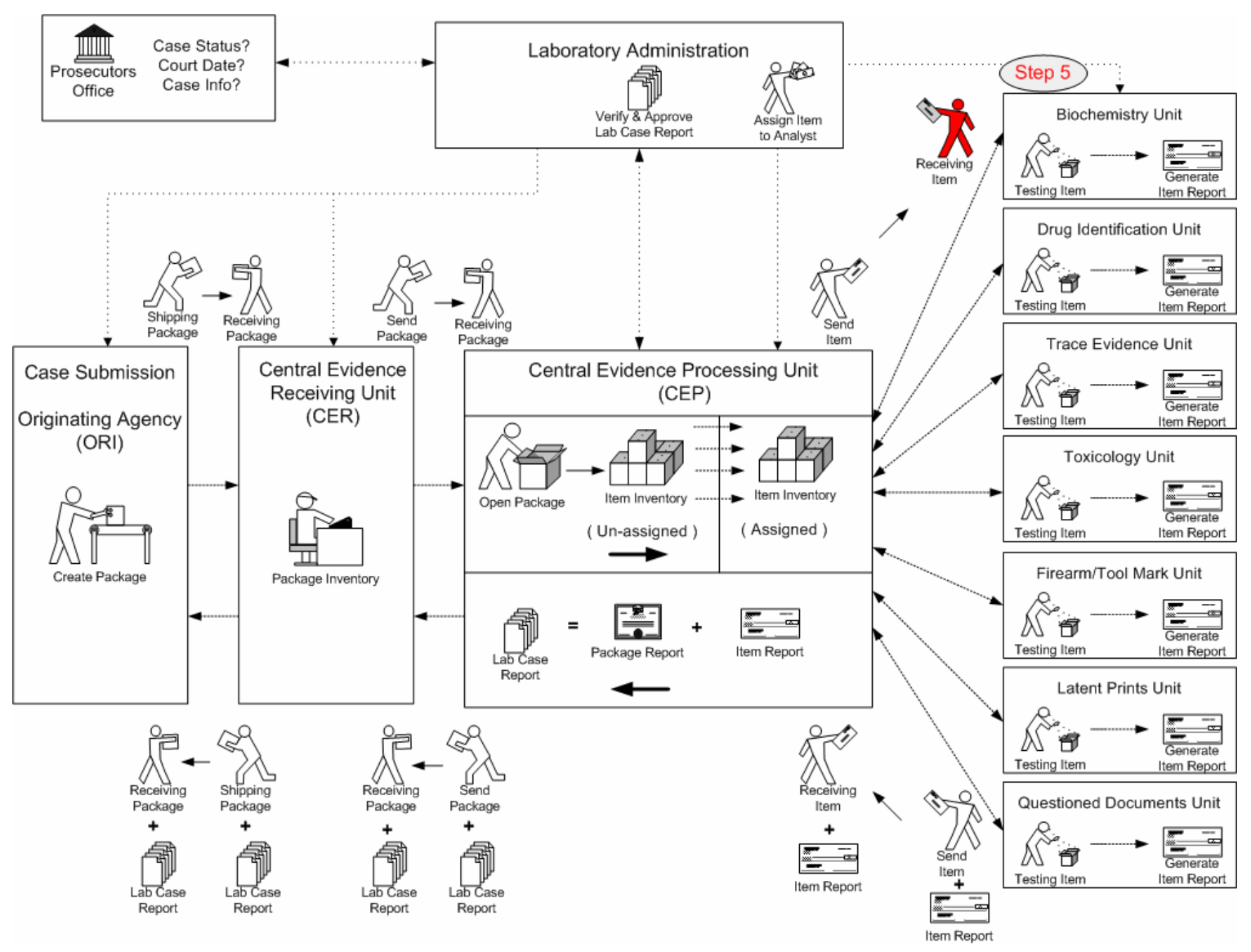

Figure 11.2: Step 5 Receive Assigned Items from CEP 
Detail procedure followed by latent prints unit for further processing and analysis is shown in Figure 11.3.

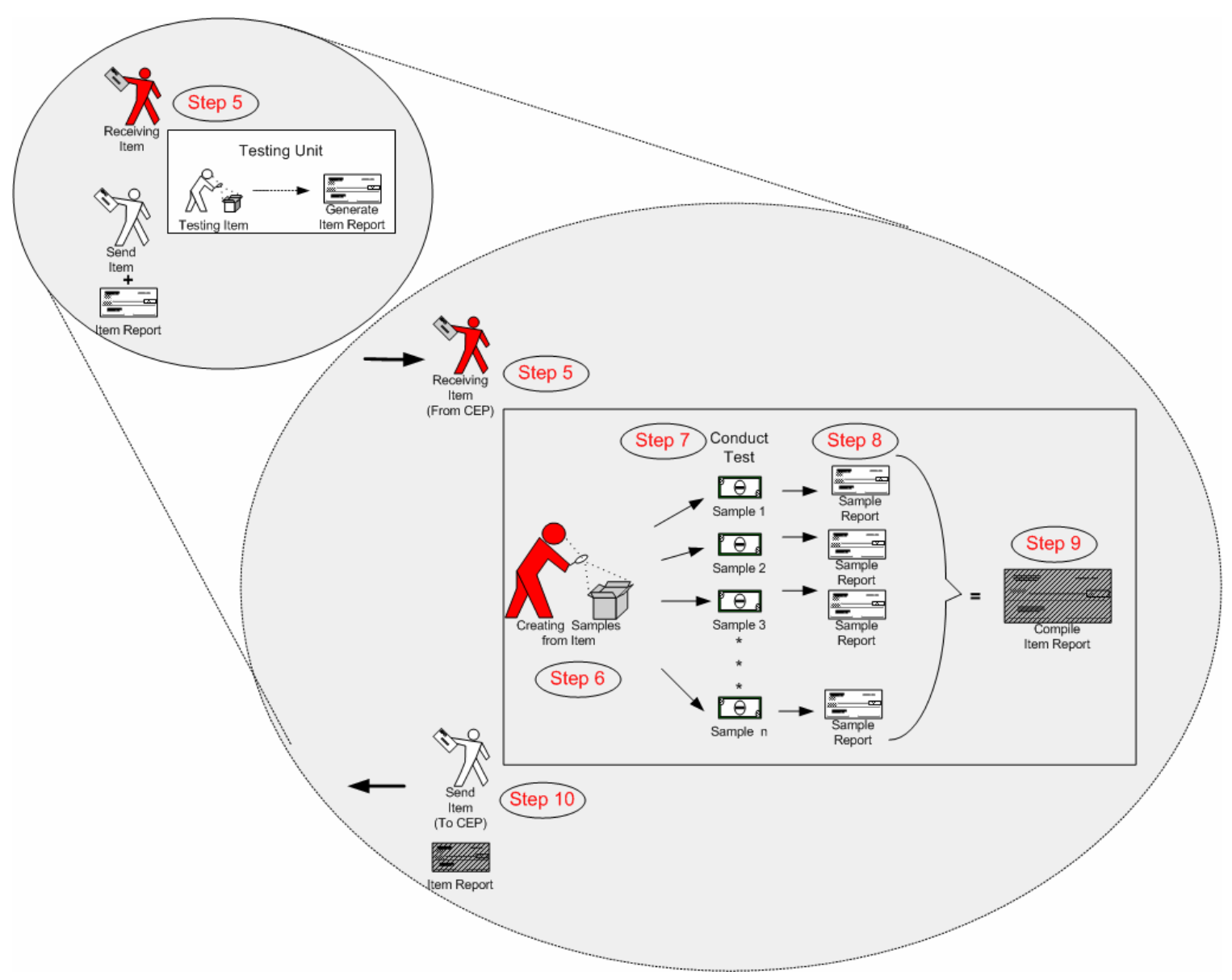

Figure 11.3: Detail Item Processing Procedure 


\subsubsection{Latent Prints Entity Relationship Diagram}

Latent prints data tables are connected with each other through relations as shown in the Figure 11.4.

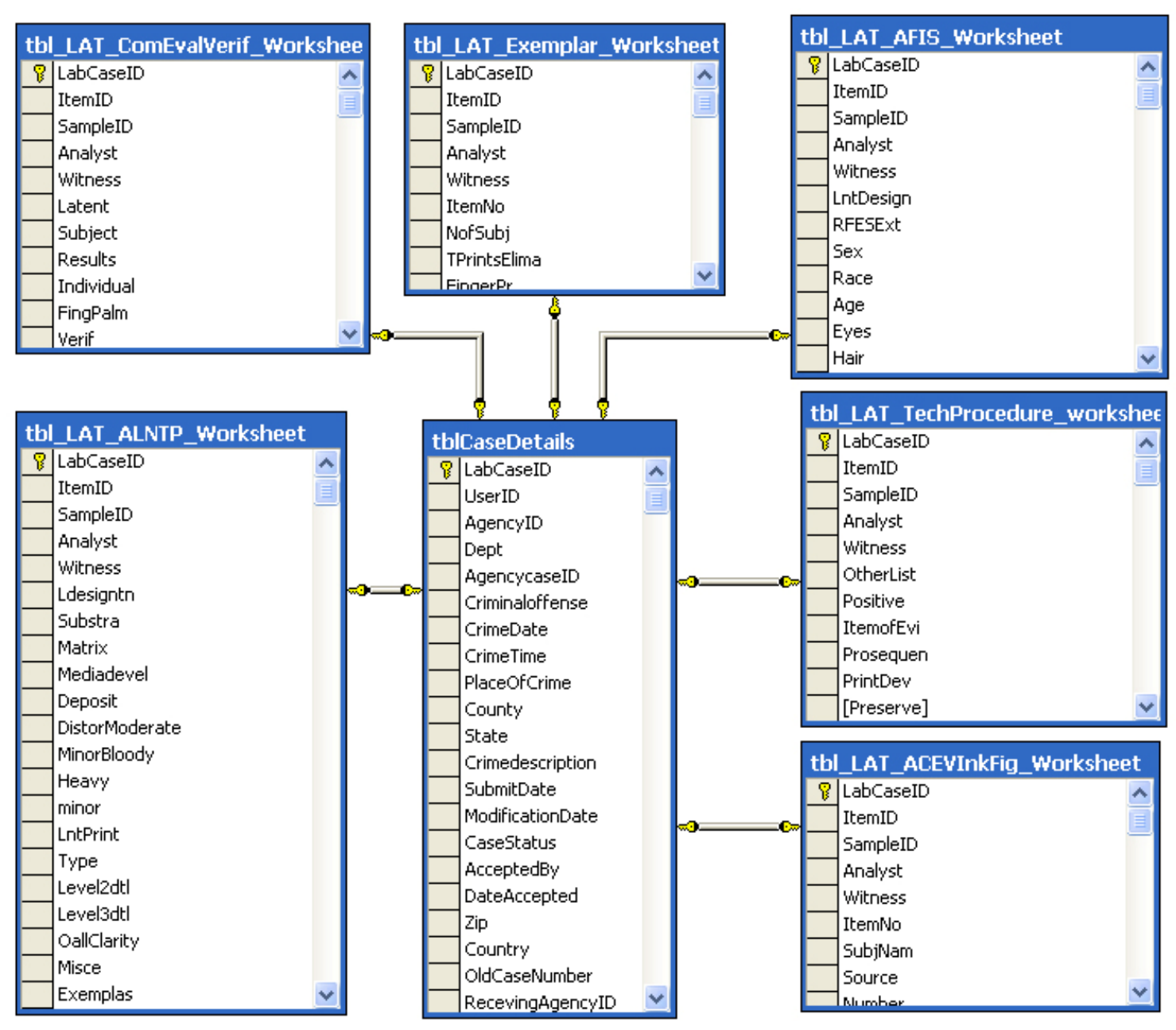

Figure 11.4: entity Relationship Diagram 


\subsubsection{Latent Prints Database Tables}

The Latent Prints unit module stores its data in the following tables

- ACE Analysis \& Comparison for Inked Finger Prints Worksheet

- $\quad$ AFIS \& IAFIS Comparison Reference Worksheet

- Analysis of Latent Prints Worksheet

- Comparison, Evaluation, \& Verification Worksheet

- Analysis Of Exemplar Prints Worksheet

- Technical Procedures Worksheet

The various fields of the ACE analysis \& comparison for inked finger prints worksheet table are shown in Table 11-1. ACE analysis \& comparison for inked finger prints worksheet data is stored and maintained in this data table

Table 11-1: ACE Analysis \& Comparison for Inked Finger Prints Worksheet Table

\begin{tabular}{|l|l|} 
tbl_LAT_ACEVInkFig_worksheet \\
\hline & LabCaseID \\
ItemID \\
SampleID \\
Analyst \\
Witness \\
ItemNo \\
SubjNam \\
Source \\
Number \\
Contri \\
\hline Dateofother \\
\hline Quality \\
\hline FigComp \\
Photoscan \\
DateCreated \\
Comments \\
TestResult
\end{tabular}


The various fields of the AFIS \& IAFIS comparison reference worksheet table are shown in Table 11-2. AFIS \& IAFIS comparison reference worksheet data is stored and maintained in this data table

Table 11-2: AFIS \& IAFIS Comparison Reference Worksheet Table

\begin{tabular}{|l|l|}
\hline tbl_LAT_AFIS_Worksheet \\
\hline \\
\hline \\
LabCaseID \\
ItemID \\
SampleID \\
Analyst \\
Witness \\
LntDesign \\
RFESExt \\
Sex \\
\hline Race \\
Age \\
Eyes \\
\hline Hair \\
\hline Height \\
\hline Weight \\
\hline POB \\
\hline databas \\
\hline Typesech \\
\hline PType \\
\hline FigPos \\
\hline Results \\
\hline Score \\
\hline SONcan \\
\hline CIBFBI \\
\hline Uif \\
\hline DateCreated \\
Comments \\
TestResult \\
\hline
\end{tabular}


The various fields of the analysis of latent prints worksheet table are shown in Table 11-3. Analysis of latent prints worksheet data is stored and maintained in this data table

Table 11-3: Analysis of Latent Prints Worksheet Table

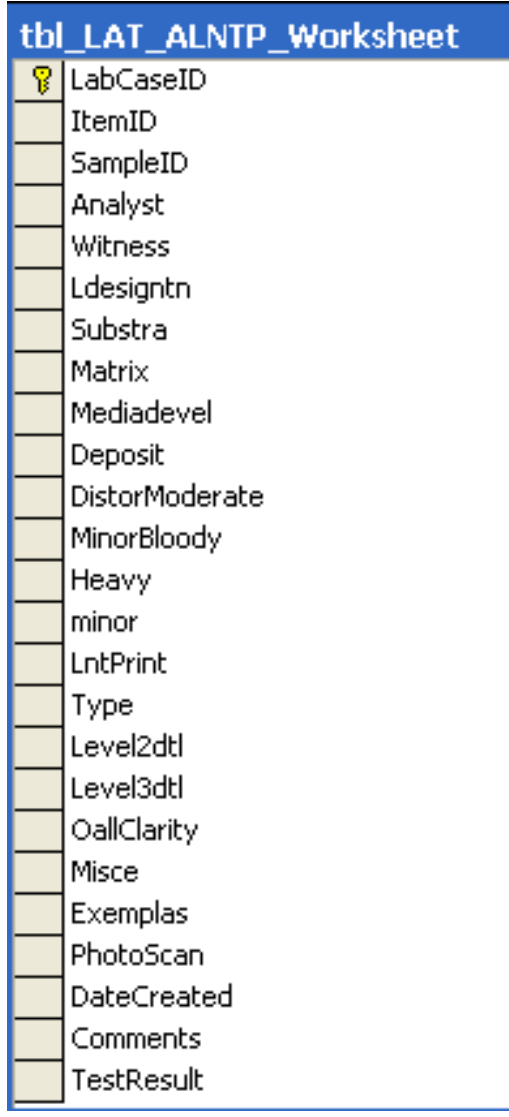


The various fields of the comparison, evaluation, \& verification worksheet table are shown in Table 11-4. Comparison, evaluation, \& verification worksheet data is stored and maintained in this data table

Table 11-4: Comparison, Evaluation, \& Verification Worksheet Table

\begin{tabular}{|l|l|}
\hline tbl_LAT_ComEvalWerif_Worksheet \\
\hline & LabCaseID \\
ItemID \\
SampleID \\
Analyst \\
Witness \\
Latent \\
Subject \\
Results \\
Individual \\
FingPalm \\
Verif \\
\hline DateCreated \\
Comments \\
TestResult \\
\hline
\end{tabular}

The various fields of the analysis of exemplar prints worksheet table are shown in Table 11-5. Analysis of exemplar prints worksheet data is stored and maintained in this data table

Table 11-5: Analysis Of Exemplar Prints Worksheet Table

\begin{tabular}{|l|l|}
\hline tbl_LAT_Exemplar_worksheet \\
\hline & LabCaseID \\
ItemID \\
SampleID \\
Analyst \\
Witness \\
\hline ItemNo \\
\hline NofSubj \\
TPrintsElima \\
\hline FingerPr \\
\hline Source \\
\hline Num \\
\hline Type \\
OverlClar \\
PhotoScan \\
DateCreated \\
Comments \\
TestResult
\end{tabular}


The various fields of the technical procedures worksheet table are shown in Table 11-6. Technical procedures worksheet data is stored and maintained in this data table

Table 11-6: Technical Procedures Worksheet Table

\begin{tabular}{|c|}
\hline Q LabCaseID \\
\hline ItemID \\
\hline SampleID \\
\hline Analyst \\
\hline Witness \\
\hline OtherList \\
\hline Positive \\
\hline ItemofEvi \\
\hline Prosequen \\
\hline PrintDev \\
\hline [Preserve] \\
\hline DateCreated \\
\hline Comments \\
\hline TestResult \\
\hline
\end{tabular}




\subsubsection{Working with Latent Prints}

Latent Prints home page requires username and password to verify user credentials, as shown in the Figure 11.5. After verifying credentials, a user is directed to list of items assigned to him/her for further processing.

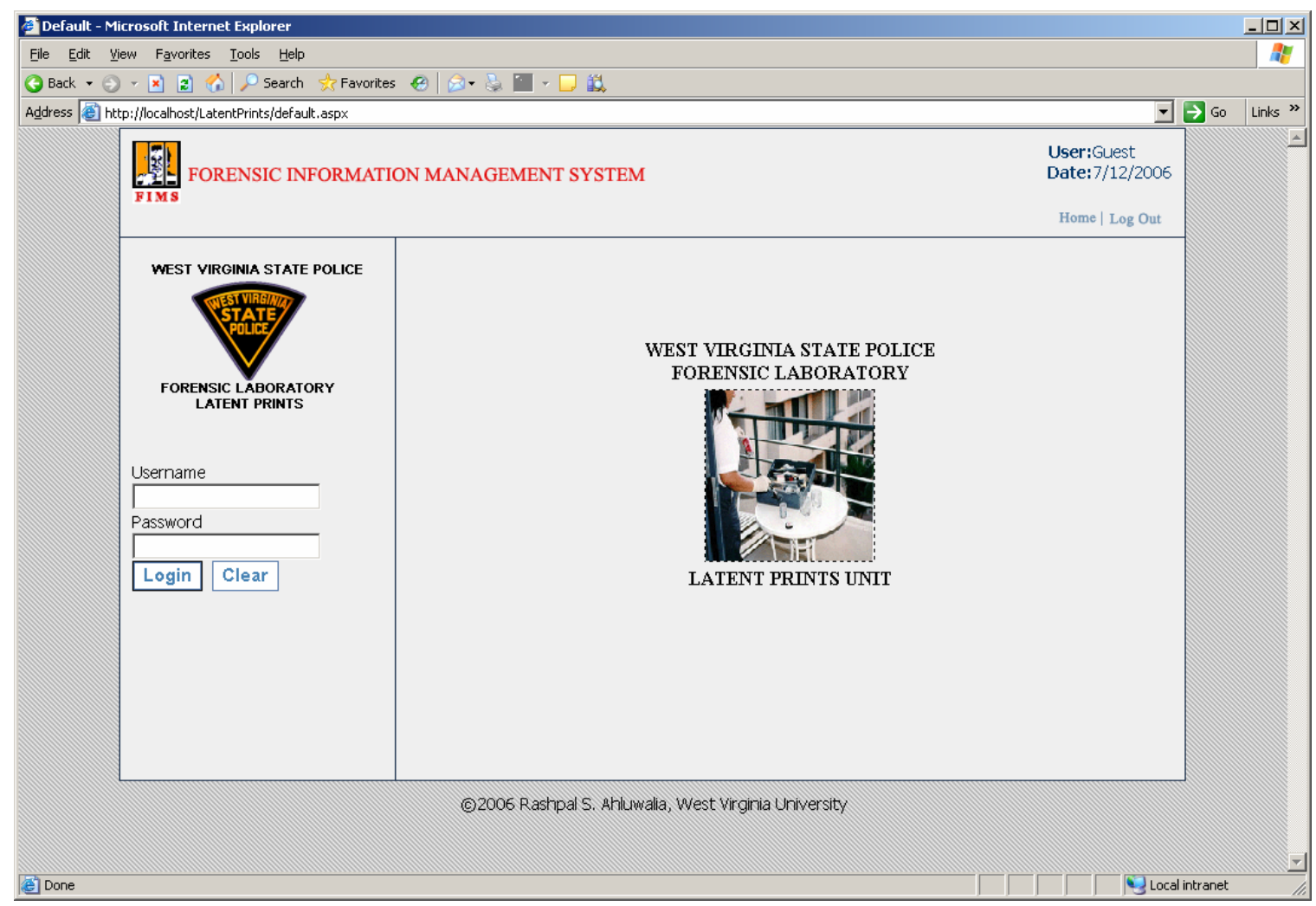

Figure 11.5: Latent Prints Home Page 


\section{View Items Assigned}

Figure 11.6 shows the list of items assigned to the analyst by the laboratory administration unit for further analysis. Initially, job status for the item assigned is set to "Job Pending". The "View Details" hyperlink enables an analyst to view the details of the case. If the list of items assigned grows long i.e. more than 15 items, the record set is divided into multiple pages. Analysts can navigate between different pages by clicking on the header and footer navigation bar of the list.

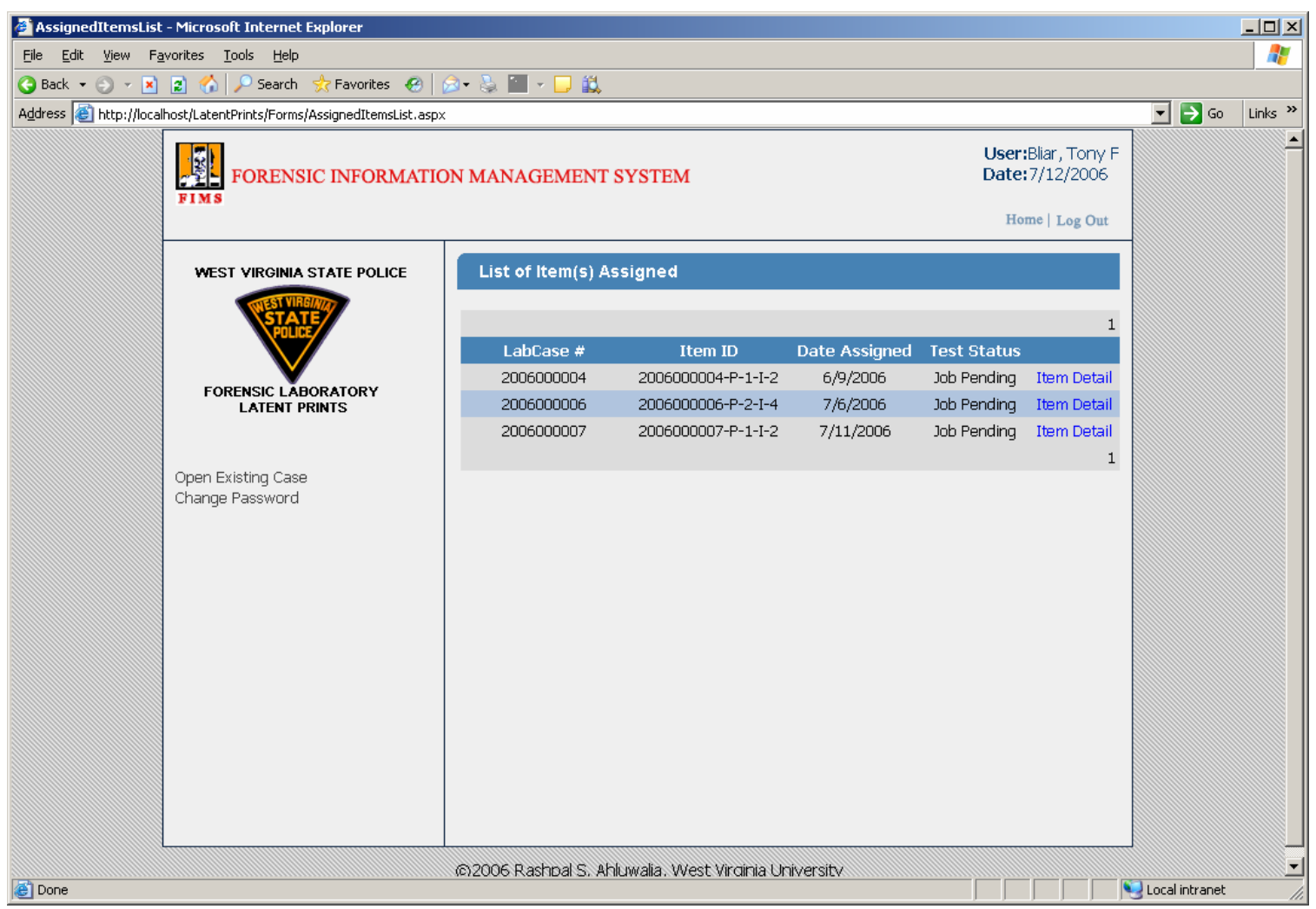

Figure 11.6: Assigned Item List 


\section{Item Picture Management}

This section describes the procedure of attaching pictures with an item. Analysts can perform two kinds of operation:

- View Pictures

- Upload Pictures

Analysts can access this section by clicking on the "View/Upload Item Photo" hyperlink in the item details section as shown inn Figure 11.9. View picture section list the pictures already attached to the item with their thumbnails as shown in Figure 11.7. Analysts can also view the full size image by clicking on the image thumbnail. Full size image opens in a new window, which can be closed after use.

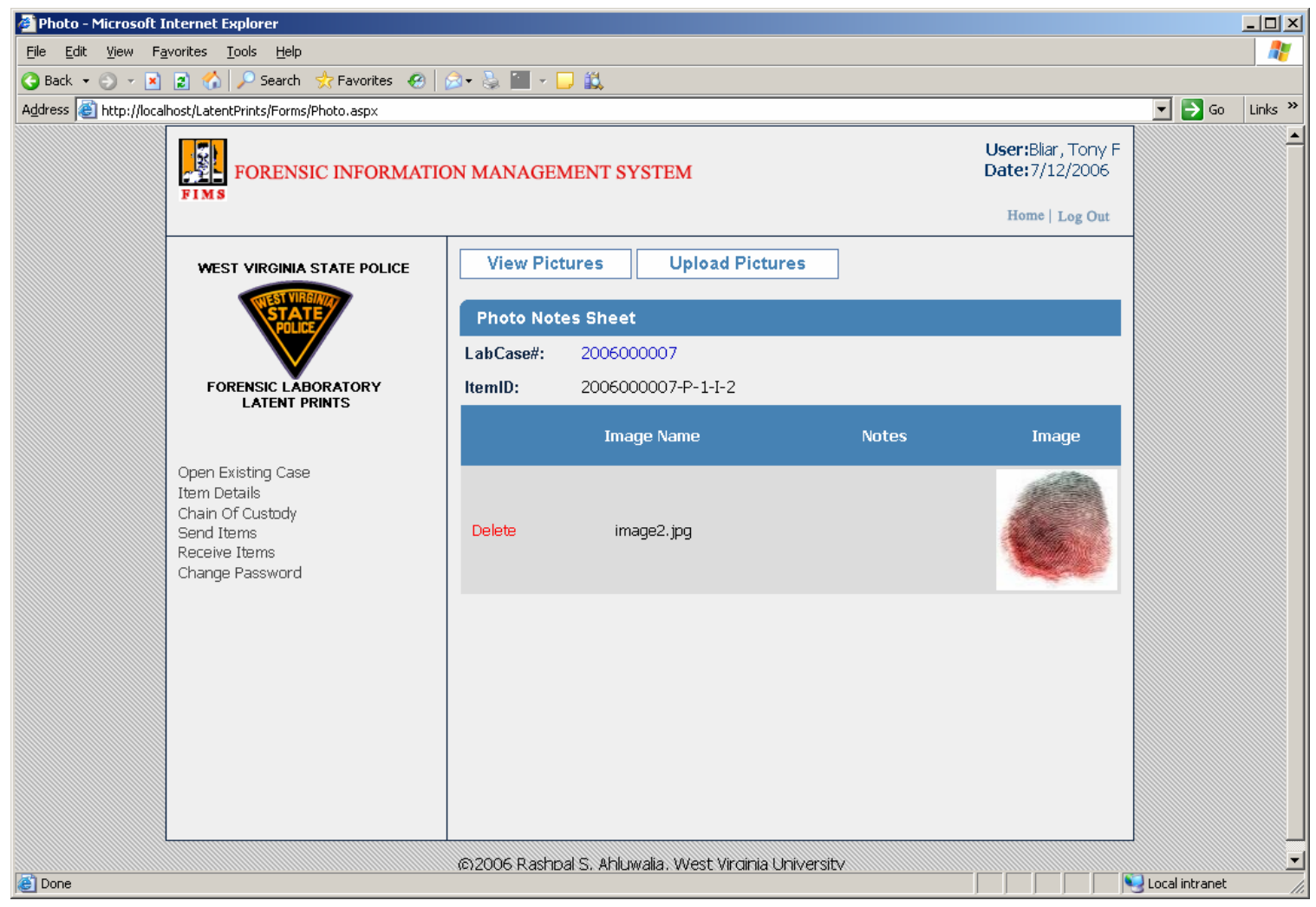

Figure 11.7: View Item Photo 
Analysts can upload any digital photo in JPG format directly into the database using this section as shown in Figure 11.8. To upload an image into database, analysts are required to browse for the image path, set other required fields and click on the "Upload the file" hyperlink. On successful upload a thumbnail appears on the bottom of the page with a success message.

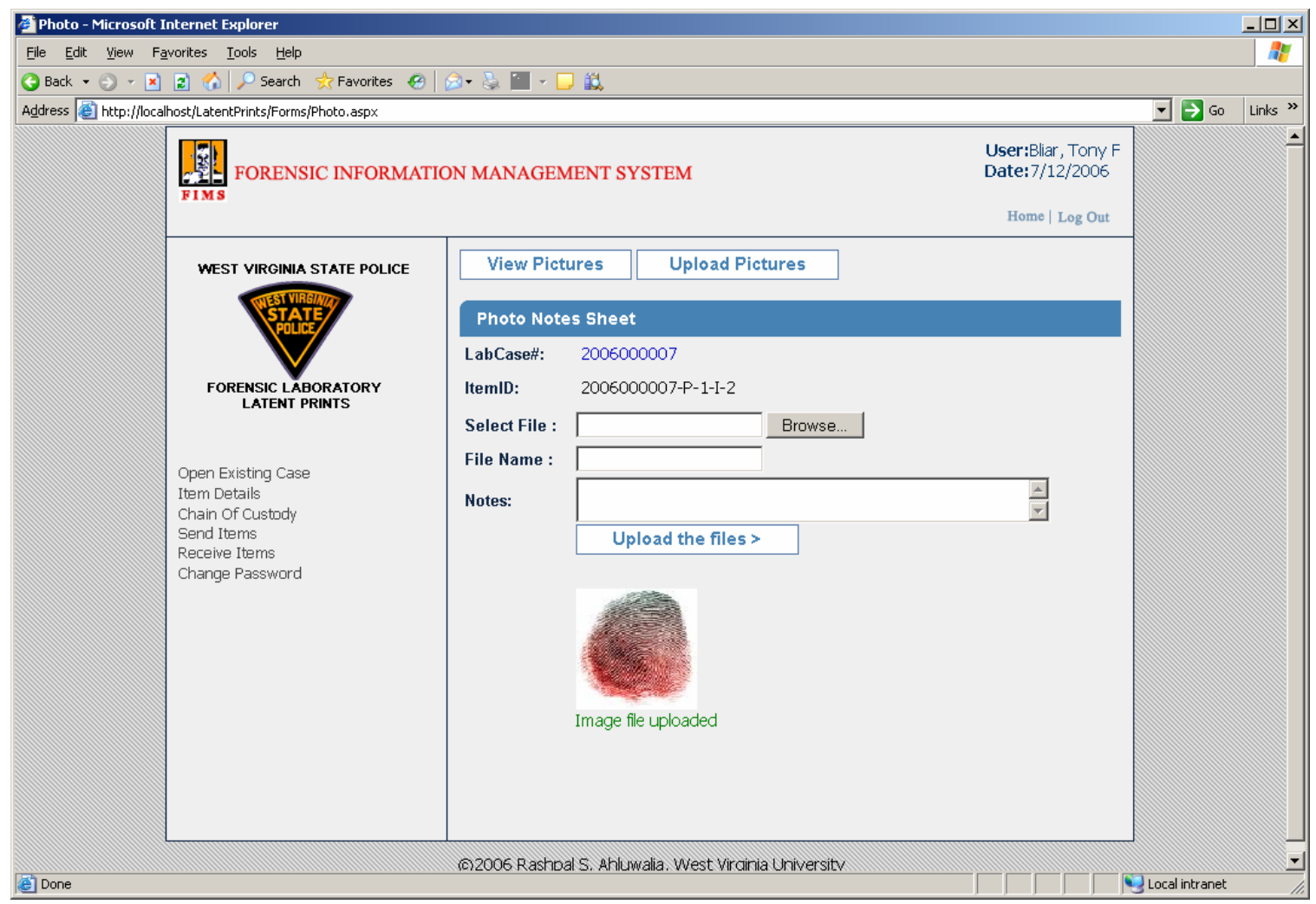

Figure 11.8: Upload Item Picture Section 


\section{Working with Samples}

Analysts receive items from the CEP unit for further analysis and processing. Once received items are sub-divided into samples for conducting requested tests. Each item can be divided into one or more samples according to the requirement and complexity of the required test.

Analysts can create new, update or delete existing samples from the item details section as shown in Figure 11.9. To add a new sample analyst is required to provide description of sample and then click on “Add Sample” hyperlink. FIMS application automatically adds the time stamp to the item created. Initially, sample test status is set to "Test Pending" for the sample. Sample information can be updated by clicking on “Edit” hyperlink and existing sample can be deleted from the system by clicking on "Delete" hyperlink.

This section also enables analysts to view the final sample, sample photo, item, and item photo report. To access the individual sample/sample photo report analyst can click on "View Report" or "Photo Sheet" hyperlink. To view comprehensive item report analysts can click on "Item Report” or "Item Photo Report” at the bottom of the page.

To complete the process for item testing, the analyst is required to click on "Item Test Complete" button, as shown in Figure 11.9. Once clicked, analyst will not be able to make any alterations to the existing item test report. 


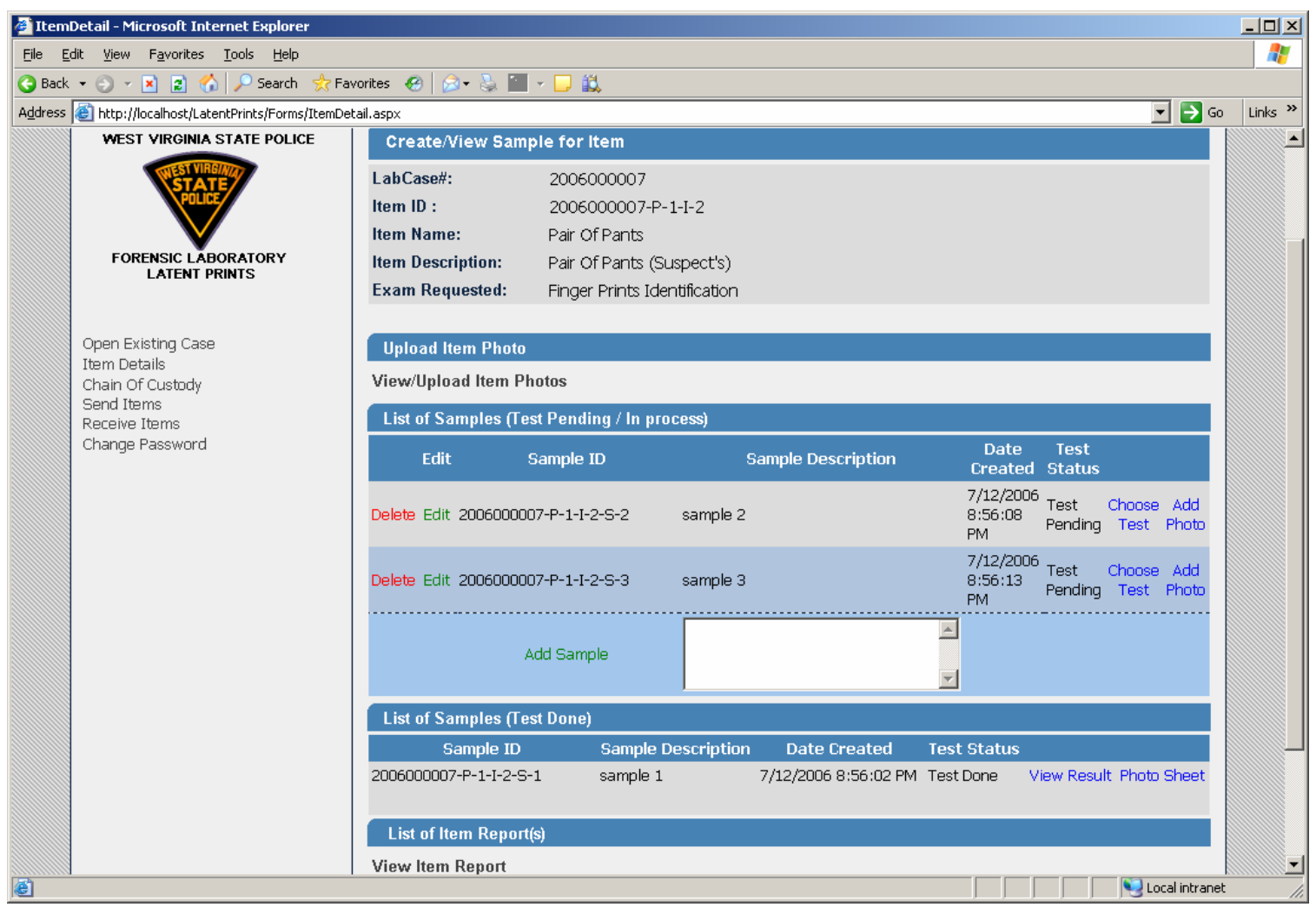

Figure 11.9: Item Detail Section 
Analysts can access this section by clicking on the "View/Upload Sample Photo" hyperlink in the item details section as shown in Figure 11.9. View picture section list the pictures already attached to the item with their thumbnails as shown in Figure 11.10. Analysts can also view the full size image by clicking on the image thumbnail. Full size image opens in a new window, which can be closed after use.

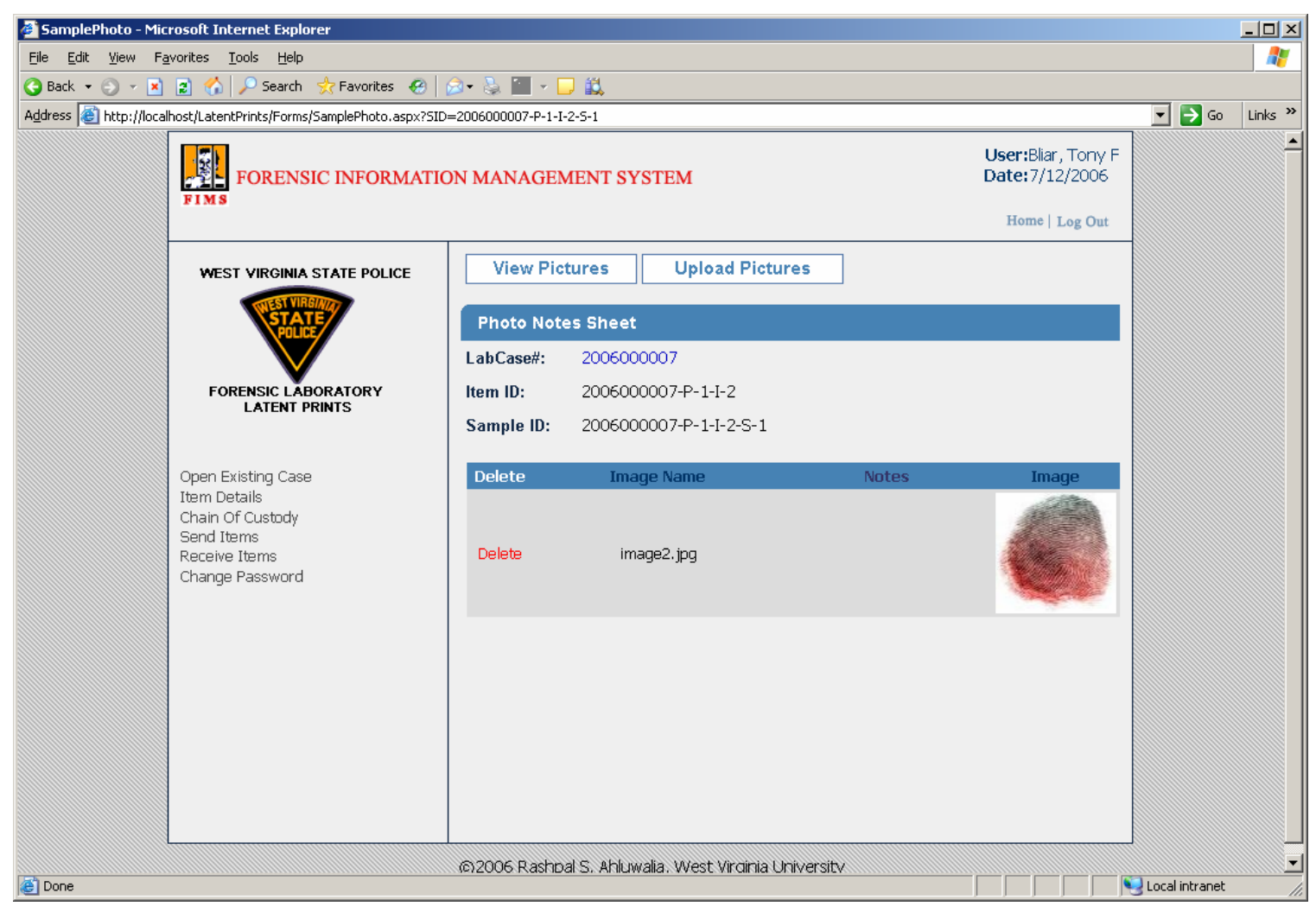

Figure 11.10: View Picture Section 
Analysts can upload any digital photo in JPG format directly into the database using this section as shown in Figure 11.11. To upload an image analysts are required to browse for the image path, set other required fields and click on the "Upload the file" hyperlink to upload the image into database. On successful upload a thumbnail appears on the bottom of the page with a success message.

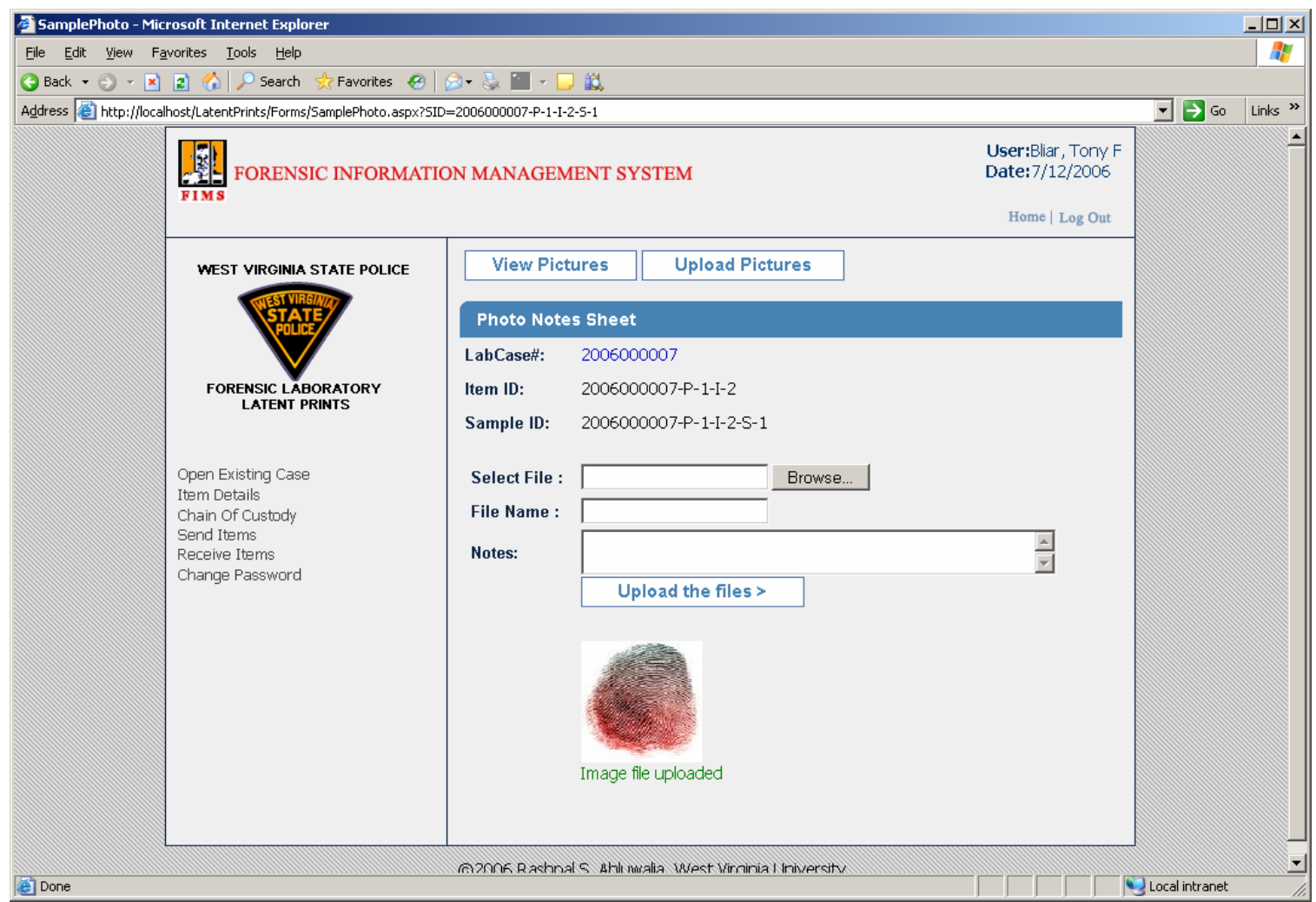

Figure 11.11: Upload Sample Picture Section 


\section{Choosing Test Worksheet}

After sample creation, analysts are required to decide what kind of tests are required? Analysts are required to make decision of choosing type of test manually. Once decision is made the analyst can see the list of tests available, and then click "Choose Test" hyperlink as shown in Figure 11.12. FIMS application remembers the chosen sample ID during the process.

The worksheet for the latent prints unit are:

- ACE Analysis \& Comparison for Inked Finger Prints

- AFIS \& IAFIS Comparison Reference

- Analysis of Latent Prints

- Comparison, Evaluation, \& Verification

- Analysis Of Exemplar Prints

- Technical Procedures

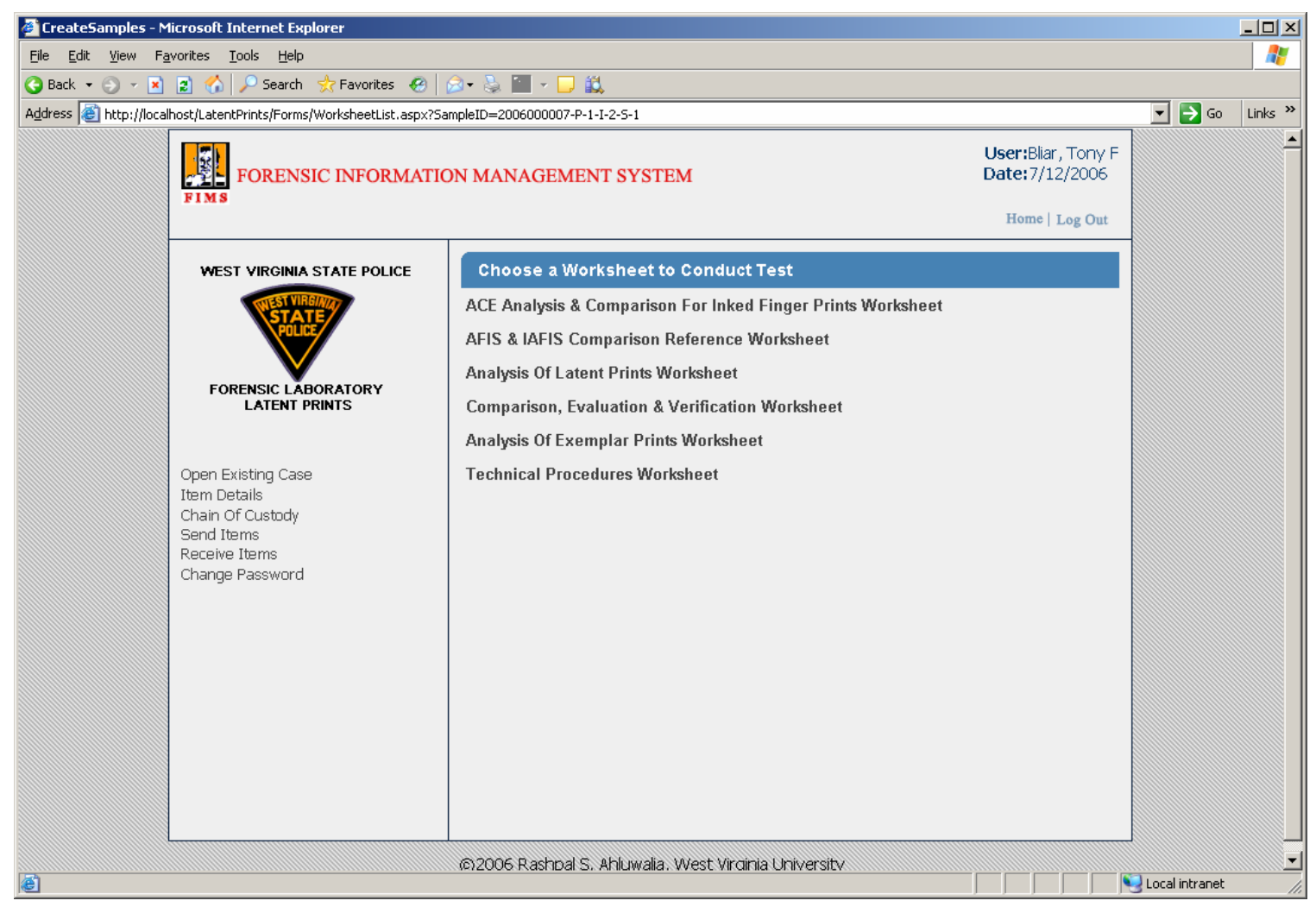

Figure 11.12: Choose Sample Test Section 


\section{ACE Analysis \& Comparison for Inked Finger Prints Worksheet}

ACE analysis \& comparison for inked finger prints worksheet is used for filling information pertaining to ACE analysis \& comparison for inked finger prints test performed on the sample by the analyst. After filling the required data fields, analyst is required to click on "Save Results" button located on top of the page, as shown in Figure 11.13. After saving the worksheet data, analyst can finalize and close the worksheet by clicking on "Test Done" button. The analyst cannot make any alterations in the worksheet once the “Test Done” button is clicked.

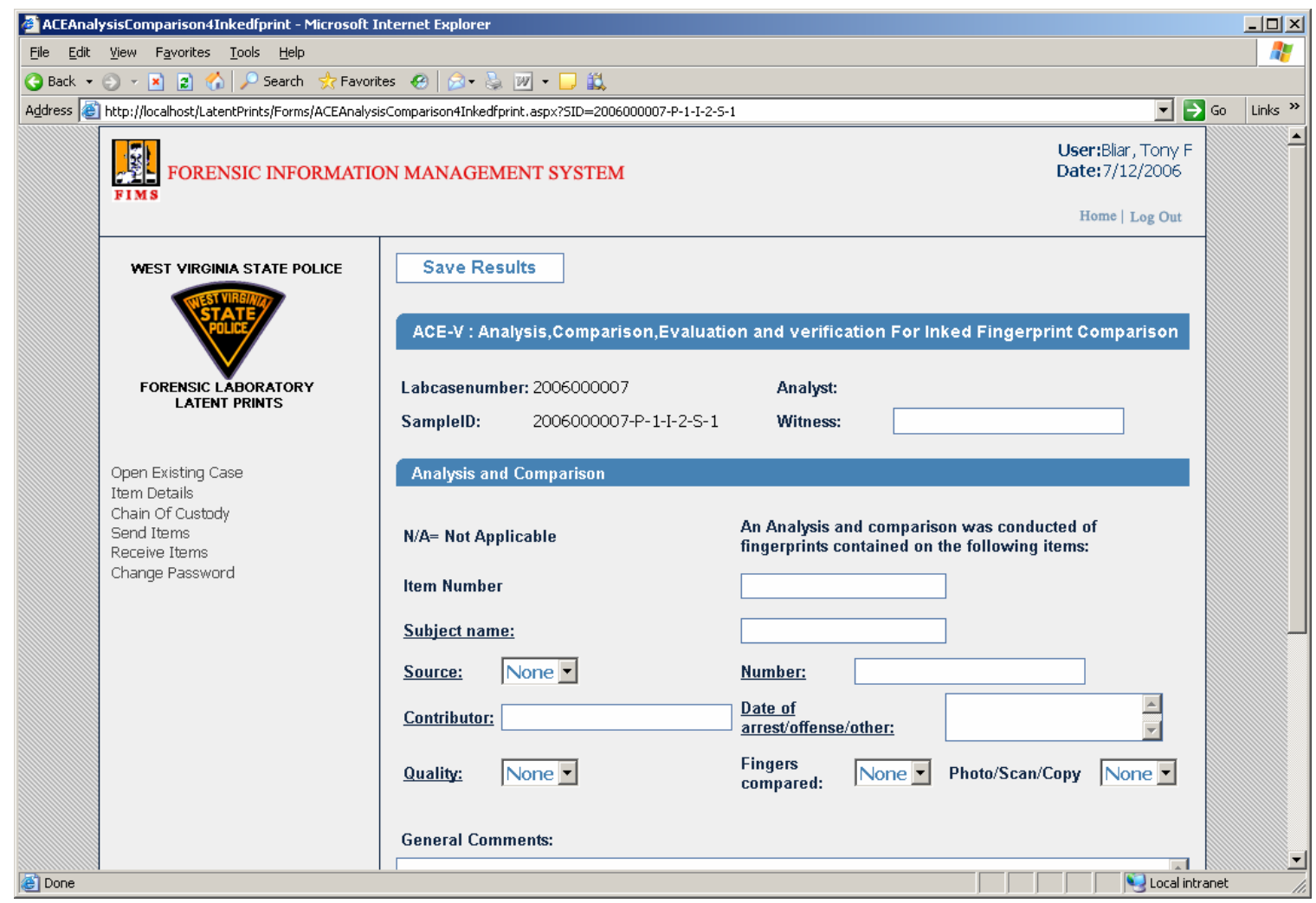

Figure 11.13: ACE Analysis \& Comparison for Inked Finger Prints Worksheet 


\section{AFIS \& IAFIS Comparison Reference Worksheet}

AFIS \& IAFIS comparison reference worksheet is used for filling information pertaining to AFIS \& IAFIS comparison reference test performed on the sample by the analyst. After filling the required data fields, analyst is required to click on "Save Results" button located on top of the page, as shown in Figure 11.14. After saving the worksheet data, analyst can finalize and close the worksheet by clicking on "Test Done" button. The analyst cannot make any alterations in the worksheet once the "Test Done" button is clicked.

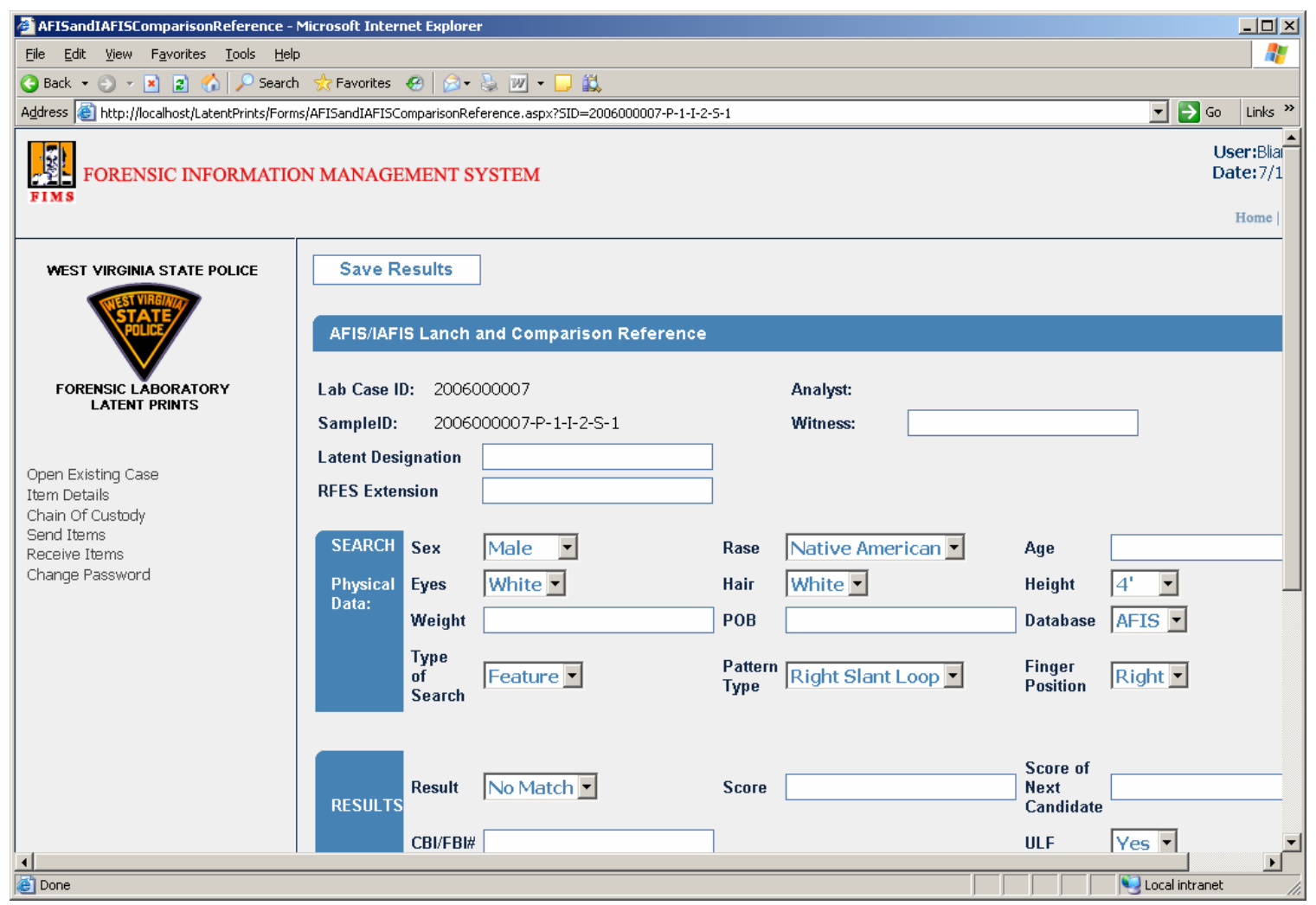

Figure 11.14: AFIS \& IAFIS Comparison Reference Worksheet 


\section{Analysis of Latent Prints Worksheet}

Analysis of latent prints worksheet is used for filling information pertaining to analysis of latent prints test performed on the sample by the analyst. After filling the required data fields, analyst is required to click on "Save Results" button located on top of the page, as shown in Figure 11.15. After saving the worksheet data, analyst can finalize and close the worksheet by clicking on “Test Done” button. The analyst cannot make any alterations in the worksheet once the "Test Done" button is clicked.

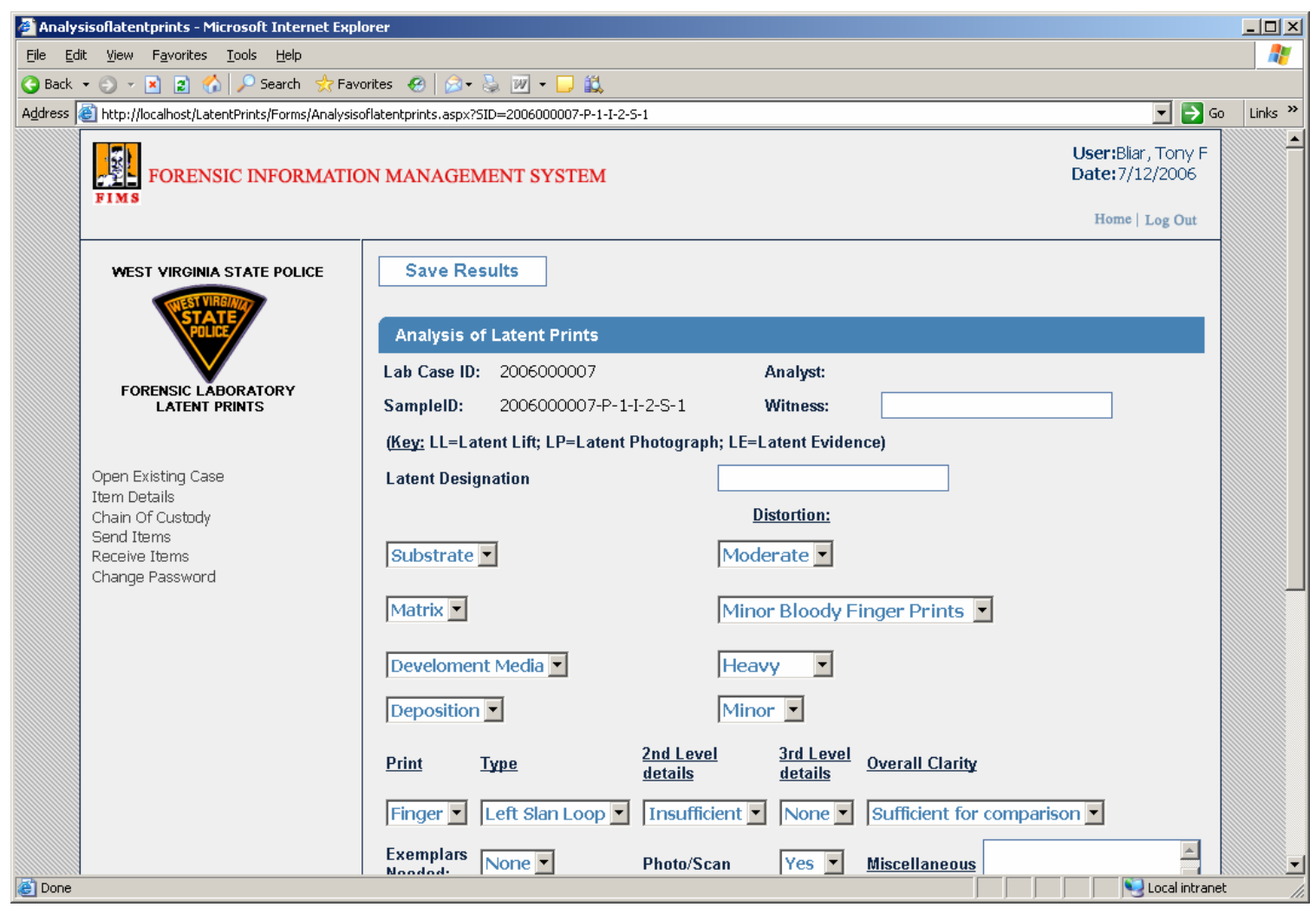

Figure 11.15: Analysis of Latent Prints Worksheet 


\section{Comparison, Evaluation, \& Verification Worksheet}

Comparison, evaluation, \& verification worksheet is used for filling information pertaining to comparison, evaluation, \& verification test performed on the sample by the analyst. After filling the required data fields, analyst is required to click on "Save Results" button located on top of the page, as shown in Figure 11.16. After saving the worksheet data, analyst can finalize and close the worksheet by clicking on "Test Done" button. The analyst cannot make any alterations in the worksheet once the "Test Done" button is clicked.

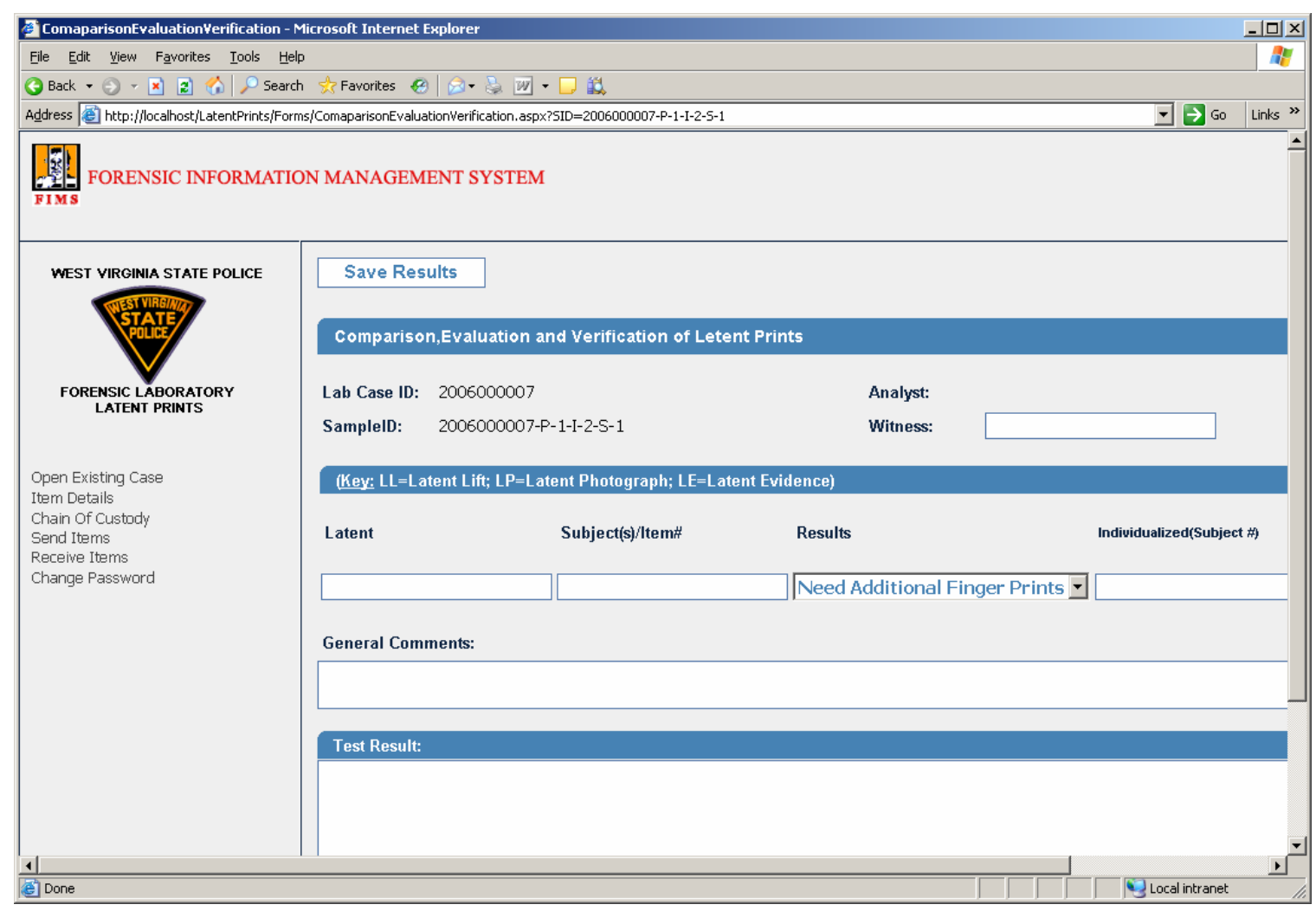

Figure 11.16: Comparison, Evaluation, \& Verification Worksheet 


\section{Analysis of Exemplar Prints Worksheet}

Analysis of exemplar prints worksheet is used for filling information pertaining to analysis of exemplar prints test performed on the sample by the analyst. After filling the required data fields, analyst is required to click on "Save Results" button located on top of the page, as shown in Figure 11.17. After saving the worksheet data, analyst can finalize and close the worksheet by clicking on “Test Done” button. The analyst cannot make any alterations in the worksheet once the "Test Done" button is clicked.

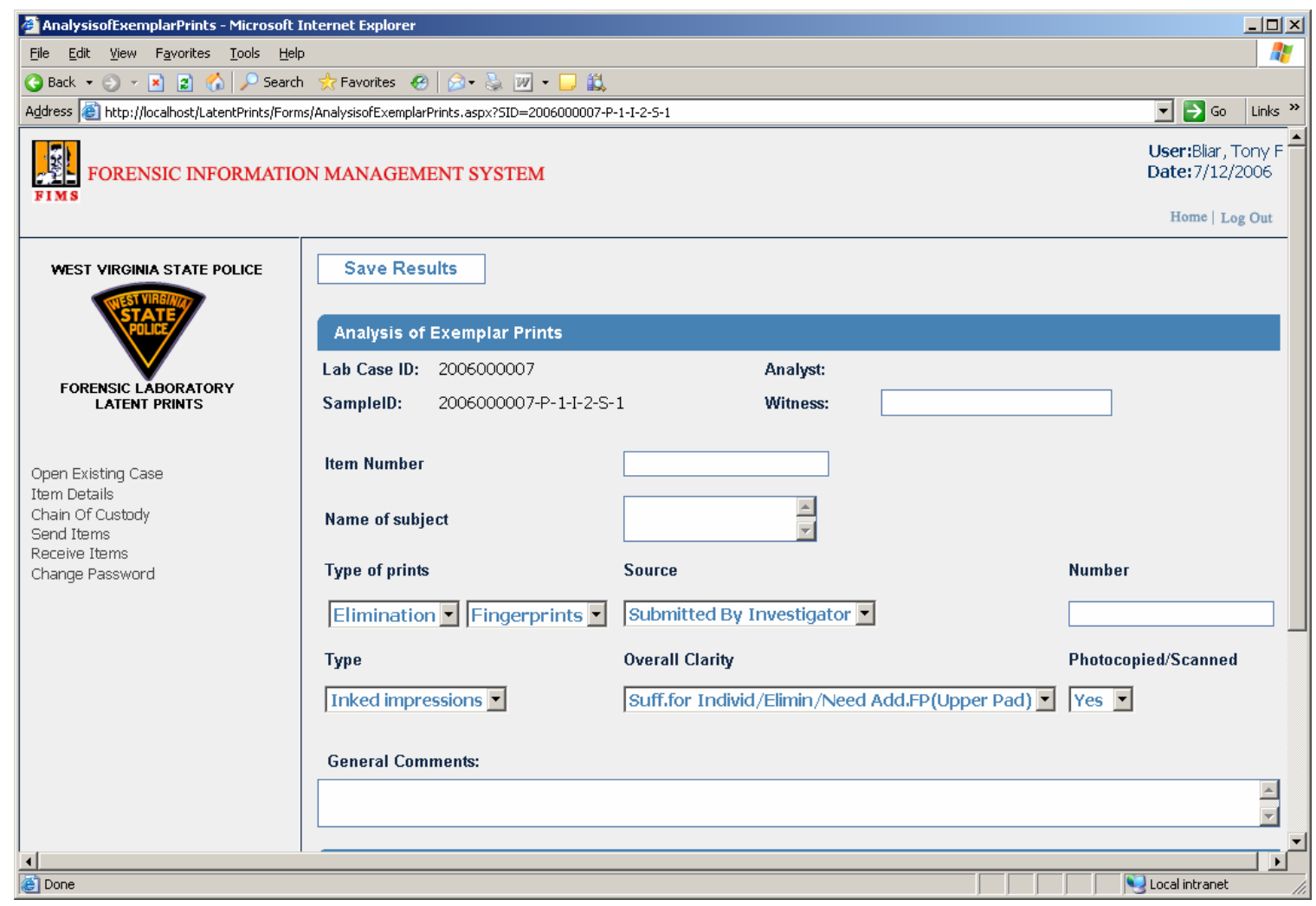

Figure 11.17: Analysis Of Exemplar Prints Worksheet 


\section{Technical Procedures Worksheet}

Technical procedures worksheet is used for filling information pertaining to analysis of Technical Procedures test performed on the sample by the analyst. After filling the required data fields, analyst is required to click on "Save Results" button located on top of the page, as shown in Figure 11.18. After saving the worksheet data, analyst can finalize and close the worksheet by clicking on "Test Done" button. The analyst cannot make any alterations in the worksheet once the "Test Done" button is clicked.

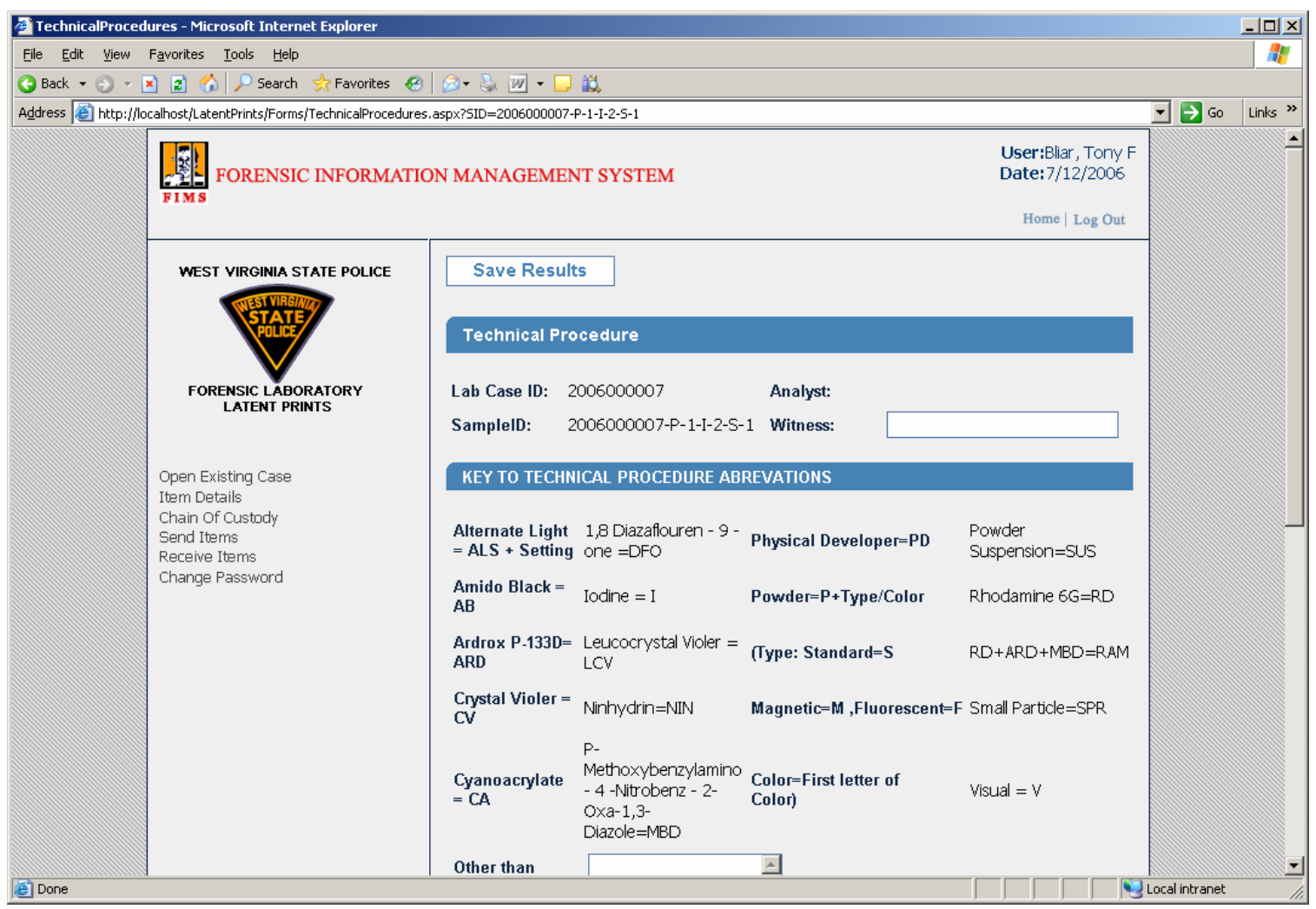

Figure 11.18: Technical Procedures Worksheet 


\section{Receive Item}

Once an item is sent by CEP unit, Latent Prints unit analyst is responsible for receiving it by clicking on "Receive" button, under receive item section, as shown in Figure 11.19. This completes the handshaking process of item transfer between units.

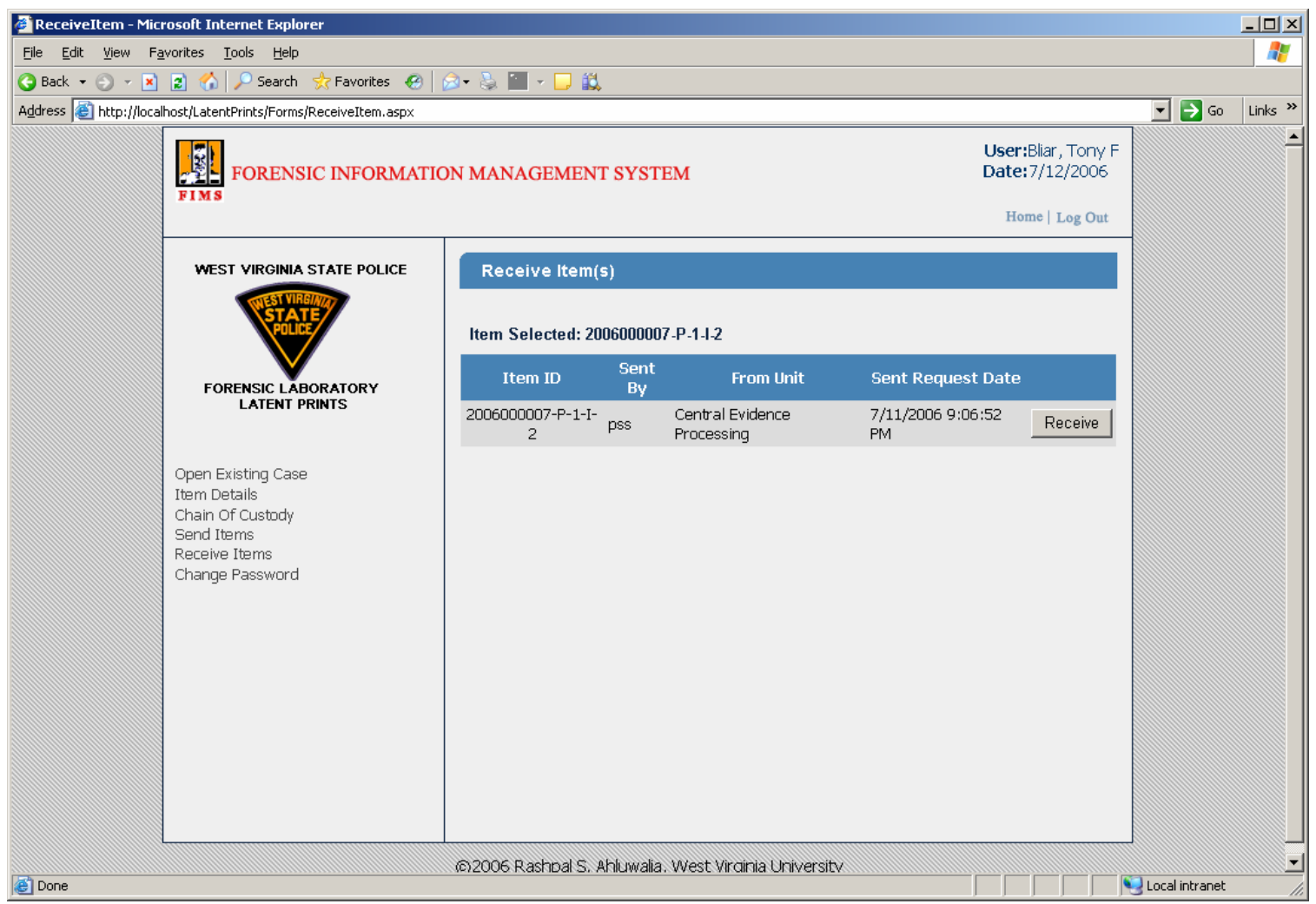

Figure 11.19: Receive Item Section 


\section{Send Item}

After completion of item testing, analyst is required to return the evidence item back to CEP unit. Figure 11.20 shows the section used to send an item back to the CEP unit. Analyst can use the drop down to select item ID and click on "Send" hyperlink, to send item back to CEP.

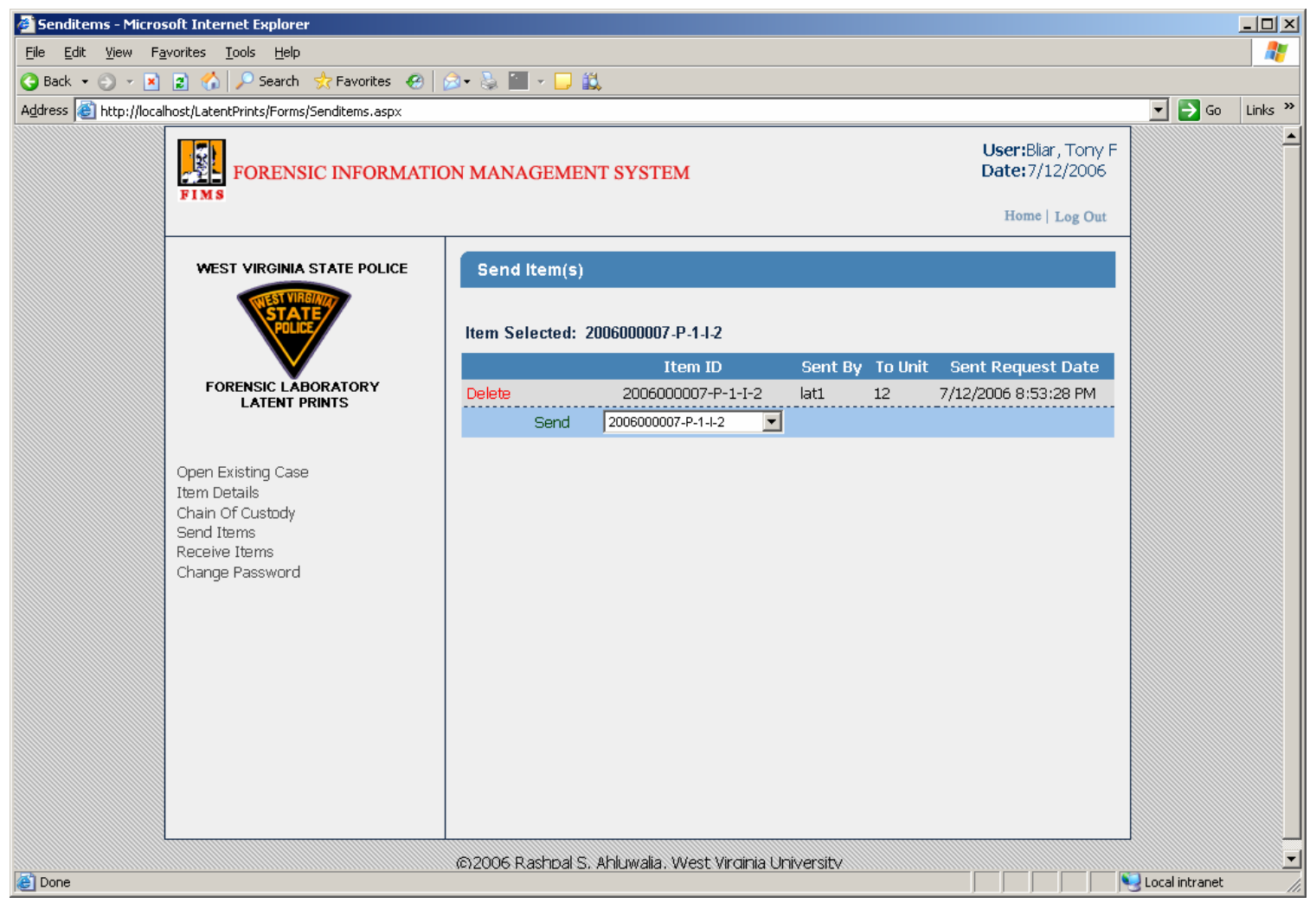

Figure 11.20: Send Item Section 


\section{Chain of Custody}

Item chain of custody information is shown in Figure 11.21. FIMS application records used ID, unit name, and time stamp for tracking item chain of custody.

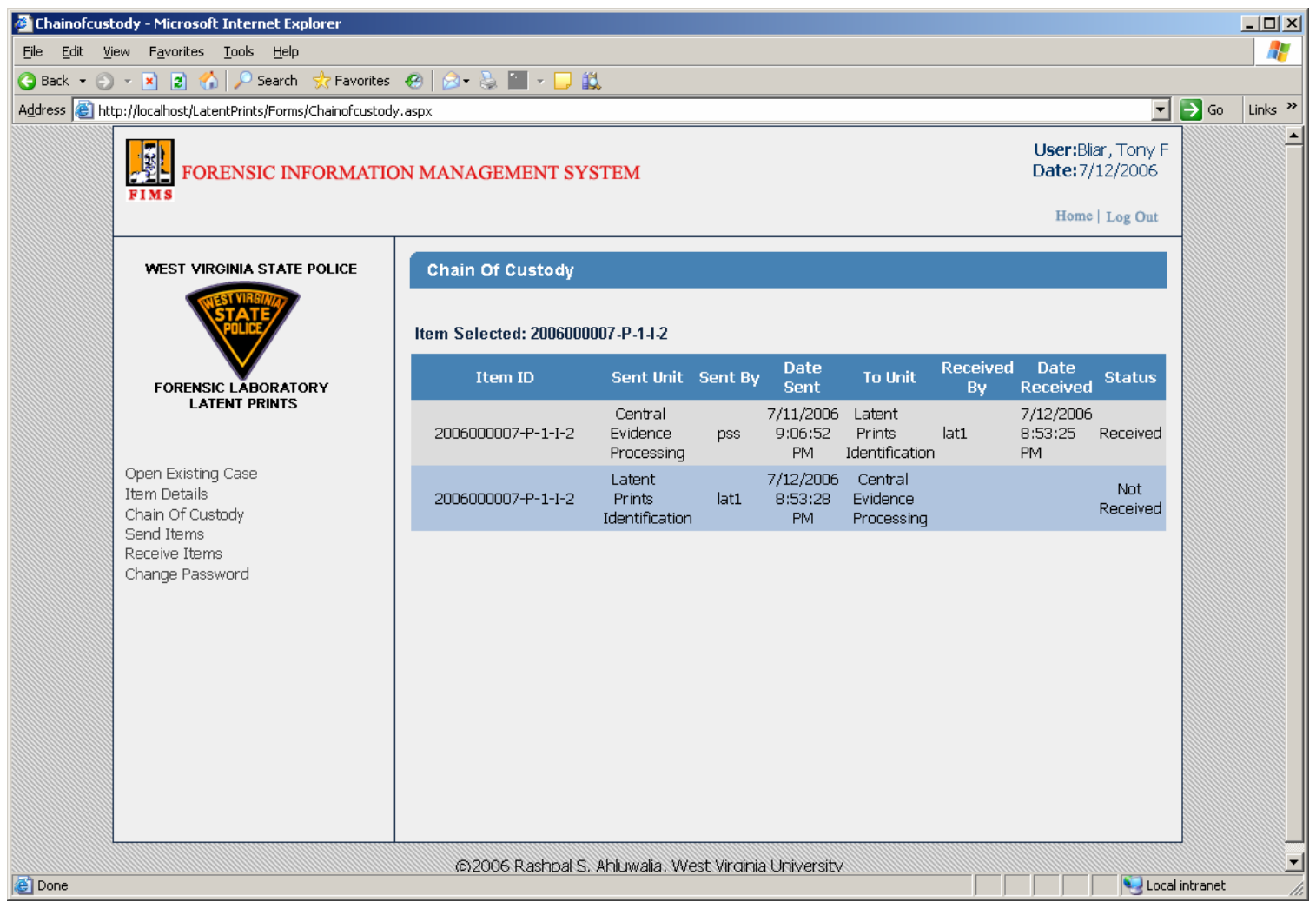

Figure 11.21: Item Chain Of Custody 


\section{Change User Password}

Analyst can change password through this section by providing appropriate credentials as shown in Figure 11.22.

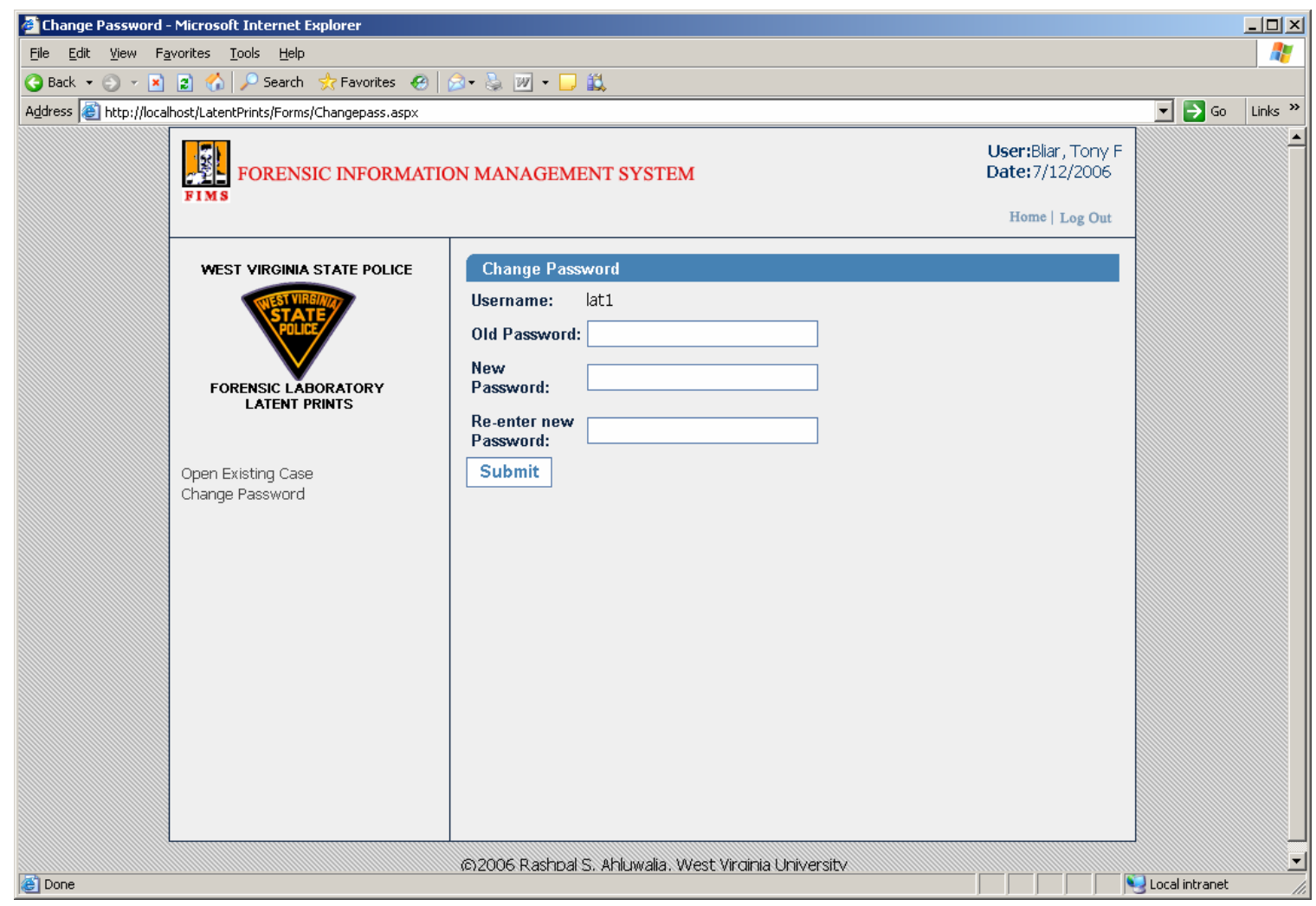

Figure 11.22: Change Password Section

\section{Exiting Latent Prints}

Users can terminate the current session by clicking on "Log Out" hyperlink on the top right corner of the page. 


\subsubsection{Generating Item and Sample Reports}

The analyst can generate the following types of reports

- Item Report

- Item Photo Report

- Sample Report

- Sample Photo Report

Figure 11.23 shows the item report, which is a cumulative report for all the samples. Figure 11.24 shows the sample report. This section also facilitate analyst to export report via PDF/MS-Word format, as shown in Figure 11.25 and Figure 11.26.

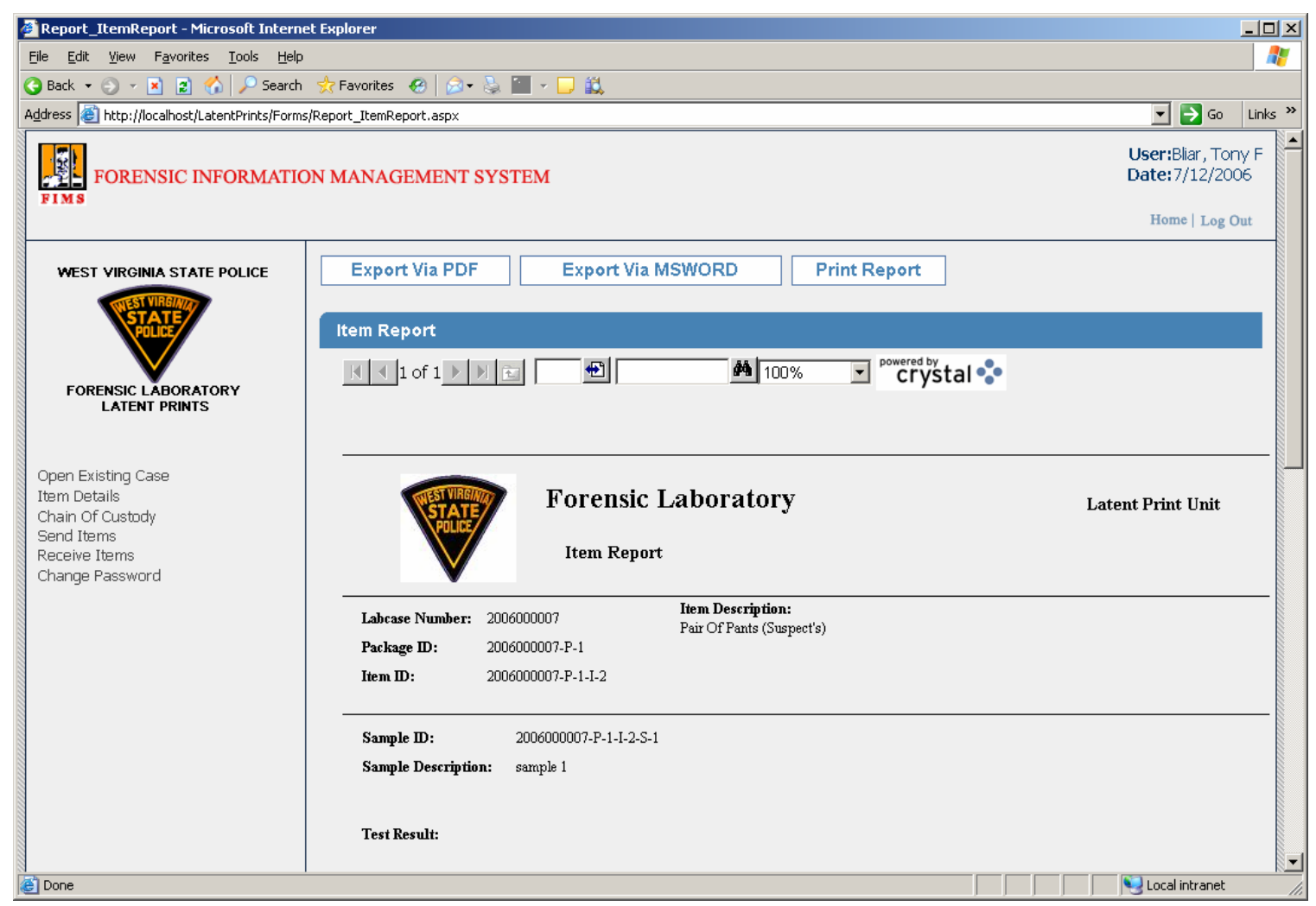

Figure 11.23: Item report 


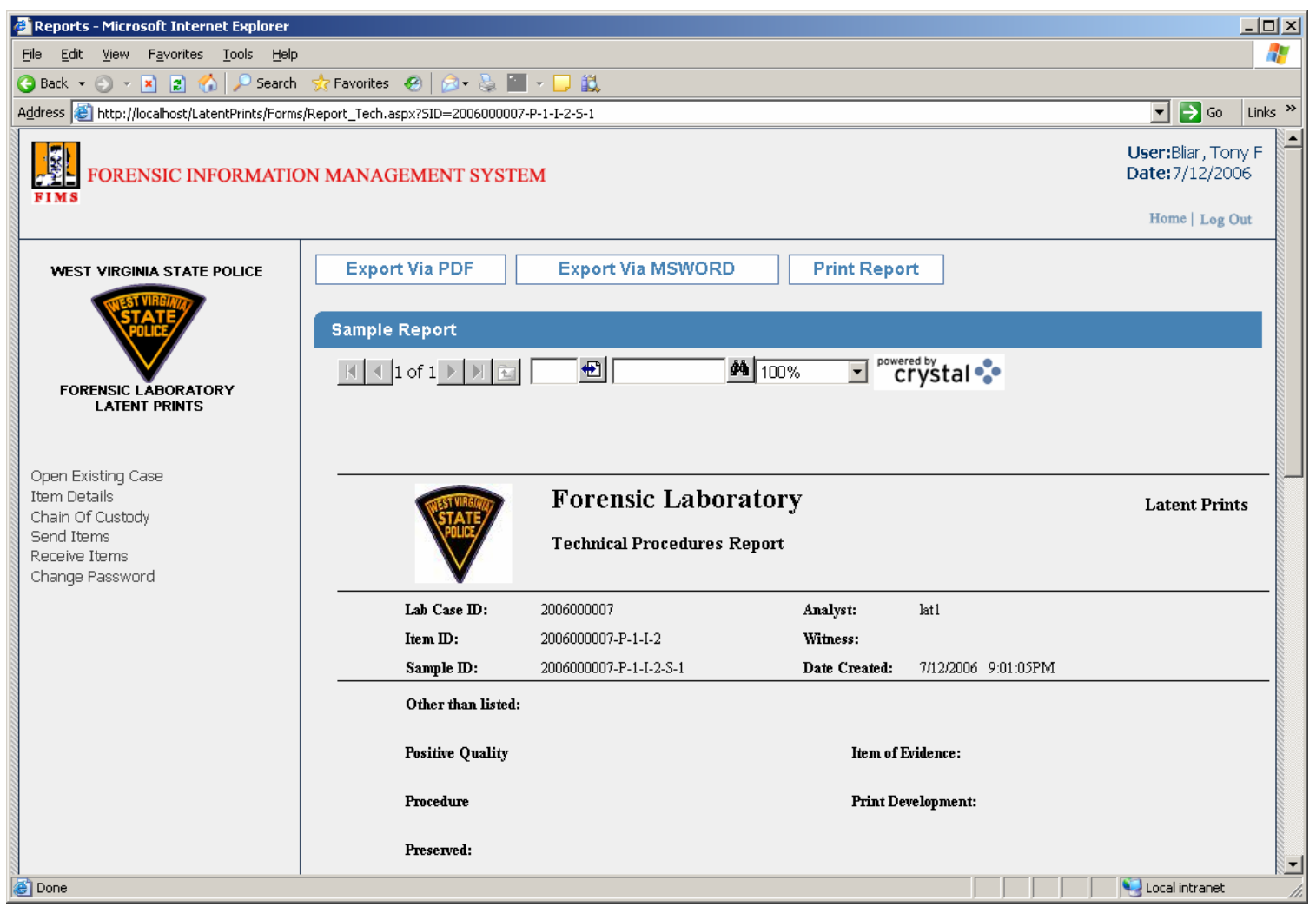

Figure 11.24: Sample Report 


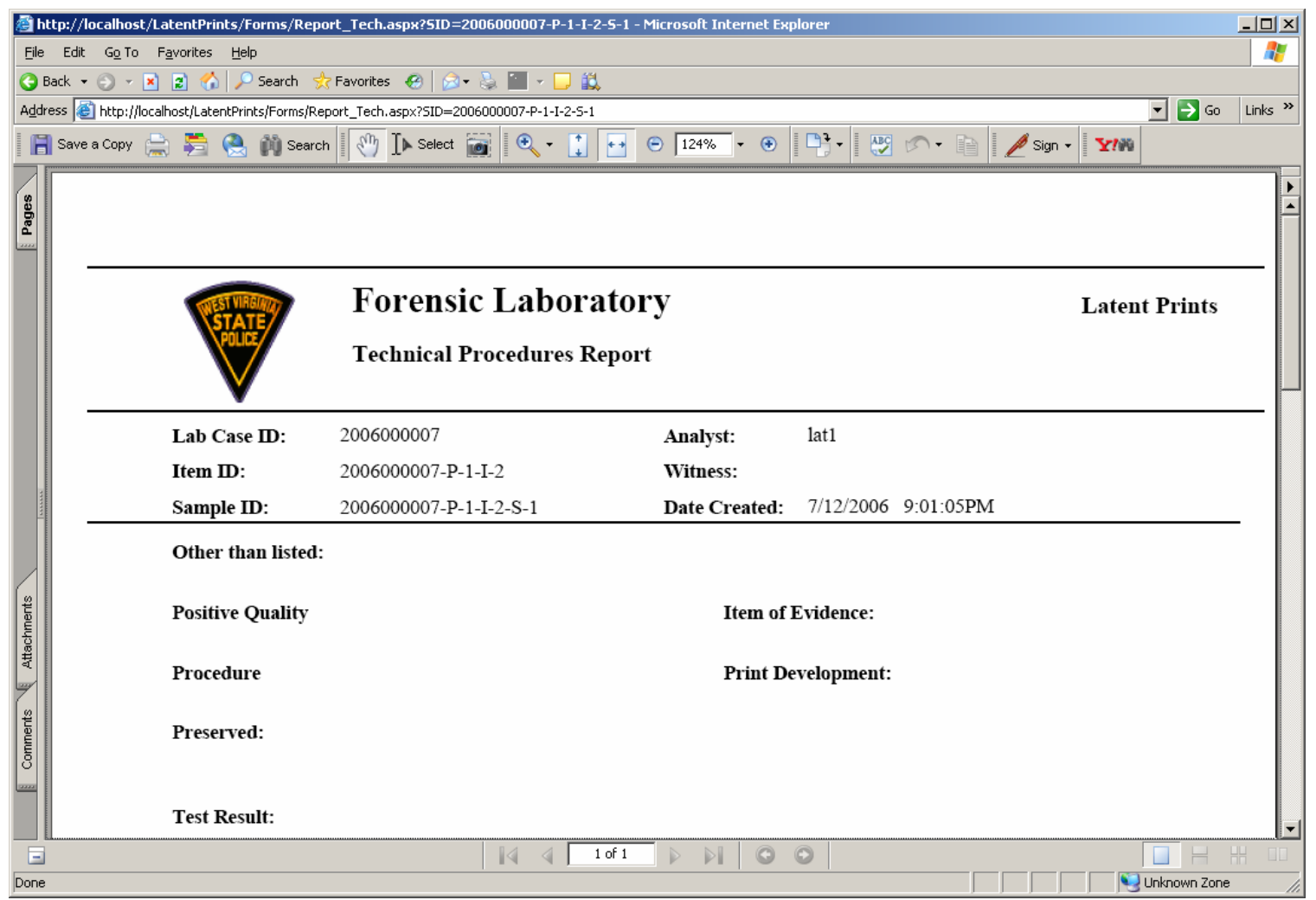

Figure 11.25: Sample Report in PDF Format

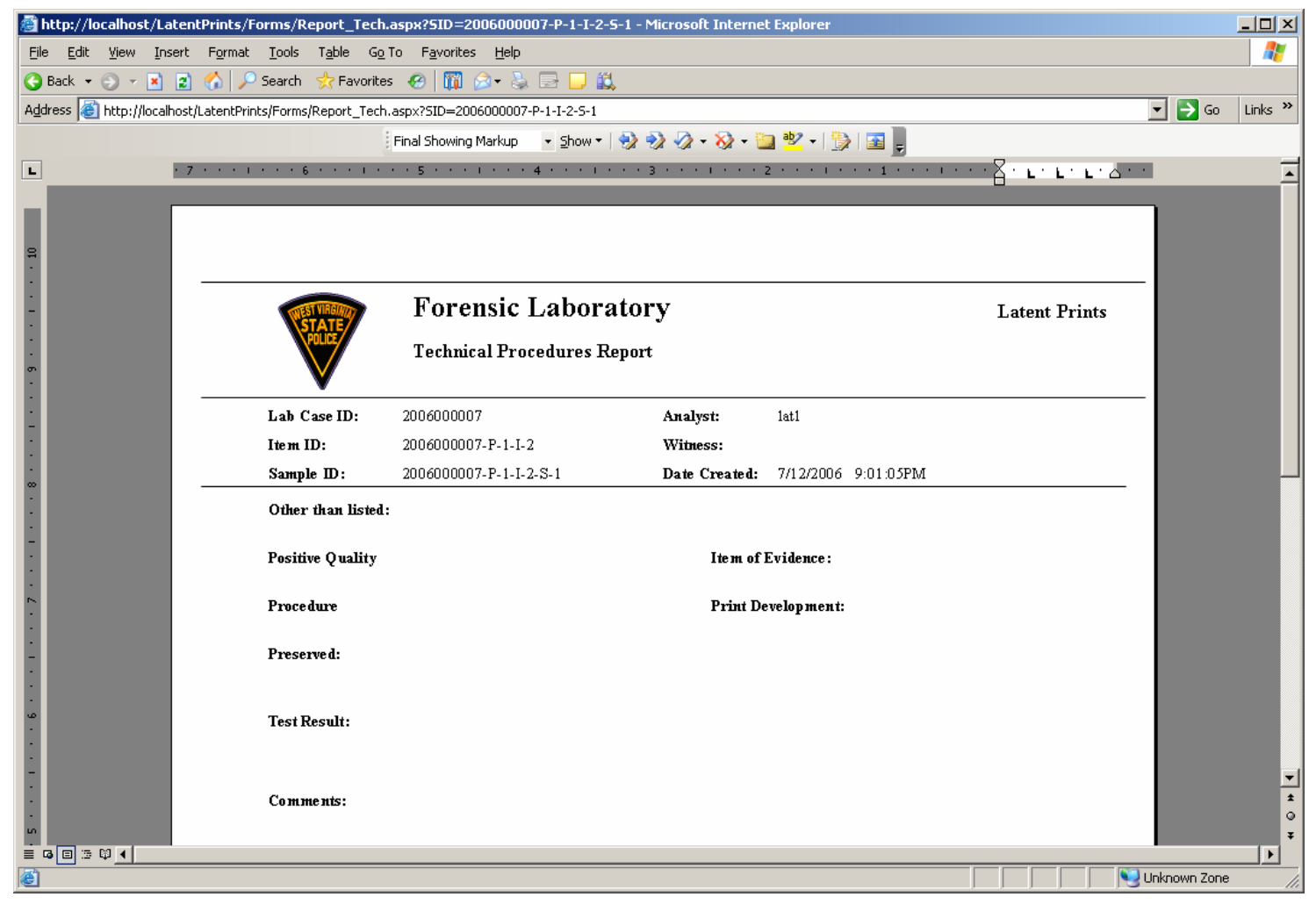

Figure 11.26: Item Report in MS Word Format 


\section{CHAPTER 12 \\ QUESTIONED DOCUMENTS}

\subsection{Existing Questioned Documents Process}

The Questioned Documents Unit is responsible for the examination of documents. The documents can be checks, withdrawal forms, credit card receipts, demand notes, suicide notes, anonymous letters, firearms transaction reports, insurance claim forms, and prescriptions. Watermarks can also be examined to determine the date and or manufacturer of the questioned paper. Questioned photocopies can be examined and linked back to a photocopier. Inks can be evaluated with differing wavelengths of light to determine if writing was added, or to visualize obliterated writing on questioned documents. Documents from computer printers can be classified as being produced from a dot matrix, ink jet or laser printer. The questioned documents unit also provides footwear and tire impression comparisons [16].

The analyst in the questioned documents unit receives the LEIF form and chain of custody form along with the evidence items. Samples are prepared from the items received from the storage. After the required tests are performed, appropriate forms are completed. The forms/worksheets used in this unit are: Footwear Examination (FE), Footwear Comparison (FC) and Tire Comparison (TC). Once the examinations are performed, evidence is returned to the CER and the secretary prepares a draft report. The draft report is reviewed by the analyst/reviewer. Upon approval, the final report is sent to CER. The existing process for this unit is shown in Figure12.1. 


\section{Questioned Documents}

Retrieve an evidence item from the storage unit.

Prepare samples from the evidence item and assign each sample a unique identification number.

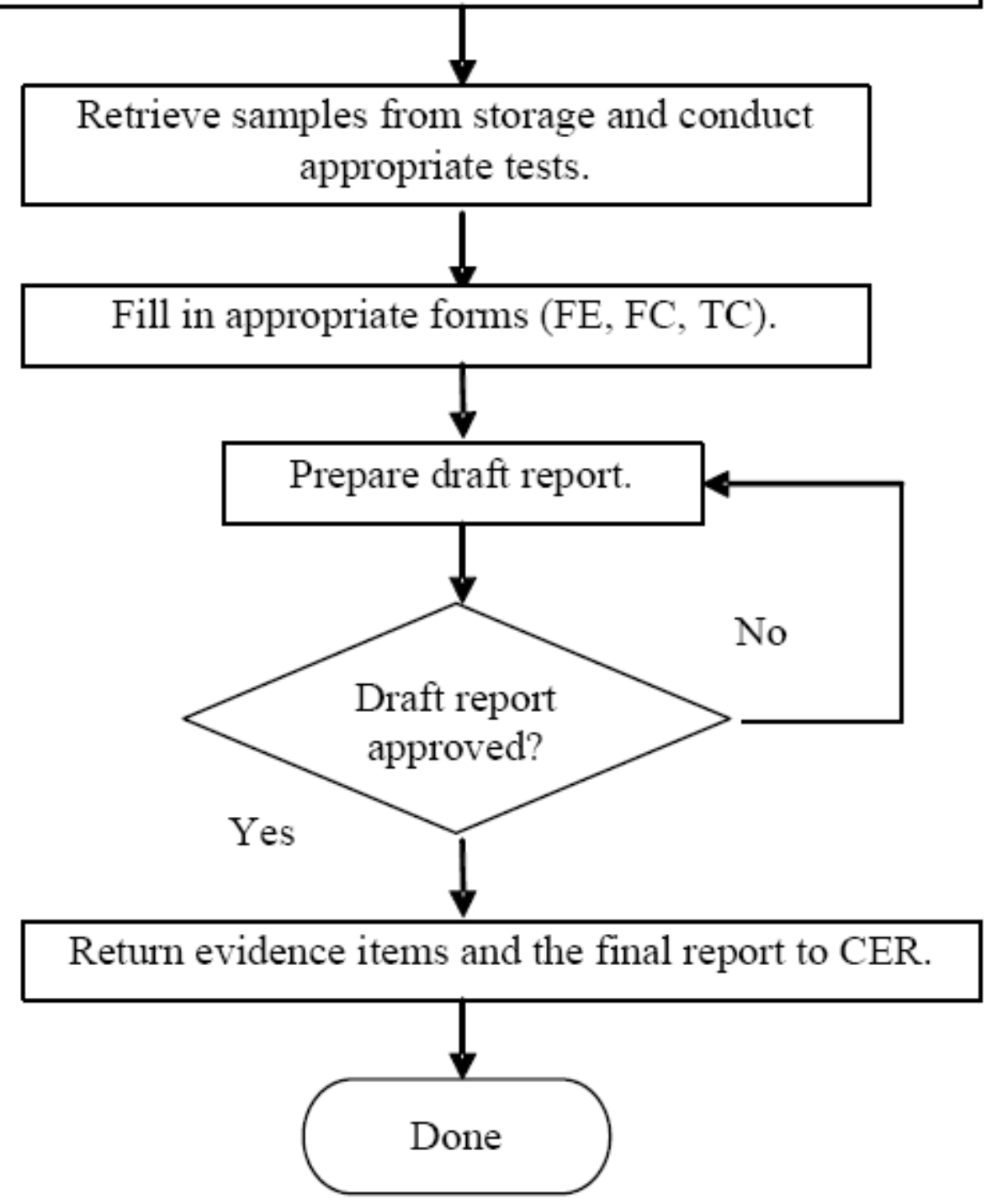

Figure 12.1: Existing Process for Questioned Documents Unit 


\subsection{Re-engineering the Questioned Documents Process}

The analyst in the questioned documents unit receives the items assigned for further processing and analysis from CEP. Received items are sub-divided into samples for conducting the requested tests. An individual item may be divided into many samples according to the requirement and complexity of the required test. A report for each sample is generated. An item report is compiled by combining sample reports. After the report is received, the reports along with the test items are sent back to CEP for further processing. The evidence and information flow diagram is shown in Figure 12.2.

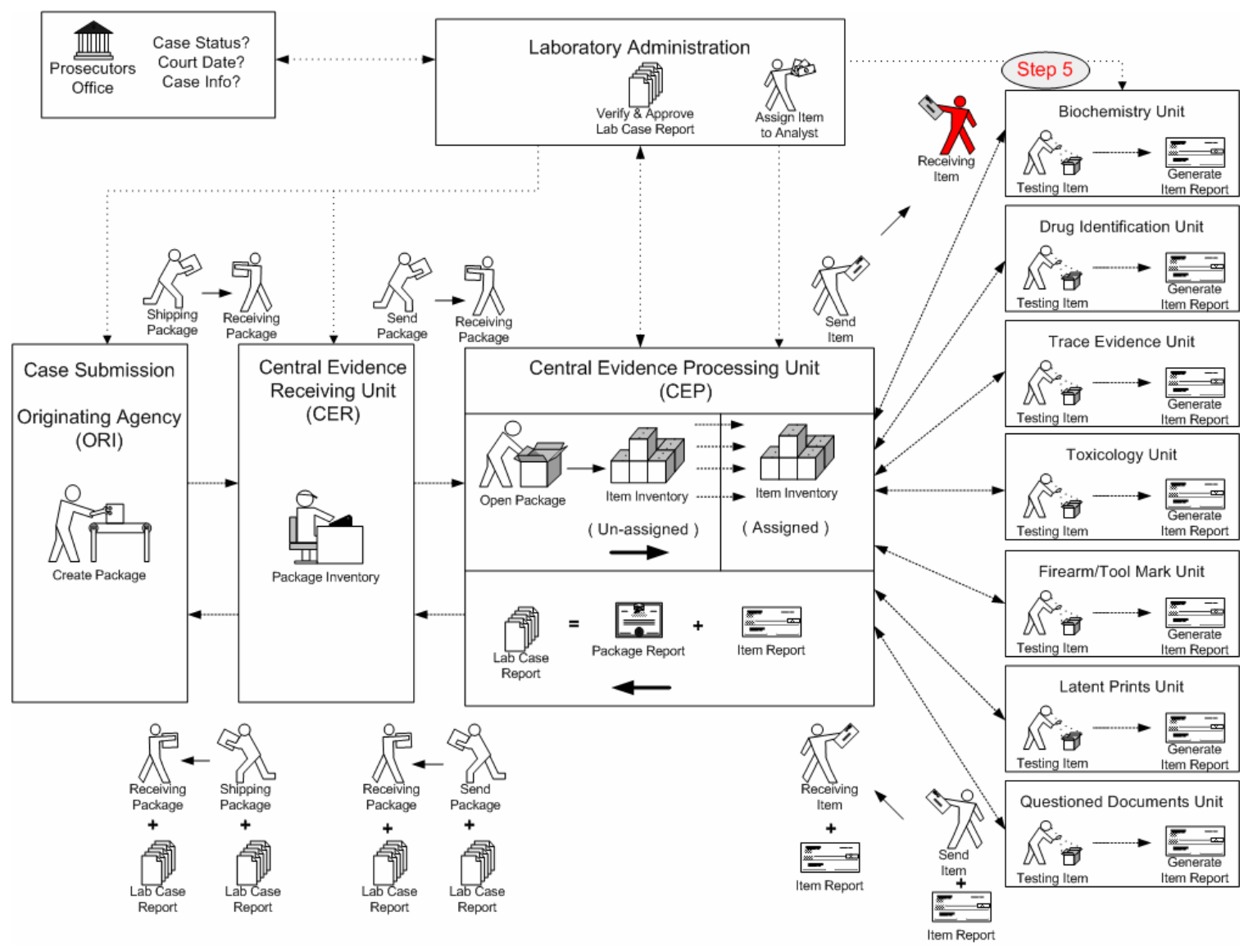

Figure 12.2: Step 5 Receive Assigned Items from CEP 
Detail procedure followed by questioned documents unit for further processing and analysis is shown in Figure 12.3.

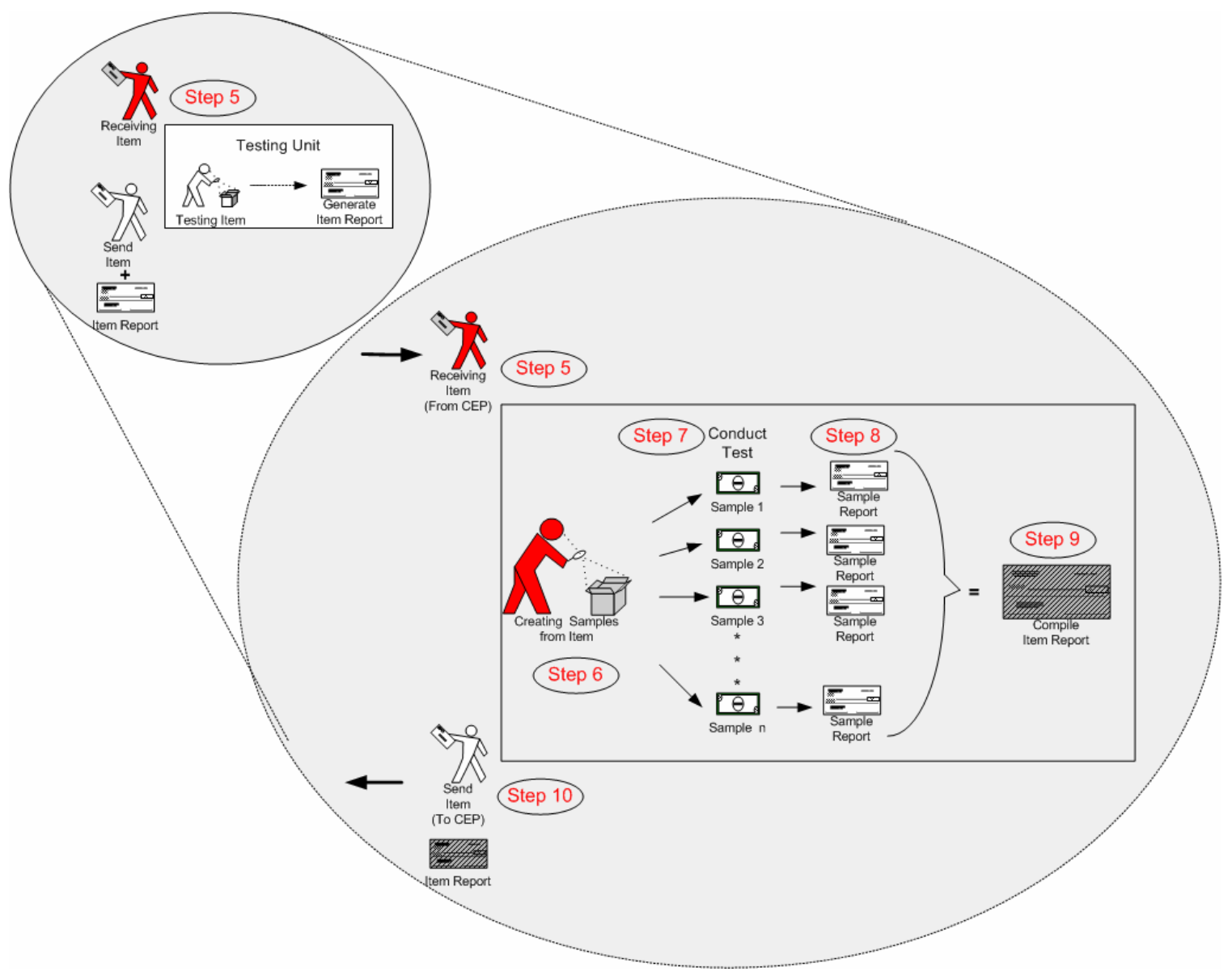

Figure 12.3: Detail Item Processing Procedure 


\subsubsection{Questioned Documents Entity Relationship Diagram}

Questioned documents data tables are connected with each other through relations as shown in the figure 12.4

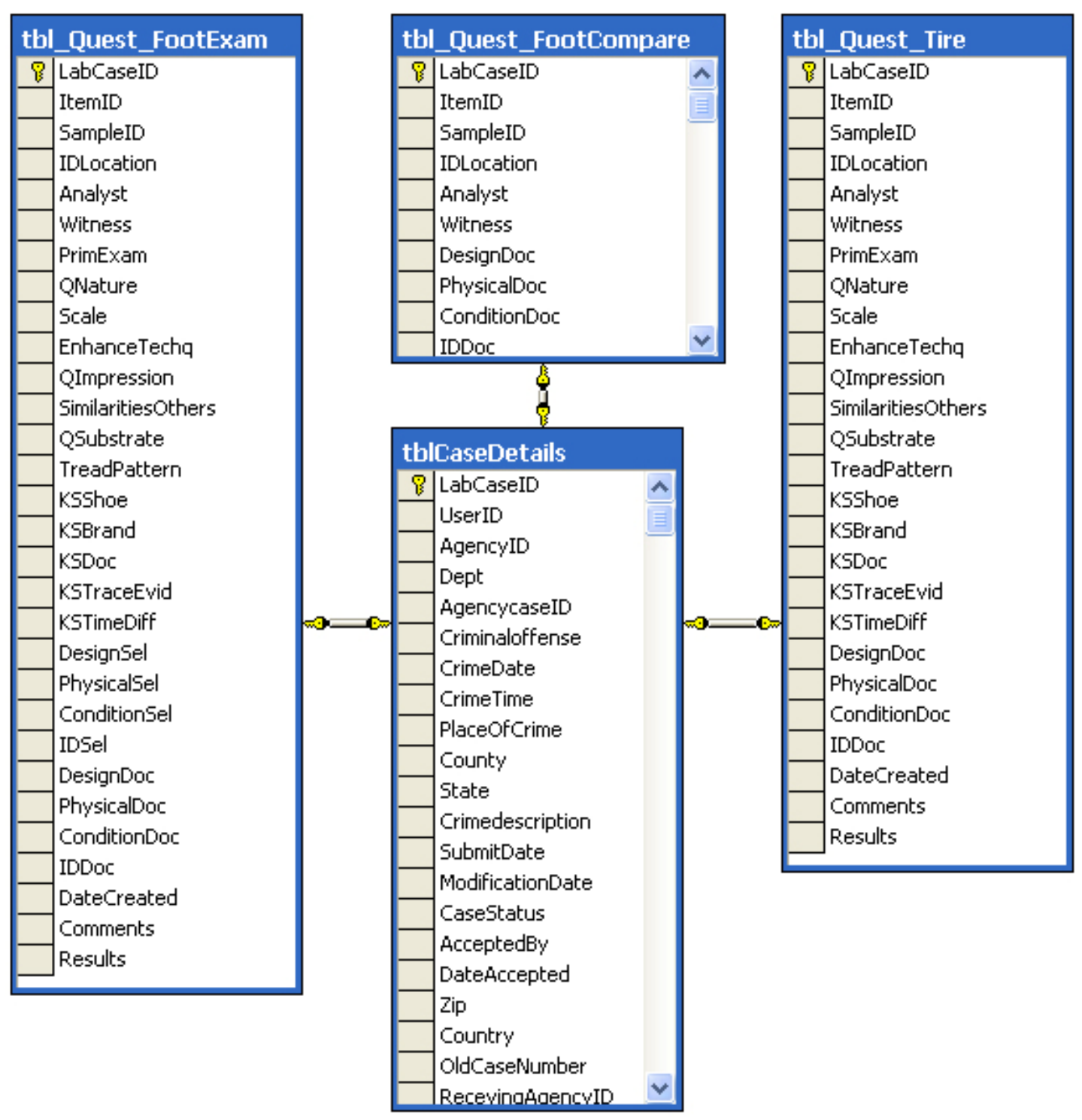

Figure 12.4: Entity Relationship Diagram 


\subsubsection{Questioned Documents Database Tables}

The Questioned Documents unit module stores its data in the following tables

- Foot Comparison(tbl_Quest_FootCompar)

- Tire Examination (tbl_Quest_Tire)

- Foot Examination (tbl_Quest_Foot_Exam)

The various fields of the foot comparison worksheet table are shown in Table 12-

1. Foot comparison worksheet data is stored and maintained in this data table

Table 12-1: Foot Comparison Table

\begin{tabular}{|l|l|}
\hline tbl_Quest_FootCompare \\
\hline B LabCaseID \\
\hline ItemID \\
SampleID \\
\hline IDLocation \\
Analyst \\
Witness \\
DesignDoc \\
PhysicalDoc \\
ConditionDoc \\
IDDoc \\
DesignSel \\
\hline PhysicalSel \\
ConditionSel \\
IDSel \\
\hline DateCreated \\
Comments \\
Results \\
\hline
\end{tabular}


The various fields of the foot examination worksheet table are shown in Table 12-

2. Foot examination worksheet data is stored and maintained in this data table

Table 12-2: Tire Table

\begin{tabular}{|l|l|}
\hline tbl_Quest_FootExam \\
\hline ItemID \\
SampleID \\
IDLocation \\
Analyst \\
Witness \\
\hline PrimExam \\
\hline QNature \\
\hline Scale \\
\hline EnhanceTechq \\
\hline QImpression \\
\hline Similaritiesothers \\
\hline QSubstrate \\
\hline TreadPattern \\
KSShoe \\
KSBrand \\
KSDoc \\
KSTraceEvid \\
KSTimeDiff \\
DesignSel \\
PhysicalSel \\
ConditionSel \\
IDSel \\
DesignDoc \\
PhysicalDoc \\
ConditionDoc \\
IDDoc \\
DateCreated \\
Comments \\
Results \\
\hline
\end{tabular}


The various fields of the tire examination worksheet table are shown in Table 123. Tire examination worksheet data is stored and maintained in this data table

Table 12-3: Foot Examination

\begin{tabular}{|l|l|}
\hline tbl_Quest_Tire \\
\hline & LabCaseID \\
\hline StemID \\
SampleID \\
IDLocation \\
Analyst \\
Witness \\
\hline PrimExam \\
QNature \\
\hline Scale \\
\hline EnhanceTechq \\
QImpression \\
SimilaritiesOthers \\
QSubstrate \\
TreadPattern \\
KSShoe \\
KSBrand \\
KSDoc \\
KSTraceEvid \\
KSTimeDiff \\
DesignDoc \\
PhysicalDoc \\
ConditionDoc \\
IDDoc \\
DateCreated \\
Comments \\
Results \\
\hline
\end{tabular}




\subsubsection{Working with Questioned Documents}

Questioned Documents home page requires username and password to verify user credentials, as shown in the Figure 12.5. After verifying credentials, a user is directed to list of items assigned to him/her for further processing.

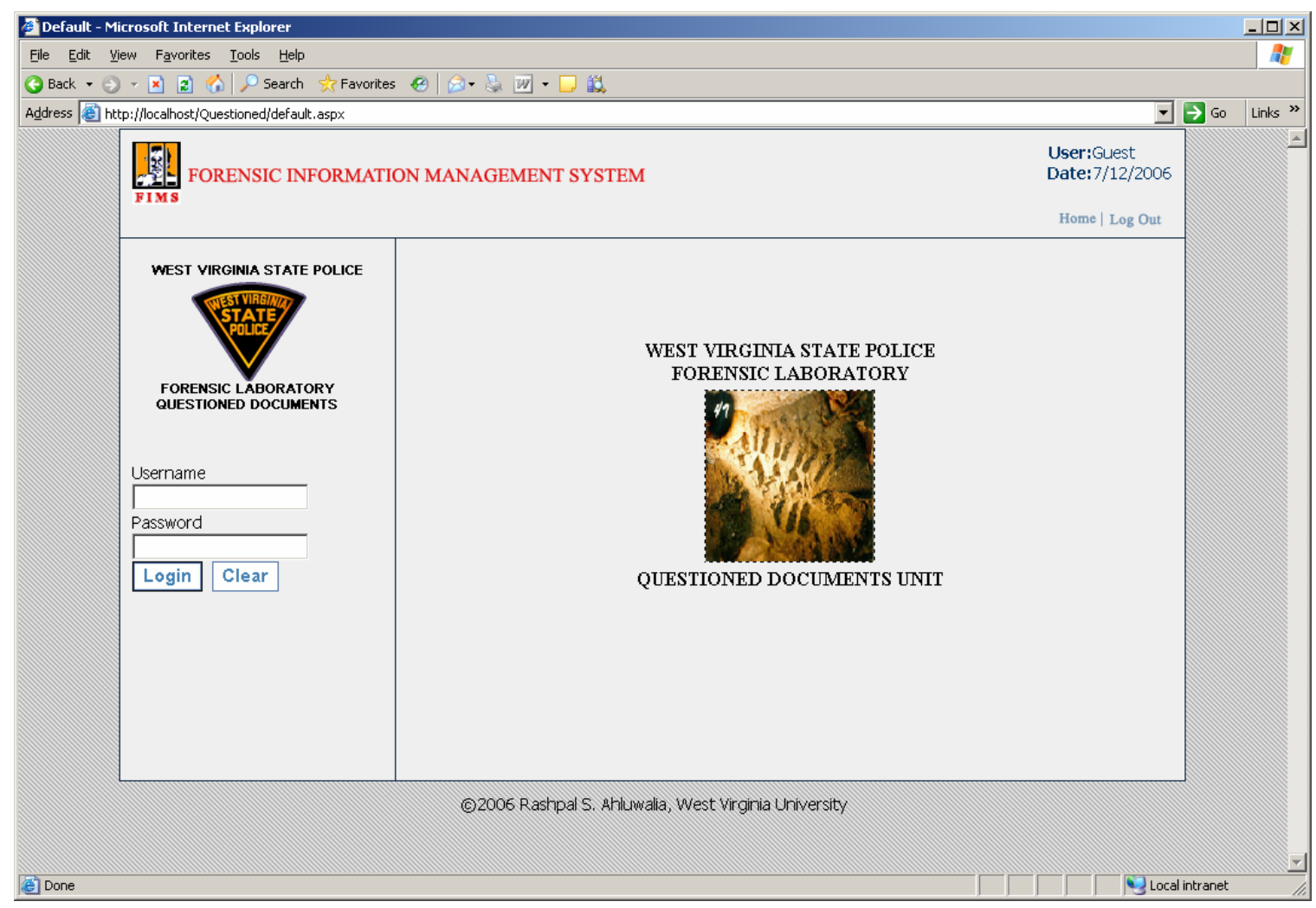

Figure 12.5: Questioned Documents Home Page 


\section{View Items Assigned}

Figure 12.6 shows the list of items assigned to the analyst by the laboratory administration unit for further analysis. Initially job status for the item assigned is set to "Job Pending". The "View Details" hyperlink enables an analyst to view the details of the case. If the list of items assigned grows long i.e. more than 15 items, the record set is divided into multiple pages. Analysts can navigate between different pages by clicking on the header and footer navigation bar of the list.

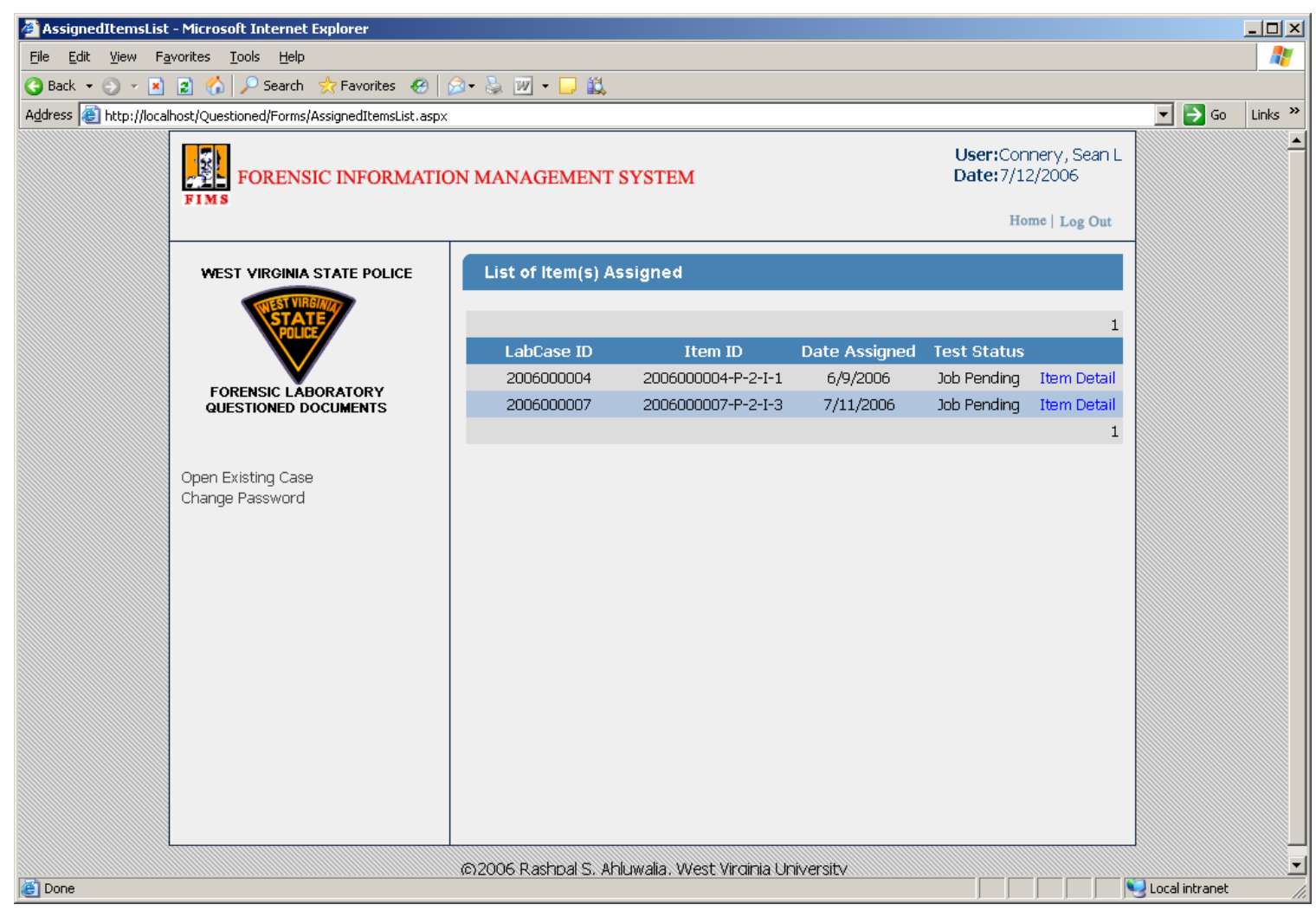

Figure 12.6: Assigned Item List 


\section{Item Picture Management}

This section describes the procedure of attaching pictures with an item. Analysts can perform two kinds of operation:

- View Pictures

- Upload Pictures

Analysts can access this section by clicking on the "View/Upload Item Photo" hyperlink in the item details section as shown in Figure 12.9. View picture section list the pictures already attached to the item with their thumbnails as shown in Figure 12.7. Analysts can also view the full size image by clicking on the image thumbnail. Full size image opens in a new window, which can be closed after use.

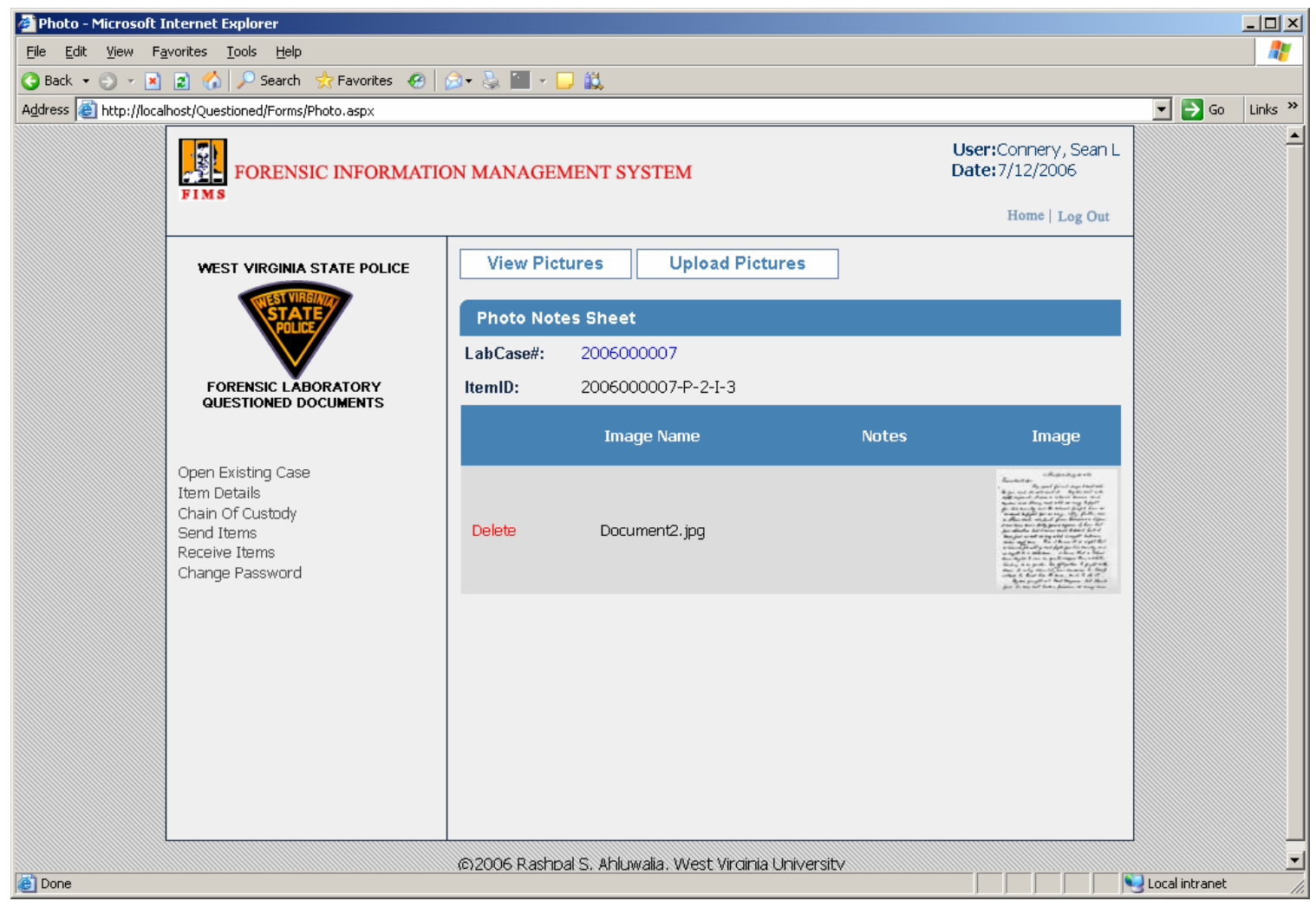

Figure 12.7: View Item Photo 
Analysts can upload any digital photo in JPG format directly into the database using this section as shown in Figure 12.8. To upload an image into database, analysts are required to browse for the image path, set other required fields and click on the "Upload the file" hyperlink. On successful upload a thumbnail appears on the bottom of the page with a success message.

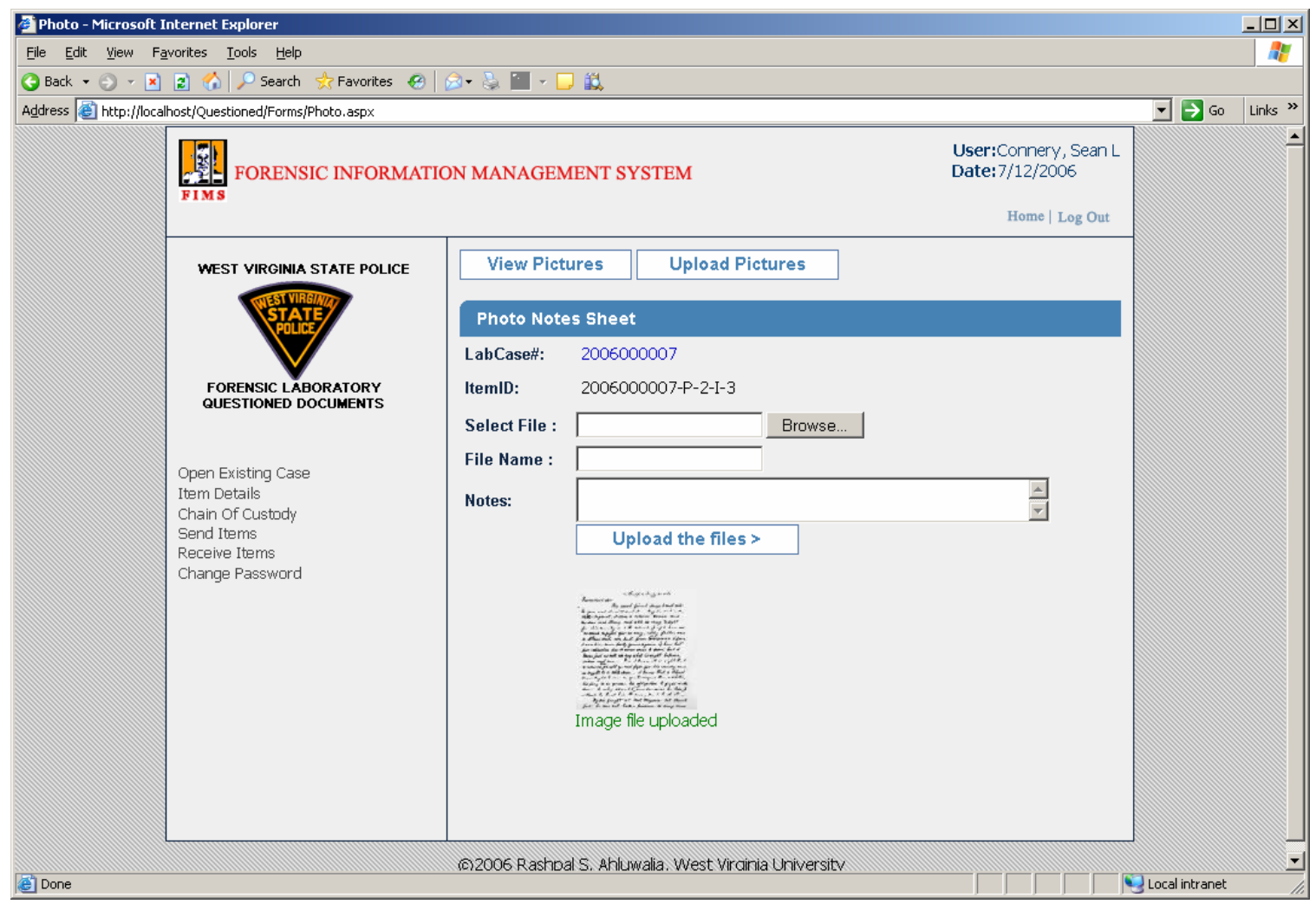

Figure 12.8: Upload Item Picture Section 


\section{Working with Samples}

Analysts receive items from the CEP unit for further analysis and processing. Once received items are sub-divided into samples for conducting requested tests. Each item can be divided into one or more samples according to the requirement and complexity of the required test.

Analysts can create new, update or delete existing samples from the item details section as shown in Figure 12.9. To add a new sample analyst is required to provide description of sample and then click on “Add Sample” hyperlink. FIMS application automatically adds the time stamp to the item created. Initially, sample test status is set to "Test Pending" for the sample. Sample information can be updated by clicking on “Edit” hyperlink and existing sample can be deleted from the system by clicking on "Delete" hyperlink.

This section also enables analysts to view the final sample, sample photo, item, and item photo report. To access the individual sample/sample photo report analyst can click on "View Report" or "Photo Sheet" hyperlink. To view comprehensive item report analysts can click on "Item Report” or "Item Photo Report” at the bottom of the page.

To complete the process for item testing, the analyst is required to click on "Item Test Complete" button, as shown in Figure 12.9. Once clicked, analyst will not be able to make any alterations to the existing item test report. 


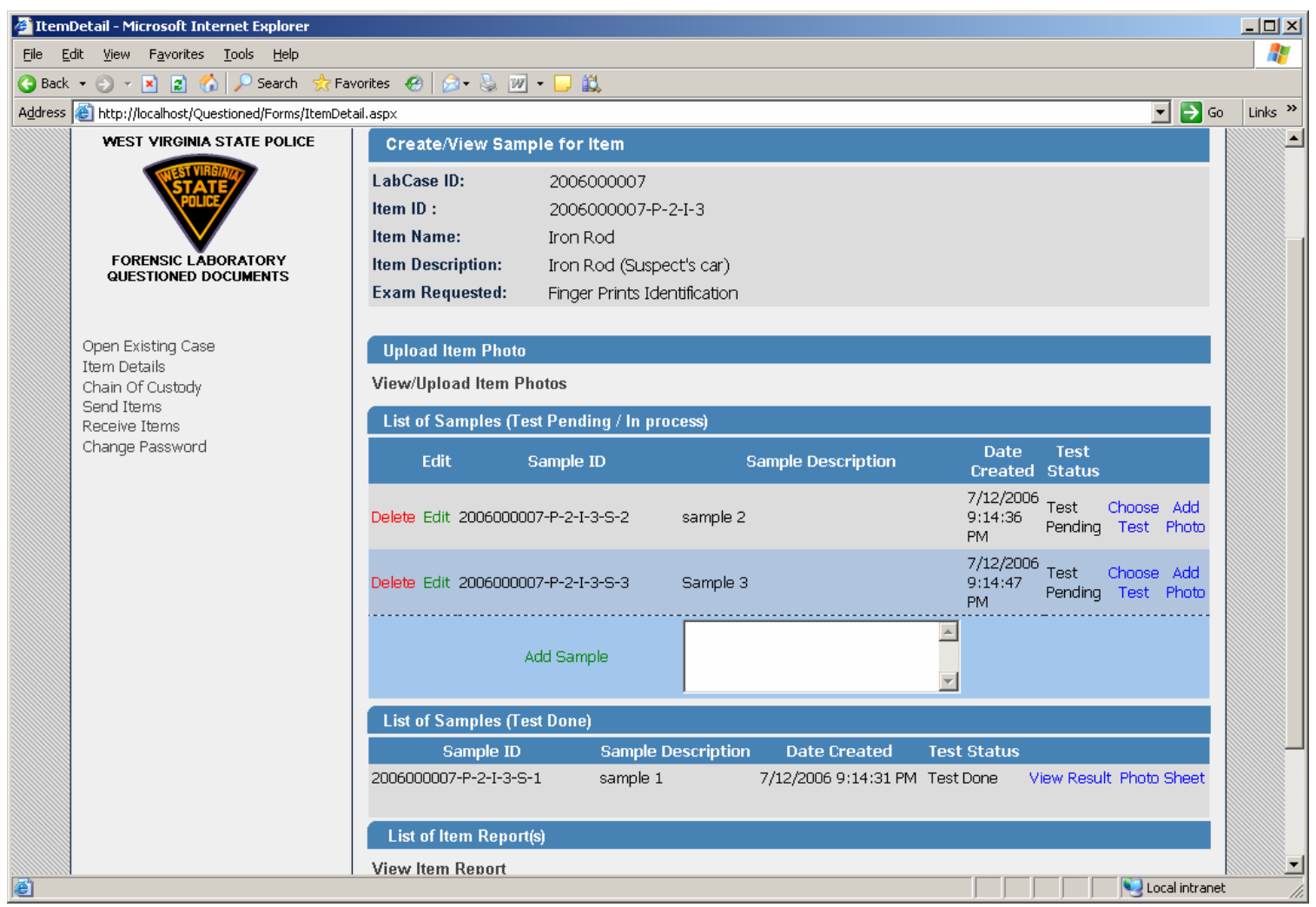

Figure 12.9: Item Detail Section 
Analysts can access this section by clicking on the "View/Upload Sample Photo" hyperlink in the item details section as shown inn Figure 12.9. View picture section list the pictures already attached to the item with their thumbnails as shown in Figure 12.10. Analysts can also view the full size image by clicking on the image thumbnail. Full size image opens in a new window, which can be closed after use.

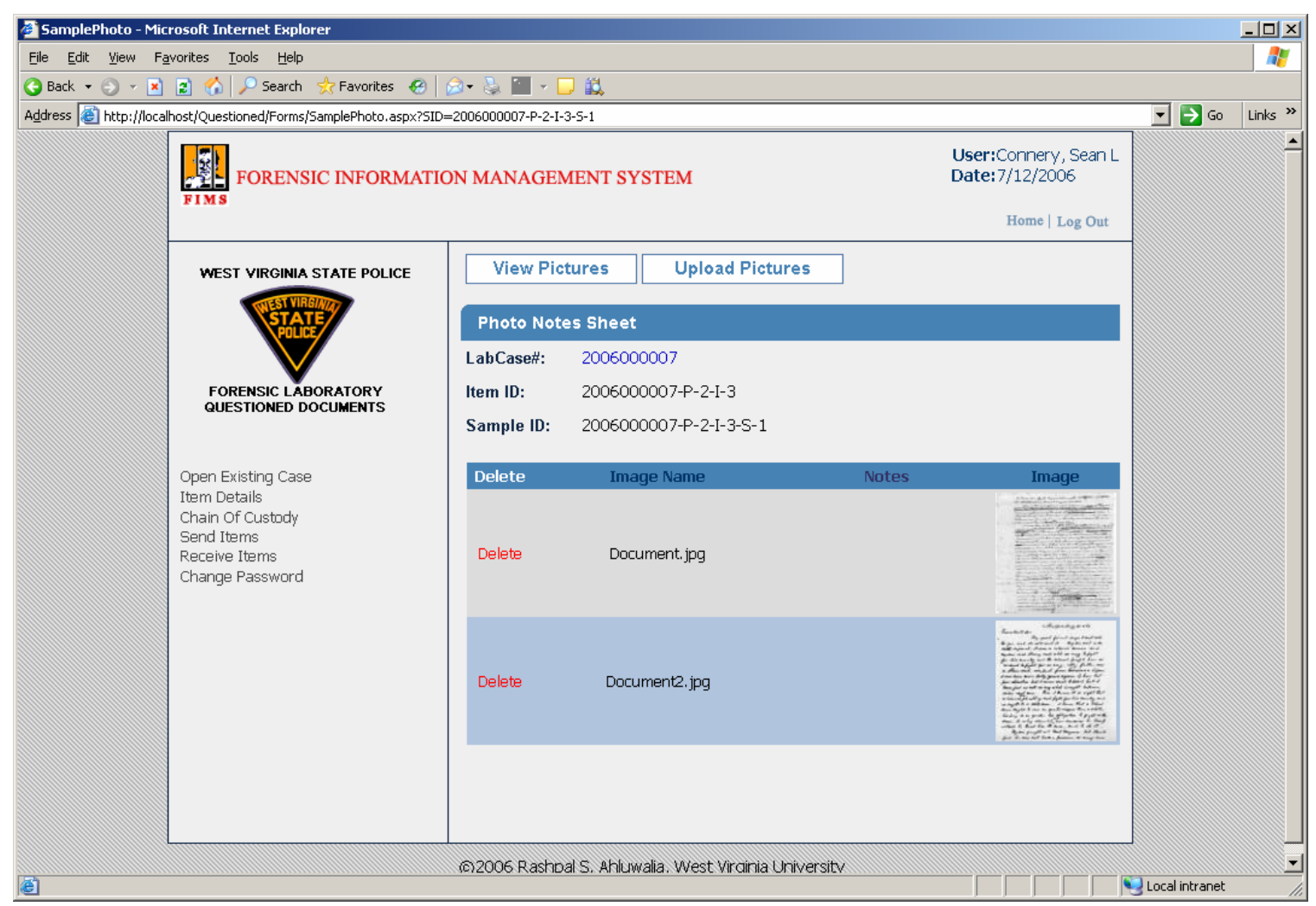

Figure 12.10: View Picture Section 
Analysts can upload any digital photo in JPG format directly into the database using this section as shown in Figure 12.11. To upload an image analysts are required to browse for the image path, set other required fields and click on the "Upload the file" hyperlink to upload the image into database. On successful upload a thumbnail appears on the bottom of the page with a success message.

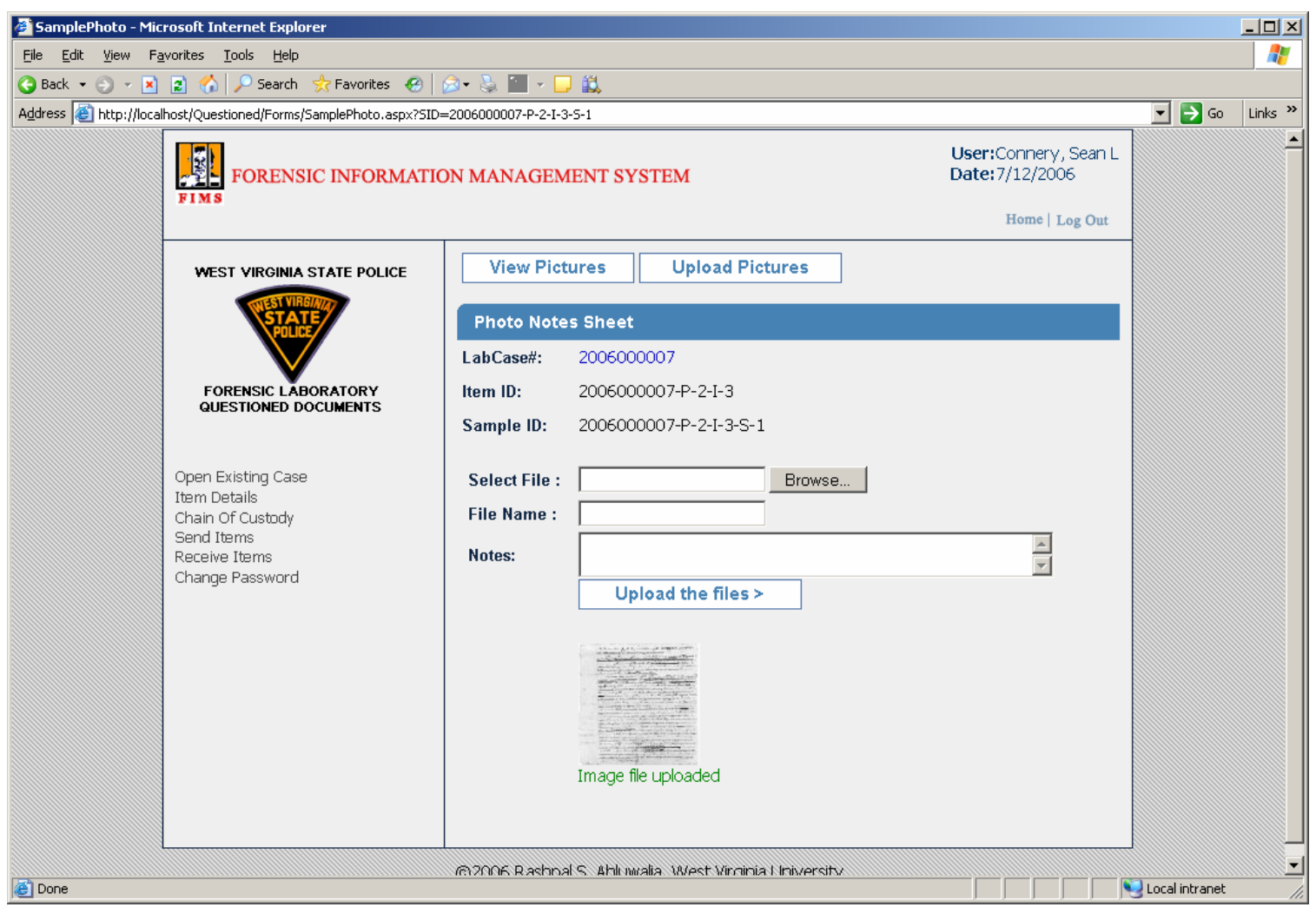

Figure 12.11: Upload Sample Picture Section 


\section{Choosing Test Worksheet}

After sample creation, analysts are required to decide what kinds of tests are required? Analysts are required to make decision of choosing type of test manually. Once the decision is made the analyst can see the list of tests available, and then click "Choose Test" hyperlink as shown in Figure 12.12. FIMS application remembers the chosen sample ID during the process.

The worksheets for the questioned documents are:

- Foot Examination

- Foot Comparison

- Tire Comparison

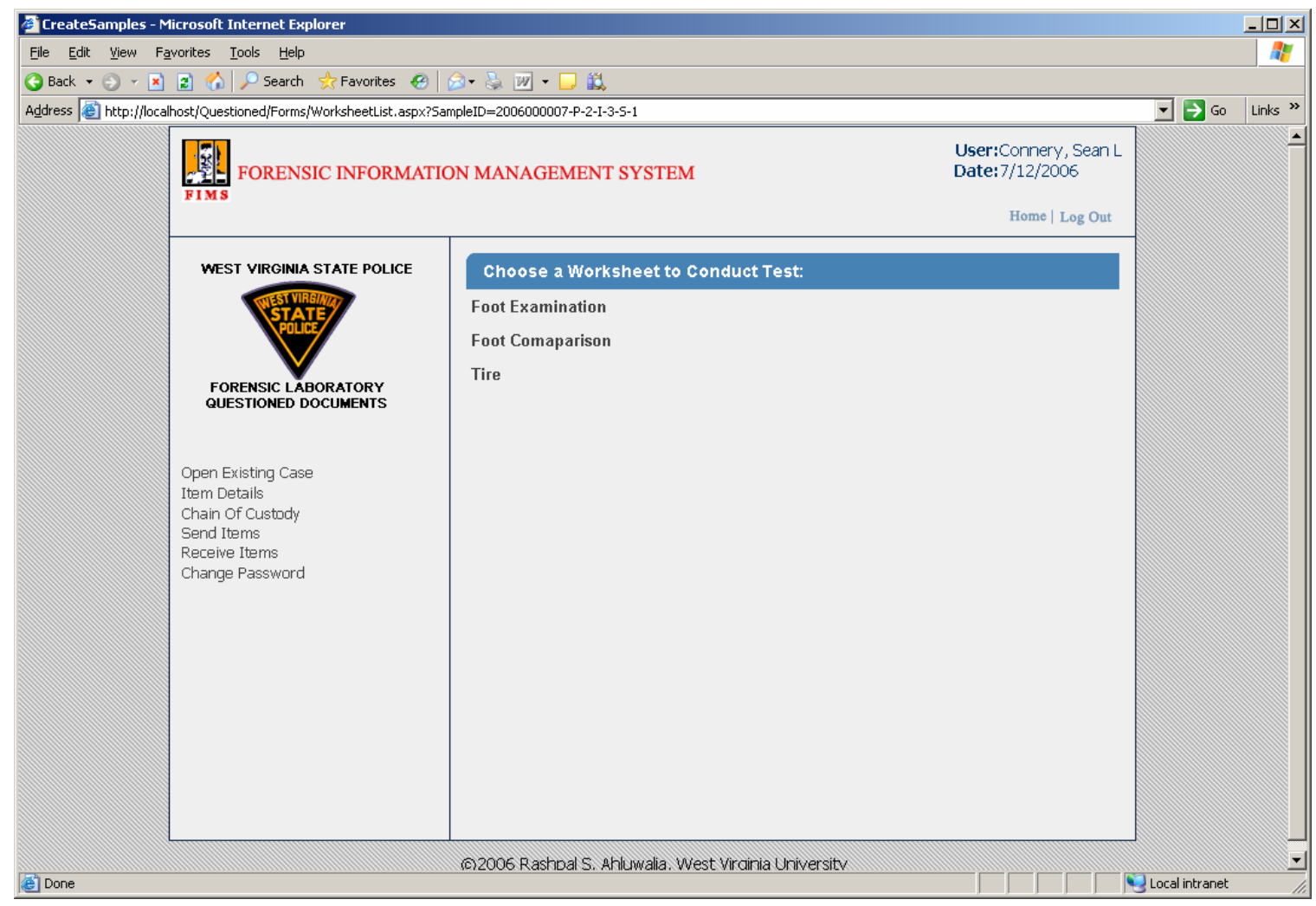

Figure 12.12: Choose Sample Test Section 


\section{Foot Examination Worksheet}

Foot Examination Worksheet is used for filling information pertaining to foot examination test performed on the sample by the analyst. After filling the required data fields, analyst is required to click on "Save Results" button located on top of the page, as shown in Figure 12.13. After saving the worksheet data, analyst can finalize and close the worksheet by clicking on "Test Done" button. The analyst cannot make any alterations in the worksheet once the "Test Done" button is clicked.

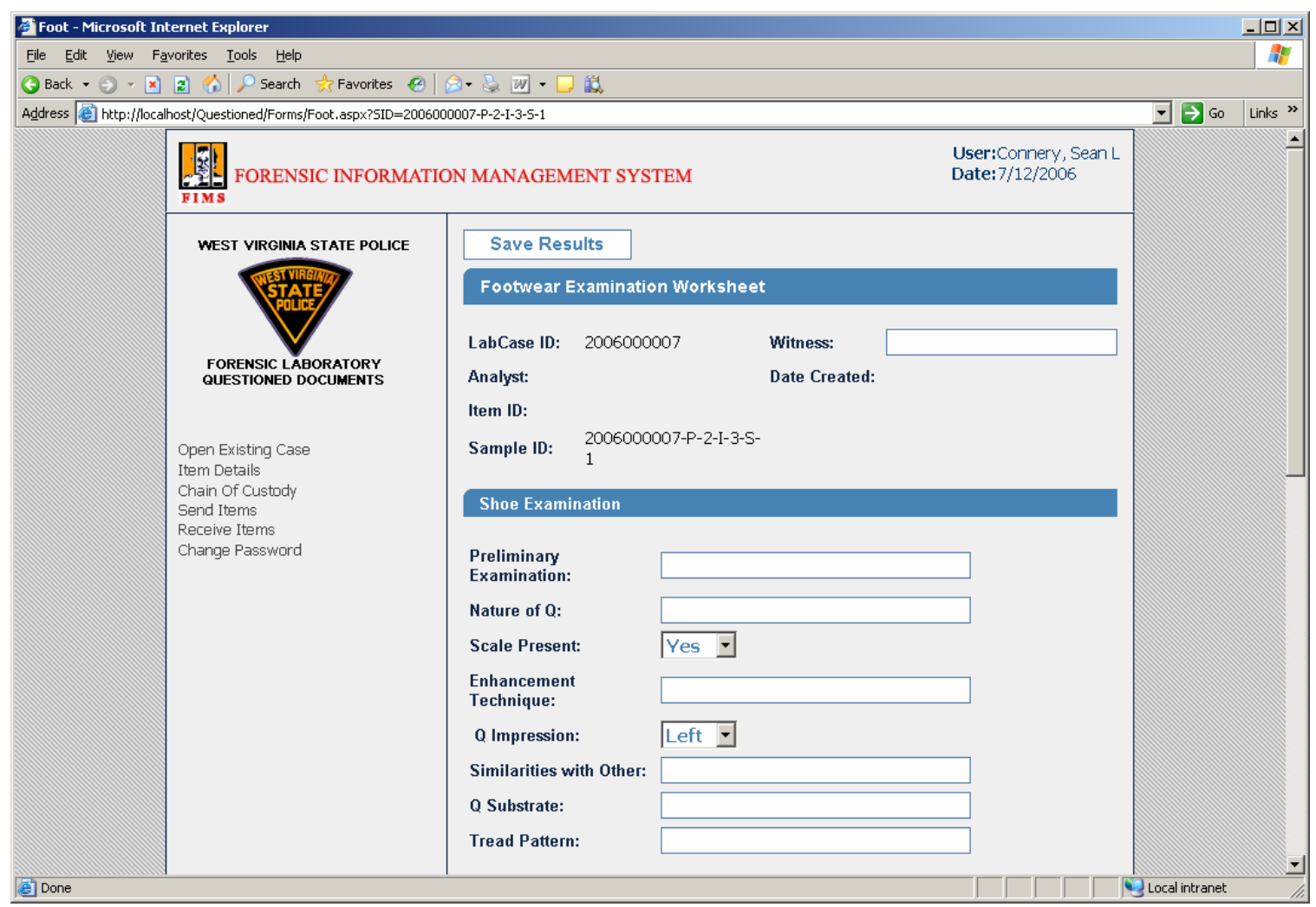

Figure 12.13: Foot Examination Worksheet 


\section{Foot Comparison Worksheet}

Foot comparison worksheet is used for filling information pertaining to foot comparison test performed on the sample by the analyst. After filling the required data fields, analyst is required to click on "Save Results" button located on top of the page, as shown in Figure 12.14. After saving the worksheet data, analyst can finalize and close the worksheet by clicking on "Test Done" button. The analyst cannot make any alterations in the worksheet once the "Test Done" button is clicked.

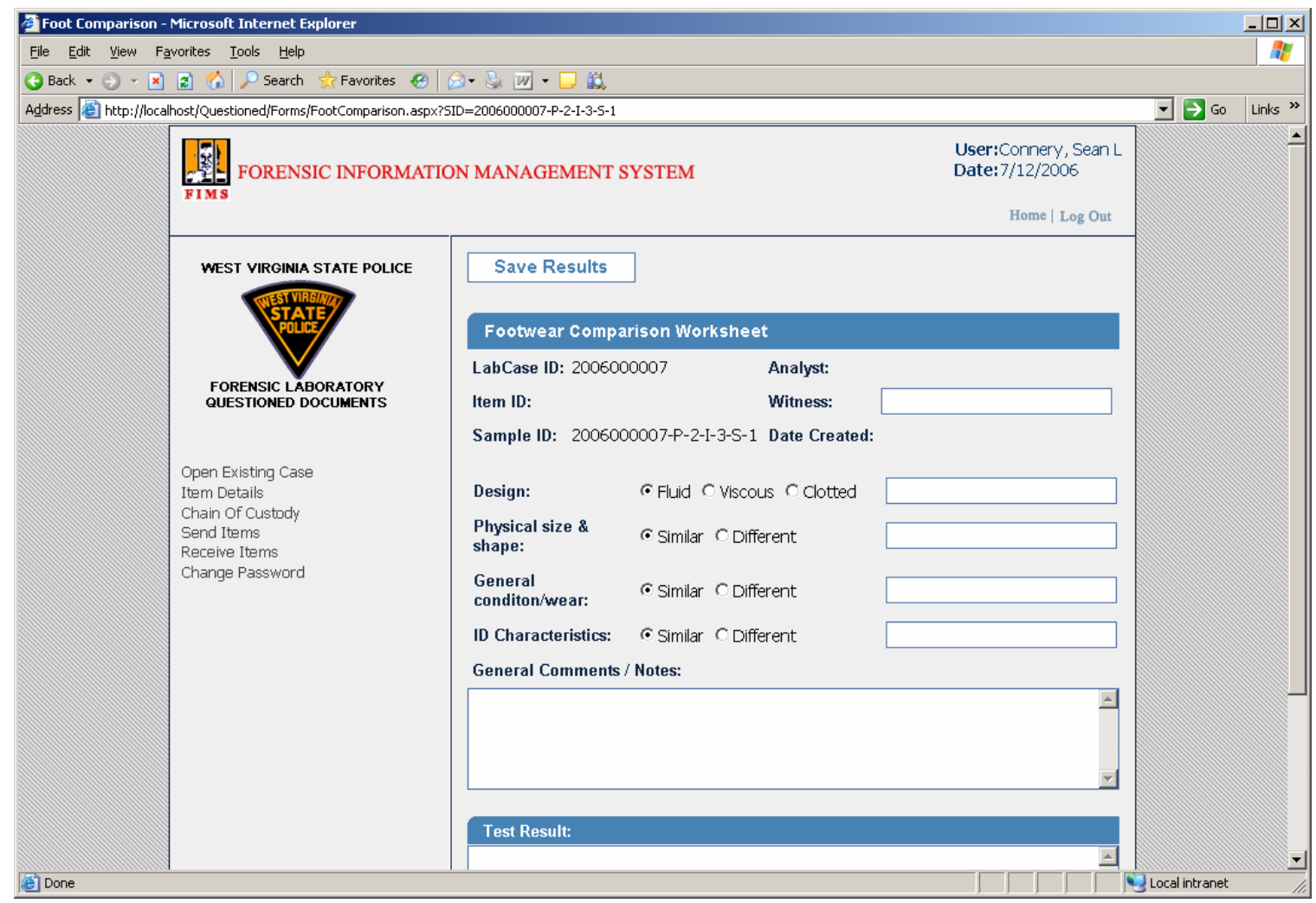

Figure 12.14: Foot Comparison Worksheet 


\section{Tire Comparison Worksheet}

Tire Comparison Worksheet is used for filling information pertaining to tire comparison test performed on the sample by the analyst. After filling the required data fields, analyst is required to click on "Save Results" button located on top of the page, as shown in Figure 12.15. After saving the worksheet data, analyst can finalize and close the worksheet by clicking on “Test Done” button. The analyst cannot make any alterations in the worksheet once the "Test Done" button is clicked.

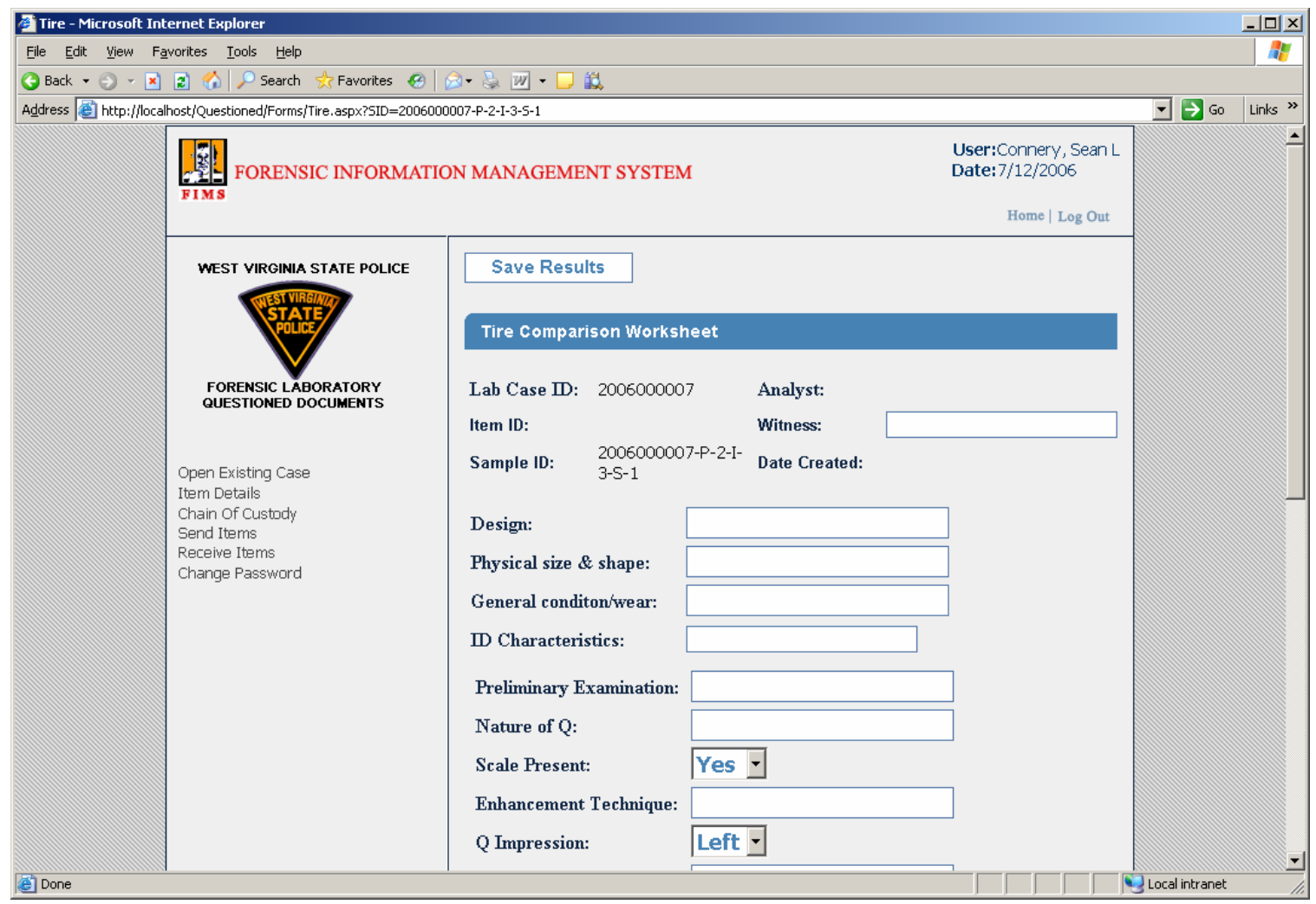

Figure 12.15: Tire Comparison Worksheet 


\section{Receive Item}

Once an item is sent by CEP unit, Questioned Documents unit analyst is responsible for receiving it by clicking on "Receive" button, under receive item section, as shown in Figure 12.16. This completes the handshaking process of item transfer between units.

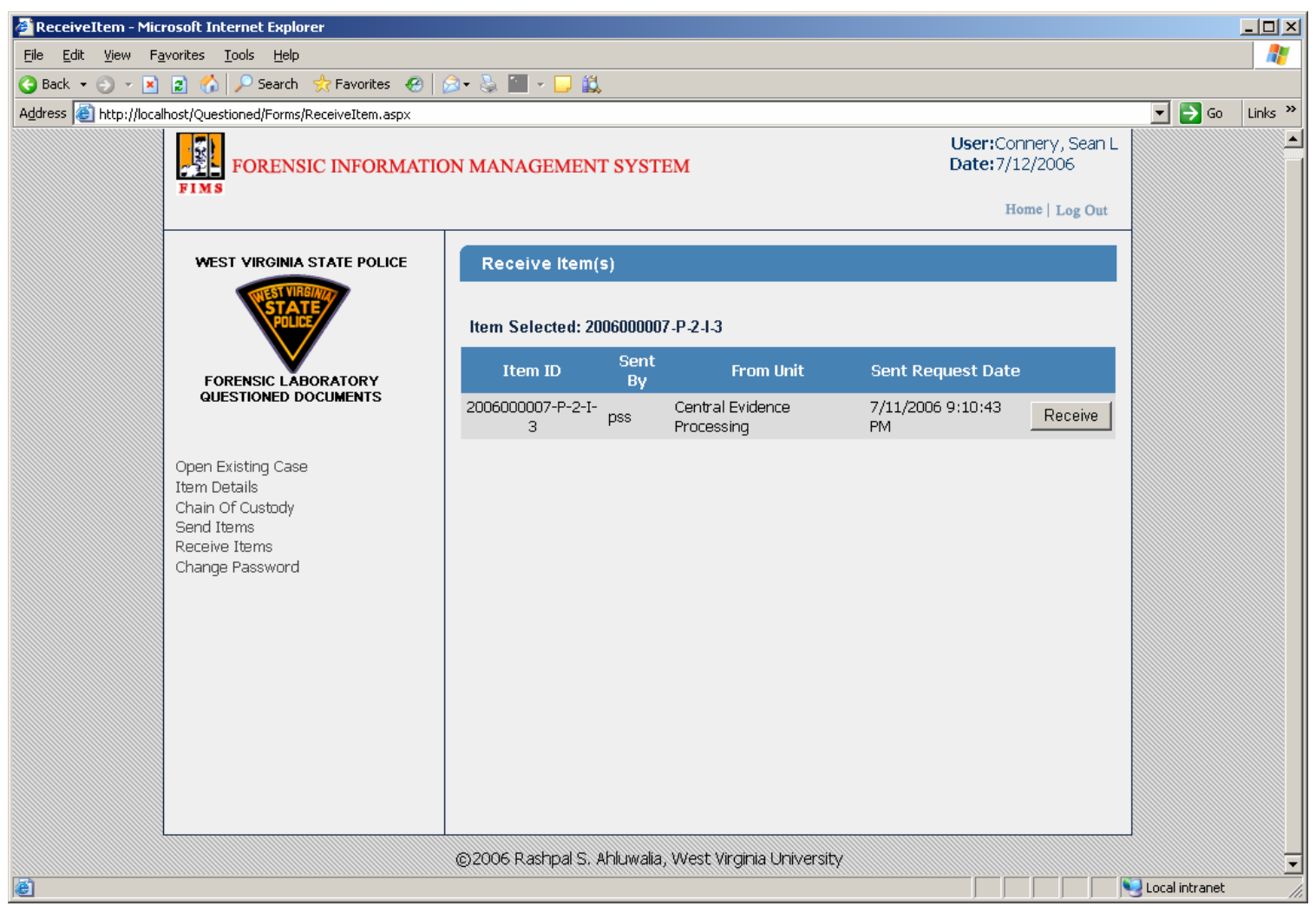

Figure 12.16: Receive Item Section 


\section{Send Item}

After completion of item testing, analyst is required to return the evidence item back to CEP unit. Figure 12.17 shows the section used to send an item back to the CEP unit. Analyst can use the drop down to select item ID and click on "Send" hyperlink, to send item back to CEP.

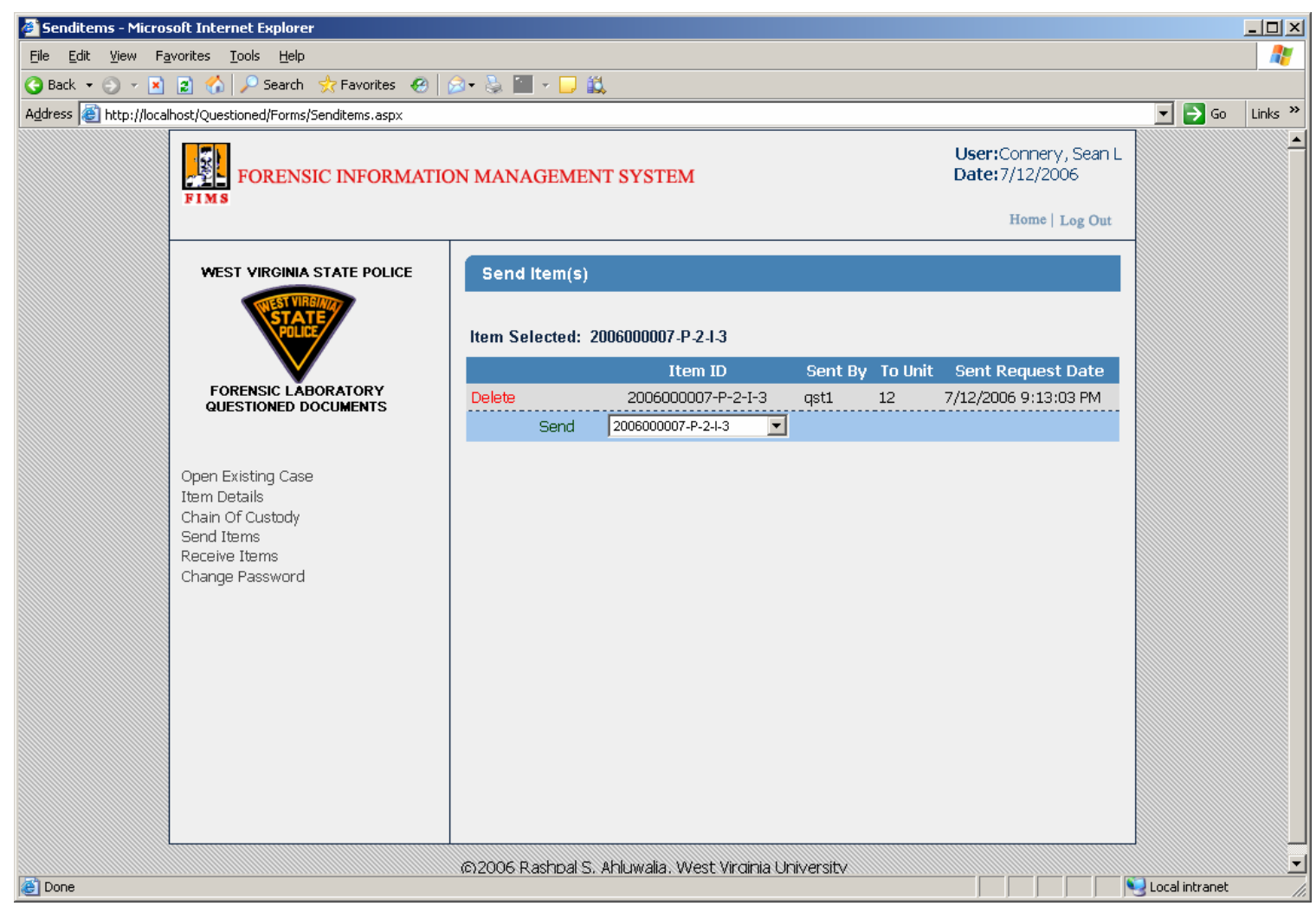

Figure 12.17: Send Item Section 


\section{Chain of Custody}

Item chain of custody information is shown in Figure 12.18. FIMS application records used ID, unit name, and time stamp for tracking item chain of custody.

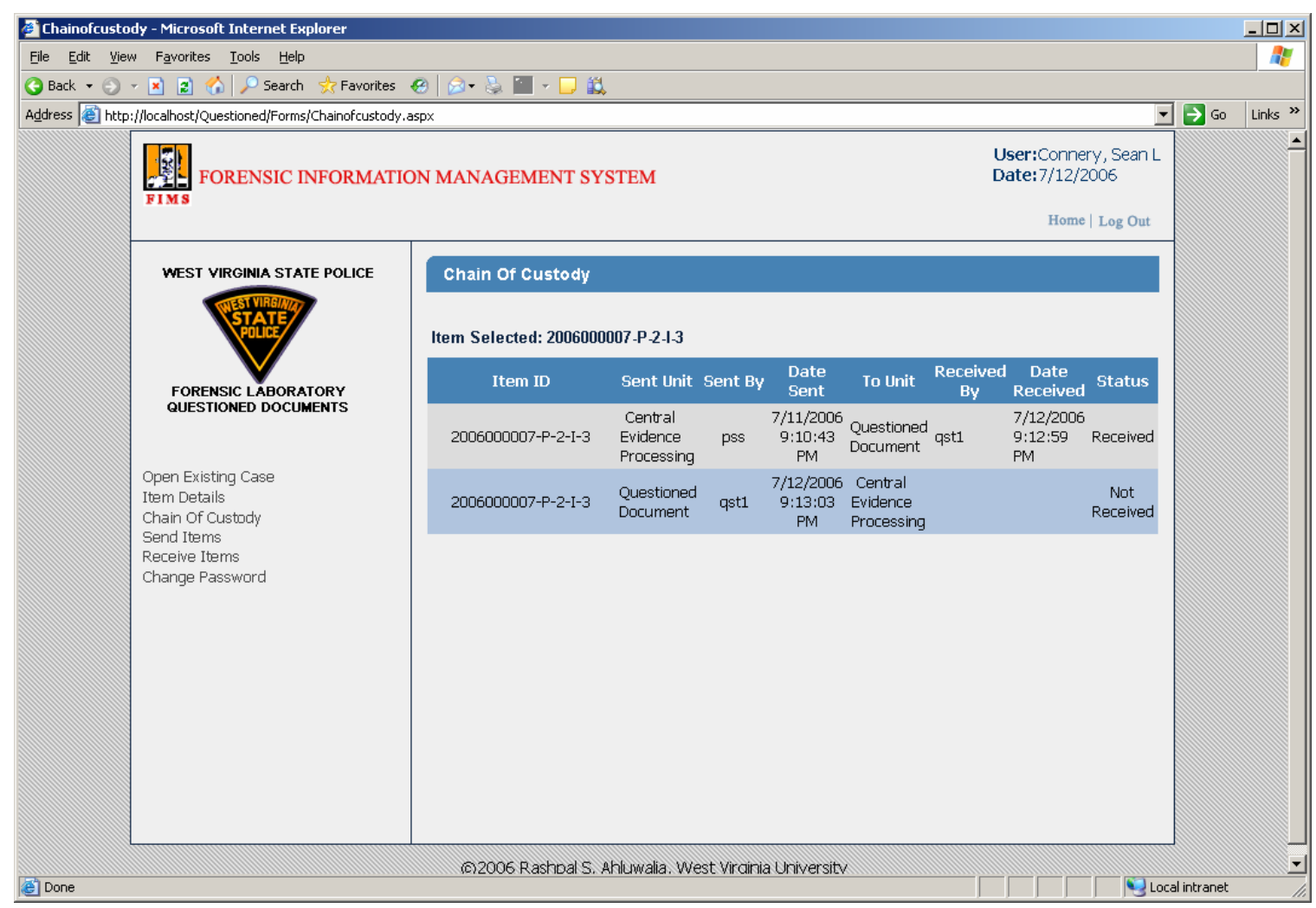

Figurer12.18: Item Chain Of Custody 


\section{Change User Password}

Analyst can change password through this section by providing appropriate credentials as shown in Figure 12.19

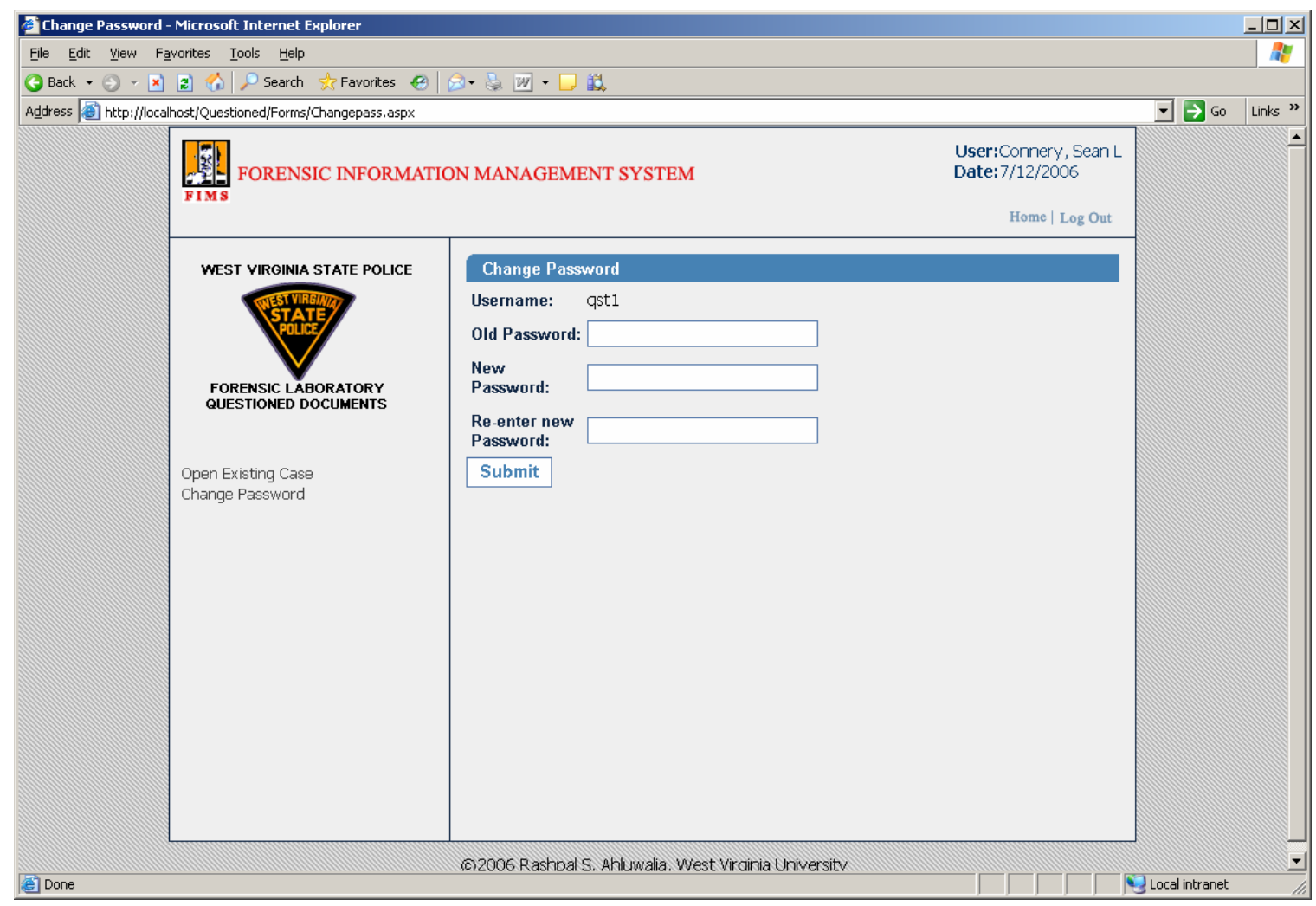

Figure 12.19: Change Password Section

\section{Exiting Questioned Documents}

Users can terminate the current session by clicking on "Log Out” hyperlink on the top right corner of the page. 


\subsubsection{Generating Item and Sample Reports}

The analyst can generate the following types of reports

- Item Report

- Item Photo Report

- Sample Report

- Sample Photo Report

Figure 12.20 shows the item report, which is a cumulative report for all the samples. Figure 12.21 shows the sample report. This section also facilitate analyst to export report via PDF/MS-Word format, as shown in Figure 12.22 and Figure 12.23.

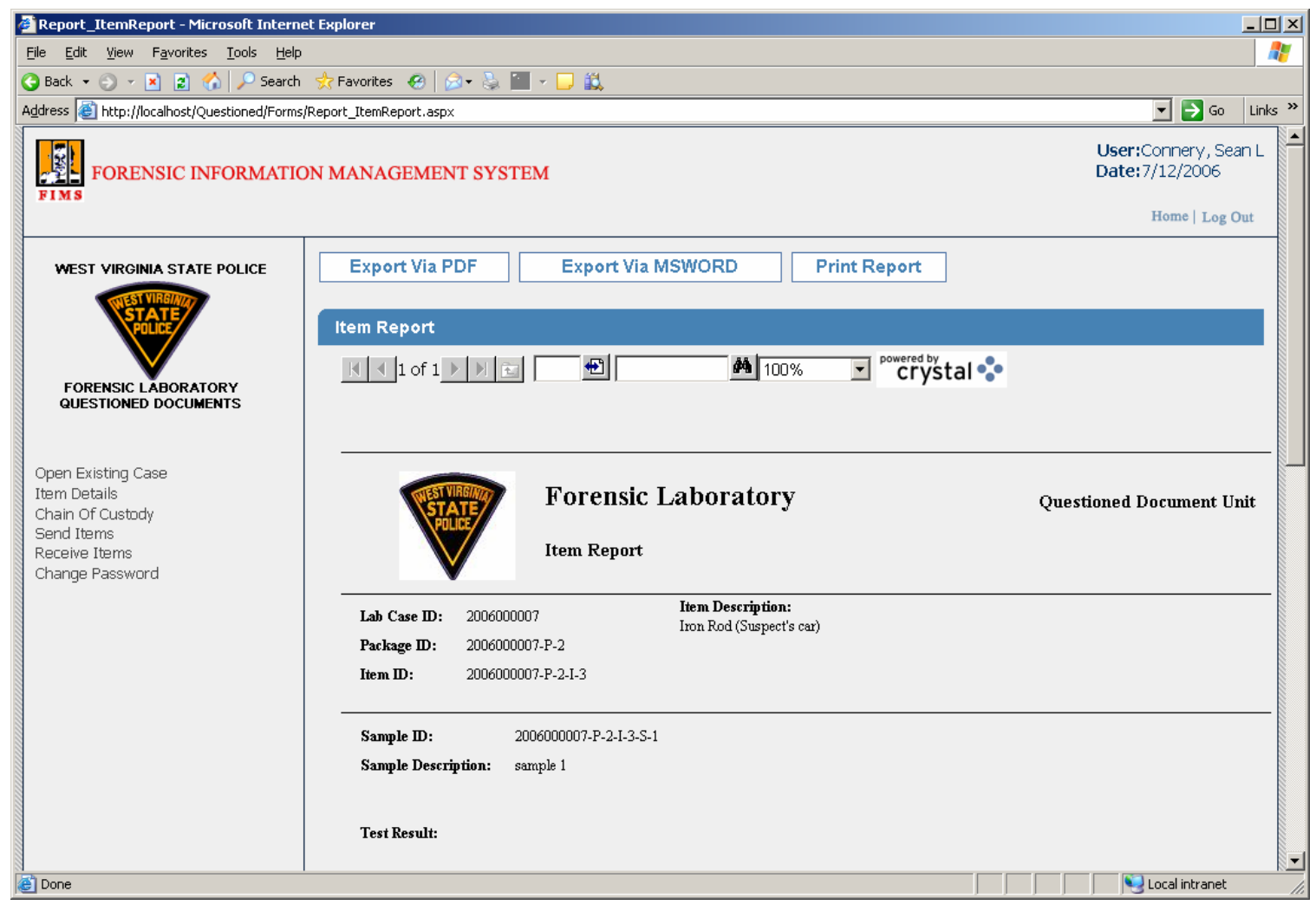

Figure 12.20: Item report 


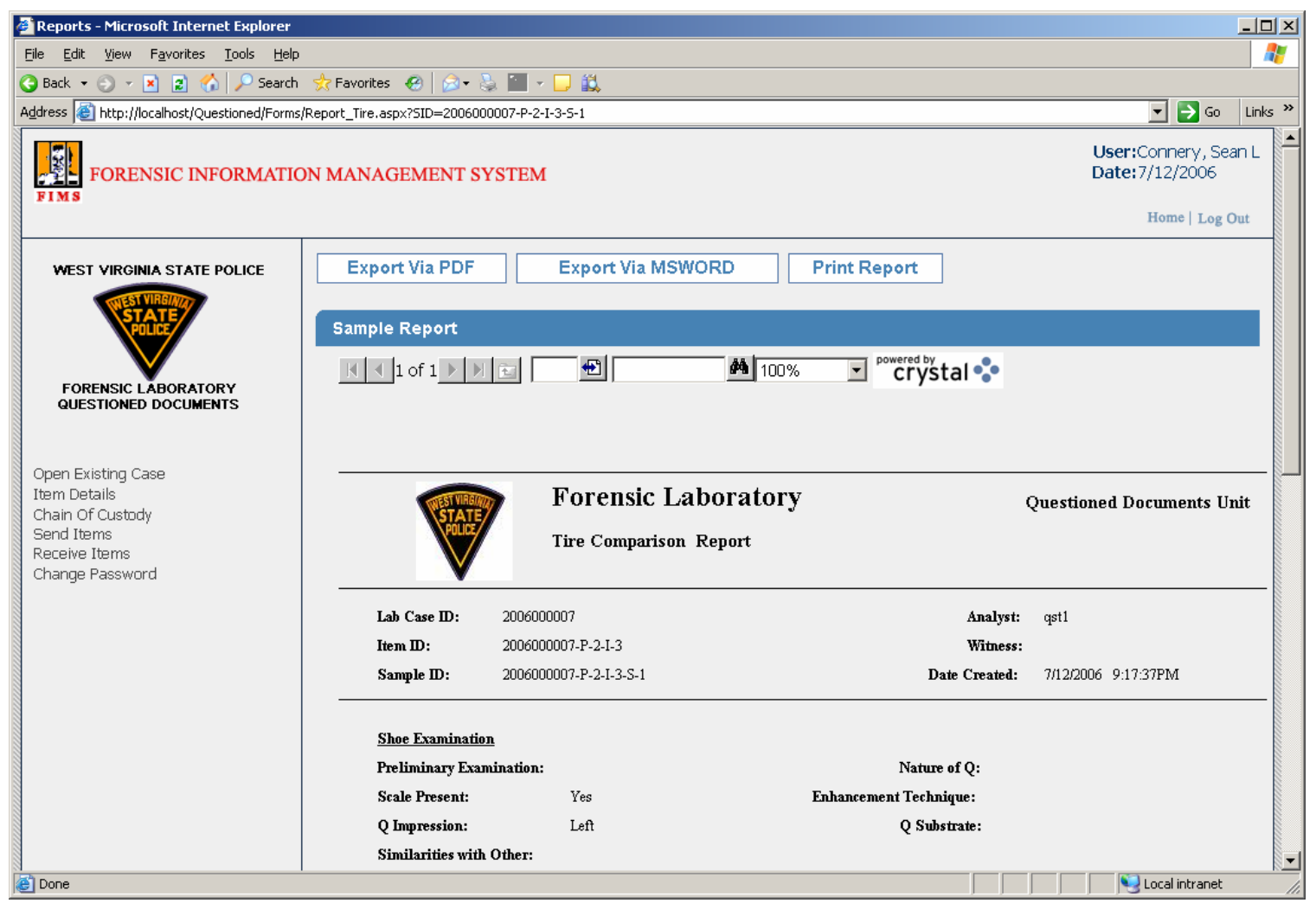

Figure 12.21: Sample Report

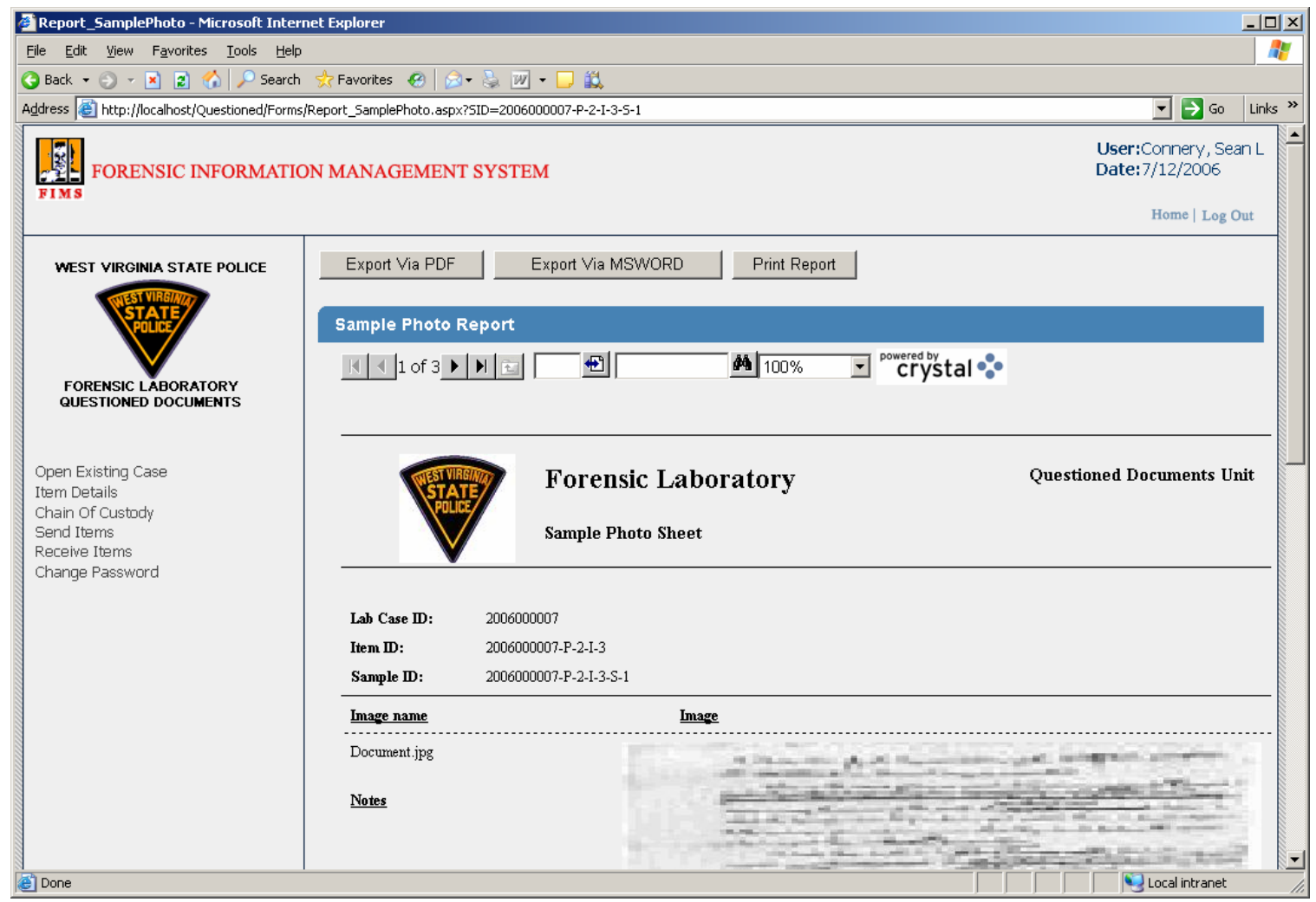

Figure 12.22 Item Photo Report 


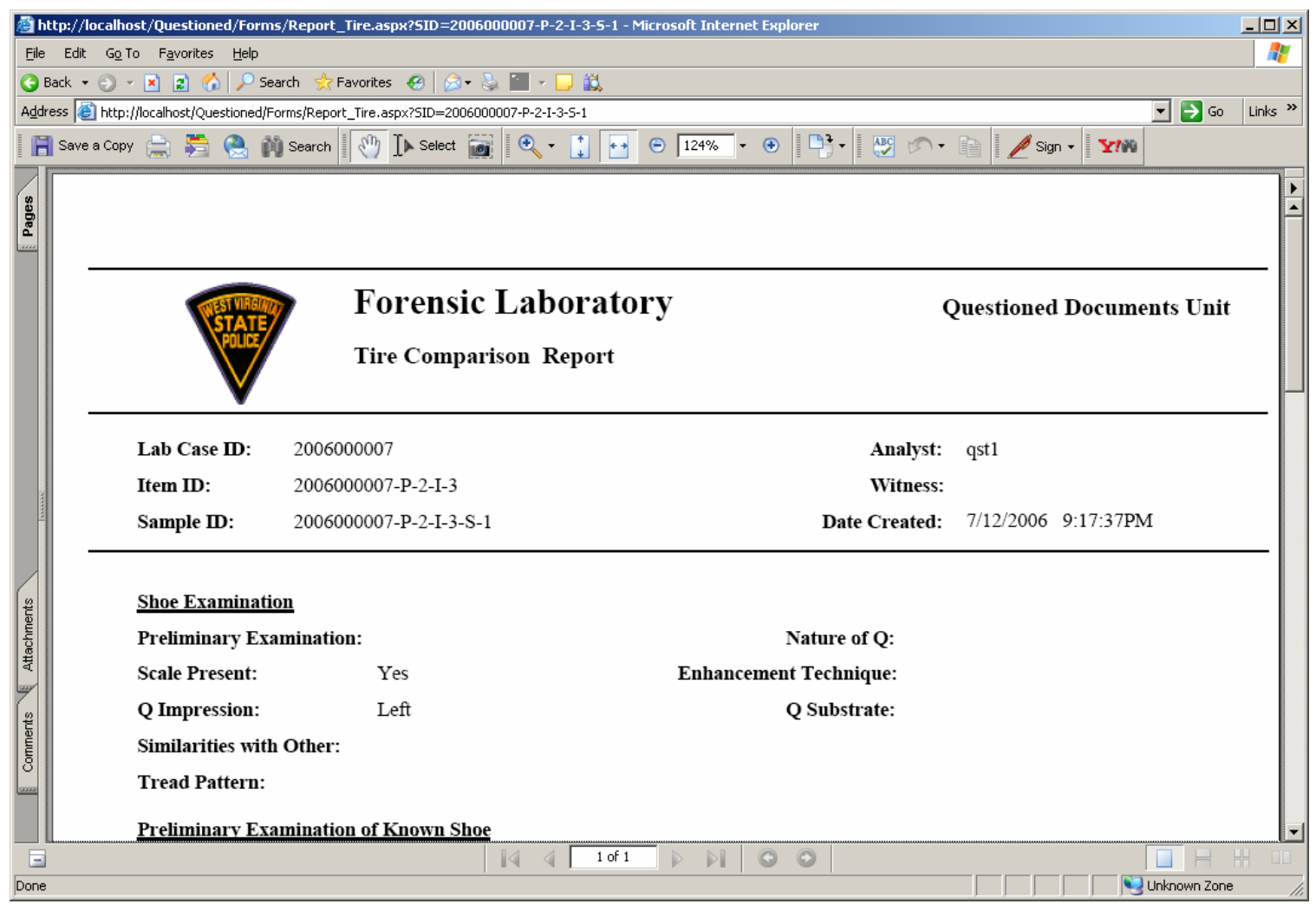

Figure 12.23: Item report in PDF Format 


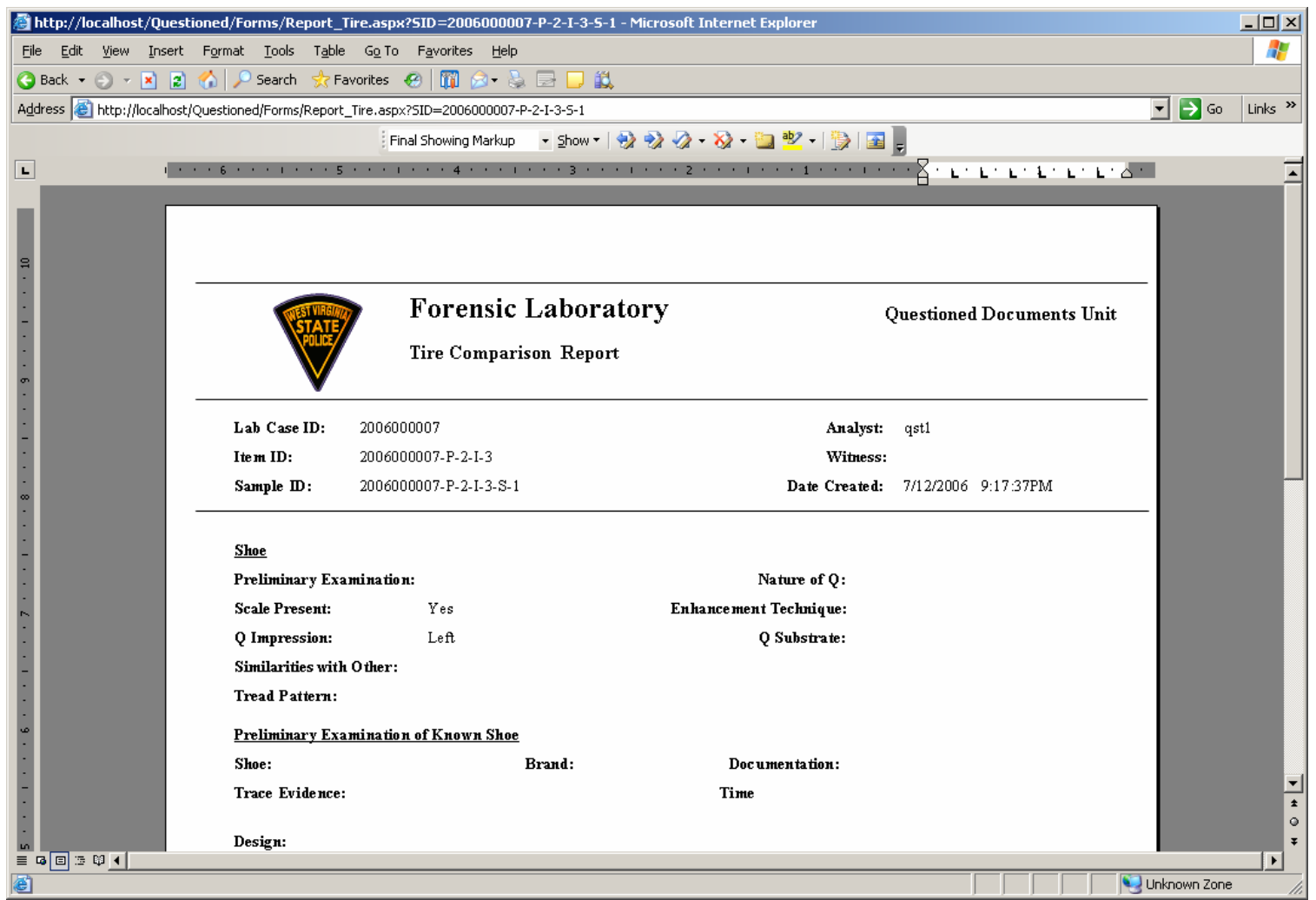

Figure 12.24: Item report in Item Format 


\section{CHAPTER 13 \\ CONCLUSIONS AND FUTURE WORK}

\subsection{Conclusions}

The focus of this research was on the design and implementation of a web based forensics information management system, FIMS 3.0 for the WVSPFL. The existing processes were examined and streamlined to develop FIMS 3.0. The software was implemented with ASP.NET (.NET Framework 1.1) as the front end and MS SQL Server 2000 as the database. The system can be accessed online from any Internet browser.

The new system of submitting cases, maintaining chain of custody, and filling appropriate test worksheets online for each unit in the laboratory provides the functionality and capability that did not exist earlier. It allows the law enforcement agencies to check the status of a case and to share data with other law enforcement agencies. Separate modules were developed for all the functional units in the forensics laboratory. A module for case submission by the ORI agencies was also developed. The system replicates the existing paper based forms and heads in the direction of making the lab operations a paperless facility. It provides information across functional levels, establishing easy communication, and improving process performance.

FIMS 3.0 software is expected to:

- $\quad$ Reduce the handwritten paperwork

- Improve data reliability.

- $\quad$ Provide a uniform approach to case reporting.

- $\quad$ Reduce case backlog.

- Eliminate duplication of work.

- Generate case statistics 
The key capabilities of the system are:

- Accessible from anywhere on the web

- Enhanced communication between the law enforcement agencies and the forensics laboratory

- Check the status of the cases submitted to the laboratory

- Easy printing of the reports and worksheets

- Maintains chain of custody records

- Store Images/Pictures in the database

\subsection{Future Work}

FIMS 30 lays a foundation upon which additional capabilities could be developed. Some of the items for future work (in arbitrary order) are listed below:

- Deployment of FIMS at ORI agencies

- Bar code printing and scanning of case numbers and item numbers

- Use of FIMS for onsite collection and cataloging of crime data

- Development of a module to store and retrieve video clips

- Development of a direct link between FIMS and other crime databases

- Development of a direct link between FIMS and various hardware and software used by the analysts in the laboratory and at the crime scene

- Design and implementation of a "Management" module to keep track of work load and training needs of the laboratory staff

- Design and implementation of a module to compute case statistics (cases submitted, cases pending, cases solved, cases unsolved, etc.)

- Development of item testing section in central processing unit.

- Deployment of FIMS on a hand held device 


\section{REFERENCES}

1. Froede, Richard C., Handbook of Forensic Pathology, College of American Pathologists, second edition, College of American Pathologists, Northfield, IL, CRC Press, 2003.

2. Roberti, J., "Sensation Seeking Characteristics and Neuroendocrine Responses to an Acute Psychological Challenge in Latent Forensic Identifiers", Dissertation, Department of Philosophy, West Virginia University, Morgantown, WV, 2002.

3. Saferstein, R., Criminalistics - An introduction to Forensic Science, $2^{\text {nd }}$ edition, Prentice Hall, 1981.

4. Langemo, M., "Records management/word processing - a needed team effort", Records Management Quarterly, vol. 14, no. 4, pp. 10-14, 1980.

5. A Guide for Project M\&E - Managing for Impact in Rural Development, International Fund for Agricultural development, Annex A, 2002.

6. Adekeye A., The Importance of Management Information Systems, Library Review, vol. 46, num. 5, pp. 318-327, 1997.

7. Schwiesow, D., "Team Management: New Web-based management systems can dramatically improve communication”, Engineering Inc., pp. 3, October 2004.

8. Pearson, M., Paynter, J., "An analysis of www-based Information Systems", Multimedia Information Systems in Practice, edited by Chow, W. S., Springer, Singapore, 1998.

9. Takahashi, K., Liang, E., “Analysis and Design of Web-based Information Systems”, Computer Networks, vol. 29, pp. 1167-1180, September, 1997.

10. Wroblewski, L., Rantanen, E., "Web-Based Information Management: An Open Portal Interface Solution”, Human Computer Interaction International Conference, 2001.

11. Strauch, B., Winter, R., "Towards a Methodology for the Development of Web-Based Systems”, Managing Business with Electronic Commerce, Idea Group Publishing, pp. 37 - 58, 2002.

12. Remington, J., "Managing your Data in a Web-based Paperless Environment", White Paper, Saber Logic, May 2003.

13. AGILE: INFOTECH - Information Sharing between Law Enforcement Agencies, Website of National Institute of Justice Solicitation, Web address: http://www.nlwctc.org/agile/research.dev

14. Srinivasan A., Forensic Information Management System, Master's Thesis, Department of Industrial and Management Systems Engineering, West Virginia University, Morgantown, WV, May 2004. 
15. N-Tier Design and the National Tech Recruiters Application Architecture Overview http://www.setfocus.com/train/NTRProject/nTierArchitectureOverview.aspx

16. Govindarajulu S , A Web Based Forensic Information Management System, Master’s Thesis, Department of Industrial and Management Systems Engineering, West Virginia University, Morgantown, WV, May 2005. 